Nevada

DOE/NV--703

Environmental

Restoration

Project

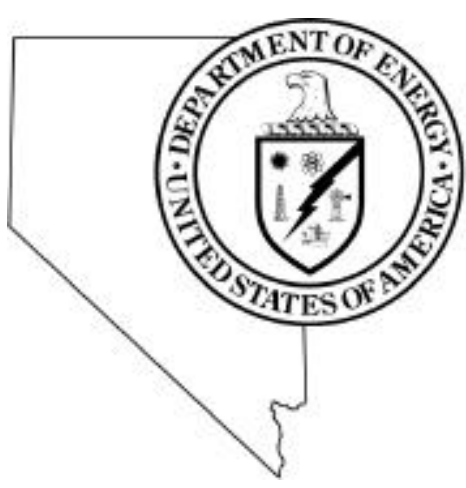

Hum an Health and Ecological R is k

Assessment W ork Plan

M ud Pit Release Sites

A mchitka Island, Alaska

Controlled Copy $\mathrm{N} 0$ ::

Revision N 0.: 0

$M \operatorname{arch} 2001$

Approved for public release; further dissemination unlimited.

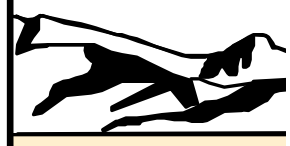

Environm ental Restoration

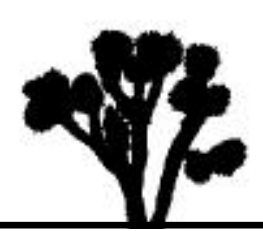

Division 
Available for public sale, in paper, from:

U.S. Department of Commerce

National Technology Information Service

5285 Port Royal Road

Springfield, VA 22161

Phone: 800.553 .6847

Fax: 703.605.6900

Email: orders@ntis.fedworld.gov

Online ordering: http//www.ntis.gov/ordering.htm

Available electronically at http://www.doe.gov/bridge.

Available for a processing fee to U.S. Department of Energy and its contractors, in paper, from:

\section{U.S. Department of Energy}

Office of Scientific and Technical Information

P.O. Box 62

Oak Ridge, TN 37831-0062

Phone: 865.576.8401

Fax: 865.576.5728

Email: reports@adonis.osti.gov

Reference herein to any specific commercial product, process, or service by trade name, trademark, manufacturer, or otherwise, does not necessarily constitute or imply its endorsement, recommendation, or favoring by the United States Government or any agency thereof or its contractors or subcontractors. 


\section{HUMAN HEALTH AND ECOLOGICAL RISK ASSESSMENT WORK PLAN MUD PIT RELEASE SITES AMCHITKA ISLAND, ALASKA}

DOE Nevada Operations Office

Las Vegas, Nevada

Controlled Copy No.:

Revision No.: 0

March 2001

Approved for public release; further dissemination unlimited. 


\title{
HUMAN HEALTH AND ECOLOGICAL RISK ASSESSMENT WORK PLAN MUD PIT RELEASE SITES AMCHITKA ISLAND, ALASKA
}

\author{
Approved by: $\quad$ Signature Approved $\quad$ Date: 3/12/2001 \\ Monica Sanchez, Offsites Project Manager \\ Environmental Restoration Division
}

Approved by: $\quad$ Signature Approved Date: 3/12/2001

Runore C. Wycoff, Division Director

Environmental Restoration Division 


\section{Table of Contents}

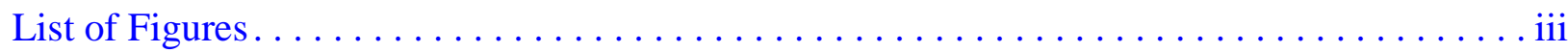

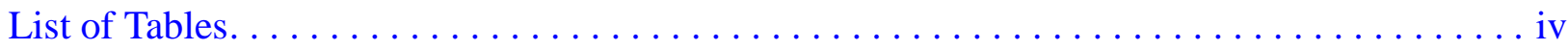

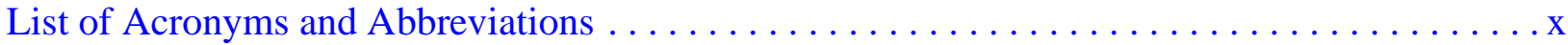

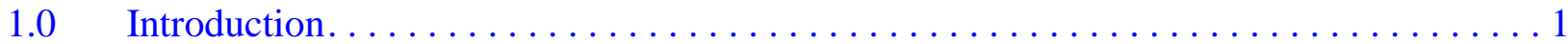

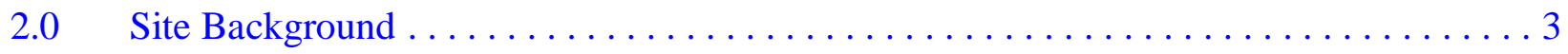

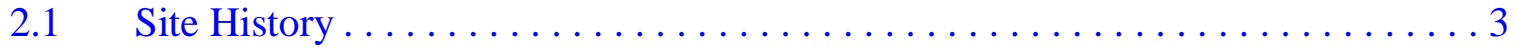

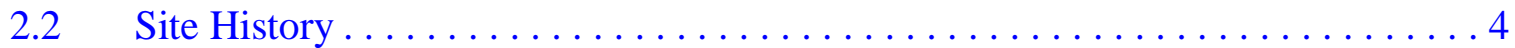

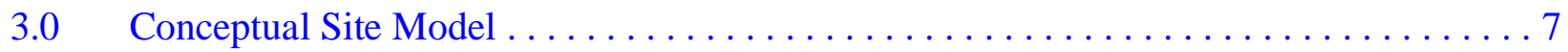

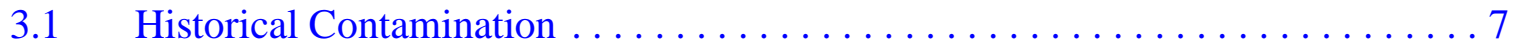

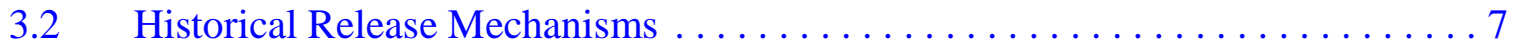

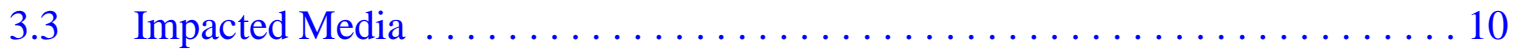

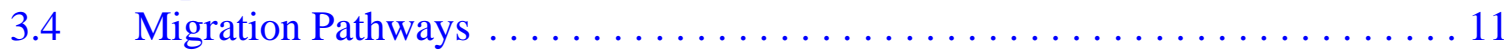

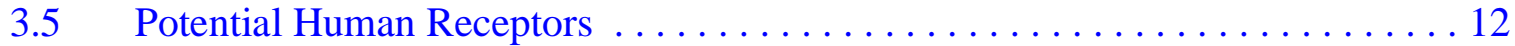

3.6 Potential Human Health Exposure Routes . . . . . . . . . . . . . . . . 13

3.7 Potential Ecological Exposure Routes . . . . . . . . . . . . . . . 13

3.8 Potential Ecological Receptors and Assessment End Points . . . . . . . . . . 14

$4.0 \quad$ Human Health Risk Assessment. . . . . . . . . . . . . . . . . . . . . . . . . . . . 18

4.1 Identification of Constituents of Concern . . . . . . . . . . . . . 18

$4.2 \quad$ Exposure Assessment . . . . . . . . . . . . . . . . . . . . 19

4.2.1 Potentially Exposed Populations . . . . . . . . . . . . . . . . 19

4.2.2 Identification of Exposure Pathways . . . . . . . . . . . . . 111

4.2.3 Exposure Pathway Dosage Estimates. . . . . . . . . . . . . . 113

4.2.3.1 Exposure Point Concentrations ............. 113

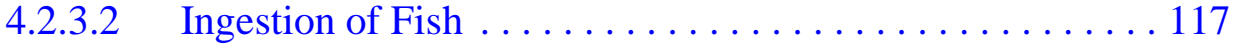

4.2.3.3 Ingestion of Surface Water . . . . . . . . . . . 118

4.2.3.4 Direct Contact With Surface Water . . . . . . . . . . . . . 118

4.2.3.5 Ingestion of Sediment . . . . . . . . . . . . . . . 119

4.2.3.6 Direct Contact With Sediment . . . . . . . . . . . . . 119

4.3 Toxicity Assessment................................. 120

4.3.1 Toxicity Assessment for Carcinogenic Effects . . . . . . . . . . . . 121

4.3.2 Toxicity Assessment of Noncarcinogenic Effects . . . . . . . . . . . . 122

4.3.3 Derivation of Dermal Toxicity Values.................. 123

$4.4 \quad$ Risk Characterization . . . . . . . . . . . . . . . . . . . . . . . . 124

4.4.1 Characterization of Carcinogenic Effects. . . . . . . . . . . . . . . 124

4.4.2 Characterization of Noncarcinogenic Effects. . . . . . . . . . . . 125 


\section{Table of Contents (Continued)}

\subsubsection{Interpretation of Calculated Cancer Risk and Noncancer}

Hazard Quotients . . . . . . . . . . . . . . . . . . . . . 127

4.5 Derivation of Human Health Risk-Based Cleanup Levels . . . . . . . . . . . . . 127

4.5.1 Sediment HHRBCLs . . . . . . . . . . . . . . . . . . . 127

4.5.2 Surface Water HHRBCLs . . . . . . . . . . . . . . . . . . . 128

4.5.3 Fish Tissue Target Levels. . . . . . . . . . . . . . . . . . . . . . . . . 129

4.6 Uncertainty Analysis. . . . . . . . . . . . . . . . . . 130

$5.0 \quad$ Ecological Risk Assessment . . . . . . . . . . . . . . . . . . . . 131

$5.1 \quad$ Ecological Screening for Compounds of Potential Concern . . . . . . . . . 131

5.1.1 Approach to Screening for Compounds of Potential Concern . . . . . . . 131

5.1.2 Identification of Compounds of Potential Ecological Concern . . . . . 141

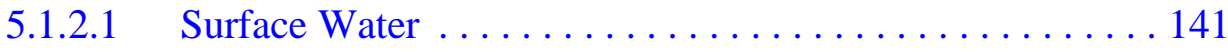

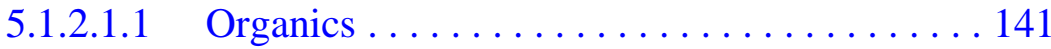

5.1.2.1.2 Inorganics $\ldots \ldots \ldots \ldots \ldots \ldots \ldots \ldots \ldots \ldots \ldots \ldots$

5.1.2.2 Sediment ......................... . 239

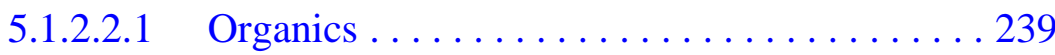

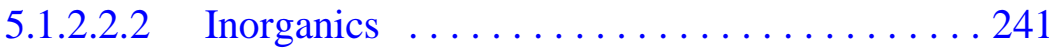

5.2 Problem Formulation and Preliminary Ecological Effects Evaluation. . . . . . . 243

5.3 Measurement End Points and Assessment Methods. . . . . . . . . . . . . . 244

5.3.1 Benthic Invertebrate Communities. . . . . . . . . . . . . . . . . . . . . 244

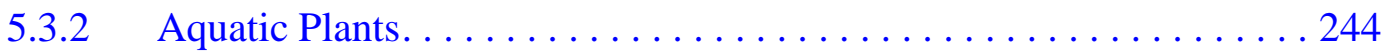

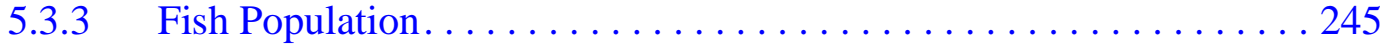

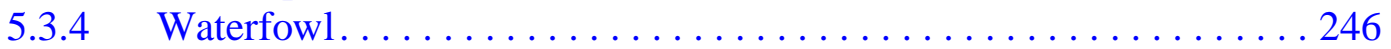

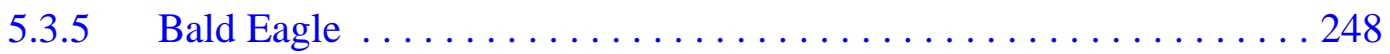

5.4 Uncertainty Analysis. . . . . . . . . . . . . . . . . . . . . . . . 249

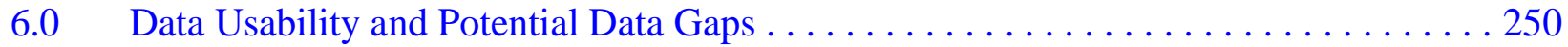

$6.1 \quad$ Data Gaps . . . . . . . . . . . . . . . . . . . . . . . 250

6.2 Detection Limits Above Ecological Screening Values. . . . . . . . . . . . . 256

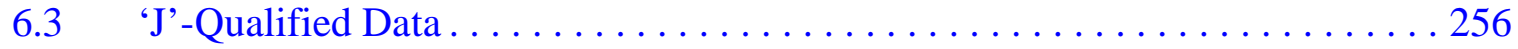

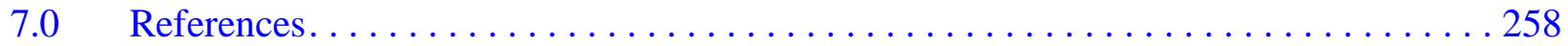




\section{List of Figures}

Number

2-1 Drill Site and Stream Locations Amchitka Island, Alaska . . . . . . . . . . . . . 5

3-1 Human Health Conceptual Site Model

Mud Pit Release Sites, Amchitka Island, Alaska . . . . . . . . . . . . . . 8

3-2 Ecological Conceptual Site Model

Mud Pit Release Sites, Amchitka Island, Alaska . . . . . . . . . . . . . . . 9 


\section{List of Tables}

Number

Title

Page

4-1 Summary of Analytical Results and Human Health Risk Assessment

Screening for Surface Water in Falls Creek, Drill Site D . . . . . . . . . . . 20

4-2 Summary of Analytical Results and Human Health Risk Assessment Screening for Sediment in Falls Creek, Drill Site D . . . . . . . . . . . . . 23

4-3 Summary of Analytical Results and Human Health Risk Assessment

Screening for Fish Tissue in Falls Creek, Drill Site D . . . . . . . . . . . . 26

4-4 Summary of Analytical Results and Human Health Risk Assessment Screening for Surface Water in Lake, Drill Site D . . . . . . . . . . . . . . 28

4-5 Summary of Analytical Results and Human Health Risk Assessment Screening for Sediment in Lake, Drill Site D . . . . . . . . . . . . . . . . . 31

4-6 Summary of Analytical Results and Human Health Risk Assessment Screening for Surface Water in Drill Site E Stream ................... 34

4-7 Summary of Analytical Results and Human Health Risk Assessment Screening for Sediment in Drill Site E Stream ...................... 37

4-8 Summary of Analytical Results and Human Health Risk Assessment Screening for Fish Tissue in Drill Site E Stream .................. 40

4-9 Summary of Analytical Results and Human Health Risk Assessment Screening for Surface Water in Limpet Creek, Drill Site F . . . . . . . . . . . 42

4-10 Summary of Analytical Results and Human Health Risk Assessment Screening for Sediment in Limpet Creek, Drill Site F . . . . . . . . . . . . . . 45

4-11 Summary of Analytical Results and Human Health Risk Assessment Screening for Fish Tissue in Limpet Creek, Drill Site F. . . . . . . . . . . . . . . 48

4-12 Summary of Analytical Results and Human Health Risk Assessment Screening for Surface Water in Bridge Creek, Long Shot Site............ 50

4-13 Summary of Analytical Results and Human Health Risk Assessment Screening for Sediment in Bridge Creek, Long Shot Site. . . . . . . . . . . . 53 


\section{List of Tables (Continued)}

Number

Title

Page

4-14 Summary of Analytical Results and Human Health Risk Assessment Screening for Fish Tissue in Bridge Creek, Long Shot Site. . . . . . . . . . . 56

4-15 Summary of Analytical Results and Human Health Risk Assessment Screening for Surface Water in Reed Pond, Long Shot Site . . . . . . . . . 58

4-16 Summary of Analytical Results and Human Health Risk Assessment Screening for Sediment in Reed Pond, Long Shot Site . . . . . . . . . . . . 61

4-17 Summary of Analytical Results and Human Health Risk Assessment Screening for Surface Water in Cloudberry Creek, Long Shot Site. . . . . . . . . 64

4-18 Summary of Analytical Results and Human Health Risk Assessment Screening for Sediment in Cloudberry Creek, Long Shot Site ........... 67

4-19 Summary of Analytical Results and Human Health Risk Assessment Screening for Fish Tissue in Cloudberry Creek, Long Shot Site.......... . 70

4-20 Summary of Analytical Results and Human Health Risk Assessment Screening for Surface Water in Rainbow Creek, Long Shot Site .......... 73

4-21 Summary of Analytical Results and Human Health Risk Assessment Screening for Sediment in Rainbow Creek, Long Shot Site . . . . . . . . . . 76

4-22 Summary of Analytical Results and Human Health Risk Assessment Screening for Fish Tissue in Rainbow Creek, Long Shot Site. . . . . . . . . . . 79

4-23 Summary of Analytical Results and Human Health Risk Assessment Screening for Surface Water in Clevenger Creek, Milrow Site............ 81

4-24 Summary of Analytical Results and Human Health Risk Assessment Screening for Sediment in Clevenger Creek, Milrow Site. . . . . . . . . . . . 84

4-25 Summary of Analytical Results and Human Health Risk Assessment Screening for Fish Tissue in Clevenger Creek, Milrow Site . . . . . . . . . . 87

4-26 Summary of Analytical Results and Human Health Risk Assessment Screening for Surface Water in Heart Lake, Milrow Site . . . . . . . . . . . . 89 


\section{List of Tables (Continued)}

Number

Title

Page

4-27 Summary of Analytical Results and Human Health Risk Assessment Screening for Sediment in Heart Lake, Milrow Site . . . . . . . . . . . . . . . 92

4-28 Summary of Analytical Results and Human Health Risk Assessment Screening for Surface Water in Cannikin Lake .................... 95

4-29 Summary of Analytical Results and Human Health Risk Assessment Screening for Sediment in Cannikin Lake . . . . . . . . . . . . . . . . 98

4-30 Summary of Analytical Results and Human Health Risk Assessment Screening for Fish Tissue in Cannikin Lake .................... 101

4-31 Summary of Analytical Results and Human Health Risk Assessment Screening for Surface Water in White Alice Creek ................. 103

4-32 Summary of Analytical Results and Human Health Risk Assessment Screening for Sediment in White Alice Creek . . . . . . . . . . . . . . . . 106

4-33 Summary of Analytical Results and Human Health Risk Assessment Screening for Fish Tissue in White Alice Creek . . . . . . . . . . . . . . . . 109

4-34 Summary of Preliminary Constituents of Concern $\ldots \ldots \ldots \ldots \ldots \ldots \ldots 11$

4-35 Parameters for On-Site USFWS Worker Exposure Pathways . . . . . . . . . . 114

4-36 Parameters for Part-Time Subsistence User Exposure Pathways. . . . . . . . . 115

5-1 Ecological Screening Values for Surface Water ................. 133

5-2 Ecological Screening Values for Sediment $\ldots \ldots \ldots \ldots \ldots \ldots \ldots \ldots \ldots$

5-3 Summary of Analytical Results and Ecological Risk Assessment Screening for Surface Water in Falls Creek, Drill Site D . . . . . . . . . . . . 142

5-4 Summary of Analytical Results and Ecological Risk Assessment Screening for Sediment in Falls Creek, Drill Site D . . . . . . . . . . . . . 145 


\section{List of Tables (Continued)}

Number

Title

Page

5-5 Summary of Analytical Results and Ecological Risk Assessment

Screening for Surface Water in Drill Site D Lake . . . . . . . . . . . . . . 148

5-6 Summary of Analytical Results and Ecological Risk Assessment

Screening for Sediment in Drill Site D Lake . . . . . . . . . . . . . . . 151

5-7 Summary of Analytical Results and Ecological Risk Assessment

Screening for Surface Water in Drill Site E Stream. . . . . . . . . . . . . 154

5-8 Summary of Analytical Results and Ecological Risk Assessment

Screening for Sediment in Drill Site E Stream. . . . . . . . . . . . . . . 157

5-9 Summary of Analytical Results and Ecological Risk Assessment

Screening for Surface Water in Limpet Creek, Drill Site F . . . . . . . . . . . 160

5-10 Summary of Analytical Results and Ecological Risk Assessment

Screening for Sediment in Limpet Creek, Drill Site F. . . . . . . . . . . . 163

5-11 Summary of Analytical Results and Ecological Risk Assessment

Screening for Surface Water in Bridge Creek, Long Shot Site . . . . . . . . . 166

5-12 Summary of Analytical Results and Ecological Risk Assessment

Screening for Sediment in Bridge Creek, Long Shot Site . . . . . . . . . . . . . 169

5-13 Summary of Analytical Results and Ecological Risk Assessment

Screening for Surface Water in Reed Pond, Long Shot Site . . . . . . . . . . 172

5-14 Summary of Analytical Results and Ecological Risk Assessment

Screening for Sediment in Reed Pond, Long Shot Site . . . . . . . . . . . . 175

5-15 Summary of Analytical Results and Ecological Risk Assessment

Screening for Surface Water in Cloudberry Creek, Long Shot Site. . . . . . . 178

5-16 Summary of Analytical Results and Ecological Risk Assessment Screening for Sediment in Cloudberry Creek, Long Shot Site $\ldots \ldots \ldots \ldots .181$

5-17 Summary of Analytical Results and Ecological Risk Assessment Screening for Surface Water in Rainbow Creek, Long Shot Site . . . . . . . 184 


\section{List of Tables (Continued)}

Number

Title

Page

5-18 Summary of Analytical Results and Ecological Risk Assessment Screening for Sediment in Rainbow Creek, Long Shot Site . . . . . . . . . . 187

5-19 Summary of Analytical Results and Ecological Risk Assessment Screening for Surface Water in Clevenger Creek, Milrow Site . . . . . . . . . 190

5-20 Summary of Analytical Results and Ecological Risk Assessment Screening for Sediment in Clevenger Creek, Milrow Site. . . . . . . . . . . 193

5-21 Summary of Analytical Results and Ecological Risk Assessment Screening for Surface Water in Heart Lake, Milrow Site . . . . . . . . . . . . 196

5-22 Summary of Analytical Results and Ecological Risk Assessment Screening for Sediment in Heart Lake, Milrow Site . . . . . . . . . . . . . . . 199

5-23 Summary of Analytical Results and Ecological Risk Assessment Screening for Surface Water in Cannikin Lake . . . . . . . . . . . . . . . . 202

5-24 Summary of Analytical Results and Ecological Risk Assessment Screening for Sediment in Cannikin Lake . . . . . . . . . . . . . . . . . . 205

5-25 Summary of Analytical Results and Ecological Risk Assessment Screening for Surface Water in White Alice Creek, Cannikin Site . . . . . . . 208

5-26 Summary of Analytical Results and Ecological Risk Assessment Screening for Sediment in White Alice Creek, Cannikin Site . . . . . . . . . 211

5-27 Summary of Analytical Results and Ecological Risk Assessment Screening for Surface Water in Reference Stream No. 1............... 214

5-28 Summary of Analytical Results and Ecological Risk Assessment Screening for Sediment in Reference Stream No. 1. . . . . . . . . . . . 217

5-29 Summary of Analytical Results and Ecological Risk Assessment Screening for Surface Water in Reference Stream No. 2 . . . . . . . . . . 220

5-30 Summary of Analytical Results and Ecological Risk Assessment Screening for Sediment in Reference Stream No. 2. . . . . . . . . . . 223 


\section{List of Tables (Continued)}

Number

Title

Page

5-31 Summary of Analytical Results and Ecological Risk Assessment

Screening for Surface Water in Reference Stream No. 3 . . . . . . . . . 226

5-32 Summary of Analytical Results and Ecological Risk Assessment Screening for Sediment in Reference Stream No. 3 . . . . . . . . . . . . . . 229

5-33 Summary of Analytical Results and Ecological Risk Assessment Screening for Surface Water in Reference Stream No. 4............ 232

5-34 Summary of Analytical Results and Ecological Risk Assessment Screening for Sediment in Reference Stream No. 4. . . . . . . . . . . . 235

6-1 Summary of Available Data......................... 251

6-2 Complete Exposure Routes for Ecological Receptors . . . . . . . . . . . . . . 254 


\section{List of Acronyms and Abbreviations}

\begin{tabular}{|c|c|}
\hline ADEC & Alaska Department of Environmental Conservation \\
\hline $\mathrm{AEC}$ & U.S. Atomic Energy Commission \\
\hline AMNWR & Alaska Maritime Natural Wildlife Refuge \\
\hline APIA & Aleutian Pribilof Island Association \\
\hline ARCS & Assessment and Remediation of Contaminated Sediments \\
\hline ATSDR & Agency for Toxic Substances and Disease Registry \\
\hline $\mathrm{BCF}$ & Bioconcentration factor \\
\hline BTEX & Benzene, toluene, ethylbenzene, xylene \\
\hline CDI & Chronic daily intake \\
\hline CFR & Code of Federal Regulations \\
\hline $\mathrm{COC}$ & Compound of concern \\
\hline COPEC & Compound of potential ecological concern \\
\hline $\mathrm{cm}^{2}$ & Square centimeter(s) \\
\hline CSM & Conceptual site model \\
\hline DoD & U.S. Department of Defense \\
\hline DOE & U.S. Department of Energy \\
\hline DRO & Diesel-range organic \\
\hline ECAO & Environmental Criteria and Assessment Office \\
\hline EPA & U.S. Environmental Protection Agency \\
\hline $\mathrm{EqP}$ & Equilibrium partitioning \\
\hline EPT & Ephemeroptera-Plecoptera-Trichoptera \\
\hline ESG & Equilibrium partitioning sediment guideline \\
\hline ESV & Ecological screening value \\
\hline FTTL & Fish tissue target level \\
\hline GRO & Gasoline-range organic \\
\hline HEAST & Health Effects Assessment Summary Tables \\
\hline
\end{tabular}




\section{List of Acronyms and Abbreviations (Continued)}

\begin{tabular}{|c|c|}
\hline HHRBCL & Human health risk-based cleanup level \\
\hline $\mathrm{HI}$ & Hazard index \\
\hline HQ & Hazard quotient \\
\hline IRIS & Integrated Risk Information System \\
\hline $\mathrm{Kt}$ & Kiloton \\
\hline $\mathrm{LC}_{50}$ & Lethal concentration - $50 \%$ \\
\hline $\mathrm{LD}_{50}$ & Lethal dose $-50 \%$ \\
\hline LEL & Lowest effect level \\
\hline LOAEL & Lowest-observed-adverse-effect-level \\
\hline LOEL & Lowest-observable-effect-level \\
\hline $\mathrm{mg} / \mathrm{kg}$ & Milligram(s) per kilogram \\
\hline $\mathrm{mg} / \mathrm{L}$ & Milligram(s) per liter \\
\hline $\mathrm{mg} / \%$ & Milligram(s) per percent \\
\hline Mt & Megaton \\
\hline NOAEL & No-observed-adverse-effect-level \\
\hline $\mathrm{OC}$ & Organic carbon \\
\hline PAH & Polyaromatic hydrocarbon \\
\hline PCB & Polychlorinated biphenyl \\
\hline RET & Residue effects threshold \\
\hline $\mathrm{RfC}$ & Reference concentration \\
\hline RfD & Reference dose \\
\hline RME & Reasonable maximum exposure \\
\hline SF & Slope factor \\
\hline TOC & Total organic carbon \\
\hline TRV & Toxicity reference value \\
\hline UCL & Upper confidence limit \\
\hline
\end{tabular}




\section{List of Acronyms and Abbreviations (Continued)}

UF Uncertainty factor

USFWS U.S. Fish and Wildlife Service

UTL Upper tolerance limit

VOC Volatile organic compound(s)

Yr Year(s)

$\mu \mathrm{g} / \mathrm{L} \quad$ Microgram(s) per liter 


\subsection{Introduction}

The U.S. Atomic Energy Commission (AEC), the predecessor of the U.S. Department of Energy (DOE), utilized Amchitka Island as an underground nuclear test site between 1965 and 1971. Deep drilling operations required large volumes of drilling mud, which is a mixture of bentonite, diesel fuel, and other compounds including chrome lignosulfate. A considerable amount of this material was left on the island in exposed mud pits after testing was completed. During the spring and summer of 2001, DOE will close and cap the remaining drilling mud pits in place by constructing a geosynthetic cap on each mud pit. Several stream, lake, and pond areas containing historic releases of drilling mud are proposed for no further action, providing that these sites do not pose an unacceptable risk to human or ecological receptors. This Work Plan describes the approach that will be used to conduct human health and ecological risk assessments for these sites.

The objectives of this Work Plan are as follows:

- To describe methods based on U.S. Environmental Protection Agency (EPA) and Alaska Department of Environmental Conservation (ADEC) guidance that will be used to conduct ecological and human health risk assessments for Amchitka mud pit release sites proposed by the DOE for no further action.

- To present human health and ecological conceptual site models (CSMs) showing potentially complete exposure pathways for human and ecological receptors.

- To propose specific exposure scenarios for human and ecological receptors.

- To present the procedures that will be used to identify contaminants of potential concern for both human and ecological receptors.

- To describe the dataset that will be used in the risk assessments, to evaluate the data in terms of quality and usability, and to identify potential data gaps.

- To present the methods and equations that will be used to calculate risk-based cleanup levels for the mud pit release sites, if necessary.

A scoping meeting for the risk assessments was held in the Anchorage, Alaska, office of the ADEC on October 24, 2000. Representatives of the DOE, ADEC, U.S. Fish and Wildlife Service (USFWS), 
and the Aleutian Pribilof Island Association (APIA) agreed at this meeting that the assessment would be limited to polychlorinated biphenyls (PCB) and known drilling mud constituents. 


\subsection{Site Background}

This section presents the geographic and ecological setting of Amchitka, and describes the activities that resulted in the present need for drilling mud pit remediation and risk assessment of historical mud pit releases.

\subsection{Site History}

Amchitka Island was inhabited by Aleuts for thousands of years before the island was first sighted by Europeans in the mid-eighteenth century. Russian promyshlenniki (hunters) exploited the Aleuts throughout the Aleutians, and the Aleut population was reduced from at least 12,000 to about 4,000 by the late 1830s (Merritt, 1977). Alaska, including the Aleutians, was purchased from Russia by the United States in 1867, and the Aleutian Islands, including Amchitka, were set aside as a wildlife preserve in 1913. The Aleuts continued to occupy and utilize Amchitka's resources through this period. Jurisdiction was transferred to the U.S. Department of the Interior in 1940, and the system was renamed the Aleutian Island National Wildlife Refuge.

Soon after the establishment of the Aleutian Island preserve, permits were issued so that individual islands could be used for fox farming. In 1920, a permit was issued to "Native residents of Atka" to farm foxes on Amchitka, and in 1921 seven blue foxes were released on the island (Merritt, 1977). The fox farmers were transients and did not reside permanently on Amchitka. Amchitka's rich avifauna provided a rich food source and the foxes multiplied rapidly, and in less than 30 years over 4,000 foxes were harvested. The foxes had a severe impact on many bird populations, and the Aleutian Canada Goose was nearly extirpated. Following collapse of the fox farming trade, the foxes were removed from Amchitka and other Aleutian islands to allow the recovery of impacted bird populations.

The regulations governing use of refuge lands provided that human occupation is permissible when necessary for national defense. Subsequent to the Japanese occupation of Kiska Island, 69 miles to the west, the U.S. Army established a forward air base on Amchitka in 1943. Since World War II, Amchitka has been used by the U.S. Department of Defense (DoD) and the AEC (now the DOE). Amchitka was selected as a nuclear test site because of its location and remoteness, and because of 
limitations that the proximity of the City of Las Vegas placed on testing large-yield devices at the Nevada Test Site.

Between 1965 and 1971, the AEC conducted two underground nuclear tests and assisted the DoD with a third test. The first test, known as Long Shot was approximately an 80-kiloton (kt) detonation at 2,350 feet underground. The purpose of this test was to determine if underground nuclear tests could be differentiated from earthquakes by seismic instruments. Two weapons-related tests were also conducted. The Milrow test was a detonation of about 1 megaton (Mt) at a depth of 4,000 feet, and was a "calibration shot" to evaluate the feasibility of conducting higher yield tests at Amchitka. The largest yield test was Cannikin. This was a detonation of less than $5 \mathrm{Mt}$ at a depth of 5,875 feet below the surface.

In addition to the three tests, emplacement holes were drilled at Sites D and F, and an exploratory hole was drilled at Site E (Figure 2-1). Most of the land disturbed by the AEC was graded, contoured, and seeded (Merritt, 1977). However, drilling mud spills occurred during the testing program and some exposed mud pits were left in place. Drilling mud may have subsequently entered nearby streams and ponds.

In 1980, the Alaska National Interest Lands Conservation Act was enacted into law and the former Aleutian National Wildlife Refuge became a subunit of the larger Alaska Maritime National Wildlife Refuge (AMNWR). Amchitka Island is presently closed to visitation except by necessary military personnel, USFWS staff engaged in management or monitoring activities, and contractors working under government contracts.

\subsection{Site History}

Amchitka Island is located 1,340 miles west-southwest of Anchorage in the western end of the Aleutian Island archipelago, in a group known as the Rat Islands. Amchitka is approximately 42 miles long and is quite narrow, ranging from about one to four miles in width. The eastern third of the island is relatively flat and is comprised of rolling terrain heavily vegetated with maritime tundra, and dotted with numerous shallow ponds. The terrain gradually becomes more rugged in the central portions of island, the tundra grasses are less dense, and there are numerous wind-eroded bare areas or "blowouts." The northwestern third of Amchitka is mountainous with elevations exceeding 


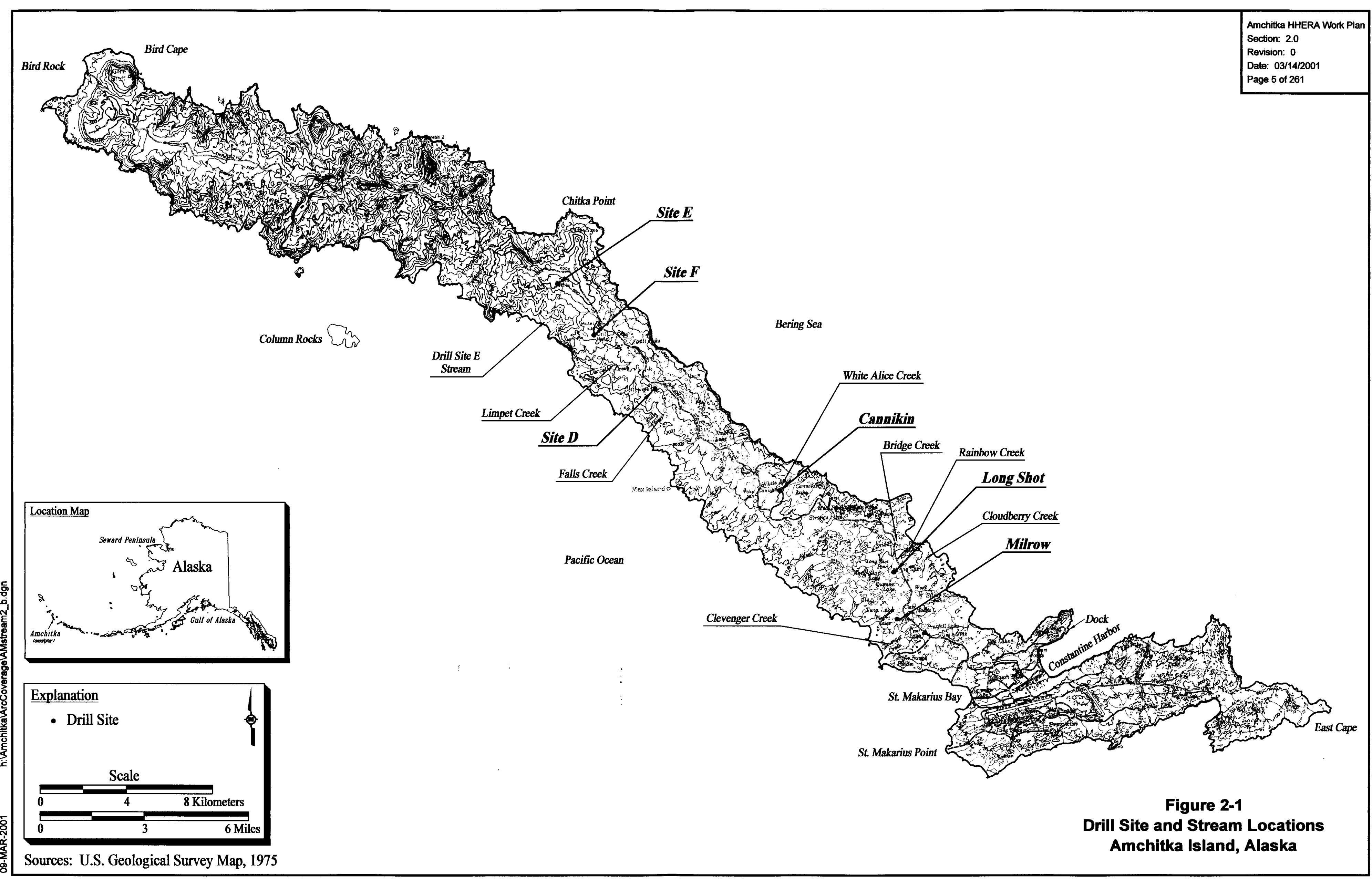


1,100 feet, with vegetation limited to areas protected from the nearly constant strong winds. The sites that will be addressed in this risk assessment are located in the eastern and central portions of the island (Figure 2-1). Most of the streams draining Amchitka originate at the island's spine and flow a short distance more or less directly to the Bering Sea to the north, or to the North Pacific Ocean to the south. Therefore, the streams are short, less than 2 miles in length, and since the watersheds they drain are small, the streams are also quite narrow. 


\subsection{Conceptual Site Model}

One of the first steps in the risk assessment process is the development of the CSMs for both the human health and ecological risk assessment process. The CSMs are diagrams or flow charts which are used to identify potential contaminant transport pathways and potential exposure routes for human health and ecological receptors. These CSMs show both actual and potential (current and future) exposure pathways. The CSMs describe the historical contamination sources and historical or potential release mechanisms from those sources, the media impacted due to releases, migration pathways and media transfers, exposure routes, and potential receptors. In the case of the ecological CSM, the assessment end points used in the risk assessment are also identified through the CSM. The following sections describe the elements of the CSMs. They are presented graphically in Figures 3-1 and 3-2.

\subsection{Historical Contamination}

Drilling muds were historically used in drilling the emplacement and/or exploratory holes at the three test sites (Milrow, Long Shot, and Cannikin) and the three drill sites (D, E, and F) (Appendix A). The boreholes were drilled using methods that employed drilling mud, some of which may have contained additives such as diesel fuel, chrome lignosulfonate, and chrome lignite to control viscosity and mitigate loss of drilling mud in the boreholes. The drilling mud was commonly stored near the drill sites in bermed pits. The walls of these mud pits were prone to leakage or outright failure. Many of the mud pits used during drilling were backfilled following use. These mud pits represent historical contaminant sources to the streams and lakes of Amchitka Island. Additionally, Milepost 13 Lake, Milepost 14 Lake, and the Rifle Range Road Mud Pits located south of the Milrow test site were reported to have been used for storing drilling mud.

\subsection{Historical Release Mechanisms}

Contaminants present in the drilling mud pits or used during the drilling operations may have been historically released to the environment and into the freshwater drainages that exist at each of these sites (Valdez et al., 1977; Fuller and Kirkwood, 1977; Shannon \& Wilson, 1999). 


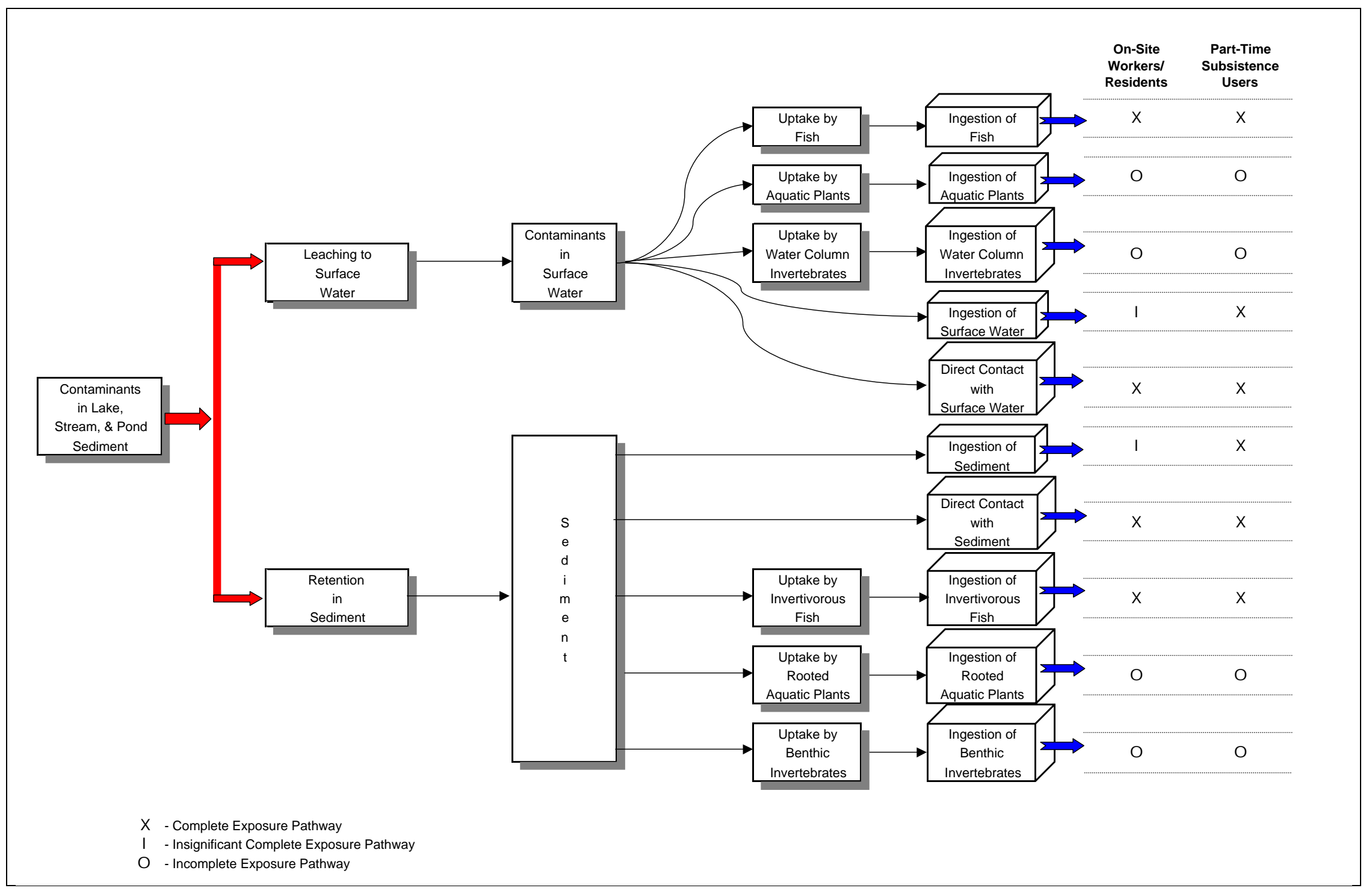

Figure 3-1

Human Health Conceptual Site Model

Mud Pit Release Sites, Amchitka Island, Alaska 


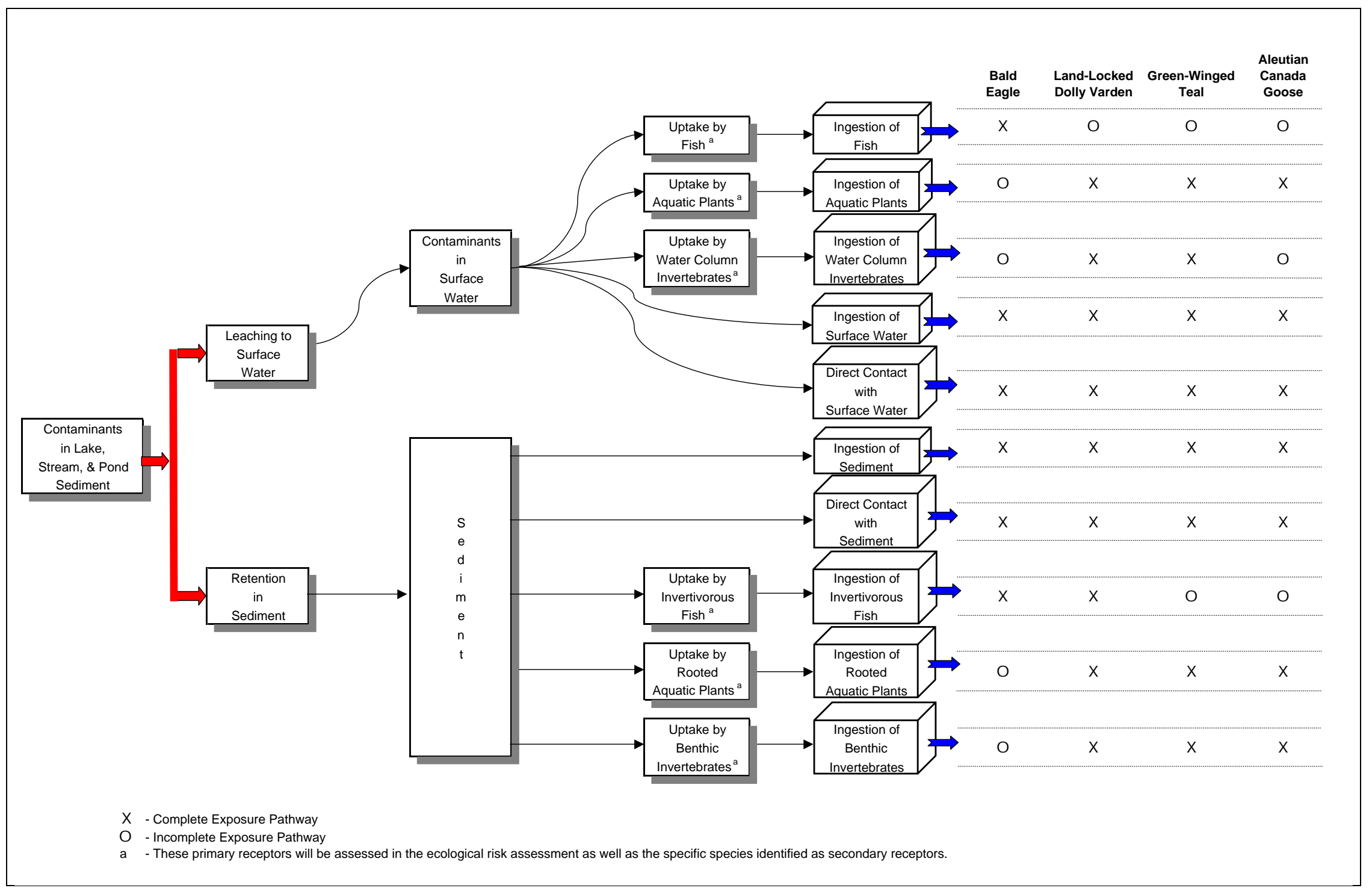

Figure 3-2

Ecological Conceptual Site Model Mud Pit Release Sites, Amchitka Island, Alaska 
Drill Site D used three large drilling mud pits. Drilling muds were also mixed at Drill Site D and piped to other drill sites as well as being used for drilling at that site. During the Milrow test, drilling mud may have splashed out of the holding ponds. It was reported that pond walls cracked and failed during the Cannikin test. Additionally, intentional releases may have resulted in releases to the freshwater drainage in the area.

Drill Site E contained one drilling mud pit near the drilling pad, and two smaller mud pits to the north and south. The Drill Site E mud pit was constructed on unstable soil, and the mud pit berm failed and drilling mud was released into the nearby stream in 1968.

Drill Site F originally contained four drilling mud pits. Mud from the sumps was reported to have escaped and resulted in releases to the Limpet Creek drainage.

Four side-by-side mud pits are present at the Long Shot test site. These mud pits were excavated into native soil in a low lying area that is drained by a shallow trench. Surface water drainage from the mud pits may have resulted in contaminant releases to streams and ponds in the area.

The Milrow test site contained four mud pits and also utilized the Rifle Range Road mud pits for drilling mud storage. Several small losses and large spills were reported to have occurred in 1968 and 1969 which released contaminants into nearby Clevenger Creek.

The Cannikin test site used four or five mud pits. Releases into White Alice Creek are reported to have occurred from 1968 to 1971 . Intentional releases occurred via drain pipes installed in mud pit walls and from trenches cut through the walls (Shannon \& Wilson, 1999; Valdez et al., 1977; Fuller and Kirkwood, 1977).

\subsection{Impacted Media}

Historical releases from the mud pits at the test sites and drill sites may have resulted in contamination of surface water and sediment in the surface water drainages from the sites. These include the following surface water bodies:

- Heart Lake

- Clevenger Creek

- Bridge Creek 
- Rainbow Creek

- Cloudberry Creek

- Reed Pond

- White Alice Creek

- Cannikin Lake

- Unnamed Lake at Site D

- Falls Creek

- Limpet Creek

- $\quad$ Site E Stream

\subsection{Migration Pathways}

Potential migration mechanisms for contaminants in impacted media include surface water and sediment transport, sediment deposition, adsorption/desorption, sediment resuspension, uptake by aquatic plants, bioaccumulation into aquatic organisms, and trophic transfer via the food web.

Surface water bodies have the potential to transport contaminants downstream to other areas of the streams or off site to the marine environment. Although the potential exists for the transport of contaminants to the marine environment, contaminants are unlikely to remain in close proximity to Amchitka. The high energy environment of the coast line combined with the lack of depositional areas precludes the deposition of site-related contaminants along the shoreline. Rapid transportation from the near vicinity of the island by strong ocean currents and thorough mixing and dilution of site-related contaminants in the marine environment render their concentrations insignificant in the marine environment.

Contaminants that are water soluble are most easily transported; these are principally metals. Contaminants that strongly adsorb to sediment or organic matter may be transported as suspended solids; such transport is most prevalent in fast-flowing waters or during high-water events. Suspended particulates with adsorbed contaminants may deposit to the sediment bed in areas of low energy. Transport routes would follow the predominant surface water-flow patterns.

Fish, shellfish, or other organisms living in the surface water bodies of Amchitka Island may bioaccumulate site-related contaminants. Most inorganics are not expected to result in significant bioaccumulation. However, mercury, in particular, has a high potential for bioaccumulation and biotic transfer. Polycyclic aromatic hydrocarbons (PAH) and PCBs have high octanol-water partitioning coefficient $\left(\mathrm{K}_{\mathrm{OW}}\right)$ values and correspondingly high bioconcentration factors $(\mathrm{BCF})$. 
Polychlorinated biphenyls in particular could be biotically transferred through the food chain. Higher trophic-level organisms are unlikely to accumulate PAHs because they possess mechanisms for metabolism and excretion of PAHs and their metabolites. Certain contaminants in surface water and sediment could be expected to bioaccumulate in invertebrates and fish. Piscivorous and invertivorous organisms could then be exposed to elevated concentrations in their diet.

Aquatic plants may accumulate contaminants via uptake from surface water and sediment. Plants are more prone to accumulate metals than high molecular weight organics.

Volatilization is considered to be an insignificant migration pathway. The compounds evaluated have relatively low vapor pressures. Low molecular weight PAHs, such as phenanthrene and pyrene, if available at the air-water interface, could volatilize.

\subsection{Potential Human Receptors}

Amchitka is currently unoccupied and is part of the AMNWR. Since 1986, Amchitka has been officially closed to all visitation except for U.S. Navy and USFWS personnel and civilian contractors working for the U.S. Government. Because of its isolated location and the aforementioned restrictions, potential human receptors at Amchitka are very limited.

The only current human receptors are crewmen from passing vessels who occasionally come ashore despite official access restrictions. On-site workers such as USFWS researchers/biota samplers may also spend time on the island. It is also possible that native Aleut fishermen and/or hunters could visit the island for relatively short periods of time.

Based on these past land uses and the expected future uses of the island, there are two potentially exposed populations at Amchitka:

- USFWS workers/residents

- Part-time subsistence users

Although trespassers may spend short periods of time on the island, the duration and magnitude of their exposures is expected to be less than either the on-site workers or the subsistence users; therefore, exposures for trespassers will not be quantified in this assessment. 


\subsection{Potential Human Health Exposure Routes}

The USFWS workers and authorized researchers from other organizations may occupy Amchitka for varying periods of time. While on the island, these workers may engage in recreational fishing, including fishing in the larger ponds and lakes. These workers could potentially come in contact with site-related contaminants through ingestion of fish, direct contact with surface water, and direct contact with sediment. Incidental ingestion of surface water and sediment are also considered complete exposure pathways for USFWS workers on the island, but these exposure pathways are likely to be insignificant and will not be quantified in this assessment.

Constantine Spring is the island's main source of potable water and is likely to be used by USFWS workers who reside on Amchitka for an extended period. This is not considered a viable contaminant exposure pathway because Constantine Spring is not impacted by drill site activities. Exposures from surface soil are not considered a viable exposure pathway because soil has not been shown to be significantly impacted from drill site activities.

Aleut subsistence fishermen and hunters may occasionally harvest food on the island. Their potential exposures result from the hunting/fishing activities and also the consumption of food items that they collect from the island. The exposure pathways by which these part-time subsistence users could come in contact with site-related contaminants include: ingestion of fish, incidental ingestion of surface water, direct contact with surface water, incidental ingestion of sediment, and direct contact with sediment. As mentioned above, ingestion of groundwater from Constantine Spring is not considered a viable contaminant exposure pathway as Constantine Spring is not impacted by drill site activities. Ingestion of groundwater and exposure from surface soil is not considered a viable exposure pathway because soil has not been shown to be significantly impacted from mud pit releases.

\subsection{Potential Ecological Exposure Routes}

Ecological receptors may be exposed to contaminants in surface water via direct contact or through consumption of water. Aquatic organisms inhabiting contaminated waters could be in constant contact with contaminants. 
Exposure to contaminants in sediment may occur through dermal contact, particularly for benthic organisms and wading birds. Some aquatic organisms consume sediment and ingest organic material from the sediment. Incidental ingestion of sediments may also occur as the result of feeding on benthic organisms and plants.

Secondary exposure pathways involve compounds that are transferred through different trophic levels of the food chain and may be bioaccumulated. This may include contaminants bioaccumulated from surface water or sediment into aquatic plants, fish, and invertebrates. These plants or animals may, in turn, be consumed by animals at higher trophic levels. Thus, these contaminants may be passed through the food chain to impact organisms at higher trophic levels.

\subsection{Potential Ecological Receptors and Assessment End Points}

For possible adverse ecological effects to occur, a site must contain contaminants in abiotic media, it must provide exposure pathways linking contaminants to receptors, and it must have ecological receptors that use the site in such a way as to come in contact with contaminants at the point of exposure. Benthic macroinvertebrates may be exposed to contaminants in both sediment and surface water through direct contact and ingestion. Aquatic macrophytes may be exposed to contaminants through direct contact and via uptake through their roots. The freshwater fish species present in the streams and lakes of Amchitka Island may be the most significant ecological receptors of mud pitrelated contaminants. Fish may be continuously exposed to contaminated surface water and sediment, and their food source is entirely composed of benthic invertebrates, fish, and plants that may have bioaccumulated contaminants. Wildlife may be exposed to contaminants from the surface water and sediment through direct contact during their daily activities, ingestion of surface water, incidental ingestion of sediment, and ingestion of plants or prey which have bioaccumulated contaminants.

Through the identification of complete exposure pathways, the following receptors that represent the trophic levels associated with mud pit release sites have been chosen as the focus of the ecological risk assessment:

- Benthic macroinvertebrate communities

- Aquatic plants (Milfoil and Aquatic Mosses)

- Freshwater fish (Landlocked Dolly Varden) 
- Omnivorous birds (Green-Winged Teal)

- Herbivorous birds (Aleutian Canada Goose)

- Piscivorous birds (Bald Eagle)

Benthic macroinvertebrates are important members of aquatic food webs, and their community health is often reflected in the overall health of the higher atrophic levels that utilize the benthic invertebrates as a food source, such as fish and waterfowl. Benthic macroinvertebrates are also important for digesting organic material and recycling nutrients in aquatic systems. Benthic macroinvertebrates may be exposed directly to contaminants within sediment, usually via the interstitial or pore water. The freshwater invertebrates on Amchitka Island include four phyla: Annelida, Nematoda, Mollusca, and Arthropoda (Valdez et al., 1977). Annelids and nematodes are common in the fine pond sediments. Bivalves and gastropods (members of the phylum Mollusca) are also common in the larger muddy ponds. Amphipods, isopods, and mysids (members of the phylum Arthropoda) are abundant in the larger ponds, and, in particular, the amphipod Hyalella sp. occurs in streams of lower current velocity. Chironomid larvae are abundant in the fine sediments of both ponds and streams, and are an important component in the diet of Landlocked Dolly Varden. The assessment end point for the ecological risk assessment is the potential for significant adverse effects on benthic invertebrate community abundance and diversity.

Twelve families of aquatic macrophytes are present in the streams and ponds of Amchitka Island (Valdez et al., 1977). The most abundant of these are the large mosses and water milfoils. Large mosses, particularly Fontinalis neomexicanus, seem to grow in every stream on the island and provide an important habitat for the benthic community, and are an important food source for young fish. It is most common in the higher gradient sections and is found attached to submerged stones in the rapidly moving waters. The water milfoils, in particular Mare's Tail (Hippuris vulgaris) and Myriophyllum spicatum, are most abundant in mud substrates and are common in the lowland streams. They provide attractive habitat for pond and stream fishes. The assessment end point for the ecological risk assessment is the potential for significant adverse effects on plant species abundance, diversity, and primary production.

Six species of fish are found in the freshwater streams and ponds of Amchitka Island (Valdez et al., 1977): Silver Salmon (Oncorhynchus kisutch), Pink Salmon (O. gorbuscha), and Sockeye Salmon (O. nerka) which are all anadromous; both anadromous and Landlocked Dolly 
Varden (Salvelinus malma); Threespine Stickleback (Gasterosteus aculeatus), and the Coast-range Sculpin (Cottus aleuticus). These fish may be exposed continuously to site contaminants through their food and direct exposure. Of these fish, the Landlocked Dolly Varden has the greatest potential for exposure to site contaminants because they spend their entire life cycle in Amchitka's freshwater creeks and ponds (i.e., they are not anadromous and do not spend part of their life in the sea). Dolly Varden spawn in gravelly areas of the streams and the juveniles remain in these areas prior to dispersal to other areas. The assessment end point for the ecological risk assessment is the potential for significant adverse effects on freshwater fish abundance.

Over 130 species of birds have been recorded on Amchitka Island, and most populations are partially or totally supported by the marine ecosystem. Waterfowl are an important component of the avifauna and use the various ponds and streams for feeding and breeding. One of the more common species of freshwater waterfowl is the Green-winged Teal (Anas crecca) which is a year-round resident on the island (White et al., 1977). Though they will spend some time on saltwater, their preference is for freshwater when not frozen. The Green-winged Teal is a surface-feeding duck that feeds by dabbling and tipping up in shallow water, often filtering through soft mud for food. They feed on seeds of aquatic plants, although they also consume aquatic invertebrates, particularly during their breeding season and as juveniles. Thus, the Green-winged Teal may be exposed to contaminants by direct contact with surface water and sediment, ingestion of surface water, sediment, or contaminated plants or invertebrates. The Aleutian Canada Goose (Branta canadensis leucopareia) is listed as an endangered species. These herbivorous waterfowl are primarily terrestrial grazers but may also consume aquatic plants and algae (White et al., 1977). They may come into contact with site contaminants through ingestion of plants, surface water, and sediment. The assessment end point to be used in the ecological risk assessment is the potential for significant adverse effects on waterfowl abundance.

The Bald Eagle (Haliaeetus leucocephalus) is common year-round resident on Amchitka Island. Although the Bald Eagle is listed as a threatened species in the conterminous lower 48 states, it is not a listed species in the northern part of its range. In most habitats, the Bald Eagle feeds primarily on fish, but it is opportunistic and will feed on mammals or birds, especially carrion (Terres, 1991). On Amchitka, birds and mammals comprise a significant percentage of the Bald Eagle's diet (White et al., 1971). The Bald Eagle is a top-level predator and may be exposed to contaminants 
Revision: 0

Date: $03 / 14 / 2001$

Page 17 of 261

which bioaccumulate in the food chain. The assessment end point to be used in the ecological risk assessment is the potential for significant adverse effects on Bald Eagle abundance. 


\subsection{Human Health Risk Assessment}

A human health risk assessment will be conducted to determine the types and magnitudes of exposures to constituents originating from the drill site on Amchitka Island and to determine the potential carcinogenic risks and noncarcinogenic health hazards posed by the estimated exposures. The procedures outlined in this Work Plan are in accordance with methods prescribed in ADEC and EPA guidance: Risk Assessment Procedures Manual (ADEC, 2000a), Guidance on Calculating Cumulative Risk (ADEC, 2000b), Risk Assessment Guidance Manual for Superfund: Volume I Human Health Evaluation Manual (EPA, 1989), and Region 10 Supplemental Risk Assessment Guidance for Superfund (EPA, 1996). The following sections describe the methodologies that will be used in conducting the human health risk assessment at the drill sites on Amchitka.

\subsection{Identification of Constituents of Concern}

The preliminary constituents of concern (COCs) for which carcinogenic risks and noncarcinogenic health hazards will be quantified in the human health risk assessment are identified in this Work Plan by first reviewing the available analytical database and then comparing these data with appropriate screening criteria. For this assessment, the following general criteria are used to identify COCs:

- The compound is positively identified in at least one sample of a given medium.

- The maximum detected concentration of a compound in a given medium is greater than the naturally occurring background concentration of the same compound.

- The maximum detected concentration of a compound in a given medium is greater than the applicable screening criteria.

- The compound is a component of drilling mud or is otherwise associated with drilling activities.

Naturally occurring background concentrations of constituents in soil, surface water, and sediment have been determined by collecting samples from locations where human activity has been minimal or nonexistent. The results of the background sampling are reported in the Draft Department of Energy 1998 Drill Site Characterization Report (Shannon \& Wilson, 1999). 
The screening criteria used to identify COCs in surface water are the federal ambient water quality criteria for the protection of human health based on ingestion of water and organisms (EPA, 1999). If an ambient water quality criterion is not available for a certain compound, then the EPA Region III risk-based concentration for tap water is used. The screening criterion for diesel-range organics (DRO) in surface water is the State of Alaska groundwater cleanup level (ADEC, 2000b).

Because there are no generally accepted human health-based screening criteria for sediment, the screening criteria used to identify COCs in sediment are the Method One and Method Two Soil Cleanup Levels presented in 18 Alaska Administrative Code (AAC) 75, Articles 3 and 9 of the Oil and Hazardous Substances Pollution Control Regulations (ADEC, 1999c). Cleanup criteria are presented for different exposure routes including ingestion, inhalation, and migration to groundwater. For this assessment, the most conservative cleanup criteria for the "under 40-inch precipitation zone" $(\mathrm{ADEC}, 1999 \mathrm{c})$ are used in the COC identification process.

The screening criteria for identifying COCs in fish tissue are the EPA Region III risk-based screening criteria for fish consumption. The data summaries for each study area and the applicable screening criteria are presented in Tables 4-1 through 4-33. The COCs that will be assessed in the human health risk assessment are also identified in these tables and summarized in Table 4-34.

\subsection{Exposure Assessment}

The objectives of the exposure assessment are to identify potentially exposed populations and quantify the type and magnitude of their exposure to COCs that are present at, or migrating from, the drill sites. A CSM has been developed (Section 3.0) to aid in the identification of contaminant migration routes and exposure pathways.

\subsubsection{Potentially Exposed Populations}

The U.S. Army, U.S. Air Force, AEC, and the U.S. Navy have utilized Amchitka periodically since the early days of World War II. The island is currently unoccupied and is part of the AMNWR. The AMNWR is under the jurisdiction of the USFWS. Although public access to Amchitka is officially restricted, crew members from passing ships have been known to ignore the restrictions and go ashore for brief periods. Researchers and other personnel from the USFWS occasionally visit the island, 
Table 4-1

\section{Summary of Analytical Results and Human Health Risk Assessment Screening for Surface Water in Falls Creek, Drill Site D}

(Page 1 of 3 )

\begin{tabular}{|c|c|c|c|c|c|c|}
\hline Analyte & $\begin{array}{l}\text { Detection } \\
\text { Frequency }\end{array}$ & $\begin{array}{c}\text { Minimum } \\
\text { Detected } \\
\text { Concentration } \\
(\mu \mathrm{g} / \mathrm{L})\end{array}$ & $\begin{array}{c}\text { Maximum } \\
\text { Detected } \\
\text { Concentration } \\
(\mu \mathrm{g} / \mathrm{L})\end{array}$ & $\begin{array}{c}\text { Upland Stream } \\
\text { Background } \\
\text { Concentration }{ }^{a} \\
(\mu \mathrm{g} / \mathrm{L})\end{array}$ & $\begin{array}{l}\text { Human Health } \\
\text { Screening } \\
\text { Value } \\
(\mu \mathrm{g} / \mathrm{L})\end{array}$ & $\begin{array}{c}\text { Human Health } \\
\text { Constituent of } \\
\text { Concern }\end{array}$ \\
\hline $\begin{array}{l}\text { Diesel-Range } \\
\text { Hydrocarbons }\end{array}$ & 0 of 5 & ND (50) & ND (84.9) & NAN & $1,500^{d}$ & $\mathrm{No}^{f}$ \\
\hline \multicolumn{7}{|c|}{ Volatile Organic Compounds } \\
\hline 1,2,4-Trimethylbenzene & 0 of 5 & ND (1.0) & ND (1.0) & NAN & $12^{\mathrm{e}}$ & $\mathrm{No}^{f}$ \\
\hline 1,3,5-Trimethylbenzene & 0 of 5 & ND (1.0) & ND (1.0) & NAN & $12^{\mathrm{e}}$ & $\mathrm{No}^{f}$ \\
\hline 2-Butanone & 0 of 5 & ND (20) & ND (20) & NAN & $1,900^{\mathrm{e}}$ & $\mathrm{No}^{f}$ \\
\hline Acetone & 0 of 5 & ND (20) & ND (20) & NAN & $3,700^{\mathrm{e}}$ & $\mathrm{No}^{f}$ \\
\hline Benzene & 0 of 5 & ND (1.0) & ND (1.0) & NAN & $1.2^{\mathrm{C}}$ & $\mathrm{No}^{f}$ \\
\hline Carbon disulfide & 0 of 5 & ND (1.0) & ND (1.0) & NAN & $1,000^{\mathrm{e}}$ & $\mathrm{No}^{f}$ \\
\hline Ethylbenzene & 0 of 5 & ND (1.0) & ND (1.0) & NAN & $3,100^{c}$ & $\mathrm{No}^{f}$ \\
\hline Isopropylbenzene & 0 of 5 & $\mathrm{ND}(1.0)$ & ND (1.0) & NAN & NAV & $\mathrm{No}^{f}$ \\
\hline m,p-Xylene & 0 of 5 & ND (2.0) & ND (2.0) & NAN & $12,000^{\mathrm{e}}$ & $\mathrm{No}^{f}$ \\
\hline Methylene chloride & 0 of 5 & ND (2.0) & ND (4.95) & NAN & $4.7^{c}$ & $\mathrm{No}^{f}$ \\
\hline n-Butylbenzene & 0 of 5 & ND (1.0) & ND (1.0) & NAN & $61^{\mathrm{e}}$ & $\mathrm{No}^{f}$ \\
\hline n-Propylbenzene & 0 of 5 & ND (1.0) & ND (1.0) & NAN & $61^{e}$ & $\mathrm{No}^{f}$ \\
\hline Naphthalene & 0 of 5 & ND (1.0) & ND (1.0) & NAN & $730^{e}$ & $\mathrm{No}^{f}$ \\
\hline o-Xylene & 0 of 5 & ND (1.0) & ND (1.0) & NAN & $12,000^{\mathrm{e}}$ & $\mathrm{No}^{f}$ \\
\hline p-Isopropyltoluene & 0 of 5 & ND (1.0) & ND (1.0) & NAN & NAV & $\mathrm{No}^{f}$ \\
\hline sec-Butylbenzene & 0 of 5 & ND (1.0) & ND (1.0) & NAN & $61^{e}$ & $\mathrm{No}^{f}$ \\
\hline Toluene & 0 of 5 & ND (1.0) & ND (1.0) & NAN & $10,000^{c}$ & $\mathrm{No}^{f}$ \\
\hline \multicolumn{7}{|c|}{ Polycyclic Aromatic Hydrocarbons } \\
\hline Acenaphthene & 0 of 5 & $\mathrm{ND}(0.1)$ & $\mathrm{ND}(0.1)$ & NAN & $1,200^{c}$ & $\mathrm{No}^{f}$ \\
\hline Acenaphthylene & 0 of 5 & $\mathrm{ND}(0.1)$ & $\mathrm{ND}(0.1)$ & NAN & $0.0028^{c}$ & $\mathrm{No}^{f}$ \\
\hline Anthracene & 0 of 5 & $\mathrm{ND}(0.1)$ & ND $(0.1)$ & NAN & $0.0028^{c}$ & $\mathrm{No}^{f}$ \\
\hline Benzo(a)anthracene & 0 of 5 & $\mathrm{ND}(0.1)$ & $\mathrm{ND}(0.1)$ & NAN & $0.0028^{c}$ & $\mathrm{No}^{f}$ \\
\hline Benzo(a)pyrene & 0 of 5 & $\mathrm{ND}(0.1)$ & $\mathrm{ND}(0.1)$ & NAN & $0.0028^{c}$ & $\mathrm{No}^{f}$ \\
\hline Benzo(b)fluoranthene & 0 of 5 & $\mathrm{ND}(0.1)$ & $\mathrm{ND}(0.1)$ & NAN & $0.092^{c}$ & $\mathrm{No}^{f}$ \\
\hline Benzo(ghi)perylene & 0 of 5 & ND $(0.1)$ & ND $(0.1)$ & NAN & $0.0028^{c}$ & $\mathrm{No}^{f}$ \\
\hline Benzo(k)fluoranthene & 0 of 5 & $\mathrm{ND}(0.1)$ & $\mathrm{ND}(0.1)$ & NAN & $0.0028^{c}$ & $\mathrm{No}^{f}$ \\
\hline Chrysene & 0 of 5 & ND $(0.1)$ & $\mathrm{ND}(0.1)$ & NAN & $0.0028^{c}$ & $\mathrm{No}^{f}$ \\
\hline
\end{tabular}


Table 4-1

\section{Summary of Analytical Results and Human Health Risk Assessment Screening for Surface Water in Falls Creek, Drill Site D}

(Page 2 of 3 )

\begin{tabular}{|c|c|c|c|c|c|c|}
\hline Analyte & $\begin{array}{l}\text { Detection } \\
\text { Frequency }\end{array}$ & $\begin{array}{c}\text { Minimum } \\
\text { Detected } \\
\text { Concentration } \\
(\mu \mathrm{g} / \mathrm{L})\end{array}$ & $\begin{array}{c}\text { Maximum } \\
\text { Detected } \\
\text { Concentration } \\
(\mu \mathrm{g} / \mathrm{L})\end{array}$ & $\begin{array}{c}\text { Upland Stream } \\
\text { Background } \\
\text { Concentration } \\
(\mu \mathrm{g} / \mathrm{L})\end{array}$ & $\begin{array}{l}\text { Human Health } \\
\text { Screening } \\
\text { Value } \\
(\mu \mathrm{g} / \mathrm{L})\end{array}$ & $\begin{array}{c}\text { Human Health } \\
\text { Constituent of } \\
\text { Concern }\end{array}$ \\
\hline Dibenzo(a,h)anthracene & 0 of 5 & $\mathrm{ND}(0.1)$ & ND $(0.1)$ & NAN & $0.0028^{c}$ & $\mathrm{No}^{\dagger}$ \\
\hline Fluoranthene & 0 of 5 & ND $(0.1)$ & ND (0.1) & NAN & $42^{c}$ & $\mathrm{No}^{f}$ \\
\hline Fluorene & 0 of 5 & $\mathrm{ND}(0.1)$ & $\mathrm{ND}(0.1)$ & NAN & $0.0028^{c}$ & $\mathrm{No}^{f}$ \\
\hline Indeno(1,2,3-cd)pyrene & 0 of 5 & $\mathrm{ND}(0.1)$ & $\mathrm{ND}(0.1)$ & NAN & $0.0028^{c}$ & $\mathrm{No}^{f}$ \\
\hline Naphthalene & 0 of 5 & $\mathrm{ND}(0.1)$ & ND $(0.1)$ & NAN & $730^{e}$ & $\mathrm{No}^{f}$ \\
\hline Phenanthrene & 0 of 5 & $\mathrm{ND}(0.1)$ & $\mathrm{ND}(0.1)$ & NAN & $0.0028^{C}$ & $\mathrm{No}^{f}$ \\
\hline Pyrene & 0 of 5 & $\mathrm{ND}(0.1)$ & $\mathrm{ND}(0.1)$ & NAN & $0.0028^{C}$ & $\mathrm{No}^{f}$ \\
\hline \multicolumn{7}{|c|}{ Polychlorinated Biphenyls } \\
\hline Aroclor 1016 & NAV & NAV & NAV & NAN & $0.000044^{\mathrm{C}}$ & $\mathrm{No}^{f}$ \\
\hline Aroclor 1221 & NAV & NAV & NAV & NAN & $0.000044^{c}$ & $\mathrm{No}^{f}$ \\
\hline Aroclor 1232 & NAV & NAV & NAV & NAN & $0.000044^{c}$ & $\mathrm{No}^{f}$ \\
\hline Aroclor 1242 & NAV & NAV & NAV & NAN & $0.000044^{c}$ & $\mathrm{No}^{f}$ \\
\hline Aroclor 1248 & NAV & NAV & NAV & NAN & $0.000044^{c}$ & $\mathrm{No}^{f}$ \\
\hline Aroclor 1254 & NAV & NAV & NAV & NAN & $0.000044^{c}$ & $\mathrm{No}^{f}$ \\
\hline Aroclor 1260 & NAV & NAV & NAV & NAN & $0.000044^{c}$ & $\mathrm{No}^{f}$ \\
\hline Aroclor 1262 & NAV & NAV & NAV & NAN & $0.000044^{c}$ & $\mathrm{No}^{f}$ \\
\hline Aroclor 1268 & NAV & NAV & NAV & NAN & $0.000044^{\mathrm{C}}$ & $\mathrm{No}^{f}$ \\
\hline \multicolumn{7}{|c|}{ Metals } \\
\hline Aluminum & 0 of 5 & ND (50) & ND (50) & $87.1^{\mathrm{b}}$ & $37,000^{e}$ & $\mathrm{No}^{f}$ \\
\hline Arsenic & 0 of 5 & ND $(0.1)$ & ND $(0.1)$ & ND (1) & $0.018^{c}$ & $\mathrm{No}^{f}$ \\
\hline Barium & 0 of 5 & ND (10) & ND (10) & ND (10) & $1,000^{c}$ & $\mathrm{No}^{f}$ \\
\hline Beryllium & 0 of 5 & ND (1.0) & ND (1.0) & ND (1) & $0.0076^{c}$ & $\mathrm{No}^{f}$ \\
\hline Boron & 0 of 5 & ND (50) & ND (50) & ND (50) & $3,300^{\mathrm{e}}$ & $\mathrm{No}^{f}$ \\
\hline Cadmium & 0 of 5 & ND (1.0) & ND (1.0) & ND (1) & $10^{\mathrm{C}}$ & $\mathrm{No}^{f}$ \\
\hline Calcium & 5 of 5 & 3,740 & 4,990 & $8,810^{b}$ & NAV & $\mathrm{No}^{\mathrm{g}}$ \\
\hline Cerium & 0 of 5 & ND (1.0) & ND (1.0) & ND (1) & NAV & $\mathrm{No}^{f}$ \\
\hline Cesium & 0 of 5 & ND (1.0) & ND (1.0) & ND (1) & NAV & $\mathrm{No}^{f}$ \\
\hline Chromium & 1 of 5 & ND (1.0) & 12 & $3.49^{b}$ & $33,000^{c}$ & $\mathrm{No}^{\mathrm{h}}$ \\
\hline Cobalt & 5 of 5 & 2.83 & 4.59 & 9.52 & $2,200^{\mathrm{e}}$ & $\mathrm{No}^{\mathrm{g}}$ \\
\hline Copper & 4 of 5 & ND (1.0) & 3.3 & $13.4^{b}$ & $1,300^{C}$ & $\mathrm{No}^{\mathrm{g}}$ \\
\hline
\end{tabular}


Table 4-1

\section{Summary of Analytical Results and Human Health Risk Assessment Screening for Surface Water in Falls Creek, Drill Site D}

(Page 3 of 3 )

\begin{tabular}{|c|c|c|c|c|c|c|}
\hline Analyte & $\begin{array}{l}\text { Detection } \\
\text { Frequency }\end{array}$ & $\begin{array}{c}\text { Minimum } \\
\text { Detected } \\
\text { Concentration } \\
(\mu \mathrm{g} / \mathrm{L})\end{array}$ & $\begin{array}{c}\text { Maximum } \\
\text { Detected } \\
\text { Concentration } \\
(\mu \mathrm{g} / \mathrm{L})\end{array}$ & $\begin{array}{c}\text { Upland Stream } \\
\text { Background } \\
\text { Concentration } \\
(\mu \mathrm{g} / \mathrm{L})\end{array}$ & $\begin{array}{c}\text { Human Health } \\
\text { Screening } \\
\text { Value } \\
(\mu \mathrm{g} / \mathrm{L})\end{array}$ & $\begin{array}{c}\text { Human Health } \\
\text { Constituent of } \\
\text { Concern }\end{array}$ \\
\hline Iron & 3 of 5 & ND (150) & 199 & $2,830^{b}$ & $300^{C}$ & $\mathrm{No}^{\mathrm{g}}$ \\
\hline Lithium & 1 of 5 & ND (1.0) & 1.04 & ND (1) & $730^{e}$ & $\mathrm{No}^{\mathrm{h}}$ \\
\hline Magnesium & 5 of 5 & 3,650 & 4,350 & 5,250 & NAV & $\mathrm{No}^{\mathrm{g}}$ \\
\hline Manganese & 5 of 5 & 10.4 & 17.1 & 748 & $50^{c}$ & $\mathrm{No}^{\mathrm{g}}$ \\
\hline Molybdenum & 1 of 5 & ND (1.0) & 1.01 & ND (1) & $180^{\mathrm{e}}$ & $\mathrm{No}^{h}$ \\
\hline Nickel & 2 of 5 & ND (1.0) & 1.59 & $1.69^{b}$ & $510^{C}$ & $\mathrm{No}^{\mathrm{g}}$ \\
\hline Potassium & 5 of 5 & 1,910 & 2,270 & $1,190^{b}$ & NAV & $\mathrm{No}^{\mathrm{i}}$ \\
\hline Selenium & 0 of 5 & ND (1.0) & ND (1.0) & ND (1) & $104^{c}$ & $\mathrm{No}^{f}$ \\
\hline Strontium & 5 of 5 & 31.9 & 36.9 & $44.1^{b}$ & $22,000^{e}$ & $\mathrm{No}^{\mathrm{g}}$ \\
\hline Thallium & 0 of 5 & ND (1.0) & ND (1.0) & $2.13^{b}$ & $1.7^{\mathrm{C}}$ & $\mathrm{No}^{f}$ \\
\hline Thorium & 0 of 5 & ND (1.0) & ND (1.0) & $1.14^{b}$ & NAV & $\mathrm{No}^{f}$ \\
\hline Titanium & 5 of 5 & 1.25 & 2.37 & $2.37^{b}$ & $150,000^{e}$ & $\mathrm{No}^{\mathrm{g}}$ \\
\hline Uranium & 0 of 5 & ND (1.0) & ND (1.0) & ND (1) & $110^{e}$ & $\mathrm{No}^{f}$ \\
\hline Vanadium & 5 of 5 & 1.62 & 2.37 & $1.44^{\mathrm{b}}$ & $260^{\mathrm{e}}$ & $\mathrm{No}^{\mathrm{h}}$ \\
\hline Zinc & 5 of 5 & 11 & 23.3 & $12.8^{b}$ & $11,000^{\mathrm{e}}$ & $\mathrm{No}^{h}$ \\
\hline
\end{tabular}

${ }^{a}$ Background concentrations are upper tolerance limits (UTL) (ADEC, 1999a) unless otherwise noted.

${ }^{b}$ Maximum detected concentration in background samples due to insufficient detection frequency to calculate UTL.

${ }^{\mathrm{c}}$ Federal ambient water quality criteria based on ingestion of water and organisms.

${ }^{d}$ State of Alaska groundwater cleanup level, Discharge Reporting, Cleanup, and Disposal of Oil and other Hazardous Substances

(18 AAC 75, Articles 3 and 9 [ADEC, 1999c]).

eU.S. EPA Region III risk-based concentrations for tap water (EPA, 2000b).

\section{COC Selection Criteria}

${ }^{\mathrm{f}}$ Constituent not detected in any samples.

${ }^{9}$ Maximum detected constituent concentration is less than local background concentration.

${ }^{\mathrm{h}}$ Maximum detected constituent concentration is less than human health screening value.

'Constituent is an essential macronutrient and only presents a risk at extremely high concentrations.

ND - Not detected (detection limit)

NAV - Not available

NAN - Not analyzed

$\mu \mathrm{g} / \mathrm{L}$ - Micrograms per liter 
Table 4-2

\section{Summary of Analytical Results and Human Health Risk Assessment Screening for Sediment in Falls Creek, Drill Site D}

(Page 1 of 3 )

\begin{tabular}{|c|c|c|c|c|c|c|}
\hline Analyte & $\begin{array}{l}\text { Detection } \\
\text { Frequency }\end{array}$ & $\begin{array}{c}\text { Minimum } \\
\text { Detected } \\
\text { Concentration } \\
(\mathrm{mg} / \mathrm{kg})\end{array}$ & $\begin{array}{c}\text { Maximum } \\
\text { Detected } \\
\text { Concentration } \\
(\mathbf{m g} / \mathbf{k g})\end{array}$ & $\begin{array}{l}\text { Upland Stream } \\
\text { Background } \\
\text { Concentration } \\
(\mathrm{mg} / \mathrm{kg})\end{array}$ & $\begin{array}{l}\text { Human Health } \\
\text { Screening } \\
\text { Value } \\
(\mathrm{mg} / \mathrm{kg})\end{array}$ & $\begin{array}{c}\text { Human Health } \\
\text { Constituent of } \\
\text { Concern }\end{array}$ \\
\hline $\begin{array}{l}\text { Diesel-Range } \\
\text { Hydrocarbons }\end{array}$ & 1 of 5 & ND (4.0) & 176 & NAN & $200^{\mathrm{C}}$ & $\mathrm{No}^{f}$ \\
\hline \multicolumn{7}{|c|}{ Volatile Organic Compounds } \\
\hline 1,2,4-Trimethylbenzene & NAV & NAV & NAV & NAN & NAV & $\mathrm{No}^{\mathrm{d}}$ \\
\hline 1,3,5-Trimethylbenzene & NAV & NAV & NAV & NAN & NAV & $\mathrm{No}^{\mathrm{d}}$ \\
\hline 2-Butanone & NAV & NAV & NAV & NAN & NAV & $\mathrm{No}^{d}$ \\
\hline Acetone & NAV & NAV & NAV & NAN & $10,100^{c}$ & No ${ }^{d}$ \\
\hline Benzene & NAV & NAV & NAV & NAN & $286^{c}$ & No ${ }^{d}$ \\
\hline Carbon disulfide & NAV & NAV & NAV & NAN & $10,100^{c}$ & $\mathrm{No}^{\mathrm{d}}$ \\
\hline Ethylbenzene & NAV & NAV & NAV & NAN & $10,100^{C}$ & No ${ }^{d}$ \\
\hline Isopropybenzene & NAV & NAV & NAV & NAN & NAV & No ${ }^{d}$ \\
\hline $\mathrm{m}, \mathrm{p}$-Xylene & NAV & NAV & NAV & NAN & $203,000^{c}$ & $\mathrm{No}^{\mathrm{d}}$ \\
\hline Methylene chloride & NAV & NAV & NAV & NAN & $1,110^{\mathrm{C}}$ & $\mathrm{No}^{d}$ \\
\hline n-Butylbenzene & NAV & NAV & NAV & NAN & NAV & $\mathrm{No}^{d}$ \\
\hline n-Propylbenzene & NAV & NAV & NAV & NAN & NAV & $\mathrm{No}^{\mathrm{d}}$ \\
\hline Naphthalene & NAV & NAV & NAV & NAN & $4,060^{\mathrm{C}}$ & $\mathrm{No}^{\mathrm{d}}$ \\
\hline o-Xylene & NAV & NAV & NAV & NAN & $203,000^{c}$ & $\mathrm{No}^{d}$ \\
\hline p-Isopropyltoluene & NAV & NAV & NAV & NAN & NAV & $\mathrm{No}^{d}$ \\
\hline sec-Butylbenzene & NAV & NAV & NAV & NAN & NAV & $\mathrm{No}^{\mathrm{d}}$ \\
\hline Toluene & NAV & NAV & NAV & NAN & $20,300^{c}$ & $\mathrm{No}^{\mathrm{d}}$ \\
\hline \multicolumn{7}{|c|}{ Polycyclic Aromatic Hydrocarbons } \\
\hline Acenaphthene & 0 of 5 & ND (0.0033) & ND $(0.0143)$ & NAN & $6,080^{c}$ & $\mathrm{No}^{\mathrm{d}}$ \\
\hline Acenaphthylene & 0 of 5 & ND (0.0033) & ND (0.0143) & NAN & NAV & $\mathrm{No}^{d}$ \\
\hline Anthracene & 1 of 5 & ND (0.0033) & 0.0161 & NAN & $30,400^{c}$ & $\mathrm{No}^{\dagger}$ \\
\hline Benzo(a)anthracene & 1 of 5 & ND (0.0033) & 0.034 & NAN & $11.4^{\mathrm{C}}$ & $\mathrm{No}^{\dagger}$ \\
\hline Benzo(a)pyrene & 1 of 5 & ND (0.0033) & 0.0501 & NAN & $1.1^{\mathrm{c}}$ & $\mathrm{No}^{f}$ \\
\hline Benzo(b)fluoranthene & 1 of 5 & ND (0.0033) & 0.0876 & NAN & $11.4^{\mathrm{C}}$ & $\mathrm{No}^{f}$ \\
\hline Benzo(ghi)perylene & 1 of 5 & ND (0.0033) & 0.0143 & NAN & $1.1^{\mathrm{c}}$ & $\mathrm{No}^{f}$ \\
\hline Benzo(k)fluoranthene & 1 of 5 & ND (0.0033) & 0.0322 & NAN & $114^{c}$ & $\mathrm{No}^{f}$ \\
\hline Chrysene & 1 of 5 & ND (0.0033) & 0.0215 & NAN & $1,140^{\mathrm{C}}$ & $\mathrm{No}^{f}$ \\
\hline
\end{tabular}


Table 4-2

\section{Summary of Analytical Results and Human Health Risk Assessment Screening for Sediment in Falls Creek, Drill Site D}

(Page 2 of 3 )

\begin{tabular}{|c|c|c|c|c|c|c|}
\hline Analyte & $\begin{array}{l}\text { Detection } \\
\text { Frequency }\end{array}$ & $\begin{array}{c}\text { Minimum } \\
\text { Detected } \\
\text { Concentration } \\
(\mathbf{m g} / \mathbf{k g})\end{array}$ & $\begin{array}{c}\text { Maximum } \\
\text { Detected } \\
\text { Concentration } \\
(\mathbf{m g} / \mathbf{k g})\end{array}$ & $\begin{array}{c}\text { Upland Stream } \\
\text { Background } \\
\text { Concentration }{ }^{a} \\
(\mathbf{m g} / \mathbf{k g})\end{array}$ & $\begin{array}{l}\text { Human Health } \\
\text { Screening } \\
\text { Value } \\
(\mathrm{mg} / \mathrm{kg})\end{array}$ & $\begin{array}{c}\text { Human Health } \\
\text { Constituent of } \\
\text { Concern }\end{array}$ \\
\hline Dibenzo(a,h)anthracene & 0 of 5 & ND (0.0033) & ND $(0.0143)$ & NAN & $1.1^{\mathrm{C}}$ & No ${ }^{d}$ \\
\hline Fluoranthene & 1 of 5 & ND (0.0033) & 0.0232 & NAN & $4,060^{\mathrm{C}}$ & $\mathrm{No}^{f}$ \\
\hline Fluorene & 1 of 5 & ND (0.0033) & 0.0125 & NAN & $4,060^{\mathrm{C}}$ & $\mathrm{No}^{f}$ \\
\hline Indeno(1,2,3-cd)pyrene & 1 of 5 & ND (0.0033) & 0.0197 & NAN & $11^{\mathrm{c}}$ & $\mathrm{No}^{\dagger}$ \\
\hline Naphthalene & 0 of 5 & ND (0.0033) & ND $(0.0143)$ & NAN & $4,060^{\mathrm{C}}$ & $\mathrm{No}^{\mathrm{d}}$ \\
\hline Phenanthrene & 1 of 5 & ND (0.0033) & 0.125 & NAN & $1.1^{\mathrm{c}}$ & $\mathrm{No}^{f}$ \\
\hline Pyrene & 1 of 5 & ND (0.0033) & 0.0554 & NAN & $3040^{c}$ & $\mathrm{No}^{f}$ \\
\hline \multicolumn{7}{|c|}{ Polychlorinated Biphenyls } \\
\hline Aroclor 1016 & NAV & NAV & NAV & NAN & $10^{c}$ & $\mathrm{No}^{\mathrm{d}}$ \\
\hline Aroclor 1221 & NAV & NAV & NAV & NAN & $10^{c}$ & $\mathrm{No}^{d}$ \\
\hline Aroclor 1232 & NAV & NAV & NAV & NAN & $10^{\mathrm{C}}$ & $\mathrm{No}^{d}$ \\
\hline Aroclor 1242 & NAV & NAV & NAV & NAN & $10^{c}$ & $\mathrm{No}^{\mathrm{d}}$ \\
\hline Aroclor 1248 & NAV & NAV & NAV & NAN & $10^{c}$ & $\mathrm{No}^{\mathrm{d}}$ \\
\hline Aroclor 1254 & NAV & NAV & NAV & NAN & $10^{c}$ & $\mathrm{No}^{d}$ \\
\hline Aroclor 1260 & 2 of 3 & ND (0.033) & 0.82 & NAN & $10^{c}$ & $\mathrm{No}^{f}$ \\
\hline Aroclor 1262 & NAV & NAV & NAV & NAN & $10^{\mathrm{C}}$ & $\mathrm{No}^{\mathrm{d}}$ \\
\hline Aroclor 1268 & NAV & NAV & NAV & NAN & $10^{c}$ & $\mathrm{No}^{d}$ \\
\hline \multicolumn{7}{|c|}{ Metals } \\
\hline Aluminum & 5 of 5 & 9,490 & 38,200 & 47,500 & NAV & $\mathrm{No}^{\mathrm{e}}$ \\
\hline Arsenic & 5 of 5 & 1.62 & 10 & 30.5 & $5.5^{c}$ & $\mathrm{No}^{\mathrm{e}}$ \\
\hline Barium & 5 of 5 & 53 & 181 & 468 & $7,100^{\mathrm{C}}$ & $\mathrm{No}^{\mathrm{e}}$ \\
\hline Beryllium & 0 of 5 & ND (0.5) & ND (2.17) & ND & $1.9^{\mathrm{C}}$ & $\mathrm{No}^{d}$ \\
\hline Boron & 5 of 5 & 17.2 & 163 & $6.42^{b}$ & NAV & $\mathrm{No}^{\mathrm{h}}$ \\
\hline Cadmium & 0 of 5 & ND (0.5) & ND (2.17) & ND & $101^{c}$ & $\mathrm{No}^{\mathrm{d}}$ \\
\hline Calcium & 5 of 5 & 1,700 & 7,410 & 7,720 & NAV & $\mathrm{No}^{\mathrm{e}}$ \\
\hline Cerium & 5 of 5 & 3.71 & 14.6 & 13 & NAV & $\mathrm{No}^{\mathrm{h}}$ \\
\hline Cesium & 0 of 5 & ND (0.5) & ND (2.17) & ND & NAV & $\mathrm{No}^{d}$ \\
\hline Chromium & 5 of 5 & 20.6 & 226 & 18.9 & $507^{c}$ & $\mathrm{No}^{f}$ \\
\hline Cobalt & 5 of 5 & 4.62 & 23.5 & 37.3 & NAV & $\mathrm{No}^{\mathrm{e}}$ \\
\hline Copper & 5 of 5 & 21.5 & 93.1 & 82.4 & NAV & $\mathrm{No}^{\mathrm{h}}$ \\
\hline
\end{tabular}


Table 4-2

\section{Summary of Analytical Results and Human Health Risk Assessment Screening for Sediment in Falls Creek, Drill Site D}

(Page 3 of 3)

\begin{tabular}{|c|c|c|c|c|c|c|}
\hline Analyte & $\begin{array}{l}\text { Detection } \\
\text { Frequency }\end{array}$ & $\begin{array}{c}\text { Minimum } \\
\text { Detected } \\
\text { Concentration } \\
(\mathbf{m g} / \mathbf{k g})\end{array}$ & $\begin{array}{c}\text { Maximum } \\
\text { Detected } \\
\text { Concentration } \\
(\mathbf{m g} / \mathbf{k g})\end{array}$ & $\begin{array}{l}\text { Upland Stream } \\
\text { Background } \\
\text { Concentration }{ }^{\mathrm{a}} \\
(\mathrm{mg} / \mathrm{kg})\end{array}$ & $\begin{array}{l}\text { Human Health } \\
\text { Screening } \\
\text { Value } \\
(\mathrm{mg} / \mathrm{kg})\end{array}$ & $\begin{array}{c}\text { Human Health } \\
\text { Constituent of } \\
\text { Concern }\end{array}$ \\
\hline Iron & 5 of 5 & 8,960 & 85,500 & 123,000 & NAV & $\mathrm{No}^{\mathrm{e}}$ \\
\hline Lithium & 5 of 5 & 2.51 & 16.6 & 22 & NAV & $\mathrm{No}^{\mathrm{e}}$ \\
\hline Magnesium & 5 of 5 & 1,660 & 17,300 & 17,000 & NAV & $\mathrm{No}^{\mathrm{g}}$ \\
\hline Manganese & 5 of 5 & 256 & 2,310 & 26,500 & NAV & $\mathrm{No}^{\mathrm{e}}$ \\
\hline Molybdenum & 1 of 5 & ND (1.34) & 0.555 & ND & NAV & $\mathrm{No}^{\mathrm{h}}$ \\
\hline Nickel & 5 of 5 & 11.4 & 21.9 & 23.8 & $2,030^{c}$ & $\mathrm{No}^{\mathrm{e}}$ \\
\hline Potassium & 5 of 5 & 175 & 1,060 & 1,290 & NAV & $\mathrm{No}^{\mathrm{e}}$ \\
\hline Selenium & 0 of 5 & ND (0.5) & ND (2.17) & $3.15^{b}$ & $507^{c}$ & $\mathrm{No}^{\mathrm{d}}$ \\
\hline Strontium & 5 of 5 & 19.8 & 93.7 & 207 & NAV & $\mathrm{No}^{\mathrm{e}}$ \\
\hline Thallium & 0 of 5 & ND (0.5) & ND (2.17) & ND & NAV & $\mathrm{No}^{d}$ \\
\hline Thorium & 0 of 5 & ND (0.5) & ND (2.17) & ND & NAV & $\mathrm{No}^{d}$ \\
\hline Titanium & 5 of 5 & 376 & 1,590 & 1480 & NAV & $\mathrm{No}^{\mathrm{e}}$ \\
\hline Uranium & 1 of 5 & ND (0.5) & 2.49 & ND & NAV & $\mathrm{No}^{\mathrm{h}}$ \\
\hline Vanadium & 5 of 5 & 49.2 & 247 & 336 & $710^{c}$ & $\mathrm{No}^{\mathrm{e}}$ \\
\hline Zinc & 5 of 5 & 82 & 256 & 185 & $30,400^{c}$ & $\mathrm{No}^{f}$ \\
\hline Total Organic Carbon & 5 of 5 & 11,600 & 82,700 & NAN & NAV & $\mathrm{No}^{h}$ \\
\hline
\end{tabular}

${ }^{a}$ Background concentrations are upper tolerance limits (UTL) (ADEC, 1999a) unless otherwise noted.

${ }^{b}$ Maximum detected concentration in background samples due to insufficient detection frequency to calculate UTL.

${ }^{\text {'}}$ State of Alaska soil cleanup level, Discharge Reporting, Cleanup, and Disposal of Oil and other Hazardous Substances

(18 AAC 75, Articles 3 and 9 [ADEC, 1999c]).

\section{COC Selection Criteria}

${ }^{\mathrm{d}}$ Constituent not detected in any samples.

${ }^{\mathrm{e}}$ Maximum detected constituent concentration is less than local background concentration.

${ }^{f}$ Maximum detected constituent concentration is less than human health screening value.

${ }^{9}$ Constituent is an essential macronutrient and only presents a risk at extremely high concentrations.

${ }^{\mathrm{h}} \mathrm{Not}$ a constituent associated with drilling mud or other drilling activities.

ND - Not detected (detection limit)

NAV - Not available

NAN - Not analyzed

$\mathrm{mg} / \mathrm{kg}$ - Milligrams per kilogram 
Table 4-3

\section{Summary of Analytical Results and Human Health Risk Assessment Screening for Fish Tissue in Falls Creek, Drill Site D}

(Page 1 of 2 )

\begin{tabular}{|c|c|c|c|c|c|c|}
\hline Analyte & $\begin{array}{l}\text { Detection } \\
\text { Frequency }\end{array}$ & $\begin{array}{c}\text { Minimum } \\
\text { Detected } \\
\text { Concentration } \\
(\mathbf{m g} / \mathbf{k g})\end{array}$ & $\begin{array}{c}\text { Maximum } \\
\text { Detected } \\
\text { Concentration } \\
(\mathrm{mg} / \mathrm{kg})\end{array}$ & $\begin{array}{c}\text { Upland Stream } \\
\text { Background } \\
\text { Concentration }{ }^{a} \\
(\mathrm{mg} / \mathrm{kg})\end{array}$ & $\begin{array}{c}\text { Human Health } \\
\text { Screening } \\
\text { Value }^{c} \\
(\mathrm{mg} / \mathrm{kg})\end{array}$ & $\begin{array}{c}\text { Human Health } \\
\text { Constituent of } \\
\text { Concern }\end{array}$ \\
\hline \multicolumn{7}{|c|}{ Polycyclic Aromatic Hydrocarbons } \\
\hline Acenaphthene & 0 of 2 & ND (0.0067) & ND (0.0335) & NAN & 81.1 & $\mathrm{No}^{\mathrm{d}}$ \\
\hline Acenaphthylene & 0 of 2 & ND (0.0067) & ND (0.0335) & NAN & NAV & $\mathrm{No}^{\mathrm{d}}$ \\
\hline Anthracene & 0 of 2 & ND (0.0335) & ND (0.0335) & NAN & 410 & $\mathrm{No}^{\mathrm{d}}$ \\
\hline Benzo (a) anthracene & 0 of 2 & ND (0.0335) & ND (0.168) & NAN & 0.0043 & $\mathrm{No}^{d}$ \\
\hline Benzo (a) pyrene & 0 of 2 & ND (0.0335) & ND (0.168) & NAN & 0.00043 & $\mathrm{No}^{d}$ \\
\hline Benzo (b) fluoranthene & 0 of 2 & ND (0.0335) & ND (0.168) & NAN & 0.0043 & $\mathrm{No}^{d}$ \\
\hline Benzo (ghi) perylene & 0 of 2 & ND (0.0335) & ND (0.168) & NAN & NAV & $\mathrm{No}^{d}$ \\
\hline Benzo (k) fluoranthene & 0 of 2 & ND (0.0335) & ND (0.168) & NAN & 0.043 & $\mathrm{No}^{d}$ \\
\hline Chrysene & 0 of 2 & ND (0.0335) & ND (0.168) & NAN & 0.43 & $\mathrm{No}^{d}$ \\
\hline $\begin{array}{l}\text { Dibenzo }(\mathrm{a}, \mathrm{h}) \\
\text { anthracene }\end{array}$ & 0 of 2 & ND (0.0335) & ND $(0.168)$ & NAN & 0.00043 & $\mathrm{No}^{d}$ \\
\hline Fluoranthene & 0 of 2 & ND (0.0335) & ND (0.0335) & NAN & 54 & $\mathrm{No}^{d}$ \\
\hline Fluorene & 0 of 2 & ND (0.0067) & ND (0.0335) & NAN & 54 & $\mathrm{No}^{d}$ \\
\hline $\begin{array}{l}\text { Indeno }(1,2,3-c d) \\
\text { pyrene }\end{array}$ & 0 of 2 & ND (0.0335) & ND (0.168) & NAN & 0.0043 & Nod \\
\hline Naphthalene & 0 of 2 & ND (0.0067) & ND (0.0335) & NAN & 27 & $\mathrm{No}^{d}$ \\
\hline Phenanthrene & 0 of 2 & ND (0.0335) & ND (0.0335) & NAN & NAV & $\mathrm{No}^{d}$ \\
\hline Pyrene & 0 of 2 & ND (0.0335) & ND (0.168) & NAN & 41 & $\mathrm{No}^{d}$ \\
\hline \multicolumn{7}{|c|}{ Polychlorinated Biphenyls } \\
\hline Aroclor 1016 & 0 of 2 & ND (0.01) & ND $(0.01)$ & NAN & 0.045 & $\mathrm{No}^{d}$ \\
\hline Aroclor 1221 & 0 of 2 & ND (0.02) & ND (0.02) & NAN & 0.0016 & $\mathrm{No}^{\mathrm{d}}$ \\
\hline Aroclor 1232 & 0 of 2 & ND (0.01) & ND (0.01) & NAN & 0.0016 & $\mathrm{No}^{\mathrm{d}}$ \\
\hline Aroclor 1242 & 0 of 2 & ND (0.01) & ND (0.01) & NAN & 0.0016 & $\mathrm{No}^{\mathrm{d}}$ \\
\hline Aroclor 1248 & 0 of 2 & ND (0.01) & ND (0.01) & NAN & 0.0016 & $\mathrm{No}^{\mathrm{d}}$ \\
\hline Aroclor 1254 & 0 of 2 & ND (0.01) & ND (0.01) & NAN & 0.0016 & $\mathrm{No}^{d}$ \\
\hline Aroclor 1260 & 2 of 2 & 0.0325 & 0.183 & NAN & 0.0016 & Yes \\
\hline \multicolumn{7}{|c|}{ Metals } \\
\hline Aluminum & 2 of 2 & 23.4 & 23.9 & 38.8 & 1,400 & $\mathrm{No}^{\mathrm{e}}$ \\
\hline Arsenic (total) & 2 of 2 & 0.038 & 0.0485 & 26.9 & 0.0021 & $\mathrm{No}^{\mathrm{e}}$ \\
\hline Arsenic (inorganic) & 2 of 2 & 0.00379 & 0.00411 & 0.0456 & 0.0021 & $\mathrm{No}^{\mathrm{e}}$ \\
\hline Barium & 2 of 2 & 0.571 & 0.722 & 0.567 & 95 & $\mathrm{No}^{f}$ \\
\hline Beryllium & 0 of 2 & ND (0.025) & ND (0.025) & ND & 2.7 & $\mathrm{No}^{d}$ \\
\hline
\end{tabular}


Table 4-3

\section{Summary of Analytical Results and Human Health Risk Assessment Screening for Fish Tissue in Falls Creek, Drill Site D}

(Page 2 of 2)

\begin{tabular}{|c|c|c|c|c|c|c|}
\hline Analyte & $\begin{array}{l}\text { Detection } \\
\text { Frequency }\end{array}$ & $\begin{array}{c}\text { Minimum } \\
\text { Detected } \\
\text { Concentration } \\
(\mathbf{m g} / \mathbf{k g})\end{array}$ & $\begin{array}{c}\text { Maximum } \\
\text { Detected } \\
\text { Concentration } \\
(\mathbf{m g} / \mathrm{kg})\end{array}$ & $\begin{array}{l}\text { Upland Stream } \\
\text { Background } \\
\text { Concentration }{ }^{\text {a }} \\
(\mathbf{m g} / \mathbf{k g})\end{array}$ & $\begin{array}{c}\text { Human Health } \\
\text { Screening } \\
\text { Value }^{c} \\
(\mathbf{m g} / \mathbf{k g})\end{array}$ & $\begin{array}{c}\text { Human Health } \\
\text { Constituent of } \\
\text { Concern }\end{array}$ \\
\hline Boron & 2 of 2 & ND (9.04) & ND (9.4) & 24.1 & 120 & $\mathrm{No}^{\mathrm{e}}$ \\
\hline Cadmium & 0 of 2 & ND (0.025) & ND (0.025) & ND & 1.4 & $\mathrm{No}^{d}$ \\
\hline Calcium & 2 of 2 & 5,650 & 8,270 & 10,300 & NAV & $\mathrm{No}^{\mathrm{e}}$ \\
\hline Cerium & 0 of 2 & ND (0.025) & ND (0.025) & ND & NAV & $\mathrm{No}^{d}$ \\
\hline Cesium & 0 of 2 & ND (0.025) & ND (0.025) & ND & NAV & $\mathrm{No}^{d}$ \\
\hline Chromium & 2 of 2 & 0.312 & 0.758 & 0.684 & 4.1 & $\mathrm{No}^{f}$ \\
\hline Cobalt & 0 of 2 & ND (0.05) & ND (0.05) & $0.0510^{b}$ & 81 & $\mathrm{No}^{d}$ \\
\hline Copper & 0 of 2 & ND (0.928) & ND (1.12) & 3.4 & 54 & $\mathrm{No}^{d}$ \\
\hline Iron & 2 of 2 & 51 & 66.5 & 73.6 & 410 & $\mathrm{No}^{\mathrm{e}}$ \\
\hline Lithium & 0 of 2 & ND (0.025) & ND (0.025) & ND & 27 & $\mathrm{No}^{d}$ \\
\hline Magnesium & 2 of 2 & 285 & 288 & 349 & NAV & $\mathrm{No}^{\mathrm{e}}$ \\
\hline Manganese & 2 of 2 & 3.84 & 4.71 & 164 & 190 & $\mathrm{No}^{\mathrm{e}}$ \\
\hline Molybdenum & 0 of 2 & ND (0.125) & ND (0.125) & $0.150^{b}$ & 6.8 & $\mathrm{No}^{d}$ \\
\hline Nickel & 2 of 2 & 0.142 & 0.184 & ND & 27 & $\mathrm{No}^{f}$ \\
\hline Potassium & 2 of 2 & 4,180 & 4,230 & 4,310 & NAV & $\mathrm{No}^{\mathrm{e}}$ \\
\hline Selenium & 2 of 2 & 0.51 & 0.803 & 2.08 & 6.8 & $\mathrm{No}^{\mathrm{e}}$ \\
\hline Strontium & 2 of 2 & 10.3 & 11.2 & $12.1^{b}$ & 810 & $\mathrm{No}^{\mathrm{e}}$ \\
\hline Thallium & 0 of 2 & ND (0.025) & ND (0.025) & $0.0755^{b}$ & 0.095 & $\mathrm{No}^{d}$ \\
\hline Thorium & 2 of 2 & ND (2.5) & 2.51 & $4.67^{b}$ & NAV & $\mathrm{No}^{\mathrm{e}}$ \\
\hline Titanium & 2 of 2 & 0.857 & 1.07 & 0.834 & 5,400 & $\mathrm{No}^{f}$ \\
\hline Uranium & 0 of 2 & $\mathrm{ND}(0.5)$ & ND (0.5) & ND & 4.1 & $\mathrm{No}^{d}$ \\
\hline Vanadium & 2 of 2 & 0.236 & 0.244 & 0.289 & 9.5 & $\mathrm{No}^{\mathrm{e}}$ \\
\hline Zinc & 2 of 2 & 32.9 & 38.5 & 78 & 410 & $\mathrm{No}^{\mathrm{e}}$ \\
\hline
\end{tabular}

${ }^{\mathrm{a} B}$ Background concentrations are upper tolerance limits (UTL) (ADEC, 1999a) unless otherwise noted.

${ }^{\mathrm{b}}$ Maximum detected concentration in background samples due to insufficient detection frequency to calculate UTL.

${ }^{\mathrm{C} U}$.S. EPA Region III risk-based screening criteria for fish consumption unless otherwise noted.

\section{COC Selection Criteria}

${ }^{\mathrm{d}}$ Constituent not detected in any samples.

${ }^{e}$ Maximum detected constituent concentration is less than local background concentration.

${ }^{f}$ Maximum detected constituent concentration is less than human health screening value.

ND - Not detected (detection limit)

NAV - Not available

NAN - Not analyzed

$\mathrm{mg} / \mathrm{kg}$ - Milligrams per kilogram 
Table 4-4

\section{Summary of Analytical Results and Human Health Risk Assessment Screening for Surface Water in Lake, Drill Site D}

(Page 1 of 3 )

\begin{tabular}{|c|c|c|c|c|c|c|}
\hline Analyte & $\begin{array}{l}\text { Detection } \\
\text { Frequency }\end{array}$ & $\begin{array}{c}\text { Minimum } \\
\text { Detected } \\
\text { Concentration } \\
(\mu \mathrm{g} / \mathrm{L})\end{array}$ & $\begin{array}{c}\text { Maximum } \\
\text { Detected } \\
\text { Concentration } \\
(\mu \mathrm{g} / \mathrm{L})\end{array}$ & $\begin{array}{c}\text { Upland Stream } \\
\text { Background } \\
\text { Concentration }{ }^{\mathrm{a}} \\
(\mu \mathrm{g} / \mathrm{L})\end{array}$ & $\begin{array}{c}\text { Human } \\
\text { Health } \\
\text { Screening } \\
\text { Value } \\
(\mu \mathrm{g} / \mathrm{L})\end{array}$ & $\begin{array}{c}\text { Human Health } \\
\text { Constituent of } \\
\text { Concern }\end{array}$ \\
\hline $\begin{array}{l}\text { Diesel-Range } \\
\text { Hydrocarbons }\end{array}$ & 3 of 3 & 56.2 & 119 & NAN & $1,500^{d}$ & $\mathrm{No}^{\mathrm{h}}$ \\
\hline \multicolumn{7}{|c|}{ Volatile Organic Compounds } \\
\hline 1,2,4-Trimethylbenzene & 0 of 3 & ND (1.0) & ND (1.0) & NAN & $12^{\mathrm{e}}$ & $\mathrm{No}^{f}$ \\
\hline 1,3,5-Trimethylbenzene & 0 of 3 & ND (1.0) & ND (1.0) & NAN & $12^{\mathrm{e}}$ & $\mathrm{No}^{\dagger}$ \\
\hline 2-Butanone & 0 of 3 & ND (20) & ND (20) & NAN & $1,900^{\mathrm{e}}$ & $\mathrm{No}^{f}$ \\
\hline Acetone & 0 of 3 & ND (20) & ND (20) & NAN & $3,700^{\mathrm{e}}$ & $\mathrm{No}^{f}$ \\
\hline Benzene & 0 of 3 & ND (1.0) & ND (1.0) & NAN & $1.2^{\mathrm{c}}$ & $\mathrm{No}^{\dagger}$ \\
\hline Carbon disulfide & 0 of 3 & ND (1.0) & ND (1.0) & NAN & $1,000^{\mathrm{e}}$ & $\mathrm{No}^{f}$ \\
\hline Ethylbenzene & 0 of 3 & ND (1.0) & ND (1.0) & NAN & $3,100^{c}$ & $\mathrm{No}^{f}$ \\
\hline Isopropylbenzene & 0 of 3 & ND (1.0) & ND (1.0) & NAN & NA & $\mathrm{No}^{f}$ \\
\hline $\mathrm{m}, \mathrm{p}$-Xylene & 0 of 3 & ND (2.0) & ND (2.0) & NAN & $12,000^{\mathrm{e}}$ & $\mathrm{No}^{f}$ \\
\hline Methylene chloride & 0 of 3 & ND (3.17) & ND (4.42) & NAN & $4.7^{\mathrm{C}}$ & $\mathrm{No}^{\dagger}$ \\
\hline n-Butylbenzene & 0 of 3 & ND (1.0) & ND (1.0) & NAN & $61^{e}$ & $\mathrm{No}^{f}$ \\
\hline n-Propylbenzene & 0 of 3 & ND (1.0) & ND (1.0) & NAN & $61^{e}$ & $\mathrm{No}^{f}$ \\
\hline Naphthalene & 0 of 3 & ND (1.0) & ND (1.0) & NAN & $730^{e}$ & $\mathrm{No}^{f}$ \\
\hline o-Xylene & 0 of 3 & ND (1.0) & ND (1.0) & NAN & $12,000^{\mathrm{e}}$ & $\mathrm{No}^{f}$ \\
\hline p-Isopropyltoluene & 0 of 3 & ND (1.0) & ND (1.0) & NAN & NA & $\mathrm{No}^{f}$ \\
\hline sec-Butylbenzene & 0 of 3 & ND (1.0) & ND (1.0) & NAN & $61^{\mathrm{e}}$ & $\mathrm{No}^{f}$ \\
\hline Toluene & 0 of 3 & ND (1.0) & ND (1.0) & NAN & $10,000^{c}$ & $\mathrm{No}^{f}$ \\
\hline \multicolumn{7}{|c|}{ Polycyclic Aromatic Hydrocarbons } \\
\hline Acenaphthene & 0 of 3 & $\mathrm{ND}(0.1)$ & ND (0.1) & NAN & $1,200^{c}$ & $\mathrm{No}^{f}$ \\
\hline Acenaphthylene & 0 of 3 & ND $(0.1)$ & ND $(0.1)$ & NAN & $0.0028^{c}$ & $\mathrm{No}^{f}$ \\
\hline Anthracene & 0 of 3 & $\mathrm{ND}(0.1)$ & ND $(0.1)$ & NAN & $0.0028^{c}$ & $\mathrm{No}^{f}$ \\
\hline Benzo(a)anthracene & 0 of 3 & $\mathrm{ND}(0.1)$ & ND $(0.1)$ & NAN & $0.0028^{c}$ & $\mathrm{No}^{f}$ \\
\hline Benzo(a)pyrene & 0 of 3 & ND (0.1) & $\mathrm{ND}(0.1)$ & NAN & $0.0028^{c}$ & $\mathrm{No}^{f}$ \\
\hline Benzo(b)fluoranthene & 0 of 3 & $\mathrm{ND}(0.1)$ & $\mathrm{ND}(0.1)$ & NAN & $0.092^{\mathrm{e}}$ & $\mathrm{No}^{f}$ \\
\hline Benzo(ghi)perylene & 0 of 3 & ND $(0.1)$ & ND $(0.1)$ & NAN & $0.0028^{c}$ & $\mathrm{No}^{f}$ \\
\hline Benzo(k)fluoranthene & 0 of 3 & ND $(0.1)$ & ND $(0.1)$ & NAN & $0.0028^{c}$ & $\mathrm{No}^{f}$ \\
\hline Chrysene & 0 of 3 & ND $(0.1)$ & ND $(0.1)$ & NAN & $0.0028^{c}$ & $\mathrm{No}^{f}$ \\
\hline
\end{tabular}


Table 4-4

\section{Summary of Analytical Results and Human Health Risk Assessment Screening for Surface Water in Lake, Drill Site D}

(Page 2 of 3 )

\begin{tabular}{|c|c|c|c|c|c|c|}
\hline Analyte & $\begin{array}{l}\text { Detection } \\
\text { Frequency }\end{array}$ & $\begin{array}{c}\text { Minimum } \\
\text { Detected } \\
\text { Concentration } \\
(\mu \mathrm{g} / \mathrm{L})\end{array}$ & $\begin{array}{c}\text { Maximum } \\
\text { Detected } \\
\text { Concentration } \\
(\mu \mathrm{g} / \mathrm{L})\end{array}$ & $\begin{array}{c}\text { Upland Stream } \\
\text { Background } \\
\text { Concentration }^{\mathrm{a}} \\
(\mu \mathrm{g} / \mathrm{L})\end{array}$ & $\begin{array}{c}\text { Human } \\
\text { Health } \\
\text { Screening } \\
\text { Value } \\
(\mu \mathrm{g} / \mathrm{L})\end{array}$ & $\begin{array}{c}\text { Human Health } \\
\text { Constituent of } \\
\text { Concern }\end{array}$ \\
\hline Dibenzo(a,h)anthracene & 0 of 3 & ND $(0.1)$ & ND $(0.1)$ & NAN & $0.0028^{C}$ & $\mathrm{No}^{f}$ \\
\hline Fluoranthene & 0 of 3 & ND (0.1) & ND $(0.1)$ & NAN & $42^{c}$ & $\mathrm{No}^{f}$ \\
\hline Fluorene & 0 of 3 & $\mathrm{ND}(0.1)$ & $\mathrm{ND}(0.1)$ & NAN & $0.0028^{c}$ & $\mathrm{No}^{f}$ \\
\hline Indeno(1,2,3-cd)pyrene & 0 of 3 & $\mathrm{ND}(0.1)$ & $\mathrm{ND}(0.1)$ & NAN & $0.0028^{C}$ & $\mathrm{No}^{f}$ \\
\hline Naphthalene & 0 of 3 & ND $(0.1)$ & $\mathrm{ND}(0.1)$ & NAN & $730^{e}$ & $\mathrm{No}^{f}$ \\
\hline Phenanthrene & 0 of 3 & ND $(0.1)$ & ND $(0.1)$ & NAN & $0.0028^{c}$ & $\mathrm{No}^{f}$ \\
\hline Pyrene & 0 of 3 & $\mathrm{ND}(0.1)$ & ND $(0.1)$ & NAN & $0.0028^{c}$ & $\mathrm{No}^{f}$ \\
\hline \multicolumn{7}{|c|}{ Polychlorinated Biphenyls } \\
\hline Aroclor 1016 & NAV & NAV & NAV & NAN & $0.000044^{c}$ & $\mathrm{No}^{f}$ \\
\hline Aroclor 1221 & NAV & NAV & NAV & NAN & $0.000044^{c}$ & $\mathrm{No}^{f}$ \\
\hline Aroclor 1232 & NAV & NAV & NAV & NAN & $0.000044^{c}$ & $\mathrm{No}^{f}$ \\
\hline Aroclor 1242 & NAV & NAV & NAV & NAN & $0.000044^{c}$ & $\mathrm{No}^{f}$ \\
\hline Aroclor 1248 & NAV & NAV & NAV & NAN & $0.000044^{c}$ & $\mathrm{No}^{f}$ \\
\hline Aroclor 1254 & NAV & NAV & NAV & NAN & $0.000044^{c}$ & $\mathrm{No}^{f}$ \\
\hline Aroclor 1260 & NAV & NAV & NAV & NAN & $0.000044^{c}$ & $\mathrm{No}^{f}$ \\
\hline Aroclor 1262 & NAV & NAV & NAV & NAN & $0.000044^{\mathrm{C}}$ & $\mathrm{No}^{f}$ \\
\hline Aroclor 1268 & NAV & NAV & NAV & NAN & $0.000044^{\mathrm{C}}$ & $\mathrm{No}^{f}$ \\
\hline \multicolumn{7}{|c|}{ Metals } \\
\hline Aluminum & 2 of 3 & ND (50) & 186 & $87.1^{b}$ & $37,000^{\mathrm{e}}$ & $\mathrm{No}^{\mathrm{h}}$ \\
\hline Arsenic & 0 of 3 & ND (1.0) & ND (1.0) & ND (1) & $0.018^{c}$ & $\mathrm{No}^{f}$ \\
\hline Barium & 0 of 3 & ND (10) & ND (10) & ND (10) & $1,000^{\mathrm{C}}$ & $\mathrm{No}^{f}$ \\
\hline Beryllium & 0 of 3 & ND (1.0) & ND (1.0) & ND (1) & $0.0076^{c}$ & $\mathrm{No}^{f}$ \\
\hline Boron & 0 of 3 & ND (50) & ND (50) & ND (50) & $3,300^{\mathrm{e}}$ & $\mathrm{No}^{f}$ \\
\hline Cadmium & 0 of 3 & ND (1.0) & ND (1.0) & ND (1) & $10^{c}$ & $\mathrm{No}^{f}$ \\
\hline Calcium & 3 of 3 & 4,700 & 5,700 & $8,810^{b}$ & NAV & $\mathrm{No}^{\mathrm{g}}$ \\
\hline Cerium & 0 of 3 & ND (1.0) & ND (1.0) & ND (1) & NAV & $\mathrm{No}^{f}$ \\
\hline Cesium & 0 of 3 & ND (1.0) & ND (1.0) & ND (1) & NAV & $\mathrm{No}^{f}$ \\
\hline Chromium & 3 of 3 & 10.5 & 11.5 & $3.49^{b}$ & $33,000^{c}$ & $\mathrm{No}^{\mathrm{h}}$ \\
\hline Cobalt & 3 of 3 & 4.66 & 5.18 & 9.52 & $2,200^{\mathrm{e}}$ & $\mathrm{No}^{\mathrm{g}}$ \\
\hline
\end{tabular}




\section{Table 4-4}

\section{Summary of Analytical Results and Human Health Risk Assessment Screening for Surface Water in Lake, Drill Site D} (Page 3 of 3 )

\begin{tabular}{|c|c|c|c|c|c|c|}
\hline Analyte & $\begin{array}{l}\text { Detection } \\
\text { Frequency }\end{array}$ & $\begin{array}{c}\text { Minimum } \\
\text { Detected } \\
\text { Concentration } \\
(\mu \mathrm{g} / \mathrm{L})\end{array}$ & $\begin{array}{c}\text { Maximum } \\
\text { Detected } \\
\text { Concentration } \\
(\mu \mathrm{g} / \mathrm{L})\end{array}$ & $\begin{array}{l}\text { Upland Stream } \\
\text { Background } \\
\text { Concentration }^{a} \\
(\mu \mathrm{g} / \mathrm{L})\end{array}$ & $\begin{array}{c}\text { Human } \\
\text { Health } \\
\text { Screening } \\
\text { Value } \\
(\mu \mathrm{g} / \mathrm{L})\end{array}$ & $\begin{array}{c}\text { Human Health } \\
\text { Constituent of } \\
\text { Concern }\end{array}$ \\
\hline Copper & 3 of 3 & 3.33 & 3.63 & $13.4^{b}$ & $1,300^{\mathrm{C}}$ & $\mathrm{No}^{\mathrm{g}}$ \\
\hline Iron & 0 of 3 & ND (150) & ND (150) & $2,830^{b}$ & $300^{c}$ & $\mathrm{No}^{f}$ \\
\hline Lithium & 3 of 3 & 1.12 & 1.18 & ND (1) & $730^{e}$ & $\mathrm{No}^{\mathrm{h}}$ \\
\hline Magnesium & 3 of 3 & 4,220 & 4,340 & 5,250 & NAV & $\mathrm{No}^{\mathrm{g}}$ \\
\hline Manganese & 3 of 3 & 17.2 & 28.9 & 748 & $50^{c}$ & $\mathrm{No}^{\mathrm{g}}$ \\
\hline Molybdenum & 0 of 3 & ND (1.0) & ND (1.0) & ND (1) & $180^{\mathrm{e}}$ & $\mathrm{No}^{f}$ \\
\hline Nickel & 3 of 3 & 1.6 & 1.92 & $1.69^{b}$ & $510^{c}$ & $\mathrm{No}^{\mathrm{h}}$ \\
\hline Potassium & 3 of 3 & 1,310 & 2,080 & $1,190^{b}$ & NAV & $\mathrm{No}^{\mathrm{i}}$ \\
\hline Selenium & 0 of 3 & ND (1.0) & ND (1.0) & ND (1) & $104^{c}$ & $\mathrm{No}^{f}$ \\
\hline Strontium & 3 of 3 & 38.4 & 39.9 & $44.1^{\mathrm{b}}$ & $22,000^{\mathrm{e}}$ & $\mathrm{No}^{\mathrm{g}}$ \\
\hline Thallium & 0 of 3 & ND (1.0) & ND (1.0) & $2.13^{b}$ & $1.7^{\mathrm{C}}$ & $\mathrm{No}^{f}$ \\
\hline Thorium & 0 of 3 & ND (1.0) & ND (1.0) & $1.14^{\mathrm{b}}$ & NAV & $\mathrm{No}^{f}$ \\
\hline Titanium & 3 of 3 & 2.46 & 8.83 & $2.37^{\mathrm{b}}$ & $150,000^{e}$ & $\mathrm{No}^{\mathrm{h}}$ \\
\hline Uranium & 0 of 3 & ND (1.0) & ND (1.0) & ND (1) & $110^{e}$ & $\mathrm{No}^{f}$ \\
\hline Vanadium & 3 of 3 & 1.44 & 1.65 & $1.44^{\mathrm{b}}$ & $260^{\mathrm{e}}$ & $\mathrm{No}^{\mathrm{h}}$ \\
\hline Zinc & 0 of 3 & ND (10) & ND (10) & $12.8^{b}$ & $11,000^{\mathrm{e}}$ & $\mathrm{No}^{\dagger}$ \\
\hline
\end{tabular}

${ }^{\mathrm{a}}$ Background concentrations are upper tolerance limits (UTL) (ADEC, 1999a) unless otherwise noted.

${ }^{b}$ Maximum detected concentration in background samples due to insufficient detection frequency to calculate UTL.

${ }^{\mathrm{C}}$ Federal ambient water quality criteria based on ingestion of water and organisms.

dState of Alaska groundwater cleanup level, Discharge Reporting, Cleanup, and Disposal of Oil and other Hazardous Substances (18 AAC 75, Articles 3 and 9 [ADEC, 1999c]).

eU.S. EPA Region III risk-based concentrations for tap water (EPA, 2000b).

\section{COC Selection Criteria}

${ }^{7}$ Constituent not detected in any samples.

${ }^{9}$ Maximum detected constituent concentration is less than local background concentration.

${ }^{h}$ Maximum detected constituent concentration is less than human health screening value.

'Constituent is an essential macronutrient and only presents a risk at extremely high concentrations.

ND - Not detected (detection limit)

NAV - Not available

NAN - Not analyzed

$\mu \mathrm{g} / \mathrm{L}$ - Micrograms per liter 
Table 4-5

\section{Summary of Analytical Results and Human Health Risk Assessment Screening for Sediment in Lake, Drill Site D}

(Page 1 of 3 )

\begin{tabular}{|c|c|c|c|c|c|c|}
\hline Analyte & $\begin{array}{l}\text { Detection } \\
\text { Frequency }\end{array}$ & $\begin{array}{c}\text { Minimum } \\
\text { Detected } \\
\text { Concentration } \\
(\mathbf{m g} / \mathbf{k g})\end{array}$ & $\begin{array}{c}\text { Maximum } \\
\text { Detected } \\
\text { Concentration } \\
(\mathbf{m g} / \mathbf{k g})\end{array}$ & $\begin{array}{c}\text { Upland Stream } \\
\text { Background } \\
\text { Concentration } \\
\text { (mg/kg) }\end{array}$ & $\begin{array}{l}\text { Human Health } \\
\text { Screening } \\
\text { Value } \\
(\mathrm{mg} / \mathrm{kg})\end{array}$ & $\begin{array}{l}\text { Human } \\
\text { Health } \\
\text { Constituent } \\
\text { of Concern }\end{array}$ \\
\hline $\begin{array}{l}\text { Diesel-Range } \\
\text { Hydrocarbons }\end{array}$ & 5 of 5 & 66.2 & 146 & NAN & $200^{c}$ & $\mathrm{No}^{f}$ \\
\hline \multicolumn{7}{|c|}{ Volatile Organic Compounds } \\
\hline 1,2,4-Trimethylbenzene & 0 of 5 & ND (0.005) & ND (0.0248) & NAN & NAV & $\mathrm{No}^{\mathrm{d}}$ \\
\hline 1,3,5-Trimethylbenzene & 0 of 5 & ND $(0.005)$ & ND (0.0248) & NAN & NAV & $\mathrm{No}^{\mathrm{d}}$ \\
\hline 2-Butanone & 1 of 5 & ND $(0.01)$ & 0.0496 & NAN & NAV & Yes \\
\hline Acetone & 0 of 5 & ND (0.4) & ND (13.9) & NAN & $10,100^{c}$ & $\mathrm{No}^{\mathrm{d}}$ \\
\hline Benzene & 0 of 5 & ND $(0.005)$ & ND $(0.0248)$ & NAN & $286^{\mathrm{C}}$ & $\mathrm{No}^{\mathrm{d}}$ \\
\hline Carbon disulfide & 0 of 5 & ND (0.018) & ND $(0.197)$ & NAN & $10,100^{c}$ & $\mathrm{No}^{f}$ \\
\hline Ethylbenzene & 0 of 5 & ND (0.005) & ND $(0.0248)$ & NAN & $10,100^{c}$ & $\mathrm{No}^{d}$ \\
\hline Isopropylbenzene & 0 of 5 & ND $(0.005)$ & ND $(0.0248)$ & NAN & NAV & $\mathrm{No}^{d}$ \\
\hline m,p-Xylene & 0 of 5 & ND $(0.005)$ & ND $(0.0248)$ & NAN & $203,000^{c}$ & $\mathrm{No}^{d}$ \\
\hline Methylene chloride & 0 of 5 & ND (0.005) & ND (0.0248) & NAN & $1,110^{\mathrm{C}}$ & $\mathrm{No}^{d}$ \\
\hline n-Butylbenzene & 0 of 5 & ND (0.005) & ND $(0.0248)$ & NAN & NAV & $\mathrm{No}^{\mathrm{d}}$ \\
\hline n-Propylbenzene & 0 of 5 & ND (0.005) & ND (0.0248) & NAN & NAV & $\mathrm{No}^{d}$ \\
\hline Naphthalene & 0 of 5 & ND (0.005) & ND (0.0248) & NAN & $4,060^{c}$ & $\mathrm{No}^{d}$ \\
\hline o-Xylene & 0 of 5 & ND (0.005) & ND (0.0248) & NAN & $203,000^{\mathrm{C}}$ & $\mathrm{No}^{d}$ \\
\hline p-Isopropyltoluene & 0 of 5 & ND (0.005) & ND (0.0248) & NAN & NAV & $\mathrm{No}^{d}$ \\
\hline sec-Butylbenzene & 0 of 5 & ND (0.005) & ND (0.0248) & NAN & NAV & $\mathrm{No}^{\mathrm{d}}$ \\
\hline Toluene & 0 of 5 & ND (0.005) & ND (0.0248) & NAN & $20,300^{c}$ & $\mathrm{No}^{d}$ \\
\hline \multicolumn{7}{|c|}{ Polycyclic Aromatic Hydrocarbons } \\
\hline Acenaphthene & 0 of 5 & ND (0.0033) & ND (0.0163) & NAN & $6,080^{c}$ & $\mathrm{No}^{\mathrm{d}}$ \\
\hline Acenaphthylene & 0 of 5 & ND (0.0033) & ND $(0.0163)$ & NAN & NAV & $\mathrm{No}^{d}$ \\
\hline Anthracene & 1 of 5 & ND $(0.0033)$ & 0.0216 & NAN & $30,400^{c}$ & $\mathrm{No}^{f}$ \\
\hline Benzo(a)anthracene & 0 of 5 & ND (0.0033) & ND $(0.0163)$ & NAN & $11.4^{\mathrm{c}}$ & $\mathrm{No}^{\mathrm{d}}$ \\
\hline Benzo(a)pyrene & 0 of 5 & ND (0.0033) & ND (0.0163) & NAN & $1.1^{\mathrm{c}}$ & $\mathrm{No}^{d}$ \\
\hline Benzo(b)fluoranthene & 0 of 5 & ND (0.0033) & ND $(0.0163)$ & NAN & $11.4^{\mathrm{c}}$ & $\mathrm{No}^{d}$ \\
\hline Benzo(ghi)perylene & 0 of 5 & ND (0.0033) & ND $(0.0163)$ & NAN & $1.1^{\mathrm{c}}$ & $\mathrm{No}^{d}$ \\
\hline Benzo(k)fluoranthene & 0 of 5 & ND (0.0033) & ND (0.0163) & NAN & $114^{\mathrm{C}}$ & $\mathrm{No}^{d}$ \\
\hline Chrysene & 0 of 5 & ND (0.0033) & ND (0.0163) & NAN & $1,140^{\mathrm{C}}$ & $\mathrm{No}^{d}$ \\
\hline
\end{tabular}


Table 4-5

\section{Summary of Analytical Results and Human Health Risk Assessment Screening for Sediment in Lake, Drill Site D}

(Page 2 of 3 )

\begin{tabular}{|c|c|c|c|c|c|c|}
\hline Analyte & $\begin{array}{l}\text { Detection } \\
\text { Frequency }\end{array}$ & $\begin{array}{c}\text { Minimum } \\
\text { Detected } \\
\text { Concentration } \\
(\mathbf{m g} / \mathbf{k g})\end{array}$ & $\begin{array}{c}\text { Maximum } \\
\text { Detected } \\
\text { Concentration } \\
(\mathbf{m g} / \mathbf{k g})\end{array}$ & $\begin{array}{c}\text { Upland Stream } \\
\text { Background } \\
\text { Concentration } \\
\text { (mg/kg) }\end{array}$ & $\begin{array}{l}\text { Human Health } \\
\text { Screening } \\
\text { Value } \\
(\mathrm{mg} / \mathrm{kg})\end{array}$ & $\begin{array}{c}\text { Human } \\
\text { Health } \\
\text { Constituent } \\
\text { of Concern }\end{array}$ \\
\hline Dibenzo(a,h)anthracene & 0 of 5 & ND (0.0033) & ND (0.0163) & NAN & $1.1^{\mathrm{c}}$ & No ${ }^{d}$ \\
\hline Fluoranthene & 0 of 5 & ND (0.0033) & ND $(0.0163)$ & NAN & $4,060^{c}$ & Nod \\
\hline Fluorene & 2 of 5 & ND (0.0033) & 0.0432 & NAN & $4,060^{c}$ & $\mathrm{No}^{f}$ \\
\hline Indeno(1,2,3-cd)pyrene & 0 of 5 & ND (0.0033) & ND (0.0163) & NAN & $11^{\mathrm{c}}$ & $\mathrm{No}^{d}$ \\
\hline Naphthalene & 0 of 5 & ND (0.0033) & ND (0.0163) & NAN & $4,060^{\mathrm{C}}$ & $\mathrm{No}^{d}$ \\
\hline Phenanthrene & 4 of 5 & ND (0.0033) & 0.207 & NAN & $1.1^{\mathrm{c}}$ & $\mathrm{No}^{f}$ \\
\hline Pyrene & 2 of 5 & ND (0.0033) & 0.036 & NAN & $3,040^{c}$ & $\mathrm{No}^{f}$ \\
\hline \multicolumn{7}{|c|}{ Polychlorinated Biphenyls } \\
\hline Aroclor 1016 & NAV & NAV & NAV & NAN & $10^{\mathrm{C}}$ & $\mathrm{No}^{\mathrm{d}}$ \\
\hline Aroclor 1221 & NAV & NAV & NAV & NAN & $10^{\mathrm{C}}$ & $\mathrm{No}^{\mathrm{d}}$ \\
\hline Aroclor 1232 & NAV & NAV & NAV & NAN & $10^{\mathrm{C}}$ & $\mathrm{No}^{d}$ \\
\hline Aroclor 1242 & NAV & NAV & NAV & NAN & $10^{\mathrm{c}}$ & $\mathrm{No}^{d}$ \\
\hline Aroclor 1248 & NAV & NAV & NAV & NAN & $10^{c}$ & No ${ }^{d}$ \\
\hline Aroclor 1254 & NAV & NAV & NAV & NAN & $10^{c}$ & $\mathrm{No}^{d}$ \\
\hline Aroclor 1260 & NAV & NAV & NAV & NAN & $10^{\mathrm{C}}$ & $\mathrm{No}^{d}$ \\
\hline Aroclor 1262 & NAV & NAV & NAV & NAN & $10^{c}$ & $\mathrm{No}^{d}$ \\
\hline Aroclor 1268 & NAV & NAV & NAV & NAN & $10^{c}$ & $\mathrm{No}^{d}$ \\
\hline \multicolumn{7}{|c|}{ Metals } \\
\hline Aluminum & 5 of 5 & 14,400 & 22,000 & 47,500 & NAV & $\mathrm{No}^{\mathrm{e}}$ \\
\hline Arsenic & 4 of 5 & ND (0.5) & 5.08 & 30.5 & $5.5^{c}$ & $\mathrm{No}^{\mathrm{e}}$ \\
\hline Barium & 5 of 5 & 38.1 & 515 & 468 & $7,100^{c}$ & $\mathrm{No}^{f}$ \\
\hline Beryllium & 0 of 5 & ND (0.5) & ND (2.48) & ND & $1.9^{\mathrm{c}}$ & $\mathrm{No}^{d}$ \\
\hline Boron & 0 of 5 & ND (2.5) & ND (12.4) & $6.42^{b}$ & NAV & $\mathrm{No}^{d}$ \\
\hline Cadmium & 0 of 5 & $\mathrm{ND}(0.5)$ & ND (2.48) & ND & $101^{\mathrm{C}}$ & No ${ }^{d}$ \\
\hline Calcium & 5 of 5 & 6,710 & 10,500 & 7,720 & NAV & $\mathrm{No}^{\mathrm{g}}$ \\
\hline Cerium & 5 of 5 & 9.45 & 61.4 & 13 & NAV & $\mathrm{No}^{\mathrm{h}}$ \\
\hline Cesium & 0 of 5 & ND (0.5) & ND (2.48) & ND & NAV & $\mathrm{No}^{d}$ \\
\hline Chromium & 5 of 5 & 16.1 & 695 & 18.9 & $507^{c}$ & Yes \\
\hline Cobalt & 5 of 5 & 2.63 & 9.83 & 37.3 & NAV & $\mathrm{No}^{\mathrm{e}}$ \\
\hline Copper & 5 of 5 & 25.7 & 98.3 & 82.4 & NAV & $\mathrm{No}^{\mathrm{h}}$ \\
\hline
\end{tabular}


Table 4-5

\section{Summary of Analytical Results and Human Health Risk Assessment Screening for Sediment in Lake, Drill Site D}

(Page 3 of 3 )

\begin{tabular}{|c|c|c|c|c|c|c|}
\hline Analyte & $\begin{array}{l}\text { Detection } \\
\text { Frequency }\end{array}$ & $\begin{array}{c}\text { Minimum } \\
\text { Detected } \\
\text { Concentration } \\
(\mathbf{m g} / \mathbf{k g})\end{array}$ & $\begin{array}{c}\text { Maximum } \\
\text { Detected } \\
\text { Concentration } \\
(\mathbf{m g} / \mathbf{k g})\end{array}$ & $\begin{array}{c}\text { Upland Stream } \\
\text { Background } \\
\text { Concentration } \\
\text { (mg/kg) }\end{array}$ & $\begin{array}{l}\text { Human Health } \\
\text { Screening } \\
\text { Value } \\
(\mathrm{mg} / \mathrm{kg})\end{array}$ & $\begin{array}{c}\text { Human } \\
\text { Health } \\
\text { Constituent } \\
\text { of Concern }\end{array}$ \\
\hline Iron & 5 of 5 & 21,600 & 32,700 & 123,000 & NAV & $\mathrm{No}^{\mathrm{e}}$ \\
\hline Lithium & 5 of 5 & 1.83 & 6.31 & 21.7 & NAV & $\mathrm{No}^{\mathrm{e}}$ \\
\hline Magnesium & 5 of 5 & 881 & 8,370 & 17,000 & NAV & $\mathrm{No}^{\mathrm{e}}$ \\
\hline Manganese & 5 of 5 & 98.7 & 783 & 26,500 & NAV & $\mathrm{No}^{\mathrm{e}}$ \\
\hline Molybdenum & 0 of 5 & ND (0.5) & ND (3.6) & ND & NAV & $\mathrm{No}^{d}$ \\
\hline Nickel & 5 of 5 & 5.78 & 15.9 & 23.8 & $2,030^{c}$ & $\mathrm{No}^{\mathrm{e}}$ \\
\hline Potassium & 5 of 5 & 216 & 2,020 & 1,290 & NAV & $\mathrm{No}^{\mathrm{g}}$ \\
\hline Selenium & 0 of 5 & ND (0.5) & ND (2.48) & $3.15^{b}$ & $507^{c}$ & $\mathrm{No}^{d}$ \\
\hline Strontium & 5 of 5 & 59.4 & 200 & 207 & NAV & $\mathrm{No}^{\mathrm{e}}$ \\
\hline Thallium & 0 of 5 & ND (0.5) & ND (2.48) & ND & NAV & $\mathrm{No}^{d}$ \\
\hline Thorium & 5 of 5 & 0.91 & 16.8 & ND & NAV & $\mathrm{No}^{h}$ \\
\hline Titanium & 5 of 5 & 416 & 1,950 & 1,480 & NAV & $\mathrm{No}^{\mathrm{h}}$ \\
\hline Uranium & 4 of 5 & ND (0.5) & 6.22 & ND & NAV & $\mathrm{No}^{\mathrm{h}}$ \\
\hline Vanadium & 5 of 5 & 32 & 99.7 & 336 & $710^{c}$ & $\mathrm{No}^{\mathrm{e}}$ \\
\hline Zinc & 5 of 5 & 13.6 & 73.4 & 185 & $30,400^{c}$ & $\mathrm{No}^{\mathrm{e}}$ \\
\hline
\end{tabular}

${ }^{a}$ Background concentrations are upper tolerance limits (UTL) (ADEC, 1999a) unless otherwise noted.

${ }^{\mathrm{b}}$ Maximum detected concentration in background samples due to insufficient detection frequency to calculate UTL.

'State of Alaska soil cleanup level, Discharge Reporting, Cleanup, and Disposal of Oil and other Hazardous Substances

(18 AAC 75, Articles 3 and 9 [ADEC, 1999c]).

\section{COC Selection Criteria}

${ }^{\mathrm{d}}$ Constituent not detected in any samples.

${ }^{\mathrm{e}}$ Maximum detected constituent concentration is less than local background concentration.

${ }^{f}$ Maximum detected constituent concentration is less than human health screening value.

${ }^{g}$ Constituent is an essential macronutrient and only presents a risk at extremely high concentrations.

${ }^{\mathrm{h}}$ Not a constituent associated with drilling mud or other drilling activities.

ND - Not detected (detection limit)

NAV - Not available

NAN - Non analyzed

$\mathrm{mg} / \mathrm{kg}$ - Milligrams per kilogram 
Table 4-6

\section{Summary of Analytical Results and Human Health Risk Assessment Screening for Surface Water in Drill Site E Stream}

(Page 1 of 3 )

\begin{tabular}{|c|c|c|c|c|c|c|}
\hline Analyte & $\begin{array}{l}\text { Detection } \\
\text { Frequency }\end{array}$ & $\begin{array}{c}\text { Minimum } \\
\text { Detected } \\
\text { Concentration } \\
(\mu \mathrm{g} / \mathrm{L})\end{array}$ & $\begin{array}{c}\text { Maximum } \\
\text { Detected } \\
\text { Concentration } \\
(\mu \mathrm{g} / \mathrm{L})\end{array}$ & $\begin{array}{c}\text { Upland Stream } \\
\text { Background } \\
\text { Concentration }{ }^{a} \\
(\mu \mathrm{g} / \mathrm{L})\end{array}$ & $\begin{array}{l}\text { Human Health } \\
\text { Screening } \\
\text { Value } \\
(\mu \mathrm{g} / \mathrm{L})\end{array}$ & $\begin{array}{l}\text { Human Health } \\
\text { Constituent of } \\
\text { Concern }\end{array}$ \\
\hline $\begin{array}{l}\text { Diesel-Range } \\
\text { Hydrocarbons }\end{array}$ & 5 of 6 & ND (50) & 104 & NAN & $1,500^{d}$ & $\mathrm{No}^{h}$ \\
\hline \multicolumn{7}{|c|}{ Volatile Organic Compounds } \\
\hline 1,2,4-Trimethylbenzene & 0 of 6 & ND (1.0) & ND (1.0) & NAN & $12^{\mathrm{e}}$ & $\mathrm{No}^{f}$ \\
\hline 1,3,5-Trimethylbenzene & 0 of 6 & ND (1.0) & ND (1.0) & NAN & $12^{\mathrm{e}}$ & $\mathrm{No}^{f}$ \\
\hline 2-Butanone & 0 of 6 & ND (20) & ND (20) & NAN & $1,900^{\mathrm{e}}$ & $\mathrm{No}^{f}$ \\
\hline Acetone & 0 of 6 & ND (20) & ND (20) & NAN & $3,700^{e}$ & $\mathrm{No}^{f}$ \\
\hline Benzene & 0 of 6 & ND (1.0) & ND (1.0) & NAN & $1.2^{\mathrm{c}}$ & $\mathrm{No}^{f}$ \\
\hline Carbon disulfide & 0 of 6 & ND (1.0) & ND (1.0) & NAN & $1,000^{\mathrm{e}}$ & $\mathrm{No}^{f}$ \\
\hline Ethylbenzene & 0 of 6 & ND (1.0) & ND (1.0) & NAN & $3,100^{c}$ & $\mathrm{No}^{f}$ \\
\hline Isopropylbenzene & 0 of 6 & ND (1.0) & ND (1.0) & NAN & NAV & $\mathrm{No}^{f}$ \\
\hline $\mathrm{m}, \mathrm{p}$-Xylene & 0 of 6 & ND (2.0) & ND (2.0) & NAN & $12,000^{\mathrm{e}}$ & $\mathrm{No}^{\dagger}$ \\
\hline Methylene chloride & 0 of 6 & ND (2.0) & ND (7.94) & NAN & $4.7^{\mathrm{C}}$ & $\mathrm{No}^{f}$ \\
\hline n-Butylbenzene & 0 of 6 & ND (1.0) & ND (1.0) & NAN & $61^{\mathrm{e}}$ & $\mathrm{No}^{f}$ \\
\hline n-Propylbenzene & 0 of 6 & ND (1.0) & ND (1.0) & NAN & $61^{e}$ & $\mathrm{No}^{\dagger}$ \\
\hline Naphthalene & 0 of 6 & ND (1.0) & ND (1.0) & NAN & $730^{e}$ & $\mathrm{No}^{\dagger}$ \\
\hline o-Xylene & 0 of 6 & ND (1.0) & ND (1.0) & NAN & $12,000^{\mathrm{e}}$ & $\mathrm{No}^{f}$ \\
\hline p-Isopropyltoluene & 0 of 6 & ND (1.0) & ND (1.0) & NAN & NAV & $\mathrm{No}^{\dagger}$ \\
\hline sec-Butylbenzene & 0 of 6 & ND (1.0) & ND (1.0) & NAN & $61^{\mathrm{e}}$ & $\mathrm{No}^{f}$ \\
\hline Toluene & 0 of 6 & ND (1.0) & ND (1.0) & NAN & $10,000^{c}$ & $\mathrm{No}^{f}$ \\
\hline \multicolumn{7}{|c|}{ Polycyclic Aromatic Hydrocarbons } \\
\hline Acenaphthene & 0 of 6 & $\mathrm{ND}(0.1)$ & $\mathrm{ND}(0.1)$ & NAN & $1,200^{c}$ & $\mathrm{No}^{\dagger}$ \\
\hline Acenaphthylene & 0 of 6 & $\mathrm{ND}(0.1)$ & $\mathrm{ND}(0.1)$ & NAN & $0.0028^{C}$ & $\mathrm{No}^{f}$ \\
\hline Anthracene & 0 of 6 & $\mathrm{ND}(0.1)$ & $\mathrm{ND}(0.1)$ & NAN & $0.0028^{c}$ & $\mathrm{No}^{\dagger}$ \\
\hline Benzo(a)anthracene & 0 of 6 & $\mathrm{ND}(0.1)$ & $\mathrm{ND}(0.1)$ & NAN & $0.0028^{c}$ & $\mathrm{No}^{f}$ \\
\hline Benzo(a)pyrene & 0 of 6 & $\mathrm{ND}(0.1)$ & $\mathrm{ND}(0.1)$ & NAN & $0.0028^{\mathrm{C}}$ & $\mathrm{No}^{f}$ \\
\hline Benzo(b)fluoranthene & 0 of 6 & $\mathrm{ND}(0.1)$ & $\mathrm{ND}(0.1)$ & NAN & $0.092^{\mathrm{e}}$ & $\mathrm{No}^{\dagger}$ \\
\hline Benzo(ghi)perylene & 0 of 6 & $\mathrm{ND}(0.1)$ & ND $(0.1)$ & NAN & $0.0028^{c}$ & $\mathrm{No}^{\dagger}$ \\
\hline Benzo(k)fluoranthene & 0 of 6 & $\mathrm{ND}(0.1)$ & $\mathrm{ND}(0.1)$ & NAN & $0.0028^{c}$ & $\mathrm{No}^{f}$ \\
\hline Chrysene & 0 of 6 & $\mathrm{ND}(0.1)$ & $\mathrm{ND}(0.1)$ & NAN & $0.0028^{c}$ & $\mathrm{No}^{f}$ \\
\hline Dibenzo(a,h)anthracene & 0 of 6 & $\mathrm{ND}(0.1)$ & $\mathrm{ND}(0.1)$ & NAN & $0.0028^{c}$ & $\mathrm{No}^{f}$ \\
\hline
\end{tabular}


Table 4-6

\section{Summary of Analytical Results and Human Health Risk Assessment Screening for Surface Water in Drill Site E Stream}

(Page 2 of 3 )

\begin{tabular}{|c|c|c|c|c|c|c|}
\hline Analyte & $\begin{array}{l}\text { Detection } \\
\text { Frequency }\end{array}$ & $\begin{array}{c}\text { Minimum } \\
\text { Detected } \\
\text { Concentration } \\
(\mu \mathrm{g} / \mathrm{L})\end{array}$ & $\begin{array}{c}\text { Maximum } \\
\text { Detected } \\
\text { Concentration } \\
(\mu \mathrm{g} / \mathrm{L})\end{array}$ & $\begin{array}{c}\text { Upland Stream } \\
\text { Background } \\
\text { Concentration } \\
(\mu \mathrm{g} / \mathrm{L})\end{array}$ & $\begin{array}{l}\text { Human Health } \\
\text { Screening } \\
\text { Value } \\
(\mu \mathrm{g} / \mathrm{L})\end{array}$ & $\begin{array}{c}\text { Human Health } \\
\text { Constituent of } \\
\text { Concern }\end{array}$ \\
\hline Fluoranthene & 0 of 6 & ND (0.1) & ND (0.1) & NAN & $42^{\mathrm{C}}$ & $\mathrm{No}^{\dagger}$ \\
\hline Fluorene & 0 of 6 & $\mathrm{ND}(0.1)$ & ND (0.1) & NAN & $0.0028^{C}$ & $\mathrm{No}^{f}$ \\
\hline Indeno(1,2,3-cd)pyrene & 0 of 6 & $\mathrm{ND}(0.1)$ & ND $(0.1)$ & NAN & $0.0028^{C}$ & $\mathrm{No}^{f}$ \\
\hline Naphthalene & 0 of 6 & ND $(0.1)$ & ND $(0.1)$ & NAN & $730^{\mathrm{e}}$ & $\mathrm{No}^{f}$ \\
\hline Phenanthrene & 0 of 6 & ND (0.1) & ND (0.1) & NAN & $0.0028^{\mathrm{C}}$ & $\mathrm{No}^{f}$ \\
\hline Pyrene & 0 of 6 & $\mathrm{ND}(0.1)$ & $\mathrm{ND}(0.1)$ & NAN & $0.0028^{c}$ & $\mathrm{No}^{f}$ \\
\hline \multicolumn{7}{|c|}{ Polychlorinated Biphenyls } \\
\hline Aroclor 1016 & NAV & NAV & NAV & NAN & $0.000044^{c}$ & $\mathrm{No}^{f}$ \\
\hline Aroclor 1221 & NAV & NAV & NAV & NAN & $0.000044^{c}$ & $\mathrm{No}^{\dagger}$ \\
\hline Aroclor 1232 & NAV & NAV & NAV & NAN & $0.000044^{c}$ & $\mathrm{No}^{f}$ \\
\hline Aroclor 1242 & NAV & NAV & NAV & NAN & $0.000044^{c}$ & $\mathrm{No}^{f}$ \\
\hline Aroclor 1248 & NAV & NAV & NAV & NAN & $0.000044^{c}$ & $\mathrm{No}^{f}$ \\
\hline Aroclor 1254 & NAV & NAV & NAV & NAN & $0.000044^{c}$ & $\mathrm{No}^{f}$ \\
\hline Aroclor 1260 & NAV & NAV & NAV & NAN & $0.000044^{c}$ & $\mathrm{No}^{f}$ \\
\hline Aroclor 1262 & NAV & NAV & NAV & NAN & $0.000044^{c}$ & $\mathrm{No}^{f}$ \\
\hline Aroclor 1268 & NAV & NAV & NAV & NAN & $0.000044^{c}$ & $\mathrm{No}^{f}$ \\
\hline \multicolumn{7}{|c|}{ Metals } \\
\hline Aluminum & 5 of 6 & ND (50) & 105 & $87.1^{b}$ & $37,000^{\mathrm{e}}$ & $\mathrm{No}^{h}$ \\
\hline Arsenic & 0 of 6 & ND (1.0) & ND (1.0) & $\mathrm{ND}(1)$ & $0.018^{c}$ & $\mathrm{No}^{f}$ \\
\hline Barium & 0 of 6 & ND (10) & ND (10) & ND (10) & $1,000^{\mathrm{C}}$ & $\mathrm{No}^{f}$ \\
\hline Beryllium & 0 of 6 & ND (1.0) & ND (1.0) & ND (1) & $0.0076^{c}$ & $\mathrm{No}^{f}$ \\
\hline Boron & 0 of 6 & ND (50) & ND (50) & ND (50) & $3,300^{\mathrm{e}}$ & $\mathrm{No}^{f}$ \\
\hline Cadmium & 0 of 6 & ND (1.0) & ND (1.0) & ND (1) & $10^{c}$ & $\mathrm{No}^{f}$ \\
\hline Calcium & 6 of 6 & 2,140 & 3,780 & $8,810^{b}$ & NAV & $\mathrm{No}^{\mathrm{g}}$ \\
\hline Cerium & 0 of 6 & ND (1.0) & ND (1.0) & ND (1) & NAV & $\mathrm{No}^{f}$ \\
\hline Cesium & 0 of 6 & ND (1.0) & ND (1.0) & ND (1) & NAV & $\mathrm{No}^{f}$ \\
\hline Chromium & 0 of 6 & ND (1.0) & ND (1.0) & $3.49^{b}$ & $33,000^{c}$ & $\mathrm{No}^{f}$ \\
\hline Cobalt & 5 of 6 & ND (1.0) & 4.74 & 9.52 & $2,200^{e}$ & $\mathrm{No}^{\mathrm{g}}$ \\
\hline Copper & 5 of 6 & ND (1.0) & 2.85 & $13.4^{b}$ & $1,300^{C}$ & $\mathrm{No}^{\mathrm{g}}$ \\
\hline Iron & 1 of 6 & ND (150) & 150 & $2,830^{b}$ & $300^{C}$ & $\mathrm{No}^{\mathrm{g}}$ \\
\hline Lithium & 0 of 6 & ND (1.0) & ND (1.0) & ND (1) & $730^{\mathrm{e}}$ & $\mathrm{No}^{f}$ \\
\hline
\end{tabular}


Table 4-6

\section{Summary of Analytical Results and Human Health Risk Assessment Screening for Surface Water in Drill Site E Stream}

(Page 3 of 3)

\begin{tabular}{|c|c|c|c|c|c|c|}
\hline Analyte & $\begin{array}{l}\text { Detection } \\
\text { Frequency }\end{array}$ & $\begin{array}{c}\text { Minimum } \\
\text { Detected } \\
\text { Concentration } \\
(\mu \mathrm{g} / \mathrm{L})\end{array}$ & $\begin{array}{c}\text { Maximum } \\
\text { Detected } \\
\text { Concentration } \\
(\mu \mathrm{g} / \mathrm{L})\end{array}$ & $\begin{array}{c}\text { Upland Stream } \\
\text { Background } \\
\text { Concentration }{ }^{\mathrm{a}} \\
(\mu \mathrm{g} / \mathrm{L})\end{array}$ & $\begin{array}{l}\text { Human Health } \\
\text { Screening } \\
\text { Value } \\
(\mu \mathrm{g} / \mathrm{L})\end{array}$ & $\begin{array}{c}\text { Human Health } \\
\text { Constituent of } \\
\text { Concern }\end{array}$ \\
\hline Magnesium & 6 of 6 & 1,690 & 2,850 & 5,250 & NAV & $\mathrm{No}^{\mathrm{g}}$ \\
\hline Manganese & 6 of 6 & 8.09 & 19.9 & 748 & $50^{c}$ & $\mathrm{No}^{\mathrm{g}}$ \\
\hline Molybdenum & 0 of 6 & ND (1.0) & ND (1.0) & ND (1) & $180^{\mathrm{e}}$ & $\mathrm{No}^{f}$ \\
\hline Nickel & 5 of 6 & ND (1.0) & 1.65 & $1.69^{b}$ & $510^{c}$ & $\mathrm{No}^{\mathrm{g}}$ \\
\hline Potassium & 6 of 6 & 947 & 1,610 & $1,190^{b}$ & NAV & $\mathrm{No}^{\mathrm{i}}$ \\
\hline Selenium & 0 of 6 & ND (1.0) & ND (1.0) & ND (1) & $104^{\mathrm{C}}$ & $\mathrm{No}^{f}$ \\
\hline Strontium & 6 of 6 & 18.7 & 29.5 & $44.1^{b}$ & $22,000^{\mathrm{e}}$ & $\mathrm{No}^{\mathrm{g}}$ \\
\hline Thallium & 0 of 6 & ND (1.0) & ND (1.0) & $2.13^{b}$ & $1.7^{\mathrm{c}}$ & $\mathrm{No}^{f}$ \\
\hline Thorium & 0 of 6 & ND (1.0) & ND (1.0) & $1.14^{b}$ & NAV & $\mathrm{No}^{f}$ \\
\hline Titanium & 5 of 6 & ND (1.0) & 7.7 & $2.37^{b}$ & $150,000^{\mathrm{e}}$ & $\mathrm{No}^{h}$ \\
\hline Uranium & 0 of 6 & ND (1.0) & ND (1.0) & ND (1) & $110^{\mathrm{e}}$ & $\mathrm{No}^{f}$ \\
\hline Vanadium & 3 of 6 & ND (1.0) & 2.25 & $1.44^{b}$ & $260^{\mathrm{e}}$ & $\mathrm{No}^{h}$ \\
\hline Zinc & 3 of 6 & ND (1.0) & 13.7 & $12.8^{\mathrm{b}}$ & $11,000^{\mathrm{e}}$ & $\mathrm{No}^{\mathrm{h}}$ \\
\hline
\end{tabular}

${ }^{\mathrm{a}}$ Background concentrations are upper tolerance limits (UTL) (ADEC, 1999a) unless otherwise noted.

${ }^{b}$ Maximum detected concentration in background samples due to insufficient detection frequency to calculate UTL.

${ }^{\mathrm{C}}$ Federal ambient water quality criteria based on ingestion of water and organisms.

${ }^{\mathrm{d}}$ State of Alaska groundwater cleanup level, Discharge Reporting, Cleanup, and Disposal of Oil and other Hazardous Substances

(18 AAC 75, Articles 3 and 9 [ADEC, 1999c]).

eU.S. EPA Region III risk-based concentrations for tap water (EPA, 2000b).

\section{COC Selection Criteria}

${ }^{7}$ Constituent not detected in any samples.

${ }^{9}$ Maximum detected constituent concentration is less than local background concentration.

${ }^{h}$ Maximum detected constituent concentration is less than human health screening value.

'Constituent is an essential macronutrient and only presents a risk at extremely high concentrations.

ND - Not detected (detection limit)

NAV - Not available

NAN - Not analyzed

$\mu \mathrm{g} / \mathrm{L}$ - Micrograms per liter 
Table 4-7

\section{Summary of Analytical Results and Human Health Risk Assessment Screening for Sediment in Drill Site E Stream}

(Page 1 of 3 )

\begin{tabular}{|c|c|c|c|c|c|c|}
\hline Analyte & $\begin{array}{l}\text { Detection } \\
\text { Frequency }\end{array}$ & $\begin{array}{c}\text { Minimum } \\
\text { Detected } \\
\text { Concentration } \\
(\mathbf{m g} / \mathbf{k g})\end{array}$ & $\begin{array}{c}\text { Maximum } \\
\text { Detected } \\
\text { Concentration } \\
(\mathbf{m g} / \mathbf{k g})\end{array}$ & $\begin{array}{c}\text { Upland Stream } \\
\text { Background } \\
\text { Concentration }{ }^{a} \\
\text { (mg/kg) }\end{array}$ & $\begin{array}{l}\text { Human Health } \\
\text { Screening } \\
\text { Value } \\
(\mathrm{mg} / \mathrm{kg})\end{array}$ & $\begin{array}{c}\text { Human Health } \\
\text { Constituent of } \\
\text { Concern }\end{array}$ \\
\hline $\begin{array}{l}\text { Diesel-Range } \\
\text { Hydrocarbons }\end{array}$ & 1 of 5 & ND (4.0) & 22.1 & NAN & $200^{c}$ & $\mathrm{No}^{f}$ \\
\hline \multicolumn{7}{|c|}{ Volatile Organic Compounds } \\
\hline 1,2,4-Trimethylbenzene & 0 of 1 & ND (0.0641) & ND (0.0641) & NAN & NAV & $\mathrm{No}^{\mathrm{d}}$ \\
\hline 1,3,5-Trimethylbenzene & 0 of 1 & ND (0.0641) & ND (0.0641) & NAN & NAV & $\mathrm{No}^{\mathrm{d}}$ \\
\hline 2-Butanone & 0 of 1 & ND (0.128) & ND (0.128) & NAN & NAV & $\mathrm{No}^{\mathrm{d}}$ \\
\hline Acetone & 0 of 1 & ND (0.107) & ND $(0.107)$ & NAN & $10,100^{c}$ & $\mathrm{No}^{\mathrm{d}}$ \\
\hline Benzene & 0 of 1 & ND $(0.0641)$ & ND $(0.0641)$ & NAN & $286^{c}$ & Nod \\
\hline Carbon disulfide & 0 of 1 & ND $(0.0641)$ & ND $(0.0641)$ & NAN & $10,100^{c}$ & $\mathrm{No}^{\mathrm{d}}$ \\
\hline Ethylbenzene & 0 of 1 & ND (0.0641) & ND $(0.0641)$ & NAN & $10,100^{c}$ & $\mathrm{No}^{d}$ \\
\hline Isopropylbenzene & 0 of 1 & ND $(0.0641)$ & ND (0.0641) & NAN & NAV & $\mathrm{No}^{d}$ \\
\hline $\mathrm{m}, \mathrm{p}$-Xylene & 0 of 1 & ND (0.0641) & ND (0.0641) & NAN & $203,000^{c}$ & $\mathrm{No}^{\mathrm{d}}$ \\
\hline Methylene chloride & 0 of 1 & ND (0.0641) & ND $(0.0641)$ & NAN & $1,110^{\mathrm{c}}$ & No ${ }^{d}$ \\
\hline n-Butylbenzene & 0 of 1 & ND (0.0641) & ND $(0.0641)$ & NAN & NAV & $\mathrm{No}^{d}$ \\
\hline n-Propylbenzene & 0 of 1 & ND $(0.0641)$ & ND $(0.0641)$ & NAN & NAV & $\mathrm{No}^{\mathrm{d}}$ \\
\hline Naphthalene & 0 of 1 & ND (0.0641) & ND (0.0641) & NAN & $4,060^{c}$ & $\mathrm{No}^{d}$ \\
\hline o-Xylene & 0 of 1 & ND (0.0641) & ND (0.0641) & NAN & $203,000^{c}$ & $\mathrm{No}^{d}$ \\
\hline p-Isopropyltoluene & 0 of 1 & ND (0.0641) & ND $(0.0641)$ & NAN & NAV & $\mathrm{No}^{d}$ \\
\hline sec-Butylbenzene & 0 of 1 & ND $(0.0641)$ & ND $(0.0641)$ & NAN & NAV & $\mathrm{No}^{d}$ \\
\hline Toluene & 0 of 1 & ND (0.0641) & ND (0.0641) & NAN & $20,300^{c}$ & $\mathrm{No}^{\mathrm{d}}$ \\
\hline \multicolumn{7}{|c|}{ Polycyclic Aromatic Hydrocarbons } \\
\hline Acenaphthene & 1 of 5 & ND (0.0033) & 0.0103 & NAN & $6,080^{\mathrm{C}}$ & $\mathrm{No}^{f}$ \\
\hline Acenaphthylene & 0 of 5 & ND (0.0033) & ND (0.0176) & NAN & NAV & Nod \\
\hline Anthracene & 0 of 5 & ND (0.0033) & ND $(0.0176)$ & NAN & $30,400^{c}$ & $\mathrm{No}^{d}$ \\
\hline Benzo(a)anthracene & 0 of 5 & ND (0.0033) & ND $(0.0176)$ & NAN & $11.4^{\mathrm{C}}$ & $\mathrm{No}^{d}$ \\
\hline Benzo(a)pyrene & 0 of 5 & ND (0.0033) & ND (0.0176) & NAN & $1.1^{\mathrm{c}}$ & $\mathrm{No}^{d}$ \\
\hline Benzo(b)fluoranthene & 0 of 5 & ND (0.0033) & ND (0.0176) & NAN & $11.4^{\mathrm{C}}$ & $\mathrm{No}^{d}$ \\
\hline Benzo(ghi)perylene & 0 of 5 & ND (0.0033) & ND (0.0176) & NAN & $1.1^{\mathrm{c}}$ & $\mathrm{No}^{d}$ \\
\hline Benzo(k)fluoranthene & 0 of 5 & ND (0.0033) & ND (0.0176) & NAN & $114^{\mathrm{C}}$ & $\mathrm{No}^{d}$ \\
\hline Chrysene & 0 of 5 & ND (0.0033) & ND $(0.0176)$ & NAN & $1,140^{\mathrm{C}}$ & $\mathrm{No}^{d}$ \\
\hline
\end{tabular}


Table 4-7

\section{Summary of Analytical Results and Human Health Risk Assessment Screening for Sediment in Drill Site E Stream}

(Page 2 of 3 )

\begin{tabular}{|c|c|c|c|c|c|c|}
\hline Analyte & $\begin{array}{l}\text { Detection } \\
\text { Frequency }\end{array}$ & $\begin{array}{c}\text { Minimum } \\
\text { Detected } \\
\text { Concentration } \\
(\mathbf{m g} / \mathbf{k g})\end{array}$ & $\begin{array}{c}\text { Maximum } \\
\text { Detected } \\
\text { Concentration } \\
(\mathrm{mg} / \mathrm{kg})\end{array}$ & $\begin{array}{c}\text { Upland Stream } \\
\text { Background } \\
\text { Concentration }{ }^{a} \\
(\mathbf{m g} / \mathbf{k g})\end{array}$ & $\begin{array}{l}\text { Human Health } \\
\text { Screening } \\
\text { Value } \\
(\mathrm{mg} / \mathrm{kg})\end{array}$ & $\begin{array}{c}\text { Human Health } \\
\text { Constituent of } \\
\text { Concern }\end{array}$ \\
\hline Dibenzo(a,h)anthracene & 0 of 5 & ND (0.0033) & ND (0.0176) & NAN & $1.1^{\mathrm{C}}$ & $\mathrm{No}^{d}$ \\
\hline Fluoranthene & 0 of 5 & ND (0.0033) & ND $(0.0176)$ & NAN & $4,060^{c}$ & Nod \\
\hline Fluorene & 1 of 5 & ND (0.0033) & 0.0274 & NAN & $4,060^{c}$ & $\mathrm{No}^{f}$ \\
\hline Indeno(1,2,3-cd)pyrene & 0 of 5 & ND (0.0033) & ND $(0.0176)$ & NAN & $11^{\mathrm{C}}$ & Nod \\
\hline Naphthalene & 0 of 5 & ND (0.00694) & ND $(0.00701)$ & NAN & $4,060^{c}$ & $\mathrm{No}^{d}$ \\
\hline Phenanthrene & 1 of 5 & ND (0.0033) & 0.0702 & NAN & $1.1^{\mathrm{c}}$ & $\mathrm{No}^{f}$ \\
\hline Pyrene & 0 of 5 & ND (0.0033) & ND (0.0176) & NAN & $3,040^{c}$ & No ${ }^{d}$ \\
\hline \multicolumn{7}{|c|}{ Polychlorinated Biphenyls } \\
\hline Aroclor 1016 & NAV & NAV & NAV & NAN & $10^{c}$ & $\mathrm{No}^{d}$ \\
\hline Aroclor 1221 & NAV & NAV & NAV & NAN & $10^{\mathrm{C}}$ & $\mathrm{No}^{d}$ \\
\hline Aroclor 1232 & NAV & NAV & NAV & NAN & $10^{c}$ & $\mathrm{No}^{d}$ \\
\hline Aroclor 1242 & NAV & NAV & NAV & NAN & $10^{c}$ & $\mathrm{No}^{\mathrm{d}}$ \\
\hline Aroclor 1248 & NAV & NAV & NAV & NAN & $10^{c}$ & $\mathrm{No}^{d}$ \\
\hline Aroclor 1254 & NAV & NAV & NAV & NAN & $10^{c}$ & $\mathrm{No}^{d}$ \\
\hline Aroclor 1260 & NAV & NAV & NAV & NAN & $10^{c}$ & $\mathrm{No}^{d}$ \\
\hline Aroclor 1262 & NAV & NAV & NAV & NAN & $10^{c}$ & $\mathrm{No}^{d}$ \\
\hline Aroclor 1268 & NAV & NAV & NAV & NAN & $10^{c}$ & $\mathrm{No}^{d}$ \\
\hline \multicolumn{7}{|c|}{ Metals } \\
\hline Aluminum & 5 of 5 & 11,700 & 25,100 & 47,500 & NAV & $\mathrm{No}^{\mathrm{e}}$ \\
\hline Arsenic & 5 of 5 & 1.25 & 3.64 & 30.5 & $5.5^{\mathrm{c}}$ & $\mathrm{No}^{\mathrm{e}}$ \\
\hline Barium & 5 of 5 & 36.5 & 76.7 & 468 & $7,100^{c}$ & $\mathrm{No}^{\mathrm{e}}$ \\
\hline Beryllium & 0 of 5 & ND (0.5) & ND (2.67) & ND & $1.9^{\mathrm{c}}$ & $\mathrm{No}^{d}$ \\
\hline Boron & 0 of 5 & ND (2.5) & ND (13.4) & $6.42^{b}$ & NAV & $\mathrm{No}^{d}$ \\
\hline Cadmium & 0 of 5 & ND (0.5) & ND (2.67) & ND & $101^{\mathrm{C}}$ & $\mathrm{No}^{d}$ \\
\hline Calcium & 5 of 5 & 1,950 & 5,370 & 7,720 & NAV & $\mathrm{No}^{\mathrm{e}}$ \\
\hline Cerium & 5 of 5 & 8.03 & 19.7 & 13 & NAV & $\mathrm{No}^{\mathrm{g}}$ \\
\hline Cesium & 0 of 5 & ND (0.5) & ND (2.67) & ND & NAV & $\mathrm{No}^{d}$ \\
\hline Chromium & 5 of 5 & 9.49 & 41.1 & 18.9 & $507^{c}$ & $\mathrm{No}^{f}$ \\
\hline Cobalt & 5 of 5 & 4.03 & 18.5 & 37.3 & NAV & $\mathrm{No}^{\mathrm{e}}$ \\
\hline Copper & 5 of 5 & 20.6 & 68 & 82.4 & NAV & $\mathrm{No}^{\mathrm{e}}$ \\
\hline
\end{tabular}


Table 4-7

\section{Summary of Analytical Results and Human Health Risk Assessment Screening for Sediment in Drill Site E Stream}

(Page 3 of 3 )

\begin{tabular}{|c|c|c|c|c|c|c|}
\hline Analyte & $\begin{array}{l}\text { Detection } \\
\text { Frequency }\end{array}$ & $\begin{array}{c}\text { Minimum } \\
\text { Detected } \\
\text { Concentration } \\
(\mathbf{m g} / \mathbf{k g})\end{array}$ & $\begin{array}{c}\text { Maximum } \\
\text { Detected } \\
\text { Concentration } \\
(\mathbf{m g} / \mathbf{k g})\end{array}$ & $\begin{array}{c}\text { Upland Stream } \\
\text { Background } \\
\text { Concentration }{ }^{\mathrm{a}} \\
(\mathrm{mg} / \mathrm{kg})\end{array}$ & $\begin{array}{l}\text { Human Health } \\
\text { Screening } \\
\text { Value } \\
(\mathrm{mg} / \mathrm{kg})\end{array}$ & $\begin{array}{c}\text { Human Health } \\
\text { Constituent of } \\
\text { Concern }\end{array}$ \\
\hline Iron & 5 of 5 & 13,700 & 31,000 & 123,000 & NAV & $\mathrm{No}^{\mathrm{e}}$ \\
\hline Lithium & 5 of 5 & 3.03 & 8.74 & 21.7 & NAV & $\mathrm{No}^{\mathrm{e}}$ \\
\hline Magnesium & 5 of 5 & 1,310 & 6,950 & 17,000 & NAV & $\mathrm{No}^{\mathrm{e}}$ \\
\hline Manganese & 5 of 5 & 82.5 & 1,260 & 26,500 & NAV & $\mathrm{No}^{\mathrm{e}}$ \\
\hline Molybdenum & 1 of 5 & ND (1.05) & 1.02 & ND & NAV & $\mathrm{No}^{\mathrm{g}}$ \\
\hline Nickel & 5 of 5 & 6.28 & 32.5 & 23.8 & $2,030^{C}$ & $\mathrm{No}^{f}$ \\
\hline Potassium & 5 of 5 & 123 & 556 & 1,290 & NAV & $\mathrm{No}^{\mathrm{e}}$ \\
\hline Selenium & 3 of 5 & ND (1.05) & 3.31 & $3.15^{b}$ & $507^{c}$ & $\mathrm{No}^{f}$ \\
\hline Strontium & 5 of 5 & 24.6 & 51.4 & 207 & NAV & $\mathrm{No}^{\mathrm{e}}$ \\
\hline Thallium & 0 of 5 & ND (0.5) & ND (2.67) & ND & NAV & $\mathrm{No}^{d}$ \\
\hline Thorium & 0 of 5 & ND (0.5) & ND (2.67) & ND & NAV & $\mathrm{No}^{d}$ \\
\hline Titanium & 5 of 5 & 471 & 1,010 & 1,480 & NAV & $\mathrm{No}^{\mathrm{e}}$ \\
\hline Uranium & 0 of 5 & ND (0.5) & ND (2.67) & ND & NAV & $\mathrm{No}^{\mathrm{d}}$ \\
\hline Vanadium & 5 of 5 & 63.3 & 90.6 & 336 & $710^{C}$ & $\mathrm{No}^{\mathrm{e}}$ \\
\hline Zinc & 4 of 5 & 36.1 & 59.3 & 185 & $30,400^{c}$ & $\mathrm{No}^{\mathrm{e}}$ \\
\hline Total Organic Carbon & 5 of 5 & 10,300 & 103,000 & NAN & NAV & No \\
\hline
\end{tabular}

${ }^{a}$ Background concentrations are upper tolerance limits (UTL) (ADEC, 1999a) unless otherwise noted.

${ }^{b}$ Maximum detected concentration in background samples due to insufficient detection frequency to calculate UTL.

'State of Alaska soil cleanup level, Discharge Reporting, Cleanup, and Disposal of Oil and other Hazardous Substances (18 AAC 75, Articles 3 and 9 [ADEC, 1999c]).

\section{COC Selection Criteria}

${ }^{\mathrm{d}}$ Constituent not detected in any samples.

${ }^{\mathrm{e}}$ Maximum detected constituent concentration is less than local background concentration.

${ }^{f}$ Maximum detected constituent concentration is less than human health screening value.

${ }^{9}$ Not a constituent associated with drilling mud or other drilling activities.

ND - Not detected (detection limit)

NAV - Not available

NAN - Not analyzed

$\mathrm{mg} / \mathrm{kg}$ - Milligrams per kilogram 
Table 4-8

\section{Summary of Analytical Results and Human Health Risk Assessment Screening for Fish Tissue in Drill Site E Stream}

(Page 1 of 2)

\begin{tabular}{|c|c|c|c|c|c|c|}
\hline Analyte & $\begin{array}{l}\text { Detection } \\
\text { Frequency }\end{array}$ & $\begin{array}{c}\text { Minimum } \\
\text { Detected } \\
\text { Concentration } \\
(\mathbf{m g} / \mathbf{k g})\end{array}$ & $\begin{array}{c}\text { Maximum } \\
\text { Detected } \\
\text { Concentration } \\
(\mathbf{m g} / \mathbf{k g})\end{array}$ & $\begin{array}{c}\text { Upland Stream } \\
\text { Background } \\
\text { Concentration } \\
\text { (mg/kg) }\end{array}$ & $\begin{array}{l}\text { Human Health } \\
\text { Screening } \\
\text { Value }^{\mathrm{c}} \\
(\mathrm{mg} / \mathrm{kg})\end{array}$ & $\begin{array}{c}\text { Human } \\
\text { Health } \\
\text { Constituent } \\
\text { of Concern }\end{array}$ \\
\hline \multicolumn{7}{|c|}{ Polycyclic Aromatic Hydrocarbons } \\
\hline Acenaphthene & 0 of 3 & ND (0.0335) & ND (0.0335) & NAN & 81.1 & $\mathrm{No}^{\mathrm{d}}$ \\
\hline Acenaphthylene & 0 of 3 & ND (0.0335) & ND (0.0335) & NAN & NAV & $\mathrm{No}^{d}$ \\
\hline Anthracene & 0 of 3 & ND (0.0335) & ND (0.0335) & NAN & 410 & $\mathrm{No}^{d}$ \\
\hline Benzo (a) anthracene & 0 of 3 & ND (0.0335) & ND (0.134) & NAN & 0.0043 & $\mathrm{No}^{\mathrm{d}}$ \\
\hline Benzo (a) pyrene & 0 of 3 & ND (0.0335) & ND (0.134) & NAN & 0.00043 & $\mathrm{No}^{d}$ \\
\hline Benzo (b) fluoranthene & 0 of 3 & ND (0.0335) & ND (0.134) & NAN & 0.0043 & $\mathrm{No}^{d}$ \\
\hline Benzo (ghi) perylene & 0 of 3 & ND (0.0335) & ND (0.134) & NAN & NAV & $\mathrm{No}^{\mathrm{d}}$ \\
\hline Benzo (k) fluoranthene & 0 of 3 & ND (0.0335) & ND (0.134) & NAN & 0.043 & $\mathrm{No}^{\mathrm{d}}$ \\
\hline Chrysene & 0 of 3 & ND (0.0335) & ND (0.134) & NAN & 0.43 & $\mathrm{No}^{\mathrm{d}}$ \\
\hline $\begin{array}{l}\text { Dibenzo }(\mathrm{a}, \mathrm{h}) \\
\text { anthracene }\end{array}$ & 0 of 3 & ND $(0.0335)$ & ND (0.134) & NAN & 0.00043 & $\mathrm{No}^{\mathrm{d}}$ \\
\hline Fluoranthene & 0 of 3 & ND (0.0335) & ND (0.0335) & NAN & 54 & $\mathrm{No}^{d}$ \\
\hline Fluorene & 0 of 3 & ND (0.0335) & ND (0.0335) & NAN & 54 & $\mathrm{No}^{\mathrm{d}}$ \\
\hline $\begin{array}{l}\text { Indeno }(1,2,3-c d) \\
\text { pyrene }\end{array}$ & 0 of 3 & ND (0.0335) & ND (0.134) & NAN & 0.0043 & $\mathrm{No}^{d}$ \\
\hline Naphthalene & 0 of 3 & ND (0.0335) & ND (0.0335) & NAN & 27 & $\mathrm{No}^{d}$ \\
\hline Phenanthrene & 0 of 3 & ND (0.0335) & ND (0.0335) & NAN & NAV & $\mathrm{No}^{d}$ \\
\hline Pyrene & 0 of 3 & ND (0.0335) & ND (0.134) & NAN & 41 & $\mathrm{No}^{d}$ \\
\hline \multicolumn{7}{|c|}{ Polychlorinated Biphenyls } \\
\hline Aroclor 1016 & 0 of 3 & ND (0.01) & ND (0.01) & NAN & 0.045 & $\mathrm{No}^{\mathrm{d}}$ \\
\hline Aroclor 1221 & 0 of 3 & ND (0.02) & ND (0.02) & NAN & 0.0016 & $\mathrm{No}^{d}$ \\
\hline Aroclor 1232 & 0 of 3 & ND (0.01) & ND (0.01) & NAN & 0.0016 & $\mathrm{No}^{d}$ \\
\hline Aroclor 1242 & 0 of 3 & ND (0.01) & ND (0.01) & NAN & 0.0016 & $\mathrm{No}^{d}$ \\
\hline Aroclor 1248 & 0 of 3 & ND (0.01) & ND (0.01) & NAN & 0.0016 & $\mathrm{No}^{d}$ \\
\hline Aroclor 1254 & 0 of 3 & ND (0.01) & ND (0.01) & NAN & 0.0016 & $\mathrm{No}^{d}$ \\
\hline Aroclor 1260 & 0 of 3 & ND $(0.01)$ & ND (0.01) & NAN & 0.0016 & $\mathrm{No}^{d}$ \\
\hline \multicolumn{7}{|c|}{ Metals } \\
\hline Aluminum & 3 of 3 & 18.2 & 21.5 & 38.8 & 1400 & $\mathrm{No}^{\mathrm{e}}$ \\
\hline Arsenic (total) & 3 of 3 & 0.047 & 0.0548 & 26.9 & 0.0021 & $\mathrm{No}^{\mathrm{e}}$ \\
\hline Arsenic (inorganic) & 3 of 3 & 0.00111 & 0.00508 & 0.0456 & 0.0021 & $\mathrm{No}^{\mathrm{e}}$ \\
\hline Barium & 3 of 3 & 0.635 & 1.08 & 0.567 & 95 & $\mathrm{No}^{f}$ \\
\hline Beryllium & 0 of 3 & ND (0.025) & ND (0.025) & ND & 2.7 & $\mathrm{No}^{d}$ \\
\hline Boron & 0 of 3 & ND (7.09) & ND (8.92) & 24.1 & 120 & $\mathrm{No}^{\mathrm{d}}$ \\
\hline
\end{tabular}


Table 4-8

\section{Summary of Analytical Results and Human Health Risk Assessment Screening for Fish Tissue in Drill Site E Stream}

(Page 2 of 2)

\begin{tabular}{|c|c|c|c|c|c|c|}
\hline Analyte & $\begin{array}{l}\text { Detection } \\
\text { Frequency }\end{array}$ & $\begin{array}{c}\text { Minimum } \\
\text { Detected } \\
\text { Concentration } \\
(\mathrm{mg} / \mathrm{kg})\end{array}$ & $\begin{array}{c}\text { Maximum } \\
\text { Detected } \\
\text { Concentration } \\
(\mathrm{mg} / \mathrm{kg})\end{array}$ & $\begin{array}{l}\text { Upland Stream } \\
\text { Background } \\
\text { Concentration }{ }^{\mathrm{a}} \\
(\mathrm{mg} / \mathrm{kg})\end{array}$ & $\begin{array}{c}\text { Human Health } \\
\text { Screening } \\
\text { Value }^{\mathrm{c}} \\
(\mathrm{mg} / \mathbf{k g})\end{array}$ & $\begin{array}{c}\text { Human } \\
\text { Health } \\
\text { Constituent } \\
\text { of Concern }\end{array}$ \\
\hline Cadmium & 0 of 3 & ND (0.025) & 0.0288 & ND & 1.4 & $\mathrm{No}^{\dagger}$ \\
\hline Calcium & 3 of 3 & 9,650 & 10,800 & 10,300 & NAV & $\mathrm{No}^{\mathrm{g}}$ \\
\hline Cerium & 0 of 3 & ND (0.025) & ND (0.025) & ND & NAV & $\mathrm{No}^{d}$ \\
\hline Cesium & 3 of 3 & 0.026 & 0.036 & ND & NAV & $\mathrm{No}^{\mathrm{h}}$ \\
\hline Chromium & 3 of 3 & 0.373 & 0.423 & 0.684 & 4.1 & $\mathrm{No}^{\mathrm{e}}$ \\
\hline Cobalt & 3 of 3 & 0.0718 & 0.15 & $0.0510^{b}$ & 81 & $\mathrm{No}^{f}$ \\
\hline Copper & 0 of 3 & ND (0.833) & ND (0.973) & 3.4 & 54 & $\mathrm{No}^{d}$ \\
\hline Iron & 3 of 3 & 35.8 & 36 & 73.6 & 410 & $\mathrm{No}^{\mathrm{e}}$ \\
\hline Lithium & 0 of 3 & ND (0.025) & ND (0.025) & ND & 27 & $\mathrm{No}^{\mathrm{d}}$ \\
\hline Magnesium & 3 of 3 & 318 & 343 & 349 & NAV & $\mathrm{No}^{\mathrm{e}}$ \\
\hline Manganese & 3 of 3 & 4.14 & 5.93 & 164 & 190 & $\mathrm{No}^{\mathrm{e}}$ \\
\hline Molybdenum & 0 of 3 & ND (0.125) & ND (0.125) & $0.150^{b}$ & 6.8 & $\mathrm{No}^{\mathrm{d}}$ \\
\hline Nickel & 1 of 3 & ND (0.125) & 0.132 & ND & 27 & $\mathrm{No}^{\dagger}$ \\
\hline Potassium & 3 of 3 & 3,500 & 4,200 & 4,310 & NAV & $\mathrm{No}^{\mathrm{e}}$ \\
\hline Selenium & 3 of 3 & 0.918 & 1.05 & 2.08 & 6.8 & $\mathrm{No}^{\mathrm{e}}$ \\
\hline Strontium & 3 of 3 & 8.94 & 13.4 & $12.1^{\mathrm{b}}$ & 810 & $\mathrm{No}^{f}$ \\
\hline Thallium & 1 of 3 & ND (0.025) & 0.0453 & $0.0755^{b}$ & 0.095 & $\mathrm{No}^{\mathrm{e}}$ \\
\hline Thorium & 0 of 3 & ND (2.5) & ND (2.5) & $4.67^{b}$ & NAV & $\mathrm{No}^{d}$ \\
\hline Titanium & 3 of 3 & 0.418 & 0.671 & 0.834 & 5,400 & $\mathrm{No}^{\mathrm{e}}$ \\
\hline Uranium & 0 of 3 & ND (0.5) & ND (0.5) & ND & 4.1 & $\mathrm{No}^{\mathrm{d}}$ \\
\hline Vanadium & 3 of 3 & 0.195 & 0.22 & 0.289 & 9.5 & $\mathrm{No}^{\mathrm{e}}$ \\
\hline Zinc & 3 of 3 & 43.4 & 54.2 & 78 & 410 & $\mathrm{No}^{\mathrm{e}}$ \\
\hline
\end{tabular}

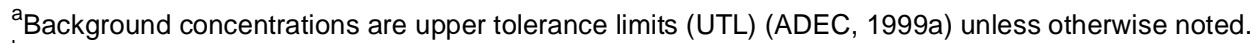

${ }^{b}$ Maximum detected concentration in background samples due to insufficient detection frequency to calculate UTL.

${ }^{\mathrm{C} U . S . ~ E P A ~ R e g i o n ~ I I I ~ r i s k-b a s e d ~ s c r e e n i n g ~ c r i t e r i a ~ f o r ~ f i s h ~ c o n s u m p t i o n ~ u n l e s s ~ o t h e r w i s e ~ n o t e d . ~}$

\section{COC Selection Criteria}

${ }^{\mathrm{D}}$ Constituent not detected in any samples.

e Maximum detected constituent concentration is less than local background concentration.

Maximum detected constituent concentration is less than human health screening value.

${ }^{g}$ Constituent is an essential macronutrient and only presents a risk at extremely high concentrations.

${ }^{h}$ Not a constituent associated with drilling mud or other drilling activities.

ND - Not detected (detection limit)

NAV - Not available

NAN - Not analyzed

$\mathrm{mg} / \mathrm{kg}$ - Milligrams per kilogram 


\section{Table 4-9}

\section{Summary of Analytical Results and Human Health Risk Assessment Screening for Surface Water in Limpet Creek, Drill Site F}

(Page 1 of 3 )

\begin{tabular}{|c|c|c|c|c|c|c|}
\hline Analyte & $\begin{array}{l}\text { Detection } \\
\text { Frequency }\end{array}$ & $\begin{array}{c}\text { Minimum } \\
\text { Detected } \\
\text { Concentration } \\
(\mu \mathrm{g} / \mathrm{L})\end{array}$ & $\begin{array}{c}\text { Maximum } \\
\text { Detected } \\
\text { Concentration } \\
(\mu \mathrm{g} / \mathrm{L})\end{array}$ & $\begin{array}{c}\text { Upland Stream } \\
\text { Background } \\
\text { Concentration }{ }^{\mathrm{a}} \\
(\mu \mathrm{g} / \mathrm{L})\end{array}$ & $\begin{array}{l}\text { Human Health } \\
\text { Screening } \\
\text { Value } \\
(\mu \mathrm{g} / \mathrm{L})\end{array}$ & $\begin{array}{l}\text { Human } \\
\text { Health } \\
\text { Constituent } \\
\text { of Concern }\end{array}$ \\
\hline $\begin{array}{l}\text { Diesel-Range } \\
\text { Hydrocarbons }\end{array}$ & 0 of 5 & ND (50) & ND (50) & NAN & $1,500^{d}$ & $\mathrm{No}^{f}$ \\
\hline \multicolumn{7}{|c|}{ Volatile Organic Compounds } \\
\hline 1,2,4-Trimethylbenzene & 0 of 5 & ND (1.0) & ND (1.0) & NAN & $12^{\mathrm{e}}$ & $\mathrm{No}^{f}$ \\
\hline 1,3,5-Trimethylbenzene & 0 of 5 & ND (1.0) & ND (1.0) & NAN & $12^{\mathrm{e}}$ & $\mathrm{No}^{f}$ \\
\hline 2-Butanone & 0 of 5 & ND (20) & ND (20) & NAN & $1,900^{\mathrm{e}}$ & $\mathrm{No}^{f}$ \\
\hline Acetone & 0 of 5 & ND (20) & ND (20) & NAN & $3,700^{\mathrm{e}}$ & $\mathrm{No}^{f}$ \\
\hline Benzene & 0 of 5 & ND (1.0) & ND (1.0) & NAN & $1.2^{\mathrm{c}}$ & $\mathrm{No}^{f}$ \\
\hline Carbon disulfide & 0 of 5 & ND (1.0) & ND (1.0) & NAN & $1,000^{\mathrm{e}}$ & $\mathrm{No}^{f}$ \\
\hline Ethylbenzene & 0 of 5 & ND (1.0) & ND (1.0) & NAN & $3,100^{c}$ & $\mathrm{No}^{f}$ \\
\hline Isopropylbenzene & 0 of 5 & ND (1.0) & ND (1.0) & NAN & NAV & $\mathrm{No}^{f}$ \\
\hline $\mathrm{m}, \mathrm{p}$-Xylene & 0 of 5 & ND (2.0) & ND (2.0) & NAN & $12,000^{\mathrm{e}}$ & $\mathrm{No}^{f}$ \\
\hline Methylene chloride & 0 of 5 & ND (2.0) & ND (4.83) & NAN & $4.7^{\mathrm{C}}$ & $\mathrm{No}^{f}$ \\
\hline n-Butylbenzene & 0 of 5 & ND (1.0) & ND (1.0) & NAN & $61^{e}$ & $\mathrm{No}^{f}$ \\
\hline n-Propylbenzene & 0 of 5 & ND (1.0) & ND (1.0) & NAN & $61^{\mathrm{e}}$ & $\mathrm{No}^{f}$ \\
\hline Naphthalene & 0 of 5 & ND (1.0) & ND (1.0) & NAN & $730^{e}$ & $\mathrm{No}^{f}$ \\
\hline o-Xylene & 0 of 5 & ND (1.0) & ND (1.0) & NAN & $12,000^{\mathrm{e}}$ & $\mathrm{No}^{f}$ \\
\hline p-Isopropyltoluene & 0 of 5 & ND (1.0) & ND (1.0) & NAN & NAV & $\mathrm{No}^{f}$ \\
\hline sec-Butylbenzene & 0 of 5 & ND (1.0) & ND (1.0) & NAN & $61^{e}$ & $\mathrm{No}^{f}$ \\
\hline Toluene & 0 of 5 & ND (1.0) & ND (1.0) & NAN & $10,000^{c}$ & $\mathrm{No}^{f}$ \\
\hline \multicolumn{7}{|c|}{ Polycyclic Aromatic Hydrocarbons } \\
\hline Acenaphthene & 0 of 5 & ND $(0.1)$ & ND $(0.1)$ & NAN & $1,200^{\mathrm{C}}$ & $\mathrm{No}^{f}$ \\
\hline Acenaphthylene & 0 of 5 & ND $(0.1)$ & $\mathrm{ND}(0.1)$ & NAN & $0.0028^{c}$ & $\mathrm{No}^{\dagger}$ \\
\hline Anthracene & 0 of 5 & ND $(0.1)$ & ND $(0.1)$ & NAN & $0.0028^{c}$ & $\mathrm{No}^{f}$ \\
\hline Benzo(a)anthracene & 0 of 5 & $\mathrm{ND}(0.1)$ & $\mathrm{ND}(0.1)$ & NAN & $0.0028^{c}$ & $\mathrm{No}^{f}$ \\
\hline Benzo(a)pyrene & 0 of 5 & $\mathrm{ND}(0.1)$ & $\mathrm{ND}(0.1)$ & NAN & $0.0028^{c}$ & $\mathrm{No}^{f}$ \\
\hline Benzo(b)fluoranthene & 0 of 5 & ND $(0.1)$ & $\mathrm{ND}(0.1)$ & NAN & $0.092^{\mathrm{e}}$ & $\mathrm{No}^{\dagger}$ \\
\hline Benzo(ghi)perylene & 0 of 5 & ND $(0.1)$ & $\mathrm{ND}(0.1)$ & NAN & $0.0028^{c}$ & $\mathrm{No}^{f}$ \\
\hline Benzo(k)fluoranthene & 0 of 5 & ND $(0.1)$ & ND $(0.1)$ & NAN & $0.0028^{c}$ & $\mathrm{No}^{\dagger}$ \\
\hline Chrysene & 0 of 5 & ND $(0.1)$ & $\mathrm{ND}(0.1)$ & NAN & $0.0028^{c}$ & $\mathrm{No}^{f}$ \\
\hline Dibenzo(a,h)anthracene & 0 of 5 & ND (0.1) & ND $(0.1)$ & NAN & $0.0028^{c}$ & $\mathrm{No}^{f}$ \\
\hline
\end{tabular}


Table 4-9

\section{Summary of Analytical Results and Human Health Risk Assessment Screening for Surface Water in Limpet Creek, Drill Site F}

(Page 2 of 3 )

\begin{tabular}{|c|c|c|c|c|c|c|}
\hline Analyte & $\begin{array}{l}\text { Detection } \\
\text { Frequency }\end{array}$ & $\begin{array}{c}\text { Minimum } \\
\text { Detected } \\
\text { Concentration } \\
(\mu \mathrm{g} / \mathrm{L})\end{array}$ & $\begin{array}{c}\text { Maximum } \\
\text { Detected } \\
\text { Concentration } \\
(\mu \mathrm{g} / \mathrm{L})\end{array}$ & $\begin{array}{c}\text { Upland Stream } \\
\text { Background } \\
\text { Concentration } \\
(\mu \mathrm{g} / \mathrm{L})\end{array}$ & $\begin{array}{l}\text { Human Health } \\
\text { Screening } \\
\text { Value } \\
(\mu \mathrm{g} / L)\end{array}$ & $\begin{array}{c}\text { Human } \\
\text { Health } \\
\text { Constituent } \\
\text { of Concern }\end{array}$ \\
\hline Fluoranthene & 0 of 5 & $\mathrm{ND}(0.1)$ & $\mathrm{ND}(0.1)$ & NAN & $42^{c}$ & $\mathrm{No}^{f}$ \\
\hline Fluorene & 0 of 5 & $\mathrm{ND}(0.1)$ & $\mathrm{ND}(0.1)$ & NAN & $0.0028^{\mathrm{C}}$ & $\mathrm{No}^{f}$ \\
\hline Indeno(1,2,3-cd)pyrene & 0 of 5 & ND $(0.1)$ & $\mathrm{ND}(0.1)$ & NAN & $0.0028^{c}$ & $\mathrm{No}^{f}$ \\
\hline Naphthalene & 0 of 5 & $\mathrm{ND}(0.1)$ & $\mathrm{ND}(0.1)$ & NAN & $730^{e}$ & $\mathrm{No}^{f}$ \\
\hline Phenanthrene & 0 of 5 & ND $(0.1)$ & ND $(0.1)$ & NAN & $0.0028^{c}$ & $\mathrm{No}^{f}$ \\
\hline Pyrene & 0 of 5 & ND $(0.1)$ & $\mathrm{ND}(0.1)$ & NAN & $0.0028^{c}$ & $\mathrm{No}^{f}$ \\
\hline \multicolumn{7}{|c|}{ Polychlorinated Biphenyls } \\
\hline Aroclor 1016 & NAV & NAV & NAV & NAN & $0.000044^{c}$ & $\mathrm{No}^{f}$ \\
\hline Aroclor 1221 & NAV & NAV & NAV & NAN & $0.000044^{c}$ & $\mathrm{No}^{f}$ \\
\hline Aroclor 1232 & NAV & NAV & NAV & NAN & $0.000044^{c}$ & $\mathrm{No}^{f}$ \\
\hline Aroclor 1242 & NAV & NAV & NAV & NAN & $0.000044^{c}$ & $\mathrm{No}^{f}$ \\
\hline Aroclor 1248 & NAV & NAV & NAV & NAN & $0.000044^{c}$ & $\mathrm{No}^{f}$ \\
\hline Aroclor 1254 & NAV & NAV & NAV & NAN & $0.000044^{c}$ & $\mathrm{No}^{f}$ \\
\hline Aroclor 1260 & NAV & NAV & NAV & NAN & $0.000044^{c}$ & $\mathrm{No}^{f}$ \\
\hline Aroclor 1262 & NAV & NAV & NAV & NAN & $0.000044^{c}$ & $\mathrm{No}^{f}$ \\
\hline Aroclor 1268 & NAV & NAV & NAV & NAN & $0.000044^{c}$ & $\mathrm{No}^{f}$ \\
\hline \multicolumn{7}{|c|}{ Metals } \\
\hline Aluminum & 0 of 5 & ND (50) & ND (50) & $87.1^{b}$ & $37,000^{\mathrm{e}}$ & $\mathrm{No}^{f}$ \\
\hline Arsenic & 0 of 5 & ND (1.0) & ND (1.0) & ND (1) & $0.018^{\mathrm{C}}$ & $\mathrm{No}^{f}$ \\
\hline Barium & 0 of 5 & ND (10) & ND (10) & ND (10) & $1,000^{c}$ & $\mathrm{No}^{f}$ \\
\hline Beryllium & 0 of 5 & ND (1.0) & ND (1.0) & ND (1) & $0.0076^{c}$ & $\mathrm{No}^{f}$ \\
\hline Boron & 0 of 5 & ND (50) & ND (50) & ND (50) & $3,300^{\mathrm{e}}$ & $\mathrm{No}^{f}$ \\
\hline Cadmium & 0 of 5 & ND (1.0) & ND (1.0) & ND (1) & $10^{c}$ & $\mathrm{No}^{f}$ \\
\hline Calcium & 5 of 5 & 3,160 & 3,540 & $8,810^{b}$ & NAV & $\mathrm{No}^{\mathrm{g}}$ \\
\hline Cerium & 0 of 5 & ND (1.0) & ND (1.0) & ND (1) & NAV & $\mathrm{No}^{f}$ \\
\hline Cesium & 0 of 5 & ND (1.0) & ND (1.0) & ND (1) & NAV & $\mathrm{No}^{f}$ \\
\hline Chromium & 0 of 5 & ND (1.0) & ND (1.0) & $3.49^{b}$ & $33,000^{c}$ & $\mathrm{No}^{f}$ \\
\hline Cobalt & 4 of 5 & ND (1.0) & 4.91 & 9.52 & $2,200^{\mathrm{e}}$ & $\mathrm{No}^{\mathrm{g}}$ \\
\hline Copper & 3 of 5 & ND (1.0) & 1.85 & $13.4^{b}$ & $1,300^{C}$ & $\mathrm{No}^{\mathrm{g}}$ \\
\hline Iron & 2 of 5 & ND (150) & 165 & $2,830^{b}$ & $300^{C}$ & $\mathrm{No}^{\mathrm{g}}$ \\
\hline Lithium & 0 of 5 & ND (1.0) & ND (1.0) & ND (1) & $730^{\mathrm{e}}$ & $\mathrm{No}^{\dagger}$ \\
\hline
\end{tabular}


Table 4-9

\section{Summary of Analytical Results and Human Health Risk Assessment Screening for Surface Water in Limpet Creek, Drill Site F} (Page 3 of 3 )

\begin{tabular}{|c|c|c|c|c|c|c|}
\hline Analyte & $\begin{array}{l}\text { Detection } \\
\text { Frequency }\end{array}$ & $\begin{array}{c}\text { Minimum } \\
\text { Detected } \\
\text { Concentration } \\
(\mu \mathrm{g} / \mathrm{L})\end{array}$ & $\begin{array}{c}\text { Maximum } \\
\text { Detected } \\
\text { Concentration } \\
(\mu \mathrm{g} / \mathrm{L})\end{array}$ & $\begin{array}{c}\text { Upland Stream } \\
\text { Background } \\
\text { Concentration }{ }^{\mathrm{a}} \\
(\mu \mathrm{g} / \mathrm{L})\end{array}$ & $\begin{array}{l}\text { Human Health } \\
\text { Screening } \\
\text { Value } \\
(\mu \mathrm{g} / L)\end{array}$ & $\begin{array}{l}\text { Human } \\
\text { Health } \\
\text { Constituent } \\
\text { of Concern }\end{array}$ \\
\hline Magnesium & 5 of 5 & 2,700 & 3,330 & 5,250 & NAV & $\mathrm{No}^{\mathrm{g}}$ \\
\hline Manganese & 5 of 5 & 2.92 & 21.3 & 748 & $50^{c}$ & $\mathrm{No}^{\mathrm{g}}$ \\
\hline Molybdenum & 0 of 5 & ND (1.0) & ND (1.0) & ND (1) & $180^{e}$ & $\mathrm{No}^{f}$ \\
\hline Nickel & 4 of 5 & ND (1.0) & 1.64 & $1.69^{b}$ & $510^{c}$ & $\mathrm{No}^{\mathrm{g}}$ \\
\hline Potassium & 5 of 5 & 1,160 & 1,640 & $1,190^{b}$ & NAV & $\mathrm{No}^{\mathrm{i}}$ \\
\hline Selenium & 0 of 5 & $\mathrm{ND}(1.0)$ & $\mathrm{ND}(1.0)$ & ND (1) & $104^{c}$ & $\mathrm{No}^{f}$ \\
\hline Strontium & 5 of 5 & 32.8 & 35.8 & $44.1^{b}$ & $22,000^{\mathrm{e}}$ & $\mathrm{No}^{\mathrm{g}}$ \\
\hline Thallium & 0 of 5 & ND (1.0) & ND (1.0) & $2.13^{b}$ & $1.7^{\mathrm{C}}$ & $\mathrm{No}^{f}$ \\
\hline Thorium & 0 of 5 & ND (1.0) & ND (1.0) & $1.14^{b}$ & NAV & $\mathrm{No}^{f}$ \\
\hline Titanium & 5 of 5 & 1.16 & 2.29 & $2.37^{b}$ & $150,000^{\mathrm{e}}$ & $\mathrm{No}^{\mathrm{g}}$ \\
\hline Uranium & 0 of 5 & ND (1.0) & ND (1.0) & ND (1) & $110^{\mathrm{e}}$ & $\mathrm{No}^{f}$ \\
\hline Vanadium & 4 of 5 & ND (1.0) & 2.02 & $1.44^{b}$ & $260^{e}$ & $\mathrm{No}^{h}$ \\
\hline Zinc & 4 of 5 & ND (1.0) & 60.8 & $12.8^{\mathrm{b}}$ & $11,000^{\mathrm{e}}$ & $\mathrm{No}^{h}$ \\
\hline
\end{tabular}

${ }^{\mathrm{a} B a c k g r o u n d}$ concentrations are upper tolerance limits (UTL) (ADEC, 1999a) unless otherwise noted.

${ }^{\mathrm{b}}$ Maximum detected concentration in background samples due to insufficient detection frequency to calculate UTL.

${ }^{\mathrm{c}}$ Federal ambient water quality criteria based on ingestion of water and organisms.

${ }^{d}$ State of Alaska groundwater cleanup level, Discharge Reporting, Cleanup, and Disposal of Oil and other Hazardous Substances

(18 AAC 75, Articles 3 and 9 [ADEC, 1999c]).

eU.S. EPA Region III risk-based concentrations for tap water (EPA, 2000b).

\section{COC Selection Criteria}

${ }^{7}$ Constituent not detected in any samples.

${ }^{9}$ Maximum detected constituent concentration is less than local background concentration.

${ }^{h}$ Maximum detected constituent concentration is less than human health screening value.

${ }^{\mathrm{i}}$ Constituent is an essential macronutrient and only presents a risk at extremely high concentrations.

ND - Not detected (detection limit)

NAV - Not available

NAN - Not analyzed

$\mu \mathrm{g} / \mathrm{L}$ - Micrograms per liter 
Table 4-10

\section{Summary of Analytical Results and Human Health Risk Assessment Screening for Sediment in Limpet Creek, Drill Site F}

(Page 1 of 3 )

\begin{tabular}{|c|c|c|c|c|c|c|}
\hline Analyte & $\begin{array}{l}\text { Detection } \\
\text { Frequency }\end{array}$ & $\begin{array}{c}\text { Minimum } \\
\text { Detected } \\
\text { Concentration } \\
(\mathbf{m g} / \mathbf{k g})\end{array}$ & $\begin{array}{c}\text { Maximum } \\
\text { Detected } \\
\text { Concentration } \\
(\mathbf{m g} / \mathbf{k g})\end{array}$ & $\begin{array}{l}\text { Upland Stream } \\
\text { Background } \\
\text { Concentration } \\
(\mathrm{mg} / \mathrm{kg})\end{array}$ & $\begin{array}{l}\text { Human Health } \\
\text { Screening } \\
\text { Value } \\
(\mathrm{mg} / \mathrm{kg})\end{array}$ & $\begin{array}{c}\text { Human } \\
\text { Health } \\
\text { Constituent } \\
\text { of Concern }\end{array}$ \\
\hline $\begin{array}{l}\text { Diesel-Range } \\
\text { Hydrocarbons }\end{array}$ & 0 of 5 & ND (10.3) & ND (19.8) & NAN & $200^{c}$ & $\mathrm{No}^{\mathrm{d}}$ \\
\hline \multicolumn{7}{|c|}{ Volatile Organic Compounds } \\
\hline 1,2,4-Trimethylbenzene & 0 of 1 & ND $(0.0244)$ & ND (0.0244) & NAN & NAV & $\mathrm{No}^{d}$ \\
\hline 1,3,5-Trimethylbenzene & 0 of 1 & ND (0.0244) & ND $(0.0244)$ & NAN & NAV & $\mathrm{No}^{\mathrm{d}}$ \\
\hline 2-Butanone & 0 of 1 & ND (0.0489) & ND (0.0489) & NAN & NAV & $\mathrm{No}^{d}$ \\
\hline Acetone & 0 of 1 & ND $(0.564)$ & ND (0.564) & NAN & $10,100^{C}$ & $\mathrm{No}^{\mathrm{d}}$ \\
\hline Benzene & 0 of 1 & ND (0.0244) & ND (0.0244) & NAN & $286^{c}$ & $\mathrm{No}^{d}$ \\
\hline Carbon disulfide & 0 of 1 & ND (0.0426) & ND (0.0426) & NAN & $10,100^{C}$ & $\mathrm{No}^{d}$ \\
\hline Ethylbenzene & 0 of 1 & ND (0.0244) & ND (0.0244) & NAN & $10,100^{C}$ & $\mathrm{No}^{d}$ \\
\hline Isopropylbenzene & 0 of 1 & ND (0.0244) & ND (0.0244) & NAN & NAV & $\mathrm{No}^{\mathrm{d}}$ \\
\hline $\mathrm{m}, \mathrm{p}$-Xylene & 0 of 1 & ND (0.0244) & ND (0.0244) & NAN & $203,000^{c}$ & $\mathrm{No}^{d}$ \\
\hline Methylene chloride & 0 of 1 & ND $(0.0244)$ & ND (0.0244) & NAN & $1,110^{c}$ & $\mathrm{No}^{d}$ \\
\hline n-Butylbenzene & 0 of 1 & ND (0.0244) & ND (0.0244) & NAN & NAV & $\mathrm{No}^{d}$ \\
\hline n-Propylbenzene & 0 of 1 & ND (0.0244) & ND (0.0244) & NAN & NAV & $\mathrm{No}^{\mathrm{d}}$ \\
\hline Naphthalene & 0 of 1 & ND (0.0244) & ND (0.0244) & NAN & $4,060^{c}$ & $\mathrm{No}^{d}$ \\
\hline o-Xylene & 0 of 1 & ND (0.0244) & ND (0.0244) & NAN & $203,000^{c}$ & $\mathrm{No}^{\mathrm{d}}$ \\
\hline p-Isopropyltoluene & 0 of 1 & ND (0.0244) & ND (0.0244) & NAN & NAV & $\mathrm{No}^{d}$ \\
\hline sec-Butylbenzene & 0 of 1 & ND (0.0244) & ND (0.0244) & NAN & NAV & $\mathrm{No}^{d}$ \\
\hline Toluene & 0 of 1 & ND (0.0244) & ND (0.0244) & NAN & $20,300^{c}$ & $\mathrm{No}^{\mathrm{d}}$ \\
\hline \multicolumn{7}{|c|}{ Polycyclic Aromatic Hydrocarbons } \\
\hline Acenaphthene & 0 of 5 & ND (0.00849) & ND (0.0163) & NAN & 6,080 & $\mathrm{No}^{d}$ \\
\hline Acenaphthylene & 0 of 5 & ND (0.00849) & ND (0.0163) & NAN & NAV & $\mathrm{No}^{\mathrm{d}}$ \\
\hline Anthracene & 0 of 5 & ND (0.00849) & ND $(0.0163)$ & NAN & $30,400^{c}$ & $\mathrm{No}^{\mathrm{d}}$ \\
\hline Benzo(a)anthracene & 0 of 5 & ND (0.00849) & ND (0.0163) & NAN & $11.4^{\mathrm{C}}$ & $\mathrm{No}^{\mathrm{d}}$ \\
\hline Benzo(a)pyrene & 0 of 5 & ND (0.00849) & ND (0.0163) & NAN & $1.1^{\mathrm{c}}$ & $\mathrm{No}^{d}$ \\
\hline Benzo(b)fluoranthene & 0 of 5 & ND (0.00849) & ND $(0.0163)$ & NAN & $11.4^{\mathrm{c}}$ & $\mathrm{No}^{d}$ \\
\hline Benzo(ghi)perylene & 0 of 5 & ND (0.00849) & ND $(0.0163)$ & NAN & $1.1^{\mathrm{c}}$ & $\mathrm{No}^{\mathrm{d}}$ \\
\hline Benzo(k)fluoranthene & 0 of 5 & ND (0.00849) & ND $(0.0163)$ & NAN & $114^{\mathrm{C}}$ & $\mathrm{No}^{\mathrm{d}}$ \\
\hline Chrysene & 0 of 5 & ND (0.00849) & ND (0.0163) & NAN & $1,140^{C}$ & $\mathrm{No}^{\mathrm{d}}$ \\
\hline
\end{tabular}


Table 4-10

\section{Summary of Analytical Results and Human Health Risk Assessment Screening for Sediment in Limpet Creek, Drill Site F}

(Page 2 of 3 )

\begin{tabular}{|c|c|c|c|c|c|c|}
\hline Analyte & $\begin{array}{l}\text { Detection } \\
\text { Frequency }\end{array}$ & $\begin{array}{c}\text { Minimum } \\
\text { Detected } \\
\text { Concentration } \\
(\mathbf{m g} / \mathbf{k g})\end{array}$ & $\begin{array}{c}\text { Maximum } \\
\text { Detected } \\
\text { Concentration } \\
(\mathbf{m g} / \mathbf{k g})\end{array}$ & $\begin{array}{l}\text { Upland Stream } \\
\text { Background } \\
\text { Concentration } \\
\text { (mg/kg) }\end{array}$ & $\begin{array}{c}\text { Human Health } \\
\text { Screening } \\
\text { Value } \\
(\mathrm{mg} / \mathrm{kg})\end{array}$ & $\begin{array}{c}\text { Human } \\
\text { Health } \\
\text { Constituent } \\
\text { of Concern }\end{array}$ \\
\hline Dibenzo(a,h)anthracene & 0 of 5 & ND (0.00849) & ND (0.0163) & NAN & $1.1^{\mathrm{C}}$ & $\mathrm{No}^{\mathrm{d}}$ \\
\hline Fluoranthene & 0 of 5 & ND (0.00849) & ND (0.0163) & NAN & $4,060^{\mathrm{C}}$ & $\mathrm{No}^{\mathrm{d}}$ \\
\hline Fluorene & 0 of 5 & ND (0.00849) & ND $(0.0163)$ & NAN & $4,060^{\mathrm{C}}$ & $\mathrm{No}^{\mathrm{d}}$ \\
\hline Indeno(1,2,3-cd)pyrene & 0 of 5 & ND (0.00849) & ND (0.0163) & NAN & $11^{\mathrm{c}}$ & $\mathrm{No}^{\mathrm{d}}$ \\
\hline Naphthalene & 0 of 5 & ND (0.00849) & ND (0.0163) & NAN & $4,060^{\mathrm{C}}$ & $\mathrm{No}^{d}$ \\
\hline Phenanthrene & 0 of 5 & ND (0.00849) & ND (0.0163) & NAN & $1.1^{\mathrm{c}}$ & $\mathrm{No}^{d}$ \\
\hline Pyrene & 0 of 5 & ND (0.00849) & ND (0.0163) & NAN & $3,040^{c}$ & No ${ }^{d}$ \\
\hline \multicolumn{7}{|c|}{ Polychlorinated Biphenyls } \\
\hline Aroclor 1016 & NAV & NAV & NAV & NAN & $10^{c}$ & $\mathrm{No}^{d}$ \\
\hline Aroclor 1221 & NAV & NAV & NAV & NAN & $10^{\mathrm{C}}$ & $\mathrm{No}^{d}$ \\
\hline Aroclor 1232 & NAV & NAV & NAV & NAN & $10^{c}$ & $\mathrm{No}^{d}$ \\
\hline Aroclor 1242 & NAV & NAV & NAV & NAN & $10^{c}$ & $\mathrm{No}^{d}$ \\
\hline Aroclor 1248 & NAV & NAV & NAV & NAN & $10^{c}$ & $\mathrm{No}^{\mathrm{d}}$ \\
\hline Aroclor 1254 & NAV & NAV & NAV & NAN & $10^{c}$ & $\mathrm{No}^{d}$ \\
\hline Aroclor 1260 & NAV & NAV & NAV & NAN & $10^{\mathrm{C}}$ & $\mathrm{No}^{\mathrm{d}}$ \\
\hline Aroclor 1262 & NAV & NAV & NAV & NAN & $10^{c}$ & $\mathrm{No}^{d}$ \\
\hline Aroclor 1268 & NAV & NAV & NAV & NAN & $10^{c}$ & $\mathrm{No}^{\mathrm{d}}$ \\
\hline \multicolumn{7}{|c|}{ Metals } \\
\hline Aluminum & 5 of 5 & 15,500 & 25,100 & 47,500 & NAV & $\mathrm{No}^{\mathrm{e}}$ \\
\hline Arsenic & 4 of 5 & ND (2.47) & 4.16 & 30.5 & $5.5^{\mathrm{c}}$ & $\mathrm{No}^{\mathrm{e}}$ \\
\hline Barium & 5 of 5 & 36.8 & 93.1 & 468 & $7,100^{\mathrm{C}}$ & $\mathrm{No}^{\mathrm{e}}$ \\
\hline Beryllium & 0 of 5 & ND (1.29) & ND (2.47) & ND & $1.9^{\mathrm{C}}$ & $\mathrm{No}^{d}$ \\
\hline Boron & 3 of 5 & ND (6.57) & 89.3 & $6.42^{b}$ & NAV & $\mathrm{No}^{\mathrm{g}}$ \\
\hline Cadmium & 0 of 5 & ND (1.29) & ND (2.47) & ND & $101^{\mathrm{c}}$ & $\mathrm{No}^{d}$ \\
\hline Calcium & 5 of 5 & 3,360 & 7,300 & 7,720 & NAV & $\mathrm{No}^{\mathrm{e}}$ \\
\hline Cerium & 5 of 5 & 5.55 & 12.9 & 13 & NAV & $\mathrm{No}^{\mathrm{e}}$ \\
\hline Cesium & 0 of 5 & ND (1.29) & ND (2.47) & ND & NAV & $\mathrm{No}^{d}$ \\
\hline Chromium & 5 of 5 & 7.66 & 9.81 & 18.9 & $507^{c}$ & $\mathrm{No}^{\mathrm{e}}$ \\
\hline Cobalt & 5 of 5 & 6.67 & 16.6 & 37.3 & NAV & $\mathrm{No}^{\mathrm{e}}$ \\
\hline Copper & 5 of 5 & 16.8 & 57.6 & 82.4 & NAV & $\mathrm{No}^{\mathrm{e}}$ \\
\hline
\end{tabular}


Table 4-10

\section{Summary of Analytical Results and Human Health Risk Assessment Screening for Sediment in Limpet Creek, Drill Site F}

(Page 3 of 3 )

\begin{tabular}{|c|c|c|c|c|c|c|}
\hline Analyte & $\begin{array}{l}\text { Detection } \\
\text { Frequency }\end{array}$ & $\begin{array}{c}\text { Minimum } \\
\text { Detected } \\
\text { Concentration } \\
(\mathbf{m g} / \mathbf{k g})\end{array}$ & $\begin{array}{c}\text { Maximum } \\
\text { Detected } \\
\text { Concentration } \\
(\mathrm{mg} / \mathrm{kg})\end{array}$ & $\begin{array}{c}\text { Upland Stream } \\
\text { Background } \\
\text { Concentration }{ }^{\mathrm{a}} \\
(\mathrm{mg} / \mathrm{kg})\end{array}$ & $\begin{array}{l}\text { Human Health } \\
\text { Screening } \\
\text { Value } \\
(\mathrm{mg} / \mathrm{kg})\end{array}$ & $\begin{array}{c}\text { Human } \\
\text { Health } \\
\text { Constituent } \\
\text { of Concern }\end{array}$ \\
\hline Iron & 5 of 5 & 10,400 & 64,800 & 123,000 & NAV & $\mathrm{No}^{\mathrm{e}}$ \\
\hline Lithium & 5 of 5 & 4.08 & 5.59 & 21.7 & NAV & $\mathrm{No}^{\mathrm{e}}$ \\
\hline Magnesium & 5 of 5 & 750 & 3,340 & 17,000 & NAV & $\mathrm{No}^{\mathrm{e}}$ \\
\hline Manganese & 5 of 5 & 91.2 & 1,250 & 26,500 & NAV & $\mathrm{No}^{\mathrm{e}}$ \\
\hline Molybdenum & 1 of 5 & ND (1.31) & 1.63 & ND & NAV & $\mathrm{No}^{\mathrm{g}}$ \\
\hline Nickel & 5 of 5 & 4.74 & 7.71 & 23.8 & $2,030^{\mathrm{C}}$ & $\mathrm{No}^{\mathrm{e}}$ \\
\hline Potassium & 5 of 5 & 201 & 260 & 1,290 & NAV & $\mathrm{No}^{\mathrm{e}}$ \\
\hline Selenium & 3 of 5 & ND (1.31) & 2.52 & $3.15^{b}$ & $507^{c}$ & $\mathrm{No}^{\mathrm{e}}$ \\
\hline Strontium & 5 of 5 & 23.6 & 37.4 & 207 & NAV & $\mathrm{No}^{\mathrm{e}}$ \\
\hline Thallium & 0 of 5 & ND (1.29) & ND (2.47) & ND & NAV & $\mathrm{No}^{d}$ \\
\hline Thorium & 0 of 5 & $\mathrm{ND}(1.29)$ & $\mathrm{ND}(2.47)$ & ND & NAV & $\mathrm{No}^{\mathrm{d}}$ \\
\hline Titanium & 5 of 5 & 865 & 1,370 & 1,480 & NAV & $\mathrm{No}^{\mathrm{e}}$ \\
\hline Uranium & 0 of 5 & ND (1.29) & ND (2.47) & ND & NAV & $\mathrm{No}^{d}$ \\
\hline Vanadium & 5 of 5 & 92.5 & 165 & 336 & $710^{c}$ & $\mathrm{No}^{\mathrm{e}}$ \\
\hline Zinc & 5 of 5 & 39.2 & 257 & 185 & $30,400^{c}$ & $\mathrm{No}^{f}$ \\
\hline Total Organic Carbon & 5 of 5 & 19,200 & 72,500 & NAN & NAV & No \\
\hline
\end{tabular}

${ }^{\mathrm{a}}$ Background concentrations are upper tolerance limits (UTL) (ADEC, 1999a) unless otherwise noted.

${ }^{\mathrm{b}}$ Maximum detected concentration in background samples due to insufficient detection frequency to calculate UTL.

'State of Alaska soil cleanup level, Discharge Reporting, Cleanup, and Disposal of Oil and other Hazardous Substances (18 AAC 75, Articles 3 and 9 [ADEC, 1999c]).

\section{COC Selection Criteria}

${ }^{\mathrm{d}}$ Constituent not detected in any samples.

${ }^{e}$ Maximum detected constituent concentration is less than local background concentration.

Maximum detected constituent concentration is less than human health screening value.

${ }^{9}$ Not a constituent associated with drilling mud or other drilling activities.

ND - Not detected (detection limit)

NAV - Not available

NAN - Not analyzed

$\mathrm{mg} / \mathrm{kg}$ - Milligrams per kilogram 


\section{Table 4-11}

\section{Summary of Analytical Results and Human Health Risk Assessment Screening for Fish Tissue in Limpet Creek, Drill Site F}

(Page 1 of 2 )

\begin{tabular}{|c|c|c|c|c|c|c|}
\hline Analyte & $\begin{array}{l}\text { Detection } \\
\text { Frequency }\end{array}$ & $\begin{array}{c}\text { Minimum } \\
\text { Detected } \\
\text { Concentration } \\
(\mathbf{m g} / \mathbf{k g})\end{array}$ & $\begin{array}{c}\text { Maximum } \\
\text { Detected } \\
\text { Concentration } \\
(\mathrm{mg} / \mathrm{kg})\end{array}$ & $\begin{array}{l}\text { Upland Stream } \\
\text { Background } \\
\text { Concentration }{ }^{\mathrm{a}} \\
(\mathrm{mg} / \mathrm{kg})\end{array}$ & $\begin{array}{l}\text { Human Health } \\
\text { Screening } \\
\text { Value }^{\mathrm{c}} \\
(\mathrm{mg} / \mathbf{k g})\end{array}$ & $\begin{array}{c}\text { Human } \\
\text { Health } \\
\text { Constituent } \\
\text { of Concern }\end{array}$ \\
\hline \multicolumn{7}{|c|}{ Polycyclic Aromatic Hydrocarbons } \\
\hline Acenaphthene & 0 of 3 & ND (0.0335) & ND (0.0335) & NAN & 81.1 & $\mathrm{No}^{d}$ \\
\hline Acenaphthylene & 0 of 3 & ND (0.0335) & ND (0.0335) & NAN & NAV & $\mathrm{No}^{\mathrm{d}}$ \\
\hline Anthracene & 0 of 3 & ND (0.0335) & ND (0.0335) & NAN & 410 & $\mathrm{No}^{\mathrm{d}}$ \\
\hline Benzo(a)anthracene & 0 of 3 & ND (0.0335) & ND (0.0335) & NAN & 0.0043 & $\mathrm{No}^{d}$ \\
\hline Benzo(a)pyrene & 0 of 3 & ND (0.0335) & ND (0.0335) & NAN & 0.00043 & $\mathrm{No}^{\mathrm{d}}$ \\
\hline Benzo(b)fluoranthene & 0 of 3 & ND (0.0335) & ND (0.0335) & NAN & 0.0043 & No ${ }^{d}$ \\
\hline Benzo(ghi)perylene & 0 of 3 & ND (0.0335) & ND (0.0335) & NAN & NAV & $\mathrm{No}^{d}$ \\
\hline Benzo(k)fluoranthene & 0 of 3 & ND (0.0335) & ND (0.0335) & NAN & 0.043 & $\mathrm{No}^{d}$ \\
\hline Chrysene & 0 of 3 & ND (0.0335) & ND (0.0335) & NAN & 0.43 & $\mathrm{No}^{d}$ \\
\hline Dibenzo(a,h)anthracene & 0 of 3 & ND (0.0335) & ND $(0.0335)$ & NAN & 0.00043 & $\mathrm{No}^{d}$ \\
\hline Fluoranthene & 0 of 3 & ND (0.0335) & ND (0.0335) & NAN & 54 & $\mathrm{No}^{d}$ \\
\hline Fluorene & 0 of 3 & ND (0.0335) & ND (0.0335) & NAN & 54 & No ${ }^{d}$ \\
\hline Indeno(1,2,3-cd)pyrene & 0 of 3 & ND (0.0335) & ND (0.0335) & NAN & 0.0043 & $\mathrm{No}^{d}$ \\
\hline Naphthalene & 0 of 3 & ND (0.0335) & ND (0.0335) & NAN & 27 & $\mathrm{No}^{d}$ \\
\hline Phenanthrene & 0 of 3 & ND (0.0335) & ND (0.0335) & NAN & NAV & $\mathrm{No}^{d}$ \\
\hline Pyrene & 0 of 3 & ND (0.0335) & ND (0.0335) & NAN & 41 & $\mathrm{No}^{d}$ \\
\hline \multicolumn{7}{|c|}{ Polychlorinated Biphenyls } \\
\hline Aroclor 1016 & 0 of 3 & $\mathrm{ND}(0.01)$ & ND (0.01) & NAN & 0.045 & No ${ }^{d}$ \\
\hline Aroclor 1221 & 0 of 3 & ND (0.02) & ND (0.02) & NAN & 0.0016 & $\mathrm{No}^{d}$ \\
\hline Aroclor 1232 & 0 of 3 & ND (0.01) & ND (0.01) & NAN & 0.0016 & $\mathrm{No}^{d}$ \\
\hline Aroclor 1242 & 0 of 3 & ND (0.01) & ND (0.01) & NAN & 0.0016 & $\mathrm{No}^{\mathrm{d}}$ \\
\hline Aroclor 1248 & 0 of 3 & ND $(0.01)$ & ND (0.01) & NAN & 0.0016 & $\mathrm{No}^{\mathrm{d}}$ \\
\hline Aroclor 1254 & 0 of 3 & ND (0.01) & ND (0.01) & NAN & 0.0016 & $\mathrm{No}^{d}$ \\
\hline Aroclor 1260 & 2 of 3 & ND $(0.01)$ & 0.0204 & NAN & 0.0016 & Yes \\
\hline \multicolumn{7}{|c|}{ Metals } \\
\hline Aluminum & 3 of 3 & 10.3 & 36 & 38.8 & 1400 & $\mathrm{No}^{\mathrm{e}}$ \\
\hline Arsenic (total) & 3 of 3 & 0.0665 & 1.37 & 26.9 & 0.0021 & $\mathrm{No}^{\mathrm{e}}$ \\
\hline Arsenic (inorganic) & 3 of 3 & 0.00172 & 0.00442 & 0.0456 & 0.0021 & $\mathrm{No}^{\mathrm{e}}$ \\
\hline Barium & 3 of 3 & 0.636 & 0.83 & 0.567 & 95 & $\mathrm{No}^{f}$ \\
\hline Beryllium & 0 of 3 & ND (0.025) & ND (0.025) & ND & 2.7 & $\mathrm{No}^{d}$ \\
\hline Boron & 0 of 3 & ND (11.4) & ND (11.9) & 24.1 & 120 & $\mathrm{No}^{d}$ \\
\hline Cadmium & 0 of 3 & ND (0.025) & ND (0.025) & ND & 1.4 & Nod \\
\hline
\end{tabular}


Table 4-11

\section{Summary of Analytical Results and Human Health Risk Assessment Screening for Fish Tissue in Limpet Creek, Drill Site F}

(Page 2 of 2)

\begin{tabular}{|c|c|c|c|c|c|c|}
\hline Analyte & $\begin{array}{l}\text { Detection } \\
\text { Frequency }\end{array}$ & $\begin{array}{c}\text { Minimum } \\
\text { Detected } \\
\text { Concentration } \\
(\mathbf{m g} / \mathbf{k g})\end{array}$ & $\begin{array}{c}\text { Maximum } \\
\text { Detected } \\
\text { Concentration } \\
(\mathbf{m g} / \mathbf{k g})\end{array}$ & $\begin{array}{l}\text { Upland Stream } \\
\text { Background } \\
\text { Concentration } \\
(\mathrm{mg} / \mathrm{kg})\end{array}$ & $\begin{array}{l}\text { Human Health } \\
\text { Screening } \\
\text { Value }^{\mathrm{c}} \\
(\mathbf{m g} / \mathbf{k g})\end{array}$ & $\begin{array}{c}\text { Human } \\
\text { Health } \\
\text { Constituent } \\
\text { of Concern }\end{array}$ \\
\hline Calcium & 3 of 3 & 6,680 & 9,480 & 10,300 & NAV & $\mathrm{No}^{\mathrm{e}}$ \\
\hline Cerium & 0 of 3 & ND (0.025) & ND (0.025) & ND & NAV & $\mathrm{No}^{\mathrm{d}}$ \\
\hline Cesium & 0 of 3 & ND (0.025) & ND (0.025) & ND & NAV & $\mathrm{No}^{\mathrm{d}}$ \\
\hline Chromium & 3 of 3 & 0.365 & 0.534 & 0.684 & 4.1 & $\mathrm{No}^{\mathrm{e}}$ \\
\hline Cobalt & 1 of 3 & ND (0.05) & 0.0523 & $0.0510^{\mathrm{b}}$ & 81 & $\mathrm{No}^{f}$ \\
\hline Copper & 0 of 3 & ND (0.736) & ND (1.34) & 3.4 & 54 & $\mathrm{No}^{d}$ \\
\hline Iron & 3 of 3 & 14.1 & 101 & 73.6 & 410 & $\mathrm{No}^{f}$ \\
\hline Lithium & 0 of 3 & ND (0.025) & ND (0.025) & ND & 27 & $\mathrm{No}^{\mathrm{d}}$ \\
\hline Magnesium & 3 of 3 & 305 & 340 & 349 & NAV & $\mathrm{No}^{\mathrm{e}}$ \\
\hline Manganese & 3 of 3 & 1.45 & 3.58 & 164 & 190 & $\mathrm{No}^{\mathrm{e}}$ \\
\hline Molybdenum & 0 of 3 & ND (0.125) & ND (0.125) & $0.150^{b}$ & 6.8 & $\mathrm{No}^{d}$ \\
\hline Nickel & 0 of 3 & ND $(0.125)$ & ND $(0.125)$ & ND & 27 & $\mathrm{No}^{\mathrm{d}}$ \\
\hline Potassium & 3 of 3 & 3,900 & 4,450 & 4,310 & NAV & $\mathrm{No}^{\mathrm{g}}$ \\
\hline Selenium & 3 of 3 & 0.669 & 1.1 & 2.08 & 6.8 & $\mathrm{No}^{\mathrm{e}}$ \\
\hline Strontium & 3 of 3 & 15.9 & 20.2 & $12.1^{\mathrm{b}}$ & 810 & $\mathrm{No}^{f}$ \\
\hline Thallium & 0 of 3 & ND (0.025) & ND (0.025) & $0.0755^{b}$ & 0.095 & $\mathrm{No}^{\mathrm{d}}$ \\
\hline Thorium & 1 of 3 & ND (2.5) & 4.98 & $4.67^{b}$ & NAV & $\mathrm{No}^{\mathrm{h}}$ \\
\hline Titanium & 3 of 3 & 0.242 & 1.25 & 0.834 & 5,400 & $\mathrm{No}^{f}$ \\
\hline Uranium & 0 of 3 & ND (0.5) & ND (0.5) & ND & 4.1 & $\mathrm{No}^{d}$ \\
\hline Vanadium & 3 of 3 & 0.169 & 0.409 & 0.289 & 9.5 & $\mathrm{No}^{f}$ \\
\hline Zinc & 3 of 3 & 34.5 & 43.8 & 78 & 410 & $\mathrm{No}^{\mathrm{e}}$ \\
\hline \% Lipids & 1 of 1 & 2.98 & 2.98 & NAN & NAV & No \\
\hline
\end{tabular}

${ }^{a}$ Background concentrations are upper tolerance limits (UTL) (ADEC, 1999a) unless otherwise noted.

${ }^{b}$ Maximum detected concentration in background samples due to insufficient detection frequency to calculate UTL.

${ }^{\mathrm{c} U}$.S. EPA Region III risk-based screening criteria for fish consumption unless otherwise noted.

coc Selection Criteria

${ }^{\mathrm{d}}$ Constituent not detected in any samples.

${ }^{\mathrm{e}}$ Maximum detected constituent concentration is less than local background concentration.

${ }^{f}$ Maximum detected constituent concentration is less than human health screening value.

${ }^{9}$ Constituent is an essential macronutrient and only presents a risk at extremely high concentrations.

${ }^{\mathrm{h}} \mathrm{Not}$ a constituent associated with drilling mud or other drilling activities.

ND - Not detected (detection limit)

NAV - Not available

NAN - Not analyzed

$\mathrm{mg} / \mathrm{kg}$ - Milligrams per kilogram 
Table 4-12

Summary of Analytical Results and Human Health Risk Assessment Screening for Surface Water in Bridge Creek, Long Shot Site

(Page 1 of 3 )

\begin{tabular}{|c|c|c|c|c|c|c|}
\hline Analyte & $\begin{array}{l}\text { Detection } \\
\text { Frequency }\end{array}$ & $\begin{array}{c}\text { Minimum } \\
\text { Detected } \\
\text { Concentration } \\
(\mu \mathrm{g} / \mathrm{L})\end{array}$ & $\begin{array}{c}\text { Maximum } \\
\text { Detected } \\
\text { Concentration } \\
(\mu \mathrm{g} / \mathrm{L})\end{array}$ & $\begin{array}{c}\text { Lowland Stream } \\
\text { Background } \\
\text { Concentration }{ }^{\mathrm{a}} \\
(\mu \mathrm{g} / \mathrm{L})\end{array}$ & $\begin{array}{l}\text { Human Health } \\
\text { Screening } \\
\text { Value } \\
(\mu \mathrm{g} / \mathrm{L})\end{array}$ & $\begin{array}{c}\text { Human } \\
\text { Health } \\
\text { Constituent } \\
\text { of Concern }\end{array}$ \\
\hline $\begin{array}{l}\text { Diesel-Range } \\
\text { Hydrocarbons }\end{array}$ & 3 of 5 & ND (50) & 77.2 & NAN & $1,500^{d}$ & $\mathrm{No}^{f}$ \\
\hline \multicolumn{7}{|c|}{ Volatile Organic Compounds } \\
\hline 1,2,4-Trimethylbenzene & 0 of 5 & ND (1.0) & ND (1.0) & NAN & $12^{\mathrm{e}}$ & $\mathrm{No}^{\mathrm{e}}$ \\
\hline 1,3,5-Trimethylbenzene & 0 of 5 & ND (1.0) & ND (1.0) & NAN & $12^{\mathrm{e}}$ & $\mathrm{No}^{\mathrm{e}}$ \\
\hline 2-Butanone & 0 of 5 & ND (20) & ND (20) & NAN & $1,900^{\mathrm{e}}$ & $\mathrm{No}^{\mathrm{e}}$ \\
\hline Acetone & 0 of 5 & ND (20) & ND (20) & NAN & $3,700^{\mathrm{e}}$ & $\mathrm{No}^{\mathrm{e}}$ \\
\hline Benzene & 0 of 5 & ND (1.0) & ND (1.0) & NAN & $1.2^{\mathrm{C}}$ & $\mathrm{No}^{\mathrm{e}}$ \\
\hline Carbon disulfide & 0 of 5 & ND (1.0) & ND (1.0) & NAN & $1,000^{\mathrm{e}}$ & $\mathrm{No}^{\mathrm{e}}$ \\
\hline Ethylbenzene & 0 of 5 & ND (1.0) & ND (1.0) & NAN & $3,100^{c}$ & $\mathrm{No}^{\mathrm{e}}$ \\
\hline Isopropylbenzene & 0 of 5 & ND (1.0) & ND (1.0) & NAN & NAV & $\mathrm{No}^{\mathrm{e}}$ \\
\hline $\mathrm{m}, \mathrm{p}$-Xylene & 0 of 5 & ND (2.0) & ND (2.0) & NAN & $12,000^{e}$ & $\mathrm{No}^{\mathrm{e}}$ \\
\hline Methylene chloride & 0 of 5 & ND (2.0) & ND (5.95) & NAN & $4.7^{c}$ & $\mathrm{No}^{\mathrm{e}}$ \\
\hline n-Butylbenzene & 0 of 5 & ND (1.0) & ND (1.0) & NAN & $61^{\mathrm{e}}$ & $\mathrm{No}^{\mathrm{e}}$ \\
\hline n-Propylbenzene & 0 of 5 & ND (1.0) & ND (1.0) & NAN & $61^{\mathrm{e}}$ & $\mathrm{No}^{\mathrm{e}}$ \\
\hline Naphthalene & 0 of 5 & ND (1.0) & ND (1.0) & NAN & $730^{e}$ & $\mathrm{No}^{\mathrm{e}}$ \\
\hline o-Xylene & 0 of 5 & ND (1.0) & ND (1.0) & NAN & $12,000^{\mathrm{e}}$ & $\mathrm{No}^{\mathrm{e}}$ \\
\hline p-Isopropyltoluene & 0 of 5 & ND (1.0) & ND (1.0) & NAN & NAV & $\mathrm{No}^{\mathrm{e}}$ \\
\hline sec-Butylbenzene & 0 of 5 & ND (1.0) & ND (1.0) & NAN & $61^{\mathrm{e}}$ & $\mathrm{No}^{\mathrm{e}}$ \\
\hline Toluene & 0 of 5 & ND (1.0) & ND (1.0) & NAN & $10,000^{C}$ & $\mathrm{No}^{\mathrm{e}}$ \\
\hline \multicolumn{7}{|c|}{ Polycyclic Aromatic Hydrocarbons } \\
\hline Acenaphthene & 0 of 5 & $\mathrm{ND}(0.1)$ & $\mathrm{ND}(0.1)$ & NAN & $1,200^{C}$ & $\mathrm{No}^{\mathrm{e}}$ \\
\hline Acenaphthylene & 0 of 5 & $\mathrm{ND}(0.1)$ & $\mathrm{ND}(0.1)$ & NAN & $0.0028^{c}$ & $\mathrm{No}^{\mathrm{e}}$ \\
\hline Anthracene & 0 of 5 & ND $(0.1)$ & $\mathrm{ND}(0.1)$ & NAN & $0.0028^{c}$ & $\mathrm{No}^{\mathrm{e}}$ \\
\hline Benzo(a)anthracene & 0 of 5 & $\mathrm{ND}(0.1)$ & $\mathrm{ND}(0.1)$ & NAN & $0.0028^{C}$ & $\mathrm{No}^{\mathrm{e}}$ \\
\hline Benzo(a)pyrene & 0 of 5 & $\mathrm{ND}(0.1)$ & ND $(0.1)$ & NAN & $0.0028^{c}$ & $\mathrm{No}^{\mathrm{e}}$ \\
\hline Benzo(b)fluoranthene & 0 of 5 & ND $(0.1)$ & ND $(0.1)$ & NAN & $0.092^{\mathrm{e}}$ & $\mathrm{No}^{\mathrm{e}}$ \\
\hline Benzo(g,h,l)perylene & 0 of 5 & ND $(0.1)$ & ND $(0.1)$ & NAN & $0.0028^{c}$ & $\mathrm{No}^{\mathrm{e}}$ \\
\hline Benzo(k)fluoranthene & 0 of 5 & ND $(0.1)$ & ND $(0.1)$ & NAN & $0.0028^{c}$ & $\mathrm{No}^{\mathrm{e}}$ \\
\hline Chrysene & 0 of 5 & ND (0.1) & $\mathrm{ND}(0.1)$ & NAN & $0.0028^{c}$ & $\mathrm{No}^{\mathrm{e}}$ \\
\hline
\end{tabular}


Table 4-12

Summary of Analytical Results and Human Health Risk Assessment Screening for Surface Water in Bridge Creek, Long Shot Site

(Page 2 of 3 )

\begin{tabular}{|c|c|c|c|c|c|c|}
\hline Analyte & $\begin{array}{l}\text { Detection } \\
\text { Frequency }\end{array}$ & $\begin{array}{c}\text { Minimum } \\
\text { Detected } \\
\text { Concentration } \\
(\mu \mathrm{g} / \mathrm{L})\end{array}$ & $\begin{array}{c}\text { Maximum } \\
\text { Detected } \\
\text { Concentration } \\
(\mu \mathrm{g} / \mathrm{L})\end{array}$ & $\begin{array}{c}\text { Lowland Stream } \\
\text { Background } \\
\text { Concentration }^{\mathrm{a}} \\
(\mu \mathrm{g} / \mathrm{L})\end{array}$ & $\begin{array}{l}\text { Human Health } \\
\text { Screening } \\
\text { Value } \\
(\mu \mathrm{g} / \mathrm{L})\end{array}$ & $\begin{array}{c}\text { Human } \\
\text { Health } \\
\text { Constituent } \\
\text { of Concern }\end{array}$ \\
\hline Dibenzo(a,h)anthracene & 0 of 5 & $\mathrm{ND}(0.1)$ & ND $(0.1)$ & NAN & $0.0028^{C}$ & $\mathrm{No}^{\mathrm{e}}$ \\
\hline Fluoranthene & 0 of 5 & $\mathrm{ND}(0.1)$ & ND $(0.1)$ & NAN & $42^{c}$ & $\mathrm{No}^{\mathrm{e}}$ \\
\hline Fluorene & 0 of 5 & $\mathrm{ND}(0.1)$ & ND $(0.1)$ & NAN & $0.0028^{C}$ & $\mathrm{No}^{\mathrm{e}}$ \\
\hline Indeno(1,2,3-cd)pyrene & 0 of 5 & $\mathrm{ND}(0.1)$ & $\mathrm{ND}(0.1)$ & NAN & $0.0028^{c}$ & $\mathrm{No}^{\mathrm{e}}$ \\
\hline Naphthalene & 0 of 5 & ND $(0.1)$ & ND $(0.1)$ & NAN & $730^{e}$ & $\mathrm{No}^{\mathrm{e}}$ \\
\hline Phenanthrene & 0 of 5 & ND $(0.1)$ & $\mathrm{ND}(0.1)$ & NAN & $0.0028^{c}$ & $\mathrm{No}^{\mathrm{e}}$ \\
\hline Pyrene & 0 of 5 & $\mathrm{ND}(0.1)$ & $\mathrm{ND}(0.1)$ & NAN & $0.0028^{c}$ & $\mathrm{No}^{\mathrm{e}}$ \\
\hline \multicolumn{7}{|c|}{ Polychlorinated Biphenyls } \\
\hline Aroclor 1016 & NAV & NAV & NAV & NAN & $0.000044^{c}$ & $\mathrm{No}^{\mathrm{e}}$ \\
\hline Aroclor 1221 & NAV & NAV & NAV & NAN & $0.000044^{c}$ & $\mathrm{No}^{\mathrm{e}}$ \\
\hline Aroclor 1232 & NAV & NAV & NAV & NAN & $0.000044^{c}$ & $\mathrm{No}^{\mathrm{e}}$ \\
\hline Aroclor 1242 & NAV & NAV & NAV & NAN & $0.000044^{c}$ & $\mathrm{No}^{\mathrm{e}}$ \\
\hline Aroclor 1248 & NAV & NAV & NAV & NAN & $0.000044^{c}$ & $\mathrm{No}^{\mathrm{e}}$ \\
\hline Aroclor 1254 & NAV & NAV & NAV & NAN & $0.000044^{c}$ & $\mathrm{No}^{\mathrm{e}}$ \\
\hline Aroclor 1260 & NAV & NAV & NAV & NAN & $0.000044^{c}$ & $\mathrm{No}^{\mathrm{e}}$ \\
\hline Aroclor 1262 & NAV & NAV & NAV & NAN & $0.000044^{c}$ & $\mathrm{No}^{\mathrm{e}}$ \\
\hline Aroclor 1268 & NAV & NAV & NAV & NAN & $0.000044^{c}$ & $\mathrm{No}^{\mathrm{e}}$ \\
\hline \multicolumn{7}{|c|}{ Metals } \\
\hline Aluminum & 4 of 5 & ND (50) & 85.1 & 269 & $37,000^{\mathrm{e}}$ & $\mathrm{No}^{f}$ \\
\hline Arsenic & 0 of 5 & ND (1.0) & ND (1.0) & ND (1) & $0.018^{c}$ & $\mathrm{No}^{\mathrm{e}}$ \\
\hline Barium & 0 of 5 & ND (10) & ND (10) & ND (10) & $1,000^{c}$ & $\mathrm{No}^{\mathrm{e}}$ \\
\hline Beryllium & 0 of 5 & ND (1.0) & ND (1.0) & ND (1) & $0.0076^{c}$ & $\mathrm{No}^{\mathrm{e}}$ \\
\hline Boron & 0 of 5 & ND (50) & ND (50) & ND (50) & $3,300^{\mathrm{e}}$ & $\mathrm{No}^{\mathrm{e}}$ \\
\hline Cadmium & 0 of 5 & ND (1.0) & ND (1.0) & ND (1) & $10^{c}$ & $\mathrm{No}^{\mathrm{e}}$ \\
\hline Calcium & 5 of 5 & 3,700 & 7,250 & $4,320^{b}$ & NAV & $\mathrm{No}^{h}$ \\
\hline Cerium & 0 of 5 & ND (1.0) & ND (1.0) & ND (1) & NAV & $\mathrm{No}^{\mathrm{e}}$ \\
\hline Cesium & 0 of 5 & ND (1.0) & ND (1.0) & ND (1) & NAV & $\mathrm{No}^{\mathrm{e}}$ \\
\hline Chromium & 0 of 5 & ND (1.0) & ND (1.0) & $2.25^{\mathrm{b}}$ & $33,000^{c}$ & $\mathrm{No}^{\mathrm{e}}$ \\
\hline Cobalt & 3 of 5 & ND (1.0) & 1.28 & 3.35 & $2,200^{\mathrm{e}}$ & $\mathrm{No}^{f}$ \\
\hline Copper & 2 of 5 & ND (1.0) & 1.78 & 10.8 & $1,300^{c}$ & $\mathrm{No}^{f}$ \\
\hline
\end{tabular}


Table 4-12

\section{Summary of Analytical Results and Human Health Risk Assessment Screening for Surface Water in Bridge Creek, Long Shot Site (Page 3 of 3 )}

\begin{tabular}{|c|c|c|c|c|c|c|}
\hline Analyte & $\begin{array}{l}\text { Detection } \\
\text { Frequency }\end{array}$ & $\begin{array}{c}\text { Minimum } \\
\text { Detected } \\
\text { Concentration } \\
(\mu \mathrm{g} / \mathrm{L})\end{array}$ & $\begin{array}{c}\text { Maximum } \\
\text { Detected } \\
\text { Concentration } \\
(\mu \mathrm{g} / \mathrm{L})\end{array}$ & $\begin{array}{c}\text { Lowland Stream } \\
\text { Background } \\
\text { Concentration } \\
(\mu \mathrm{g} / \mathrm{L})\end{array}$ & $\begin{array}{l}\text { Human Health } \\
\text { Screening } \\
\text { Value } \\
(\mu \mathrm{g} / \mathrm{L})\end{array}$ & $\begin{array}{l}\text { Human } \\
\text { Health } \\
\text { Constituent } \\
\text { of Concern }\end{array}$ \\
\hline Iron & 5 of 5 & 199 & 535 & 993 & $300^{c}$ & $\mathrm{No}^{\mathrm{h}}$ \\
\hline Lithium & 0 of 5 & ND (1.0) & ND (1.0) & $1.17^{b}$ & $730^{e}$ & $\mathrm{No}^{\mathrm{e}}$ \\
\hline Magnesium & 5 of 5 & 3,170 & 4,750 & $4,690^{b}$ & NAV & $\mathrm{No}^{\mathrm{h}}$ \\
\hline Manganese & 5 of 5 & 7.24 & 52.1 & 267 & $50^{c}$ & $\mathrm{No}^{f}$ \\
\hline Molybdenum & 0 of 5 & ND (1.0) & ND (1.0) & ND (1) & $180^{e}$ & $\mathrm{No}^{\mathrm{e}}$ \\
\hline Nickel & 4 of 5 & ND (1.0) & 1.32 & ND (1) & $510^{c}$ & $\mathrm{No}^{\mathrm{g}}$ \\
\hline Potassium & 4 of 5 & ND (200) & 1,100 & 2,620 & NAV & $\mathrm{No}^{\mathrm{h}}$ \\
\hline Selenium & 0 of 5 & ND (1.0) & ND (1.0) & ND (1) & $104^{c}$ & $\mathrm{No}^{\mathrm{e}}$ \\
\hline Strontium & 5 of 5 & 22.1 & 33.7 & 36.1 & $22,000^{\mathrm{e}}$ & $\mathrm{No}^{f}$ \\
\hline Thallium & 0 of 5 & ND (1.0) & ND (1.0) & $7.07^{b}$ & $1.7^{\mathrm{C}}$ & $\mathrm{No}^{\mathrm{e}}$ \\
\hline Thorium & 0 of 5 & ND (1.0) & ND (1.0) & ND (1) & NAV & $\mathrm{No}^{\mathrm{e}}$ \\
\hline Titanium & 5 of 5 & 1.38 & 3.85 & 6.13 & $150,000^{\mathrm{e}}$ & $\mathrm{No}^{f}$ \\
\hline Uranium & 0 of 5 & ND (1.0) & ND (1.0) & ND (1) & $110^{e}$ & $\mathrm{No}^{\mathrm{e}}$ \\
\hline Vanadium & 1 of 5 & ND (1.0) & 1.03 & $4.43^{b}$ & $260^{e}$ & $\mathrm{No}^{f}$ \\
\hline Zinc & 1 of 5 & ND (10) & 12.2 & $50.3^{b}$ & $11,000^{\mathrm{e}}$ & $\mathrm{No}^{f}$ \\
\hline
\end{tabular}

${ }^{\mathrm{a}}$ Background concentrations are upper tolerance limits (UTL) (ADEC, 1999a) unless otherwise noted.

${ }^{b}$ Maximum detected concentration in background samples due to insufficient detection frequency to calculate UTL.

${ }^{\mathrm{c}}$ Federal ambient water quality criteria based on ingestion of water and organisms.

dState of Alaska groundwater cleanup level, Discharge Reporting, Cleanup, and Disposal of Oil and other Hazardous Substances (18 AAC 75, Articles 3 and 9 [ADEC, 1999c]).

eU.S. EPA Region III risk-based concentrations for tap water (EPA, 2000b).

\section{COC Selection Criteria}

${ }^{\mathrm{f}}$ Constituent not detected in any samples.

${ }^{9}$ Maximum detected constituent concentration is less than local background concentration.

${ }^{h}$ Maximum detected constituent concentration is less than human health screening value.

ND - Not detected (detection limit)

NAV - Not available

NAN - Not analyzed

$\mu \mathrm{g} / \mathrm{L}$ - Micrograms per liter 
Table 4-13

Summary of Analytical Results and Human Health Risk Assessment
Screening for Sediment in Bridge Creek, Long Shot Site

(Page 1 of 3 )

\begin{tabular}{|c|c|c|c|c|c|c|}
\hline Analyte & $\begin{array}{l}\text { Detection } \\
\text { Frequency }\end{array}$ & $\begin{array}{c}\text { Minimum } \\
\text { Detected } \\
\text { Concentration } \\
(\mathbf{m g} / \mathbf{k g})\end{array}$ & $\begin{array}{c}\text { Maximum } \\
\text { Detected } \\
\text { Concentration } \\
(\mathbf{m g} / \mathbf{k g})\end{array}$ & $\begin{array}{l}\text { Lowland Stream } \\
\text { Background } \\
\text { Concentration } \\
\text { (mg/kg) }\end{array}$ & $\begin{array}{l}\text { Human Health } \\
\text { Screening } \\
\text { Value } \\
(\mathrm{mg} / \mathrm{kg})\end{array}$ & $\begin{array}{c}\text { Human } \\
\text { Health } \\
\text { Constituent } \\
\text { of Concern }\end{array}$ \\
\hline $\begin{array}{l}\text { Diesel-Range } \\
\text { Hydrocarbons }\end{array}$ & 0 of 5 & ND (15.2) & ND (64.5) & NAN & $200^{\mathrm{C}}$ & No ${ }^{d}$ \\
\hline \multicolumn{7}{|c|}{ Volatile Organic Compounds } \\
\hline 1,2,4-Trimethylbenzene & 0 of 1 & ND $(0.071)$ & $\mathrm{ND}(0.071)$ & NAN & NAV & $\mathrm{No}^{\mathrm{d}}$ \\
\hline 1,3,5-Trimethylbenzene & 0 of 1 & ND $(0.071)$ & ND $(0.071)$ & NAN & NAV & $\mathrm{No}^{d}$ \\
\hline 2-Butanone & 0 of 1 & $\mathrm{ND}(0.142)$ & $\mathrm{ND}(0.142)$ & NAN & NAV & $\mathrm{No}^{\mathrm{d}}$ \\
\hline Acetone & 0 of 1 & ND (1.01) & ND (1.01) & NAN & $10,100^{c}$ & $\mathrm{No}^{d}$ \\
\hline Benzene & 0 of 1 & ND $(0.071)$ & ND $(0.071)$ & NAN & $286^{\mathrm{C}}$ & $\mathrm{No}^{\mathrm{d}}$ \\
\hline Carbon disulfide & 0 of 1 & ND $(0.071)$ & ND $(0.071)$ & NAN & $10,100^{c}$ & $\mathrm{No}^{\mathrm{d}}$ \\
\hline Ethylbenzene & 0 of 1 & ND $(0.071)$ & ND $(0.071)$ & NAN & $10,100^{C}$ & $\mathrm{No}^{\mathrm{d}}$ \\
\hline Isopropylbenzene & 0 of 1 & ND $(0.071)$ & ND $(0.071)$ & NAN & NAV & $\mathrm{No}^{d}$ \\
\hline $\mathrm{m}, \mathrm{p}$-Xylene & 0 of 1 & ND $(0.071)$ & ND $(0.071)$ & NAN & $203,000^{c}$ & $\mathrm{No}^{d}$ \\
\hline Methylene chloride & 0 of 1 & ND $(0.071)$ & ND $(0.071)$ & NAN & $1,110^{\mathrm{C}}$ & $\mathrm{No}^{d}$ \\
\hline n-Butylbenzene & 0 of 1 & ND $(0.071)$ & ND $(0.071)$ & NAN & NAV & $\mathrm{No}^{\mathrm{d}}$ \\
\hline n-Propylbenzene & 0 of 1 & ND $(0.071)$ & ND $(0.071)$ & NAN & NAV & $\mathrm{No}^{d}$ \\
\hline Naphthalene & 0 of 1 & ND $(0.071)$ & ND $(0.071)$ & NAN & $4,060^{c}$ & $\mathrm{No}^{d}$ \\
\hline o-Xylene & 0 of 1 & ND $(0.071)$ & ND $(0.071)$ & NAN & $203,000^{c}$ & $\mathrm{No}^{d}$ \\
\hline p-Isopropyltoluene & 0 of 1 & ND $(0.071)$ & ND $(0.071)$ & NAN & NAV & $\mathrm{No}^{d}$ \\
\hline sec-Butylbenzene & 0 of 1 & ND $(0.071)$ & ND $(0.071)$ & NAN & NAV & $\mathrm{No}^{d}$ \\
\hline Toluene & 0 of 1 & ND $(0.071)$ & ND $(0.071)$ & NAN & $20,300^{c}$ & $\mathrm{No}^{\mathrm{d}}$ \\
\hline \multicolumn{7}{|c|}{ Polycyclic Aromatic Hydrocarbons } \\
\hline Acenaphthene & 0 of 5 & ND $(0.0126)$ & ND (0.0532) & NAN & $6,080^{c}$ & $\mathrm{No}^{d}$ \\
\hline Acenaphthylene & 0 of 5 & ND $(0.0126)$ & ND (0.0532) & NAN & NAV & $\mathrm{No}^{\mathrm{d}}$ \\
\hline Anthracene & 0 of 5 & ND $(0.0126)$ & ND (0.0532) & NAN & $30,400^{c}$ & $\mathrm{No}^{d}$ \\
\hline Benzo(a)anthracene & 0 of 5 & ND $(0.0126)$ & ND (0.0532) & NAN & $11.4^{\mathrm{C}}$ & $\mathrm{No}^{d}$ \\
\hline Benzo(a)pyrene & 0 of 5 & ND $(0.0126)$ & ND (0.0532) & NAN & $1.1^{\mathrm{c}}$ & $\mathrm{No}^{d}$ \\
\hline Benzo(b)fluoranthene & 0 of 5 & ND $(0.0126)$ & ND (0.0532) & NAN & $11.4^{\mathrm{C}}$ & $\mathrm{No}^{d}$ \\
\hline Benzo(g,h,i)perylene & 0 of 5 & ND $(0.0126)$ & ND (0.0532) & NAN & $1.1^{\mathrm{c}}$ & $\mathrm{No}^{d}$ \\
\hline Benzo(k)fluoranthene & 0 of 5 & ND (0.0126) & ND (0.0532) & NAN & $114^{\mathrm{c}}$ & No ${ }^{d}$ \\
\hline
\end{tabular}


Table 4-13

\section{Summary of Analytical Results and Human Health Risk Assessment Screening for Sediment in Bridge Creek, Long Shot Site}

(Page 2 of 3 )

\begin{tabular}{|c|c|c|c|c|c|c|}
\hline Analyte & $\begin{array}{l}\text { Detection } \\
\text { Frequency }\end{array}$ & $\begin{array}{c}\text { Minimum } \\
\text { Detected } \\
\text { Concentration } \\
(\mathbf{m g} / \mathbf{k g})\end{array}$ & $\begin{array}{c}\text { Maximum } \\
\text { Detected } \\
\text { Concentration } \\
(\mathbf{m g} / \mathbf{k g})\end{array}$ & $\begin{array}{l}\text { Lowland Stream } \\
\text { Background } \\
\text { Concentration }{ }^{\mathrm{a}} \\
(\mathrm{mg} / \mathrm{kg})\end{array}$ & $\begin{array}{l}\text { Human Health } \\
\text { Screening } \\
\text { Value } \\
(\mathrm{mg} / \mathrm{kg})\end{array}$ & $\begin{array}{c}\text { Human } \\
\text { Health } \\
\text { Constituent } \\
\text { of Concern }\end{array}$ \\
\hline Chrysene & 0 of 5 & ND (0.0126) & ND (0.0532) & NAN & $1,140^{\mathrm{C}}$ & $\mathrm{No}^{\mathrm{d}}$ \\
\hline Dibenzo(a,h)anthracene & 0 of 5 & ND (0.0126) & ND (0.0532) & NAN & $1.1^{\mathrm{c}}$ & $\mathrm{No}^{d}$ \\
\hline Fluoranthene & 0 of 5 & ND (0.0126) & ND (0.0532) & NAN & $4,060^{c}$ & $\mathrm{No}^{d}$ \\
\hline Fluorene & 0 of 5 & ND (0.0126) & ND (0.0532) & NAN & $4,060^{c}$ & $\mathrm{No}^{\mathrm{d}}$ \\
\hline Indeno(1,2,3-cd)pyrene & 0 of 5 & ND (0.0126) & ND (0.0532) & NAN & $11^{\mathrm{c}}$ & $\mathrm{No}^{\mathrm{d}}$ \\
\hline Naphthalene & 0 of 5 & ND (0.0126) & ND (0.0532) & NAN & $4,060^{c}$ & $\mathrm{No}^{\mathrm{d}}$ \\
\hline Phenanthrene & 0 of 5 & ND (0.0126) & ND (0.0532) & NAN & $1.1^{\mathrm{c}}$ & $\mathrm{No}^{d}$ \\
\hline Pyrene & 0 of 5 & ND (0.0126) & ND (0.0532) & NAN & $3,040^{c}$ & $\mathrm{No}^{d}$ \\
\hline \multicolumn{7}{|c|}{ Polychlorinated Biphenyls } \\
\hline Aroclor 1016 & NAV & NAV & NAV & NAN & $10^{c}$ & $\mathrm{No}^{d}$ \\
\hline Aroclor 1221 & NAV & NAV & NAV & NAN & $10^{c}$ & Nod \\
\hline Aroclor 1232 & NAV & NAV & NAV & NAN & $10^{c}$ & $\mathrm{No}^{\mathrm{d}}$ \\
\hline Aroclor 1242 & NAV & NAV & NAV & NAN & $10^{c}$ & $\mathrm{No}^{d}$ \\
\hline Aroclor 1248 & NAV & NAV & NAV & NAN & $10^{c}$ & $\mathrm{No}^{d}$ \\
\hline Aroclor 1254 & NAV & NAV & NAV & NAN & $10^{c}$ & $\mathrm{No}^{d}$ \\
\hline Aroclor 1260 & NAV & NAV & NAV & NAN & $10^{c}$ & $\mathrm{No}^{d}$ \\
\hline Aroclor 1262 & NAV & NAV & NAV & NAN & $10^{c}$ & $\mathrm{No}^{d}$ \\
\hline Aroclor 1268 & NAV & NAV & NAV & NAN & $10^{c}$ & $\mathrm{No}^{d}$ \\
\hline \multicolumn{7}{|c|}{ Metals } \\
\hline Aluminum & 5 of 5 & 11,200 & 63,900 & 45,400 & NAV & $\mathrm{No}^{h}$ \\
\hline Arsenic & 5 of 5 & 5.42 & 16.7 & 100 & $5.5^{\mathrm{c}}$ & $\mathrm{No}^{\mathrm{e}}$ \\
\hline Barium & 4 of 5 & ND (80.6) & 271 & 469 & $7,100^{\mathrm{C}}$ & $\mathrm{No}^{\mathrm{e}}$ \\
\hline Beryllium & 0 of 5 & ND (1.9) & ND (8.06) & ND & $1.9^{\mathrm{C}}$ & $\mathrm{No}^{d}$ \\
\hline Boron & 0 of 5 & ND (9.51) & ND (40.3) & $7.18^{b}$ & NAV & $\mathrm{No}^{d}$ \\
\hline Cadmium & 0 of 5 & ND (1.9) & ND (8.06) & $0.608^{b}$ & $101^{c}$ & $\mathrm{No}^{d}$ \\
\hline Calcium & 5 of 5 & 3,930 & 8,770 & 7,300 & NAV & $\mathrm{No}^{\mathrm{g}}$ \\
\hline Cerium & 5 of 5 & 7.11 & 11.9 & 14.2 & NAV & $\mathrm{No}^{\mathrm{e}}$ \\
\hline Cesium & 0 of 5 & ND (1.9) & ND (8.06) & ND & NAV & $\mathrm{No}^{d}$ \\
\hline Chromium & 4 of 5 & 5.82 & 10 & 11.8 & $507^{c}$ & $\mathrm{No}^{\mathrm{e}}$ \\
\hline
\end{tabular}




\section{Table 4-13}

\section{Summary of Analytical Results and Human Health Risk Assessment Screening for Sediment in Bridge Creek, Long Shot Site}

(Page 3 of 3 )

\begin{tabular}{|c|c|c|c|c|c|c|}
\hline Analyte & $\begin{array}{l}\text { Detection } \\
\text { Frequency }\end{array}$ & $\begin{array}{c}\text { Minimum } \\
\text { Detected } \\
\text { Concentration } \\
(\mathbf{m g} / \mathbf{k g})\end{array}$ & $\begin{array}{c}\text { Maximum } \\
\text { Detected } \\
\text { Concentration } \\
(\mathbf{m g} / \mathbf{k g})\end{array}$ & $\begin{array}{l}\text { Lowland Stream } \\
\text { Background } \\
\text { Concentration }{ }^{\mathrm{a}} \\
(\mathrm{mg} / \mathrm{kg})\end{array}$ & $\begin{array}{l}\text { Human Health } \\
\text { Screening } \\
\text { Value } \\
(\mathrm{mg} / \mathrm{kg})\end{array}$ & $\begin{array}{l}\text { Human } \\
\text { Health } \\
\text { Constituent } \\
\text { of Concern }\end{array}$ \\
\hline Cobalt & 5 of 5 & 8.35 & 20.3 & 43.7 & NAV & $\mathrm{No}^{\mathrm{e}}$ \\
\hline Copper & 5 of 5 & 32.2 & 68.4 & 71.1 & NAV & $\mathrm{No}^{\mathrm{e}}$ \\
\hline Iron & 5 of 5 & 39,400 & 98,600 & 155,000 & NAV & $\mathrm{No}^{\mathrm{g}}$ \\
\hline Lithium & 4 of 5 & ND (8.06) & 18 & 48.8 & NAV & $\mathrm{No}^{\mathrm{e}}$ \\
\hline Magnesium & 5 of 5 & 1,930 & 12,300 & 9,320 & NAV & $\mathrm{No}^{\mathrm{g}}$ \\
\hline Manganese & 5 of 5 & 373 & 6,320 & 20,700 & NAV & $\mathrm{No}^{\mathrm{e}}$ \\
\hline Molybdenum & 1 of 5 & ND (1.9) & 8.47 & $5.5^{b}$ & NAV & $\mathrm{No}^{\mathrm{h}}$ \\
\hline Nickel & 4 of 5 & 6.83 & 9.8 & 13.6 & $2,030^{c}$ & $\mathrm{No}^{\mathrm{e}}$ \\
\hline Potassium & 5 of 5 & 432 & 4,050 & 1,390 & NAV & $\mathrm{No}^{\mathrm{g}}$ \\
\hline Selenium & 2 of 5 & ND (1.9) & 8.86 & $5.44^{b}$ & $507^{c}$ & $\mathrm{No}^{f}$ \\
\hline Strontium & 5 of 5 & 66.4 & 178 & 164 & NAV & $\mathrm{No}^{\mathrm{h}}$ \\
\hline Thallium & 0 of 5 & ND (1.9) & ND (8.06) & $1^{b}$ & NAV & $\mathrm{No}^{d}$ \\
\hline Thorium & 0 of 5 & ND (1.9) & ND (8.06) & $0.5^{\mathrm{b}}$ & NAV & $\mathrm{No}^{d}$ \\
\hline Titanium & 5 of 5 & 440 & 1,460 & 3,010 & NAV & $\mathrm{No}^{\mathrm{e}}$ \\
\hline Uranium & 0 of 5 & ND (1.9) & ND (8.06) & $4.13^{b}$ & NAV & $\mathrm{No}^{d}$ \\
\hline Vanadium & 5 of 5 & 109 & 294 & 734 & $710^{c}$ & $\mathrm{No}^{\mathrm{e}}$ \\
\hline Zinc & 5 of 5 & 98.6 & 138 & 434 & $30,400^{c}$ & $\mathrm{No}^{\mathrm{e}}$ \\
\hline Total Organic Carbon & 5 of 5 & 43,300 & 194,000 & NAN & NAV & No \\
\hline
\end{tabular}

${ }^{a}$ Background concentrations are upper tolerance limits (UTL) (ADEC, 1999a) unless otherwise noted.

${ }^{\mathrm{b}}$ Maximum detected concentration in background samples due to insufficient detection frequency to calculate UTL.

${ }^{\mathrm{c}}$ State of Alaska soil cleanup level, Discharge Reporting, Cleanup, and Disposal of Oil and other Hazardous Substances

(18 AAC 75, Articles 3 and 9 [ADEC, 1999c]).

\section{coc Selection Criteria}

${ }^{\mathrm{d}}$ Constituent not detected in any samples.

${ }^{\mathrm{e}}$ Maximum detected constituent concentration is less than local background concentration.

${ }^{f}$ Maximum detected constituent concentration is less than human health screening value.

${ }^{g}$ Constituent is an essential macronutrient and only presents a risk at extremely high concentrations.

${ }^{\mathrm{h}}$ Not a constituent associated with drilling mud or other drilling activities.

ND - Not detected (detection limit)

NAV - Not available

NAN - Not analyzed

$\mathrm{mg} / \mathrm{kg}$ - Milligrams per kilogram 
Table 4-14

\section{Summary of Analytical Results and Human Health Risk Assessment Screening for Fish Tissue in Bridge Creek, Long Shot Site}

(Page 1 of 2 )

\begin{tabular}{|c|c|c|c|c|c|c|}
\hline Analyte & $\begin{array}{l}\text { Detection } \\
\text { Frequency }\end{array}$ & $\begin{array}{c}\text { Minimum } \\
\text { Detected } \\
\text { Concentration } \\
(\mathrm{mg} / \mathrm{kg})\end{array}$ & $\begin{array}{c}\text { Maximum } \\
\text { Detected } \\
\text { Concentration } \\
(\mathbf{m g} / \mathrm{kg})\end{array}$ & $\begin{array}{c}\text { Lowland } \\
\text { Stream } \\
\text { Background } \\
\text { Concentration }{ }^{\mathrm{a}} \\
(\mathrm{mg} / \mathbf{k g})\end{array}$ & $\begin{array}{l}\text { Human Health } \\
\text { Screening } \\
\text { Value }^{\mathrm{c}} \\
(\mathrm{mg} / \mathbf{k g})\end{array}$ & $\begin{array}{c}\text { Human } \\
\text { Health } \\
\text { Constituent } \\
\text { of Concern }\end{array}$ \\
\hline \multicolumn{7}{|c|}{ Polycyclic Aromatic Hydrocarbons } \\
\hline Acenaphthene & 0 of 2 & ND $(0.067)$ & ND $(0.067)$ & NAN & 81.1 & $\mathrm{No}^{\mathrm{d}}$ \\
\hline Acenaphthylene & 0 of 2 & ND (0.067) & ND (0.067) & NAN & NAV & $\mathrm{No}^{\mathrm{d}}$ \\
\hline Anthracene & 0 of 2 & ND (0.067) & ND $(0.067)$ & NAN & 410 & $\mathrm{No}^{d}$ \\
\hline Benzo(a)anthracene & 0 of 2 & ND (0.067) & ND (0.067) & NAN & 0.0043 & No ${ }^{d}$ \\
\hline Benzo(a)pyrene & 0 of 2 & ND $(0.067)$ & ND $(0.067)$ & NAN & 0.00043 & No ${ }^{d}$ \\
\hline Benzo(b)fluoranthene & 0 of 2 & ND $(0.067)$ & ND $(0.067)$ & NAN & 0.0043 & $\mathrm{No}^{\mathrm{d}}$ \\
\hline Benzo(ghi)perylene & 0 of 2 & ND (0.067) & ND $(0.067)$ & NAN & NAV & $\mathrm{No}^{\mathrm{d}}$ \\
\hline Benzo(k)fluoranthene & 0 of 2 & ND (0.067) & ND $(0.067)$ & NAN & 0.043 & No ${ }^{d}$ \\
\hline Chrysene & 0 of 2 & ND $(0.067)$ & ND $(0.067)$ & NAN & 0.43 & $\mathrm{No}^{d}$ \\
\hline Dibenzo(a,h)anthracene & 0 of 2 & ND $(0.067)$ & ND $(0.067)$ & NAN & 0.00043 & $\mathrm{No}^{\mathrm{d}}$ \\
\hline Fluoranthene & 0 of 2 & ND (0.067) & ND (0.168) & NAN & 54 & $\mathrm{No}^{d}$ \\
\hline Fluorene & 0 of 2 & ND (0.0067) & ND (0.067) & NAN & 54 & $\mathrm{No}^{d}$ \\
\hline Indeno(1,2,3-cd)pyrene & 0 of 2 & ND $(0.067)$ & ND $(0.067)$ & NAN & 0.0043 & $\mathrm{No}^{d}$ \\
\hline Naphthalene & 0 of 2 & ND (0.0067) & ND (0.067) & NAN & 27 & $\mathrm{No}^{d}$ \\
\hline Phenanthrene & 0 of 2 & ND (0.067) & ND $(0.067)$ & NAN & NAV & $\mathrm{No}^{d}$ \\
\hline Pyrene & 0 of 2 & ND $(0.067)$ & ND (0.067) & NAN & 41 & No ${ }^{d}$ \\
\hline \multicolumn{7}{|c|}{ Polychlorinated Biphenyls } \\
\hline Aroclor 1016 & 0 of 2 & ND (0.01) & ND $(0.027)$ & NAN & 0.045 & $\mathrm{No}^{d}$ \\
\hline Aroclor 1221 & 0 of 2 & ND (0.02) & ND (0.054) & NAN & 0.0016 & $\mathrm{No}^{\mathrm{d}}$ \\
\hline Aroclor 1232 & 0 of 2 & ND (0.01) & ND (0.027) & NAN & 0.0016 & $\mathrm{No}^{\mathrm{d}}$ \\
\hline Aroclor 1242 & 0 of 2 & ND $(0.01)$ & ND $(0.027)$ & NAN & 0.0016 & $\mathrm{No}^{d}$ \\
\hline Aroclor 1248 & 0 of 2 & ND (0.01) & ND $(0.027)$ & NAN & 0.0016 & $\mathrm{No}^{d}$ \\
\hline Aroclor 1254 & 0 of 2 & ND (0.01) & ND $(0.027)$ & NAN & 0.0016 & No ${ }^{d}$ \\
\hline Aroclor 1260 & 0 of 2 & ND (0.01) & ND (0.027) & NAN & 0.0016 & No ${ }^{d}$ \\
\hline \multicolumn{7}{|c|}{ Metals } \\
\hline Aluminum & 2 of 2 & 57 & 425 & 475 & 1,400 & $\mathrm{No}^{\mathrm{e}}$ \\
\hline Arsenic & 2 of 2 & 0.0508 & 0.257 & 0.127 & 0.0021 & $\mathrm{No}^{\dagger}$ \\
\hline Inorganic Arsenic & 2 of 2 & 0.0109 & 0.154 & 0.0208 & 0.0021 & $\mathrm{No}^{\mathrm{h}}$ \\
\hline Barium & 2 of 2 & 0.261 & 1.62 & 1.03 & 95 & $\mathrm{No}^{f}$ \\
\hline Beryllium & 0 of 2 & ND (0.025) & ND (0.025) & ND & 2.7 & $\mathrm{No}^{d}$ \\
\hline Boron & 0 of 2 & ND (6.23) & ND (8.65) & 28.6 & 120 & $\mathrm{No}^{\mathrm{d}}$ \\
\hline Cadmium & 0 of 2 & ND (0.025) & ND (0.025) & $0.0373^{b}$ & 1.4 & $\mathrm{No}^{d}$ \\
\hline
\end{tabular}


Table 4-14

\section{Summary of Analytical Results and Human Health Risk Assessment Screening for Fish Tissue in Bridge Creek, Long Shot Site}

(Page 2 of 2 )

\begin{tabular}{|c|c|c|c|c|c|c|}
\hline Analyte & $\begin{array}{l}\text { Detection } \\
\text { Frequency }\end{array}$ & $\begin{array}{c}\text { Minimum } \\
\text { Detected } \\
\text { Concentration } \\
(\mathbf{m g} / \mathbf{k g})\end{array}$ & $\begin{array}{c}\text { Maximum } \\
\text { Detected } \\
\text { Concentration } \\
(\mathrm{mg} / \mathrm{kg})\end{array}$ & $\begin{array}{c}\text { Lowland } \\
\text { Stream } \\
\text { Background } \\
\text { Concentration } \\
\text { (mg/kg) }\end{array}$ & $\begin{array}{l}\text { Human Health } \\
\text { Screening } \\
\text { Value }^{\mathrm{c}} \\
(\mathrm{mg} / \mathrm{kg})\end{array}$ & $\begin{array}{c}\text { Human } \\
\text { Health } \\
\text { Constituent } \\
\text { of Concern }\end{array}$ \\
\hline Calcium & 2 of 2 & 7,280 & 8,080 & 19,200 & NAV & $\mathrm{No}^{\mathrm{e}}$ \\
\hline Cerium & 1 of 2 & ND (0.025) & 0.088 & $0.0280^{b}$ & NAV & $\mathrm{No}^{\mathrm{h}}$ \\
\hline Cesium & 1 of 2 & ND (0.025) & 0.0305 & $0.0258^{b}$ & NAV & $\mathrm{No}^{h}$ \\
\hline Chromium & 2 of 2 & 0.39 & 0.395 & 1.03 & 4.1 & $\mathrm{No}^{\mathrm{e}}$ \\
\hline Cobalt & 1 of 2 & ND (0.05) & 0.257 & $0.0780^{b}$ & 81 & $\mathrm{No}^{f}$ \\
\hline Copper & 0 of 2 & ND (0.864) & ND (1.57) & $1.20^{\mathrm{b}}$ & 54 & $\mathrm{No}^{d}$ \\
\hline Iron & 2 of 2 & 57 & 10,900 & 310 & 410 & $\mathrm{No}^{\mathrm{g}}$ \\
\hline Lithium & 2 of 2 & 0.0255 & 0.17 & $0.0993^{b}$ & 27 & $\mathrm{No}^{f}$ \\
\hline Magnesium & 2 of 2 & 273 & 473 & 408 & NAV & $\mathrm{No}^{\mathrm{g}}$ \\
\hline Manganese & 2 of 2 & 10.2 & 93.6 & 160 & 190 & $\mathrm{No}^{\mathrm{e}}$ \\
\hline Molybdenum & 0 of 2 & ND (0.125) & ND (0.163) & $0.173^{b}$ & 6.8 & $\mathrm{No}^{d}$ \\
\hline Nickel & 1 of 2 & ND (0.125) & 0.217 & ND & 27 & $\mathrm{No}^{\dagger}$ \\
\hline Potassium & 2 of 2 & 4,330 & 4,730 & $4,780^{b}$ & NAV & $\mathrm{No}^{\mathrm{e}}$ \\
\hline Selenium & 2 of 2 & 0.397 & 0.473 & 0.936 & 6.8 & $\mathrm{No}^{\mathrm{e}}$ \\
\hline Strontium & 2 of 2 & 5.91 & 9.93 & 22.1 & 810 & $\mathrm{No}^{\mathrm{e}}$ \\
\hline Thallium & 1 of 2 & ND (0.025) & 0.0503 & $0.0283^{b}$ & 0.095 & $\mathrm{No}^{\dagger}$ \\
\hline Thorium & 1 of 2 & ND (2.5) & 4.06 & $3.43^{b}$ & NAV & $\mathrm{No}^{\mathrm{h}}$ \\
\hline Titanium & 2 of 2 & 2.03 & 12.1 & 32.1 & 5,400 & $\mathrm{No}^{\mathrm{e}}$ \\
\hline Uranium & 0 of 2 & ND (0.5) & ND (0.5) & ND & 4.1 & $\mathrm{No}^{\mathrm{d}}$ \\
\hline Vanadium & 2 of 2 & 0.389 & 2.6 & 1.05 & 9.5 & $\mathrm{No}^{f}$ \\
\hline Zinc & 2 of 2 & 28.6 & 33 & 48.8 & 410 & $\mathrm{No}^{\mathrm{e}}$ \\
\hline
\end{tabular}

${ }^{\mathrm{a} B a c k g r o u n d}$ concentrations are upper tolerance limits (UTL) (ADEC, 1999a) unless otherwise noted.

${ }^{b}$ Maximum detected concentration in background samples due to insufficient detection frequency to calculate UTL.

${ }^{\mathrm{C} U}$.S. EPA Region III risk-based screening criteria for fish consumption unless otherwise noted.

Coc Selection Criteria

${ }^{\mathrm{d}}$ Constituent not detected in any samples.

${ }^{\mathrm{e}}$ Maximum detected constituent concentration is less than local background concentration.

${ }^{f}$ Maximum detected constituent concentration is less than human health screening value.

${ }^{9}$ Constituent is an essential macronutrient and only presents a risk at extremely high concentrations.

${ }^{\mathrm{h}} \mathrm{Not}$ a constituent associated with drilling mud or other drilling activities.

ND - Not detected (detection limit)

NAV - Not available

NAN - Not analyzed

$\mathrm{mg} / \mathrm{kg}$ - Milligrams per kilogram 
Table 4-15

Summary of Analytical Results and Human Health Risk Assessment Screening for Surface Water in Reed Pond, Long Shot Site

(Page 1 of 3 )

\begin{tabular}{|c|c|c|c|c|c|c|}
\hline Analyte & $\begin{array}{l}\text { Detection } \\
\text { Frequency }\end{array}$ & $\begin{array}{c}\text { Minimum } \\
\text { Detected } \\
\text { Concentration } \\
(\mu \mathrm{g} / \mathrm{L})\end{array}$ & $\begin{array}{c}\text { Maximum } \\
\text { Detected } \\
\text { Concentration } \\
(\mu \mathrm{g} / \mathrm{L})\end{array}$ & $\begin{array}{c}\text { Lowland } \\
\text { Stream } \\
\text { Background } \\
\text { Concentration } \\
(\mu \mathrm{g} / \mathrm{L})\end{array}$ & $\begin{array}{l}\text { Human Health } \\
\text { Screening } \\
\text { Value } \\
(\mu \mathrm{g} / \mathrm{L})\end{array}$ & $\begin{array}{c}\text { Human Health } \\
\text { Constituent of } \\
\text { Concern }\end{array}$ \\
\hline $\begin{array}{l}\text { Diesel-Range } \\
\text { Hydrocarbons }\end{array}$ & 2 of 2 & 113 & 137 & NAN & $1,500^{d}$ & $\mathrm{No}^{\mathrm{h}}$ \\
\hline \multicolumn{7}{|c|}{ Volatile Organic Compounds } \\
\hline 1,2,4-Trimethylbenzene & 0 of 2 & ND (1.0) & ND (1.0) & NAN & $12^{\mathrm{e}}$ & $\mathrm{No}^{\dagger}$ \\
\hline 1,3,5-Trimethylbenzene & 0 of 2 & ND (1.0) & ND (1.0) & NAN & $12^{\mathrm{e}}$ & $\mathrm{No}^{f}$ \\
\hline 2-Butanone & 0 of 2 & ND (20) & ND (20) & NAN & $1,900^{\mathrm{e}}$ & $\mathrm{No}^{f}$ \\
\hline Acetone & 0 of 2 & ND (20) & ND (20) & NAN & $3,700^{\mathrm{e}}$ & $\mathrm{No}^{f}$ \\
\hline Benzene & 0 of 2 & ND (1.0) & ND (1.0) & NAN & $1.2^{c}$ & $\mathrm{No}^{\dagger}$ \\
\hline Carbon disulfide & 0 of 2 & ND (1.0) & ND (1.0) & NAN & $1,000^{\mathrm{C}}$ & $\mathrm{No}^{\dagger}$ \\
\hline Ethylbenzene & 0 of 2 & ND (1.0) & ND (1.0) & NAN & $3,100^{c}$ & $\mathrm{No}^{f}$ \\
\hline Isopropylbenzene & 0 of 2 & ND (1.0) & ND (1.0) & NAN & NAV & $\mathrm{No}^{f}$ \\
\hline m,p-Xylene & 0 of 2 & ND (2.0) & ND (2.0) & NAN & $12,000^{\mathrm{e}}$ & $\mathrm{No}^{f}$ \\
\hline Methylene chloride & 0 of 2 & ND (2.47) & ND (3.04) & NAN & $4.7^{\mathrm{C}}$ & $\mathrm{No}^{f}$ \\
\hline n-Butylbenzene & 0 of 2 & ND (1.0) & ND (1.0) & NAN & $61^{e}$ & $\mathrm{No}^{f}$ \\
\hline n-Propylbenzene & 0 of 2 & ND (1.0) & ND (1.0) & NAN & $61^{\mathrm{e}}$ & $\mathrm{No}^{f}$ \\
\hline Naphthalene & 0 of 2 & ND (1.0) & ND (1.0) & NAN & $730^{e}$ & $\mathrm{No}^{f}$ \\
\hline o-Xylene & 0 of 2 & ND (1.0) & ND (1.0) & NAN & $12,000^{\mathrm{e}}$ & $\mathrm{No}^{f}$ \\
\hline p-Isopropyltoluene & 0 of 2 & ND (1.0) & ND (1.0) & NAN & NAV & $\mathrm{No}^{f}$ \\
\hline sec-Butylbenzene & 0 of 2 & ND (1.0) & ND (1.0) & NAN & $61^{e}$ & $\mathrm{No}^{f}$ \\
\hline Toluene & 0 of 2 & ND (1.0) & ND (1.0) & NAN & $10,000^{c}$ & $\mathrm{No}^{f}$ \\
\hline \multicolumn{7}{|c|}{ Polycyclic Aromatic Hydrocarbons } \\
\hline Acenaphthene & 0 of 2 & ND $(0.1)$ & ND $(0.1)$ & NAN & $1,200^{C}$ & $\mathrm{No}^{\dagger}$ \\
\hline Acenaphthylene & 0 of 2 & ND $(0.1)$ & ND $(0.1)$ & NAN & $0.0028^{c}$ & $\mathrm{No}^{\dagger}$ \\
\hline Anthracene & 0 of 2 & $\mathrm{ND}(0.1)$ & $\mathrm{ND}(0.1)$ & NAN & $0.0028^{\mathrm{C}}$ & $\mathrm{No}^{\dagger}$ \\
\hline Benzo(a)anthracene & 0 of 2 & $\mathrm{ND}(0.1)$ & $\mathrm{ND}(0.1)$ & NAN & $0.0028^{\mathrm{C}}$ & $\mathrm{No}^{f}$ \\
\hline Benzo(a)pyrene & 0 of 2 & $\mathrm{ND}(0.1)$ & $\mathrm{ND}(0.1)$ & NAN & $0.0028^{\mathrm{C}}$ & $\mathrm{No}^{\dagger}$ \\
\hline Benzo(b)fluoranthene & 0 of 2 & ND $(0.1)$ & ND $(0.1)$ & NAN & $0.092^{\mathrm{e}}$ & $\mathrm{No}^{\dagger}$ \\
\hline Benzo(ghi)perylene & 0 of 2 & ND $(0.1)$ & ND $(0.1)$ & NAN & $0.0028^{c}$ & $\mathrm{No}^{f}$ \\
\hline Benzo(k)fluoranthene & 0 of 2 & $\mathrm{ND}(0.1)$ & $\mathrm{ND}(0.1)$ & NAN & $0.0028^{\mathrm{C}}$ & $\mathrm{No}^{f}$ \\
\hline Chrysene & 0 of 2 & $\mathrm{ND}(0.1)$ & ND $(0.1)$ & NAN & $0.0028^{c}$ & $\mathrm{No}^{f}$ \\
\hline
\end{tabular}


Table 4-15

Summary of Analytical Results and Human Health Risk Assessment Screening for Surface Water in Reed Pond, Long Shot Site

(Page 2 of 3 )

\begin{tabular}{|c|c|c|c|c|c|c|}
\hline Analyte & $\begin{array}{l}\text { Detection } \\
\text { Frequency }\end{array}$ & $\begin{array}{c}\text { Minimum } \\
\text { Detected } \\
\text { Concentration } \\
(\mu \mathrm{g} / \mathrm{L})\end{array}$ & $\begin{array}{c}\text { Maximum } \\
\text { Detected } \\
\text { Concentration } \\
(\mu \mathrm{g} / \mathrm{L})\end{array}$ & $\begin{array}{c}\text { Lowland } \\
\text { Stream } \\
\text { Background } \\
\text { Concentration }{ }^{\mathrm{a}} \\
(\mu \mathrm{g} / \mathrm{L})\end{array}$ & $\begin{array}{l}\text { Human Health } \\
\text { Screening } \\
\text { Value } \\
(\mu \mathrm{g} / \mathrm{L})\end{array}$ & $\begin{array}{l}\text { Human Health } \\
\text { Constituent of } \\
\text { Concern }\end{array}$ \\
\hline Dibenzo(a,h)anthracene & 0 of 2 & ND $(0.1)$ & ND $(0.1)$ & NAN & $0.0028^{c}$ & $\mathrm{No}^{f}$ \\
\hline Fluoranthene & 0 of 2 & ND $(0.1)$ & ND $(0.1)$ & NAN & $42^{c}$ & $\mathrm{No}^{\dagger}$ \\
\hline Fluorene & 0 of 2 & $\mathrm{ND}(0.1)$ & $\mathrm{ND}(0.1)$ & NAN & $0.0028^{c}$ & $\mathrm{No}^{\dagger}$ \\
\hline Indeno(1,2,3-cd)pyrene & 0 of 2 & $\mathrm{ND}(0.1)$ & $\mathrm{ND}(0.1)$ & NAN & $0.0028^{c}$ & $\mathrm{No}^{\dagger}$ \\
\hline Naphthalene & 0 of 2 & ND $(0.1)$ & $\mathrm{ND}(0.1)$ & NAN & $730^{e}$ & $\mathrm{No}^{f}$ \\
\hline Phenanthrene & 0 of 2 & $\mathrm{ND}(0.1)$ & $\mathrm{ND}(0.1)$ & NAN & $0.0028^{c}$ & $\mathrm{No}^{f}$ \\
\hline Pyrene & 0 of 2 & $\mathrm{ND}(0.1)$ & $\mathrm{ND}(0.1)$ & NAN & $0.0028^{c}$ & $\mathrm{No}^{\dagger}$ \\
\hline \multicolumn{7}{|c|}{ Polychlorinated Biphenyls } \\
\hline Aroclor 1016 & NAV & NAV & NAV & NAN & $0.000044^{c}$ & $\mathrm{No}^{\dagger}$ \\
\hline Aroclor 1221 & NAV & NAV & NAV & NAN & $0.000044^{c}$ & $\mathrm{No}^{f}$ \\
\hline Aroclor 1232 & NAV & NAV & NAV & NAN & $0.000044^{c}$ & $\mathrm{No}^{\dagger}$ \\
\hline Aroclor 1242 & NAV & NAV & NAV & NAN & $0.000044^{c}$ & $\mathrm{No}^{f}$ \\
\hline Aroclor 1248 & NAV & NAV & NAV & NAN & $0.000044^{c}$ & $\mathrm{No}^{f}$ \\
\hline Aroclor 1254 & NAV & NAV & NAV & NAN & $0.000044^{c}$ & $\mathrm{No}^{\dagger}$ \\
\hline Aroclor 1260 & NAV & NAV & NAV & NAN & $0.000044^{c}$ & $\mathrm{No}^{f}$ \\
\hline Aroclor 1262 & NAV & NAV & NAV & NAN & $0.000044^{c}$ & $\mathrm{No}^{f}$ \\
\hline Aroclor 1268 & NAV & NAV & NAV & NAN & $0.000044^{c}$ & $\mathrm{No}^{f}$ \\
\hline \multicolumn{7}{|c|}{ Metals } \\
\hline Aluminum & 1 of 2 & ND (50) & 82.2 & 269 & $37,000^{\mathrm{e}}$ & $\mathrm{No}^{\mathrm{g}}$ \\
\hline Arsenic & 0 of 2 & ND (1.0) & ND (1.0) & ND (1) & $0.018^{c}$ & $\mathrm{No}^{f}$ \\
\hline Barium & 2 of 2 & 49.4 & 53 & ND (10) & $1,000^{c}$ & $\mathrm{No}^{\mathrm{h}}$ \\
\hline Beryllium & 0 of 2 & ND (1.0) & ND (1.0) & ND (1) & $0.0076^{c}$ & $\mathrm{No}^{f}$ \\
\hline Boron & 0 of 2 & ND (50) & ND (50) & ND (50) & $3,300^{\mathrm{e}}$ & $\mathrm{No}^{f}$ \\
\hline Cadmium & 0 of 2 & ND (1.0) & ND (1.0) & ND (1) & $10^{c}$ & $\mathrm{No}^{f}$ \\
\hline Calcium & 2 of 2 & 3,420 & 7,330 & $4,320 \mathrm{~b}$ & NAV & $\mathrm{No}^{i}$ \\
\hline Cerium & 0 of 2 & ND (1.0) & ND (1.0) & ND (1) & NAV & $\mathrm{No}^{f}$ \\
\hline Cesium & 0 of 2 & ND (1.0) & ND (1.0) & ND (1) & NAV & $\mathrm{No}^{f}$ \\
\hline Chromium & 2 of 2 & 1.15 & 1.4 & $2.25^{b}$ & $33,000^{c}$ & $\mathrm{No}^{\mathrm{g}}$ \\
\hline Cobalt & 0 of 2 & ND (1.0) & ND (1.0) & 3.35 & $2,200^{\mathrm{e}}$ & $\mathrm{No}^{\dagger}$ \\
\hline
\end{tabular}


Table 4-15

\section{Summary of Analytical Results and Human Health Risk Assessment Screening for Surface Water in Reed Pond, Long Shot Site}

(Page 3 of 3 )

\begin{tabular}{|c|c|c|c|c|c|c|}
\hline Analyte & $\begin{array}{l}\text { Detection } \\
\text { Frequency }\end{array}$ & $\begin{array}{c}\text { Minimum } \\
\text { Detected } \\
\text { Concentration } \\
(\mu \mathrm{g} / \mathrm{L})\end{array}$ & $\begin{array}{c}\text { Maximum } \\
\text { Detected } \\
\text { Concentration } \\
(\mu \mathrm{g} / \mathrm{L})\end{array}$ & $\begin{array}{c}\text { Lowland } \\
\text { Stream } \\
\text { Background } \\
\text { Concentration }{ }^{\mathrm{a}} \\
(\mu \mathrm{g} / \mathrm{L})\end{array}$ & $\begin{array}{l}\text { Human Health } \\
\text { Screening } \\
\text { Value } \\
(\mu \mathrm{g} / \mathrm{L})\end{array}$ & $\begin{array}{c}\text { Human Health } \\
\text { Constituent of } \\
\text { Concern }\end{array}$ \\
\hline Copper & 1 of 2 & ND (1.0) & 1.04 & 10.8 & $1,300^{c}$ & $\mathrm{No}^{\mathrm{g}}$ \\
\hline Iron & 2 of 2 & 370 & 571 & 993 & $300^{c}$ & $\mathrm{No}^{\mathrm{g}}$ \\
\hline Lithium & 0 of 2 & ND (1.0) & ND (1.0) & $1.17^{\mathrm{b}}$ & $730^{e}$ & $\mathrm{No}^{\dagger}$ \\
\hline Magnesium & 2 of 2 & 2,310 & 3,870 & $4,690^{b}$ & NAV & $\mathrm{No}^{\mathrm{g}}$ \\
\hline Manganese & 2 of 2 & 6.85 & 42.4 & 267 & $50^{c}$ & $\mathrm{No}^{\mathrm{g}}$ \\
\hline Molybdenum & 0 of 2 & ND (1.0) & ND (1.0) & ND (1) & $180^{\mathrm{e}}$ & $\mathrm{No}^{f}$ \\
\hline Nickel & 0 of 2 & ND (1.0) & ND (1.0) & ND (1) & $510^{c}$ & $\mathrm{No}^{\dagger}$ \\
\hline Potassium & 2 of 2 & 1,300 & 1,360 & 2,620 & NAV & $\mathrm{No}^{\mathrm{g}}$ \\
\hline Selenium & 0 of 2 & ND (1.0) & ND (1.0) & ND (1) & $104^{c}$ & $\mathrm{No}^{\dagger}$ \\
\hline Strontium & 2 of 2 & 19.5 & 25.9 & 36.1 & $22,000^{\mathrm{e}}$ & $\mathrm{No}^{\mathrm{g}}$ \\
\hline Thallium & 0 of 2 & ND (1.0) & ND (1.0) & $7.07^{b}$ & $1.7^{\mathrm{c}}$ & $\mathrm{No}^{\dagger}$ \\
\hline Thorium & 0 of 2 & ND (1.0) & ND (1.0) & ND (1) & NAV & $\mathrm{No}^{f}$ \\
\hline Titanium & 2 of 2 & 1.47 & 2.1 & 6.13 & $150,000^{e}$ & $\mathrm{No}^{\mathrm{g}}$ \\
\hline Uranium & 0 of 2 & ND (1.0) & ND (1.0) & ND (1) & $110^{\mathrm{e}}$ & $\mathrm{No}^{f}$ \\
\hline Vanadium & 0 of 2 & ND (1.0) & ND (1.0) & $4.43^{b}$ & $260^{e}$ & $\mathrm{No}^{f}$ \\
\hline Zinc & 0 of 2 & ND (10) & ND (10) & $50.3^{b}$ & $11,000^{\mathrm{e}}$ & $\mathrm{No}^{f}$ \\
\hline
\end{tabular}

${ }^{\mathrm{a} B a c k g r o u n d}$ concentrations are upper tolerance limits (UTL) (ADEC, 1999a) unless otherwise noted.

${ }^{\mathrm{b}}$ Maximum detected concentration in background samples due to insufficient detection frequency to calculate UTL.

${ }^{\mathrm{c}}$ Federal ambient water quality criteria based on ingestion of water and organisms.

d State of Alaska groundwater cleanup level, Discharge Reporting, Cleanup, and Disposal of Oil and other Hazardous Substances (18 AAC 75, Articles 3 and 9 [ADEC, 1999c]).

eU.S. EPA Region III risk-based concentrations for tap water (EPA, 2000b).

\section{COC Selection Criteria}

${ }^{f}$ Constituent not detected in any samples.

${ }^{9}$ Maximum detected constituent concentration is less than local background concentration.

${ }^{h}$ Maximum detected constituent concentration is less than human health screening value.

i'Constituent is an essential macronutrient and only presents a risk at extremely high concentrations.

ND - Not detected (detection limit)

NAV - Not available

NAN - Not analyzed

$\mu \mathrm{g} / \mathrm{L}$ - Micrograms per liter 
Table 4-16

\section{Summary of Analytical Results and Human Health Risk Assessment Screening for Sediment in Reed Pond, Long Shot Site}

(Page 1 of 3 )

\begin{tabular}{|c|c|c|c|c|c|c|}
\hline Analyte & $\begin{array}{l}\text { Detection } \\
\text { Frequency }\end{array}$ & $\begin{array}{c}\text { Minimum } \\
\text { Detected } \\
\text { Concentration } \\
(\mathrm{mg} / \mathrm{kg})\end{array}$ & $\begin{array}{c}\text { Maximum } \\
\text { Detected } \\
\text { Concentration } \\
(\mathbf{m g} / \mathbf{k g})\end{array}$ & $\begin{array}{c}\text { Lowland } \\
\text { Stream } \\
\text { Background } \\
\text { Concentration }{ }^{\mathrm{a}} \\
(\mathbf{m g} / \mathbf{k g})\end{array}$ & $\begin{array}{l}\text { Human Health } \\
\text { Screening } \\
\text { Value } \\
(\mathrm{mg} / \mathrm{kg})\end{array}$ & $\begin{array}{c}\text { Human } \\
\text { Health } \\
\text { Constituent } \\
\text { of Concern }\end{array}$ \\
\hline $\begin{array}{l}\text { Diesel-Range } \\
\text { Hydrocarbons }\end{array}$ & 1 of 2 & ND (27.4) & 42.6 & NAN & $200^{C}$ & $\mathrm{No}^{\dagger}$ \\
\hline \multicolumn{7}{|c|}{ Volatile Organic Compounds } \\
\hline 1,2,4-Trimethylbenzene & 0 of 2 & ND (0.0188) & ND (0.0533) & NAN & NAV & $\mathrm{No}^{\mathrm{d}}$ \\
\hline 1,3,5-Trimethylbenzene & 0 of 2 & ND (0.0188) & ND (0.0533) & NAN & NAV & No ${ }^{d}$ \\
\hline 2-Butanone & 2 of 2 & 0.0575 & 0.136 & NAN & NAV & Yes \\
\hline Acetone & 2 of 2 & 0.38 & 1.02 & NAN & $10,100^{c}$ & $\mathrm{No}^{f}$ \\
\hline Benzene & 0 of 2 & ND (0.0188) & ND (0.0533) & NAN & $286^{\mathrm{C}}$ & $\mathrm{No}^{\mathrm{d}}$ \\
\hline Carbon disulfide & 0 of 2 & ND (0.0188) & ND (0.0533) & NAN & $10,100^{c}$ & $\mathrm{No}^{\mathrm{d}}$ \\
\hline Ethylbenzene & 0 of 2 & ND (0.0188) & ND $(0.0533)$ & NAN & $10,100^{c}$ & $\mathrm{No}^{d}$ \\
\hline Isopropylbenzene & 0 of 2 & ND (0.0188) & ND (0.0533) & NAN & NAV & $\mathrm{No}^{d}$ \\
\hline $\mathrm{m}, \mathrm{p}$-Xylene & 0 of 2 & ND (0.0188) & ND (0.0533) & NAN & $203,000^{c}$ & $\mathrm{No}^{d}$ \\
\hline Methylene chloride & 0 of 2 & ND (0.0188) & ND (0.0533) & NAN & $1,110^{\mathrm{C}}$ & $\mathrm{No}^{d}$ \\
\hline n-Butylbenzene & 0 of 2 & ND (0.0188) & ND (0.0533) & NAN & NAV & $\mathrm{No}^{d}$ \\
\hline n-Propylbenzene & 0 of 2 & ND (0.0188) & ND (0.0533) & NAN & NAV & $\mathrm{No}^{d}$ \\
\hline Naphthalene & 0 of 2 & ND (0.0188) & ND (0.0533) & NAN & $4,060^{c}$ & $\mathrm{No}^{d}$ \\
\hline o-Xylene & 0 of 2 & ND (0.0188) & ND (0.0533) & NAN & $203,000^{c}$ & $\mathrm{No}^{d}$ \\
\hline p-Isopropyltoluene & 0 of 2 & ND (0.0188) & ND (0.0533) & NAN & NAV & $\mathrm{No}^{d}$ \\
\hline sec-Butylbenzene & 0 of 2 & ND (0.0188) & ND (0.0533) & NAN & NAV & $\mathrm{No}^{d}$ \\
\hline Toluene & 1 of 2 & ND (0.0533) & 0.0434 & NAN & $20,300^{c}$ & $\mathrm{No}^{\mathrm{d}}$ \\
\hline \multicolumn{7}{|c|}{ Polycyclic Aromatic Hydrocarbons } \\
\hline Acenaphthene & 0 of 2 & ND (0.0226) & ND (0.0333) & NAN & $6,080^{c}$ & $\mathrm{No}^{d}$ \\
\hline Acenaphthylene & 0 of 2 & ND (0.0226) & ND (0.0333) & NAN & NAV & $\mathrm{No}^{d}$ \\
\hline Anthracene & 0 of 2 & ND (0.0226) & ND (0.0333) & NAN & $30,400^{c}$ & $\mathrm{No}^{\mathrm{d}}$ \\
\hline Benzo(a)anthracene & 0 of 2 & ND (0.0226) & ND (0.0333) & NAN & $11.4^{\mathrm{C}}$ & $\mathrm{No}^{\mathrm{d}}$ \\
\hline Benzo(a)pyrene & 0 of 2 & ND (0.0226) & ND (0.0333) & NAN & $1.1^{\mathrm{c}}$ & $\mathrm{No}^{d}$ \\
\hline Benzo(b)fluoranthene & 0 of 2 & ND (0.0226) & ND (0.0333) & NAN & $11.4^{\mathrm{c}}$ & $\mathrm{No}^{d}$ \\
\hline Benzo(ghi)perylene & 0 of 2 & ND (0.0226) & ND (0.0333) & NAN & $1.1^{\mathrm{c}}$ & $\mathrm{No}^{\mathrm{d}}$ \\
\hline Benzo(k)fluoranthene & 0 of 2 & ND (0.0226) & ND (0.0333) & NAN & $114^{\mathrm{C}}$ & $\mathrm{No}^{\mathrm{d}}$ \\
\hline Chrysene & 0 of 2 & ND (0.0226) & ND (0.0333) & NAN & $1,140^{\mathrm{C}}$ & $\mathrm{No}^{\mathrm{d}}$ \\
\hline
\end{tabular}


Table 4-16

\section{Summary of Analytical Results and Human Health Risk Assessment Screening for Sediment in Reed Pond, Long Shot Site}

(Page 2 of 3 )

\begin{tabular}{|c|c|c|c|c|c|c|}
\hline Analyte & $\begin{array}{l}\text { Detection } \\
\text { Frequency }\end{array}$ & $\begin{array}{c}\text { Minimum } \\
\text { Detected } \\
\text { Concentration } \\
(\mathbf{m g} / \mathbf{k g})\end{array}$ & $\begin{array}{c}\text { Maximum } \\
\text { Detected } \\
\text { Concentration } \\
(\mathrm{mg} / \mathrm{kg})\end{array}$ & $\begin{array}{c}\text { Lowland } \\
\text { Stream } \\
\text { Background } \\
\text { Concentration }{ }^{\mathrm{a}} \\
(\mathrm{mg} / \mathrm{kg})\end{array}$ & $\begin{array}{l}\text { Human Health } \\
\text { Screening } \\
\text { Value } \\
(\mathrm{mg} / \mathrm{kg})\end{array}$ & $\begin{array}{c}\text { Human } \\
\text { Health } \\
\text { Constituent } \\
\text { of Concern }\end{array}$ \\
\hline Dibenzo(a,h)anthracene & 0 of 2 & ND (0.0226) & ND (0.0333) & NAN & $1.1^{\mathrm{c}}$ & $\mathrm{No}^{\mathrm{d}}$ \\
\hline Fluoranthene & 0 of 2 & ND (0.0226) & ND (0.0333) & NAN & $4,060^{c}$ & $\mathrm{No}^{\mathrm{d}}$ \\
\hline Fluorene & 0 of 2 & ND (0.0226) & ND $(0.0333)$ & NAN & $4,060^{c}$ & $\mathrm{No}^{\mathrm{d}}$ \\
\hline Indeno(1,2,3-cd)pyrene & 0 of 2 & ND (0.0226) & ND (0.0333) & NAN & $11^{\mathrm{c}}$ & $\mathrm{No}^{\mathrm{d}}$ \\
\hline Naphthalene & 0 of 2 & ND (0.0226) & ND (0.0333) & NAN & $4,060^{c}$ & $\mathrm{No}^{\mathrm{d}}$ \\
\hline Phenanthrene & 0 of 2 & ND (0.0226) & ND (0.0333) & NAN & $1.1^{\mathrm{c}}$ & Nod \\
\hline Pyrene & 0 of 2 & ND $(0.0226)$ & ND $(0.0333)$ & NAN & $3,040^{\mathrm{C}}$ & $\mathrm{No}^{\mathrm{d}}$ \\
\hline \multicolumn{7}{|c|}{ Polychlorinated Biphenyls } \\
\hline Aroclor 1016 & NAV & NAV & NAV & NAN & $10^{c}$ & $\mathrm{No}^{\mathrm{d}}$ \\
\hline Aroclor 1221 & NAV & NAV & NAV & NAN & $10^{c}$ & $\mathrm{No}^{\mathrm{d}}$ \\
\hline Aroclor 1232 & NAV & NAV & NAV & NAN & $10^{\mathrm{C}}$ & $\mathrm{No}^{d}$ \\
\hline Aroclor 1242 & NAV & NAV & NAV & NAN & $10^{c}$ & $\mathrm{No}^{d}$ \\
\hline Aroclor 1248 & NAV & NAV & NAV & NAN & $10^{c}$ & $\mathrm{No}^{d}$ \\
\hline Aroclor 1254 & NAV & NAV & NAV & NAN & $10^{c}$ & $\mathrm{No}^{d}$ \\
\hline Aroclor 1260 & NAV & NAV & NAV & NAN & $10^{\mathrm{C}}$ & $\mathrm{No}^{d}$ \\
\hline Aroclor 1262 & NAV & NAV & NAV & NAN & $10^{c}$ & $\mathrm{No}^{d}$ \\
\hline Aroclor 1268 & NAV & NAV & NAV & NAN & $10^{c}$ & $\mathrm{No}^{d}$ \\
\hline \multicolumn{7}{|c|}{ Metals } \\
\hline Aluminum & 2 of 2 & 10,000 & 13,100 & 45,400 & NAV & $\mathrm{No}^{\mathrm{e}}$ \\
\hline Arsenic & 1 of 2 & ND (3.42) & 7.92 & 100 & $5.5^{c}$ & $\mathrm{No}^{\mathrm{e}}$ \\
\hline Barium & 1 of 2 & ND (50.5) & 43.6 & 469 & $7,100^{C}$ & $\mathrm{No}^{\mathrm{e}}$ \\
\hline Beryllium & 0 of 2 & ND (3.42) & ND (5.05) & ND & $1.9^{\mathrm{c}}$ & $\mathrm{No}^{d}$ \\
\hline Boron & 0 of 2 & ND (17.1) & ND (25.3) & $7.18^{b}$ & NAV & $\mathrm{No}^{d}$ \\
\hline Cadmium & 0 of 2 & ND (3.42) & ND (5.05) & $0.608^{b}$ & $101^{c}$ & $\mathrm{No}^{\mathrm{d}}$ \\
\hline Calcium & 2 of 2 & 3,570 & 9,230 & 7,300 & NAV & $\mathrm{No}^{\mathrm{g}}$ \\
\hline Cerium & 2 of 2 & 3.87 & 5.72 & 14.2 & NAV & $\mathrm{No}^{\mathrm{e}}$ \\
\hline Cesium & 1 of 2 & ND (3.42) & 5.72 & ND & NAV & $\mathrm{No}^{h}$ \\
\hline Chromium & 1 of 2 & ND (3.42) & 9.71 & 11.8 & $507^{c}$ & $\mathrm{No}^{\mathrm{e}}$ \\
\hline Cobalt & 2 of 2 & 3.99 & 7.57 & 43.7 & NAV & $\mathrm{No}^{\mathrm{e}}$ \\
\hline
\end{tabular}


Table 4-16

\section{Summary of Analytical Results and Human Health Risk Assessment Screening for Sediment in Reed Pond, Long Shot Site} (Page 3 of 3)

\begin{tabular}{|c|c|c|c|c|c|c|}
\hline Analyte & $\begin{array}{l}\text { Detection } \\
\text { Frequency }\end{array}$ & $\begin{array}{c}\text { Minimum } \\
\text { Detected } \\
\text { Concentration } \\
(\mathrm{mg} / \mathrm{kg})\end{array}$ & $\begin{array}{c}\text { Maximum } \\
\text { Detected } \\
\text { Concentration } \\
(\mathrm{mg} / \mathrm{kg})\end{array}$ & $\begin{array}{c}\text { Lowland } \\
\text { Stream } \\
\text { Background } \\
\text { Concentration }{ }^{\mathrm{a}} \\
(\mathrm{mg} / \mathbf{k g})\end{array}$ & $\begin{array}{l}\text { Human Health } \\
\text { Screening } \\
\text { Value } \\
(\mathbf{m g} / \mathbf{k g})\end{array}$ & $\begin{array}{c}\text { Human } \\
\text { Health } \\
\text { Constituent } \\
\text { of Concern }\end{array}$ \\
\hline Copper & 2 of 2 & 27.4 & 47.5 & 71.1 & NAV & $\mathrm{No}^{\mathrm{e}}$ \\
\hline Iron & 2 of 2 & 4,910 & 5,890 & 155,000 & NAV & $\mathrm{No}^{\mathrm{e}}$ \\
\hline Lithium & 1 of 2 & ND (5.05) & 3.53 & 48.8 & NAV & $\mathrm{No}^{\mathrm{e}}$ \\
\hline Magnesium & 2 of 2 & 1,480 & 1,790 & 9,320 & NAV & $\mathrm{No}^{\mathrm{e}}$ \\
\hline Manganese & 2 of 2 & 97.7 & 221 & 20,700 & NAV & $\mathrm{No}^{\mathrm{e}}$ \\
\hline Molybdenum & 0 of 2 & ND (3.42) & ND (5.05) & $5.5^{\mathrm{b}}$ & NAV & $\mathrm{No}^{d}$ \\
\hline Nickel & 1 of 2 & ND (3.42) & 8.04 & 13.6 & $2,030^{c}$ & $\mathrm{No}^{\mathrm{e}}$ \\
\hline Potassium & 2 of 2 & 108 & 145 & 1,390 & NAV & $\mathrm{No}^{\mathrm{e}}$ \\
\hline Selenium & 0 of 2 & ND (3.42) & ND (5.05) & $5.44^{b}$ & $507^{c}$ & $\mathrm{No}^{\mathrm{d}}$ \\
\hline Strontium & 2 of 2 & 41 & 46.9 & 164 & NAV & $\mathrm{No}^{\mathrm{e}}$ \\
\hline Thallium & 0 of 2 & ND (3.42) & ND (5.05) & $1^{\mathrm{b}}$ & NAV & $\mathrm{No}^{d}$ \\
\hline Thorium & 0 of 2 & ND (3.42) & ND (5.05) & $0.5^{\mathrm{b}}$ & NAV & $\mathrm{No}^{d}$ \\
\hline Titanium & 2 of 2 & 752 & 1,280 & 3,010 & NAV & $\mathrm{No}^{\mathrm{e}}$ \\
\hline Uranium & 0 of 2 & ND (3.42) & ND (5.05) & $4.13^{b}$ & NAV & $\mathrm{No}^{d}$ \\
\hline Vanadium & 2 of 2 & 46.6 & 340 & 734 & $710^{c}$ & $\mathrm{No}^{\mathrm{e}}$ \\
\hline Zinc & 0 of 2 & ND (34.2) & ND (50.5) & 434 & $30,400^{c}$ & $\mathrm{No}^{d}$ \\
\hline
\end{tabular}

${ }^{\mathrm{a}}$ Background concentrations are upper tolerance limits (UTL) (ADEC, 1999a) unless otherwise noted.

${ }^{\mathrm{b}}$ Maximum detected concentration in background samples due to insufficient detection frequency to calculate UTL.

'State of Alaska soil cleanup level, Discharge Reporting, Cleanup, and Disposal of Oil and other Hazardous Substances (18 AAC 75, Articles 3 and 9 [ADEC, 1999c]).

\section{COC Selection Criteria}

${ }^{\mathrm{d}}$ Constituent not detected in any samples.

${ }^{\mathrm{e}}$ Maximum detected constituent concentration is less than local background concentration.

${ }^{f}$ Maximum detected constituent concentration is less than human health screening value.

${ }^{9}$ Constituent is an essential macronutrient and only presents a risk at extremely high concentrations.

${ }^{h}$ Not a constituent associated with drilling mud or other drilling activities.

ND - Not detected (detection limit)

NAV - Not available

NAN - Not analyzed

$\mathrm{mg} / \mathrm{kg}$ - Milligrams per kilogram 
Table 4-17

\section{Summary of Analytical Results and Human Health Risk Assessment Screening for Surface Water in Cloudberry Creek, Long Shot Site} (Page 1 of 3 )

\begin{tabular}{|c|c|c|c|c|c|c|}
\hline Analyte & $\begin{array}{l}\text { Detection } \\
\text { Frequency }\end{array}$ & $\begin{array}{c}\text { Minimum } \\
\text { Detected } \\
\text { Concentration } \\
(\mu \mathrm{g} / \mathrm{L})\end{array}$ & $\begin{array}{c}\text { Maximum } \\
\text { Detected } \\
\text { Concentration } \\
(\mu \mathrm{g} / \mathrm{L})\end{array}$ & $\begin{array}{c}\text { Lowland } \\
\text { Stream } \\
\text { Background } \\
\text { Concentration }{ }^{\mathrm{a}} \\
(\mu \mathrm{g} / \mathrm{L})\end{array}$ & $\begin{array}{l}\text { Human Health } \\
\text { Screening } \\
\text { Value } \\
(\mu \mathrm{g} / \mathrm{L})\end{array}$ & $\begin{array}{c}\text { Human Health } \\
\text { Constituent of } \\
\text { Concern }\end{array}$ \\
\hline $\begin{array}{l}\text { Diesel-Range } \\
\text { Hydrocarbons }\end{array}$ & 2 of 5 & ND (50) & 54.6 & NAN & $1,500^{d}$ & No ${ }^{h}$ \\
\hline \multicolumn{7}{|c|}{ Volatile Organic Compounds } \\
\hline 1,2,4-Trimethylbenzene & 0 of 5 & ND (1.0) & ND (1.0) & NAN & $12^{\mathrm{e}}$ & $\mathrm{No}^{f}$ \\
\hline 1,3,5-Trimethylbenzene & 0 of 5 & ND (1.0) & ND (1.0) & NAN & $12^{\mathrm{e}}$ & $\mathrm{No}^{f}$ \\
\hline 2-Butanone & 0 of 5 & ND (20) & ND (20) & NAN & $1,900^{\mathrm{e}}$ & $\mathrm{No}^{f}$ \\
\hline Acetone & 0 of 5 & ND (20) & ND (20) & NAN & $3,700^{\mathrm{e}}$ & $\mathrm{No}^{f}$ \\
\hline Benzene & 0 of 5 & ND (1.0) & ND (1.0) & NAN & $1.2^{\mathrm{c}}$ & $\mathrm{No}^{f}$ \\
\hline Carbon disulfide & 0 of 5 & ND (1.0) & ND (1.0) & NAN & $1,000^{\mathrm{e}}$ & $\mathrm{No}^{f}$ \\
\hline Ethylbenzene & 0 of 5 & ND (1.0) & ND (1.0) & NAN & $3,100^{c}$ & $\mathrm{No}^{f}$ \\
\hline Isopropylbenzene & 0 of 5 & ND (1.0) & ND (1.0) & NAN & NAV & $\mathrm{No}^{f}$ \\
\hline m,p-Xylene & 0 of 5 & ND (2.0) & ND (2.0) & NAN & $12,000^{\mathrm{e}}$ & $\mathrm{No}^{f}$ \\
\hline Methylene chloride & 0 of 5 & ND (2.0) & ND (5.14) & NAN & $4.7^{\mathrm{C}}$ & $\mathrm{No}^{f}$ \\
\hline n-Butylbenzene & 0 of 5 & ND (1.0) & ND (1.0) & NAN & $61^{\mathrm{e}}$ & $\mathrm{No}^{f}$ \\
\hline n-Propylbenzene & 0 of 5 & ND (1.0) & ND (1.0) & NAN & $61^{\mathrm{e}}$ & $\mathrm{No}^{f}$ \\
\hline Naphthalene & 0 of 5 & ND (1.0) & ND (1.0) & NAN & $730^{e}$ & $\mathrm{No}^{f}$ \\
\hline o-Xylene & 0 of 5 & ND (1.0) & ND (1.0) & NAN & $12,000^{\mathrm{e}}$ & $\mathrm{No}^{f}$ \\
\hline p-Isopropyltoluene & 0 of 5 & ND (1.0) & ND (1.0) & NAN & NAV & $\mathrm{No}^{f}$ \\
\hline sec-Butylbenzene & 0 of 5 & $\mathrm{ND}(1.0)$ & ND (1.0) & NAN & $61^{\mathrm{e}}$ & $\mathrm{No}^{f}$ \\
\hline Toluene & 0 of 5 & ND (1.0) & ND (1.0) & NAN & $10,000^{c}$ & $\mathrm{No}^{f}$ \\
\hline \multicolumn{7}{|c|}{ Polycyclic Aromatic Hydrocarbons } \\
\hline Acenaphthene & 0 of 5 & ND (0.1) & $\mathrm{ND}(0.1)$ & NAN & $1,200^{C}$ & $\mathrm{No}^{f}$ \\
\hline Acenaphthylene & 0 of 5 & ND $(0.1)$ & ND $(0.1)$ & NAN & $0.0028^{c}$ & $\mathrm{No}^{f}$ \\
\hline Anthracene & 0 of 5 & $\mathrm{ND}(0.1)$ & $\mathrm{ND}(0.1)$ & NAN & $0.0028^{c}$ & $\mathrm{No}^{f}$ \\
\hline Benzo(a)anthracene & 0 of 5 & ND $(0.1)$ & ND $(0.1)$ & NAN & $0.0028^{c}$ & $\mathrm{No}^{f}$ \\
\hline Benzo(a)pyrene & 0 of 5 & $\mathrm{ND}(0.1)$ & $\mathrm{ND}(0.1)$ & NAN & $0.0028^{C}$ & $\mathrm{No}^{f}$ \\
\hline Benzo(b)fluoranthene & 0 of 5 & ND $(0.1)$ & ND $(0.1)$ & NAN & $0.092^{\mathrm{e}}$ & $\mathrm{No}^{f}$ \\
\hline Benzo(ghi)perylene & 0 of 5 & ND $(0.1)$ & $\mathrm{ND}(0.1)$ & NAN & $0.0028^{c}$ & $\mathrm{No}^{f}$ \\
\hline Benzo(k)fluoranthene & 0 of 5 & ND $(0.1)$ & ND $(0.1)$ & NAN & $0.0028^{c}$ & $\mathrm{No}^{f}$ \\
\hline Chrysene & 0 of 5 & ND (0.1) & ND (0.1) & NAN & $0.0028^{c}$ & $\mathrm{No}^{f}$ \\
\hline
\end{tabular}


Table 4-17

\section{Summary of Analytical Results and Human Health Risk Assessment Screening for Surface Water in Cloudberry Creek, Long Shot Site} (Page 2 of 3 )

\begin{tabular}{|c|c|c|c|c|c|c|}
\hline Analyte & $\begin{array}{l}\text { Detection } \\
\text { Frequency }\end{array}$ & $\begin{array}{c}\text { Minimum } \\
\text { Detected } \\
\text { Concentration } \\
(\mu \mathrm{g} / \mathrm{L})\end{array}$ & $\begin{array}{c}\text { Maximum } \\
\text { Detected } \\
\text { Concentration } \\
(\mu \mathrm{g} / \mathrm{L})\end{array}$ & $\begin{array}{c}\text { Lowland } \\
\text { Stream } \\
\text { Background } \\
\text { Concentration }{ }^{\mathrm{a}} \\
(\mu \mathrm{g} / \mathrm{L})\end{array}$ & $\begin{array}{l}\text { Human Health } \\
\text { Screening } \\
\text { Value } \\
(\mu \mathrm{g} / \mathrm{L})\end{array}$ & $\begin{array}{c}\text { Human Health } \\
\text { Constituent of } \\
\text { Concern }\end{array}$ \\
\hline Dibenzo(a,h)anthracene & 0 of 5 & ND $(0.1)$ & ND $(0.1)$ & NAN & $0.0028^{C}$ & $\mathrm{No}^{f}$ \\
\hline Fluoranthene & 0 of 5 & $\mathrm{ND}(0.1)$ & $\mathrm{ND}(0.1)$ & NAN & $42^{c}$ & $\mathrm{No}^{f}$ \\
\hline Fluorene & 0 of 5 & $\mathrm{ND}(0.1)$ & ND (0.1) & NAN & $0.0028^{C}$ & $\mathrm{No}^{f}$ \\
\hline Indeno(1,2,3-cd)pyrene & 0 of 5 & ND $(0.1)$ & ND $(0.1)$ & NAN & $0.0028^{c}$ & $\mathrm{No}^{f}$ \\
\hline Naphthalene & 0 of 5 & $\mathrm{ND}(0.1)$ & $\mathrm{ND}(0.1)$ & NAN & $730^{e}$ & $\mathrm{No}^{f}$ \\
\hline Phenanthrene & 0 of 5 & $\mathrm{ND}(0.1)$ & ND $(0.1)$ & NAN & $0.0028^{c}$ & $\mathrm{No}^{f}$ \\
\hline Pyrene & 0 of 5 & $\mathrm{ND}(0.1)$ & $\mathrm{ND}(0.1)$ & NAN & $0.0028^{c}$ & $\mathrm{No}^{f}$ \\
\hline \multicolumn{7}{|c|}{ Polychlorinated Biphenyls } \\
\hline Aroclor 1016 & NAV & NAV & NAV & NAN & $0.000044^{c}$ & $\mathrm{No}^{f}$ \\
\hline Aroclor 1221 & NAV & NAV & NAV & NAN & $0.000044^{c}$ & $\mathrm{No}^{f}$ \\
\hline Aroclor 1232 & NAV & NAV & NAV & NAN & $0.000044^{c}$ & $\mathrm{No}^{f}$ \\
\hline Aroclor 1242 & NAV & NAV & NAV & NAN & $0.000044^{c}$ & $\mathrm{No}^{f}$ \\
\hline Aroclor 1248 & NAV & NAV & NAV & NAN & $0.000044^{c}$ & $\mathrm{No}^{f}$ \\
\hline Aroclor 1254 & NAV & NAV & NAV & NAN & $0.000044^{c}$ & $\mathrm{No}^{f}$ \\
\hline Aroclor 1260 & NAV & NAV & NAV & NAN & $0.000044^{c}$ & $\mathrm{No}^{f}$ \\
\hline Aroclor 1262 & NAV & NAV & NAV & NAN & $0.000044^{c}$ & $\mathrm{No}^{f}$ \\
\hline Aroclor 1268 & NAV & NAV & NAV & NAN & $0.000044^{c}$ & $\mathrm{No}^{f}$ \\
\hline \multicolumn{7}{|c|}{ Metals } \\
\hline Aluminum & 3 of 5 & ND (50) & 77.1 & 269 & $37,000^{\mathrm{e}}$ & $\mathrm{No}^{\mathrm{g}}$ \\
\hline Arsenic & 0 of 5 & ND (1.0) & ND (1.0) & $\mathrm{ND}(1)$ & $0.018^{C}$ & $\mathrm{No}^{f}$ \\
\hline Barium & 0 of 5 & ND (10) & ND (10) & ND (10) & $1,000^{\mathrm{C}}$ & $\mathrm{No}^{f}$ \\
\hline Beryllium & 0 of 5 & ND (1.0) & ND (1.0) & ND (1) & $0.0076^{c}$ & $\mathrm{No}^{f}$ \\
\hline Boron & 0 of 5 & ND (50) & ND (50) & ND (50) & $3,300^{\mathrm{e}}$ & $\mathrm{No}^{f}$ \\
\hline Cadmium & 0 of 5 & ND (1.0) & ND (1.0) & ND (1) & $10^{c}$ & $\mathrm{No}^{f}$ \\
\hline Calcium & 5 of 5 & 3,490 & 4,300 & $4,320^{b}$ & NAV & $\mathrm{No}^{\mathrm{g}}$ \\
\hline Cerium & 0 of 5 & ND (1.0) & ND (1.0) & ND (1) & NAV & $\mathrm{No}^{f}$ \\
\hline Cesium & 0 of 5 & ND (1.0) & ND (1.0) & ND (1) & NAV & $\mathrm{No}^{f}$ \\
\hline Chromium & 0 of 5 & ND (1.0) & ND (1.0) & $2.25^{b}$ & $33,000^{c}$ & $\mathrm{No}^{f}$ \\
\hline Cobalt & 3 of 5 & ND (1.0) & 2 & 3.35 & $2,200^{e}$ & $\mathrm{No}^{\mathrm{g}}$ \\
\hline
\end{tabular}


Table 4-17

\section{Summary of Analytical Results and Human Health Risk Assessment Screening for Surface Water in Cloudberry Creek, Long Shot Site} (Page 3 of 3 )

\begin{tabular}{|c|c|c|c|c|c|c|}
\hline Analyte & $\begin{array}{l}\text { Detection } \\
\text { Frequency }\end{array}$ & $\begin{array}{c}\text { Minimum } \\
\text { Detected } \\
\text { Concentration } \\
(\mu \mathrm{g} / \mathrm{L})\end{array}$ & $\begin{array}{c}\text { Maximum } \\
\text { Detected } \\
\text { Concentration } \\
(\mu \mathrm{g} / \mathrm{L})\end{array}$ & $\begin{array}{c}\text { Lowland } \\
\text { Stream } \\
\text { Background } \\
\text { Concentration }^{\mathrm{a}} \\
(\mu \mathrm{g} / \mathrm{L})\end{array}$ & $\begin{array}{c}\text { Human Health } \\
\text { Screening } \\
\text { Value } \\
(\mu \mathrm{g} / \mathrm{L})\end{array}$ & $\begin{array}{c}\text { Human Health } \\
\text { Constituent of } \\
\text { Concern }\end{array}$ \\
\hline Copper & 5 of 5 & 1.79 & 3.19 & 10.8 & $1,300^{C}$ & $\mathrm{No}^{\mathrm{g}}$ \\
\hline Iron & 5 of 5 & 196 & 349 & 993 & $300^{c}$ & $\mathrm{No}^{\mathrm{g}}$ \\
\hline Lithium & 1 of 5 & ND (1.0) & 1.21 & $1.17^{\mathrm{b}}$ & $730^{\mathrm{e}}$ & $\mathrm{No}^{\mathrm{h}}$ \\
\hline Magnesium & 5 of 5 & 3,610 & 3,910 & $4,690^{b}$ & NAV & $\mathrm{No}^{\mathrm{g}}$ \\
\hline Manganese & 5 of 5 & 5.84 & 35.4 & 267 & $50^{c}$ & $\mathrm{No}^{\mathrm{g}}$ \\
\hline Molybdenum & 0 of 5 & ND (1.0) & ND (1.0) & ND (1) & $180^{e}$ & $\mathrm{No}^{f}$ \\
\hline Nickel & 3 of 5 & ND (1.0) & 1.27 & ND (1) & $510^{c}$ & No ${ }^{h}$ \\
\hline Potassium & 5 of 5 & 915 & 1,480 & 2,620 & NAV & $\mathrm{No}^{\mathrm{g}}$ \\
\hline Selenium & 0 of 5 & ND (1.0) & ND (1.0) & ND (1) & $104^{c}$ & $\mathrm{No}^{f}$ \\
\hline Strontium & 5 of 5 & 1.85 & 20.8 & 36.1 & $22,000^{\mathrm{e}}$ & $\mathrm{No}^{\mathrm{g}}$ \\
\hline Thallium & 0 of 5 & ND (1.0) & ND (1.0) & $7.07^{b}$ & $1.7^{\mathrm{C}}$ & $\mathrm{No}^{f}$ \\
\hline Thorium & 0 of 5 & ND (1.0) & ND (1.0) & ND (1) & NAV & $\mathrm{No}^{f}$ \\
\hline Titanium & 5 of 5 & 1.72 & 3.13 & 6.13 & $150,000^{e}$ & $\mathrm{No}^{\mathrm{g}}$ \\
\hline Uranium & 0 of 5 & ND (1.0) & ND (1.0) & ND (1) & $110^{\mathrm{e}}$ & $\mathrm{No}^{f}$ \\
\hline Vanadium & 4 of 5 & ND (1.0) & 2.87 & $4.43^{b}$ & $260^{\mathrm{e}}$ & $\mathrm{No}^{\mathrm{g}}$ \\
\hline Zinc & 1 of 5 & ND (10) & 17 & $50.3^{b}$ & $11,000^{\mathrm{e}}$ & $\mathrm{No}^{\mathrm{g}}$ \\
\hline
\end{tabular}

${ }^{\mathrm{a} B a c k g r o u n d}$ concentrations are upper tolerance limits (UTL) (ADEC, 1999a) unless otherwise noted.

${ }^{b}$ Maximum detected concentration in background samples due to insufficient detection frequency to calculate UTL.

${ }^{\mathrm{c}}$ Federal ambient water quality criteria based on ingestion of water and organisms.

dState of Alaska groundwater cleanup level, Discharge Reporting, Cleanup, and Disposal of Oil and other Hazardous Substances

(18 AAC 75, Articles 3 and 9 [ADEC, 1999c]).

eU.S. EPA Region III risk-based concentrations for tap water (EPA, 2000b).

\section{COC Selection Criteria}

${ }^{7}$ Constituent not detected in any samples.

${ }^{9}$ Maximum detected constituent concentration is less than local background concentration.

${ }^{h}$ Maximum detected constituent concentration is less than human health screening value.

ND - Not detected (detection limit)

NAV - Not available

NAN - Not analyzed

$\mu \mathrm{g} / \mathrm{L}$ - Micrograms per liter 
Table 4-18

\section{Summary of Analytical Results and Human Health Risk Assessment Screening for Sediment in Cloudberry Creek, Long Shot Site}

(Page 1 of 3 )

\begin{tabular}{|c|c|c|c|c|c|c|}
\hline Analyte & $\begin{array}{l}\text { Detection } \\
\text { Frequency }\end{array}$ & $\begin{array}{c}\text { Minimum } \\
\text { Detected } \\
\text { Concentration } \\
(\mathrm{mg} / \mathrm{kg})\end{array}$ & $\begin{array}{c}\text { Maximum } \\
\text { Detected } \\
\text { Concentration } \\
(\mathrm{mg} / \mathrm{kg})\end{array}$ & $\begin{array}{c}\text { Lowland } \\
\text { Stream } \\
\text { Background } \\
\text { Concentration } \\
(\mathbf{m g} / \mathbf{k g})\end{array}$ & $\begin{array}{l}\text { Human Health } \\
\text { Screening } \\
\text { Value } \\
(\mathrm{mg} / \mathrm{kg})\end{array}$ & $\begin{array}{c}\text { Human } \\
\text { Health } \\
\text { Constituent } \\
\text { of Concern }\end{array}$ \\
\hline $\begin{array}{l}\text { Diesel-Range } \\
\text { Hydrocarbons }\end{array}$ & 0 of 6 & ND (12.1) & ND (50.5) & NAN & $200^{c}$ & $\mathrm{No}^{d}$ \\
\hline \multicolumn{7}{|c|}{ Volatile Organic Compounds } \\
\hline 1,2,4-Trimethylbenzene & 0 of 1 & ND (0.0389) & ND (0.0389) & NAN & NAV & $\mathrm{No}^{d}$ \\
\hline 1,3,5-Trimethylbenzene & 0 of 1 & ND (0.0389) & ND $(0.0389)$ & NAN & NAV & $\mathrm{No}^{\mathrm{d}}$ \\
\hline 2-Butanone & 0 of 1 & ND (0.0778) & ND (0.0778) & NAN & NAV & $\mathrm{No}^{d}$ \\
\hline Acetone & 0 of 1 & ND $(0.907)$ & ND (0.907) & NAN & $10,100^{c}$ & $\mathrm{No}^{d}$ \\
\hline Benzene & 0 of 1 & ND (0.0389) & ND (0.0389) & NAN & $286^{\mathrm{C}}$ & $\mathrm{No}^{\mathrm{d}}$ \\
\hline Carbon disulfide & 0 of 1 & ND (0.0494) & ND $(0.0494)$ & NAN & $10,100^{c}$ & $\mathrm{No}^{d}$ \\
\hline Ethylbenzene & 0 of 1 & ND (0.0389) & ND (0.0389) & NAN & $10,100^{c}$ & $\mathrm{No}^{\mathrm{d}}$ \\
\hline Isopropylbenzene & 0 of 1 & ND (0.0389) & ND (0.0389) & NAN & NAV & $\mathrm{No}^{d}$ \\
\hline m,p-Xylene & 0 of 1 & ND (0.0389) & ND (0.0389) & NAN & $203,000^{C}$ & $\mathrm{No}^{d}$ \\
\hline Methylene chloride & 0 of 1 & ND (0.0389) & ND (0.0389) & NAN & $1,110^{\mathrm{c}}$ & $\mathrm{No}^{d}$ \\
\hline n-Butylbenzene & 0 of 1 & ND (0.0389) & ND (0.0389) & NAN & NAV & $\mathrm{No}^{d}$ \\
\hline n-Propylbenzene & 0 of 1 & ND (0.0389) & ND (0.0389) & NAN & NAV & $\mathrm{No}^{d}$ \\
\hline Naphthalene & 0 of 1 & ND (0.0389) & ND (0.0389) & NAN & $4,060^{\mathrm{C}}$ & $\mathrm{No}^{d}$ \\
\hline o-Xylene & 0 of 1 & ND (0.0389) & ND (0.0389) & NAN & $203,000^{c}$ & $\mathrm{No}^{d}$ \\
\hline p-Isopropyltoluene & 0 of 1 & ND (0.0389) & ND (0.0389) & NAN & NAV & $\mathrm{No}^{\mathrm{d}}$ \\
\hline sec-Butylbenzene & 0 of 1 & ND (0.0389) & ND (0.0389) & NAN & NAV & $\mathrm{No}^{d}$ \\
\hline Toluene & 0 of 1 & ND (0.0389) & ND (0.0389) & NAN & $20,300^{c}$ & $\mathrm{No}^{d}$ \\
\hline \multicolumn{7}{|c|}{ Polycyclic Aromatic Hydrocarbons } \\
\hline Acenaphthene & 0 of 6 & ND (0.00996) & ND $(0.0417)$ & NAN & $6,080^{c}$ & $\mathrm{No}^{\mathrm{d}}$ \\
\hline Acenaphthylene & 0 of 6 & ND (0.00996) & ND $(0.0417)$ & NAN & NAV & $\mathrm{No}^{\mathrm{d}}$ \\
\hline Anthracene & 0 of 6 & ND (0.00996) & ND $(0.0417)$ & NAN & $30,400^{c}$ & $\mathrm{No}^{\mathrm{d}}$ \\
\hline Benzo(a)anthracene & 0 of 6 & ND $(0.00996)$ & ND $(0.0417)$ & NAN & $11.4^{\mathrm{C}}$ & $\mathrm{No}^{d}$ \\
\hline Benzo(a)pyrene & 0 of 6 & ND $(0.00996)$ & ND $(0.0417)$ & NAN & $1.1^{\mathrm{c}}$ & $\mathrm{No}^{d}$ \\
\hline Benzo(b)fluoranthene & 0 of 6 & ND $(0.00996)$ & ND $(0.0417)$ & NAN & $11.4^{\mathrm{C}}$ & $\mathrm{No}^{\mathrm{d}}$ \\
\hline Benzo(ghi)perylene & 0 of 6 & ND (0.00996) & ND $(0.0417)$ & NAN & $1.1^{\mathrm{c}}$ & $\mathrm{No}^{\mathrm{d}}$ \\
\hline Benzo(k)fluoranthene & 0 of 6 & ND $(0.00996)$ & ND $(0.0417)$ & NAN & $114^{\mathrm{C}}$ & $\mathrm{No}^{\mathrm{d}}$ \\
\hline Chrysene & 0 of 6 & ND (0.00996) & ND (0.0417) & NAN & $1,140^{c}$ & $\mathrm{No}^{\mathrm{d}}$ \\
\hline
\end{tabular}


Table 4-18

\section{Summary of Analytical Results and Human Health Risk Assessment Screening for Sediment in Cloudberry Creek, Long Shot Site}

(Page 2 of 3 )

\begin{tabular}{|c|c|c|c|c|c|c|}
\hline Analyte & $\begin{array}{l}\text { Detection } \\
\text { Frequency }\end{array}$ & $\begin{array}{c}\text { Minimum } \\
\text { Detected } \\
\text { Concentration } \\
(\mathbf{m g} / \mathbf{k g})\end{array}$ & $\begin{array}{c}\text { Maximum } \\
\text { Detected } \\
\text { Concentration } \\
(\mathbf{m g} / \mathbf{k g})\end{array}$ & $\begin{array}{c}\text { Lowland } \\
\text { Stream } \\
\text { Background } \\
\text { Concentration }{ }^{\mathrm{a}} \\
(\mathrm{mg} / \mathrm{kg})\end{array}$ & $\begin{array}{l}\text { Human Health } \\
\text { Screening } \\
\text { Value } \\
(\mathrm{mg} / \mathrm{kg})\end{array}$ & $\begin{array}{c}\text { Human } \\
\text { Health } \\
\text { Constituent } \\
\text { of Concern }\end{array}$ \\
\hline Dibenzo(a,h)anthracene & 0 of 6 & ND (0.00996) & ND $(0.0417)$ & NAN & $1.1^{\mathrm{C}}$ & $\mathrm{No}^{\mathrm{d}}$ \\
\hline Fluoranthene & 0 of 6 & ND (0.00996) & ND $(0.0417)$ & NAN & $4,060^{\mathrm{C}}$ & $\mathrm{No}^{d}$ \\
\hline Fluorene & 0 of 6 & ND (0.00996) & $\mathrm{ND}(0.0417)$ & NAN & $4,060^{\mathrm{C}}$ & $\mathrm{No}^{\mathrm{d}}$ \\
\hline Indeno(1,2,3-cd)pyrene & 0 of 6 & ND (0.00996) & ND $(0.0417)$ & NAN & $11^{\mathrm{c}}$ & $\mathrm{No}^{\mathrm{d}}$ \\
\hline Naphthalene & 0 of 6 & ND (0.00996) & ND $(0.0417)$ & NAN & $4,060^{C}$ & $\mathrm{No}^{\mathrm{d}}$ \\
\hline Phenanthrene & 0 of 6 & ND (0.00996) & ND $(0.0417)$ & NAN & $1.1^{\mathrm{c}}$ & $\mathrm{No}^{d}$ \\
\hline Pyrene & 0 of 6 & ND (0.00996) & ND $(0.0417)$ & NAN & $3,040^{c}$ & $\mathrm{No}^{\mathrm{d}}$ \\
\hline \multicolumn{7}{|c|}{ Polychlorinated Biphenyls } \\
\hline Aroclor 1016 & NAV & NAV & NAV & NAN & $10^{C}$ & $\mathrm{No}^{\mathrm{d}}$ \\
\hline Aroclor 1221 & NAV & NAV & NAV & NAN & $10^{\mathrm{C}}$ & $\mathrm{No}^{\mathrm{d}}$ \\
\hline Aroclor 1232 & NAV & NAV & NAV & NAN & $10^{C}$ & $\mathrm{No}^{\mathrm{d}}$ \\
\hline Aroclor 1242 & NAV & NAV & NAV & NAN & $10^{c}$ & $\mathrm{No}^{d}$ \\
\hline Aroclor 1248 & NAV & NAV & NAV & NAN & $10^{c}$ & $\mathrm{No}^{d}$ \\
\hline Aroclor 1254 & NAV & NAV & NAV & NAN & $10^{c}$ & $\mathrm{No}^{\mathrm{d}}$ \\
\hline Aroclor 1260 & NAV & NAV & NAV & NAN & $10^{c}$ & $\mathrm{No}^{\mathrm{d}}$ \\
\hline Aroclor 1262 & NAV & NAV & NAV & NAN & $10^{c}$ & $\mathrm{No}^{d}$ \\
\hline Aroclor 1268 & NAV & NAV & NAV & NAN & $10^{c}$ & $\mathrm{No}^{d}$ \\
\hline \multicolumn{7}{|c|}{ Metals } \\
\hline Aluminum & 6 of 6 & 17,100 & 41,800 & 45,400 & NAV & $\mathrm{No}^{\mathrm{e}}$ \\
\hline Arsenic & 5 of 6 & ND (6.31) & 9.89 & 100 & $5.5^{\mathrm{c}}$ & $\mathrm{No}^{\mathrm{e}}$ \\
\hline Barium & 6 of 6 & 68.5 & 151 & 469 & $7,100^{\mathrm{C}}$ & $\mathrm{No}^{\mathrm{e}}$ \\
\hline Beryllium & 1 of 6 & ND (1.51) & 2.9 & ND & $1.9^{C}$ & $\mathrm{No}^{h}$ \\
\hline Boron & 1 of 6 & ND (10) & 159 & $7.18^{b}$ & NAV & $\mathrm{No}^{\mathrm{h}}$ \\
\hline Cadmium & 1 of 6 & ND (1.51) & 3.17 & $0.608^{b}$ & $101^{\mathrm{C}}$ & $\mathrm{No}^{f}$ \\
\hline Calcium & 6 of 6 & 5,460 & 8,010 & 7,300 & NAV & $\mathrm{No}^{\mathrm{g}}$ \\
\hline Cerium & 6 of 6 & 6.83 & 12 & 14.2 & NAV & $\mathrm{No}^{\mathrm{e}}$ \\
\hline Cesium & 1 of 6 & $\mathrm{ND}(1.51)$ & 3.51 & ND & NAV & $\mathrm{No}^{h}$ \\
\hline Chromium & 5 of 6 & ND (6.31) & 11.3 & 11.8 & $507^{\mathrm{C}}$ & $\mathrm{No}^{\mathrm{e}}$ \\
\hline Cobalt & 6 of 6 & 8.33 & 24.6 & 43.7 & NAV & $\mathrm{No}^{\mathrm{e}}$ \\
\hline
\end{tabular}




\section{Table 4-18 \\ Summary of Analytical Results and Human Health Risk Assessment Screening for Sediment in Cloudberry Creek, Long Shot Site}

(Page 3 of 3 )

\begin{tabular}{|c|c|c|c|c|c|c|}
\hline Analyte & $\begin{array}{l}\text { Detection } \\
\text { Frequency }\end{array}$ & $\begin{array}{c}\text { Minimum } \\
\text { Detected } \\
\text { Concentration } \\
(\mathrm{mg} / \mathrm{kg})\end{array}$ & $\begin{array}{c}\text { Maximum } \\
\text { Detected } \\
\text { Concentration } \\
(\mathrm{mg} / \mathrm{kg})\end{array}$ & $\begin{array}{c}\text { Lowland } \\
\text { Stream } \\
\text { Background } \\
\text { Concentration }^{\mathrm{a}} \\
(\mathrm{mg} / \mathrm{kg})\end{array}$ & $\begin{array}{l}\text { Human Health } \\
\text { Screening } \\
\text { Value } \\
(\mathrm{mg} / \mathrm{kg})\end{array}$ & $\begin{array}{c}\text { Human } \\
\text { Health } \\
\text { Constituent } \\
\text { of Concern }\end{array}$ \\
\hline Copper & 6 of 6 & 52.6 & 71.7 & 71.1 & NAV & $\mathrm{No}^{\mathrm{e}}$ \\
\hline Iron & 6 of 6 & 18,700 & 79,900 & 155,000 & NAV & $\mathrm{No}^{\mathrm{e}}$ \\
\hline Lithium & 6 of 6 & 10.2 & 17 & 48.8 & NAV & $\mathrm{No}^{\mathrm{e}}$ \\
\hline Magnesium & 6 of 6 & 7,490 & 16,800 & 9,320 & NAV & $\mathrm{No}^{\mathrm{g}}$ \\
\hline Manganese & 6 of 6 & 140 & 5,160 & 20,700 & NAV & $\mathrm{No}^{\mathrm{e}}$ \\
\hline Molybdenum & 1 of 6 & ND (1.51) & 3.79 & $5.5^{\mathrm{b}}$ & NAV & $\mathrm{No}^{\mathrm{e}}$ \\
\hline Nickel & 6 of 6 & 6.86 & 9.67 & 13.6 & $2,030^{\mathrm{C}}$ & $\mathrm{No}^{\mathrm{e}}$ \\
\hline Potassium & 6 of 6 & 537 & 2,630 & 1,390 & NAV & $\mathrm{No}^{\mathrm{g}}$ \\
\hline Selenium & 1 of 6 & ND (1.51) & 4.55 & $5.44^{\mathrm{b}}$ & $507^{C}$ & $\mathrm{No}^{\mathrm{e}}$ \\
\hline Strontium & 6 of 6 & 50.8 & 221 & 164 & NAV & $\mathrm{No}^{\mathrm{h}}$ \\
\hline Thallium & 1 of 6 & ND (1.51) & 4.08 & $1^{b}$ & NAV & $\mathrm{No}^{\mathrm{h}}$ \\
\hline Thorium & 0 of 6 & ND (1.51) & ND (6.31) & $0.5^{\mathrm{b}}$ & NAV & $\mathrm{No}^{\mathrm{d}}$ \\
\hline Titanium & 6 of 6 & 639 & 1,640 & 3,010 & NAV & $\mathrm{No}^{\mathrm{e}}$ \\
\hline Uranium & 0 of 6 & ND (1.51) & ND (6.31) & $4.13^{b}$ & NAV & $\mathrm{No}^{\mathrm{d}}$ \\
\hline Vanadium & 6 of 6 & 121 & 230 & 734 & $710^{c}$ & $\mathrm{No}^{\mathrm{e}}$ \\
\hline Zinc & 6 of 6 & 57.8 & 106 & 434 & $30,400^{c}$ & $\mathrm{No}^{\mathrm{e}}$ \\
\hline Total Organic Carbon & 6 of 6 & 29,900 & 220,000 & NAN & NAV & No \\
\hline
\end{tabular}

${ }^{\mathrm{a}}$ Background concentrations are upper tolerance limits (UTL) (ADEC, 1999a) unless otherwise noted.

${ }^{\mathrm{b}}$ Maximum detected concentration in background samples due to insufficient detection frequency to calculate UTL.

'State of Alaska soil cleanup level, Discharge Reporting, Cleanup, and Disposal of Oil and other Hazardous Substances

(18 AAC 75, Articles 3 and 9 [ADEC, 1999c]).

\section{COC Selection Criteria}

${ }^{\mathrm{d}}$ Constituent not detected in any samples.

${ }^{e}$ Maximum detected constituent concentration is less than local background concentration.

${ }^{\mathrm{f}}$ Maximum detected constituent concentration is less than human health screening value.

${ }^{9}$ Constituent is an essential macronutrient and only presents a risk at extremely high concentrations.

${ }^{\mathrm{h}}$ Not a constituent associated with drilling mud or other drilling activities.

ND - Not detected (detection limit)

NAV - Not available

NAN - Not analyzed

$\mathrm{mg} / \mathrm{kg}$ - Milligrams per kilogram 
Table 4-19

\section{Summary of Analytical Results and Human Health Risk Assessment Screening for Fish Tissue in Cloudberry Creek, Long Shot Site} (Page 1 of 3 )

\begin{tabular}{|c|c|c|c|c|c|c|}
\hline Analyte & $\begin{array}{l}\text { Detection } \\
\text { Frequency }\end{array}$ & $\begin{array}{c}\text { Minimum } \\
\text { Detected } \\
\text { Concentration } \\
(\mathbf{m g} / \mathbf{k g})\end{array}$ & $\begin{array}{c}\text { Maximum } \\
\text { Detected } \\
\text { Concentration } \\
(\mathbf{m g} / \mathbf{k g})\end{array}$ & $\begin{array}{c}\text { Lowland } \\
\text { Stream } \\
\text { Background } \\
\text { Concentration }{ }^{\mathrm{a}} \\
(\mathrm{mg} / \mathbf{k g})\end{array}$ & $\begin{array}{l}\text { Human Health } \\
\text { Screening } \\
\text { Value } \\
(\mathbf{m g} / \mathbf{k g})\end{array}$ & $\begin{array}{c}\text { Human Health } \\
\text { Constituent of } \\
\text { Concern }\end{array}$ \\
\hline \multicolumn{7}{|c|}{ Polycyclic Aromatic Hydrocarbons } \\
\hline Acenaphthene & 0 of 3 & ND $(0.0067)$ & ND $(0.0067)$ & NAN & 81.1 & No ${ }^{d}$ \\
\hline Acenaphthylene & 0 of 3 & ND (0.0067) & ND (0.0067) & NAN & NAV & No ${ }^{d}$ \\
\hline Anthracene & 0 of 3 & ND (0.0335) & ND $(0.067)$ & NAN & 410 & $\mathrm{No}^{d}$ \\
\hline Benzo(a)anthracene & 0 of 3 & ND (0.0335) & ND $(0.067)$ & NAN & 0.0043 & No ${ }^{d}$ \\
\hline Benzo(a)pyrene & 0 of 3 & ND (0.0335) & ND $(0.067)$ & NAN & 0.00043 & $\mathrm{No}^{d}$ \\
\hline Benzo(b)fluoranthene & 0 of 3 & ND (0.0335) & ND $(0.067)$ & NAN & 0.0043 & No ${ }^{d}$ \\
\hline Benzo(ghi)perylene & 0 of 3 & ND (0.0335) & ND $(0.067)$ & NAN & NAV & $\mathrm{No}^{d}$ \\
\hline Benzo(k)fluoranthene & 0 of 3 & ND (0.0335) & ND $(0.067)$ & NAN & 0.043 & $\mathrm{No}^{d}$ \\
\hline Chrysene & 0 of 3 & ND (0.0335) & ND $(0.067)$ & NAN & 0.43 & $\mathrm{No}^{d}$ \\
\hline Dibenzo(a,h)anthracene & 0 of 3 & ND (0.0335) & ND $(0.067)$ & NAN & 0.00043 & No ${ }^{d}$ \\
\hline Fluoranthene & 0 of 3 & ND (0.0335) & ND $(0.067)$ & NAN & 54 & $\mathrm{No}^{d}$ \\
\hline Fluorene & 0 of 3 & ND (0.0067) & ND (0.0067) & NAN & 54 & $\mathrm{No}^{d}$ \\
\hline Indeno(1,2,3-cd)pyrene & 0 of 3 & ND (0.0335) & ND $(0.067)$ & NAN & 0.0043 & $\mathrm{No}^{d}$ \\
\hline Naphthalene & 0 of 3 & ND (0.0067) & ND (0.0067) & NAN & 27 & No ${ }^{d}$ \\
\hline Phenanthrene & 0 of 3 & ND (0.0335) & ND $(0.067)$ & NAN & NAV & No ${ }^{d}$ \\
\hline Pyrene & 0 of 3 & ND (0.0335) & ND $(0.067)$ & NAN & 41 & $\mathrm{No}^{d}$ \\
\hline \multicolumn{7}{|c|}{ Polychlorinated Biphenyls } \\
\hline Aroclor 1016 & 0 of 3 & ND $(0.01)$ & ND $(0.01)$ & NAN & 0.045 & $\mathrm{No}^{d}$ \\
\hline Aroclor 1221 & 0 of 3 & ND (0.02) & ND (0.02) & NAN & 0.0016 & No ${ }^{d}$ \\
\hline Aroclor 1232 & 0 of 3 & ND (0.01) & ND $(0.01)$ & NAN & 0.0016 & No ${ }^{d}$ \\
\hline Aroclor 1242 & 0 of 3 & ND $(0.01)$ & ND $(0.01)$ & NAN & 0.0016 & $\mathrm{No}^{d}$ \\
\hline Aroclor 1248 & 0 of 3 & ND $(0.01)$ & ND $(0.01)$ & NAN & 0.0016 & No ${ }^{d}$ \\
\hline Aroclor 1254 & 0 of 3 & ND $(0.01)$ & ND $(0.01)$ & NAN & 0.0016 & $\mathrm{No}^{d}$ \\
\hline
\end{tabular}


Table 4-19

\section{Summary of Analytical Results and Human Health Risk Assessment Screening for Fish Tissue in Cloudberry Creek, Long Shot Site} (Page 2 of 3 )

\begin{tabular}{|c|c|c|c|c|c|c|}
\hline Analyte & $\begin{array}{l}\text { Detection } \\
\text { Frequency }\end{array}$ & $\begin{array}{c}\text { Minimum } \\
\text { Detected } \\
\text { Concentration } \\
(\mathrm{mg} / \mathrm{kg})\end{array}$ & $\begin{array}{c}\text { Maximum } \\
\text { Detected } \\
\text { Concentration } \\
(\mathbf{m g} / \mathbf{k g})\end{array}$ & $\begin{array}{c}\text { Lowland } \\
\text { Stream } \\
\text { Background } \\
\text { Concentration }{ }^{\mathrm{a}} \\
(\mathrm{mg} / \mathbf{k g})\end{array}$ & $\begin{array}{l}\text { Human Health } \\
\text { Screening } \\
\text { Value } \\
(\mathrm{mg} / \mathrm{kg})\end{array}$ & $\begin{array}{c}\text { Human Health } \\
\text { Constituent of } \\
\text { Concern }\end{array}$ \\
\hline Aroclor 1260 & 0 of 3 & ND (0.01) & ND (0.01) & NAN & 0.0016 & $\mathrm{No}^{d}$ \\
\hline \multicolumn{7}{|c|}{ Metals } \\
\hline Aluminum & 3 of 3 & 10 & 166 & 475 & 1,400 & $\mathrm{No}^{\mathrm{e}}$ \\
\hline Arsenic & 2 of 3 & ND (0.025) & 0.0413 & 0.127 & 0.0021 & $\mathrm{No}^{\mathrm{e}}$ \\
\hline Inorganic Arsenic & 3 of 3 & 0.000521 & 0.0152 & 0.0208 & 0.0021 & $\mathrm{No}^{\mathrm{e}}$ \\
\hline Barium & 3 of 3 & 0.0448 & 0.224 & 1.03 & 95 & $\mathrm{No}^{\mathrm{e}}$ \\
\hline Beryllium & 0 of 3 & ND (0.025) & ND $(0.025)$ & ND & 2.7 & $\mathrm{No}^{d}$ \\
\hline Boron & 0 of 3 & ND (6.45) & ND (6.75) & 28.6 & 120 & $\mathrm{No}^{d}$ \\
\hline Cadmium & 0 of 3 & ND (0.025) & ND (0.025) & $0.0373^{b}$ & 1.4 & $\mathrm{No}^{d}$ \\
\hline Calcium & 3 of 3 & 5,430 & 9,800 & 19,200 & NAV & $\mathrm{No}^{\mathrm{e}}$ \\
\hline Cerium & 1 of 3 & ND $(0.025)$ & 0.031 & $0.0280^{\mathrm{b}}$ & NAV & $\mathrm{No}^{\mathrm{g}}$ \\
\hline Cesium & 0 of 3 & ND (0.025) & ND (0.025) & $0.0258^{b}$ & NAV & $\mathrm{No}^{d}$ \\
\hline Chromium & 3 of 3 & 0.3 & 0.337 & 1.03 & 4.1 & $\mathrm{No}^{\mathrm{e}}$ \\
\hline Cobalt & 1 of 3 & ND (0.05) & 0.111 & $0.0780^{b}$ & 81 & $\mathrm{No}^{f}$ \\
\hline Copper & 0 of 3 & ND (0.678) & ND (1.52) & $1.20^{\mathrm{b}}$ & 54 & $\mathrm{No}^{d}$ \\
\hline Iron & 3 of 3 & 19.8 & 260 & 310 & 410 & $\mathrm{No}^{\mathrm{e}}$ \\
\hline Lithium & 1 of 3 & ND (0.025) & 0.0575 & $0.0993^{b}$ & 27 & $\mathrm{No}^{\mathrm{e}}$ \\
\hline Magnesium & 3 of 3 & 315 & 373 & 408 & NAV & $\mathrm{No}^{\mathrm{e}}$ \\
\hline Manganese & 3 of 3 & 1.83 & 20.3 & 160 & 190 & $\mathrm{No}^{\mathrm{e}}$ \\
\hline Molybdenum & 0 of 3 & ND (0.125) & ND (0.125) & $0.173^{\mathrm{b}}$ & 6.8 & $\mathrm{No}^{d}$ \\
\hline Nickel & 1 of 3 & ND $(0.125)$ & 0.135 & ND & 27 & $\mathrm{No}^{f}$ \\
\hline Potassium & 3 of 3 & 3,050 & 4,600 & $4,780^{b}$ & NAV & $\mathrm{No}^{\mathrm{e}}$ \\
\hline Selenium & 3 of 3 & 0.322 & 0.637 & 0.936 & 6.8 & $\mathrm{No}^{\mathrm{e}}$ \\
\hline Strontium & 3 of 3 & 7.47 & 14.3 & 22.1 & 810 & $\mathrm{No}^{\mathrm{e}}$ \\
\hline
\end{tabular}


Table 4-19

\section{Summary of Analytical Results and Human Health Risk Assessment Screening for Fish Tissue in Cloudberry Creek, Long Shot Site} (Page 3 of 3 )

\begin{tabular}{|c|c|c|c|c|c|c|}
\hline Analyte & $\begin{array}{l}\text { Detection } \\
\text { Frequency }\end{array}$ & $\begin{array}{c}\text { Minimum } \\
\text { Detected } \\
\text { Concentration } \\
(\mathbf{m g} / \mathbf{k g})\end{array}$ & $\begin{array}{c}\text { Maximum } \\
\text { Detected } \\
\text { Concentration } \\
(\mathbf{m g} / \mathrm{kg})\end{array}$ & $\begin{array}{c}\text { Lowland } \\
\text { Stream } \\
\text { Background } \\
\text { Concentration } \\
(\mathrm{mg} / \mathrm{kg})\end{array}$ & $\begin{array}{l}\text { Human Health } \\
\text { Screening } \\
\text { Value } \\
(\mathrm{mg} / \mathrm{kg})\end{array}$ & $\begin{array}{c}\text { Human Health } \\
\text { Constituent of } \\
\text { Concern }\end{array}$ \\
\hline Thallium & 0 of 3 & ND (0.025) & ND (0.025) & $0.0283^{b}$ & 0.095 & $\mathrm{No}^{\mathrm{d}}$ \\
\hline Thorium & 1 of 3 & ND (2.5) & 4.17 & $3.43^{\mathrm{b}}$ & NAV & $\mathrm{No}^{\mathrm{g}}$ \\
\hline Titanium & 0 of 3 & 0.227 & 4.64 & 32.1 & 5,400 & $\mathrm{No}^{\mathrm{e}}$ \\
\hline Uranium & 0 of 3 & ND (0.5) & ND (0.5) & ND & 4.1 & $\mathrm{No}^{d}$ \\
\hline Vanadium & 3 of 3 & 0.166 & 0.853 & 1.05 & 9.5 & $\mathrm{No}^{\mathrm{e}}$ \\
\hline Zinc & 3 of 3 & 33.9 & 37.3 & 48.8 & 410 & $\mathrm{No}^{\mathrm{e}}$ \\
\hline
\end{tabular}

${ }^{\mathrm{a} B}$ Background concentrations are upper tolerance limits (UTL) (ADEC, 1999a) unless otherwise noted.

${ }^{b}$ Maximum detected concentration in background samples due to insufficient detection frequency to calculate UTL.

${ }^{\mathrm{c} U}$.S. EPA Region III risk-based screening criteria for fish consumption unless otherwise noted.

\section{COC Selection Criteria}

${ }^{\mathrm{d} C o n s t i t u e n t}$ not detected in any samples.

${ }^{\mathrm{e}}$ Maximum detected constituent concentration is less than local background concentration.

${ }^{f}$ Maximum detected constituent concentration is less than human health screening value.

${ }^{9}$ Not a constituent associated with drilling mud or other drilling activities.

ND - Not detected (detection limit)

NAV - Not available

NAN - Not analyzed

$\mathrm{mg} / \mathrm{kg}$ - Milligrams per kilogram 
Table 4-20

Summary of Analytical Results and Human Health Risk Assessment Screening for Surface Water in Rainbow Creek, Long Shot Site

(Page 1 of 3 )

\begin{tabular}{|c|c|c|c|c|c|c|}
\hline Analyte & $\begin{array}{l}\text { Detection } \\
\text { Frequency }\end{array}$ & $\begin{array}{c}\text { Minimum } \\
\text { Detected } \\
\text { Concentration } \\
(\mu \mathrm{g} / \mathrm{L})\end{array}$ & $\begin{array}{c}\text { Maximum } \\
\text { Detected } \\
\text { Concentration } \\
(\mu \mathrm{g} / \mathrm{L})\end{array}$ & $\begin{array}{c}\text { Lowland } \\
\text { Stream } \\
\text { Background } \\
\text { Concentration }{ }^{\mathrm{a}} \\
(\mu \mathrm{g} / \mathrm{L})\end{array}$ & $\begin{array}{l}\text { Human Health } \\
\text { Screening } \\
\text { Value } \\
(\mu \mathrm{g} / \mathrm{L})\end{array}$ & $\begin{array}{c}\text { Human Health } \\
\text { Constituent of } \\
\text { Concern }\end{array}$ \\
\hline $\begin{array}{l}\text { Diesel-Range } \\
\text { Hydrocarbons }\end{array}$ & 4 of 5 & ND (50) & 121 & NAN & $1,500^{d}$ & $\mathrm{No}^{\mathrm{h}}$ \\
\hline \multicolumn{7}{|c|}{ Volatile Organic Compounds } \\
\hline 1,2,4-Trimethylbenzene & 0 of 5 & ND (1.0) & ND (1.0) & NAN & $12^{\mathrm{e}}$ & $\mathrm{No}^{\dagger}$ \\
\hline 1,3,5-Trimethylbenzene & 0 of 5 & ND (1.0) & $\mathrm{ND}(1.0)$ & NAN & $12^{\mathrm{e}}$ & $\mathrm{No}^{\dagger}$ \\
\hline 2-Butanone & 0 of 5 & ND (20) & ND (20) & NAN & $1,900^{\mathrm{e}}$ & $\mathrm{No}^{\dagger}$ \\
\hline Acetone & 0 of 5 & ND (20) & ND (20) & NAN & $3,700^{\mathrm{e}}$ & $\mathrm{No}^{\dagger}$ \\
\hline Benzene & 0 of 5 & ND (1.0) & $\mathrm{ND}(1.0)$ & NAN & $1.2^{\mathrm{c}}$ & $\mathrm{No}^{\dagger}$ \\
\hline Carbon disulfide & 0 of 5 & ND (1.0) & ND (1.0) & NAN & $1,000^{\mathrm{e}}$ & $\mathrm{No}^{\dagger}$ \\
\hline Ethylbenzene & 0 of 5 & ND (1.0) & ND (1.0) & NAN & $3,100^{c}$ & $\mathrm{No}^{f}$ \\
\hline Isopropylbenzene & 0 of 5 & ND (1.0) & ND (1.0) & NAN & NAV & $\mathrm{No}^{\dagger}$ \\
\hline $\mathrm{m}, \mathrm{p}$-Xylene & 0 of 5 & ND (2.0) & $\mathrm{ND}(2.0)$ & NAN & $12,000^{\mathrm{e}}$ & $\mathrm{No}^{\dagger}$ \\
\hline Methylene chloride & 0 of 5 & ND (4.64) & ND (10.3) & NAN & $4.7^{c}$ & $\mathrm{No}^{\dagger}$ \\
\hline n-Butylbenzene & 0 of 5 & ND (1.0) & ND (1.0) & NAN & $61^{e}$ & $\mathrm{No}^{f}$ \\
\hline n-Propylbenzene & 0 of 5 & ND (1.0) & ND (1.0) & NAN & $61^{e}$ & $\mathrm{No}^{\dagger}$ \\
\hline Naphthalene & 0 of 5 & ND (1.0) & ND (1.0) & NAN & $730^{e}$ & $\mathrm{No}^{\dagger}$ \\
\hline o-Xylene & 0 of 5 & ND (1.0) & ND (1.0) & NAN & $12,000^{\mathrm{e}}$ & $\mathrm{No}^{\dagger}$ \\
\hline p-Isopropyltoluene & 0 of 5 & ND (1.0) & ND (1.0) & NAN & NAV & $\mathrm{No}^{f}$ \\
\hline sec-Butylbenzene & 0 of 5 & ND (1.0) & ND (1.0) & NAN & $61^{e}$ & $\mathrm{No}^{\dagger}$ \\
\hline Toluene & 0 of 5 & ND (1.0) & ND (1.0) & NAN & $10,000^{c}$ & $\mathrm{No}^{\dagger}$ \\
\hline \multicolumn{7}{|c|}{ Polycyclic Aromatic Hydrocarbons } \\
\hline Acenaphthene & 0 of 5 & ND (0.1) & $\mathrm{ND}(0.1)$ & NAN & $1,200^{c}$ & $\mathrm{No}^{f}$ \\
\hline Acenaphthylene & 0 of 5 & $\mathrm{ND}(0.1)$ & $\mathrm{ND}(0.1)$ & NAN & $0.0028^{c}$ & $\mathrm{No}^{\dagger}$ \\
\hline Anthracene & 0 of 5 & $\mathrm{ND}(0.1)$ & $\mathrm{ND}(0.1)$ & NAN & $0.0028^{c}$ & $\mathrm{No}^{\dagger}$ \\
\hline Benzo(a)anthracene & 0 of 5 & ND $(0.1)$ & ND $(0.1)$ & NAN & $0.0028^{c}$ & $\mathrm{No}^{f}$ \\
\hline Benzo(a)pyrene & 0 of 5 & $\mathrm{ND}(0.1)$ & $\mathrm{ND}(0.1)$ & NAN & $0.0028^{c}$ & $\mathrm{No}^{\dagger}$ \\
\hline Benzo(b)fluoranthene & 0 of 5 & ND $(0.1)$ & $\mathrm{ND}(0.1)$ & NAN & $0.092^{\mathrm{e}}$ & $\mathrm{No}^{f}$ \\
\hline Benzo(ghi)perylene & 0 of 5 & ND $(0.1)$ & $\mathrm{ND}(0.1)$ & NAN & $0.0028^{c}$ & $\mathrm{No}^{f}$ \\
\hline Benzo(k)fluoranthene & 0 of 5 & ND $(0.1)$ & $\mathrm{ND}(0.1)$ & NAN & $0.0028^{\mathrm{C}}$ & $\mathrm{No}^{\dagger}$ \\
\hline
\end{tabular}


Table 4-20

Summary of Analytical Results and Human Health Risk Assessment Screening for Surface Water in Rainbow Creek, Long Shot Site

(Page 2 of 3 )

\begin{tabular}{|c|c|c|c|c|c|c|}
\hline Analyte & $\begin{array}{l}\text { Detection } \\
\text { Frequency }\end{array}$ & $\begin{array}{c}\text { Minimum } \\
\text { Detected } \\
\text { Concentration } \\
(\mu \mathrm{g} / \mathrm{L})\end{array}$ & $\begin{array}{c}\text { Maximum } \\
\text { Detected } \\
\text { Concentration } \\
(\mu \mathrm{g} / \mathrm{L})\end{array}$ & $\begin{array}{c}\text { Lowland } \\
\text { Stream } \\
\text { Background } \\
\text { Concentration }{ }^{\mathrm{a}} \\
(\mu \mathrm{g} / \mathrm{L})\end{array}$ & $\begin{array}{l}\text { Human Health } \\
\text { Screening } \\
\text { Value } \\
(\mu \mathrm{g} / \mathrm{L})\end{array}$ & $\begin{array}{l}\text { Human Health } \\
\text { Constituent of } \\
\text { Concern }\end{array}$ \\
\hline Chrysene & 0 of 5 & $\mathrm{ND}(0.1)$ & $\mathrm{ND}(0.1)$ & NAN & $0.0028^{c}$ & $\mathrm{No}^{f}$ \\
\hline Dibenzo(a,h)anthracene & 0 of 5 & $\mathrm{ND}(0.1)$ & $\mathrm{ND}(0.1)$ & NAN & $0.0028^{c}$ & $\mathrm{No}^{\dagger}$ \\
\hline Fluoranthene & 0 of 5 & ND $(0.1)$ & $\mathrm{ND}(0.1)$ & NAN & $42^{c}$ & $\mathrm{No}^{\dagger}$ \\
\hline Fluorene & 0 of 5 & $\mathrm{ND}(0.1)$ & $\mathrm{ND}(0.1)$ & NAN & $0.0028^{c}$ & $\mathrm{No}^{\dagger}$ \\
\hline Indeno(1,2,3-cd)pyrene & 0 of 5 & $\mathrm{ND}(0.1)$ & $\mathrm{ND}(0.1)$ & NAN & $0.0028^{C}$ & $\mathrm{No}^{\dagger}$ \\
\hline Naphthalene & 0 of 5 & $\mathrm{ND}(0.1)$ & $\mathrm{ND}(0.1)$ & NAN & $730^{e}$ & $\mathrm{No}^{f}$ \\
\hline Phenanthrene & 0 of 5 & $\mathrm{ND}(0.1)$ & $\mathrm{ND}(0.1)$ & NAN & $0.0028^{c}$ & $\mathrm{No}^{\dagger}$ \\
\hline Pyrene & 0 of 5 & $\mathrm{ND}(0.1)$ & $\mathrm{ND}(0.1)$ & NAN & $0.0028^{c}$ & $\mathrm{No}^{\dagger}$ \\
\hline \multicolumn{7}{|c|}{ Polychlorinated Biphenyls } \\
\hline Aroclor 1016 & NAV & NAV & NAV & NAN & $0.000044^{c}$ & $\mathrm{No}^{\dagger}$ \\
\hline Aroclor 1221 & NAV & NAV & NAV & NAN & $0.000044^{c}$ & $\mathrm{No}^{f}$ \\
\hline Aroclor 1232 & NAV & NAV & NAV & NAN & $0.000044^{c}$ & $\mathrm{No}^{\dagger}$ \\
\hline Aroclor 1242 & NAV & NAV & NAV & NAN & $0.000044^{c}$ & $\mathrm{No}^{f}$ \\
\hline Aroclor 1248 & NAV & NAV & NAV & NAN & $0.000044^{c}$ & $\mathrm{No}^{\dagger}$ \\
\hline Aroclor 1254 & NAV & NAV & NAV & NAN & $0.000044^{c}$ & $\mathrm{No}^{\dagger}$ \\
\hline Aroclor 1260 & NAV & NAV & NAV & NAN & $0.000044^{c}$ & $\mathrm{No}^{f}$ \\
\hline Aroclor 1262 & NAV & NAV & NAV & NAN & $0.000044^{c}$ & $\mathrm{No}^{\dagger}$ \\
\hline Aroclor 1268 & NAV & NAV & NAV & NAN & $0.000044^{c}$ & $\mathrm{No}^{\dagger}$ \\
\hline \multicolumn{7}{|c|}{ Metals } \\
\hline Aluminum & 0 of 5 & ND (50) & ND (50) & 269 & $37,000^{\mathrm{e}}$ & $\mathrm{No}^{f}$ \\
\hline Arsenic & 0 of 5 & ND (1.0) & ND (1.0) & ND (1) & $0.018^{c}$ & $\mathrm{No}^{\dagger}$ \\
\hline Barium & 0 of 5 & ND (10) & ND (10) & ND (10) & $1,000^{c}$ & $\mathrm{No}^{f}$ \\
\hline Beryllium & 0 of 5 & ND (1.0) & ND (1.0) & ND (1) & $0.0076^{c}$ & $\mathrm{No}^{\dagger}$ \\
\hline Boron & 0 of 5 & ND (50) & ND (50) & ND (50) & $3,300^{\mathrm{e}}$ & $\mathrm{No}^{\dagger}$ \\
\hline Cadmium & 0 of 5 & ND (1.0) & ND (1.0) & ND (1) & $10^{c}$ & $\mathrm{No}^{\dagger}$ \\
\hline Calcium & 5 of 5 & 5,530 & 8,220 & $4,320 \mathrm{~b}$ & NAV & $\mathrm{No}^{\mathrm{i}}$ \\
\hline Cerium & 0 of 5 & ND (1.0) & ND (1.0) & ND (1) & NAV & $\mathrm{No}^{f}$ \\
\hline Cesium & 0 of 5 & ND (1.0) & ND (1.0) & ND (1) & NAV & $\mathrm{No}^{\dagger}$ \\
\hline
\end{tabular}


Table 4-20

\section{Summary of Analytical Results and Human Health Risk Assessment Screening for Surface Water in Rainbow Creek, Long Shot Site}

(Page 3 of 3 )

\begin{tabular}{|c|c|c|c|c|c|c|}
\hline Analyte & $\begin{array}{l}\text { Detection } \\
\text { Frequency }\end{array}$ & $\begin{array}{c}\text { Minimum } \\
\text { Detected } \\
\text { Concentration } \\
(\mu \mathrm{g} / \mathrm{L})\end{array}$ & $\begin{array}{c}\text { Maximum } \\
\text { Detected } \\
\text { Concentration } \\
(\mu \mathrm{g} / \mathrm{L})\end{array}$ & $\begin{array}{c}\text { Lowland } \\
\text { Stream } \\
\text { Background } \\
\text { Concentration }{ }^{\mathrm{a}} \\
(\mu \mathrm{g} / \mathrm{L})\end{array}$ & $\begin{array}{l}\text { Human Health } \\
\text { Screening } \\
\text { Value } \\
(\mu \mathrm{g} / \mathrm{L})\end{array}$ & $\begin{array}{c}\text { Human Health } \\
\text { Constituent of } \\
\text { Concern }\end{array}$ \\
\hline Chromium & 0 of 5 & ND (1.0) & ND (1.0) & $2.25^{b}$ & $33,000^{c}$ & $\mathrm{No}^{\dagger}$ \\
\hline Cobalt & 2 of 5 & ND (1.0) & 2.48 & 3.35 & $2,200^{\mathrm{e}}$ & $\mathrm{No}^{\mathrm{g}}$ \\
\hline Copper & 3 of 5 & ND (1.0) & 17.7 & 10.8 & $1,300^{C}$ & $\mathrm{No}^{h}$ \\
\hline Iron & 4 of 5 & ND (150) & 321 & 993 & $300^{c}$ & $\mathrm{No}^{\mathrm{g}}$ \\
\hline Lithium & 0 of 5 & ND (1.0) & ND (1.0) & $1.17^{\mathrm{b}}$ & $730^{e}$ & $\mathrm{No}^{\dagger}$ \\
\hline Magnesium & 5 of 5 & 4,160 & 5,350 & $4,690^{b}$ & NAV & $\mathrm{No}^{\mathrm{i}}$ \\
\hline Manganese & 5 of 5 & 7.74 & 44.4 & 267 & $50^{c}$ & $\mathrm{No}^{\mathrm{g}}$ \\
\hline Molybdenum & 0 of 5 & ND (1.0) & ND (1.0) & ND (1) & $180^{e}$ & $\mathrm{No}^{\dagger}$ \\
\hline Nickel & 0 of 5 & ND (1.0) & ND (1.0) & ND (1) & $510^{c}$ & $\mathrm{No}^{\dagger}$ \\
\hline Potassium & 5 of 5 & 898 & 1,270 & 2,620 & NAV & $\mathrm{No}^{\mathrm{g}}$ \\
\hline Selenium & 0 of 5 & ND (1.0) & ND (1.0) & ND (1) & $104^{\mathrm{C}}$ & $\mathrm{No}^{\dagger}$ \\
\hline Strontium & 5 of 5 & 35 & 58.3 & 36.1 & $22,000^{\mathrm{e}}$ & $\mathrm{No}^{\mathrm{h}}$ \\
\hline Thallium & 0 of 5 & ND (1.0) & ND (1.0) & $7.07^{b}$ & $1.7^{\mathrm{C}}$ & $\mathrm{No}^{f}$ \\
\hline Thorium & 0 of 5 & ND (1.0) & ND (1.0) & ND (1) & NAV & $\mathrm{No}^{f}$ \\
\hline Titanium & 5 of 5 & 1.09 & 1.94 & 6.13 & $150,000^{e}$ & $\mathrm{No}^{\mathrm{g}}$ \\
\hline Uranium & 0 of 5 & ND (1.0) & ND (1.0) & ND (1) & $110^{e}$ & $\mathrm{No}^{\dagger}$ \\
\hline Vanadium & 1 of 5 & ND (1.0) & 2.12 & $4.43^{b}$ & $260^{\mathrm{e}}$ & $\mathrm{No}^{\mathrm{g}}$ \\
\hline Zinc & 1 of 5 & ND (10) & 23.3 & $50.3^{b}$ & $11,000^{\mathrm{e}}$ & $\mathrm{No}^{\mathrm{g}}$ \\
\hline
\end{tabular}

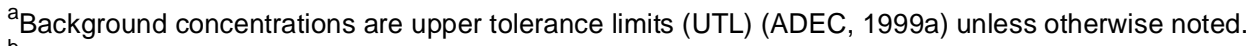

${ }^{\mathrm{b}}$ Maximum detected concentration in background samples due to insufficient detection frequency to calculate UTL.

${ }^{c}$ Federal ambient water quality criteria based on ingestion of water and organisms.

${ }^{\mathrm{d}}$ State of Alaska groundwater cleanup level, Discharge Reporting, Cleanup, and Disposal of Oil and other Hazardous Substances

(18 AAC 75, Articles 3 and 9 [ADEC, 1999c]).

eU.S. EPA Region III risk-based concentrations for tap water (EPA, 2000b).

\section{COC Selection Criteria}

Constituent not detected in any samples.

${ }^{9}$ Maximum detected constituent concentration is less than local background concentration.

${ }^{h}$ Maximum detected constituent concentration is less than human health screening value.

i'Constituent is an essential macronutrient and only presents a risk at extremely high concentrations.

ND - Not detected (detection limit)

NAV - Not available

NAN - Not analyzed

$\mu \mathrm{g} / \mathrm{L}$ - Micrograms per liter 
Table 4-21

Summary of Analytical Results and Human Health Risk Assessment Screening for Sediment in Rainbow Creek, Long Shot Site

(Page 1 of 3 )

\begin{tabular}{|c|c|c|c|c|c|c|}
\hline Analyte & $\begin{array}{l}\text { Detection } \\
\text { Frequency }\end{array}$ & $\begin{array}{c}\text { Minimum } \\
\text { Detected } \\
\text { Concentration } \\
(\mathrm{mg} / \mathrm{kg})\end{array}$ & $\begin{array}{c}\text { Maximum } \\
\text { Detected } \\
\text { Concentration } \\
(\mathrm{mg} / \mathrm{kg})\end{array}$ & $\begin{array}{c}\text { Lowland } \\
\text { Stream } \\
\text { Background } \\
\text { Concentration } \\
(\mathbf{m g} / \mathbf{k g})\end{array}$ & $\begin{array}{l}\text { Human Health } \\
\text { Screening } \\
\text { Value } \\
(\mathrm{mg} / \mathrm{kg})\end{array}$ & $\begin{array}{c}\text { Human Health } \\
\text { Constituent of } \\
\text { Concern }\end{array}$ \\
\hline $\begin{array}{l}\text { Diesel-Range } \\
\text { Hydrocarbons }\end{array}$ & 4 of 5 & ND (19.9) & 2,110 & NAN & $200^{c}$ & Yes \\
\hline \multicolumn{7}{|c|}{ Volatile Organic Compounds } \\
\hline 1,2,4-Trimethylbenzene & NAV & NAV & NAV & NAN & NAV & $\mathrm{No}^{d}$ \\
\hline 1,3,5-Trimethylbenzene & NAV & NAV & NAV & NAN & NAV & $\mathrm{No}^{\mathrm{d}}$ \\
\hline 2-Butanone & NAV & NAV & NAV & NAN & NAV & $\mathrm{No}^{d}$ \\
\hline Acetone & NAV & NAV & NAV & NAN & $10,100^{c}$ & $\mathrm{No}^{\mathrm{d}}$ \\
\hline Benzene & NAV & NAV & NAV & NAN & $286^{\mathrm{C}}$ & $\mathrm{No}^{\mathrm{d}}$ \\
\hline Carbon disulfide & NAV & NAV & NAV & NAN & $10,100^{c}$ & $\mathrm{No}^{\mathrm{d}}$ \\
\hline Ethylbenzene & NAV & NAV & NAV & NAN & $10,100^{c}$ & $\mathrm{No}^{\mathrm{d}}$ \\
\hline Isopropylbenzene & NAV & NAV & NAV & NAN & NAV & $\mathrm{No}^{d}$ \\
\hline $\mathrm{m}, \mathrm{p}$-Xylene & NAV & NAV & NAV & NAN & $203,000^{C}$ & $\mathrm{No}^{\mathrm{d}}$ \\
\hline Methylene chloride & NAV & NAV & NAV & NAN & $1,110^{\mathrm{C}}$ & $\mathrm{No}^{d}$ \\
\hline n-Butylbenzene & NAV & NAV & NAV & NAN & NAV & $\mathrm{No}^{\mathrm{d}}$ \\
\hline n-Propylbenzene & NAV & NAV & NAV & NAN & NAV & $\mathrm{No}^{d}$ \\
\hline Naphthalene & NAV & NAV & NAV & NAN & $4,060^{\mathrm{C}}$ & $\mathrm{No}^{d}$ \\
\hline o-Xylene & NAV & NAV & NAV & NAN & $203,000^{\mathrm{C}}$ & $\mathrm{No}^{d}$ \\
\hline p-Isopropyltoluene & NAV & NAV & NAV & NAN & NAV & $\mathrm{No}^{d}$ \\
\hline sec-Butylbenzene & NAV & NAV & NAV & NAN & NAV & $\mathrm{No}^{d}$ \\
\hline Toluene & NAV & NAV & NAV & NAN & $20,300^{c}$ & $\mathrm{No}^{d}$ \\
\hline \multicolumn{7}{|c|}{ Polycyclic Aromatic Hydrocarbons } \\
\hline Acenaphthene & 0 of 5 & ND (0.0164) & ND (0.0454) & NAN & $6,080^{\mathrm{C}}$ & $\mathrm{No}^{d}$ \\
\hline Acenaphthylene & 0 of 5 & ND (0.0164) & ND (0.0454) & NAN & NAV & $\mathrm{No}^{d}$ \\
\hline Anthracene & 0 of 5 & ND $(0.0164)$ & ND $(0.0454)$ & NAN & $30,400^{c}$ & $\mathrm{No}^{\mathrm{d}}$ \\
\hline Benzo(a)anthracene & 0 of 5 & ND (0.0164) & ND (0.0454) & NAN & $11.4^{\mathrm{C}}$ & $\mathrm{No}^{d}$ \\
\hline Benzo(a)pyrene & 0 of 5 & ND (0.0164) & ND (0.0454) & NAN & $1.1^{\mathrm{C}}$ & $\mathrm{No}^{\mathrm{d}}$ \\
\hline Benzo(b)fluoranthene & 0 of 5 & ND (0.0164) & ND $(0.0454)$ & NAN & $11.4^{\mathrm{C}}$ & $\mathrm{No}^{d}$ \\
\hline Benzo(ghi)perylene & 0 of 5 & ND (0.0164) & ND (0.0454) & NAN & $1.1^{\mathrm{c}}$ & $\mathrm{No}^{\mathrm{d}}$ \\
\hline Benzo(k)fluoranthene & 0 of 5 & ND $(0.0164)$ & ND (0.0454) & NAN & $114^{\mathrm{C}}$ & $\mathrm{No}^{d}$ \\
\hline Chrysene & 0 of 5 & ND (0.0164) & ND (0.0454) & NAN & $1140^{c}$ & $\mathrm{No}^{\mathrm{d}}$ \\
\hline
\end{tabular}


Table 4-21

Summary of Analytical Results and Human Health Risk Assessment Screening for Sediment in Rainbow Creek, Long Shot Site

(Page 2 of 3)

\begin{tabular}{|c|c|c|c|c|c|c|}
\hline Analyte & $\begin{array}{l}\text { Detection } \\
\text { Frequency }\end{array}$ & $\begin{array}{c}\text { Minimum } \\
\text { Detected } \\
\text { Concentration } \\
(\mathbf{m g} / \mathrm{kg})\end{array}$ & $\begin{array}{c}\text { Maximum } \\
\text { Detected } \\
\text { Concentration } \\
(\mathrm{mg} / \mathrm{kg})\end{array}$ & $\begin{array}{c}\text { Lowland } \\
\text { Stream } \\
\text { Background } \\
\text { Concentration }{ }^{\mathrm{a}} \\
(\mathbf{m g} / \mathbf{k g})\end{array}$ & $\begin{array}{l}\text { Human Health } \\
\text { Screening } \\
\text { Value } \\
(\mathrm{mg} / \mathrm{kg})\end{array}$ & $\begin{array}{c}\text { Human Health } \\
\text { Constituent of } \\
\text { Concern }\end{array}$ \\
\hline Dibenzo(a,h)anthracene & 0 of 5 & ND (0.0164) & ND (0.0454) & NAN & $1.1^{\mathrm{C}}$ & $\mathrm{No}^{\mathrm{d}}$ \\
\hline Fluoranthene & 0 of 5 & ND $(0.0164)$ & ND (0.0454) & NAN & $4,060^{C}$ & $\mathrm{No}^{\mathrm{d}}$ \\
\hline Fluorene & 0 of 5 & ND (0.0164) & ND (0.0454) & NAN & $4,060^{\mathrm{C}}$ & $\mathrm{No}^{\mathrm{d}}$ \\
\hline Indeno(1,2,3-cd)pyrene & 0 of 5 & ND (0.0164) & ND (0.0454) & NAN & $11^{\mathrm{c}}$ & $\mathrm{No}^{\mathrm{d}}$ \\
\hline Naphthalene & 0 of 5 & ND (0.0164) & ND (0.0454) & NAN & $4,060^{C}$ & $\mathrm{No}^{\mathrm{d}}$ \\
\hline Phenanthrene & 0 of 5 & ND $(0.0164)$ & ND $(0.0454)$ & NAN & $1.1^{\mathrm{c}}$ & $\mathrm{No}^{\mathrm{d}}$ \\
\hline Pyrene & 0 of 5 & ND $(0.0164)$ & ND (0.0454) & NAN & $3,040^{c}$ & Nod \\
\hline \multicolumn{7}{|c|}{ Polychlorinated Biphenyls } \\
\hline Aroclor 1016 & NAV & NAV & NAV & NAN & $10^{\mathrm{C}}$ & $\mathrm{No}^{\mathrm{d}}$ \\
\hline Aroclor 1221 & NAV & NAV & NAV & NAN & $10^{\mathrm{C}}$ & $\mathrm{No}^{\mathrm{d}}$ \\
\hline Aroclor 1232 & NAV & NAV & NAV & NAN & $10^{c}$ & $\mathrm{No}^{\mathrm{d}}$ \\
\hline Aroclor 1242 & NAV & NAV & NAV & NAN & $10^{c}$ & $\mathrm{No}^{d}$ \\
\hline Aroclor 1248 & NAV & NAV & NAV & NAN & $10^{c}$ & $\mathrm{No}^{\mathrm{d}}$ \\
\hline Aroclor 1254 & NAV & NAV & NAV & NAN & $10^{\mathrm{C}}$ & $\mathrm{No}^{d}$ \\
\hline Aroclor 1260 & 1 of 2 & ND (0.056) & 0.46 & NAN & $10^{c}$ & $\mathrm{No}^{f}$ \\
\hline Aroclor 1262 & NAV & NAV & NAV & NAN & $10^{\mathrm{C}}$ & $\mathrm{No}^{d}$ \\
\hline Aroclor 1268 & NAV & NAV & NAV & NAN & $10^{c}$ & $\mathrm{No}^{d}$ \\
\hline \multicolumn{7}{|c|}{ Metals } \\
\hline Aluminum & 5 of 5 & 3,990 & 35,400 & 45,400 & NAV & $\mathrm{No}^{\mathrm{e}}$ \\
\hline Arsenic & 3 of 5 & ND (3.67) & 32.1 & 100 & $5.5^{\mathrm{C}}$ & $\mathrm{No}^{\mathrm{e}}$ \\
\hline Barium & 4 of 5 & ND (68.3) & 266 & 469 & $7,100^{c}$ & $\mathrm{No}^{\mathrm{e}}$ \\
\hline Beryllium & 0 of 5 & ND (2.48) & ND (6.87) & ND & $1.9^{\mathrm{C}}$ & $\mathrm{No}^{\mathrm{d}}$ \\
\hline Boron & 1 of 5 & ND (18.3) & 18.2 & $7.18^{b}$ & NAV & $\mathrm{No}^{h}$ \\
\hline Cadmium & 0 of 5 & ND (2.48) & ND (6.87) & $0.608^{b}$ & $101^{c}$ & $\mathrm{No}^{d}$ \\
\hline Calcium & 5 of 5 & 2,880 & 7,300 & 7,300 & NAV & $\mathrm{No}^{\mathrm{e}}$ \\
\hline Cerium & 4 of 5 & ND (6.83) & 13.1 & 14.2 & NAV & $\mathrm{No}^{\mathrm{e}}$ \\
\hline Cesium & 0 of 5 & ND (2.48) & ND (6.87) & ND & NAV & $\mathrm{No}^{d}$ \\
\hline Chromium & 4 of 5 & ND (6.83) & 38.8 & 11.8 & $507^{c}$ & $\mathrm{No}^{\dagger}$ \\
\hline Cobalt & 3 of 5 & ND (6.83) & 28 & 43.7 & NAV & $\mathrm{No}^{\mathrm{e}}$ \\
\hline
\end{tabular}


Table 4-21

\section{Summary of Analytical Results and Human Health Risk Assessment Screening for Sediment in Rainbow Creek, Long Shot Site}

(Page 3 of 3 )

\begin{tabular}{|c|c|c|c|c|c|c|}
\hline Analyte & $\begin{array}{l}\text { Detection } \\
\text { Frequency }\end{array}$ & $\begin{array}{c}\text { Minimum } \\
\text { Detected } \\
\text { Concentration } \\
(\mathrm{mg} / \mathrm{kg})\end{array}$ & $\begin{array}{c}\text { Maximum } \\
\text { Detected } \\
\text { Concentration } \\
(\mathrm{mg} / \mathrm{kg})\end{array}$ & $\begin{array}{c}\text { Lowland } \\
\text { Stream } \\
\text { Background } \\
\text { Concentration }^{\mathrm{a}} \\
(\mathrm{mg} / \mathrm{kg})\end{array}$ & $\begin{array}{c}\text { Human Health } \\
\text { Screening } \\
\text { Value } \\
(\mathrm{mg} / \mathrm{kg})\end{array}$ & $\begin{array}{c}\text { Human Health } \\
\text { Constituent of } \\
\text { Concern }\end{array}$ \\
\hline Copper & 5 of 5 & 13 & 61.4 & 71.1 & NAV & $\mathrm{No}^{\mathrm{e}}$ \\
\hline Iron & 5 of 5 & 8,070 & 192,000 & 155,000 & NAV & $\mathrm{No}^{\mathrm{g}}$ \\
\hline Lithium & 2 of 5 & ND (5.13) & 9.52 & 48.8 & NAV & $\mathrm{No}^{\mathrm{e}}$ \\
\hline Magnesium & 5 of 5 & 1,890 & 7,650 & 9,320 & NAV & $\mathrm{No}^{\mathrm{e}}$ \\
\hline Manganese & 5 of 5 & 196 & 14,700 & 20,700 & NAV & $\mathrm{No}^{\mathrm{e}}$ \\
\hline Molybdenum & 0 of 5 & ND (2.48) & ND (6.87) & $5.5^{b}$ & NAV & $\mathrm{No}^{d}$ \\
\hline Nickel & 3 of 5 & ND (6.83) & 9.66 & 13.6 & $2,030^{C}$ & $\mathrm{No}^{\mathrm{e}}$ \\
\hline Potassium & 5 of 5 & 324 & 748 & 1,390 & NAV & $\mathrm{No}^{\mathrm{e}}$ \\
\hline Selenium & 0 of 5 & ND (2.48) & ND (6.87) & $5.44^{b}$ & $507^{C}$ & $\mathrm{No}^{\mathrm{d}}$ \\
\hline Strontium & 5 of 5 & 59.5 & 94.1 & 164 & NAV & $\mathrm{No}^{\mathrm{e}}$ \\
\hline Thallium & 0 of 5 & ND (2.48) & ND (6.87) & $1^{b}$ & NAV & $\mathrm{No}^{d}$ \\
\hline Thorium & 0 of 5 & ND (2.48) & ND (6.87) & $0.5^{b}$ & NAV & $\mathrm{No}^{d}$ \\
\hline Titanium & 5 of 5 & 285 & 1,550 & 3,010 & NAV & $\mathrm{No}^{\mathrm{e}}$ \\
\hline Uranium & 0 of 5 & ND (2.48) & ND (6.87) & $4.13^{b}$ & NAV & $\mathrm{No}^{\mathrm{d}}$ \\
\hline Vanadium & 5 of 5 & 54.7 & 296 & 734 & $710^{c}$ & $\mathrm{No}^{\mathrm{e}}$ \\
\hline Zinc & 2 of 5 & ND (36.7) & 142 & 434 & $30,400^{c}$ & $\mathrm{No}^{\mathrm{e}}$ \\
\hline Total Organic Carbon & 5 of 5 & 63,300 & 284,000 & NAN & NAV & $\mathrm{No}^{h}$ \\
\hline
\end{tabular}

${ }^{\mathrm{a}}$ Background concentrations are upper tolerance limits (UTL) (ADEC, 1999a) unless otherwise noted.

${ }^{b}$ Maximum detected concentration in background samples due to insufficient detection frequency to calculate UTL.

'State of Alaska soil cleanup level, Discharge Reporting, Cleanup, and Disposal of Oil and other Hazardous Substances

(18 AAC 75, Articles 3 and 9 [ADEC, 1999c]).

\section{COC Selection Criteria}

${ }^{\mathrm{d}}$ Constituent not detected in any samples.

e Maximum detected constituent concentration is less than local background concentration.

${ }^{f}$ Maximum detected constituent concentration is less than human health screening value.

${ }^{9}$ Constituent is an essential macronutrient and only presents a risk at extremely high concentrations.

${ }^{\mathrm{h}}$ Not a constituent associated with drilling mud or other drilling activities.

ND - Not detected (detection limit)

NAV - Not available

NAN - Not analyzed

$\mathrm{mg} / \mathrm{kg}$ - Milligrams per kilogram 
Table 4-22

Summary of Analytical Results and Human Health Risk Assessment Screening for Fish Tissue in Rainbow Creek, Long Shot Site

(Page 1 of 2)

\begin{tabular}{|c|c|c|c|c|c|c|}
\hline Analyte & $\begin{array}{l}\text { Detection } \\
\text { Frequency }\end{array}$ & $\begin{array}{c}\text { Minimum } \\
\text { Detected } \\
\text { Concentration } \\
(\mathrm{mg} / \mathrm{kg})\end{array}$ & $\begin{array}{c}\text { Maximum } \\
\text { Detected } \\
\text { Concentration } \\
(\mathrm{mg} / \mathrm{kg})\end{array}$ & $\begin{array}{c}\text { Lowland } \\
\text { Stream } \\
\text { Background } \\
\text { Concentration }{ }^{\mathrm{a}} \\
(\mathrm{mg} / \mathrm{kg})\end{array}$ & $\begin{array}{c}\text { Human Health } \\
\text { Screening } \\
\text { Value }^{c} \\
(\mathrm{mg} / \mathrm{kg})\end{array}$ & $\begin{array}{c}\text { Human Health } \\
\text { Constituent of } \\
\text { Concern }\end{array}$ \\
\hline \multicolumn{7}{|c|}{ Polycyclic Aromatic Hydrocarbons } \\
\hline Acenaphthene & 0 of 1 & ND (0.0335) & ND (0.0335) & NAN & 81.1 & $\mathrm{No}^{d}$ \\
\hline Acenaphthylene & 0 of 1 & ND (0.0335) & ND (0.0335) & NAN & NAV & $\mathrm{No}^{d}$ \\
\hline Anthracene & 0 of 1 & ND (0.0335) & ND (0.0335) & NAN & 410 & $\mathrm{No}^{d}$ \\
\hline Benzo(a)anthracene & 0 of 1 & ND (0.0335) & ND (0.0335) & NAN & 0.0043 & $\mathrm{No}^{d}$ \\
\hline Benzo(a)pyrene & 0 of 1 & ND (0.0335) & ND (0.0335) & NAN & 0.00043 & $\mathrm{No}^{d}$ \\
\hline Benzo(b)fluoranthene & 0 of 1 & ND (0.0335) & ND (0.0335) & NAN & 0.0043 & $\mathrm{No}^{d}$ \\
\hline Benzo(ghi)perylene & 0 of 1 & ND (0.0335) & ND (0.0335) & NAN & NAV & $\mathrm{No}^{d}$ \\
\hline Benzo(k)fluoranthene & 0 of 1 & ND (0.0335) & ND (0.0335) & NAN & 0.043 & $\mathrm{No}^{d}$ \\
\hline Chrysene & 0 of 1 & ND (0.0335) & ND (0.0335) & NAN & 0.43 & $\mathrm{No}^{d}$ \\
\hline Dibenzo(a,h)anthracene & 0 of 1 & ND (0.0335) & ND (0.0335) & NAN & 0.00043 & $\mathrm{No}^{d}$ \\
\hline Fluoranthene & 0 of 1 & ND (0.0335) & ND (0.0335) & NAN & 54 & $\mathrm{No}^{d}$ \\
\hline Fluorene & 0 of 1 & ND (0.0335) & ND (0.0335) & NAN & 54 & $\mathrm{No}^{\mathrm{d}}$ \\
\hline Indeno(1,2,3-cd)pyrene & 0 of 1 & ND (0.0335) & ND (0.0335) & NAN & 0.0043 & $\mathrm{No}^{d}$ \\
\hline Naphthalene & 0 of 1 & ND (0.0335) & ND (0.0335) & NAN & 27 & $\mathrm{No}^{d}$ \\
\hline Phenanthrene & 0 of 1 & ND (0.0335) & ND (0.0335) & NAN & NAV & $\mathrm{No}^{\mathrm{d}}$ \\
\hline Pyrene & 0 of 1 & ND (0.0335) & ND (0.0335) & NAN & 41 & $\mathrm{No}^{\mathrm{d}}$ \\
\hline \multicolumn{7}{|c|}{ Polychlorinated Biphenyls } \\
\hline Aroclor 1016 & 0 of 1 & ND (0.01) & ND (0.01) & NAN & 0.045 & $\mathrm{No}^{d}$ \\
\hline Aroclor 1221 & 0 of 1 & ND (0.02) & ND (0.02) & NAN & 0.0016 & $\mathrm{No}^{d}$ \\
\hline Aroclor 1232 & 0 of 1 & ND (0.01) & ND (0.01) & NAN & 0.0016 & $\mathrm{No}^{d}$ \\
\hline Aroclor 1242 & 0 of 1 & ND (0.01) & ND (0.01) & NAN & 0.0016 & $\mathrm{No}^{d}$ \\
\hline Aroclor 1248 & 0 of 1 & ND $(0.01)$ & ND (0.01) & NAN & 0.0016 & $\mathrm{No}^{d}$ \\
\hline Aroclor 1254 & 0 of 1 & ND (0.01) & ND (0.01) & NAN & 0.0016 & $\mathrm{No}^{d}$ \\
\hline Aroclor 1260 & 0 of 1 & 0.0161 & 0.0161 & NAN & 0.0016 & Yes \\
\hline \multicolumn{7}{|c|}{ Metals } \\
\hline Aluminum & 2 of 2 & 13.5 & 32.3 & 475 & 1,400 & $\mathrm{No}^{\mathrm{e}}$ \\
\hline Arsenic & 1 of 2 & ND (0.025) & 0.049 & 0.127 & 0.0021 & $\mathrm{No}^{\mathrm{e}}$ \\
\hline Inorganic Arsenic & 2 of 2 & 0.00521 & 0.0236 & 0.0208 & 0.0021 & $\mathrm{No}^{\dagger}$ \\
\hline Barium & 2 of 2 & 0.158 & 0.233 & 1.03 & 95 & $\mathrm{No}^{\mathrm{e}}$ \\
\hline Beryllium & 0 of 2 & ND (0.025) & ND (0.025) & ND & 2.7 & $\mathrm{No}^{\mathrm{d}}$ \\
\hline Boron & 0 of 2 & ND (10.3) & ND (21.5) & 28.6 & 120 & $\mathrm{No}^{d}$ \\
\hline
\end{tabular}


Table 4-22

Summary of Analytical Results and Human Health Risk Assessment
Screening for Fish Tissue in Rainbow Creek, Long Shot Site (Page 2 of 2)

\begin{tabular}{|c|c|c|c|c|c|c|}
\hline Analyte & $\begin{array}{l}\text { Detection } \\
\text { Frequency }\end{array}$ & $\begin{array}{c}\text { Minimum } \\
\text { Detected } \\
\text { Concentration } \\
(\mathrm{mg} / \mathrm{kg})\end{array}$ & $\begin{array}{c}\text { Maximum } \\
\text { Detected } \\
\text { Concentration } \\
(\mathrm{mg} / \mathrm{kg})\end{array}$ & $\begin{array}{l}\text { Lowland } \\
\text { Stream } \\
\text { Background } \\
\text { Concentration }{ }^{\mathrm{a}} \\
(\mathrm{mg} / \mathrm{kg})\end{array}$ & $\begin{array}{l}\text { Human Health } \\
\text { Screening } \\
\text { Value }^{c} \\
(\mathrm{mg} / \mathrm{kg})\end{array}$ & $\begin{array}{c}\text { Human Health } \\
\text { Constituent of } \\
\text { Concern }\end{array}$ \\
\hline Cadmium & 0 of 2 & ND (0.025) & ND (0.025) & $0.0373^{b}$ & 1.4 & $\mathrm{No}^{\mathrm{d}}$ \\
\hline Calcium & 2 of 2 & 4,130 & 6,950 & 19,200 & NAV & $\mathrm{No}^{\mathrm{e}}$ \\
\hline Cerium & 0 of 2 & ND (0.025) & ND (0.025) & $0.0280^{b}$ & NAV & $\mathrm{No}^{\mathrm{d}}$ \\
\hline Cesium & 0 of 2 & ND (0.025) & ND (0.025) & $0.0258^{b}$ & NAV & $\mathrm{No}^{\mathrm{d}}$ \\
\hline Chromium & 1 of 2 & ND (0.05) & 0.353 & 1.03 & 4.1 & $\mathrm{No}^{\mathrm{e}}$ \\
\hline Cobalt & 1 of 2 & ND (0.05) & 0.053 & $0.0780^{b}$ & 81 & $\mathrm{No}^{\mathrm{e}}$ \\
\hline Copper & 2 of 2 & 0.979 & 1.08 & $1.20^{b}$ & 54 & $\mathrm{No}^{\mathrm{e}}$ \\
\hline Iron & 2 of 2 & 73 & 81.5 & 310 & 410 & $\mathrm{No}^{\mathrm{e}}$ \\
\hline Lithium & 0 of 2 & ND (0.025) & ND (0.025) & $0.0993^{b}$ & 27 & $\mathrm{No}^{d}$ \\
\hline Magnesium & 2 of 2 & 244 & 298 & 408 & NAV & $\mathrm{No}^{\mathrm{e}}$ \\
\hline Manganese & 2 of 2 & 22.3 & 23.9 & 160 & 190 & $\mathrm{No}^{\mathrm{e}}$ \\
\hline Molybdenum & 0 of 2 & ND (.125) & ND (.125) & $0.173^{b}$ & 6.8 & $\mathrm{No}^{d}$ \\
\hline Nickel & 0 of 2 & ND (.125) & ND (.125) & ND & 27 & $\mathrm{No}^{\mathrm{d}}$ \\
\hline Potassium & 2 of 2 & 3,030 & 3,450 & $4,780^{b}$ & NAV & $\mathrm{No}^{\mathrm{e}}$ \\
\hline Selenium & 2 of 2 & 0.372 & 0.401 & 0.936 & 6.8 & $\mathrm{No}^{\mathrm{e}}$ \\
\hline Strontium & 2 of 2 & 5.29 & 8.47 & 22.1 & 810 & $\mathrm{No}^{\mathrm{e}}$ \\
\hline Thallium & 0 of 2 & ND $(0.025)$ & ND (0.025) & $0.0283^{b}$ & 0.095 & $\mathrm{No}^{d}$ \\
\hline Thorium & 1 of 2 & ND (2.5) & 5.4 & $3.43^{b}$ & NAV & $\mathrm{No}^{\dagger}$ \\
\hline Titanium & 2 of 2 & 0.358 & 0.763 & 32.1 & 5,400 & $\mathrm{No}^{\mathrm{e}}$ \\
\hline Uranium & 0 of 2 & ND (0.5) & ND $(0.5)$ & ND & 4.1 & $\mathrm{No}^{\mathrm{d}}$ \\
\hline Vanadium & 2 of 2 & 0.314 & 0.359 & 1.05 & 9.5 & $\mathrm{No}^{\mathrm{e}}$ \\
\hline Zinc & 2 of 2 & 38.2 & 41.9 & 48.8 & 410 & $\mathrm{No}^{\mathrm{e}}$ \\
\hline
\end{tabular}

${ }^{a}$ Background concentrations are upper tolerance limits (UTL) (ADEC, 1999a) unless otherwise noted.

${ }^{b}$ Maximum detected concentration in background samples due to insufficient detection frequency to calculate UTL.

${ }^{\mathrm{c} U . S . ~ E P A ~ R e g i o n ~ I I I ~ r i s k-b a s e d ~ s c r e e n i n g ~ c r i t e r i a ~ f o r ~ f i s h ~ c o n s u m p t i o n ~ u n l e s s ~ o t h e r w i s e ~ n o t e d . ~}$

\section{COC Selection Criteria}

${ }^{\mathrm{d}}$ Constituent not detected in any samples.

e Maximum detected constituent concentration is less than local background concentration.

${ }^{f}$ Not a constituent associated with drilling mud or other drilling activities.

ND - Not detected (detection limit)

NAV - Not available

NAN - Not analyzed

$\mathrm{mg} / \mathrm{kg}$ - Milligrams per kilogram 
Table 4-23

Summary of Analytical Results and Human Health Risk Assessment Screening for Surface Water in Clevenger Creek, Milrow Site

(Page 1 of 3 )

\begin{tabular}{|c|c|c|c|c|c|c|}
\hline Analyte & $\begin{array}{l}\text { Detection } \\
\text { Frequency }\end{array}$ & $\begin{array}{c}\text { Minimum } \\
\text { Detected } \\
\text { Concentration } \\
(\mu \mathrm{g} / \mathrm{L})\end{array}$ & $\begin{array}{c}\text { Maximum } \\
\text { Detected } \\
\text { Concentration } \\
(\mu \mathrm{g} / \mathrm{L})\end{array}$ & $\begin{array}{c}\text { Lowland } \\
\text { Stream } \\
\text { Background } \\
\text { Concentration } \\
(\mu \mathrm{g} / \mathrm{L})\end{array}$ & $\begin{array}{l}\text { Human Health } \\
\text { Screening } \\
\text { Value } \\
(\mu \mathrm{g} / L)\end{array}$ & $\begin{array}{c}\text { Human Health } \\
\text { Constituent of } \\
\text { Concern }\end{array}$ \\
\hline $\begin{array}{l}\text { Diesel-Range } \\
\text { Hydrocarbons }\end{array}$ & 0 of 5 & ND (98.9) & ND (225) & NAN & $1,500^{d}$ & $\mathrm{No}^{\dagger}$ \\
\hline \multicolumn{7}{|c|}{ Volatile Organic Compounds } \\
\hline 1,2,4-Trimethylbenzene & 0 of 5 & ND (1.0) & ND (1.0) & NAN & $12^{\mathrm{e}}$ & $\mathrm{No}^{\dagger}$ \\
\hline 1,3,5-Trimethylbenzene & 0 of 5 & ND (1.0) & ND (1.0) & NAN & $12^{\mathrm{e}}$ & $\mathrm{No}^{\dagger}$ \\
\hline 2-Butanone & 0 of 5 & ND (20) & ND (20) & NAN & $1,900^{\mathrm{e}}$ & $\mathrm{No}^{\dagger}$ \\
\hline Acetone & 0 of 5 & ND (20) & ND (20) & NAN & $3,700^{\mathrm{e}}$ & $\mathrm{No}^{\dagger}$ \\
\hline Benzene & 0 of 5 & ND (1.0) & $\mathrm{ND}(1.0)$ & NAN & $1.2^{\mathrm{c}}$ & $\mathrm{No}^{\dagger}$ \\
\hline Carbon disulfide & 0 of 5 & ND (1.0) & ND (1.0) & NAN & $1,000^{\mathrm{e}}$ & $\mathrm{No}^{f}$ \\
\hline Ethylbenzene & 0 of 5 & ND (1.0) & ND (1.0) & NAN & $3,100^{c}$ & $\mathrm{No}^{\dagger}$ \\
\hline Isopropylbenzene & 0 of 5 & ND (1.0) & ND (1.0) & NAN & NAV & $\mathrm{No}^{\dagger}$ \\
\hline $\mathrm{m}, \mathrm{p}$-Xylene & 0 of 5 & ND (2.0) & ND (2.0) & NAN & $12,000^{\mathrm{e}}$ & $\mathrm{No}^{\dagger}$ \\
\hline Methylene chloride & 0 of 5 & ND (2.0) & ND (4.51) & NAN & $4.7^{\mathrm{C}}$ & $\mathrm{No}^{\dagger}$ \\
\hline n-Butylbenzene & 0 of 5 & ND (1.0) & ND (1.0) & NAN & $61^{\mathrm{e}}$ & $\mathrm{No}^{\dagger}$ \\
\hline n-Propylbenzene & 0 of 5 & ND (1.0) & ND (1.0) & NAN & $61^{\mathrm{e}}$ & $\mathrm{No}^{\dagger}$ \\
\hline Naphthalene & 0 of 5 & ND (1.0) & $\mathrm{ND}(1.0)$ & NAN & $730^{\mathrm{e}}$ & $\mathrm{No}^{\dagger}$ \\
\hline o-Xylene & 0 of 5 & ND (1.0) & ND (1.0) & NAN & $12,000^{\mathrm{e}}$ & $\mathrm{No}^{\dagger}$ \\
\hline p-Isopropyltoluene & 0 of 5 & $\mathrm{ND}(1.0)$ & $\mathrm{ND}(1.0)$ & NAN & NAV & $\mathrm{No}^{\dagger}$ \\
\hline sec-Butylbenzene & 0 of 5 & ND (1.0) & ND (1.0) & NAN & $61^{e}$ & $\mathrm{No}^{\dagger}$ \\
\hline Toluene & 0 of 5 & ND (1.0) & ND (1.0) & NAN & $10,000^{c}$ & $\mathrm{No}^{\dagger}$ \\
\hline \multicolumn{7}{|c|}{ Polycyclic Aromatic Hydrocarbons } \\
\hline Acenaphthene & 0 of 5 & $\mathrm{ND}(0.1)$ & $\mathrm{ND}(0.1)$ & NAN & $1,200^{c}$ & $\mathrm{No}^{\dagger}$ \\
\hline Acenaphthylene & 0 of 5 & ND $(0.1)$ & $\mathrm{ND}(0.1)$ & NAN & $0.0028^{c}$ & $\mathrm{No}^{\dagger}$ \\
\hline Anthracene & 0 of 5 & $\mathrm{ND}(0.1)$ & $\mathrm{ND}(0.1)$ & NAN & $0.0028^{C}$ & $\mathrm{No}^{\dagger}$ \\
\hline Benzo(a)anthracene & 0 of 5 & $\mathrm{ND}(0.1)$ & $\mathrm{ND}(0.1)$ & NAN & $0.0028^{C}$ & $\mathrm{No}^{\dagger}$ \\
\hline Benzo(a)pyrene & 0 of 5 & ND $(0.1)$ & ND $(0.1)$ & NAN & $0.0028^{c}$ & $\mathrm{No}^{\dagger}$ \\
\hline Benzo(b)fluoranthene & 0 of 5 & ND $(0.1)$ & ND $(0.1)$ & NAN & $0.092^{\mathrm{e}}$ & $\mathrm{No}^{\dagger}$ \\
\hline Benzo(g,h,l)perylene & 0 of 5 & ND $(0.1)$ & $\mathrm{ND}(0.1)$ & NAN & $0.0028^{c}$ & $\mathrm{No}^{\dagger}$ \\
\hline Benzo(k)fluoranthene & 0 of 5 & $\mathrm{ND}(0.1)$ & $\mathrm{ND}(0.1)$ & NAN & $0.0028^{C}$ & $\mathrm{No}^{\dagger}$ \\
\hline Chrysene & 0 of 5 & $\mathrm{ND}(0.1)$ & $\mathrm{ND}(0.1)$ & NAN & $0.0028^{c}$ & $\mathrm{No}^{\dagger}$ \\
\hline
\end{tabular}


Table 4-23

Summary of Analytical Results and Human Health Risk Assessment Screening for Surface Water in Clevenger Creek, Milrow Site

(Page 2 of 3 )

\begin{tabular}{|c|c|c|c|c|c|c|}
\hline Analyte & $\begin{array}{l}\text { Detection } \\
\text { Frequency }\end{array}$ & $\begin{array}{c}\text { Minimum } \\
\text { Detected } \\
\text { Concentration } \\
(\mu \mathrm{g} / \mathrm{L})\end{array}$ & $\begin{array}{c}\text { Maximum } \\
\text { Detected } \\
\text { Concentration } \\
(\mu \mathrm{g} / \mathrm{L})\end{array}$ & $\begin{array}{c}\text { Lowland } \\
\text { Stream } \\
\text { Background } \\
\text { Concentration }{ }^{\mathrm{a}} \\
(\mu \mathrm{g} / \mathrm{L})\end{array}$ & $\begin{array}{l}\text { Human Health } \\
\text { Screening } \\
\text { Value } \\
(\mu \mathrm{g} / L)\end{array}$ & $\begin{array}{c}\text { Human Health } \\
\text { Constituent of } \\
\text { Concern }\end{array}$ \\
\hline Dibenzo(a,h)anthracene & 0 of 5 & ND $(0.1)$ & ND $(0.1)$ & NAN & $0.0028^{c}$ & $\mathrm{No}^{\dagger}$ \\
\hline Fluoranthene & 0 of 5 & ND $(0.1)$ & $\mathrm{ND}(0.1)$ & NAN & $42^{c}$ & $\mathrm{No}^{\dagger}$ \\
\hline Fluorene & 0 of 5 & $\mathrm{ND}(0.1)$ & $\mathrm{ND}(0.1)$ & NAN & $0.0028^{c}$ & $\mathrm{No}^{\dagger}$ \\
\hline Indeno(1,2,3-cd)pyrene & 0 of 5 & ND $(0.1)$ & $\mathrm{ND}(0.1)$ & NAN & $0.0028^{c}$ & $\mathrm{No}^{\dagger}$ \\
\hline Naphthalene & 0 of 5 & ND $(0.1)$ & $\mathrm{ND}(0.1)$ & NAN & $730^{\mathrm{e}}$ & $\mathrm{No}^{\dagger}$ \\
\hline Phenanthrene & 0 of 5 & $\mathrm{ND}(0.1)$ & $\mathrm{ND}(0.1)$ & NAN & $0.0028^{c}$ & $\mathrm{No}^{\dagger}$ \\
\hline Pyrene & 0 of 5 & $\mathrm{ND}(0.1)$ & $\mathrm{ND}(0.1)$ & NAN & $0.0028^{c}$ & $\mathrm{No}^{\dagger}$ \\
\hline \multicolumn{7}{|c|}{ Polychlorinated Biphenyls } \\
\hline Aroclor 1016 & NAV & NAV & NAV & NAN & $0.000044^{c}$ & $\mathrm{No}^{\dagger}$ \\
\hline Aroclor 1221 & NAV & NAV & NAV & NAN & $0.000044^{\mathrm{c}}$ & $\mathrm{No}^{\dagger}$ \\
\hline Aroclor 1232 & NAV & NAV & NAV & NAN & $0.000044^{\mathrm{c}}$ & $\mathrm{No}^{\dagger}$ \\
\hline Aroclor 1242 & NAV & NAV & NAV & NAN & $0.000044^{c}$ & $\mathrm{No}^{\dagger}$ \\
\hline Aroclor 1248 & NAV & NAV & NAV & NAN & $0.000044^{c}$ & $\mathrm{No}^{\dagger}$ \\
\hline Aroclor 1254 & NAV & NAV & NAV & NAN & $0.000044^{\mathrm{c}}$ & $\mathrm{No}^{\dagger}$ \\
\hline Aroclor 1260 & NAV & NAV & NAV & NAN & $0.000044^{c}$ & $\mathrm{No}^{\dagger}$ \\
\hline Aroclor 1262 & NAV & NAV & NAV & NAN & $0.000044^{\mathrm{c}}$ & $\mathrm{No}^{\dagger}$ \\
\hline Aroclor 1268 & NAV & NAV & NAV & NAN & $0.000044^{c}$ & $\mathrm{No}^{\dagger}$ \\
\hline \multicolumn{7}{|c|}{ Metals } \\
\hline Aluminum & 5 of 5 & 75.8 & 625 & 269 & $37,000^{\mathrm{e}}$ & $\mathrm{No}^{\mathrm{h}}$ \\
\hline Arsenic & 3 of 5 & ND (1.0) & 1.85 & ND (1) & $0.018^{\mathrm{C}}$ & $\mathrm{No}^{\mathrm{j}}$ \\
\hline Barium & 0 of 5 & ND (10) & ND (10) & ND (10) & $1000^{c}$ & $\mathrm{No}^{\dagger}$ \\
\hline Beryllium & 0 of 5 & ND (1.0) & ND (1.0) & ND (1) & $0.0076^{c}$ & $\mathrm{No}^{f}$ \\
\hline Boron & 2 of 5 & ND (50) & 88.7 & ND (50) & $3,300^{\mathrm{e}}$ & $\mathrm{No}^{h}$ \\
\hline Cadmium & 0 of 5 & ND (1.0) & ND (1.0) & ND (1) & $10^{c}$ & $\mathrm{No}^{\dagger}$ \\
\hline Calcium & 5 of 5 & 3,340 & 4,470 & $4,320^{b}$ & NAV & $\mathrm{No}^{\mathrm{i}}$ \\
\hline Cerium & 0 of 5 & ND (1.0) & ND (1.0) & ND (1) & NAV & $\mathrm{No}^{\dagger}$ \\
\hline Cesium & 0 of 5 & ND (1.0) & ND (1.0) & ND (1) & NAV & $\mathrm{No}^{f}$ \\
\hline Chromium & 1 of 5 & ND (1.0) & 1.25 & $2.25^{b}$ & $33,000^{c}$ & $\mathrm{No}^{\mathrm{g}}$ \\
\hline Cobalt & 3 of 5 & ND (1.0) & 4.32 & 3.35 & $2,200^{\mathrm{e}}$ & $\mathrm{No}^{\mathrm{h}}$ \\
\hline
\end{tabular}


Table 4-23

\section{Summary of Analytical Results and Human Health Risk Assessment Screening for Surface Water in Clevenger Creek, Milrow Site}

(Page 3 of 3 )

\begin{tabular}{|c|c|c|c|c|c|c|}
\hline Analyte & $\begin{array}{l}\text { Detection } \\
\text { Frequency }\end{array}$ & $\begin{array}{c}\text { Minimum } \\
\text { Detected } \\
\text { Concentration } \\
(\mu \mathrm{g} / \mathrm{L})\end{array}$ & $\begin{array}{c}\text { Maximum } \\
\text { Detected } \\
\text { Concentration } \\
(\mu \mathrm{g} / \mathrm{L})\end{array}$ & $\begin{array}{c}\text { Lowland } \\
\text { Stream } \\
\text { Background } \\
\text { Concentration }{ }^{\mathrm{a}} \\
(\mu \mathrm{g} / \mathrm{L})\end{array}$ & $\begin{array}{l}\text { Human Health } \\
\text { Screening } \\
\text { Value } \\
(\mu \mathrm{g} / L)\end{array}$ & $\begin{array}{c}\text { Human Health } \\
\text { Constituent of } \\
\text { Concern }\end{array}$ \\
\hline Copper & 5 of 5 & 3.7 & 11.8 & 10.8 & $1,300^{C}$ & $\mathrm{No}^{\mathrm{h}}$ \\
\hline Iron & 5 of 5 & 336 & 1,070 & 993 & $300^{c}$ & $\mathrm{No}^{\mathrm{i}}$ \\
\hline Lithium & 1 of 5 & ND (1.0) & 1.14 & $1.17^{b}$ & $730^{e}$ & $\mathrm{No}^{\mathrm{g}}$ \\
\hline Magnesium & 5 of 5 & 2,110 & 3,250 & $4,690^{b}$ & NAV & $\mathrm{N}^{g}$ \\
\hline Manganese & 5 of 5 & 12.6 & 27.2 & 267 & $50^{c}$ & $\mathrm{No}^{\mathrm{g}}$ \\
\hline Molybdenum & 0 of 5 & ND (1.0) & ND (1.0) & ND (1) & $180^{e}$ & $\mathrm{No}^{\dagger}$ \\
\hline Nickel & 2 of 5 & ND (1.0) & 1.97 & ND (1) & $510^{c}$ & $\mathrm{No}^{h}$ \\
\hline Potassium & 5 of 5 & 1,570 & 2,730 & 2,620 & NAV & $\mathrm{No}^{\mathrm{i}}$ \\
\hline Selenium & 0 of 5 & ND (1.0) & ND (1.0) & ND (1) & $104^{c}$ & $\mathrm{No}^{f}$ \\
\hline Strontium & 5 of 5 & 10.5 & 18.9 & 36.1 & $22,000^{\mathrm{e}}$ & $\mathrm{No}^{\mathrm{g}}$ \\
\hline Thallium & 0 of 5 & ND (1.0) & ND (1.0) & $7.07^{b}$ & $1.7^{\mathrm{C}}$ & $\mathrm{No}^{\dagger}$ \\
\hline Thorium & 0 of 5 & ND (1.0) & ND (1.0) & ND (1) & NAV & $\mathrm{No}^{f}$ \\
\hline Titanium & 5 of 5 & 2.79 & 123 & 6.13 & $150,000^{\mathrm{e}}$ & $\mathrm{No}^{\mathrm{h}}$ \\
\hline Uranium & 0 of 5 & ND (1.0) & ND (1.0) & ND (1) & $110^{e}$ & $\mathrm{No}^{f}$ \\
\hline Vanadium & 4 of 5 & ND (1.0) & 4.44 & $4.43^{b}$ & $260^{e}$ & $\mathrm{No}^{\mathrm{h}}$ \\
\hline Zinc & 4 of 5 & ND (10) & 44.9 & $50.3^{b}$ & $11,000^{\mathrm{e}}$ & $\mathrm{No}^{\mathrm{g}}$ \\
\hline
\end{tabular}

${ }^{\mathrm{a} B a c k g r o u n d}$ concentrations are upper tolerance limits (UTL) (ADEC, 1999a) unless otherwise noted.

${ }^{\mathrm{b}}$ Maximum detected concentration in background samples due to insufficient detection frequency to calculate UTL.

${ }^{\mathrm{C}}$ Federal ambient water quality criteria based on ingestion of water and organisms.

d State of Alaska groundwater cleanup level, Discharge Reporting, Cleanup, and Disposal of Oil and other Hazardous Substances

(18 AAC 75, Articles 3 and 9 [ADEC, 1999c]).

eU.S. EPA Region III risk-based concentrations for tap water (EPA, 2000b).

\section{COC Selection Criteria}

${ }^{f}$ Constituent not detected in any samples.

${ }^{9}$ Maximum detected constituent concentration is less than local background concentration.

${ }^{h}$ Maximum detected constituent concentration is less than human health screening value.

iConstituent is an essential macronutrient and only presents a risk at extremely high concentrations.

${ }^{j}$ Not a constituent associated with drilling mud or other drilling activities.

ND - Not detected (detection limit)

NAV - Not available

NAN - Not analyzed

$\mu \mathrm{g} / \mathrm{L}$ - Micrograms per liter 
Table 4-24

Summary of Analytical Results and Human Health Risk Assessment Screening for Sediment in Clevenger Creek, Milrow Site

(Page 1 of 3 )

\begin{tabular}{|c|c|c|c|c|c|c|}
\hline Analyte & $\begin{array}{l}\text { Detection } \\
\text { Frequency }\end{array}$ & $\begin{array}{c}\text { Minimum } \\
\text { Detected } \\
\text { Concentration } \\
(\mathbf{m g} / \mathbf{k g})\end{array}$ & $\begin{array}{c}\text { Maximum } \\
\text { Detected } \\
\text { Concentration } \\
(\mathrm{mg} / \mathrm{kg})\end{array}$ & $\begin{array}{c}\text { Lowland } \\
\text { Stream } \\
\text { Background } \\
\text { Concentration } \\
(\mathbf{m g} / \mathbf{k g})\end{array}$ & $\begin{array}{l}\text { Human Health } \\
\text { Screening } \\
\text { Value } \\
(\mathrm{mg} / \mathrm{kg})\end{array}$ & $\begin{array}{l}\text { Human Health } \\
\text { Constituent of } \\
\text { Concern }\end{array}$ \\
\hline $\begin{array}{l}\text { Diesel-Range } \\
\text { Hydrocarbons }\end{array}$ & 4 of 6 & 29.6 & 542 & NAN & $200^{c}$ & Yes \\
\hline \multicolumn{7}{|c|}{ Volatile Organic Compounds } \\
\hline 1,2,4-Trimethylbenzene & 0 of 2 & ND (0.0085) & ND (0.045) & NAN & NAV & $\mathrm{No}^{d}$ \\
\hline 1,3,5-Trimethylbenzene & 0 of 2 & ND (0.0085) & ND (0.045) & NAN & NAV & $\mathrm{No}^{\mathrm{d}}$ \\
\hline 2-Butanone & 0 of 2 & ND $(0.017)$ & ND (0.09) & NAN & NAV & $\mathrm{No}^{d}$ \\
\hline Acetone & 0 of 2 & ND (1.64) & ND (0.04) & NAN & $10,100^{c}$ & $\mathrm{No}^{\mathrm{d}}$ \\
\hline Benzene & 0 of 2 & ND $(0.0085)$ & ND (0.045) & NAN & $286^{\mathrm{C}}$ & $\mathrm{No}^{\mathrm{d}}$ \\
\hline Carbon disulfide & 0 of 2 & ND $(0.0901)$ & ND $(0.318)$ & NAN & $10,100^{c}$ & $\mathrm{No}^{\mathrm{d}}$ \\
\hline Ethylbenzene & 0 of 2 & ND $(0.0085)$ & ND $(0.045)$ & NAN & $10,100^{c}$ & $\mathrm{No}^{\mathrm{d}}$ \\
\hline Isopropylbenzene & 0 of 2 & ND (0.0085) & ND (0.045) & NAN & NAV & $\mathrm{No}^{d}$ \\
\hline $\mathrm{m}, \mathrm{p}$-Xylene & 0 of 2 & ND (0.0085) & ND $(0.045)$ & NAN & $203,000^{c}$ & $\mathrm{No}^{\mathrm{d}}$ \\
\hline Methylene chloride & 0 of 2 & ND (0.0085) & ND (0.045) & NAN & $1,110^{\mathrm{C}}$ & $\mathrm{No}^{d}$ \\
\hline n-Butylbenzene & 0 of 2 & ND $(0.0085)$ & ND (0.045) & NAN & NAV & $\mathrm{No}^{\mathrm{d}}$ \\
\hline n-Propylbenzene & 0 of 2 & ND (0.0085) & ND (0.045) & NAN & NAV & $\mathrm{No}^{d}$ \\
\hline Naphthalene & 0 of 2 & ND (0.0085) & ND $(0.045)$ & NAN & $4,060^{\mathrm{C}}$ & $\mathrm{No}^{d}$ \\
\hline o-Xylene & 0 of 2 & ND (0.0085) & ND $(0.045)$ & NAN & $203,000^{\mathrm{C}}$ & $\mathrm{No}^{d}$ \\
\hline p-Isopropyltoluene & 0 of 2 & ND (0.0085) & ND (0.045) & NAN & NAV & $\mathrm{No}^{d}$ \\
\hline sec-Butylbenzene & 0 of 2 & ND (0.0085) & ND (0.045) & NAN & NAV & $\mathrm{No}^{d}$ \\
\hline Toluene & 0 of 2 & ND (0.0085) & ND (0.045) & NAN & $20,300^{c}$ & $\mathrm{No}^{d}$ \\
\hline \multicolumn{7}{|c|}{ Polycyclic Aromatic Hydrocarbons } \\
\hline Acenaphthene & 0 of 6 & ND (0.00702) & ND (0.0232) & NAN & $6,080^{c}$ & $\mathrm{No}^{d}$ \\
\hline Acenaphthylene & 0 of 6 & ND (0.00702) & ND (0.0232) & NAN & NAV & $\mathrm{No}^{d}$ \\
\hline Anthracene & 0 of 6 & ND (0.00702) & ND (0.0232) & NAN & $30,400^{C}$ & $\mathrm{No}^{d}$ \\
\hline Benzo(a)anthracene & 0 of 6 & ND (0.00702) & ND (0.0232) & NAN & $11.4^{\mathrm{C}}$ & $\mathrm{No}^{d}$ \\
\hline Benzo(a)pyrene & 0 of 6 & ND $(0.00702)$ & ND (0.0232) & NAN & $1.1^{\mathrm{c}}$ & $\mathrm{No}^{d}$ \\
\hline Benzo(b)fluoranthene & 0 of 6 & ND (0.00702) & ND (0.0232) & NAN & $11.4^{\mathrm{C}}$ & $\mathrm{No}^{d}$ \\
\hline Benzo(g,h,l)perylene & 0 of 6 & ND $(0.00702)$ & ND (0.0232) & NAN & $1.1^{\mathrm{c}}$ & $\mathrm{No}^{\mathrm{d}}$ \\
\hline Benzo(k)fluoranthene & 0 of 6 & ND (0.00702) & ND (0.0232) & NAN & $114^{\mathrm{C}}$ & $\mathrm{No}^{d}$ \\
\hline Chrysene & 0 of 6 & ND (0.00702) & ND (0.0232) & NAN & $1,140^{\mathrm{C}}$ & $\mathrm{No}^{\mathrm{d}}$ \\
\hline
\end{tabular}


Table 4-24

Summary of Analytical Results and Human Health Risk Assessment Screening for Sediment in Clevenger Creek, Milrow Site

(Page 2 of 3 )

\begin{tabular}{|c|c|c|c|c|c|c|}
\hline Analyte & $\begin{array}{l}\text { Detection } \\
\text { Frequency }\end{array}$ & $\begin{array}{c}\text { Minimum } \\
\text { Detected } \\
\text { Concentration } \\
(\mathrm{mg} / \mathrm{kg})\end{array}$ & $\begin{array}{c}\text { Maximum } \\
\text { Detected } \\
\text { Concentration } \\
(\mathrm{mg} / \mathrm{kg})\end{array}$ & $\begin{array}{c}\text { Lowland } \\
\text { Stream } \\
\text { Background } \\
\text { Concentration }{ }^{\mathrm{a}} \\
(\mathbf{m g} / \mathbf{k g})\end{array}$ & $\begin{array}{l}\text { Human Health } \\
\text { Screening } \\
\text { Value } \\
(\mathrm{mg} / \mathrm{kg})\end{array}$ & $\begin{array}{c}\text { Human Health } \\
\text { Constituent of } \\
\text { Concern }\end{array}$ \\
\hline Dibenzo(a,h)anthracene & 0 of 6 & ND (0.00702) & ND (0.0232) & NAN & $1.1^{\mathrm{C}}$ & $\mathrm{No}^{\mathrm{d}}$ \\
\hline Fluoranthene & 0 of 6 & ND (0.00702) & ND (0.0232) & NAN & $4,060^{C}$ & $\mathrm{No}^{\mathrm{d}}$ \\
\hline Fluorene & 0 of 6 & ND (0.00702) & ND (0.0232) & NAN & $4,060^{\mathrm{C}}$ & $\mathrm{No}^{\mathrm{d}}$ \\
\hline Indeno(1,2,3-cd)pyrene & 0 of 6 & ND (0.00702) & ND (0.0232) & NAN & $11^{\mathrm{c}}$ & $\mathrm{No}^{\mathrm{d}}$ \\
\hline Naphthalene & 0 of 6 & ND (0.00702) & ND (0.0232) & NAN & $4,060^{C}$ & $\mathrm{No}^{\mathrm{d}}$ \\
\hline Phenanthrene & 0 of 6 & ND (0.00702) & ND $(0.0232)$ & NAN & $1.1^{\mathrm{c}}$ & $\mathrm{No}^{\mathrm{d}}$ \\
\hline Pyrene & 0 of 6 & ND (0.00702) & ND (0.0232) & NAN & $3,040^{c}$ & Nod \\
\hline \multicolumn{7}{|c|}{ Polychlorinated Biphenyls } \\
\hline Aroclor 1016 & NAV & NAV & NAV & NAN & $10^{\mathrm{C}}$ & $\mathrm{No}^{\mathrm{d}}$ \\
\hline Aroclor 1221 & NAV & NAV & NAV & NAN & $10^{\mathrm{C}}$ & $\mathrm{No}^{\mathrm{d}}$ \\
\hline Aroclor 1232 & NAV & NAV & NAV & NAN & $10^{c}$ & $\mathrm{No}^{\mathrm{d}}$ \\
\hline Aroclor 1242 & NAV & NAV & NAV & NAN & $10^{c}$ & $\mathrm{No}^{d}$ \\
\hline Aroclor 1248 & NAV & NAV & NAV & NAN & $10^{c}$ & $\mathrm{No}^{\mathrm{d}}$ \\
\hline Aroclor 1254 & NAV & NAV & NAV & NAN & $10^{\mathrm{C}}$ & $\mathrm{No}^{d}$ \\
\hline Aroclor 1260 & NAV & NAV & NAV & NAN & $10^{c}$ & $\mathrm{No}^{\mathrm{d}}$ \\
\hline Aroclor 1262 & NAV & NAV & NAV & NAN & $10^{\mathrm{C}}$ & $\mathrm{No}^{d}$ \\
\hline Aroclor 1268 & NAV & NAV & NAV & NAN & $10^{c}$ & $\mathrm{No}^{d}$ \\
\hline \multicolumn{7}{|c|}{ Metals } \\
\hline Aluminum & 6 of 6 & 16,000 & 90,600 & 45,400 & NAV & $\mathrm{No}^{\mathrm{h}}$ \\
\hline Arsenic & 6 of 6 & 6.05 & 48.9 & 100 & $5.5^{\mathrm{C}}$ & $\mathrm{No}^{\mathrm{e}}$ \\
\hline Barium & 6 of 6 & 104 & 355 & 469 & $7,100^{\mathrm{C}}$ & $\mathrm{No}^{\mathrm{e}}$ \\
\hline Beryllium & 0 of 6 & ND (1.06) & ND (4.02) & ND & $1.9^{\mathrm{C}}$ & $\mathrm{No}^{\mathrm{d}}$ \\
\hline Boron & 4 of 6 & 76.8 & 198 & $7.18^{b}$ & NAV & $\mathrm{No}^{h}$ \\
\hline Cadmium & 0 of 6 & ND (1.06) & ND (4.02) & $0.608^{b}$ & $101^{c}$ & $\mathrm{No}^{d}$ \\
\hline Calcium & 6 of 6 & 2,450 & 20,200 & 7,300 & NAV & $\mathrm{No}^{\mathrm{g}}$ \\
\hline Cerium & 6 of 6 & 7.58 & 30.9 & 14.2 & NAV & $\mathrm{No}^{h}$ \\
\hline Cesium & 2 of 6 & 1.8 & 4.26 & ND & NAV & $\mathrm{No}^{\mathrm{h}}$ \\
\hline Chromium & 6 of 6 & 9.8 & 24.7 & 11.8 & $507^{c}$ & $\mathrm{No}^{\dagger}$ \\
\hline Cobalt & 6 of 6 & 13.5 & 38.3 & 43.7 & NAV & $\mathrm{No}^{\mathrm{e}}$ \\
\hline
\end{tabular}


Table 4-24

\section{Summary of Analytical Results and Human Health Risk Assessment Screening for Sediment in Clevenger Creek, Milrow Site}

(Page 3 of 3 )

\begin{tabular}{|c|c|c|c|c|c|c|}
\hline Analyte & $\begin{array}{l}\text { Detection } \\
\text { Frequency }\end{array}$ & $\begin{array}{c}\text { Minimum } \\
\text { Detected } \\
\text { Concentration } \\
(\mathbf{m g} / \mathbf{k g})\end{array}$ & $\begin{array}{c}\text { Maximum } \\
\text { Detected } \\
\text { Concentration } \\
(\mathrm{mg} / \mathrm{kg})\end{array}$ & $\begin{array}{c}\text { Lowland } \\
\text { Stream } \\
\text { Background } \\
\text { Concentration } \\
(\mathbf{m g} / \mathbf{k g})\end{array}$ & $\begin{array}{l}\text { Human Health } \\
\text { Screening } \\
\text { Value } \\
(\mathrm{mg} / \mathrm{kg})\end{array}$ & $\begin{array}{c}\text { Human Health } \\
\text { Constituent of } \\
\text { Concern }\end{array}$ \\
\hline Copper & 6 of 6 & 40.6 & 247 & 71.1 & NAV & $\mathrm{No}^{\mathrm{h}}$ \\
\hline Iron & 6 of 6 & 36,000 & 108,000 & 155,000 & NAV & $\mathrm{No}^{\mathrm{e}}$ \\
\hline Lithium & 6 of 6 & 5.84 & 41.7 & 48.8 & NAV & $\mathrm{No}^{\mathrm{e}}$ \\
\hline Magnesium & 6 of 6 & 5,280 & 37,500 & 9,320 & NAV & $\mathrm{No}^{\mathrm{g}}$ \\
\hline Manganese & 6 of 6 & 297 & 9,020 & 20,700 & NAV & $\mathrm{No}^{\mathrm{e}}$ \\
\hline Molybdenum & 0 of 6 & ND (1.06) & ND (4.02) & $5.5^{b}$ & NAV & $\mathrm{No}^{d}$ \\
\hline Nickel & 6 of 6 & 5.47 & 18.9 & 13.6 & $2,030^{c}$ & $\mathrm{No}^{\dagger}$ \\
\hline Potassium & 6 of 6 & 359 & 3,560 & 1,390 & NAV & $\mathrm{No}^{\mathrm{g}}$ \\
\hline Selenium & 0 of 6 & ND (1.06) & ND (4.02) & $5.44^{b}$ & $507^{c}$ & $\mathrm{No}^{d}$ \\
\hline Strontium & 6 of 6 & 44.4 & 609 & 164 & NAV & $\mathrm{No}^{\mathrm{h}}$ \\
\hline Thallium & 0 of 6 & ND (1.06) & ND (4.02) & $1^{b}$ & NAV & $\mathrm{No}^{d}$ \\
\hline Thorium & 0 of 6 & ND (1.06) & ND (4.02) & $0.5^{b}$ & NAV & $\mathrm{No}^{\mathrm{d}}$ \\
\hline Titanium & 6 of 6 & 552 & 5,760 & 3,010 & NAV & $\mathrm{No}^{\mathrm{h}}$ \\
\hline Uranium & 0 of 6 & ND (1.06) & ND (4.02) & $4.13^{b}$ & NAV & $\mathrm{No}^{d}$ \\
\hline Vanadium & 6 of 6 & 151 & 420 & 734 & $710^{c}$ & $\mathrm{No}^{\mathrm{e}}$ \\
\hline Zinc & 6 of 6 & 90.5 & 208 & 434 & $30,400^{c}$ & $\mathrm{No}^{\mathrm{e}}$ \\
\hline Total Organic Carbon & 6 of 6 & 44,900 & 178,000 & NAN & NAV & No \\
\hline
\end{tabular}

${ }^{\mathrm{a}}$ Background concentrations are upper tolerance limits (UTL) (ADEC, 1999a) unless otherwise noted.

${ }^{\mathrm{b}}$ Maximum detected concentration in background samples due to insufficient detection frequency to calculate UTL.

'State of Alaska soil cleanup level, Discharge Reporting, Cleanup, and Disposal of Oil and other Hazardous Substances

(18 AAC 75, Articles 3 and 9 [ADEC, 1999c]).

\section{COC Selection Criteria}

${ }^{\mathrm{d} C o n s t i t u e n t}$ not detected in any samples.

${ }^{e}$ Maximum detected constituent concentration is less than local background concentration.

${ }^{\mathrm{f}}$ Maximum detected constituent concentration is less than human health screening value.

${ }^{9}$ Constituent is an essential macronutrient and only presents a risk at extremely high concentrations.

${ }^{\mathrm{h}}$ Not a constituent associated with drilling mud or other drilling activities.

ND - Not detected (detection limit)

NAV - Not available

NAN - Not analyzed

$\mathrm{mg} / \mathrm{kg}$ - Milligrams per kilogram 
Table 4-25

Summary of Analytical Results and Human Health Risk Assessment
Screening for Fish Tissue in Clevenger Creek, Milrow Site

(Page 1 of 2)

\begin{tabular}{|c|c|c|c|c|c|c|}
\hline Analyte & $\begin{array}{l}\text { Detection } \\
\text { Frequency }\end{array}$ & $\begin{array}{c}\text { Minimum } \\
\text { Detected } \\
\text { Concentration } \\
(\mathrm{mg} / \mathrm{kg})\end{array}$ & $\begin{array}{c}\text { Maximum } \\
\text { Detected } \\
\text { Concentration } \\
(\mathrm{mg} / \mathrm{kg})\end{array}$ & $\begin{array}{c}\text { Lowland } \\
\text { Stream } \\
\text { Background } \\
\text { Concentration }{ }^{\mathrm{a}} \\
(\mathrm{mg} / \mathrm{kg})\end{array}$ & $\begin{array}{l}\text { Human Health } \\
\text { Screening } \\
\text { Value }^{c} \\
(\mathrm{mg} / \mathrm{kg})\end{array}$ & $\begin{array}{c}\text { Human Health } \\
\text { Constituent of } \\
\text { Concern }\end{array}$ \\
\hline \multicolumn{7}{|c|}{ Polycyclic Aromatic Hydrocarbons } \\
\hline Acenaphthene & 0 of 3 & ND (0.0067) & ND (0.067) & NAN & 81.1 & No ${ }^{d}$ \\
\hline Acenaphthylene & 0 of 3 & ND $(0.0067)$ & ND $(0.067)$ & NAN & NAV & $\mathrm{No}^{\mathrm{d}}$ \\
\hline Anthracene & 0 of 3 & ND (0.067) & ND $(0.168)$ & NAN & 410 & $\mathrm{No}^{\mathrm{d}}$ \\
\hline Benzo(a)anthracene & 0 of 3 & ND $(0.067)$ & ND (0.168) & NAN & 0.0043 & $\mathrm{No}^{d}$ \\
\hline Benzo(a)pyrene & 0 of 3 & ND (0.067) & ND $(0.168)$ & NAN & 0.00043 & $\mathrm{No}^{\mathrm{d}}$ \\
\hline Benzo(b)fluoranthene & 0 of 3 & ND (0.067) & ND $(0.168)$ & NAN & 0.0043 & $\mathrm{No}^{d}$ \\
\hline Benzo(ghi)perylene & 0 of 3 & ND (0.067) & ND (0.168) & NAN & NAV & $\mathrm{No}^{d}$ \\
\hline Benzo(k)fluoranthene & 0 of 3 & ND $(0.067)$ & ND (0.168) & NAN & 0.043 & $\mathrm{No}^{d}$ \\
\hline Chrysene & 0 of 3 & ND (0.067) & ND (0.168) & NAN & 0.43 & $\mathrm{No}^{\mathrm{d}}$ \\
\hline Dibenzo(a,h)anthracene & 0 of 3 & ND (0.067) & ND $(0.168)$ & NAN & 0.00043 & $\mathrm{No}^{d}$ \\
\hline Fluoranthene & 0 of 3 & ND (0.067) & ND (0.168) & NAN & 54 & $\mathrm{No}^{\mathrm{d}}$ \\
\hline Fluorene & 0 of 3 & ND (0.0067) & ND (0.067) & NAN & 54 & $\mathrm{No}^{\mathrm{d}}$ \\
\hline Indeno(1,2,3-cd)pyrene & 0 of 3 & ND $(0.067)$ & ND (0.168) & NAN & 0.0043 & $\mathrm{No}^{\mathrm{d}}$ \\
\hline Naphthalene & 1 of 3 & ND (0.0067) & 0.0155 & NAN & 27 & $\mathrm{No}^{f}$ \\
\hline Phenanthrene & 0 of 3 & ND (0.067) & ND $(0.168)$ & NAN & NAV & $\mathrm{No}^{\mathrm{d}}$ \\
\hline Pyrene & 0 of 3 & ND (0.067) & ND (0.168) & NAN & 41 & $\mathrm{No}^{\mathrm{d}}$ \\
\hline \multicolumn{7}{|c|}{ Polychlorinated Biphenyls } \\
\hline Aroclor 1016 & 0 of 3 & ND (0.01) & ND $(0.01)$ & NAN & 0.045 & $\mathrm{No}^{\mathrm{d}}$ \\
\hline Aroclor 1221 & 0 of 3 & ND (0.02) & ND (0.02) & NAN & 0.0016 & $\mathrm{No}^{\mathrm{d}}$ \\
\hline Aroclor 1232 & 0 of 3 & ND (0.01) & ND $(0.01)$ & NAN & 0.0016 & $\mathrm{No}^{d}$ \\
\hline Aroclor 1242 & 0 of 3 & ND (0.01) & ND $(0.01)$ & NAN & 0.0016 & $\mathrm{No}^{d}$ \\
\hline Aroclor 1248 & 0 of 3 & ND (0.01) & ND (0.01) & NAN & 0.0016 & $\mathrm{No}^{d}$ \\
\hline Aroclor 1254 & 0 of 3 & ND (0.01) & ND (0.01) & NAN & 0.0016 & $\mathrm{No}^{d}$ \\
\hline Aroclor 1260 & 2 of 3 & ND (0.01) & 0.0094 & NAN & 0.0016 & Yes \\
\hline \multicolumn{7}{|c|}{ Metals } \\
\hline Aluminum & 3 of 3 & 16.1 & 61 & 475 & 1,400 & $\mathrm{No}^{\mathrm{e}}$ \\
\hline Arsenic & 3 of 3 & 0.0533 & 0.225 & 0.127 & 0.0021 & $\mathrm{No}^{\mathrm{g}}$ \\
\hline Inorganic Arsenic & 3 of 3 & 0.0138 & 0.0551 & 0.0208 & 0.0021 & $\mathrm{No}^{\mathrm{h}}$ \\
\hline Barium & 3 of 3 & 0.209 & 0.46 & 1.03 & 95 & $\mathrm{No}^{\mathrm{e}}$ \\
\hline Beryllium & 0 of 3 & ND (0.025) & ND (0.025) & ND & 2.7 & $\mathrm{No}^{d}$ \\
\hline Boron & 0 of 3 & ND (6.83) & ND (8.74) & 28.6 & 120 & $\mathrm{No}^{\mathrm{d}}$ \\
\hline Cadmium & 0 of 3 & ND (0.025) & ND $(0.025)$ & $0.0373^{b}$ & 1.4 & $\mathrm{No}^{d}$ \\
\hline
\end{tabular}


Table 4-25

\section{Summary of Analytical Results and Human Health Risk Assessment Screening for Fish Tissue in Clevenger Creek, Milrow Site}

(Page 2 of 2 )

\begin{tabular}{|c|c|c|c|c|c|c|}
\hline Analyte & $\begin{array}{l}\text { Detection } \\
\text { Frequency }\end{array}$ & $\begin{array}{c}\text { Minimum } \\
\text { Detected } \\
\text { Concentration } \\
(\mathrm{mg} / \mathrm{kg})\end{array}$ & $\begin{array}{c}\text { Maximum } \\
\text { Detected } \\
\text { Concentration } \\
(\mathbf{m g} / \mathbf{k g})\end{array}$ & $\begin{array}{c}\text { Lowland } \\
\text { Stream } \\
\text { Background } \\
\text { Concentration }{ }^{\mathrm{a}} \\
(\mathrm{mg} / \mathrm{kg})\end{array}$ & $\begin{array}{c}\text { Human Health } \\
\text { Screening } \\
\text { Value }^{\mathrm{c}} \\
(\mathrm{mg} / \mathrm{kg})\end{array}$ & $\begin{array}{c}\text { Human Health } \\
\text { Constituent of } \\
\text { Concern }\end{array}$ \\
\hline Calcium & 3 of 3 & 7,500 & 8,800 & 19,200 & NAV & $\mathrm{No}^{\mathrm{e}}$ \\
\hline Cerium & 1 of 3 & ND (0.025) & 0.0278 & $0.0280^{b}$ & NAV & $\mathrm{No}^{\mathrm{e}}$ \\
\hline Cesium & 2 of 3 & ND (0.025) & 0.0285 & $0.0258^{b}$ & NAV & $\mathrm{No}^{\mathrm{g}}$ \\
\hline Chromium & 3 of 3 & 0.326 & 0.509 & 1.03 & 4.1 & $\mathrm{No}^{\mathrm{e}}$ \\
\hline Cobalt & 2 of 3 & ND (0.05) & 0.053 & $0.0780^{b}$ & 81 & $\mathrm{No}^{\mathrm{e}}$ \\
\hline Copper & 0 of 3 & ND (0.95) & ND (1.27) & $1.20^{\mathrm{b}}$ & 54 & $\mathrm{No}^{d}$ \\
\hline Iron & 3 of 3 & 50 & 206 & 310 & 410 & $\mathrm{No}^{\mathrm{e}}$ \\
\hline Lithium & 1 of 3 & ND (0.025) & 0.028 & $0.0993^{b}$ & 27 & $\mathrm{No}^{\mathrm{e}}$ \\
\hline Magnesium & 3 of 3 & 310 & 330 & 408 & NAV & $\mathrm{No}^{\mathrm{e}}$ \\
\hline Manganese & 3 of 3 & 10.5 & 25 & 160 & 190 & $\mathrm{No}^{\mathrm{e}}$ \\
\hline Molybdenum & 0 of 3 & ND (0.125) & ND $(0.16)$ & $0.173^{b}$ & 6.8 & $\mathrm{No}^{d}$ \\
\hline Nickel & 1 of 3 & ND (0.125) & 0.127 & ND & 27 & $\mathrm{No}^{f}$ \\
\hline Potassium & 3 of 3 & 4,150 & 4,580 & $4,780^{b}$ & NAV & $\mathrm{No}^{\mathrm{e}}$ \\
\hline Selenium & 3 of 3 & 0.337 & 0.359 & 0.936 & 6.8 & $\mathrm{No}^{\mathrm{e}}$ \\
\hline Strontium & 3 of 3 & 5.89 & 12.6 & 22.1 & 810 & $\mathrm{No}^{\mathrm{e}}$ \\
\hline Thallium & 0 of 3 & ND (0.025) & ND (0.025) & $0.0283^{b}$ & 0.095 & $\mathrm{No}^{d}$ \\
\hline Thorium & 0 of 3 & ND (2.5) & ND (2.5) & $3.43^{b}$ & NAV & $\mathrm{No}^{d}$ \\
\hline Titanium & 3 of 3 & 0.498 & 3.76 & 32.1 & 5,400 & $\mathrm{No}^{\mathrm{e}}$ \\
\hline Uranium & 0 of 3 & ND (0.5) & ND (0.5) & ND & 4.1 & $\mathrm{No}^{\mathrm{d}}$ \\
\hline Vanadium & 3 of 3 & 0.279 & 0.597 & 1.05 & 9.5 & $\mathrm{No}^{\mathrm{e}}$ \\
\hline Zinc & 3 of 3 & 32.7 & 38.7 & 48.8 & 410 & $\mathrm{No}^{\mathrm{e}}$ \\
\hline$\%$ Lipids & 1 of 1 & 2.21 & 2.21 & NAN & NAV & No \\
\hline
\end{tabular}

${ }^{a}$ Background concentrations are upper tolerance limits (UTL) (ADEC, 1999a) unless otherwise noted.

${ }^{b}$ Maximum detected concentration in background samples due to insufficient detection frequency to calculate UTL.

${ }^{\mathrm{C} U . S . ~ E P A ~ R e g i o n ~ I I I ~ r i s k-b a s e d ~ s c r e e n i n g ~ c r i t e r i a ~ f o r ~ f i s h ~ c o n s u m p t i o n ~ u n l e s s ~ o t h e r w i s e ~ n o t e d . ~}$

\section{COC Selection Criteria}

${ }^{\mathrm{d}}$ Constituent not detected in any samples.

${ }^{\mathrm{e}}$ Maximum detected constituent concentration is less than local background concentration.

${ }^{f}$ Maximum detected constituent concentration is less than human health screening value.

${ }^{9}$ Not a constituent associated with drilling mud or other drilling activities.

ND - Not detected (detection limit)

NAV - Not available

NAN - Not analyzed

$\mathrm{mg} / \mathrm{kg}$ - Milligrams per kilogram 
Table 4-26

Summary of Analytical Results and Human Health Risk Assessment Screening for Surface Water in Heart Lake, Milrow Site

(Page 1 of 3 )

\begin{tabular}{|c|c|c|c|c|c|c|}
\hline Analyte & $\begin{array}{l}\text { Detection } \\
\text { Frequency }\end{array}$ & $\begin{array}{c}\text { Minimum } \\
\text { Detected } \\
\text { Concentration } \\
(\mu \mathrm{g} / \mathrm{L})\end{array}$ & $\begin{array}{c}\text { Maximum } \\
\text { Detected } \\
\text { Concentration } \\
(\mu \mathrm{g} / \mathrm{L})\end{array}$ & $\begin{array}{c}\text { Lowland } \\
\text { Stream } \\
\text { Background } \\
\text { Concentration }{ }^{\mathrm{a}} \\
(\mu \mathrm{g} / \mathrm{L})\end{array}$ & $\begin{array}{l}\text { Human Health } \\
\text { Screening } \\
\text { Value } \\
(\mu \mathrm{g} / \mathrm{L})\end{array}$ & $\begin{array}{c}\text { Human Health } \\
\text { Constituent of } \\
\text { Concern }\end{array}$ \\
\hline $\begin{array}{l}\text { Diesel-Range } \\
\text { Hydrocarbons }\end{array}$ & 1 of 3 & ND (220) & 56.8 & NAN & $1,500^{d}$ & $\mathrm{No}^{\mathrm{h}}$ \\
\hline \multicolumn{7}{|c|}{ Volatile Organic Compounds } \\
\hline 1,2,4-Trimethylbenzene & 0 of 3 & ND (1.0) & ND (1.0) & NAN & $12^{\mathrm{e}}$ & $\mathrm{No}^{\dagger}$ \\
\hline 1,3,5-Trimethylbenzene & 0 of 3 & ND (1.0) & $\mathrm{ND}(1.0)$ & NAN & $12^{\mathrm{e}}$ & $\mathrm{No}^{\dagger}$ \\
\hline 2-Butanone & 0 of 3 & ND (20) & ND (20) & NAN & $1,900^{\mathrm{e}}$ & $\mathrm{No}^{\dagger}$ \\
\hline Acetone & 0 of 3 & ND (20) & ND (20) & NAN & $3,700^{\mathrm{e}}$ & $\mathrm{No}^{\dagger}$ \\
\hline Benzene & 0 of 3 & ND (1.0) & $\mathrm{ND}(1.0)$ & NAN & $1.2^{\mathrm{c}}$ & $\mathrm{No}^{\dagger}$ \\
\hline Carbon disulfide & 0 of 3 & ND (1.0) & ND (1.0) & NAN & $1,000^{\mathrm{e}}$ & $\mathrm{No}^{\dagger}$ \\
\hline Ethylbenzene & 0 of 3 & ND (1.0) & ND (1.0) & NAN & $3,100^{c}$ & $\mathrm{No}^{f}$ \\
\hline Isopropylbenzene & 0 of 3 & ND (1.0) & ND (1.0) & NAN & NAV & $\mathrm{No}^{\dagger}$ \\
\hline $\mathrm{m}, \mathrm{p}$-Xylene & 0 of 3 & ND (2.0) & $\mathrm{ND}(2.0)$ & NAN & $12,000^{\mathrm{e}}$ & $\mathrm{No}^{\dagger}$ \\
\hline Methylene chloride & 0 of 3 & ND (2.0) & ND (5.48) & NAN & $4.7^{c}$ & $\mathrm{No}^{\dagger}$ \\
\hline n-Butylbenzene & 0 of 3 & ND (1.0) & ND (1.0) & NAN & $61^{\mathrm{e}}$ & $\mathrm{No}^{f}$ \\
\hline n-Propylbenzene & 0 of 3 & ND (1.0) & ND (1.0) & NAN & $61^{e}$ & $\mathrm{No}^{\dagger}$ \\
\hline Naphthalene & 0 of 3 & ND (1.0) & ND (1.0) & NAN & $730^{e}$ & $\mathrm{No}^{\dagger}$ \\
\hline o-Xylene & 0 of 3 & ND (2.0) & ND (2.0) & NAN & $12,000^{\mathrm{e}}$ & $\mathrm{No}^{\dagger}$ \\
\hline p-Isopropyltoluene & 0 of 3 & ND (1.0) & ND (1.0) & NAN & NAV & $\mathrm{No}^{f}$ \\
\hline sec-Butylbenzene & 0 of 3 & ND (1.0) & ND (1.0) & NAN & $61^{e}$ & $\mathrm{No}^{\dagger}$ \\
\hline Toluene & 0 of 3 & ND (1.0) & ND (1.0) & NAN & $10,000^{c}$ & $\mathrm{No}^{\dagger}$ \\
\hline \multicolumn{7}{|c|}{ Polycyclic Aromatic Hydrocarbons } \\
\hline Acenaphthene & 0 of 3 & ND (0.1) & $\mathrm{ND}(0.1)$ & NAN & $1,200^{c}$ & $\mathrm{No}^{f}$ \\
\hline Acenaphthylene & 0 of 3 & $\mathrm{ND}(0.1)$ & $\mathrm{ND}(0.1)$ & NAN & $0.0028^{c}$ & $\mathrm{No}^{\dagger}$ \\
\hline Anthracene & 0 of 3 & $\mathrm{ND}(0.1)$ & $\mathrm{ND}(0.1)$ & NAN & $0.0028^{c}$ & $\mathrm{No}^{\dagger}$ \\
\hline Benzo(a)anthracene & 0 of 3 & ND $(0.1)$ & ND $(0.1)$ & NAN & $0.0028^{c}$ & $\mathrm{No}^{f}$ \\
\hline Benzo(a)pyrene & 0 of 3 & $\mathrm{ND}(0.1)$ & $\mathrm{ND}(0.1)$ & NAN & $0.0028^{c}$ & $\mathrm{No}^{\dagger}$ \\
\hline Benzo(b)fluoranthene & 0 of 3 & ND $(0.1)$ & $\mathrm{ND}(0.1)$ & NAN & $0.092^{\mathrm{e}}$ & $\mathrm{No}^{f}$ \\
\hline Benzo(ghi)perylene & 0 of 3 & ND $(0.1)$ & $\mathrm{ND}(0.1)$ & NAN & $0.0028^{c}$ & $\mathrm{No}^{f}$ \\
\hline Benzo(k)fluoranthene & 0 of 3 & ND $(0.1)$ & $\mathrm{ND}(0.1)$ & NAN & $0.0028^{\mathrm{C}}$ & $\mathrm{No}^{\dagger}$ \\
\hline
\end{tabular}


Table 4-26

Summary of Analytical Results and Human Health Risk Assessment Screening for Surface Water in Heart Lake, Milrow Site

(Page 2 of 3 )

\begin{tabular}{|c|c|c|c|c|c|c|}
\hline Analyte & $\begin{array}{l}\text { Detection } \\
\text { Frequency }\end{array}$ & $\begin{array}{c}\text { Minimum } \\
\text { Detected } \\
\text { Concentration } \\
(\mu \mathrm{g} / \mathrm{L})\end{array}$ & $\begin{array}{c}\text { Maximum } \\
\text { Detected } \\
\text { Concentration } \\
(\mu \mathrm{g} / \mathrm{L})\end{array}$ & $\begin{array}{c}\text { Lowland } \\
\text { Stream } \\
\text { Background } \\
\text { Concentration }{ }^{\mathrm{a}} \\
(\mu \mathrm{g} / \mathrm{L})\end{array}$ & $\begin{array}{l}\text { Human Health } \\
\text { Screening } \\
\text { Value } \\
(\mu \mathrm{g} / \mathrm{L})\end{array}$ & $\begin{array}{l}\text { Human Health } \\
\text { Constituent of } \\
\text { Concern }\end{array}$ \\
\hline Chrysene & 0 of 3 & $\mathrm{ND}(0.1)$ & $\mathrm{ND}(0.1)$ & NAN & $0.0028^{c}$ & $\mathrm{No}^{f}$ \\
\hline Dibenzo(a,h)anthracene & 0 of 3 & $\mathrm{ND}(0.1)$ & $\mathrm{ND}(0.1)$ & NAN & $0.0028^{c}$ & $\mathrm{No}^{\dagger}$ \\
\hline Fluoranthene & 0 of 3 & ND $(0.1)$ & $\mathrm{ND}(0.1)$ & NAN & $42^{c}$ & $\mathrm{No}^{\dagger}$ \\
\hline Fluorene & 0 of 3 & $\mathrm{ND}(0.1)$ & $\mathrm{ND}(0.1)$ & NAN & $0.0028^{c}$ & $\mathrm{No}^{\dagger}$ \\
\hline Indeno(1,2,3-cd)pyrene & 0 of 3 & ND $(0.1)$ & ND $(0.1)$ & NAN & $0.0028^{c}$ & $\mathrm{No}^{\dagger}$ \\
\hline Naphthalene & 0 of 3 & $\mathrm{ND}(0.1)$ & $\mathrm{ND}(0.1)$ & NAN & $730^{e}$ & $\mathrm{No}^{\dagger}$ \\
\hline Phenanthrene & 0 of 3 & $\mathrm{ND}(0.1)$ & $\mathrm{ND}(0.1)$ & NAN & $0.0028^{c}$ & $\mathrm{No}^{\dagger}$ \\
\hline Pyrene & 0 of 3 & $\mathrm{ND}(0.1)$ & $\mathrm{ND}(0.1)$ & NAN & $0.0028^{c}$ & $\mathrm{No}^{\dagger}$ \\
\hline \multicolumn{7}{|c|}{ Polychlorinated Biphenyls } \\
\hline Aroclor 1016 & NAV & NAV & NAV & NAN & $0.000044^{c}$ & $\mathrm{No}^{\dagger}$ \\
\hline Aroclor 1221 & NAV & NAV & NAV & NAN & $0.000044^{c}$ & $\mathrm{No}^{f}$ \\
\hline Aroclor 1232 & NAV & NAV & NAV & NAN & $0.000044^{c}$ & $\mathrm{No}^{\dagger}$ \\
\hline Aroclor 1242 & NAV & NAV & NAV & NAN & $0.000044^{c}$ & $\mathrm{No}^{f}$ \\
\hline Aroclor 1248 & NAV & NAV & NAV & NAN & $0.000044^{c}$ & $\mathrm{No}^{\dagger}$ \\
\hline Aroclor 1254 & NAV & NAV & NAV & NAN & $0.000044^{c}$ & $\mathrm{No}^{\dagger}$ \\
\hline Aroclor 1260 & NAV & NAV & NAV & NAN & $0.000044^{c}$ & $\mathrm{No}^{f}$ \\
\hline Aroclor 1262 & NAV & NAV & NAV & NAN & $0.000044^{c}$ & $\mathrm{No}^{\dagger}$ \\
\hline Aroclor 1268 & NAV & NAV & NAV & NAN & $0.000044^{c}$ & $\mathrm{No}^{\dagger}$ \\
\hline \multicolumn{7}{|c|}{ Metals } \\
\hline Aluminum & 3 of 3 & 231 & 261 & 269 & $37,000^{\mathrm{e}}$ & $\mathrm{No}^{\mathrm{g}}$ \\
\hline Arsenic & 0 of 3 & ND (1.0) & ND (1.0) & ND (1) & $0.018^{C}$ & $\mathrm{No}^{\dagger}$ \\
\hline Barium & 0 of 3 & ND (10) & ND (10) & ND (10) & $1,000^{c}$ & $\mathrm{No}^{f}$ \\
\hline Beryllium & 0 of 3 & ND (1.0) & ND (1.0) & ND (1) & $0.0076^{c}$ & $\mathrm{No}^{\dagger}$ \\
\hline Boron & 0 of 3 & ND (50) & ND (50) & ND (50) & $3,300^{\mathrm{e}}$ & $\mathrm{No}^{\dagger}$ \\
\hline Cadmium & 0 of 3 & ND (1.0) & ND (1.0) & ND (1) & $10^{c}$ & $\mathrm{No}^{\dagger}$ \\
\hline Calcium & 3 of 3 & 1,390 & 1,490 & $4,320^{b}$ & NAV & $\mathrm{No}^{\mathrm{g}}$ \\
\hline Cerium & 0 of 3 & ND (1.0) & ND (1.0) & ND (1) & NAV & $\mathrm{No}^{f}$ \\
\hline Cesium & 0 of 3 & ND (1.0) & ND (1.0) & ND (1) & NAV & $\mathrm{No}^{\dagger}$ \\
\hline
\end{tabular}


Table 4-26

\section{Summary of Analytical Results and Human Health Risk Assessment Screening for Surface Water in Heart Lake, Milrow Site}

(Page 3 of 3 )

\begin{tabular}{|c|c|c|c|c|c|c|}
\hline Analyte & $\begin{array}{l}\text { Detection } \\
\text { Frequency }\end{array}$ & $\begin{array}{c}\text { Minimum } \\
\text { Detected } \\
\text { Concentration } \\
(\mu \mathrm{g} / \mathrm{L})\end{array}$ & $\begin{array}{c}\text { Maximum } \\
\text { Detected } \\
\text { Concentration } \\
(\mu \mathrm{g} / \mathrm{L})\end{array}$ & $\begin{array}{c}\text { Lowland } \\
\text { Stream } \\
\text { Background } \\
\text { Concentration } \\
(\mu \mathrm{g} / L)\end{array}$ & $\begin{array}{l}\text { Human Health } \\
\text { Screening } \\
\text { Value } \\
(\mu \mathrm{g} / \mathrm{L})\end{array}$ & $\begin{array}{c}\text { Human Health } \\
\text { Constituent of } \\
\text { Concern }\end{array}$ \\
\hline Chromium & 0 of 3 & ND (1.0) & ND (1.0) & $2.25^{b}$ & $33,000^{c}$ & $\mathrm{No}^{\dagger}$ \\
\hline Cobalt & 2 of 3 & ND (1.0) & 1.82 & 3.35 & $2,200^{\mathrm{e}}$ & $\mathrm{No}^{\mathrm{g}}$ \\
\hline Copper & 3 of 3 & 3.23 & 4.47 & 10.8 & $1,300^{\mathrm{C}}$ & $\mathrm{No}^{\mathrm{g}}$ \\
\hline Iron & 3 of 3 & 232 & 243 & 993 & $300^{c}$ & $\mathrm{No}^{\mathrm{g}}$ \\
\hline Lithium & 0 of 3 & ND (1.0) & ND (1.0) & $1.17^{\mathrm{b}}$ & $730^{e}$ & $\mathrm{No}^{f}$ \\
\hline Magnesium & 3 of 3 & 1,460 & 1,480 & $4,690^{b}$ & NAV & $\mathrm{No}^{\mathrm{g}}$ \\
\hline Manganese & 3 of 3 & 6.54 & 6.98 & 267 & $50^{c}$ & $\mathrm{No}^{\mathrm{g}}$ \\
\hline Molybdenum & 0 of 3 & ND (1.0) & ND (1.0) & ND (1) & $180^{e}$ & $\mathrm{No}^{f}$ \\
\hline Nickel & 3 of 3 & 1.41 & 1.81 & ND (1) & $510^{c}$ & $\mathrm{No}^{h}$ \\
\hline Potassium & 3 of 3 & 1,110 & 1,210 & 2,620 & NAV & $\mathrm{No}^{\mathrm{g}}$ \\
\hline Selenium & 0 of 3 & ND (1.0) & ND (1.0) & ND (1) & $104^{c}$ & $\mathrm{No}^{f}$ \\
\hline Strontium & 3 of 3 & 11.6 & 12.4 & 36.1 & $22,000^{\mathrm{e}}$ & $\mathrm{No}^{\mathrm{g}}$ \\
\hline Thallium & 0 of 3 & $\mathrm{ND}(1.0)$ & ND (1.0) & $7.07^{b}$ & $1.7^{\mathrm{C}}$ & $\mathrm{No}^{f}$ \\
\hline Thorium & 0 of 3 & ND (1.0) & ND (1.0) & ND (1) & NAV & $\mathrm{No}^{f}$ \\
\hline Titanium & 3 of 3 & 7.94 & 8.21 & 6.13 & $150,000^{e}$ & $\mathrm{No}^{\mathrm{h}}$ \\
\hline Uranium & 0 of 3 & $\mathrm{ND}(1.0)$ & ND (1.0) & ND (1) & $110^{\mathrm{e}}$ & $\mathrm{No}^{f}$ \\
\hline Vanadium & 0 of 3 & ND (1.0) & ND (1.0) & $4.43^{b}$ & $260^{\mathrm{e}}$ & $\mathrm{No}^{f}$ \\
\hline Zinc & 0 of 3 & ND (10) & ND (10) & $50.3^{b}$ & $11,000^{\mathrm{e}}$ & $\mathrm{No}^{\dagger}$ \\
\hline
\end{tabular}

${ }^{\mathrm{a}}$ Background concentrations are upper tolerance limits (UTL) (ADEC, 1999a) unless otherwise noted.

${ }^{\mathrm{b}}$ Maximum detected concentration in background samples due to insufficient detection frequency to calculate UTL.

${ }^{\mathrm{c}}$ Federal ambient water quality criteria based on ingestion of water and organisms.

${ }^{\mathrm{d}}$ State of Alaska groundwater cleanup level, Discharge Reporting, Cleanup, and Disposal of Oil and other Hazardous Substances

(18 AAC 75, Articles 3 and 9 [ADEC, 1999c]).

'U.S. EPA Region III risk-based concentrations for tap water (EPA, 2000b).

\section{COC Selection Criteria}

Constituent not detected in any samples.

${ }^{g}$ Maximum detected constituent concentration is less than local background concentration.

${ }^{h}$ Maximum detected constituent concentration is less than human health screening value.

ND - Not detected (detection limit)

NAV - Not available

NAN - Not analyzed

$\mu \mathrm{g} / \mathrm{L}$ - Micrograms per liter 
Table 4-27

Summary of Analytical Results and Human Health Risk Assessment Screening for Sediment in Heart Lake, Milrow Site

(Page 1 of 3 )

\begin{tabular}{|c|c|c|c|c|c|c|}
\hline Analyte & $\begin{array}{l}\text { Detection } \\
\text { Frequency }\end{array}$ & $\begin{array}{c}\text { Minimum } \\
\text { Detected } \\
\text { Concentration } \\
(\mathrm{mg} / \mathrm{L})\end{array}$ & $\begin{array}{c}\text { Maximum } \\
\text { Detected } \\
\text { Concentration } \\
(\mathrm{mg} / \mathrm{L})\end{array}$ & $\begin{array}{c}\text { Lowland } \\
\text { Stream } \\
\text { Background } \\
\text { Concentration }{ }^{\mathrm{a}} \\
(\mathrm{mg} / \mathrm{L})\end{array}$ & $\begin{array}{l}\text { Human Health } \\
\text { Screening } \\
\text { Value } \\
(\mathrm{mg} / \mathrm{L})\end{array}$ & $\begin{array}{c}\text { Human Health } \\
\text { Constituent of } \\
\text { Concern }\end{array}$ \\
\hline $\begin{array}{l}\text { Diesel-Range } \\
\text { Hydrocarbons }\end{array}$ & 0 of 5 & ND (4.0) & ND (32.7) & NAN & $200^{c}$ & $\mathrm{No}^{d}$ \\
\hline \multicolumn{7}{|c|}{ Volatile Organic Compounds } \\
\hline 1,2,4-Trimethylbenzene & 0 of 5 & ND (0.005) & ND (0.0409) & NAN & NAV & $\mathrm{No}^{\mathrm{d}}$ \\
\hline 1,3,5-Trimethylbenzene & 0 of 5 & ND (0.005) & ND (0.0409) & NAN & NAV & $\mathrm{No}^{d}$ \\
\hline 2-Butanone & 3 of 5 & ND $(0.01)$ & 0.127 & NAN & NAV & Yes \\
\hline Acetone & 0 of 5 & ND (0.07) & ND (2.24) & NAN & $10,100^{\mathrm{C}}$ & $\mathrm{No}^{d}$ \\
\hline Benzene & 0 of 5 & ND (0.005) & ND (0.0409) & NAN & $286^{\mathrm{C}}$ & $\mathrm{No}^{d}$ \\
\hline Carbon disulfide & 0 of 5 & ND (0.005) & ND (0.0409) & NAN & $10,100^{C}$ & $\mathrm{No}^{d}$ \\
\hline Ethylbenzene & 0 of 5 & ND (0.005) & ND (0.0409) & NAN & $10,100^{c}$ & $\mathrm{No}^{d}$ \\
\hline Isopropylbenzene & 0 of 5 & ND (0.005) & ND (0.0409) & NAN & NAV & $\mathrm{No}^{d}$ \\
\hline $\mathrm{m}, \mathrm{p}$-Xylene & 0 of 5 & ND (0.005) & ND (0.0409) & NAN & $203,000^{c}$ & No ${ }^{d}$ \\
\hline Methylene chloride & 0 of 5 & ND (0.005) & ND (0.0409) & NAN & $1,110^{\mathrm{C}}$ & $\mathrm{No}^{d}$ \\
\hline n-Butylbenzene & 0 of 5 & ND (0.005) & ND (0.0409) & NAN & NAV & $\mathrm{No}^{\mathrm{d}}$ \\
\hline n-Propylbenzene & 0 of 5 & ND (0.005) & ND (0.0409) & NAN & NAV & $\mathrm{No}^{d}$ \\
\hline Naphthalene & 0 of 5 & ND (0.005) & ND (0.0409) & NAN & $4,060^{c}$ & $\mathrm{No}^{d}$ \\
\hline o-Xylene & 0 of 5 & ND (0.005) & ND (0.0409) & NAN & $203,000^{c}$ & $\mathrm{No}^{d}$ \\
\hline p-Isopropyltoluene & 0 of 5 & ND $(0.005)$ & ND (0.0409) & NAN & NAV & $\mathrm{No}^{d}$ \\
\hline sec-Butylbenzene & 0 of 5 & ND (0.005) & ND (0.0409) & NAN & NAV & $\mathrm{No}^{d}$ \\
\hline Toluene & 0 of 5 & ND (0.005) & ND (0.0409) & NAN & $20,300^{c}$ & $\mathrm{No}^{\mathrm{d}}$ \\
\hline \multicolumn{7}{|c|}{ Polycyclic Aromatic Hydrocarbons } \\
\hline Acenaphthene & 0 of 5 & ND (0.0033) & ND (0.027) & NAN & $6,080^{c}$ & $\mathrm{No}^{d}$ \\
\hline Acenaphthylene & 0 of 5 & ND (0.0033) & ND (0.027) & NAN & NAV & $\mathrm{No}^{d}$ \\
\hline Anthracene & 0 of 5 & ND (0.0033) & ND $(0.027)$ & NAN & $30,400^{c}$ & $\mathrm{No}^{d}$ \\
\hline Benzo(a)anthracene & 0 of 5 & ND $(0.0033)$ & ND $(0.027)$ & NAN & $11.4^{\mathrm{C}}$ & $\mathrm{No}^{d}$ \\
\hline Benzo(a)pyrene & 0 of 5 & ND (0.0033) & ND (0.027) & NAN & $1.1^{\mathrm{c}}$ & $\mathrm{No}^{d}$ \\
\hline Benzo(b)fluoranthene & 0 of 5 & ND (0.0033) & ND (0.027) & NAN & $11.4^{\mathrm{C}}$ & $\mathrm{No}^{d}$ \\
\hline Benzo(ghi)perylene & 0 of 5 & ND (0.0033) & ND (0.027) & NAN & $1.1^{\mathrm{c}}$ & $\mathrm{No}^{d}$ \\
\hline Benzo(k)fluoranthene & 0 of 5 & ND (0.0033) & ND (0.027) & NAN & $114^{\mathrm{c}}$ & $\mathrm{No}^{\mathrm{d}}$ \\
\hline
\end{tabular}


Table 4-27

Summary of Analytical Results and Human Health Risk Assessment Screening for Sediment in Heart Lake, Milrow Site

(Page 2 of 3 )

\begin{tabular}{|c|c|c|c|c|c|c|}
\hline Analyte & $\begin{array}{l}\text { Detection } \\
\text { Frequency }\end{array}$ & $\begin{array}{c}\text { Minimum } \\
\text { Detected } \\
\text { Concentration } \\
(\mathrm{mg} / \mathrm{L})\end{array}$ & $\begin{array}{c}\text { Maximum } \\
\text { Detected } \\
\text { Concentration } \\
(\mathrm{mg} / \mathrm{L})\end{array}$ & $\begin{array}{c}\text { Lowland } \\
\text { Stream } \\
\text { Background } \\
\text { Concentration } \\
(\mathrm{mg} / \mathrm{L})\end{array}$ & $\begin{array}{l}\text { Human Health } \\
\text { Screening } \\
\text { Value } \\
(\mathrm{mg} / \mathrm{L})\end{array}$ & $\begin{array}{c}\text { Human Health } \\
\text { Constituent of } \\
\text { Concern }\end{array}$ \\
\hline Chrysene & 0 of 5 & ND $(0.0033)$ & ND (0.027) & NAN & $1,140^{\mathrm{C}}$ & $\mathrm{No}^{\mathrm{d}}$ \\
\hline Dibenzo(a,h)anthracene & 0 of 5 & ND (0.0033) & ND (0.027) & NAN & $1.1^{\mathrm{c}}$ & $\mathrm{No}^{\mathrm{d}}$ \\
\hline Fluoranthene & 0 of 5 & ND $(0.0033)$ & ND $(0.027)$ & NAN & $4,060^{\mathrm{C}}$ & $\mathrm{No}^{\mathrm{d}}$ \\
\hline Fluorene & 0 of 5 & ND (0.0033) & ND $(0.027)$ & NAN & $4,060^{\mathrm{C}}$ & $\mathrm{No}^{\mathrm{d}}$ \\
\hline Indeno(1,2,3-cd)pyrene & 0 of 5 & ND (0.0033) & ND $(0.027)$ & NAN & $11^{\mathrm{c}}$ & $\mathrm{No}^{\mathrm{d}}$ \\
\hline Naphthalene & 0 of 5 & ND (0.0033) & ND (0.027) & NAN & $4,060^{\mathrm{C}}$ & $\mathrm{No}^{\mathrm{d}}$ \\
\hline Phenanthrene & 0 of 5 & ND (0.0033) & ND $(0.027)$ & NAN & $1.1^{\mathrm{C}}$ & $\mathrm{No}^{\mathrm{d}}$ \\
\hline Pyrene & 0 of 5 & ND $(0.0033)$ & ND $(0.027)$ & NAN & $3,040^{\mathrm{C}}$ & $\mathrm{No}^{\mathrm{d}}$ \\
\hline \multicolumn{7}{|c|}{ Polychlorinated Biphenyls } \\
\hline Aroclor 1016 & NAV & NAV & NAV & NAN & $10^{\mathrm{C}}$ & $\mathrm{No}^{\mathrm{d}}$ \\
\hline Aroclor 1221 & NAV & NAV & NAV & NAN & $10^{c}$ & $\mathrm{No}^{\mathrm{d}}$ \\
\hline Aroclor 1232 & NAV & NAV & NAV & NAN & $10^{c}$ & $\mathrm{No}^{d}$ \\
\hline Aroclor 1242 & NAV & NAV & NAV & NAN & $10^{\mathrm{C}}$ & $\mathrm{No}^{\mathrm{d}}$ \\
\hline Aroclor 1248 & NAV & NAV & NAV & NAN & $10^{\mathrm{C}}$ & $\mathrm{No}^{d}$ \\
\hline Aroclor 1254 & NAV & NAV & NAV & NAN & $10^{\mathrm{C}}$ & $\mathrm{No}^{d}$ \\
\hline Aroclor 1260 & NAV & NAV & NAV & NAN & $10^{c}$ & $\mathrm{No}^{d}$ \\
\hline Aroclor 1262 & NAV & NAV & NAV & NAN & $10^{\mathrm{C}}$ & $\mathrm{No}^{d}$ \\
\hline Aroclor 1268 & NAV & NAV & NAV & NAN & $10^{\mathrm{C}}$ & $\mathrm{No}^{\mathrm{d}}$ \\
\hline \multicolumn{7}{|c|}{ Metals } \\
\hline Aluminum & 5 of 5 & 29,500 & 77,500 & 45,400 & NAV & $\mathrm{No}^{h}$ \\
\hline Arsenic & 0 of 5 & ND (0.5) & ND (4.09) & 100 & $5.5^{\mathrm{c}}$ & $\mathrm{No}^{d}$ \\
\hline Barium & 5 of 5 & 60.3 & 188 & 469 & $7,100^{c}$ & $\mathrm{No}^{\mathrm{e}}$ \\
\hline Beryllium & 0 of 5 & ND (0.5) & ND (4.09) & ND & $1.9^{c}$ & $\mathrm{No}^{\mathrm{d}}$ \\
\hline Boron & 0 of 5 & ND (2.5) & ND (20.4) & $7.18^{b}$ & NAV & $\mathrm{No}^{d}$ \\
\hline Cadmium & 0 of 5 & ND (0.5) & ND (4.09) & $0.608^{b}$ & $101^{c}$ & $\mathrm{No}^{d}$ \\
\hline Calcium & 5 of 5 & 1,390 & 8,130 & 7,300 & NAV & $\mathrm{No}^{\mathrm{g}}$ \\
\hline Cerium & 5 of 5 & 8.29 & 16.3 & 14.2 & NAV & $\mathrm{No}^{\mathrm{h}}$ \\
\hline Cesium & 0 of 5 & ND (0.5) & ND (4.09) & ND & NAV & $\mathrm{No}^{d}$ \\
\hline
\end{tabular}


Table 4-27

\section{Summary of Analytical Results and Human Health Risk Assessment Screening for Sediment in Heart Lake, Milrow Site}

(Page 3 of 3 )

\begin{tabular}{|c|c|c|c|c|c|c|}
\hline Analyte & $\begin{array}{l}\text { Detection } \\
\text { Frequency }\end{array}$ & $\begin{array}{c}\text { Minimum } \\
\text { Detected } \\
\text { Concentration } \\
(\mathrm{mg} / \mathrm{L})\end{array}$ & $\begin{array}{c}\text { Maximum } \\
\text { Detected } \\
\text { Concentration } \\
(\mathrm{mg} / \mathrm{L})\end{array}$ & $\begin{array}{c}\text { Lowland } \\
\text { Stream } \\
\text { Background } \\
\text { Concentration }{ }^{\mathrm{a}} \\
(\mathrm{mg} / \mathrm{L})\end{array}$ & $\begin{array}{l}\text { Human Health } \\
\text { Screening } \\
\text { Value } \\
\text { (mg/L) }\end{array}$ & $\begin{array}{c}\text { Human Health } \\
\text { Constituent of } \\
\text { Concern }\end{array}$ \\
\hline Chromium & 5 of 5 & 7.76 & 21.7 & 11.8 & $507^{\mathrm{C}}$ & $\mathrm{No}^{\dagger}$ \\
\hline Cobalt & 5 of 5 & 5.74 & 27 & 43.7 & NAV & $\mathrm{No}^{\mathrm{e}}$ \\
\hline Copper & 5 of 5 & 66.7 & 192 & 71.1 & NAV & $\mathrm{No}^{h}$ \\
\hline Iron & 5 of 5 & 7,660 & 66,400 & 155,000 & NAV & $\mathrm{No}^{\mathrm{e}}$ \\
\hline Lithium & 5 of 5 & 10.9 & 19.1 & 48.8 & NAV & $\mathrm{No}^{\mathrm{e}}$ \\
\hline Magnesium & 5 of 5 & 2,510 & 29,400 & 9,320 & NAV & $\mathrm{No}^{\mathrm{g}}$ \\
\hline Manganese & 5 of 5 & 28.4 & 309 & 20,700 & NAV & $\mathrm{No}^{\mathrm{e}}$ \\
\hline Molybdenum & 0 of 5 & ND (0.5) & ND (4.09) & $5.5^{\mathrm{b}}$ & NAV & $\mathrm{No}^{d}$ \\
\hline Nickel & 5 of 5 & 6.51 & 13.6 & 13.6 & $2,030^{C}$ & $\mathrm{No}^{\mathrm{e}}$ \\
\hline Potassium & 5 of 5 & 258 & 1,580 & 1,390 & NAV & $\mathrm{No}^{\mathrm{g}}$ \\
\hline Selenium & 1 of 5 & ND $(0.5)$ & 4.67 & $5.44^{b}$ & $507^{c}$ & $\mathrm{No}^{\mathrm{e}}$ \\
\hline Strontium & 5 of 5 & 30.7 & 196 & 164 & NAV & $\mathrm{No}^{\mathrm{h}}$ \\
\hline Thallium & 0 of 5 & ND (0.5) & ND (4.09) & $1^{b}$ & NAV & $\mathrm{No}^{d}$ \\
\hline Thorium & 1 of 5 & ND (3.06) & 0.855 & $0.5^{b}$ & NAV & $\mathrm{No}^{\mathrm{h}}$ \\
\hline Titanium & 5 of 5 & 1,460 & 1,830 & 3,010 & NAV & $\mathrm{No}^{\mathrm{e}}$ \\
\hline Uranium & 0 of 5 & $\mathrm{ND}(0.5)$ & ND (4.09) & $4.13^{b}$ & NAV & $\mathrm{No}^{\mathrm{d}}$ \\
\hline Vanadium & 5 of 5 & 118 & 212 & 734 & $710^{c}$ & $\mathrm{No}^{\mathrm{e}}$ \\
\hline Zinc & 4 of 5 & 33.6 & 71 & 434 & $30,400^{c}$ & $\mathrm{No}^{\mathrm{e}}$ \\
\hline
\end{tabular}

${ }^{\mathrm{a} B a c k g r o u n d}$ concentrations are upper tolerance limits (UTL) (ADEC, 1999a) unless otherwise noted.

${ }^{b}$ Maximum detected concentration in background samples due to insufficient detection frequency to calculate UTL.

'State of Alaska soil cleanup level, Discharge Reporting, Cleanup, and Disposal of Oil and other Hazardous Substances

(18 AAC 75, Articles 3 and 9 [ADEC, 1999c]).

\section{COC Selection Criteria}

${ }^{\mathrm{d}}$ Constituent not detected in any samples.

${ }^{\mathrm{e}}$ Maximum detected constituent concentration is less than local background concentration.

${ }^{f}$ Maximum detected constituent concentration is less than human health screening value.

${ }^{9}$ Constituent is an essential macronutrient and only presents a risk at extremely high concentrations.

${ }^{\mathrm{h}}$ Not a constituent associated with drilling mud or other drilling activities.

ND - Not detected (detection limit)

NAV - Not available

NAN - Not analyzed

$\mathrm{mg} / \mathrm{L}$ - Milligrams per liter 
Table 4-28 Summary of Analytical Results and Human Health Risk Assessment
Screening for Surface Water in Cannikin Lake

(Page 1 of 3 )

\begin{tabular}{|c|c|c|c|c|c|c|}
\hline Analyte & $\begin{array}{l}\text { Detection } \\
\text { Frequency }\end{array}$ & $\begin{array}{c}\text { Minimum } \\
\text { Detected } \\
\text { Concentration } \\
(\mu \mathrm{g} / \mathrm{L})\end{array}$ & $\begin{array}{c}\text { Maximum } \\
\text { Detected } \\
\text { Concentration } \\
(\mu \mathrm{g} / \mathrm{L})\end{array}$ & $\begin{array}{c}\text { Lowland } \\
\text { Stream } \\
\text { Background } \\
\text { Concentration } \\
(\mu \mathrm{g} / \mathrm{L})\end{array}$ & $\begin{array}{l}\text { Human Health } \\
\text { Screening } \\
\text { Value } \\
(\mu \mathrm{g} / L)\end{array}$ & $\begin{array}{c}\text { Human Health } \\
\text { Constituent of } \\
\text { Concern }\end{array}$ \\
\hline $\begin{array}{l}\text { Diesel-Range } \\
\text { Hydrocarbons }\end{array}$ & 3 of 3 & 57.8 & 72.2 & NAN & $1,500^{d}$ & $\mathrm{No}^{\mathrm{h}}$ \\
\hline \multicolumn{7}{|c|}{ Volatile Organic Compounds } \\
\hline 1,2,4-Trimethylbenzene & 0 of 3 & ND (1.0) & ND (1.0) & NAN & $12^{\mathrm{e}}$ & $\mathrm{No}^{\dagger}$ \\
\hline 1,3,5-Trimethylbenzene & 0 of 3 & ND (1.0) & ND (1.0) & NAN & $12^{\mathrm{e}}$ & $\mathrm{No}^{\dagger}$ \\
\hline 2-Butanone & 0 of 3 & ND (20) & ND (20) & NAN & $1,900^{\mathrm{e}}$ & $\mathrm{No}^{\dagger}$ \\
\hline Acetone & 0 of 3 & ND (20) & ND (20) & NAN & $3,700^{\mathrm{e}}$ & $\mathrm{No}^{\dagger}$ \\
\hline Benzene & 0 of 3 & ND (1.0) & ND (1.0) & NAN & $1.2^{\mathrm{C}}$ & $\mathrm{No}^{\dagger}$ \\
\hline Carbon disulfide & 0 of 3 & ND (1.0) & ND (1.0) & NAN & $1,000^{\mathrm{e}}$ & $\mathrm{No}^{\dagger}$ \\
\hline Ethylbenzene & 0 of 3 & ND (1.0) & ND (1.0) & NAN & $3,100^{\mathrm{C}}$ & $\mathrm{No}^{\dagger}$ \\
\hline Isopropylbenzene & 0 of 3 & ND (1.0) & ND (1.0) & NAN & NAV & $\mathrm{No}^{\dagger}$ \\
\hline $\mathrm{m}, \mathrm{p}$-Xylene & 0 of 3 & ND (2.0) & ND (2.0) & NAN & $12,000^{\mathrm{e}}$ & $\mathrm{No}^{\dagger}$ \\
\hline Methylene chloride & 0 of 3 & ND (2.03) & ND (5.34) & NAN & $4.7^{\mathrm{C}}$ & $\mathrm{No}^{\dagger}$ \\
\hline n-Butylbenzene & 0 of 3 & ND (1.0) & ND (1.0) & NAN & $61^{\mathrm{e}}$ & $\mathrm{No}^{\dagger}$ \\
\hline n-Propylbenzene & 0 of 3 & ND (1.0) & ND (1.0) & NAN & $61^{e}$ & $\mathrm{No}^{\dagger}$ \\
\hline Naphthalene & 0 of 3 & ND (1.0) & ND (1.0) & NAN & $730^{e}$ & $\mathrm{No}^{f}$ \\
\hline o-Xylene & 0 of 3 & ND (1.0) & ND (1.0) & NAN & $12,000^{\mathrm{e}}$ & $\mathrm{No}^{\dagger}$ \\
\hline p-Isopropyltoluene & 0 of 3 & ND (1.0) & ND (1.0) & NAN & NAV & $\mathrm{No}^{\dagger}$ \\
\hline sec-Butylbenzene & 0 of 3 & ND (1.0) & ND (1.0) & NAN & $61^{e}$ & $\mathrm{No}^{\dagger}$ \\
\hline Toluene & 0 of 3 & ND (1.0) & ND (1.0) & NAN & $10,000^{c}$ & $\mathrm{No}^{f}$ \\
\hline \multicolumn{7}{|c|}{ Polycyclic Aromatic Hydrocarbons } \\
\hline Acenaphthene & 0 of 3 & ND $(0.1)$ & ND $(0.1)$ & NAN & $1,200^{C}$ & $\mathrm{No}^{\dagger}$ \\
\hline Acenaphthylene & 0 of 3 & $\mathrm{ND}(0.1)$ & $\mathrm{ND}(0.1)$ & NAN & $0.0028^{c}$ & $\mathrm{No}^{\dagger}$ \\
\hline Anthracene & 0 of 3 & $\mathrm{ND}(0.1)$ & $\mathrm{ND}(0.1)$ & NAN & $0.0028^{c}$ & $\mathrm{No}^{\dagger}$ \\
\hline Benzo(a)anthracene & 0 of 3 & $\mathrm{ND}(0.1)$ & $\mathrm{ND}(0.1)$ & NAN & $0.0028^{C}$ & $\mathrm{No}^{\dagger}$ \\
\hline Benzo(a)pyrene & 0 of 3 & $\mathrm{ND}(0.1)$ & $\mathrm{ND}(0.1)$ & NAN & $0.0028^{c}$ & $\mathrm{No}^{\dagger}$ \\
\hline Benzo(b)fluoranthene & 0 of 3 & ND $(0.1)$ & $\mathrm{ND}(0.1)$ & NAN & $0.092^{\mathrm{e}}$ & $\mathrm{No}^{\dagger}$ \\
\hline Benzo(g,h,l)perylene & 0 of 3 & $\mathrm{ND}(0.1)$ & ND $(0.1)$ & NAN & $0.0028^{c}$ & $\mathrm{No}^{f}$ \\
\hline Benzo(k)fluoranthene & 0 of 3 & ND $(0.1)$ & ND $(0.1)$ & NAN & $0.0028^{c}$ & $\mathrm{No}^{\dagger}$ \\
\hline Chrysene & 0 of 3 & $\mathrm{ND}(0.1)$ & $\mathrm{ND}(0.1)$ & NAN & $0.0028^{c}$ & $\mathrm{No}^{\dagger}$ \\
\hline
\end{tabular}


Table 4-28 Summary of Analytical Results and Human Health Risk Assessment
Screening for Surface Water in Cannikin Lake

(Page 2 of 3 )

\begin{tabular}{|c|c|c|c|c|c|c|}
\hline Analyte & $\begin{array}{l}\text { Detection } \\
\text { Frequency }\end{array}$ & $\begin{array}{c}\text { Minimum } \\
\text { Detected } \\
\text { Concentration } \\
(\mu \mathrm{g} / \mathrm{L})\end{array}$ & $\begin{array}{c}\text { Maximum } \\
\text { Detected } \\
\text { Concentration } \\
(\mu \mathrm{g} / \mathrm{L})\end{array}$ & $\begin{array}{c}\text { Lowland } \\
\text { Stream } \\
\text { Background } \\
\text { Concentration }{ }^{\mathrm{a}} \\
(\mu \mathrm{g} / \mathrm{L})\end{array}$ & $\begin{array}{l}\text { Human Health } \\
\text { Screening } \\
\text { Value } \\
(\mu \mathrm{g} / \mathrm{L})\end{array}$ & $\begin{array}{c}\text { Human Health } \\
\text { Constituent of } \\
\text { Concern }\end{array}$ \\
\hline Dibenzo(a,h)anthracene & 0 of 3 & $\mathrm{ND}(0.1)$ & $\mathrm{ND}(0.1)$ & NAN & $0.0028^{C}$ & $\mathrm{No}^{f}$ \\
\hline Fluoranthene & 0 of 3 & $\mathrm{ND}(0.1)$ & ND $(0.1)$ & NAN & $42^{c}$ & $\mathrm{No}^{\dagger}$ \\
\hline Fluorene & 0 of 3 & $\mathrm{ND}(0.1)$ & $\mathrm{ND}(0.1)$ & NAN & $0.0028^{C}$ & $\mathrm{No}^{\dagger}$ \\
\hline Indeno(1,2,3-cd0pyrene & 0 of 3 & $\mathrm{ND}(0.1)$ & $\mathrm{ND}(0.1)$ & NAN & $0.0028^{c}$ & $\mathrm{No}^{f}$ \\
\hline Naphthalene & 0 of 3 & $\mathrm{ND}(0.1)$ & $\mathrm{ND}(0.1)$ & NAN & $730^{\mathrm{e}}$ & $\mathrm{No}^{\dagger}$ \\
\hline Phenanthrene & 0 of 3 & $\mathrm{ND}(0.1)$ & $\mathrm{ND}(0.1)$ & NAN & $0.0028^{c}$ & $\mathrm{No}^{\dagger}$ \\
\hline Pyrene & 0 of 3 & $\mathrm{ND}(0.1)$ & $\mathrm{ND}(0.1)$ & NAN & $0.0028^{c}$ & $\mathrm{No}^{f}$ \\
\hline \multicolumn{7}{|c|}{ Polychlorinated Biphenyls } \\
\hline Aroclor 1016 & 0 of 3 & ND (0.5) & ND (0.5) & NAN & $0.000044^{c}$ & $\mathrm{No}^{f}$ \\
\hline Aroclor 1221 & 0 of 3 & $\mathrm{ND}(0.5)$ & ND (0.5) & NAN & $0.000044^{c}$ & $\mathrm{No}^{\dagger}$ \\
\hline Aroclor 1232 & 0 of 3 & ND $(0.5)$ & ND $(0.5)$ & NAN & $0.000044^{c}$ & $\mathrm{No}^{f}$ \\
\hline Aroclor 1242 & 0 of 3 & ND (0.5) & ND (0.5) & NAN & $0.000044^{c}$ & $\mathrm{No}^{f}$ \\
\hline Aroclor 1248 & 0 of 3 & $\mathrm{ND}(0.5)$ & $\mathrm{ND}(0.5)$ & NAN & $0.000044^{c}$ & $\mathrm{No}^{f}$ \\
\hline Aroclor 1254 & 0 of 3 & ND (0.5) & $\mathrm{ND}(0.5)$ & NAN & $0.000044^{c}$ & $\mathrm{No}^{\dagger}$ \\
\hline Aroclor 1260 & 0 of 3 & ND (0.5) & ND (0.5) & NAN & $0.000044^{c}$ & $\mathrm{No}^{f}$ \\
\hline Aroclor 1262 & 0 of 3 & ND (0.5) & ND (0.5) & NAN & $0.000044^{c}$ & $\mathrm{No}^{\dagger}$ \\
\hline Aroclor 1268 & 0 of 3 & ND (0.5) & ND (0.5) & NAN & $0.000044^{c}$ & $\mathrm{No}^{\dagger}$ \\
\hline \multicolumn{7}{|c|}{ Metals } \\
\hline Aluminum & 3 of 3 & 77.8 & 102 & 269 & $37,000^{\mathrm{e}}$ & $\mathrm{No}^{\mathrm{g}}$ \\
\hline Arsenic & 3 of 3 & 6.81 & 7.53 & ND (1) & $0.018^{C}$ & $\mathrm{No}^{\mathrm{j}}$ \\
\hline Barium & 0 of 3 & ND (10) & ND (10) & ND (10) & $1,000^{\mathrm{C}}$ & $\mathrm{No}^{\dagger}$ \\
\hline Beryllium & 0 of 3 & ND (1.0) & ND (1.0) & ND (1) & $0.0076^{c}$ & $\mathrm{No}^{f}$ \\
\hline Boron & 3 of 3 & 429 & 468 & ND (50) & $3,300^{\mathrm{e}}$ & $\mathrm{No}^{h}$ \\
\hline Cadmium & 0 of 3 & ND (1) & ND (1) & ND (1) & $10^{c}$ & $\mathrm{No}^{\dagger}$ \\
\hline Calcium & 3 of 3 & 5,250 & 5,500 & $4,320^{b}$ & NAV & $\mathrm{No}^{\mathrm{i}}$ \\
\hline Cerium & 0 of 3 & ND (1) & ND (1) & ND (1) & NAV & $\mathrm{No}^{\dagger}$ \\
\hline Cesium & 0 of 3 & ND (1) & ND (1) & ND (1) & NAV & $\mathrm{No}^{f}$ \\
\hline Chromium & 3 of 3 & 1.91 & 2.05 & $2.25^{b}$ & $33,000^{c}$ & $\mathrm{No}^{\mathrm{g}}$ \\
\hline Cobalt & 2 of 3 & ND (1.0) & 4.81 & 3.35 & $2,200^{\mathrm{e}}$ & $\mathrm{No}^{\mathrm{h}}$ \\
\hline
\end{tabular}


Table 4-28

Summary of Analytical Results and Human Health Risk Assessment
Screening for Surface Water in Cannikin Lake (Page 3 of 3 )

\begin{tabular}{|c|c|c|c|c|c|c|}
\hline Analyte & $\begin{array}{l}\text { Detection } \\
\text { Frequency }\end{array}$ & $\begin{array}{c}\text { Minimum } \\
\text { Detected } \\
\text { Concentration } \\
(\mu \mathrm{g} / \mathrm{L})\end{array}$ & $\begin{array}{c}\text { Maximum } \\
\text { Detected } \\
\text { Concentration } \\
(\mu \mathrm{g} / \mathrm{L})\end{array}$ & $\begin{array}{c}\text { Lowland } \\
\text { Stream } \\
\text { Background } \\
\text { Concentration }{ }^{\mathrm{a}} \\
(\mu \mathrm{g} / \mathrm{L})\end{array}$ & $\begin{array}{l}\text { Human Health } \\
\text { Screening } \\
\text { Value } \\
(\mu \mathrm{g} / L)\end{array}$ & $\begin{array}{c}\text { Human Health } \\
\text { Constituent of } \\
\text { Concern }\end{array}$ \\
\hline Copper & 3 of 3 & 1.66 & 2.5 & 10.8 & $1,300^{c}$ & $\mathrm{No}^{\mathrm{g}}$ \\
\hline Iron & 3 of 3 & 222 & 242 & 993 & $300^{c}$ & $\mathrm{No}^{\mathrm{g}}$ \\
\hline Lithium & 0 of 3 & ND (1.0) & ND (1.0) & $1.17^{\mathrm{b}}$ & $730^{e}$ & $\mathrm{No}^{\dagger}$ \\
\hline Magnesium & 3 of 3 & 1,840 & 1,890 & $4,690^{b}$ & NAV & $\mathrm{No}^{\mathrm{g}}$ \\
\hline Manganese & 3 of 3 & 17.4 & 28.2 & 267 & $50^{c}$ & $\mathrm{No}^{\mathrm{g}}$ \\
\hline Molybdenum & 0 of 3 & ND (1.0) & ND (1.0) & ND (1) & $180^{\mathrm{e}}$ & $\mathrm{No}^{f}$ \\
\hline Nickel & 3 of 3 & ND (1) & 1.2 & ND (1) & $510^{c}$ & $\mathrm{No}^{h}$ \\
\hline Potassium & 3 of 3 & 689 & 773 & 2,620 & NAV & $\mathrm{No}^{\mathrm{g}}$ \\
\hline Selenium & 0 of 3 & ND (1) & ND (1) & ND (1) & $104^{c}$ & $\mathrm{No}^{\dagger}$ \\
\hline Strontium & 3 of 3 & 28.8 & 29.8 & 36.1 & $22,000^{\mathrm{e}}$ & $\mathrm{No}^{\mathrm{g}}$ \\
\hline Thallium & 1 of 3 & ND (1.0) & 1.27 & $7.07^{b}$ & $1.7^{\mathrm{C}}$ & $\mathrm{No}^{\mathrm{g}}$ \\
\hline Thorium & 0 of 3 & ND (1) & ND (1) & ND (1) & NAV & $\mathrm{No}^{\dagger}$ \\
\hline Titanium & 3 of 3 & 2.81 & 4.1 & 6.13 & $150,000^{e}$ & $\mathrm{No}^{\mathrm{g}}$ \\
\hline Uranium & 0 of 3 & ND (1) & ND (1) & ND (1) & $110^{e}$ & $\mathrm{No}^{f}$ \\
\hline Vanadium & 3 of 3 & 5.04 & 5.31 & $4.43^{b}$ & $260^{e}$ & $\mathrm{No}^{\mathrm{h}}$ \\
\hline Zinc & 0 of 3 & ND (10) & ND (10) & $50.3^{b}$ & $11,000^{\mathrm{e}}$ & $\mathrm{No}^{f}$ \\
\hline
\end{tabular}

${ }^{\mathrm{a}}$ Background concentrations are upper tolerance limits (UTL) (ADEC, 1999a) unless otherwise noted.

${ }^{\mathrm{b}}$ Maximum detected concentration in background samples due to insufficient detection frequency to calculate UTL.

${ }^{\mathrm{c}}$ Federal ambient water quality criteria based on ingestion of water and organisms.

${ }^{d}$ State of Alaska groundwater cleanup level, Discharge Reporting, Cleanup, and Disposal of Oil and other Hazardous Substances (18 AAC 75, Articles 3 and 9 [ADEC, 1999c]).

eU.S. EPA Region III risk-based concentrations for tap water (EPA, 2000b).

\section{COC Selection Criteria}

${ }^{7}$ Constituent not detected in any samples.

${ }^{9}$ Maximum detected constituent concentration is less than local background concentration.

${ }^{h}$ Maximum detected constituent concentration is less than human health screening value.

i'Constituent is an essential macronutrient and only presents a risk at extremely high concentrations.

${ }^{\mathrm{j}}$ Not a constituent associated with drilling mud or other drilling activities.

ND - Not detected (detection limit)

NAV - Not available

NAN - Not analyzed

$\mu \mathrm{g} / \mathrm{L}$ - Micrograms per liter 
Table 4-29

Summary of Analytical Results and Human Health Risk Assessment Screening for Sediment in Cannikin Lake

(Page 1 of 3 )

\begin{tabular}{|c|c|c|c|c|c|c|}
\hline Analyte & $\begin{array}{l}\text { Detection } \\
\text { Frequency }\end{array}$ & $\begin{array}{c}\text { Minimum } \\
\text { Detected } \\
\text { Concentration } \\
(\mathrm{mg} / \mathrm{L})\end{array}$ & $\begin{array}{c}\text { Maximum } \\
\text { Detected } \\
\text { Concentration } \\
(\mathrm{mg} / \mathrm{L})\end{array}$ & $\begin{array}{c}\text { Lowland } \\
\text { Stream } \\
\text { Background } \\
\text { Concentration }{ }^{\mathrm{a}} \\
(\mathrm{mg} / \mathrm{L})\end{array}$ & $\begin{array}{l}\text { Human Health } \\
\text { Screening } \\
\text { Value } \\
\text { (mg/L) }\end{array}$ & $\begin{array}{c}\text { Human Health } \\
\text { Constituent of } \\
\text { Concern }\end{array}$ \\
\hline \multicolumn{7}{|c|}{ Petroleum Hydrocarbons } \\
\hline $\begin{array}{l}\text { Gasoline-Range } \\
\text { Hydrocarbons }\end{array}$ & 1 of 3 & ND (5.0) & 25.9 & NAN & $200^{c}$ & $\mathrm{No}^{\dagger}$ \\
\hline $\begin{array}{l}\text { Diesel-Range } \\
\text { Hydrocarbons }\end{array}$ & 3 of 3 & 110 & 4,710 & NAN & $100^{\mathrm{C}}$ & Yes \\
\hline \multicolumn{7}{|c|}{ Volatile Organic Compounds } \\
\hline 1,2,4-Trimethylbenzene & 2 of 3 & ND (0.493) & 5.16 & NAN & NAV & Yes \\
\hline 1,3,5-Trimethylbenzene & 1 of 3 & ND $(0.15)$ & 1.21 & NAN & NAV & Yes \\
\hline 2-Butanone & 0 of 3 & ND (3.5) & ND (12.3) & NAN & NAV & $\mathrm{No}^{\mathrm{d}}$ \\
\hline Acetone & 0 of 3 & ND (3.5) & ND (12.3) & NAN & $10,100^{C}$ & No ${ }^{d}$ \\
\hline Benzene & 0 of 3 & ND $(0.14)$ & ND (0.493) & NAN & $286^{\mathrm{C}}$ & Nod \\
\hline Carbon disulfide & 0 of 3 & $\mathrm{ND}(0.7)$ & ND (2.47) & NAN & $10,100^{c}$ & $\mathrm{No}^{\mathrm{d}}$ \\
\hline Ethylbenzene & 1 of 3 & ND $(0.15)$ & 0.186 & NAN & $10,100^{c}$ & $\mathrm{No}^{\dagger}$ \\
\hline Isopropylbenzene & 0 of 3 & ND $(0.14)$ & ND $(0.493)$ & NAN & NAV & $\mathrm{No}^{d}$ \\
\hline m,p-Xylene & 1 of 3 & $\mathrm{ND}(0.3)$ & 0.836 & NAN & $203,000^{C}$ & $\mathrm{No}^{f}$ \\
\hline Methylene chloride & 0 of 3 & $\mathrm{ND}(0.7)$ & ND (2.47) & NAN & $1,110^{\mathrm{C}}$ & $\mathrm{No}^{d}$ \\
\hline n-Butylbenzene & 2 of 3 & 0.311 & 0.496 & NAN & NAV & Yes \\
\hline n-Propylbenzene & 2 of 3 & 0.15 & 0.369 & NAN & NAV & Yes \\
\hline Naphthalene & 1 of 3 & ND $(0.15)$ & 5.53 & NAN & $4,060^{c}$ & $\mathrm{No}^{\dagger}$ \\
\hline o-Xylene & 1 of 3 & ND (0.15) & 0.498 & NAN & $203,000^{c}$ & $\mathrm{No}^{f}$ \\
\hline p-Isopropyltoluene & 2 of 3 & 0.282 & 0.304 & NAN & NAV & Yes \\
\hline sec-Butylbenzene & 2 of 3 & 0.202 & 0.233 & NAN & NAV & Yes \\
\hline Toluene & 0 of 3 & ND $(0.14)$ & ND (0.493) & NAN & $20,300^{c}$ & $\mathrm{No}^{d}$ \\
\hline \multicolumn{7}{|c|}{ Polycyclic Aromatic Hydrocarbons } \\
\hline Acenaphthene & 2 of 3 & ND (0.00798) & 1.89 & NAN & $6,080^{c}$ & $\mathrm{No}^{f}$ \\
\hline Acenaphthylene & 0 of 3 & ND $(0.00798)$ & $\mathrm{ND}(1.0)$ & NAN & NAV & No ${ }^{d}$ \\
\hline Anthracene & 2 of 3 & ND (0.00798) & 0.403 & NAN & $30,400^{c}$ & $\mathrm{No}^{f}$ \\
\hline Benzo(a)anthracene & 0 of 3 & ND (0.00798) & ND (0.033) & NAN & $11.4^{\mathrm{C}}$ & $\mathrm{No}^{d}$ \\
\hline Benzo(a)pyrene & 0 of 3 & ND (0.00798) & ND (0.033) & NAN & $1.1^{\mathrm{c}}$ & $\mathrm{No}^{d}$ \\
\hline Benzo(b)fluoranthene & 0 of 3 & ND (0.00798) & ND (0.033) & NAN & $11.4^{\mathrm{C}}$ & Nod \\
\hline
\end{tabular}


Table 4-29

Summary of Analytical Results and Human Health Risk Assessment Screening for Sediment in Cannikin Lake

(Page 2 of 3 )

\begin{tabular}{|c|c|c|c|c|c|c|}
\hline Analyte & $\begin{array}{l}\text { Detection } \\
\text { Frequency }\end{array}$ & $\begin{array}{c}\text { Minimum } \\
\text { Detected } \\
\text { Concentration } \\
(\mathrm{mg} / \mathrm{L})\end{array}$ & $\begin{array}{c}\text { Maximum } \\
\text { Detected } \\
\text { Concentration } \\
(\mathrm{mg} / \mathrm{L})\end{array}$ & $\begin{array}{c}\text { Lowland } \\
\text { Stream } \\
\text { Background } \\
\text { Concentration }{ }^{\mathrm{a}} \\
(\mathrm{mg} / \mathrm{L})\end{array}$ & $\begin{array}{l}\text { Human Health } \\
\text { Screening } \\
\text { Value } \\
(\mathrm{mg} / \mathrm{L})\end{array}$ & $\begin{array}{c}\text { Human Health } \\
\text { Constituent of } \\
\text { Concern }\end{array}$ \\
\hline Benzo(g,h,i)perylene & 0 of 3 & ND (0.00798) & ND (0.033) & NAN & $1.1^{\mathrm{c}}$ & $\mathrm{No}^{d}$ \\
\hline Benzo(k)fluoranthene & 0 of 3 & ND (0.00798) & ND (0.033) & NAN & $114^{\mathrm{C}}$ & $\mathrm{No}^{\mathrm{d}}$ \\
\hline Chrysene & 0 of 3 & ND (0.00798) & ND (0.033) & NAN & $1,140^{\mathrm{C}}$ & $\mathrm{No}^{d}$ \\
\hline Dibenzo(a,h)anthracene & 0 of 3 & ND (0.00798) & ND (0.033) & NAN & $1.1^{\mathrm{C}}$ & $\mathrm{No}^{\mathrm{d}}$ \\
\hline Fluoranthene & 2 of 3 & ND $(0.00798)$ & 0.227 & NAN & $4,060^{c}$ & $\mathrm{No}^{\dagger}$ \\
\hline Fluorene & 2 of 3 & ND $(0.00798)$ & 4.87 & NAN & $4,060^{\mathrm{C}}$ & $\mathrm{No}^{f}$ \\
\hline Indeno(1,2,3-cd)pyrene & 0 of 3 & ND (0.00798) & ND (0.033) & NAN & $11^{\mathrm{c}}$ & $\mathrm{No}^{d}$ \\
\hline Naphthalene & 2 of 3 & ND (0.00798) & 1.4 & NAN & $4,060^{c}$ & $\mathrm{No}^{\dagger}$ \\
\hline Phenanthrene & 2 of 3 & ND (0.00798) & 12.1 & NAN & $1.1^{\mathrm{c}}$ & Yes \\
\hline Pyrene & 2 of 3 & ND $(0.00798)$ & 0.34 & NAN & $3,040^{c}$ & $\mathrm{No}^{f}$ \\
\hline \multicolumn{7}{|c|}{ Polychlorinated Biphenyls } \\
\hline Aroclor 1016 & 0 of 3 & ND (0.05) & ND $(0.121)$ & NAN & $10^{c}$ & $\mathrm{No}^{d}$ \\
\hline Aroclor 1221 & 0 of 3 & ND (0.05) & ND $(0.121)$ & NAN & $10^{\mathrm{C}}$ & $\mathrm{No}^{d}$ \\
\hline Aroclor 1232 & 0 of 3 & ND (0.05) & ND $(0.121)$ & NAN & $10^{\mathrm{C}}$ & $\mathrm{No}^{d}$ \\
\hline Aroclor 1242 & 0 of 3 & ND (0.05) & ND $(0.121)$ & NAN & $10^{\mathrm{C}}$ & $\mathrm{No}^{d}$ \\
\hline Aroclor 1248 & 1 of 3 & ND (0.05) & 0.185 & NAN & $10^{c}$ & $\mathrm{No}^{f}$ \\
\hline Aroclor 1254 & 0 of 3 & ND (0.05) & ND (0.121) & NAN & $10^{c}$ & $\mathrm{No}^{d}$ \\
\hline Aroclor 1260 & 2 of 3 & ND (0.05) & 0.107 & NAN & $10^{\mathrm{C}}$ & $\mathrm{No}^{\dagger}$ \\
\hline Aroclor 1262 & 0 of 3 & ND (0.05) & ND (0.05) & NAN & $10^{c}$ & $\mathrm{No}^{d}$ \\
\hline Aroclor 1268 & 0 of 3 & ND (0.05) & ND (0.05) & NAN & $10^{c}$ & $\mathrm{No}^{d}$ \\
\hline \multicolumn{7}{|c|}{ Metals } \\
\hline Aluminum & 3 of 3 & 29,300 & 55,900 & 45,400 & NAV & $\mathrm{No}^{\mathrm{h}}$ \\
\hline Arsenic & 3 of 3 & 9.15 & 20.4 & 100 & $5.5^{c}$ & $\mathrm{No}^{\mathrm{e}}$ \\
\hline Barium & 3 of 3 & 60 & 198 & 469 & $7,100^{c}$ & $\mathrm{No}^{\mathrm{e}}$ \\
\hline Beryllium & 0 of 3 & ND (1.21) & ND (2.5) & ND (1) & $1.9^{c}$ & $\mathrm{No}^{d}$ \\
\hline Boron & 3 of 3 & 7.01 & 15.6 & $7.18^{b}$ & NAV & $\mathrm{No}^{\mathrm{h}}$ \\
\hline Cadmium & 1 of 3 & ND (0.5) & 0.876 & $0.608^{b}$ & $101^{c}$ & $\mathrm{No}^{\dagger}$ \\
\hline Calcium & 3 of 3 & 3,300 & 25,900 & 7,300 & NAV & $\mathrm{No}^{\mathrm{g}}$ \\
\hline Cerium & 3 of 3 & 4.73 & 21.1 & 14.2 & NAV & $\mathrm{No}^{h}$ \\
\hline
\end{tabular}


Table 4-29 Summary of Analytical Results and Human Health Risk Assessment
Screening for Sediment in Cannikin Lake

(Page 3 of 3 )

\begin{tabular}{|c|c|c|c|c|c|c|}
\hline Analyte & $\begin{array}{l}\text { Detection } \\
\text { Frequency }\end{array}$ & $\begin{array}{c}\text { Minimum } \\
\text { Detected } \\
\text { Concentration } \\
(\mathrm{mg} / \mathrm{L})\end{array}$ & $\begin{array}{c}\text { Maximum } \\
\text { Detected } \\
\text { Concentration } \\
(\mathrm{mg} / \mathrm{L})\end{array}$ & $\begin{array}{c}\text { Lowland } \\
\text { Stream } \\
\text { Background } \\
\text { Concentration } \\
\text { (mg/L) }\end{array}$ & $\begin{array}{l}\text { Human Health } \\
\text { Screening } \\
\text { Value } \\
(\mathrm{mg} / \mathrm{L})\end{array}$ & $\begin{array}{c}\text { Human Health } \\
\text { Constituent of } \\
\text { Concern }\end{array}$ \\
\hline Cesium & 1 of 3 & $\mathrm{ND}(0.5)$ & 1.11 & ND & NAV & $\mathrm{No}^{\mathrm{h}}$ \\
\hline Chromium & 3 of 3 & 13.8 & 257 & 11.8 & $507^{c}$ & $\mathrm{No}^{\dagger}$ \\
\hline Cobalt & 3 of 3 & 8.62 & 21.6 & 43.7 & NAV & $\mathrm{No}^{\mathrm{e}}$ \\
\hline Copper & 3 of 3 & 65.6 & 203 & 71.1 & NAV & $\mathrm{No}^{h}$ \\
\hline Iron & 3 of 3 & 36,900 & 55,900 & 155,000 & NAV & $\mathrm{No}^{\mathrm{g}}$ \\
\hline Lithium & 3 of 3 & 14.7 & 15.8 & 48.8 & NAV & $\mathrm{No}^{\mathrm{e}}$ \\
\hline Magnesium & 3 of 3 & 11,400 & 23,300 & 9,320 & NAV & $\mathrm{No}^{\mathrm{g}}$ \\
\hline Manganese & 3 of 3 & 357 & 956 & 20,700 & NAV & $\mathrm{No}^{\mathrm{e}}$ \\
\hline Molybdenum & 0 of 3 & ND (1.21) & ND (2.5) & $5.5^{b}$ & NAV & $\mathrm{No}^{d}$ \\
\hline Nickel & 3 of 3 & 17.5 & 66.9 & 13.6 & $2,030^{\mathrm{C}}$ & $\mathrm{No}^{\dagger}$ \\
\hline Potassium & 3 of 3 & 379 & 1,290 & 1,390 & NAV & $\mathrm{No}^{\mathrm{e}}$ \\
\hline Selenium & 3 of 3 & 0.707 & 1.64 & $5.44^{b}$ & $507^{c}$ & $\mathrm{No}^{\mathrm{e}}$ \\
\hline Strontium & 3 of 3 & 32.3 & 251 & 164 & NAV & $\mathrm{No}^{\mathrm{h}}$ \\
\hline Thallium & 0 of 3 & ND (0.5) & ND (1.21) & $1^{b}$ & NAV & $\mathrm{No}^{d}$ \\
\hline Thorium & 2 of 3 & ND (1.21) & 2.81 & $0.5^{\mathrm{b}}$ & NAV & $\mathrm{No}^{\mathrm{h}}$ \\
\hline Titanium & 3 of 3 & 798 & 1,360 & 3,010 & NAV & $\mathrm{No}^{\mathrm{e}}$ \\
\hline Uranium & 2 of 3 & ND (1.21) & 1.32 & $4.13^{b}$ & NAV & $\mathrm{No}^{\mathrm{e}}$ \\
\hline Vanadium & 3 of 3 & 118 & 164 & 734 & $710^{c}$ & $\mathrm{No}^{\mathrm{e}}$ \\
\hline Zinc & 3 of 3 & 53.9 & 78.9 & 434 & $30,400^{c}$ & $\mathrm{No}^{\mathrm{e}}$ \\
\hline
\end{tabular}

a Background concentrations are upper tolerance limits (UTL) (ADEC, 1999a) unless otherwise noted.

${ }^{b}$ Maximum detected concentration in background samples due to insufficient detection frequency to calculate UTL.

${ }^{\mathrm{c}}$ State of Alaska soil cleanup level, Discharge Reporting, Cleanup, and Disposal of Oil and other Hazardous Substances (18 AAC 75, Articles 3 and 9 [ADEC, 1999c]).

\section{COC Selection Criteria}

${ }^{\mathrm{d}}$ Constituent not detected in any samples.

${ }^{\mathrm{e}}$ Maximum detected constituent concentration is less than local background concentration.

${ }^{f}$ Maximum detected constituent concentration is less than human health screening value.

${ }^{9}$ Constituent is an essential macronutrient and only presents a risk at extremely high concentrations.

${ }^{h}$ Not a constituent associated with drilling mud or other drilling activities.

ND - Not detected (detection limit)

NAV - Not available

NAN - Not analyzed

$\mathrm{mg} / \mathrm{L}$ - Milligrams per liter 
Table 4-30

Summary of Analytical Results and Human Health Risk Assessment Screening for Fish Tissue in Cannikin Lake

(Page 1 of 2)

\begin{tabular}{|c|c|c|c|c|c|c|}
\hline Analyte & $\begin{array}{l}\text { Detection } \\
\text { Frequency }\end{array}$ & $\begin{array}{c}\text { Minimum } \\
\text { Detected } \\
\text { Concentration } \\
(\mathrm{mg} / \mathrm{kg})\end{array}$ & $\begin{array}{c}\text { Maximum } \\
\text { Detected } \\
\text { Concentration } \\
(\mathbf{m g} / \mathrm{kg})\end{array}$ & $\begin{array}{c}\text { Lowland } \\
\text { Stream } \\
\text { Background } \\
\text { Concentration } \\
\text { (mg/kg) }\end{array}$ & $\begin{array}{c}\text { Human Health } \\
\text { Screening } \\
\text { Value }^{c} \\
(\mathbf{m g} / \mathbf{k g})\end{array}$ & $\begin{array}{c}\text { Human Health } \\
\text { Constituent of } \\
\text { Concern }\end{array}$ \\
\hline \multicolumn{7}{|c|}{ Polycyclic Aromatic Hydrocarbons } \\
\hline Acenaphthene & 0 of 2 & ND (0.0223) & ND $(0.0223)$ & NAN & 81.1 & $\mathrm{No}^{\mathrm{d}}$ \\
\hline Acenaphthylene & 0 of 2 & ND (0.0223) & ND (0.0223) & NAN & NAV & $\mathrm{No}^{\mathrm{d}}$ \\
\hline Anthracene & 0 of 2 & ND (0.223) & ND (0.223) & NAN & 410 & $\mathrm{No}^{d}$ \\
\hline Benzo(a)anthracene & 0 of 2 & ND (0.223) & ND (0.223) & NAN & 0.0043 & $\mathrm{No}^{d}$ \\
\hline Benzo(a)pyrene & 0 of 2 & ND (0.223) & ND (0.223) & NAN & 0.00043 & $\mathrm{No}^{d}$ \\
\hline Benzo(b)fluoranthene & 0 of 2 & ND $(0.223)$ & ND $(0.223)$ & NAN & 0.0043 & $\mathrm{No}^{\mathrm{d}}$ \\
\hline Benzo(ghi)perylene & 0 of 2 & ND (0.223) & ND (0.223) & NAN & NAV & $\mathrm{No}^{\mathrm{d}}$ \\
\hline Benzo(k)fluoranthene & 0 of 2 & ND (0.223) & ND (0.223) & NAN & 0.043 & $\mathrm{No}^{d}$ \\
\hline Chrysene & 0 of 2 & ND (0.223) & ND (0.223) & NAN & 0.43 & $\mathrm{No}^{\mathrm{d}}$ \\
\hline Dibenzo(a,h)anthracene & 0 of 2 & ND $(0.223)$ & ND $(0.223)$ & NAN & 0.00043 & $\mathrm{No}^{\mathrm{d}}$ \\
\hline Fluoranthene & 0 of 2 & ND (0.223) & ND (0.446) & NAN & 54 & $\mathrm{No}^{\mathrm{d}}$ \\
\hline Fluorene & 0 of 2 & ND (0.0223) & ND (0.0223) & NAN & 54 & $\mathrm{No}^{d}$ \\
\hline Indeno(1,2,3-cd)pyrene & 0 of 2 & ND (0.223) & ND (0.223) & NAN & 0.0043 & $\mathrm{No}^{d}$ \\
\hline Naphthalene & 0 of 2 & ND (0.0223) & ND (0.0223) & NAN & 27 & $\mathrm{No}^{d}$ \\
\hline Phenanthrene & 0 of 2 & ND (0.223) & ND (0.446) & NAN & NAV & $\mathrm{No}^{\mathrm{d}}$ \\
\hline Pyrene & 0 of 2 & ND (0.223) & ND (0.223) & NAN & 41 & $\mathrm{No}^{d}$ \\
\hline \multicolumn{7}{|c|}{ Polychlorinated Biphenyls } \\
\hline Aroclor 1016 & 0 of 2 & ND (0.025) & ND (0.025) & NAN & 0.045 & $\mathrm{No}^{\mathrm{d}}$ \\
\hline Aroclor 1221 & 0 of 2 & ND (0.05) & ND (0.05) & NAN & 0.0016 & $\mathrm{No}^{\mathrm{d}}$ \\
\hline Aroclor 1232 & 0 of 2 & ND (0.025) & ND (0.025) & NAN & 0.0016 & $\mathrm{No}^{d}$ \\
\hline Aroclor 1242 & 0 of 2 & ND (0.025) & ND (0.025) & NAN & 0.0016 & $\mathrm{No}^{d}$ \\
\hline Aroclor 1248 & 0 of 2 & ND (0.025) & ND (0.025) & NAN & 0.0016 & $\mathrm{No}^{d}$ \\
\hline Aroclor 1254 & 0 of 2 & ND (0.025) & ND (0.025) & NAN & 0.0016 & $\mathrm{No}^{\mathrm{d}}$ \\
\hline Aroclor 1260 & 2 of 2 & 0.019 & 0.0248 & NAN & 0.0016 & Yes \\
\hline \multicolumn{7}{|c|}{ Metals } \\
\hline Aluminum & 1 of 2 & ND (2.5) & 66.5 & 475 & 1,400 & $\mathrm{No}^{\mathrm{e}}$ \\
\hline Arsenic (total) & 2 of 2 & 0.12 & 0.564 & 0.127 & 0.0021 & $\mathrm{No}^{\mathrm{g}}$ \\
\hline Arsenic (inorganic) & NAV & NAV & NAV & 0.0208 & 0.0021 & $\mathrm{No}^{d}$ \\
\hline Barium & 2 of 2 & 0.1 & 0.365 & 1.03 & 95 & $\mathrm{No}^{\mathrm{e}}$ \\
\hline Beryllium & 0 of 2 & ND (0.05) & ND (0.05) & ND & 2.7 & $\mathrm{No}^{\mathrm{d}}$ \\
\hline Boron & 2 of 2 & 3.53 & 6.6 & 28.6 & 120 & $\mathrm{No}^{\mathrm{e}}$ \\
\hline Cadmium & 1 of 2 & ND (0.05) & 0.0535 & $0.0373^{b}$ & 1.4 & $\mathrm{No}^{\dagger}$ \\
\hline
\end{tabular}


Table 4-30 Summary of Analytical Results and Human Health Risk Assessment
Screening for Fish Tissue in Cannikin Lake

(Page 2 of 2)

\begin{tabular}{|c|c|c|c|c|c|c|}
\hline Analyte & $\begin{array}{l}\text { Detection } \\
\text { Frequency }\end{array}$ & $\begin{array}{c}\text { Minimum } \\
\text { Detected } \\
\text { Concentration } \\
(\mathrm{mg} / \mathrm{kg})\end{array}$ & $\begin{array}{c}\text { Maximum } \\
\text { Detected } \\
\text { Concentration } \\
(\mathrm{mg} / \mathrm{kg})\end{array}$ & $\begin{array}{c}\text { Lowland } \\
\text { Stream } \\
\text { Background } \\
\text { Concentration } \\
\text { (mg/kg) }\end{array}$ & $\begin{array}{l}\text { Human Health } \\
\text { Screening } \\
\text { Value }^{c} \\
(\mathrm{mg} / \mathrm{kg})\end{array}$ & $\begin{array}{c}\text { Human Health } \\
\text { Constituent of } \\
\text { Concern }\end{array}$ \\
\hline Calcium & 2 of 2 & 1,850 & 3,080 & 19,200 & NAV & $\mathrm{No}^{\mathrm{e}}$ \\
\hline Cerium & 0 of 2 & ND (0.025) & ND (0.025) & $0.0280^{b}$ & NAV & $\mathrm{No}^{d}$ \\
\hline Cesium & 0 of 2 & ND (0.025) & ND (0.025) & $0.0258^{b}$ & NAV & $\mathrm{No}^{d}$ \\
\hline Chromium & 2 of 2 & 0.226 & 0.499 & 1.03 & 4.1 & $\mathrm{No}^{\mathrm{e}}$ \\
\hline Cobalt & 0 of 2 & ND (0.05) & ND (0.05) & $0.0780^{b}$ & 81 & $\mathrm{No}^{\mathrm{d}}$ \\
\hline Copper & 2 of 2 & 1.22 & 1.86 & $1.20^{b}$ & 54 & $\mathrm{No}^{\dagger}$ \\
\hline Iron & 2 of 2 & 8.3 & 104 & 310 & 410 & $\mathrm{No}^{\mathrm{e}}$ \\
\hline Lithium & 0 of 2 & ND (0.05) & ND (0.05) & $0.0993^{b}$ & 27 & $\mathrm{No}^{\mathrm{d}}$ \\
\hline Magnesium & 2 of 2 & 290 & 295 & 408 & NAV & $\mathrm{No}^{\mathrm{e}}$ \\
\hline Manganese & 2 of 2 & 2 & 18.2 & 160 & 190 & $\mathrm{No}^{\mathrm{e}}$ \\
\hline Molybdenum & 0 of 2 & ND (1.0) & ND (1.0) & $0.173^{b}$ & 6.8 & $\mathrm{No}^{\mathrm{d}}$ \\
\hline Nickel & 2 of 2 & 0.117 & 0.21 & ND & 27 & $\mathrm{No}^{f}$ \\
\hline Potassium & 2 of 2 & 3,330 & 3,550 & $4,780^{b}$ & NAV & $\mathrm{No}^{\mathrm{e}}$ \\
\hline Selenium & 2 of 2 & 0.717 & 0.72 & 0.936 & 6.8 & $\mathrm{No}^{\mathrm{e}}$ \\
\hline Strontium & 2 of 2 & 5.18 & 7.6 & 22.1 & 810 & $\mathrm{No}^{\mathrm{e}}$ \\
\hline Thallium & 1 of 2 & ND (0.05) & 0.05 & $0.0283^{b}$ & 0.095 & $\mathrm{No}^{f}$ \\
\hline Thorium & 0 of 2 & ND (0.5) & ND (0.5) & $3.43^{b}$ & NAV & $\mathrm{No}^{\mathrm{d}}$ \\
\hline Titanium & 2 of 2 & 3.18 & 5.48 & 32.1 & 5,400 & $\mathrm{No}^{\mathrm{e}}$ \\
\hline Uranium & 0 of 2 & $\mathrm{ND}(0.5)$ & $\mathrm{ND}(0.5)$ & ND & 4.1 & $\mathrm{No}^{\mathrm{d}}$ \\
\hline Vanadium & 2 of 2 & 0.166 & 0.475 & 1.05 & 9.5 & $\mathrm{No}^{\mathrm{e}}$ \\
\hline Zinc & 2 of 2 & 22 & 23.2 & 48.8 & 410 & $\mathrm{No}^{\mathrm{e}}$ \\
\hline$\%$ Lipids & 2 of 2 & 5.75 & 7 & NAN & NAV & No \\
\hline
\end{tabular}

${ }^{\mathrm{a} B}$ Background concentrations are upper tolerance limits (UTL) (ADEC, 1999a) unless otherwise noted.

${ }^{\mathrm{b}}$ Maximum detected concentration in background samples due to insufficient detection frequency to calculate UTL.

${ }^{\mathrm{C} U . S . ~ E P A ~ R e g i o n ~ I I I ~ r i s k-b a s e d ~ s c r e e n i n g ~ c r i t e r i a ~ f o r ~ f i s h ~ c o n s u m p t i o n ~ u n l e s s ~ o t h e r w i s e ~ n o t e d . ~}$

\section{COC Selection Criteria}

${ }^{\mathrm{d}}$ Constituent not detected in any samples.

e Maximum detected constituent concentration is less than local background concentration.

${ }^{f}$ Maximum detected constituent concentration is less than human health screening value.

${ }^{9}$ Not a constituent associated with drilling mud or other drilling activities.

ND - Not detected (detection limit)

NAV - Not available

NAN - Not analyzed

$\mathrm{mg} / \mathrm{kg}$ - Milligrams per kilogram 
Table 4-31

Summary of Analytical Results and Human Health Risk Assessment Screening for Surface Water in White Alice Creek

(Page 1 of 3 )

\begin{tabular}{|c|c|c|c|c|c|c|}
\hline Analyte & $\begin{array}{l}\text { Detection } \\
\text { Frequency }\end{array}$ & $\begin{array}{c}\text { Minimum } \\
\text { Detected } \\
\text { Concentration } \\
(\mu \mathrm{g} / \mathrm{L})\end{array}$ & $\begin{array}{c}\text { Maximum } \\
\text { Detected } \\
\text { Concentration } \\
(\mu \mathrm{g} / \mathrm{L})\end{array}$ & $\begin{array}{c}\text { Lowland } \\
\text { Stream } \\
\text { Background } \\
\text { Concentration } \\
(\mu \mathrm{g} / \mathrm{L})\end{array}$ & $\begin{array}{l}\text { Human Health } \\
\text { Screening } \\
\text { Value } \\
(\mu \mathrm{g} / L)\end{array}$ & $\begin{array}{l}\text { Human Health } \\
\text { Constituent of } \\
\text { Concern }\end{array}$ \\
\hline $\begin{array}{l}\text { Diesel-Range } \\
\text { Hydrocarbons }\end{array}$ & 5 of 5 & 65.6 & 95.3 & NAN & $1,500^{d}$ & $\mathrm{No}^{\mathrm{h}}$ \\
\hline \multicolumn{7}{|c|}{ Volatile Organic Compounds } \\
\hline 1,2,4-Trimethylbenzene & 0 of 5 & ND (1.0) & ND (1.0) & NAN & $12^{\mathrm{e}}$ & $\mathrm{No}^{\dagger}$ \\
\hline 1,3,5-Trimethylbenzene & 0 of 5 & ND (1.0) & ND (1.0) & NAN & $12^{\mathrm{e}}$ & $\mathrm{No}^{\dagger}$ \\
\hline 2-Butanone & 0 of 5 & ND (20) & ND (20) & NAN & $1,900^{\mathrm{e}}$ & $\mathrm{No}^{\dagger}$ \\
\hline Acetone & 0 of 5 & ND (20) & ND (20) & NAN & $3,700^{\mathrm{e}}$ & $\mathrm{No}^{\dagger}$ \\
\hline Benzene & 0 of 5 & ND (1.0) & $\mathrm{ND}(1.0)$ & NAN & $1.2^{\mathrm{c}}$ & $\mathrm{No}^{\dagger}$ \\
\hline Carbon disulfide & 0 of 5 & ND (1.0) & ND (1.0) & NAN & $1,000^{\mathrm{e}}$ & $\mathrm{No}^{f}$ \\
\hline Ethylbenzene & 0 of 5 & ND (1.0) & ND (1.0) & NAN & $3,100^{c}$ & $\mathrm{No}^{\dagger}$ \\
\hline Isopropylbenzene & 0 of 5 & ND (1.0) & ND (1.0) & NAN & NAV & $\mathrm{No}^{\dagger}$ \\
\hline $\mathrm{m}, \mathrm{p}$-Xylene & 0 of 5 & ND (2.0) & ND (2.0) & NAN & $12,000^{\mathrm{e}}$ & $\mathrm{No}^{\dagger}$ \\
\hline Methylene chloride & 0 of 5 & ND (2.0) & ND (5.59) & NAN & $4.7^{\mathrm{C}}$ & $\mathrm{No}^{\dagger}$ \\
\hline n-Butylbenzene & 0 of 5 & ND (1.0) & ND (1.0) & NAN & $61^{\mathrm{e}}$ & $\mathrm{No}^{\dagger}$ \\
\hline n-Propylbenzene & 0 of 5 & ND (1.0) & ND (1.0) & NAN & $61^{\mathrm{e}}$ & $\mathrm{No}^{\dagger}$ \\
\hline Naphthalene & 0 of 5 & ND (1.0) & $\mathrm{ND}(1.0)$ & NAN & $730^{\mathrm{e}}$ & $\mathrm{No}^{\dagger}$ \\
\hline o-Xylene & 0 of 5 & ND (1.0) & ND (1.0) & NAN & $12,000^{\mathrm{e}}$ & $\mathrm{No}^{\dagger}$ \\
\hline p-Isopropyltoluene & 0 of 5 & $\mathrm{ND}(1.0)$ & $\mathrm{ND}(1.0)$ & NAN & NAV & $\mathrm{No}^{\dagger}$ \\
\hline sec-Butylbenzene & 0 of 5 & ND (1.0) & ND (1.0) & NAN & $61^{e}$ & $\mathrm{No}^{\dagger}$ \\
\hline Toluene & 0 of 5 & ND (1.0) & ND (1.0) & NAN & $10,000^{c}$ & $\mathrm{No}^{\dagger}$ \\
\hline \multicolumn{7}{|c|}{ Polycyclic Aromatic Hydrocarbons } \\
\hline Acenaphthene & 0 of 5 & $\mathrm{ND}(0.1)$ & $\mathrm{ND}(0.1)$ & NAN & $1,200^{c}$ & $\mathrm{No}^{\dagger}$ \\
\hline Acenaphthylene & 0 of 5 & ND $(0.1)$ & $\mathrm{ND}(0.1)$ & NAN & $0.0028^{c}$ & $\mathrm{No}^{\dagger}$ \\
\hline Anthracene & 0 of 5 & $\mathrm{ND}(0.1)$ & $\mathrm{ND}(0.1)$ & NAN & $0.0028^{C}$ & $\mathrm{No}^{\dagger}$ \\
\hline Benzo(a)anthracene & 0 of 5 & $\mathrm{ND}(0.1)$ & $\mathrm{ND}(0.1)$ & NAN & $0.0028^{C}$ & $\mathrm{No}^{\dagger}$ \\
\hline Benzo(a)pyrene & 0 of 5 & ND $(0.1)$ & ND $(0.1)$ & NAN & $0.0028^{c}$ & $\mathrm{No}^{\dagger}$ \\
\hline Benzo(b)fluoranthene & 0 of 5 & ND $(0.1)$ & ND $(0.1)$ & NAN & $0.092^{\mathrm{e}}$ & $\mathrm{No}^{\dagger}$ \\
\hline Benzo(g,h,i)perylene & 0 of 5 & ND $(0.1)$ & $\mathrm{ND}(0.1)$ & NAN & $0.0028^{c}$ & $\mathrm{No}^{\dagger}$ \\
\hline Benzo(k)fluoranthene & 0 of 5 & $\mathrm{ND}(0.1)$ & $\mathrm{ND}(0.1)$ & NAN & $0.0028^{C}$ & $\mathrm{No}^{\dagger}$ \\
\hline Chrysene & 0 of 5 & $\mathrm{ND}(0.1)$ & $\mathrm{ND}(0.1)$ & NAN & $0.0028^{c}$ & $\mathrm{No}^{\dagger}$ \\
\hline
\end{tabular}


Table 4-31

Summary of Analytical Results and Human Health Risk Assessment Screening for Surface Water in White Alice Creek

(Page 2 of 3 )

\begin{tabular}{|c|c|c|c|c|c|c|}
\hline Analyte & $\begin{array}{l}\text { Detection } \\
\text { Frequency }\end{array}$ & $\begin{array}{c}\text { Minimum } \\
\text { Detected } \\
\text { Concentration } \\
(\mu \mathrm{g} / \mathrm{L})\end{array}$ & $\begin{array}{c}\text { Maximum } \\
\text { Detected } \\
\text { Concentration } \\
(\mu \mathrm{g} / \mathrm{L})\end{array}$ & $\begin{array}{c}\text { Lowland } \\
\text { Stream } \\
\text { Background } \\
\text { Concentration }{ }^{\mathrm{a}} \\
(\mu \mathrm{g} / \mathrm{L})\end{array}$ & $\begin{array}{l}\text { Human Health } \\
\text { Screening } \\
\text { Value } \\
(\mu \mathrm{g} / L)\end{array}$ & $\begin{array}{c}\text { Human Health } \\
\text { Constituent of } \\
\text { Concern }\end{array}$ \\
\hline Dibenzo(a,h)anthracene & 0 of 5 & ND $(0.1)$ & ND $(0.1)$ & NAN & $0.0028^{c}$ & $\mathrm{No}^{\dagger}$ \\
\hline Fluoranthene & 0 of 5 & ND $(0.1)$ & $\mathrm{ND}(0.1)$ & NAN & $42^{c}$ & $\mathrm{No}^{\dagger}$ \\
\hline Fluorene & 0 of 5 & $\mathrm{ND}(0.1)$ & $\mathrm{ND}(0.1)$ & NAN & $0.0028^{c}$ & $\mathrm{No}^{\dagger}$ \\
\hline Indeno(1,2,3-cd)pyrene & 0 of 5 & ND $(0.1)$ & $\mathrm{ND}(0.1)$ & NAN & $0.0028^{c}$ & $\mathrm{No}^{\dagger}$ \\
\hline Naphthalene & 0 of 5 & $\mathrm{ND}(0.1)$ & $\mathrm{ND}(0.1)$ & NAN & $730^{\mathrm{e}}$ & $\mathrm{No}^{\dagger}$ \\
\hline Phenanthrene & 0 of 5 & $\mathrm{ND}(0.1)$ & $\mathrm{ND}(0.1)$ & NAN & $0.0028^{c}$ & $\mathrm{No}^{\dagger}$ \\
\hline Pyrene & 0 of 5 & $\mathrm{ND}(0.1)$ & $\mathrm{ND}(0.1)$ & NAN & $0.0028^{c}$ & $\mathrm{No}^{\dagger}$ \\
\hline \multicolumn{7}{|c|}{ Polychlorinated Biphenyls } \\
\hline Aroclor 1016 & NAV & NAV & NAV & NAN & $0.000044^{c}$ & $\mathrm{No}^{\dagger}$ \\
\hline Aroclor 1221 & NAV & NAV & NAV & NAN & $0.000044^{\mathrm{c}}$ & $\mathrm{No}^{\dagger}$ \\
\hline Aroclor 1232 & NAV & NAV & NAV & NAN & $0.000044^{\mathrm{c}}$ & $\mathrm{No}^{\dagger}$ \\
\hline Aroclor 1242 & NAV & NAV & NAV & NAN & $0.000044^{c}$ & $\mathrm{No}^{\dagger}$ \\
\hline Aroclor 1248 & NAV & NAV & NAV & NAN & $0.000044^{c}$ & $\mathrm{No}^{\dagger}$ \\
\hline Aroclor 1254 & NAV & NAV & NAV & NAN & $0.000044^{\mathrm{c}}$ & $\mathrm{No}^{\dagger}$ \\
\hline Aroclor 1260 & NAV & NAV & NAV & NAN & $0.000044^{c}$ & $\mathrm{No}^{\dagger}$ \\
\hline Aroclor 1262 & NAV & NAV & NAV & NAN & $0.000044^{\mathrm{c}}$ & $\mathrm{No}^{\dagger}$ \\
\hline Aroclor 1268 & NAV & NAV & NAV & NAN & $0.000044^{c}$ & $\mathrm{No}^{\dagger}$ \\
\hline \multicolumn{7}{|c|}{ Metals } \\
\hline Aluminum & 4 of 5 & ND (50) & 97.4 & 269 & $37,000^{\mathrm{e}}$ & $\mathrm{No}^{\mathrm{g}}$ \\
\hline Arsenic & 2 of 5 & ND (1.0) & 6.42 & ND (1) & $0.018^{\mathrm{C}}$ & $\mathrm{No}^{\mathrm{j}}$ \\
\hline Barium & 0 of 5 & ND (10) & ND (10) & ND (10) & $1,000^{\mathrm{C}}$ & $\mathrm{No}^{\dagger}$ \\
\hline Beryllium & 0 of 5 & ND (1.0) & ND (1.0) & ND (1) & $0.0076^{c}$ & $\mathrm{No}^{f}$ \\
\hline Boron & 2 of 5 & ND (50) & 424 & ND (50) & $3,300^{e}$ & $\mathrm{No}^{h}$ \\
\hline Cadmium & 0 of 5 & ND (1.0) & ND (1.0) & ND (1) & $10^{c}$ & $\mathrm{No}^{\dagger}$ \\
\hline Calcium & 5 of 5 & 3,280 & 5,550 & $4,320^{b}$ & NAV & $\mathrm{No}^{\mathrm{i}}$ \\
\hline Cerium & 0 of 5 & ND (1.0) & ND (1.0) & ND (1) & NAV & $\mathrm{No}^{\dagger}$ \\
\hline Cesium & 0 of 5 & ND (1.0) & ND (1.0) & ND (1) & NAV & $\mathrm{No}^{f}$ \\
\hline Chromium & 0 of 5 & ND (1.0) & ND (1.0) & $2.25^{b}$ & $33,000^{c}$ & $\mathrm{No}^{f}$ \\
\hline Cobalt & 5 of 5 & 1.09 & 1.54 & 3.35 & $2,200^{\mathrm{e}}$ & $\mathrm{No}^{\mathrm{g}}$ \\
\hline
\end{tabular}


Table 4-31

\section{Summary of Analytical Results and Human Health Risk Assessment Screening for Surface Water in White Alice Creek}

(Page 3 of 3 )

\begin{tabular}{|c|c|c|c|c|c|c|}
\hline Analyte & $\begin{array}{l}\text { Detection } \\
\text { Frequency }\end{array}$ & $\begin{array}{c}\text { Minimum } \\
\text { Detected } \\
\text { Concentration } \\
(\mu \mathrm{g} / \mathrm{L})\end{array}$ & $\begin{array}{c}\text { Maximum } \\
\text { Detected } \\
\text { Concentration } \\
(\mu \mathrm{g} / \mathrm{L})\end{array}$ & $\begin{array}{c}\text { Lowland } \\
\text { Stream } \\
\text { Background } \\
\text { Concentration }{ }^{\mathrm{a}} \\
(\mu \mathrm{g} / \mathrm{L})\end{array}$ & $\begin{array}{l}\text { Human Health } \\
\text { Screening } \\
\text { Value } \\
(\mu \mathrm{g} / L)\end{array}$ & $\begin{array}{c}\text { Human Health } \\
\text { Constituent of } \\
\text { Concern }\end{array}$ \\
\hline Copper & 5 of 5 & 1.2 & 2.01 & 10.8 & $1,300^{c}$ & $\mathrm{No}^{\mathrm{g}}$ \\
\hline Iron & 4 of 5 & ND (150) & 266 & 993 & $300^{c}$ & $\mathrm{No}^{\mathrm{g}}$ \\
\hline Lithium & 0 of 5 & ND (1.0) & ND (1.0) & $1.17^{\mathrm{b}}$ & $730^{e}$ & $\mathrm{No}^{\dagger}$ \\
\hline Magnesium & 5 of 5 & 1,880 & 3,090 & $4,690^{b}$ & NAV & $\mathrm{No}^{\mathrm{g}}$ \\
\hline Manganese & 5 of 5 & 7.3 & 21.5 & 267 & $50^{c}$ & $\mathrm{No}^{\mathrm{g}}$ \\
\hline Molybdenum & 0 of 5 & ND (1.0) & ND (1.0) & ND (1) & $180^{e}$ & $\mathrm{No}^{f}$ \\
\hline Nickel & 0 of 5 & ND (1.0) & ND (1.0) & ND (1) & $510^{c}$ & $\mathrm{No}^{\dagger}$ \\
\hline Potassium & 5 of 5 & 217 & 564 & 2,620 & NAV & $\mathrm{No}^{\mathrm{g}}$ \\
\hline Selenium & 1 of 5 & ND (1.0) & 1.1 & ND (1) & $104^{c}$ & $\mathrm{No}^{\mathrm{h}}$ \\
\hline Strontium & 5 of 5 & 22.4 & 33 & 36.1 & $22,000^{\mathrm{e}}$ & $\mathrm{No}^{\mathrm{g}}$ \\
\hline Thallium & 0 of 5 & ND (1.0) & ND (1.0) & $7.07^{b}$ & $1.7^{\mathrm{C}}$ & $\mathrm{No}^{\dagger}$ \\
\hline Thorium & 0 of 5 & ND (1.0) & ND (1.0) & ND (1) & NAV & $\mathrm{No}^{\dagger}$ \\
\hline Titanium & 5 of 5 & 1.41 & 2.96 & 6.13 & $150,000^{\mathrm{e}}$ & $\mathrm{No}^{\mathrm{g}}$ \\
\hline Uranium & 0 of 5 & ND (1.0) & ND (1.0) & ND (1) & $110^{\mathrm{e}}$ & $\mathrm{No}^{f}$ \\
\hline Vanadium & 4 of 5 & ND (1.0) & 4.94 & $4.43^{b}$ & $260^{e}$ & $\mathrm{No}^{\mathrm{h}}$ \\
\hline Zinc & 0 of 5 & ND (10) & ND (10) & $50.3^{b}$ & $11,000^{\mathrm{e}}$ & $\mathrm{No}^{\dagger}$ \\
\hline
\end{tabular}

${ }^{\mathrm{a}}$ Background concentrations are upper tolerance limits (UTL) (ADEC, 1999a) unless otherwise noted.

${ }^{\mathrm{b}}$ Maximum detected concentration in background samples due to insufficient detection frequency to calculate UTL.

${ }^{\mathrm{C}}$ Federal ambient water quality criteria based on ingestion of water and organisms.

d State of Alaska groundwater cleanup level, Discharge Reporting, Cleanup, and Disposal of Oil and other Hazardous Substances

(18 AAC 75, Articles 3 and 9 [ADEC, 1999c]).

eU.S. EPA Region III risk-based concentrations for tap water (EPA, 2000b).

\section{COC Selection Criteria}

${ }^{f}$ Constituent not detected in any samples.

${ }^{9}$ Maximum detected constituent concentration is less than local background concentration.

${ }^{h}$ Maximum detected constituent concentration is less than human health screening value.

i Constituent is an essential macronutrient and only presents a risk at extremely high concentrations.

${ }^{j}$ Not a constituent associated with drilling mud or other drilling activities.

ND - Not detected (detection limit)

NAV - Not available

NAN - Not analyzed

$\mu \mathrm{g} / \mathrm{L}$ - Micrograms per liter 
Table 4-32

Summary of Analytical Results and Human Health Risk Assessment Screening for Sediment in White Alice Creek

(Page 1 of 3 )

\begin{tabular}{|c|c|c|c|c|c|c|}
\hline Analyte & $\begin{array}{l}\text { Detection } \\
\text { Frequency }\end{array}$ & $\begin{array}{c}\text { Minimum } \\
\text { Detected } \\
\text { Concentration } \\
(\mathrm{mg} / \mathrm{kg})\end{array}$ & $\begin{array}{c}\text { Maximum } \\
\text { Detected } \\
\text { Concentration } \\
(\mathrm{mg} / \mathrm{kg})\end{array}$ & $\begin{array}{c}\text { Lowland } \\
\text { Stream } \\
\text { Background } \\
\text { Concentration } \\
(\mathbf{m g} / \mathbf{k g})\end{array}$ & $\begin{array}{l}\text { Human Health } \\
\text { Screening } \\
\text { Value } \\
(\mathrm{mg} / \mathrm{kg})\end{array}$ & $\begin{array}{c}\text { Human Health } \\
\text { Constituent of } \\
\text { Concern }\end{array}$ \\
\hline \multicolumn{7}{|c|}{ Petroleum Hydrocarbons } \\
\hline $\begin{array}{l}\text { Diesel-Range } \\
\text { Hydrocarbons }\end{array}$ & 5 of 5 & 32.6 & 116 & NAN & $200^{c}$ & $\mathrm{No}^{\dagger}$ \\
\hline \multicolumn{7}{|c|}{ Volatile Organic Compounds } \\
\hline 1,2,4-Trimethylbenzene & 0 of 1 & ND (0.078) & ND $(0.078)$ & NAN & NAV & $\mathrm{No}^{\mathrm{d}}$ \\
\hline 1,3,5-Trimethylbenzene & 0 of 1 & ND $(0.078)$ & ND $(0.078)$ & NAN & NAV & $\mathrm{No}^{\mathrm{d}}$ \\
\hline 2-Butanone & 0 of 1 & ND (0.156) & ND $(0.156)$ & NAN & NAV & $\mathrm{No}^{d}$ \\
\hline Acetone & 0 of 1 & ND (8.91) & ND (8.91) & NAN & $10,100^{c}$ & $\mathrm{No}^{\mathrm{d}}$ \\
\hline Benzene & 0 of 1 & ND (0.078) & ND $(0.078)$ & NAN & $286^{\mathrm{C}}$ & $\mathrm{No}^{\mathrm{d}}$ \\
\hline Carbon disulfide & 0 of 1 & ND $(0.078)$ & ND $(0.078)$ & NAN & $10,100^{c}$ & $\mathrm{No}^{\mathrm{d}}$ \\
\hline Ethylbenzene & 0 of 1 & ND (0.078) & ND (0.078) & NAN & $10,100^{c}$ & $\mathrm{No}^{\mathrm{d}}$ \\
\hline Isopropylbenzene & 0 of 1 & ND (0.078) & ND (0.078) & NAN & NAV & $\mathrm{No}^{d}$ \\
\hline $\mathrm{m}, \mathrm{p}$-Xylene & 0 of 1 & ND $(0.078)$ & ND (0.078) & NAN & $203,000^{\mathrm{C}}$ & $\mathrm{No}^{d}$ \\
\hline Methylene chloride & 0 of 1 & ND $(0.078)$ & ND (0.078) & NAN & $1,110^{\mathrm{c}}$ & $\mathrm{No}^{d}$ \\
\hline n-Butylbenzene & 0 of 1 & ND (0.078) & ND (0.078) & NAN & NAV & $\mathrm{No}^{d}$ \\
\hline n-Propylbenzene & 0 of 1 & ND $(0.078)$ & ND (0.078) & NAN & NAV & $\mathrm{No}^{d}$ \\
\hline Naphthalene & 0 of 1 & ND (0.078) & ND $(0.078)$ & NAN & $4,060^{c}$ & $\mathrm{No}^{d}$ \\
\hline o-Xylene & 0 of 1 & ND (0.078) & ND $(0.078)$ & NAN & $203,000^{C}$ & $\mathrm{No}^{\mathrm{d}}$ \\
\hline p-Isopropyltoluene & 0 of 1 & ND (0.078) & ND (0.078) & NAN & NAV & $\mathrm{No}^{d}$ \\
\hline sec-Butylbenzene & 0 of 1 & ND (0.078) & ND (0.078) & NAN & NAV & $\mathrm{No}^{\mathrm{d}}$ \\
\hline Toluene & 0 of 1 & ND (0.078) & ND (0.078) & NAN & $20,300^{\mathrm{C}}$ & $\mathrm{No}^{d}$ \\
\hline \multicolumn{7}{|c|}{ Polycyclic Aromatic Hydrocarbons } \\
\hline Acenaphthene & 0 of 5 & ND $(0.0125)$ & ND $(0.0387)$ & NAN & $6,080^{\mathrm{C}}$ & $\mathrm{No}^{d}$ \\
\hline Acenaphthylene & 0 of 5 & ND (0.0125) & ND $(0.0387)$ & NAN & NAV & $\mathrm{No}^{d}$ \\
\hline Anthracene & 0 of 5 & ND (0.0125) & ND (0.0387) & NAN & $30,400^{c}$ & $\mathrm{No}^{\mathrm{d}}$ \\
\hline Benzo(a)anthracene & 0 of 5 & ND (0.0125) & ND (0.0387) & NAN & $11.4^{\mathrm{C}}$ & $\mathrm{No}^{\mathrm{d}}$ \\
\hline Benzo(a)pyrene & 0 of 5 & ND (0.0125) & ND $(0.0387)$ & NAN & $1.1^{\mathrm{c}}$ & $\mathrm{No}^{d}$ \\
\hline Benzo(b)fluoranthene & 0 of 5 & ND (0.0125) & ND $(0.0387)$ & NAN & $11.4^{\mathrm{C}}$ & $\mathrm{No}^{d}$ \\
\hline Benzo(g,h,i)perylene & 0 of 5 & ND (0.0125) & ND $(0.0387)$ & NAN & $1.1^{\mathrm{c}}$ & $\mathrm{No}^{d}$ \\
\hline Benzo(k)fluoranthene & 0 of 5 & ND (0.0125) & ND (0.0387) & NAN & $114^{\mathrm{C}}$ & No ${ }^{d}$ \\
\hline
\end{tabular}


Table 4-32

Summary of Analytical Results and Human Health Risk Assessment Screening for Sediment in White Alice Creek

(Page 2 of 3 )

\begin{tabular}{|c|c|c|c|c|c|c|}
\hline Analyte & $\begin{array}{l}\text { Detection } \\
\text { Frequency }\end{array}$ & $\begin{array}{c}\text { Minimum } \\
\text { Detected } \\
\text { Concentration } \\
(\mathrm{mg} / \mathrm{kg})\end{array}$ & $\begin{array}{c}\text { Maximum } \\
\text { Detected } \\
\text { Concentration } \\
(\mathrm{mg} / \mathrm{kg})\end{array}$ & $\begin{array}{c}\text { Lowland } \\
\text { Stream } \\
\text { Background } \\
\text { Concentration } \\
\text { (mg/kg) }\end{array}$ & $\begin{array}{l}\text { Human Health } \\
\text { Screening } \\
\text { Value } \\
(\mathrm{mg} / \mathrm{kg})\end{array}$ & $\begin{array}{c}\text { Human Health } \\
\text { Constituent of } \\
\text { Concern }\end{array}$ \\
\hline Chrysene & 0 of 5 & ND (0.0125) & ND (0.0387) & NAN & $1,140^{\mathrm{C}}$ & $\mathrm{No}^{\mathrm{d}}$ \\
\hline Dibenzo(a,h)anthracene & 0 of 5 & ND $(0.0125)$ & ND (0.0387) & NAN & $1.1^{\mathrm{C}}$ & $\mathrm{No}^{\mathrm{d}}$ \\
\hline Fluoranthene & 0 of 5 & ND $(0.0125)$ & ND (0.0387) & NAN & $4,060^{\mathrm{C}}$ & $\mathrm{No}^{\mathrm{d}}$ \\
\hline Fluorene & 0 of 5 & ND (0.0125) & ND (0.0387) & NAN & $4,060^{\mathrm{C}}$ & $\mathrm{No}^{\mathrm{d}}$ \\
\hline Indeno(1,2,3-cd)pyrene & 0 of 5 & ND $(0.0125)$ & ND (0.0387) & NAN & $11^{\mathrm{c}}$ & $\mathrm{No}^{\mathrm{d}}$ \\
\hline Naphthalene & 0 of 5 & ND $(0.0125)$ & ND $(0.0387)$ & NAN & $4,060^{\mathrm{C}}$ & $\mathrm{No}^{d}$ \\
\hline Phenanthrene & 0 of 5 & ND $(0.0125)$ & ND (0.0387) & NAN & $1.1^{\mathrm{C}}$ & $\mathrm{No}^{\mathrm{d}}$ \\
\hline Pyrene & 0 of 5 & ND $(0.0125)$ & ND (0.0387) & NAN & $3,040^{C}$ & $\mathrm{No}^{\mathrm{d}}$ \\
\hline \multicolumn{7}{|c|}{ Polychlorinated Biphenyls } \\
\hline Aroclor 1016 & 0 of 5 & ND $(0.19)$ & ND $(0.586)$ & NAN & $10^{\mathrm{C}}$ & $\mathrm{No}^{\mathrm{d}}$ \\
\hline Aroclor 1221 & 0 of 5 & ND (0.19) & ND (0.586) & NAN & $10^{C}$ & $\mathrm{No}^{\mathrm{d}}$ \\
\hline Aroclor 1232 & 0 of 5 & ND (0.19) & ND (0.586) & NAN & $10^{\mathrm{C}}$ & $\mathrm{No}^{d}$ \\
\hline Aroclor 1242 & 0 of 5 & ND (0.19) & ND (0.586) & NAN & $10^{c}$ & $\mathrm{No}^{d}$ \\
\hline Aroclor 1248 & 0 of 5 & ND (0.19) & ND (0.586) & NAN & $10^{c}$ & $\mathrm{No}^{d}$ \\
\hline Aroclor 1254 & 0 of 5 & ND (0.19) & ND (0.586) & NAN & $10^{C}$ & $\mathrm{No}^{\mathrm{d}}$ \\
\hline Aroclor 1260 & 0 of 5 & ND (0.19) & ND (0.586) & NAN & $10^{C}$ & $\mathrm{No}^{d}$ \\
\hline Aroclor 1262 & 0 of 5 & ND (0.19) & ND (0.586) & NAN & $10^{\mathrm{C}}$ & $\mathrm{No}^{d}$ \\
\hline Aroclor 1268 & 0 of 5 & ND (0.19) & ND (0.586) & NAN & $10^{C}$ & $\mathrm{No}^{\mathrm{d}}$ \\
\hline \multicolumn{7}{|c|}{ Metals } \\
\hline Aluminum & 5 of 5 & 6,480 & 68,300 & 45,400 & NAV & $\mathrm{No}^{\mathrm{h}}$ \\
\hline Arsenic & 4 of 5 & ND (5.86) & 109 & 100 & $5.5^{\mathrm{C}}$ & $\mathrm{No}^{\mathrm{h}}$ \\
\hline Barium & 3 of 5 & ND (58.6) & 377 & 469 & $7,100^{C}$ & $\mathrm{No}^{\mathrm{e}}$ \\
\hline Beryllium & 0 of 5 & ND (5.86) & ND (94.8) & ND & $1.9^{\mathrm{C}}$ & $\mathrm{No}^{\mathrm{d}}$ \\
\hline Boron & 0 of 5 & ND (9.48) & ND (29.3) & $7.18^{b}$ & NAV & $\mathrm{No}^{\mathrm{d}}$ \\
\hline Cadmium & 0 of 5 & ND (1.9) & ND (5.86) & $0.608^{b}$ & $101^{\mathrm{C}}$ & $\mathrm{No}^{d}$ \\
\hline Calcium & 5 of 5 & 5,540 & 10,800 & 7,300 & NAV & $\mathrm{No}^{\mathrm{g}}$ \\
\hline Cerium & 4 of 5 & ND (5.86) & 20.9 & 14.2 & NAV & $\mathrm{No}^{h}$ \\
\hline Cesium & 0 of 5 & ND (1.9) & ND (5.86) & ND & NAV & $\mathrm{No}^{\mathrm{d}}$ \\
\hline Chromium & 5 of 5 & 13.1 & 27.5 & 11.8 & $507^{C}$ & $\mathrm{No}^{f}$ \\
\hline
\end{tabular}


Table 4-32

\section{Summary of Analytical Results and Human Health Risk Assessment Screening for Sediment in White Alice Creek}

(Page 3 of 3 )

\begin{tabular}{|c|c|c|c|c|c|c|}
\hline Analyte & $\begin{array}{l}\text { Detection } \\
\text { Frequency }\end{array}$ & $\begin{array}{c}\text { Minimum } \\
\text { Detected } \\
\text { Concentration } \\
(\mathbf{m g} / \mathbf{k g})\end{array}$ & $\begin{array}{c}\text { Maximum } \\
\text { Detected } \\
\text { Concentration } \\
(\mathbf{m g} / \mathbf{k g})\end{array}$ & $\begin{array}{c}\text { Lowland } \\
\text { Stream } \\
\text { Background } \\
\text { Concentration }{ }^{\mathrm{a}} \\
(\mathbf{m g} / \mathbf{k g})\end{array}$ & $\begin{array}{l}\text { Human Health } \\
\text { Screening } \\
\text { Value } \\
(\mathrm{mg} / \mathrm{kg})\end{array}$ & $\begin{array}{c}\text { Human Health } \\
\text { Constituent of } \\
\text { Concern }\end{array}$ \\
\hline Cobalt & 4 of 5 & ND (5.86) & 38.2 & 43.7 & NAV & $\mathrm{No}^{\mathrm{e}}$ \\
\hline Copper & 5 of 5 & 28.7 & 168 & 71.1 & NAV & $\mathrm{No}^{\mathrm{h}}$ \\
\hline Iron & 5 of 5 & 6,900 & 96,800 & 155,000 & NAV & $\mathrm{No}^{\mathrm{e}}$ \\
\hline Lithium & 4 of 5 & ND (5.86) & 22.7 & 48.8 & NAV & $\mathrm{No}^{\mathrm{e}}$ \\
\hline Magnesium & 5 of 5 & 1,530 & 36,600 & 9,320 & NAV & $\mathrm{No}^{\mathrm{g}}$ \\
\hline Manganese & 5 of 5 & 442 & 10,300 & 20,700 & NAV & $\mathrm{No}^{\mathrm{e}}$ \\
\hline Molybdenum & 0 of 5 & ND (1.9) & ND (5.86) & $5.5^{\mathrm{b}}$ & NAV & $\mathrm{No}^{\mathrm{d}}$ \\
\hline Nickel & 5 of 5 & 6.22 & 24.9 & 13.6 & $2,030^{c}$ & $\mathrm{No}^{f}$ \\
\hline Potassium & 5 of 5 & 249 & 3,880 & 1,390 & NAV & $\mathrm{No}^{\mathrm{g}}$ \\
\hline Selenium & 2 of 5 & ND (3.29) & 4.52 & $5.44^{\mathrm{b}}$ & $507^{C}$ & $\mathrm{No}^{\mathrm{e}}$ \\
\hline Strontium & 5 of 5 & 49.5 & 547 & 164 & NAV & $\mathrm{No}^{\mathrm{h}}$ \\
\hline Thallium & 0 of 5 & ND (1.9) & ND (5.86) & $1^{b}$ & NAV & $\mathrm{No}^{d}$ \\
\hline Thorium & 0 of 5 & ND (1.9) & ND (5.86) & $0.5^{b}$ & NAV & $\mathrm{No}^{d}$ \\
\hline Titanium & 5 of 5 & 228 & 2,010 & 3,010 & NAV & $\mathrm{No}^{\mathrm{e}}$ \\
\hline Uranium & 0 of 5 & ND (1.9) & ND (5.86) & $4.13^{b}$ & NAV & $\mathrm{No}^{\mathrm{d}}$ \\
\hline Vanadium & 5 of 5 & 40.6 & 645 & 734 & $710^{c}$ & $\mathrm{No}^{\mathrm{e}}$ \\
\hline Zinc & 4 of 5 & ND (58.6) & 248 & 434 & $30,400^{c}$ & $\mathrm{No}^{\mathrm{e}}$ \\
\hline Total Organic Carbon & 5 of 5 & 8,250 & 158,000 & NAN & NAV & No \\
\hline
\end{tabular}

${ }^{\mathrm{a} B a c k g r o u n d}$ concentrations are upper tolerance limits (UTL) (ADEC, 1999a) unless otherwise noted.

${ }^{\mathrm{b}}$ Maximum detected concentration in background samples due to insufficient detection frequency to calculate UTL.

'State of Alaska soil cleanup level, Discharge Reporting, Cleanup, and Disposal of Oil and other Hazardous Substances (18 AAC 75, Articles 3 and 9 [ADEC, 1999c]).

\section{COC Selection Criteria}

${ }^{\mathrm{d}}$ Constituent not detected in any samples.

${ }^{\mathrm{e}}$ Maximum detected constituent concentration is less than local background concentration.

'Maximum detected constituent concentration is less than human health screening value.

${ }^{9}$ Constituent is an essential macronutrient and only presents a risk at extremely high concentrations.

${ }^{h}$ Not a constituent associated with drilling mud or other drilling activities.

ND - Not detected (detection limit)

NAV - Not available

NAN - Non analyzed

$\mathrm{mg} / \mathrm{kg}$ - Milligrams per kilogram 
Table 4-33

Summary of Analytical Results and Human Health Risk Assessment
Screening for Fish Tissue in White Alice Creek

(Page 1 of 2)

\begin{tabular}{|c|c|c|c|c|c|c|}
\hline Analyte & $\begin{array}{l}\text { Detection } \\
\text { Frequency }\end{array}$ & $\begin{array}{c}\text { Minimum } \\
\text { Detected } \\
\text { Concentration } \\
(\mathrm{mg} / \mathrm{kg})\end{array}$ & $\begin{array}{c}\text { Maximum } \\
\text { Detected } \\
\text { Concentration } \\
(\mathbf{m g} / \mathrm{kg})\end{array}$ & $\begin{array}{c}\text { Lowland } \\
\text { Stream } \\
\text { Background } \\
\text { Concentration }{ }^{\mathrm{a}} \\
(\mathrm{mg} / \mathrm{kg})\end{array}$ & $\begin{array}{l}\text { Human Health } \\
\text { Screening } \\
\text { Value } \\
(\mathrm{mg} / \mathrm{kg})\end{array}$ & $\begin{array}{c}\text { Human Health } \\
\text { Constituent of } \\
\text { Concern }\end{array}$ \\
\hline \multicolumn{7}{|c|}{ Polycyclic Aromatic Hydrocarbons } \\
\hline Acenaphthene & 0 of 5 & ND (0.0335) & ND (0.0335) & NAN & 81.1 & $\mathrm{No}^{\mathrm{d}}$ \\
\hline Acenaphthylene & 0 of 5 & ND (0.0335) & ND (0.0335) & NAN & NAV & $\mathrm{No}^{\mathrm{d}}$ \\
\hline Anthracene & 0 of 5 & ND (0.0335) & ND (0.0335) & NAN & 410 & $\mathrm{No}^{d}$ \\
\hline Benzo(a)anthracene & 0 of 5 & ND (0.0335) & ND (0.0335) & NAN & 0.0043 & $\mathrm{No}^{d}$ \\
\hline Benzo(a)pyrene & 0 of 5 & ND (0.0335) & ND (0.0335) & NAN & 0.00043 & $\mathrm{No}^{d}$ \\
\hline Benzo(b)fluoranthene & 0 of 5 & ND (0.0335) & ND (0.0335) & NAN & 0.0043 & $\mathrm{No}^{d}$ \\
\hline Benzo(ghi)perylene & 0 of 5 & ND (0.0335) & ND (0.0335) & NAN & NAV & $\mathrm{No}^{\mathrm{d}}$ \\
\hline Benzo(k)fluoranthene & 0 of 5 & ND (0.0335) & ND (0.0335) & NAN & 0.043 & $\mathrm{No}^{d}$ \\
\hline Chrysene & 0 of 5 & ND (0.0335) & ND (0.0335) & NAN & 0.43 & $\mathrm{No}^{\mathrm{d}}$ \\
\hline Dibenzo(a,h)anthracene & 0 of 5 & ND (0.0335) & ND (0.0335) & NAN & 0.00043 & $\mathrm{No}^{d}$ \\
\hline Fluoranthene & 0 of 5 & ND (0.0335) & ND (0.0335) & NAN & 54 & $\mathrm{No}^{\mathrm{d}}$ \\
\hline Fluorene & 0 of 5 & ND (0.0335) & ND (0.0335) & NAN & 54 & $\mathrm{No}^{d}$ \\
\hline Indeno(1,2,3-cd)pyrene & 0 of 5 & ND (0.0335) & ND (0.0335) & NAN & 0.0043 & $\mathrm{No}^{d}$ \\
\hline Naphthalene & 0 of 5 & ND (0.0335) & ND (0.0335) & NAN & 27 & $\mathrm{No}^{d}$ \\
\hline Phenanthrene & 0 of 5 & ND (0.0335) & ND (0.0335) & NAN & NAV & $\mathrm{No}^{\mathrm{d}}$ \\
\hline Pyrene & 0 of 5 & ND (0.0335) & ND (0.0335) & NAN & 41 & $\mathrm{No}^{\mathrm{d}}$ \\
\hline \multicolumn{7}{|c|}{ Polychlorinated Biphenyls } \\
\hline Aroclor 1016 & 0 of 5 & ND $(0.01)$ & ND (0.01) & NAN & 0.045 & $\mathrm{No}^{\mathrm{d}}$ \\
\hline Aroclor 1221 & 0 of 5 & ND (0.02) & ND (0.02) & NAN & 0.0016 & $\mathrm{No}^{\mathrm{d}}$ \\
\hline Aroclor 1232 & 0 of 5 & ND (0.01) & ND $(0.01)$ & NAN & 0.0016 & $\mathrm{No}^{d}$ \\
\hline Aroclor 1242 & 0 of 5 & ND $(0.01)$ & ND (0.01) & NAN & 0.0016 & $\mathrm{No}^{d}$ \\
\hline Aroclor 1248 & 0 of 5 & ND (0.01) & ND (0.01) & NAN & 0.0016 & $\mathrm{No}^{d}$ \\
\hline Aroclor 1254 & 0 of 5 & ND (0.01) & ND (0.01) & NAN & 0.0016 & $\mathrm{No}^{d}$ \\
\hline Aroclor 1260 & 5 of 5 & 0.0115 & 0.0394 & NAN & 0.0016 & Yes \\
\hline \multicolumn{7}{|c|}{ Metals } \\
\hline Aluminum & 5 of 5 & 10.6 & 99.3 & 475 & 1,400 & $\mathrm{No}^{\mathrm{e}}$ \\
\hline Arsenic (total) & 4 of 5 & ND (0.025) & 0.135 & 0.127 & 0.0021 & $\mathrm{No}^{f}$ \\
\hline Arsenic (inorganic) & 4 of 5 & ND (0.000158) & 0.0457 & 0.0208 & 0.0021 & $\mathrm{No}^{\mathrm{g}}$ \\
\hline Barium & 5 of 5 & 0.053 & 0.135 & 1.03 & 95 & $\mathrm{No}^{\mathrm{e}}$ \\
\hline Beryllium & 0 of 5 & ND (0.025) & ND (0.025) & ND & 2.7 & $\mathrm{No}^{\mathrm{d}}$ \\
\hline Boron & 0 of 5 & ND (9.08) & ND (37.2) & 28.6 & 120 & $\mathrm{No}^{\dagger}$ \\
\hline Cadmium & 0 of 5 & ND (0.025) & ND (0.025) & $0.0373^{b}$ & 1.4 & $\mathrm{No}^{d}$ \\
\hline
\end{tabular}


Table 4-33

Summary of Analytical Results and Human Health Risk Assessment
Screening for Fish Tissue in White Alice Creek (Page 2 of 2 )

\begin{tabular}{|c|c|c|c|c|c|c|}
\hline Analyte & $\begin{array}{l}\text { Detection } \\
\text { Frequency }\end{array}$ & $\begin{array}{c}\text { Minimum } \\
\text { Detected } \\
\text { Concentration } \\
(\mathbf{m g} / \mathbf{k g})\end{array}$ & $\begin{array}{c}\text { Maximum } \\
\text { Detected } \\
\text { Concentration } \\
(\mathbf{m g} / \mathbf{k g})\end{array}$ & $\begin{array}{c}\text { Lowland } \\
\text { Stream } \\
\text { Background } \\
\text { Concentration } \\
\text { (mg/kg) }\end{array}$ & $\begin{array}{l}\text { Human Health } \\
\text { Screening } \\
\text { Value } \\
(\mathrm{mg} / \mathrm{kg})\end{array}$ & $\begin{array}{l}\text { Human Health } \\
\text { Constituent of } \\
\text { Concern }\end{array}$ \\
\hline Calcium & 5 of 5 & 3,680 & 6,000 & 19,200 & NAV & $\mathrm{No}^{\mathrm{e}}$ \\
\hline Cerium & 0 of 5 & ND (0.025) & ND (0.025) & $0.0280^{b}$ & NAV & $\mathrm{No}^{d}$ \\
\hline Cesium & 0 of 5 & ND (0.025) & ND (0.025) & $0.0258^{b}$ & NAV & $\mathrm{No}^{d}$ \\
\hline Chromium & 5 of 5 & 0.289 & 0.426 & 1.03 & 4.1 & $\mathrm{No}^{\mathrm{e}}$ \\
\hline Cobalt & 1 of 5 & ND (0.05) & 0.0508 & $0.0780^{b}$ & 81 & $\mathrm{No}^{d}$ \\
\hline Copper & 4 of 5 & ND (0.0857) & 1.31 & $1.20^{b}$ & 54 & $\mathrm{No}^{f}$ \\
\hline Iron & 5 of 5 & 17 & 110 & 310 & 410 & $\mathrm{No}^{\mathrm{e}}$ \\
\hline Lithium & 1 of 5 & ND (0.025) & 0.0368 & $0.0993^{b}$ & 27 & $\mathrm{No}^{\mathrm{e}}$ \\
\hline Magnesium & 5 of 5 & 260 & 383 & 408 & NAV & $\mathrm{No}^{\mathrm{e}}$ \\
\hline Manganese & 5 of 5 & 2.22 & 7.41 & 160 & 190 & $\mathrm{No}^{\mathrm{e}}$ \\
\hline Molybdenum & 0 of 5 & ND (0.125) & ND (0.125) & $0.173^{b}$ & 6.8 & $\mathrm{No}^{d}$ \\
\hline Nickel & 0 of 5 & ND (0.125) & ND (0.125) & ND & 27 & $\mathrm{No}^{\mathrm{d}}$ \\
\hline Potassium & 5 of 5 & 3,350 & 3,680 & $4,780^{b}$ & NAV & $\mathrm{No}^{\mathrm{e}}$ \\
\hline Selenium & 5 of 5 & 0.446 & 0.686 & 0.936 & 6.8 & $\mathrm{No}^{\mathrm{e}}$ \\
\hline Strontium & 5 of 5 & 6.73 & 10.6 & 22.1 & 810 & $\mathrm{No}^{\mathrm{e}}$ \\
\hline Thallium & 0 of 5 & ND (0.025) & ND (0.025) & $0.0283^{b}$ & 0.095 & $\mathrm{No}^{\mathrm{d}}$ \\
\hline Thorium & 0 of 5 & ND (2.5) & ND (2.5) & $3.43^{b}$ & NAV & $\mathrm{No}^{d}$ \\
\hline Titanium & 5 of 5 & 0.241 & 1.64 & 32.1 & 5,400 & $\mathrm{No}^{\mathrm{e}}$ \\
\hline Uranium & 0 of 5 & ND (0.5) & $\mathrm{ND}(0.5)$ & ND & 4.1 & $\mathrm{No}^{d}$ \\
\hline Vanadium & 5 of 5 & 0.148 & 0.545 & 1.05 & 9.5 & $\mathrm{No}^{\mathrm{e}}$ \\
\hline Zinc & 5 of 5 & 23.9 & 29.6 & 48.8 & 410 & $\mathrm{No}^{\mathrm{e}}$ \\
\hline$\%$ Lipids & 1 of 1 & 5.67 & 5.67 & NAN & NAV & No \\
\hline
\end{tabular}

${ }^{a}$ Background concentrations are upper tolerance limits (UTL) (ADEC, 1999a) unless otherwise noted.

${ }^{\mathrm{b}}$ Maximum detected concentration in background samples due to insufficient detection frequency to calculate UTL.

${ }^{\mathrm{C} U . S . ~ E P A ~ R e g i o n ~ I I I ~ r i s k-b a s e d ~ s c r e e n i n g ~ c r i t e r i a ~ f o r ~ f i s h ~ c o n s u m p t i o n ~ u n l e s s ~ o t h e r w i s e ~ n o t e d . ~}$

\section{coc Selection Criteria}

${ }^{\mathrm{d}}$ Constituent not detected in any samples.

e Maximum detected constituent concentration is less than local background concentration.

${ }^{f}$ Maximum detected constituent concentration is less than human health screening value.

${ }^{9}$ Not a constituent associated with drilling mud or other drilling activities.

ND - Not detected (detection limit)

NAV - Not available

NAN - Not analyzed

$\mathrm{mg} / \mathrm{kg}$ - Milligrams per kilogram 
Table 4-34

Summary of Preliminary Constituents of Concern

\begin{tabular}{|c|c|c|c|}
\hline Upland Streams/Lakes & Surface Water & Sediment & Fish Tissue \\
\hline Falls Creek (Drill Site D) & None & None & Aroclor-1260 \\
\hline Drill Site D Lake & None & 2-butanone, chromium & NAN \\
\hline Drill Site E Stream & None & None & None \\
\hline Limpet Creek (Drill Site F) & None & None & Aroclor-1260 \\
\hline Lowland Streams/Lakes & Surface Water & Sediment & Fish Tissue \\
\hline Bridge Creek (Long Shot Site) & None & None & None \\
\hline Reed Pond (Long Shot Site) & None & 2-butanone & NAN \\
\hline Cloudberry Creek (Long Shot Site) & None & None & None \\
\hline Rainbow Creek (Long Shot Site) & None & DRO & Aroclor-1260 \\
\hline Clevenger Creek (Milrow Site) & None & DRO & Aroclor-1260 \\
\hline Heart Lake (Milrow Site) & None & 2-butanone & NAN \\
\hline Cannikin Lake (Cannikin Site) & None & $\begin{array}{l}\text { DRO, 1,2,4-trimethylbenzene, } \\
\text { 1,3,5-trimethylbenzene, n-butylbenzene, } \\
\text { n-propylbenzene, p-isopropyltoluene, } \\
\text { sec-butylbenzene, phenanthrene }\end{array}$ & Aroclor-1260 \\
\hline White Alice Creek (Cannikin Site) & None & None & Aroclor-1260 \\
\hline
\end{tabular}

NAN - No samples from this medium were analyzed.

generally, for brief periods of time. It is also possible that Aleut subsistence fishermen and/or hunters could visit the island for relatively short periods of time.

As discussed in Section 3.5, there are two potentially exposed populations at Amchitka:

- USFWS workers/residents

- Part-time subsistence users

Although trespassers may spend short periods of time on the island, the duration of their exposures is expected to be less than either the on-site workers or the subsistence users; therefore, exposures for trespassers are not quantified in this assessment.

\subsubsection{Identification of Exposure Pathways}

The USFWS workers and other authorized researchers from other organizations may work on Amchitka from time to time. Some of these personnel may reside on ships during their work 
assignments or they may establish on-site living quarters. During their work assignment period, these workers may also engage in recreational fishing activities. The exposure pathways by which these workers could come in contact with site-related contaminants are the following:

- Ingestion of fish

- Direct contact with surface water

- Direct contact with sediment

Incidental ingestion of surface water and sediment are also considered complete exposure pathways for the USFWS worker, but the magnitude of these two exposure pathways is expected to be insignificant compared to the other pathways and will not be quantified in this assessment.

Ingestion of groundwater from Constantine Spring is not considered a viable exposure pathway because Constantine Spring is not impacted by drill site activities. Additionally, exposures to surface soil (e.g., incidental ingestion, inhalation, dermal contact) are not considered viable exposure pathways because soil has not been shown to be significantly impacted by contaminants released from mud pits.

Local subsistence fishermen and hunters may utilize the island as a source of food from time to time. Their potential exposures consist of the activities that they engage in while on the island and also the consumption of food items that they collect from the island. The exposure pathways by which these part-time subsistence users could come in contact with site-related contaminants are the following:

- Ingestion of fish

- Incidental ingestion of surface water

- Direct contact with surface water

- Incidental ingestion of sediment

- Direct contact with sediment

As is the case with the USFWS worker, ingestion of groundwater from Constantine Spring is not considered a viable exposure pathway because Constantine Spring is not impacted by drilling activities. Additionally, exposures to surface soil (e.g., incidental ingestion, inhalation, dermal contact) are not considered viable exposure pathways because soil has not been shown to be significantly impacted from mud pit releases. 


\subsubsection{Exposure Pathway Dosage Estimates}

Environmental medium-specific exposure algorithms have been developed for each of the identified exposure pathways. These exposure algorithms are used to estimate the chronic daily intake (CDI) of COCs by receptor populations (i.e., on-site workers or subsistence users).

For each exposure activity, the CDI, expressed as milligrams per kilogram per day ( $\mathrm{mg} / \mathrm{kg} / \mathrm{day})$, is an averaged daily dose of a COC ingested or absorbed by a receptor. The averaged dose received by a receptor is the critical point estimate for determining the extent of health risk/hazard associated with exposure to each COC. The exposure parameters that influence receptor intake or absorption of COCs, including exposure duration and frequency, can and do vary in the exposure algorithms used to estimate the CDI by different exposure routes to the same medium. For each identified pathway, a reasonable maximum exposure (RME) scenario has been developed. The exposure parameters used in the RME assessments are upper-bound $\left(90^{\text {th }}\right.$ to $95^{\text {th }}$ percentile) point estimates for each parameter and present a maximal conservative exposure assessment. The parameters that will be used to quantify exposures in the human health risk assessment are presented in Tables 4-35 and 4-36. The algorithms for each complete exposure pathway are presented in the following sections.

\subsubsection{Exposure Point Concentrations}

For quantitative human health risk assessments, the concentration term (exposure point concentration) in the exposure equations is an estimate of the arithmetic average concentration for a contaminant based on a set of sampling results. Because of the uncertainty associated with estimating the true average concentration at a site, the 95 percent upper confidence limit (UCL) of the arithmetic mean is used for this variable. The 95 percent UCL provides reasonable confidence that the true site average concentration will not be underestimated.

To calculate the 95 percent UCL of the arithmetic mean for a log-normally distributed dataset, the data are transformed using the natural logarithm function. After transforming the data, the 95 percent UCL for the dataset is calculated using the following steps:

- Calculate the arithmetic mean of the log-transformed data

- Calculate the standard deviation of the log-transformed data

- Determine the H-statistic (Gilbert, 1987)

- Calculate the UCL using the following equation 
Table 4-35

Parameters for On-Site USFWS Worker Exposure Pathways

\begin{tabular}{|c|c|c|c|c|}
\hline $\begin{array}{l}\text { Environmental } \\
\text { Medium and } \\
\text { Exposure Route }\end{array}$ & Exposure Parameter & Units & $\begin{array}{l}\text { RME } \\
\text { Value }^{1}\end{array}$ & Rationale \\
\hline & Body Weight (BW) & $\mathrm{kg}$ & $70^{\mathrm{a}}$ & Default value \\
\hline & Exposure Duration (ED) & years & $1^{\mathrm{a}}$ & Default value \\
\hline & $\begin{array}{l}\text { Averaging Time (AT) } \\
\text { Cancer } \\
\text { Noncancer }\end{array}$ & days & $\begin{array}{l}25,550^{\mathrm{a}} \\
365^{\mathrm{a}}\end{array}$ & $\begin{array}{l}\text { Default value } \\
\text { Equal to exposure duration }\end{array}$ \\
\hline \multicolumn{5}{|c|}{ Surface Water (Dermal Contact): } \\
\hline & $\begin{array}{l}\text { Surface Area of Exposed } \\
\text { Skin (SA) }\end{array}$ & $\mathrm{cm}^{2} /$ event & $5,700^{\mathrm{b}}$ & $\begin{array}{l}\text { 95th percentile area of head, arms, forearms, } \\
\text { and hands of adult males and females }\end{array}$ \\
\hline & $\begin{array}{l}\text { Dermal Permeability Constant } \\
\left(K_{p}\right)\end{array}$ & $\mathrm{cm} / \mathrm{hr}$ & e & Constituent-specific value \\
\hline & Exposure Time $\left(\mathrm{ET}_{\mathrm{SW}}\right)$ & $\mathrm{hrs} /$ day & $10^{\mathrm{C}}$ & Activity requires an entire work-day to complete \\
\hline & Exposure Frequency $\left(\mathrm{EF}_{\mathrm{SW}}\right)$ & days/year & $365^{\mathrm{c}}$ & Daily routine maintenance/monitoring activities \\
\hline \multicolumn{5}{|c|}{ Sediment (Dermal Contact): } \\
\hline & $\begin{array}{l}\text { Surface Area of Exposed } \\
\text { Skin (SA) }\end{array}$ & $\mathrm{cm}^{2} /$ event & $5,700^{\mathrm{b}}$ & $\begin{array}{l}\text { 95th percentile area of head, arms, forearms, } \\
\text { and hands of adult males and females }\end{array}$ \\
\hline & $\begin{array}{l}\text { Sediment Adherence Factor } \\
\left(\mathrm{AF}_{\text {sed }}\right)\end{array}$ & $\mathrm{mg} / \mathrm{cm}^{2}$ & $0.08^{d}$ & Recommended value for adult soil contact \\
\hline & $\begin{array}{l}\text { Dermal Absorption Factor } \\
\left(\text { ABS }_{\text {dermal }}\right) \\
\text { Other Organics } \\
\text { Other Inorganics }\end{array}$ & unitless & $\begin{array}{c}d \\
0.1^{d} \\
0.01^{d}\end{array}$ & $\begin{array}{l}\text { Constituent-specific value } \\
\text { Default value if chemical-specific value is } \\
\text { unavailable } \\
\text { Default value if chemical-specific value is } \\
\text { unavailable }\end{array}$ \\
\hline & Exposure Frequency $\left(\mathrm{EF}_{\text {sed }}\right)$ & events/year & $365^{c}$ & Daily routine maintenance/monitoring activities \\
\hline \multicolumn{5}{|c|}{ Fish Tissue (Ingestion): } \\
\hline & Ingestion Rate $\left(\mathrm{IR}_{\mathrm{fish}}\right)$ & $\mathrm{kg} /$ day & $0.0068^{\mathrm{b}}$ & $\begin{array}{l}\text { 95th percentile intake of recreational anglers in } \\
\text { Pacific region }\end{array}$ \\
\hline & Exposure Frequency $\left(\mathrm{EF}_{\text {fish }}\right)$ & days/year & $365^{\mathrm{b}}$ & Default. Ingestion rate based on annual average \\
\hline & Absorption Factor $\left(\mathrm{ABS}_{\text {ingest }}\right)$ & unitless & $1^{a}$ & Default value \\
\hline
\end{tabular}

Notes:

1. Reasonable Maximum Exposure (RME) parameters are based on upper bound (e.g., 90-95th percentile) exposure values.

${ }^{a}$ U.S. EPA, 1991. Human Health Evaluation Manual, Supplemental Guidance, Standard Default Exposure Factors. OSWER Directive 9285.6-03.

U.S. EPA, 1997. Exposure Factors Handbook. EPA/600/P-95/002F.

Site-specific risk assessment assumption.

U.S. EPA, 1998, Risk Assessment Guidance for Superfund, Volume I: Human Health Evaluation Manual, Supplemental Guidance, Dermal Risk Assessment, Interim Guidance.

e U.S. EPA, 1992. Dermal Exposure Assessment: Principles and Applications, EPA/600/8-91/011B. 
Table 4-36

Parameters for Part-Time Subsistence User Exposure Pathways (Page 1 of 2)

\begin{tabular}{|c|c|c|c|c|}
\hline $\begin{array}{l}\text { Environmental } \\
\text { Medium and } \\
\text { Exposure Route }\end{array}$ & Exposure Parameter & Units & $\begin{array}{c}\text { RME } \\
\text { Value }^{1}\end{array}$ & Rationale \\
\hline & $\begin{array}{l}\text { Body Weight (BW) } \\
\text { Adult } \\
\text { Child }\end{array}$ & $\mathrm{kg}$ & $\begin{array}{l}70^{\mathrm{a}} \\
15^{\mathrm{a}}\end{array}$ & $\begin{array}{l}\text { Default value } \\
\text { Default value }\end{array}$ \\
\hline & $\begin{array}{l}\text { Exposure Duration (ED) } \\
\text { Adult } \\
\text { Child }\end{array}$ & years & $\begin{array}{c}30^{\mathrm{a}} \\
6^{\mathrm{a}}\end{array}$ & $\begin{array}{l}\text { 95th percentile for population mobility } \\
\text { Default value }\end{array}$ \\
\hline & $\begin{array}{l}\text { Averaging Time (AT) } \\
\text { Adult } \\
\text { Cancer } \\
\text { Noncancer } \\
\text { Child } \\
\text { Cancer } \\
\text { Noncancer }\end{array}$ & days & $\begin{array}{l}25,550^{\mathrm{a}} \\
10,950^{\mathrm{a}} \\
25,550^{\mathrm{a}} \\
2,190^{\mathrm{a}}\end{array}$ & $\begin{array}{l}\text { Default value } \\
\text { Equal to exposure duration } \\
\text { Default value } \\
\text { Equal to exposure duration }\end{array}$ \\
\hline \multicolumn{5}{|c|}{ Surface Water (Incidental Ingestion): } \\
\hline & Absorption Factor $\left(\mathrm{ABS}_{\text {ingest }}\right)$ & unitless & $1^{a}$ & Default value \\
\hline & Ingestion Rate $\left(\mathrm{IR}_{\mathrm{SW}}\right)$ & L/day & $0.4^{\mathrm{C}}$ & $50 \mathrm{ml}$ per hour for 8 hours a day \\
\hline & Exposure Frequency $\left(\mathrm{EF}_{\mathrm{sW}}\right)$ & days/year & $18^{g}$ & Three day stay every other month \\
\hline \multicolumn{5}{|c|}{ Surface Water (Dermal Contact) } \\
\hline & $\begin{array}{l}\text { Surface Area of Exposed } \\
\text { Skin (SA) }\end{array}$ & $\mathrm{cm}^{2} /$ event & $5,700^{\mathrm{b}}$ & $\begin{array}{l}\text { 95th percentile area of head, arms, forearms, } \\
\text { and hands of adult males and females }\end{array}$ \\
\hline & $\begin{array}{l}\text { Dermal Permeability } \\
\text { Constant }\left(\mathrm{K}_{\mathrm{p}}\right)\end{array}$ & $\mathrm{cm} / \mathrm{hr}$ & e & Constituent-specific value \\
\hline & Exposure Time $\left(\mathrm{ET}_{\mathrm{SW}}\right)$ & hrs/day & $8^{g}$ & $\begin{array}{l}\text { Time spent in direct contact with surface water } \\
\text { during each day }\end{array}$ \\
\hline & Exposure Frequency $\left(\mathrm{EF}_{\mathrm{SW}}\right)$ & days/year & $18^{\mathrm{g}}$ & Three day stay every other month \\
\hline \multicolumn{5}{|c|}{ Sediment (Incidental Ingestion): } \\
\hline & Absorption Factor $\left(\right.$ ABS $\left._{\text {ingest }}\right)$ & unitless & $1^{a}$ & Default value \\
\hline & Ingestion Rate $\left(\mathrm{IR}_{\text {sed }}\right)$ & $\mathrm{mg} /$ day & $100^{f}$ & Upper percentile estimate for adult soil ingestion \\
\hline & Exposure Frequency $\left(\mathrm{EF}_{\text {sed }}\right)$ & days/year & $18^{g}$ & Three day stay every other month \\
\hline
\end{tabular}


Table 4-36

Parameters for Part-Time Subsistence User Exposure Pathways (Page 2 of 2)

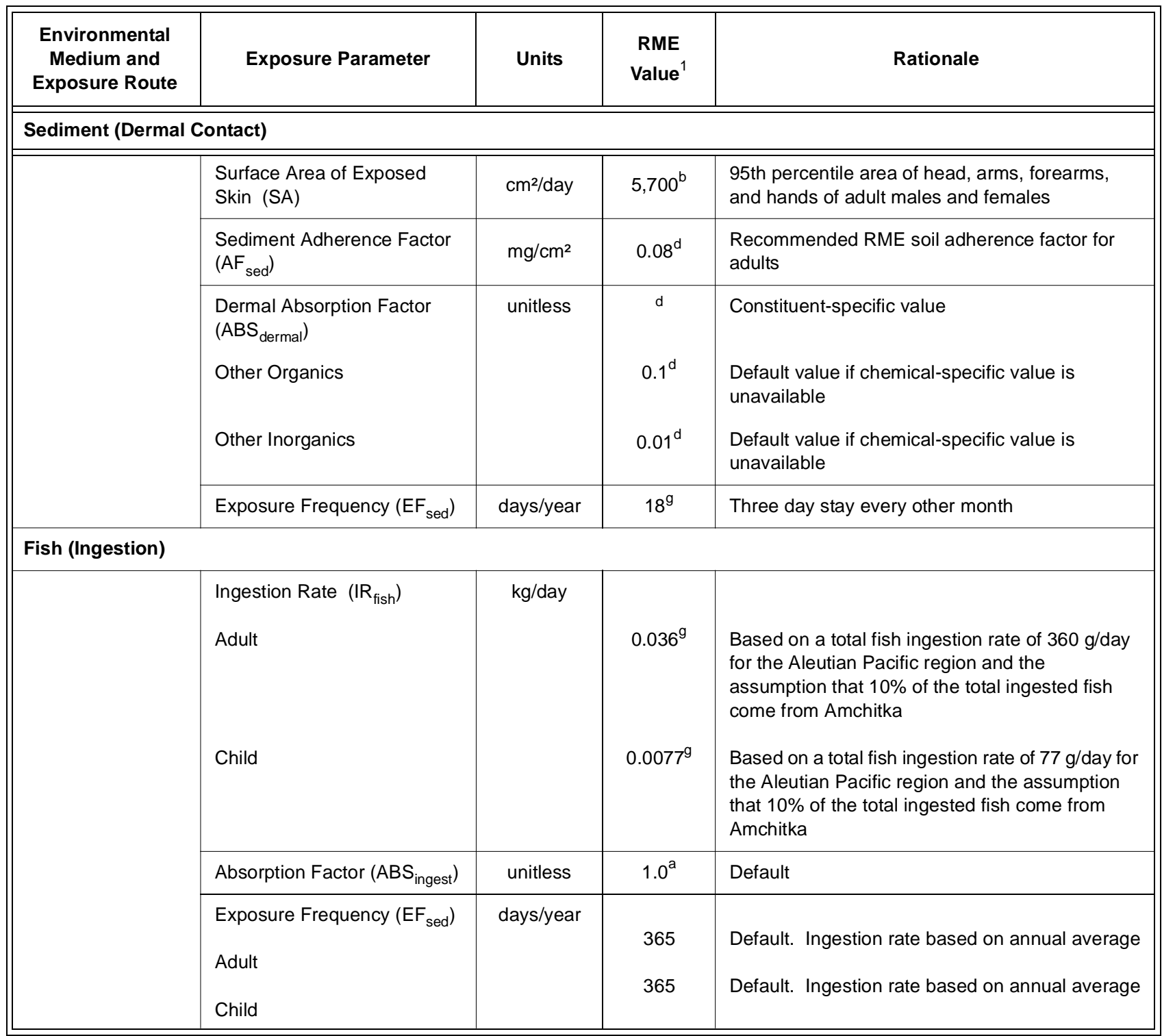

Notes:

1. Reasonable Maximum Exposure (RME) parameters are based on upper bound (e.g., 90-95th percentile) exposure values

aU.S. EPA, 1991. Human Health Evaluation Manual, Supplemental Guidance, Standard Default Exposure Factors. OSWER Directive 9285.6-03.

b.S. EPA, 1997. Exposure Factors Handbook. EPA/600/P-95/002F.

CU.S. EPA, 1989. Risk Assessment Guidance for Superfund, Volume I: Human Health Evaluation Manual, EPA/540/1-89/002.

U.S. EPA, 1998a. Risk Assessment Guidance for Superfund, Volume I: Human Health Evaluation Manual, Supplemental Guidance, Dermal Risk Assessment, Interim Guidance.

EU.S. EPA, 1992. Dermal Exposure Assessment: Principles and Applications, EPA/600/8-91/011B.

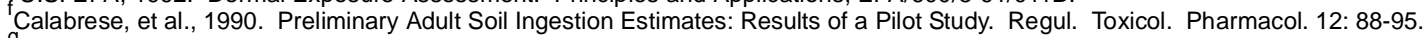

${ }^{9}$ Site-specific risk assessment assumption. 


$$
\mathrm{UCL}=e^{\left(\overline{\mathrm{x}}+0.5 \mathrm{~s}^{2}+\sqrt[\mathrm{sH} /]{\mathrm{n}-1}\right)}
$$

where:

$$
\begin{array}{ll}
\mathrm{UCL} & =\text { upper confidence limit } \\
e & =\text { base of the natural log, equal to } 2.718 \\
\overline{\mathrm{X}} & =\text { arithmetic mean of the log transformed data } \\
\mathrm{s} & =\text { standard deviation of the log transformed data } \\
\mathrm{H} & =\text { H-statistic (from Gilbert, 1987) } \\
\mathrm{n} & =\text { number of samples }
\end{array}
$$

If the 95 percent UCL concentration is greater than the maximum detected concentration, then the maximum detected compound concentration will be used to represent the exposure point concentration of the particular contaminant.

\subsubsection{Ingestion of Fish}

The CDI of compounds due to ingestion of fish caught on the island by on-site workers and part-time subsistence users is calculated by the following formula:

$$
\mathrm{CDI}_{\mathrm{F}}=\frac{\mathrm{C}_{\mathrm{F}} \times \mathrm{CR}_{\mathrm{F}} \times \mathrm{CF} \times \mathrm{ABS} \times \mathrm{EF} \times \mathrm{ED}}{\mathrm{BW} \times \mathrm{AT}}
$$

where:

$\begin{array}{ll}\mathrm{CDI}_{\mathrm{F}} & =\text { Chronic daily intake of fish }(\mathrm{mg} / \mathrm{kg} / \mathrm{day}) \\ \mathrm{C}_{\mathrm{F}} & =\text { Constituent concentration in fish }(\mathrm{mg} / \mathrm{kg}) \\ \mathrm{CR}_{\mathrm{F}} & =\text { Consumption rate of fish }(\mathrm{kg} / \mathrm{day}) \\ \mathrm{CF} & =\text { Conversion factor }(1.0) \\ \mathrm{ABS} & =\text { Absorption factor }(1.0, \text { unitless) } \\ \mathrm{EF} & =\text { Exposure frequency (days/year }[\mathrm{yr}]) \\ \mathrm{ED} & =\text { Exposure duration (years) } \\ \mathrm{BW} & =\text { Body weight }(\mathrm{kg}) \\ \mathrm{AT} & =\text { Averaging time (days). }\end{array}$


It should be noted that although fish tissue samples have been collected from several streams and ponds associated with the drill sites, the vast majority of the fish collected were very small in size (1 to 6 inches in total length) and not reasonably considered large enough for human consumption. The only water body that supports fish of sufficient size for human consumption is Cannikin Lake. Therefore, for this assessment, the exposure point concentrations of COCs in fish tissue will be determined from samples collected from Cannikin Lake only.

\subsubsection{Ingestion of Surface Water}

The CDI of compounds due to incidental ingestion of surface water on the island by part-time subsistence users is calculated by the following formula:

$$
\mathrm{CDI}_{\mathrm{SW}}=\frac{\mathrm{C}_{\mathrm{SW}} \times \mathrm{CR}_{\mathrm{SW}} \times \mathrm{ABS} \times \mathrm{EF} \times \mathrm{ED}}{\mathrm{BW} \times \mathrm{AT}}
$$

where:

$\mathrm{CDI}_{\mathrm{SW}} \quad=$ Chronic daily intake of surface water $(\mathrm{mg} / \mathrm{kg} /$ day $)$

$\mathrm{C}_{\mathrm{SW}} \quad=$ Constituent concentration in surface water $(\mathrm{mg} / \mathrm{L})$

$\mathrm{CR}_{\mathrm{SW}} \quad=$ Consumption rate of surface water $(\mathrm{L} /$ day $)$

$\mathrm{ABS} \quad=$ Absorption factor (1.0, unitless)

$\mathrm{EF} \quad=$ Exposure frequency $($ day/yr $)$

$\mathrm{ED} \quad=$ Exposure duration $(\mathrm{yrs})$

$\mathrm{BW} \quad=$ Body weight $(\mathrm{kg})$

AT $\quad=$ Averaging time (days)

\subsubsection{Direct Contact With Surface Water}

The CDI of compounds due to absorption of contaminants in surface water via direct dermal contact by on-site workers and part-time subsistence users is calculated by the following formula:

$$
\mathrm{CDI}_{\mathrm{SW}}=\frac{\mathrm{C}_{\mathrm{SW}} \times \mathrm{SA} \times \mathrm{PC} \times \mathrm{ET} \times \mathrm{EF} \times \mathrm{ED} \times \mathrm{CF}}{\mathrm{BW} \times \mathrm{AT}}
$$


where:

$\mathrm{CDI}_{\mathrm{SW}}=$ Chronic daily intake of surface water $(\mathrm{mg} / \mathrm{kg} /$ day $)$

$\mathrm{C}_{\mathrm{SW}} \quad=$ Constituent concentration in surface water $(\mathrm{mg} / \mathrm{L})$

SA $\quad=$ Surface area of skin exposed to surface water $\left(\mathrm{cm}^{2}\right)$

PC $=$ Dermal permeability constant $(\mathrm{cm} /$ hour $)$

ET $\quad=$ Exposure time (hours/day)

$\mathrm{EF} \quad=$ Exposure frequency $($ day/yr)

$\mathrm{ED} \quad=$ Exposure duration (yrs)

$\mathrm{CF}=$ Conversion factor $\left(1.0 \times 10^{-3} \mathrm{~L} / \mathrm{cm}^{3}\right)$

$\mathrm{BW} \quad=$ Body weight $(\mathrm{kg})$

AT $\quad=$ Averaging time (days)

\subsubsection{Ingestion of Sediment}

The CDI of compounds due to incidental ingestion of sediment on the island by part-time subsistence users is calculated by the following formula:

$$
\mathrm{CDI}_{\mathrm{Sed}}=\frac{\mathrm{C}_{\mathrm{Sed}} \times \mathrm{CR}_{\mathrm{Sed}} \times \mathrm{CF} \times \mathrm{ABS} \times \mathrm{EF} \times \mathrm{ED}}{\mathrm{BW} \times \mathrm{AT}}
$$

where:

$\mathrm{CDI}_{\text {Sed }} \quad=$ Chronic daily intake $(\mathrm{mg} / \mathrm{kg} /$ day $)$

$\mathrm{C}_{\mathrm{Sed}} \quad=$ Constituent concentration in sediment $(\mathrm{mg} / \mathrm{kg})$

$\mathrm{CR}_{\mathrm{Sed}} \quad=$ Consumption rate of sediment $(\mathrm{kg} /$ day $)$

$\mathrm{CF} \quad=$ Conversion factor $\left(1.0 \times 10^{-6} \mathrm{~kg} / \mathrm{mg}\right)$

$\mathrm{ABS}=$ Absorption factor (1.0, unitless)

$\mathrm{EF} \quad=$ Exposure frequency $($ days/yr)

$\mathrm{ED} \quad=$ Exposure duration $(\mathrm{yrs})$

$\mathrm{BW} \quad=$ Body weight $(\mathrm{kg})$

AT $\quad=$ Averaging time (days)

\subsubsection{Direct Contact With Sediment}

The CDI of compounds due to absorption of contaminants in sediment via direct dermal contact by on-site workers and part-time subsistence workers is calculated by the following formula:

$$
\mathrm{CDI}_{\mathrm{Sed}}=\frac{\mathrm{C}_{\mathrm{Sed}} \times \mathrm{SA} \times \mathrm{ABS} \times \mathrm{AF} \times \mathrm{EF} \times \mathrm{ED} \times \mathrm{CF}}{\mathrm{BW} \times \mathrm{AT}}
$$


where:

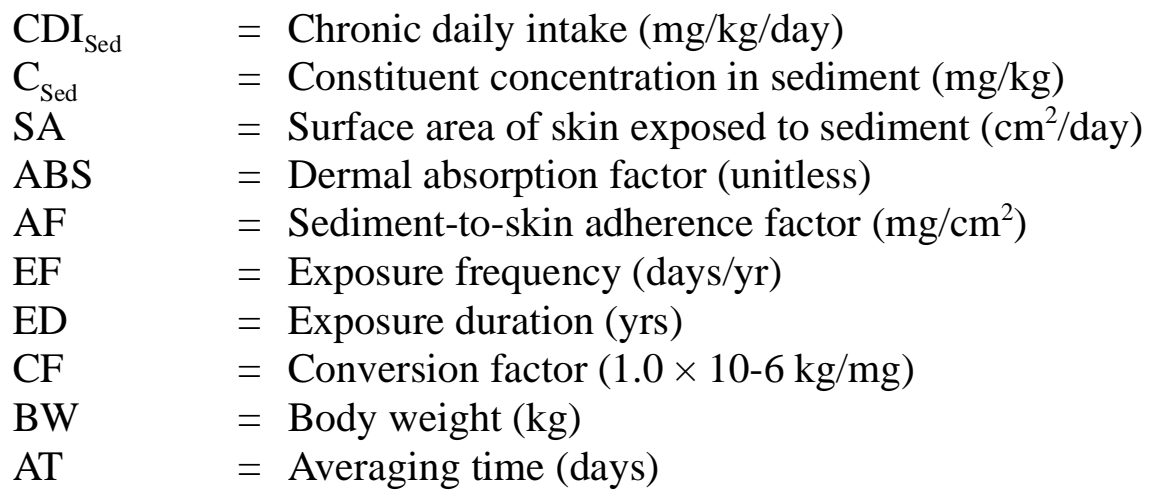

\subsection{Toxicity Assessment}

The toxicity assessment weighs the available evidence regarding the potential for particular compounds to cause adverse effects in exposed individuals (receptors) and provides, where possible, an estimate of the relationship between the extent of exposure to a compound and the increased likelihood and/or severity of induced adverse health effects. Two broad categories of chemically-induced disease states (e.g., carcinogenic effects and noncarcinogenic effects) are evaluated in the toxicity assessment of each identified compound.

As the exposure assessment attempts to define the chronic lifetime dosage of COCs received by an individual in a given scenario, the toxicity assessment links adverse effects associated with exposure to the particular COC. Establishing an association between exposure to a compound with possible adverse effects is the major tenet of toxicology. The dose received determines the magnitude of any anticipated adverse effects related to the compound's inherent toxicity.

Toxicity values are used in risk characterization to quantify the probability of observing cancer and noncancer effects in a potentially exposed population. Two types of toxicity values are used to express a COC's dose-response-effect relationship:

- A slope factor (SF) for estimating the likelihood of carcinogenic effects.

- A reference dose (RfD) or reference concentration ( $R f C)$ for estimating possible noncarcinogenic effects. 
In general, SF and RfD values, expressed in the units of $(\mathrm{mg} / \mathrm{kg} / \mathrm{day})^{-1}$ and $\mathrm{mg} / \mathrm{kg} /$ day, respectively, are derived from long-term animal studies and incorporate uncertainty factors to compensate for extrapolation of observed adverse effects in laboratory animals to estimate possible adverse effects in humans. If adequate human data from epidemiological studies are available, these data are used to reduce uncertainty in deriving toxicity values.

\subsubsection{Toxicity Assessment for Carcinogenic Effects}

For carcinogens, risks are estimated as the incremental probability of an individual developing cancer over a lifetime as a result of exposure to the potential carcinogen (i.e., incremental or excess individual lifetime cancer risk). Based on the extent to which a compound has been shown to be a carcinogen in animal studies, in humans, or in both, the agent is given a provisional weight-ofevidence classification. The EPA's current classification of the overall weight of evidence has the following five categories:

- Group A: Human Carcinogen - Sufficient evidence from epidemiological studies substantiated by causal association between exposure and carcinogenicity.

- Group B1: Probable Human Carcinogen - Limited evidence of carcinogenicity in humans from available epidemiological data.

- Group B2: Probable Human Carcinogen - Sufficient evidence of carcinogenicity in animals, but inadequate or no evidence in humans.

- Group C: Possible Human Carcinogen - Limited evidence of carcinogenicity in animals.

- Group D: Not Classified - Inadequate evidence of carcinogenicity in animals to support classification.

- Group E: Not a Human Carcinogen - No evidence of carcinogenicity in at least two adequate animal tests in different species or in both epidemiological and animal studies.

Based on the evidence that a compound is a known or probable human carcinogen, a toxicity value that defines a quantitative relationship between dose and response (i.e., SF) is calculated by the EPA. An SF converts estimated daily intakes averaged over a lifetime of exposure directly to incremental risk of an individual developing cancer. 


\subsubsection{Toxicity Assessment of Noncarcinogenic Effects}

A chronic RfD or RfC is an estimate of the daily exposure to a human population, including sensitive subpopulations, that is unlikely to cause an increased incidence of deleterious health effects during a lifetime of exposure. Chronic RfD or RfC values are specifically developed to be protective for long-term exposure to a compound.

Two toxicity assessment terms are used to characterize low dose exposure effects. The no-observed-adverse-effect-level (NOAEL) is an exposure level where there are no statistically or biologically significant increases in the frequency or severity of adverse effects in the exposed population. The lowest-observed-adverse-effect-level (LOAEL) is the lowest exposure dose in a dose-response experiment at which there are statistically or biologically significant increases in severity or frequency of adverse effects in the exposed population.

In arriving at RfD or RfC toxicity values, the NOAEL or LOAEL is divided by additional factors to account for uncertainties in extrapolation from subchronic to chronic exposures and from uncertain species-to-species toxicity relationships. These uncertainty factors can range from 1 to 10,000.

The toxicity assessment component of this human health risk assessment will be mainly dependent upon the use of EPA-derived toxicity values. The resource hierarchy for selection of the most current and appropriate toxicity values will be, in descending order:

- Integrated Risk Information System (IRIS), an on-line database maintained by the EPA (IRIS, 2000)

- Health Effects Assessment Summary Tables (HEAST), published annually by the EPA (EPA, 1997a)

- Agency for Toxic Substances and Disease Registry (ATSDR) toxicological profiles, compound-specific literature reviews of the use/production, chemical properties, toxicology, analytical methodology, and regulatory status of a compound

- EPA Environmental Criteria and Assessment Office (ECAO) guidance documents, general toxicology information, extrapolations, and guidance for compounds without toxicity values 


\subsubsection{Derivation of Dermal Toxicity Values}

Because there are few, if any, toxicity reference values for dermal exposure, oral values are frequently used to assess risks from dermal exposure. Most RfDs and some slope factors are expressed as the amount of substance administered per unit time and unit body weight, whereas exposure estimates for the dermal route of exposure are expressed as absorbed doses. Thus, for dermal exposure to contaminants, it may be necessary to adjust an oral toxicity value from an administered to a dermally absorbed dose. The methodology used to adjust oral toxicity values to dermally absorbed toxicity values is presented in Appendix A of the EPA's Risk Assessment Guidance Manual for Superfund: Vol. 1, Human Health Evaluation Manual (Part A) (EPA, 1989).

In order to adjust oral toxicity values to dermal toxicity values, the orally administered absorption efficiency must be known. This information is available for some compounds in their respective toxicological profiles prepared by ATSDR or in the IRIS database. However, for a number of compounds, this information is not available. For compounds without compound-specific absorption efficiencies, default values suggested by EPA Region VI (5 percent for metals and 100 percent for organics) are used to adjust the oral toxicity values to dermally absorbed toxicity values in the following manner:

$$
\mathrm{RfD}_{\text {derm }}=\mathrm{RfD}_{\text {oral }} \times \mathrm{AE}_{\text {oral }}
$$

where:

$\mathrm{RfD}_{\text {derm }} \quad=$ dermally absorbed noncancer reference dose

$\mathrm{RfD}_{\text {oral }} \quad=$ orally administered noncancer reference dose

$\mathrm{AE}_{\text {oral }} \quad=$ absorption efficiency of orally administered dose

and

$$
\mathrm{CSF}_{\text {derm }}=\frac{\mathrm{CSF}_{\text {oral }}}{\mathrm{AE}_{\text {oral }}}
$$


where:

$\mathrm{CSF}_{\text {derm }}=$ dermally absorbed cancer slope factor

$\mathrm{CSF}_{\text {oral }} \quad=$ orally administered cancer slope factor

$\mathrm{AE}_{\text {oral }} \quad=$ absorption efficiency of orally administered dose

\subsection{Risk Characterization}

The risk characterization will combine the information presented in the exposure assessment with the information presented in the toxicity assessment to describe the type and magnitude of potential carcinogenic risks and noncarcinogenic hazards due to exposure to contaminants originating from the drill sites on Amchitka Island. The magnitude and types of risks depend on the nature, duration, and frequency of exposure to COCs, and the characteristics of the exposed populations.

\subsubsection{Characterization of Carcinogenic Effects}

For carcinogens, risks are estimated as the incremental probability of an individual developing cancer over a lifetime as a result of exposure to the potential carcinogen (i.e., excess individual lifetime cancer risk).

Based on the evidence that a compound is a known or probable human carcinogen, a toxicity value that defines a quantitative relationship between dose and response (i.e., SF) is calculated. A SF converts estimated daily intakes averaged over a lifetime of exposure directly to incremental risk of an individual developing cancer. A critical assumption of this approach is that the dose-response relationship is a linear relationship in the low-dose portion of the dose-response curve. Under this assumption, the SF is a constant and risk will be directly related to intake. Thus, the linear form of the carcinogenic risk equation is usually applicable for estimating site risks. This linear low-dose equation is defined as:

$$
\text { Risk }=\mathrm{CDI} \times \mathrm{SF}
$$

where:

Risk = a unitless probability (e.g., $1 \times 10^{-6}$ ) of an individual developing cancer over a lifetime; 
CDI = chronic daily intake averaged over 70 years $(\mathrm{mg} / \mathrm{kg} /$ day $)$

$\mathrm{SF} \quad=$ slope factor, expressed in $(\mathrm{mg} / \mathrm{kg} / \mathrm{day})^{-1}$

The aggregate carcinogenic risk from exposure to several compounds is calculated by summation of the individual cancer risks for each compound in each appropriate exposure pathway, and then summing the total carcinogen risk for all relevant exposure pathways:

$$
\text { Risk }_{\mathrm{T}}=\Sigma \text { Risk }_{\mathrm{ij}}
$$

where:

Risk $_{\mathrm{T}} \quad=$ the total cancer risk, expressed as a unitless probability

Risk $_{\mathrm{ij}} \quad=$ the risk estimate for the $\mathrm{i}^{\text {th }}$ compound in the $\mathrm{j}^{\text {th }}$ exposure medium pathway.

The resultant summation of compound-specific cancer risks is a very conservative upper bound estimate of cancer risk for the following reason. Each SF is an upper $95^{\text {th }}$ percentile estimate of potency, and because percentiles of probability distributions are not strictly additive, the total cancer risk estimate becomes more conservative as the number of cancer risk estimates are summed. However, this method is used to ensure that carcinogenic risks will not be underestimated.

\subsubsection{Characterization of Noncarcinogenic Effects}

A chronic RfD or RfC is an estimate of the daily exposure to a human population, including sensitive subpopulations, that is unlikely to cause an increased incidence of deleterious health effects during a lifetime of exposure. Chronic RfD or RfC values are specifically developed to be protective for long-term exposure to a compound.

For noncarcinogenic compounds, the measure used to describe the potential for noncarcinogenic toxicity to occur in an individual is evaluated by comparing the estimated exposure level over a specified time period (e.g., lifetime) with the appropriate noncancer toxicity value (i.e., RfD or RfC).

This ratio of exposure to toxicity is called a noncancer hazard quotient (HQ):

$$
H Q=\frac{C D I}{R f D}
$$


where:

HQ $\quad=$ hazard quotient

CDI $\quad=$ chronic daily intake $(\mathrm{mg} / \mathrm{kg} /$ day $)$

RfD $\quad=$ Reference dose.

The noncarcinogenic hazard quotient assumes that there is a level of exposure (e.g., RfD or RfC) below which it is unlikely for even sensitive subpopulations to experience adverse health effects.

For assessing the health impacts of several noncarcinogenic compounds, RfDs or RfCs are compared to exposure-specific intake rates of each COC. A summation of these hazard quotients is termed the hazard index $(\mathrm{HI})$. The aggregate $\mathrm{HI}$ is expressed as:

$$
\mathrm{HI}_{\mathrm{T}}=\Sigma_{\mathrm{k}} \frac{\mathrm{CDI}_{j}}{\operatorname{RfD}_{\mathrm{j}}}
$$

where:

$\mathrm{HI}_{\mathrm{T}} \quad=$ total hazard index for cumulative exposure scenarios for an individual

$\mathrm{k} \quad=\mathrm{kth}$ exposure pathway

$\mathrm{CDI}_{\mathrm{j}} \quad=$ chronic daily exposure for the $\mathrm{jth}$ compound in each exposure medium

$\operatorname{RfD}_{\mathrm{j}} \quad=$ Reference dose for the $\mathrm{j}^{\mathrm{th}}$ compound.

If this ratio of the daily intake to the RfD or RfC exceeds 1.0 (unity) for the defined exposure scenario, this provides an indication that the exposed receptor may be subject to an adverse health impact and that further investigation should be undertaken. If the ratio is below unity, then it is generally assumed that no adverse impact to human health has or will occur.

The HI approach does have limitations and should be interpreted carefully based on the known aspects of additive toxic effects from exposure to mixtures of chemicals. First, because both the HQ and $\mathrm{HI}$ are ratios, after unity has been exceeded, the magnitude of the index has little bearing on the potential severity of adverse effects that may be anticipated. An HI of five does not indicate the noncancer hazard is greater than a HI of three. Secondly, it is inappropriate to sum noncancer hazard quotients for compounds that do not have similar toxic modes of action or that do not affect the same organ system. Therefore, hazard quotients are only added for compounds that act on the same target organ or system. 


\subsubsection{Interpretation of Calculated Cancer Risk and Noncancer Hazard Quotients}

Current EPA guidance indicates that a site presents acceptable risk if the calculated cumulative cancer risk to a potentially exposed population is within the range of 1 in 10,000 to 1 in 1,000,000 $\left(1.0 \times 10^{-4}\right.$ to $\left.1.0 \times 10^{-6}\right)$ and the $\mathrm{HI}$ for noncancer adverse health effects is less than 1.0 (EPA, 1991). Per ADEC guidance (18 AAC 75.325 [ADEC, 1999b]), action may be warranted at a site if the cumulative carcinogenic risk is greater than 1 in $100,000\left(1.0 \times 10^{-5}\right)$ or if the cumulative noncarcinogenic $\mathrm{HI}$ is greater than 1.0. However, under certain circumstances, “. . . the department will, in its discretion, consider a risk standard consistent with the range acceptable under 40 CFR 300.430, revised as of July 1, 1996. ..” (ADEC, 2000c).

\subsection{Derivation of Human Health Risk-Based Cleanup Levels}

If the results of the human health risk assessment indicate impacted media at the site require remediation, site-specific human health risk-based cleanup levels (HHRBCLs) will be derived. A target cancer risk of $10^{-6}$ for individual carcinogens, and a target hazard quotient of 0.1 for individual noncarcinogens, will be used in the calculation of cleanup levels in order to account for potential cumulative effects and ensure that the cumulative carcinogenic risk is less than $1.0 \times 10^{-5}$ and the cumulative noncancer health hazard is less than 1.0. The HHRBCLs for a given chemical will be the more conservative of those based on carcinogenic effects and those based on noncarcinogenic effects.

The methodologies for calculating site-specific HHRBCLs are presented in the following sections.

\subsubsection{Sediment HHRBCLs}

The HHRBCLs for carcinogens in sediment will be estimated as follows:

$$
\mathrm{HHRBCL}_{\mathrm{Sed}}=\frac{\mathrm{CR}_{\mathrm{T}}}{\left(\left[\frac{\left[\mathrm{R}_{\mathrm{S}} \times \mathrm{EF} \times \mathrm{ED} \times \mathrm{SF}_{\mathrm{o}}\right.}{\mathrm{BW} \times \mathrm{AT}_{\mathrm{c}}}\right]+\left[\frac{\mathrm{CF} \times \mathrm{AF} \times \mathrm{SA} \times \mathrm{ABS} \times \mathrm{EF} \times \mathrm{ED} \times \mathrm{SF}_{\mathrm{d}}}{\mathrm{BW} \times \mathrm{AT}_{\mathrm{c}}}\right]\right)}
$$

HHRBCLs for noncarcinogens in sediment will be estimated as follows: 


$$
\mathrm{HHRBCL}_{\text {Sed }}=\frac{\mathrm{HQ}_{\mathrm{T}}}{\left(\left[\frac{\left(\mathrm{IR}_{\mathrm{S}} \times \mathrm{EF} \times \mathrm{ED}\right) /\left(\mathrm{BW} \times \mathrm{AT}_{\mathrm{n}}\right)}{\mathrm{RFD}_{\mathrm{o}}}\right]+\left[\frac{(\mathrm{CF} \times \mathrm{AF} \times \mathrm{SA} \times \mathrm{ABS} \times \mathrm{EF} \times \mathrm{ED}) /\left(\mathrm{BW} \times \mathrm{AT}_{\mathrm{n}}\right)}{\mathrm{RFD}_{\mathrm{d}}}\right]\right.}
$$

where:

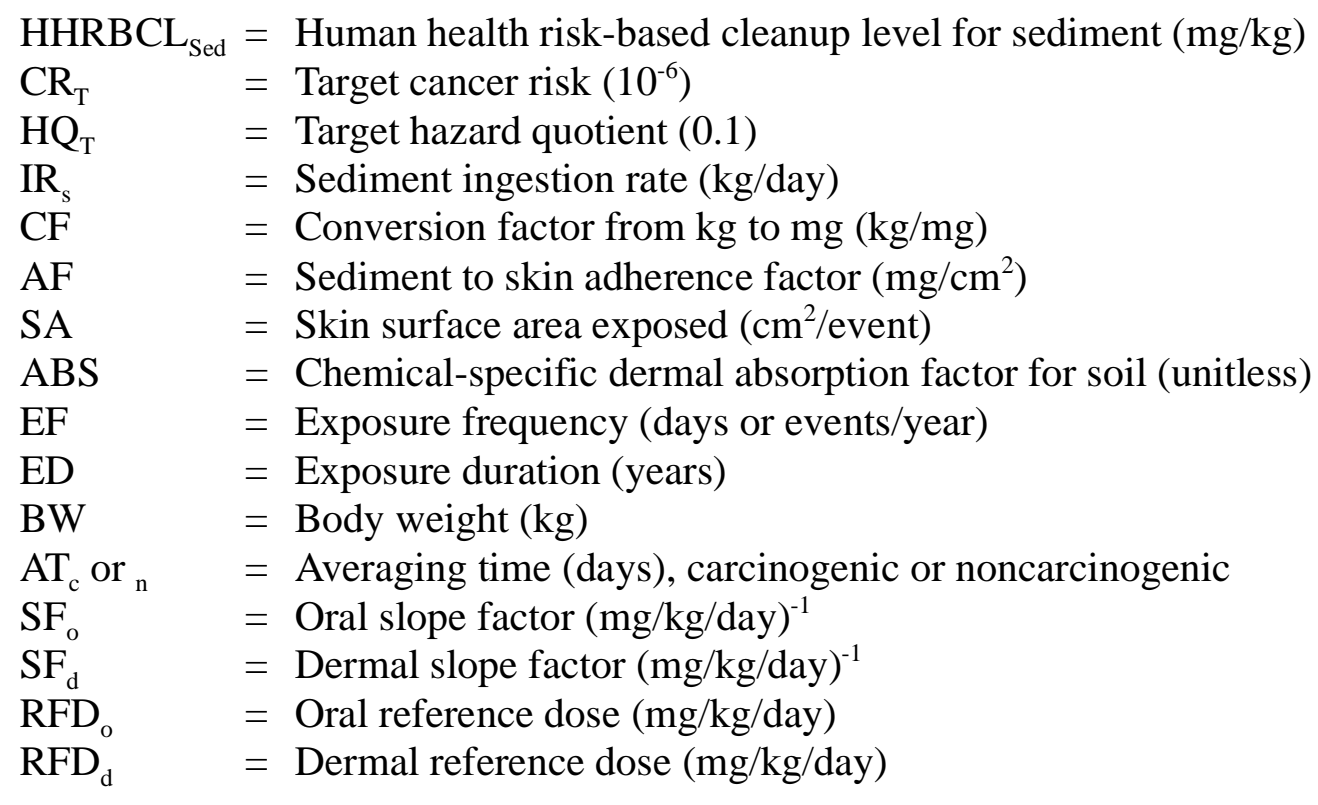

\subsubsection{Surface Water HHRBCLs}

HHRBCLs for carcinogens in fresh surface water will be calculated as follows:

$$
\text { HHRBCL }_{\mathrm{SW}}=\frac{\mathrm{CR}_{\mathrm{T}}}{\left(\left[\frac{\mathrm{CF} \times \mathrm{SA} \times \mathrm{P}_{\mathrm{c}} \times \mathrm{ET} \times \mathrm{ED} \times \mathrm{SF}_{\mathrm{d}}}{\mathrm{BW} \times \mathrm{AT}_{\mathrm{c}}}\right]+\left[\frac{\mathrm{IR}_{\mathrm{SW}} \times \mathrm{EF} \times \mathrm{ED} \times \mathrm{SF}_{\mathrm{d}}}{\mathrm{BW} \times \mathrm{AT}_{\mathrm{c}}}\right]\right)}
$$

HHRBSCs for noncarcinogens in fresh surface water will be calculated as follows:

$$
\mathrm{HHRBCL}_{\mathrm{SW}}=\frac{\mathrm{HQ}_{\mathrm{T}}}{\left(\left[\frac{\left(\mathrm{CF} \times \mathrm{SA} \times \mathrm{P}_{\mathrm{c}} \times \mathrm{ET} \times \mathrm{EF} \times \mathrm{ED}\right) /\left(\mathrm{BW} \times \mathrm{AT}_{\mathrm{n}}\right)}{\mathrm{RfD}_{\mathrm{d}}}\right]+\left[\frac{\left(\mathrm{IR}_{\mathrm{SW}} \times \mathrm{EF} \times \mathrm{ED}\right) /\left(\mathrm{BW} \times \mathrm{AT}_{\mathrm{n}}\right)}{\mathrm{RfD}_{\mathrm{c}}}\right]\right)}
$$


where:

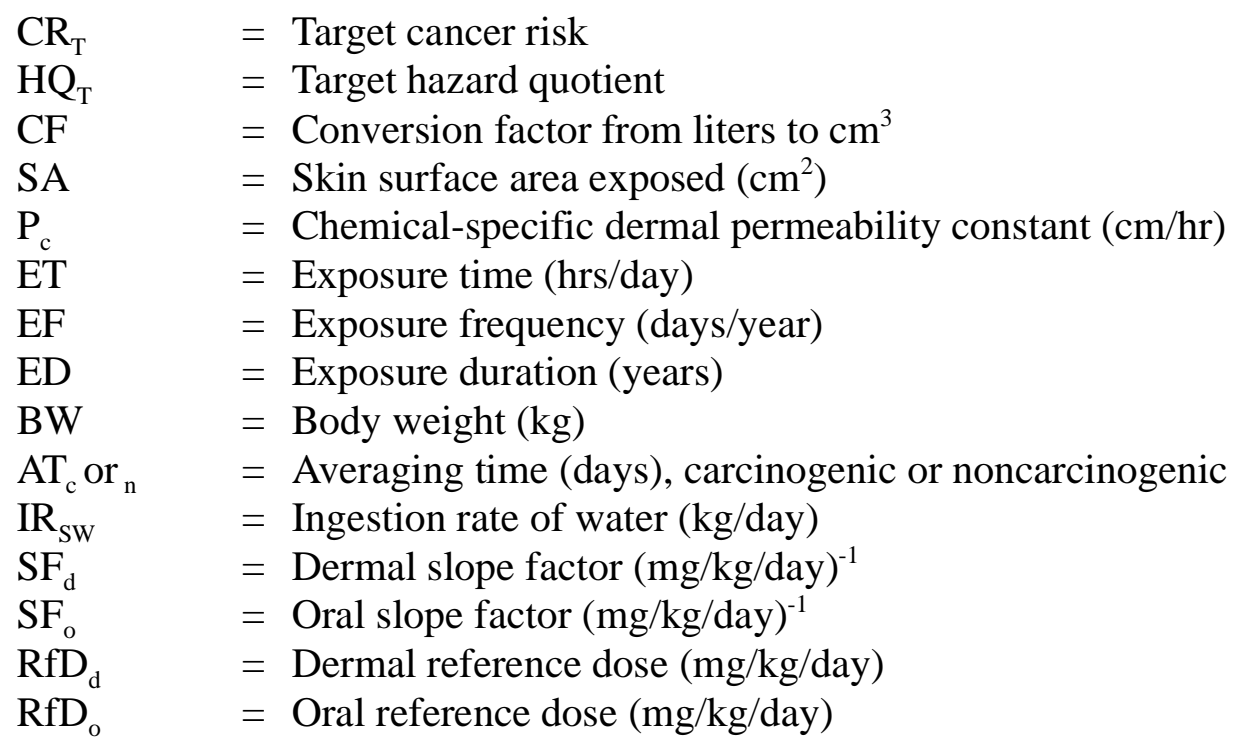

\subsubsection{Fish Tissue Target Levels}

Unlike abiotic media (surface water and sediment), fish tissue cannot be "cleaned up." Therefore, tissue concentrations that result in target cancer risks and noncancer hazards will be calculated. These calculated risk-based tissue concentrations can then be related back to surface water and sediment via bioconcentration and bioaccumulation factors for use in making risk management decisions. Fish tissue target levels (FTTLs) for carcinogens in fish will be calculated as follows:

$$
\text { FFTL }_{\mathrm{SW}}=\frac{\mathrm{CR}_{\mathrm{T}}}{\left[\frac{\mathrm{IR}_{\mathrm{Fish}} \times \mathrm{EF} \times \mathrm{ED} \times \mathrm{ABS} \times \mathrm{SF}_{\mathrm{o}}}{\mathrm{BW} \times \mathrm{AT}_{\mathrm{c}}}\right]}
$$

FTTLs for noncarcinogens in fish will be calculated as follows:

$$
\text { FFTL }_{\text {Fish }}=\frac{\mathrm{HQ}_{\mathrm{T}}}{\left[\frac{\left(\mathrm{IR}_{\mathrm{Fish}} \times \mathrm{EF} \times \mathrm{ED} \times \mathrm{ABS}\right) /\left(\mathrm{BW} \times \mathrm{AT}_{\mathrm{n}}\right)}{\mathrm{RfD}_{\mathrm{o}}}\right]}
$$


where:

$\mathrm{CR}_{\mathrm{T}} \quad=$ Target cancer risk

$\mathrm{HQ}_{\mathrm{T}} \quad=$ Target hazard quotient

$\mathrm{EF} \quad=$ Exposure frequency (days/year)

$\mathrm{ED} \quad=$ Exposure duration (years)

ABS $=$ Absorption factor (unitless)

$\mathrm{BW} \quad=$ Body weight $(\mathrm{kg})$

$\mathrm{AT}_{\mathrm{c}}$ or $\quad=$ Averaging time (days), carcinogenic or noncarcinogenic

$\mathrm{IR}_{\text {Fish }} \quad=$ Ingestion rate of fish ( $\mathrm{kg} /$ day)

$\mathrm{SF}_{\mathrm{o}} \quad=$ Oral slope factor $(\mathrm{mg} / \mathrm{kg} / \text { day })^{-1}$

$\mathrm{RfD}_{\mathrm{o}} \quad=$ Oral reference dose $(\mathrm{mg} / \mathrm{kg} /$ day $)$

\subsection{Uncertainty Analysis}

The uncertainty analysis will consist of a qualitative discussion of uncertainties involved in each step of the risk assessment process. Where possible, a qualitative description of the magnitude (e.g., high, medium, low) and direction (i.e., over-estimation, under-estimation) of the bias associated with the uncertainty will be specified. 


\subsection{Ecological Risk Assessment}

An ecological risk assessment will be conducted to determine the types and magnitudes of exposures to compounds originating from the drill sites on Amchitka Island and to determine the potential risks posed by the estimated exposures to ecological receptors. The procedures outlined in this Work Plan are in accordance with methods prescribed in ADEC and EPA guidance: Risk Assessment Procedures Manual (ADEC, 2000a), Framework for Ecological Risk Assessment (EPA, 1992), Region 10 Supplemental Ecological Risk Assessment Guidance for Superfund (EPA, 1997b), Ecological Risk Assessment Guidance for Superfund: Process for Designing and Conducting Ecological Risk Assessments (EPA, 1997c), and EPA Proposed Guidelines for Ecological Risk Assessment (EPA, 1998b). The following sections describe the methodologies that will be used in conducting the ecological risk assessment on Amchitka Island.

\subsection{Ecological Screening for Compounds of Potential Concern}

Similar to the human health risk assessment, the compounds for which environmental media at the site are screened in order to determine if those compounds are of potential ecological concern and will be addressed in the ecological risk assessment. In this section, the approach to screening for compounds of potential ecological concern (COPEC) is presented, followed by the screening conducted for the site. The result is a set of compounds that will be addressed in the ecological risk assessment.

\subsubsection{Approach to Screening for Compounds of Potential Concern}

The screening for compounds of potential ecological concern is based on the following four factors:

- Presence in abiotic media at sufficient frequency to be of potential concern

- Presence in abiotic media at sufficient concentrations to be of potential concern

- Presence of contaminants related to site activities

- Ability of contaminants to bioaccumulate in the food chain

If a chemical is identified as a COPEC, that does not imply that a risk exists or that even a risk is possible, but that the chemical is present such that it should be evaluated to determine if a risk is possible. 
Chemicals detected in either surface water or sediment samples were compared to ecological benchmarks to determine if they should be considered COPECs. If chemicals were not detected above appropriate benchmarks, they were not considered to be COPECs. The ecological benchmarks used are presented in Table 5-1 and Table 5-2, respectively. The following sources were used, in the order of preference listed, to identify appropriate ecological benchmarks for surface water:

- Alaska Department of Environmental Conservation Water Quality Standards (ADEC, 1999a) State surface water quality criteria represent a quality of water that supports a particular use. A total aqueous hydrocarbon standard is provided for the protection of freshwater systems for growth and propagation of fish, shellfish, other aquatic life, and wildlife. This was used as a conservative ecological benchmark for both DRO and gasoline-range organics (GRO).

- The EPA Ambient Water Quality Criteria (EPA, 1999) - Federal ambient water quality criteria have been developed by the EPA to prevent unacceptable acute or chronic effects to sensitive families of aquatic vertebrates, invertebrates, and plants. Chronic criteria, when available, were used as ecological benchmarks.

- Suter and Tsao (1996) Tier II Screening Values - Suter and Tsao refined toxicological benchmarks for aquatic receptors in order to screen chemicals at DOE sites. These benchmarks were developed for chemicals lacking sufficient data to derive ambient water quality criteria, and are concentrations that would be expected to be higher than criteria in no more than 20 percent of the cases. Suter and Tsao also presented chronic values for fish, daphnids, and aquatic plants. The lowest of these chronic values was used as a benchmark if a Tier II screening value was not presented.

- Final Chronic Values for Individual PAHs (EPA, 2000a) - In their draft guidance on the determination of PAH benchmarks for the protection of benthic organisms using the equilibrium partitioning (EqP) approach, the EPA developed final chronic values for water only exposures based on toxicity tests, determination of acute-to-chronic ratios, and extrapolation between PAH compounds. These final chronic values were used as benchmarks when not available in other sources.

- New York State Ambient Water Quality Standards (NYSDEC, 2000) - New York State has developed ambient water quality standards for freshwater quality for fish survival and propagation. Some of NYSDEC's standards are for compounds not found in other sources (e.g., trimethylbenzene and isopropylbenzene). These standards were used as ecological benchmarks for such compounds.

- AQUIRE database (AQUIRE, 2000) - The EPA provides an on-line database of aquatic toxicity data. This database was queried for toxicity data on freshwater fish and invertebrates for ecologically relevant end points (e.g., survival, reproduction, and growth). The lowest effect or no-effect levels were used as benchmarks after application of appropriate safety factors. A screening benchmark was derived from an acute lethal concentration-50\% $\left(\mathrm{LC}_{50}\right)$ by 
Table 5-1

Ecological Screening Values for Surface Water (Page 1 of 3 )

\begin{tabular}{|c|c|c|}
\hline Parameter & Screening Concentration $(\mu \mathrm{g} / \mathrm{L})$ & Source \\
\hline \multicolumn{3}{|c|}{ Inorganics } \\
\hline Aluminum & 87 & EPA, 1999 \\
\hline Arsenic & $150^{\mathrm{a}}$ & EPA, 1999 \\
\hline Barium & $4.0^{b}$ & Suter and Tsao, 1996 \\
\hline Beryllium & $0.66^{\mathrm{b}}$ & Suter and Tsao, 1996 \\
\hline Boron & $1.6^{\mathrm{b}}$ & Suter and Tsao, 1996 \\
\hline Cadmium & $2.2^{\mathrm{a}, \mathrm{c}}$ & EPA, 1999 \\
\hline Calcium & $116,000^{d}$ & Suter and Tsao, 1996 \\
\hline Cerium & $0.5^{\mathrm{e}}$ & AQUIRE, 2000 \\
\hline Cesium & $30^{f}$ & AQUIRE, 2000 \\
\hline Chromium & $74^{\mathrm{a}, \mathrm{c}}$ & EPA, 1999 \\
\hline Chromium III & $74^{\mathrm{a}, \mathrm{c}}$ & EPA, 1999 \\
\hline Chromium VI & $11^{\mathrm{a}}$ & EPA, 1999 \\
\hline Cobalt & $23^{b}$ & Suter and Tsao, 1996 \\
\hline Copper & $9.0^{\mathrm{a}, \mathrm{c}}$ & EPA, 1999 \\
\hline Iron & 1,000 & EPA, 1999 \\
\hline Lithium & $14^{\mathrm{b}}$ & Suter and Tsao, 1996 \\
\hline Magnesium & $82,000^{d}$ & Suter and Tsao, 1996 \\
\hline Manganese & $120^{\mathrm{b}}$ & Suter and Tsao, 1996 \\
\hline Molybdenum & $370^{\mathrm{b}}$ & Suter and Tsao, 1996 \\
\hline Nickel & $52^{a, c}$ & EPA, 1999 \\
\hline Potassium & $53,000^{d}$ & Suter and Tsao, 1996 \\
\hline Selenium & 5.0 & EPA, 1999 \\
\hline Strontium & $1,500^{b}$ & Suter and Tsao, 1996 \\
\hline Thallium & $12^{\mathrm{b}}$ & Suter and Tsao, 1996 \\
\hline Thorium & NAV & NAV \\
\hline Titanium & $29^{f}$ & AQUIRE, 2000 \\
\hline Uranium & $2.6^{\mathrm{b}}$ & Suter and Tsao, 1996 \\
\hline Vanadium & $20^{\mathrm{b}}$ & Suter and Tsao, 1996 \\
\hline Zinc & $120^{\mathrm{a}, \mathrm{c}}$ & EPA, 1999 \\
\hline
\end{tabular}


Table 5-1

Ecological Screening Values for Surface Water (Page 2 of 3 )

\begin{tabular}{|c|c|c|}
\hline Parameter & Screening Concentration $(\mu \mathrm{g} / \mathrm{L})$ & Source \\
\hline \multicolumn{3}{|c|}{ Volatile Organic Compounds } \\
\hline 1,2,4-Trimethylbenzene & 33 & NYSDEC, 2000 \\
\hline 1,3,5-Trimethylbenzene & $33^{9}$ & NYSDEC, 2000 \\
\hline 2-Butanone & $14,000^{b}$ & Suter and Tsao, 1996 \\
\hline Acetone & $1,500^{b}$ & Suter and Tsao, 1996 \\
\hline Benzene & $130^{b}$ & Suter and Tsao, 1996 \\
\hline Carbon Disulfide & $0.92^{b}$ & Suter and Tsao, 1996 \\
\hline Ethylbenzene & $7.3^{\mathrm{b}}$ & Suter and Tsao, 1996 \\
\hline Isopropylbenzene & $26^{f}$ & NYSDEC, 2000 \\
\hline $\mathrm{m}, \mathrm{p}$-Xylene & $13^{\mathrm{b}}$ & Suter and Tsao, 1996 \\
\hline Methylene chloride & $2,200^{b}$ & Suter and Tsao, 1996 \\
\hline n-Butylbenzene & $164^{\mathrm{f}, \mathrm{h}}$ & AQUIRE, 2000 \\
\hline n-Propylbenzene & $6^{f}$ & AQUIRE, 2000 \\
\hline o-Xylene & $13^{b}$ & Suter and Tsao, 1996 \\
\hline p-Isopropyltoluene & $26^{f}$ & AQUIRE, 2000 \\
\hline sec-Butylbenzene & $164^{\mathrm{f}, \mathrm{h}}$ & AQUIRE, 2000 \\
\hline Toluene & $9.8^{b}$ & Suter and Tsao, 1996 \\
\hline Xylene & $13^{\mathrm{b}}$ & Suter and Tsao, 1996 \\
\hline \multicolumn{3}{|c|}{ PAHs } \\
\hline Acenaphthene & $74^{d}$ & Suter and Tsao, 1996 \\
\hline Acenaphthylene & $307^{i}$ & EPA, 2000a \\
\hline Anthracene & $0.73^{b}$ & Suter and Tsao, 1996 \\
\hline Benzo(a)anthracene & $0.027^{b}$ & Suter and Tsao, 1996 \\
\hline Benzo(b)fluoranthene & $0.68^{f}$ & EPA, 2000a \\
\hline Benzo(k)fluoranthene & $0.64^{i}$ & EPA, 2000a \\
\hline Benzo(a)pyrene & $0.014^{b}$ & Suter and Tsao, 1996 \\
\hline Benzo(g,h,i)perylene & $0.44^{i}$ & EPA, 2000a \\
\hline Chrysene & $2.0^{i}$ & EPA, 2000a \\
\hline Dibenzo(a,h)anthracene & $0.28^{\mathrm{i}}$ & EPA, 2000a \\
\hline Fluoranthene & $15^{\mathrm{d}}$ & Suter and Tsao, 1996 \\
\hline
\end{tabular}


Table 5-1

Ecological Screening Values for Surface Water (Page 3 of 3 )

\begin{tabular}{|c|c|c|}
\hline Parameter & Screening Concentration $(\mu \mathrm{g} / \mathrm{L})$ & Source \\
\hline Fluorene & $3.9^{b}$ & Suter and Tsao, 1996 \\
\hline Indeno(1,2,3-c,d)pyrene & $0.28^{\mathrm{i}}$ & EPA, 2000a \\
\hline Naphthalene & $12^{\mathrm{b}}$ & EPA, 2000a \\
\hline Phenanthrene & $200^{d}$ & Suter and Tsao, 1996 \\
\hline Pyrene & $10^{i}$ & EPA, 2000a \\
\hline \multicolumn{3}{|c|}{ PCBs } \\
\hline Aroclor 1016 & 0.014 & EPA, 1999 \\
\hline Aroclor 1221 & 0.014 & EPA, 1999 \\
\hline Aroclor 1232 & 0.014 & EPA, 1999 \\
\hline Aroclor 1242 & 0.014 & EPA, 1999 \\
\hline Aroclor 1248 & 0.014 & EPA, 1999 \\
\hline Aroclor 1254 & 0.014 & EPA, 1999 \\
\hline Aroclor 1260 & 0.014 & EPA, 1999 \\
\hline Aroclor 1262 & 0.014 & EPA, 1999 \\
\hline Aroclor 1268 & 0.014 & EPA, 1999 \\
\hline \multicolumn{3}{|c|}{ Petroleum Hydrocarbons } \\
\hline Diesel-Range Organics & $15^{\mathrm{j}}$ & ADEC, 1999a \\
\hline
\end{tabular}

${ }^{\mathrm{a}}$ Expressed in terms of dissolved metal in water.

${ }^{\mathrm{b}}$ Tier II Secondary Chronic Value.

${ }^{\mathrm{c}}$ Screening concentration is a function of hardness. Number presented is for a hardness of $100 \mathrm{mg} / \mathrm{L}$ as $\mathrm{CaCO}_{3}$.

${ }^{\mathrm{d}}$ Lowest chronic value.

${ }^{\mathrm{e}}$ Value is derived by dividing acute LOEL by 100 .

${ }^{f} V a l u e$ is derived by dividing lowest $L_{50}$ by 250 .

${ }^{9}$ Value for 1,2,4-Trimethylbenzene used as a surrogate.

${ }^{\mathrm{h}}$ Value for tert-butylbenzene used as a surrogate.

${ }^{i}$ Final Chronic Values calculated for derivation of equilibrium partitioning sediment guidelines (ESGs).

${ }^{\mathrm{j}}$ Measured as total aqueous hydrocarbons.

NAV - Not available

$\mu \mathrm{g} / \mathrm{L}$ - Micrograms per liter 
Table 5-2

Ecological Screening Values for Sediment

(Page 1 of 3 )

\begin{tabular}{|c|c|c|}
\hline Parameter & $\begin{array}{l}\text { Screening Concentration } \\
(\mathrm{mg} / \mathrm{kg})\end{array}$ & Source \\
\hline \multicolumn{3}{|c|}{ Inorganics } \\
\hline Aluminum & $58,000^{d}$ & Jones et al., 1997 \\
\hline Arsenic & 6 & Persaud et al., 1992 \\
\hline Barium & NAV & - \\
\hline Beryllium & NAV & - \\
\hline Boron & NAV & - \\
\hline Cadmium & 0.6 & Persaud et al., 1992 \\
\hline Calcium & NAV & - \\
\hline Cerium & NAV & - \\
\hline Cesium & NAV & - \\
\hline Chromium & 26 & Persaud et al., 1992 \\
\hline Cobalt & NAV & - \\
\hline Copper & 16 & Persaud et al., 1992 \\
\hline Iron & 20,000 & Persaud et al., 1992 \\
\hline Lithium & NAV & - \\
\hline Magnesium & NAV & - \\
\hline Manganese & 460 & Persaud et al., 1992 \\
\hline Molybdenum & NAV & - \\
\hline Nickel & 16 & Persaud et al., 1992 \\
\hline Potassium & NAV & - \\
\hline Selenium & NAV & - \\
\hline Strontium & NAV & - \\
\hline Thallium & NAV & - \\
\hline Thorium & NAV & - \\
\hline Titanium & NAV & - \\
\hline Uranium & NAV & - \\
\hline Vanadium & NAV & - \\
\hline Zinc & 120 & Persaud et al., 1992 \\
\hline
\end{tabular}


Table 5-2

Ecological Screening Values for Sediment

(Page 2 of 3 )

\begin{tabular}{|c|c|c|}
\hline Parameter & $\begin{array}{l}\text { Screening Concentration } \\
(\mathrm{mg} / \mathrm{kg})\end{array}$ & Source \\
\hline \multicolumn{3}{|c|}{ Volatile Organic Compounds } \\
\hline 1,2,4-Trimethylbenzene & $1.2^{\mathrm{a}}$ & EqP Approach (EPA, 2000a) \\
\hline 1,3,5-Trimethylbenzene & $0.76^{a}$ & EqP Approach (EPA, 2000a) \\
\hline 2-Butanone & $14^{\mathrm{C}}$ & \\
\hline Acetone & $1.5^{\mathrm{c}}$ & \\
\hline Benzene & $0.34^{\mathrm{a}}$ & MacDonald et al., 1992 \\
\hline Carbon disulfide & $0.001^{\mathrm{C}}$ & \\
\hline Ethylbenzene & $1.4^{\mathrm{a}}$ & MacDonald et al., 1992 \\
\hline Isopropylbenzene & $0.10^{\mathrm{a}}$ & EqP Approach (EPA, 2000a) \\
\hline $\mathrm{m}, \mathrm{p}$-Xylene & $0.12^{\mathrm{a}}$ & MacDonald et al., 1992 \\
\hline Methylene chloride & $0.5^{\mathrm{a}}$ & MacDonald et al., 1992 \\
\hline n-Butylbenzene & $22^{a}$ & EqP Approach (EPA, 2000a) \\
\hline n-Propylbenzene & $0.25^{\mathrm{a}}$ & EqP Approach (EPA, 2000a) \\
\hline o-Xylene & $0.12^{\mathrm{a}}$ & MacDonald et al., 1992 \\
\hline p-Isopropyltoluene & $2.8^{\mathrm{a}}$ & EqP Approach (EPA, 2000a) \\
\hline sec-Butylbenzene & $24^{\mathrm{a}}$ & EqP Approach (EPA, 2000a) \\
\hline Toluene & $2.5^{\mathrm{a}}$ & MacDonald et al., 1992 \\
\hline Xylene & $0.12^{\mathrm{a}}$ & MacDonald et al., 1992 \\
\hline \multicolumn{3}{|c|}{ PAHs } \\
\hline Acenaphthene & $4.91^{a}$ & EPA, 2000a \\
\hline Acenaphthylene & $4.52^{\mathrm{a}}$ & EPA, 2000a \\
\hline Anthracene & $5.94^{\mathrm{a}}$ & EPA, 2000a \\
\hline Benzo(a)anthracene & $8.41^{a}$ & EPA, 2000a \\
\hline Benzo(a)pyrene & $9.65^{a}$ & EPA, 2000a \\
\hline Benzo(b)fluoranthene & $9.79^{\mathrm{a}}$ & EPA, 2000a \\
\hline Benzo(g,h,i)perylene & $10.95^{\mathrm{a}}$ & EPA, 2000a \\
\hline Benzo(k)fluoranthene & $9.81^{a}$ & EPA, 2000a \\
\hline Chrysene & $8.44^{\mathrm{a}}$ & EPA, 2000a \\
\hline Dibenzo(a,h)anthracene & $11.23^{\mathrm{a}}$ & EPA, 2000a \\
\hline Fluoranthene & $7.07^{\mathrm{a}}$ & EPA, 2000a \\
\hline
\end{tabular}


Table 5-2

Ecological Screening Values for Sediment

(Page 3 of 3 )

\begin{tabular}{|c|c|c|}
\hline Parameter & $\begin{array}{c}\text { Screening Concentration } \\
(\mathrm{mg} / \mathrm{kg})\end{array}$ & Source \\
\hline Fluorene & $5.38^{\mathrm{a}}$ & EPA, 2000a \\
\hline Indeno(1,2,3-c,d)pyrene & $11.15^{\mathrm{a}}$ & EPA, 2000a \\
\hline Naphthalene & $3.85^{\mathrm{a}}$ & EPA, 2000a \\
\hline Phenanthrene & $5.96^{\mathrm{a}}$ & EPA, 2000a \\
\hline Pyrene & $6.97^{\mathrm{a}}$ & EPA, 2000a \\
\hline \multicolumn{3}{|c|}{ PCBs } \\
\hline Aroclor 1016 & $0.07^{\mathrm{a}, \mathrm{b}}$ & Persaud et al., 1992 \\
\hline Aroclor 1221 & $0.07^{\mathrm{a}, \mathrm{b}}$ & Persaud et al., 1992 \\
\hline Aroclor 1232 & $0.07^{\mathrm{a}, \mathrm{b}}$ & Persaud et al., 1992 \\
\hline Aroclor 1242 & $0.07^{\mathrm{a}, \mathrm{b}}$ & Persaud et al., 1992 \\
\hline Aroclor 1248 & $0.07^{\mathrm{a}, \mathrm{b}}$ & Persaud et al., 1992 \\
\hline Aroclor 1254 & $0.07^{\mathrm{a}, \mathrm{b}}$ & Persaud et al., 1992 \\
\hline Aroclor 1260 & $0.07^{a, b}$ & Persaud et al., 1992 \\
\hline Aroclor 1262 & $0.07^{\mathrm{a}, \mathrm{b}}$ & Persaud et al., 1992 \\
\hline Aroclor 1268 & $0.07^{\mathrm{a}, \mathrm{b}}$ & Persaud et al., 1992 \\
\hline \multicolumn{3}{|c|}{ Petroleum Hydrocarbons } \\
\hline Diesel-Range Organics & $640^{\mathrm{a}, \mathrm{e}}$ & EqP Approach (EPA, 2000a) \\
\hline Gasoline-Range Organics & $1.2^{\mathrm{a}, \mathrm{e}}$ & EqP Approach (EPA, 2000a) \\
\hline
\end{tabular}

${ }^{\text {a }}$ Screening concentration is based on $1 \%$ organic carbon.

${ }^{b}$ Screening concentration is for total PCBs.

${ }^{\mathrm{c}}$ Since Log KOC < 2 assumes VOC partitions completely to pore water and conservatively assumes $50 \%$ moisture content.

${ }^{\mathrm{d}}$ Probable Effects Concentration from Assessment and Remediation of Contaminated Sediments Program.

${ }^{e}$ Equilibrium Partitioning Approach using $15 \mu \mathrm{g} / \mathrm{L}$ total aqueous hydrocarbon standard, and KOC values and percent aromatics and aliphatics from ADEC (2000b).

NAV - Not available

$\mathrm{mg} / \mathrm{kg}$ - Milligrams per kilogram 
dividing it by a safety factor of 250 , whereas a screening benchmark was derived from an acute LOEL by dividing it by a safety factor of 100 (ADEC, 2000a).

Similarly, the following sources were used, in the order of preference listed, to identify appropriate ecological benchmarks for sediment:

- Equilibrium Partitioning Sediment Guidelines (ESG) - EPA (2000) has issued draft guidelines for the protection of benthic organisms from chronic effects due to PAH mixtures in sediment based on the EqP approach. These values were used as ecological benchmarks for sediment.

- Ontario Ministry of Environment and Energy Lowest Effect Levels (LEL) (Persaud et al., 1992) - The Ontario Ministry of Environment and Energy derived guidelines for the protection and management of aquatic sediment quality in freshwater of Ontario. The LEL is the concentration at which the majority of sediment-dwelling organisms are not affected. The LELs were derived using an effects-based approach that used field data on the co-occurrence of benthic infaunal species and different concentrations of chemicals in sediment. The derivation of the LELs used organic carbon (OC) normalized concentrations of nonionic organic chemicals and present the LELs based on 1 percent organic carbon. Thus, sample-specific LELs were used by adjusting for sample-specific OC.

- Canadian Marine Sediment Quality Guidelines (MacDonald et al., 1992) - Sediment quality guidelines for several volatile organic compounds were developed for marine sediments as part of the Canadian Sediment Quality Guidelines. Though these are for marine sediments, they are assumed to be equally protective of freshwater organisms (MacDonald et al, 1992). These are based on equilibrium partitioning and are presented for 1 percent OC. Thus, sample-specific LELs were used by adjusting for sample-specific OC.

- Probable Effects Concentration (EPA, 1996) - Jones et al. (1997) present a compilation of sediment quality guidelines. Included in this compilation is a probable effects concentration for aluminum which was determined from toxicity testing with both the amphipod, Hyalella azteca, and the midge, Chironomus riparius, as part of the Assessment and Remediation of Contaminated Sediments (ARCS) program.

- EqP-based benchmark values - For those nonionic organic compounds for which no sediment benchmarks could be found, the EqP approach was used to derive sediment benchmarks from the appropriate surface water benchmarks. For organic compounds for which the $\log \mathrm{K}_{\mathrm{OC}}$ is greater than 2, the following equation was used to derive the OC-normalized sediment quality criterion based on 1 percent OC:

$$
\mathrm{SQC}_{\% \mathrm{OC}}=\mathrm{AWQC} \times \mathrm{K}_{\mathrm{OC}} / 100
$$


If the $\log \mathrm{K}_{\mathrm{OC}}$ is less than 2, then the compound is assumed to partition completely to the pore water and to be completely bioavailable. The moisture content of the sediment is conservatively assumed to be 50 percent. Thus, the following equation is used to derive the OC-normalized sediment quality criterion based on 1 percent OC:

$$
\mathrm{SQC}=\mathrm{AWQC} \times \frac{\% \text { Moisture }}{\% \text { Solids }}=\mathrm{AWQC} \times \frac{50 \%}{50 \%}=\mathrm{AWQC}
$$

If a chemical is not detected in abiotic media, then it was not considered a COPEC unless the detection limits were above the ecological benchmarks.

Inorganic compound concentrations were also compared to site-specific background levels. In order to compare site concentrations to background concentrations, the site mean should be compared to the 95 percent UCL of the background mean (ADEC, 1998). However, due to the small background dataset and small datasets for individual streams and ponds, this approach was not deemed sufficiently conservative for screening for ecological COPECs due to two factors: (1) the 95 percent UCL for the background mean was often greater than the maximum detected background concentration, and (2) based on the small dataset for the individual streams and ponds, the mean concentration has a large degree of uncertainty. Thus, this mean may considerably underestimate (or considerably overestimate) the true mean. In order to compensate for this uncertainty, all sample concentrations were compared individually to the range of background concentrations. The range of background concentrations was defined by the upper tolerance limit (UTL) as described in Appendix D of the site characterization report (Shannon \& Wilson, 1999). An inorganic analyte at a site was considered to be in the range of background if all site concentrations were below the UTL background concentration.

All compounds which have the potential to bioaccumulate were considered for inclusion as COPECs. Compounds were considered bioaccumulatable if the Log BCF is greater than 2.

Finally, all compounds which were being considered as COPECs were evaluated for site relatedness. Only PCBs and constituents known to be associated with drilling fluids were considered for inclusion as COPECs. 


\subsubsection{Identification of Compounds of Potential Ecological Concern}

The surface water and sediment data for the individual streams and ponds are summarized in Tables 5-3 through 5-34. Information contained in these tables are the detection frequency, the minimum and maximum detected concentrations, the inorganic background concentration, the ecological screening value (ESV), and the frequency of detection above the ESV.

\subsubsection{Surface Water}

Surface water samples were collected from Falls Creek, Drill Site D Lake, Drill Site E Stream, Limpet Creek, Bridge Creek, Reed Pond, Cloudberry Creek, Rainbow Creek, Clevenger Creek, Heart Lake, Cannikin Lake, White Alice Creek, and four reference streams. Analyses conducted on some or all of these samples were inorganics, PAHs, volatile organic compounds (VOCs), PCBs, and DRO. The identification of COPECs for surface water is presented below.

\subsection{Organics}

The DROs were detected in Bridge Creek, Cannikin Lake, Cloudberry Creek, Drill Site D Lake, Drill Site E Stream, Falls Creek, Heart Lake, Rainbow Creek, Reed Pond, and White Alice Creek. All detected concentrations were above the ESV of 0.28 micrograms per liter $(\mu \mathrm{g} / \mathrm{L})$. The DROs were also detected in most of the reference streams, which may indicate some biogenic origin to these organics. However, since DROs are associated with drilling fluids they could be site-related, especially where they are elevated above the background levels, and DROs are considered to be COPECs for the ecological risk assessment. Where DROs were not detected, the detection limit was significantly greater than the ESV. Therefore, DROs were considered COPECs for all surface water bodies.

Volatile organic compounds were not detected in any of the surface water samples on site. Detection limits for VOCs were lower than the ESVs except for carbon disulfide. The detection limit for carbon disulfide of $1.0 \mu \mathrm{g} / \mathrm{L}$ is just marginally greater than the ESV of $0.92 \mu \mathrm{g} / \mathrm{L}$. Since the detection limit was nearly equal to the ESV, and carbon disulfide is not expected as a site-related contaminant, no VOCs were considered COPECs for surface water. 


\section{Table 5-3}

\section{Summary of Analytical Results and Ecological Risk Assessment Screening for Surface Water in Falls Creek, Drill Site D}

(Page 1 of 3 )

\begin{tabular}{|c|c|c|c|c|c|c|}
\hline Analyte & $\begin{array}{l}\text { Detection } \\
\text { Frequency }\end{array}$ & $\begin{array}{c}\text { Minimum } \\
\text { Detected } \\
\text { Concentration } \\
(\mu \mathrm{g} / \mathrm{L})\end{array}$ & $\begin{array}{c}\text { Maximum } \\
\text { Detected } \\
\text { Concentration } \\
(\mu \mathrm{g} / \mathrm{L})\end{array}$ & $\begin{array}{c}\text { Upland Stream } \\
\text { Background } \\
\text { Concentration } \\
(\mu \mathrm{g} / \mathrm{L})\end{array}$ & $\begin{array}{c}\text { Ecological } \\
\text { Screening } \\
\text { Value } \\
(\mu \mathrm{g} / \mathrm{L})\end{array}$ & $\begin{array}{l}\text { Detection } \\
\text { Frequency } \\
\text { Above ESV }\end{array}$ \\
\hline $\begin{array}{l}\text { Diesel-Range } \\
\text { Hydrocarbons }\end{array}$ & 0 of 5 & ND (50) & ND (84.9) & NAN & 15 & 0 of 5 \\
\hline \multicolumn{7}{|c|}{ Volatile Organic Compounds } \\
\hline 1,2,4-Trimethylbenzene & 0 of 5 & ND (1.0) & ND (1.0) & NAN & 33 & 0 of 5 \\
\hline 1,3,5-Trimethylbenzene & 0 of 5 & ND (1.0) & ND (1.0) & NAN & 33 & 0 of 5 \\
\hline 2-Butanone & 0 of 5 & ND (20) & ND (20) & NAN & 14,000 & 0 of 5 \\
\hline Acetone & 0 of 5 & ND (20) & ND (20) & NAN & 1,500 & 0 of 5 \\
\hline Benzene & 0 of 5 & ND (1.0) & ND (1.0) & NAN & 130 & 0 of 5 \\
\hline Carbon disulfide & 0 of 5 & ND (1.0) & ND (1.0) & NAN & 0.92 & 0 of 5 \\
\hline Ethylbenzene & 0 of 5 & ND (1.0) & ND (1.0) & NAN & 7.3 & 0 of 5 \\
\hline Isopropylbenzene & 0 of 5 & ND (1.0) & ND (1.0) & NAN & 2.6 & 0 of 5 \\
\hline $\mathrm{m}, \mathrm{p}$-Xylene & 0 of 5 & ND (2.0) & ND (2.0) & NAN & 13 & 0 of 5 \\
\hline Methylene chloride & 0 of 5 & ND (2.0) & ND (4.95) & NAN & 2,200 & 0 of 5 \\
\hline n-Butylbenzene & 0 of 5 & ND (1.0) & ND (1.0) & NAN & 164 & 0 of 5 \\
\hline n-Propylbenzene & 0 of 5 & ND (1.0) & ND (1.0) & NAN & 6.0 & 0 of 5 \\
\hline Naphthalene & 0 of 5 & ND (1.0) & ND (1.0) & NAN & 12 & 0 of 5 \\
\hline o-Xylene & 0 of 5 & ND (1.0) & ND (1.0) & NAN & 13 & 0 of 5 \\
\hline p-Isopropyltoluene & 0 of 5 & ND (1.0) & ND (1.0) & NAN & 26 & 0 of 5 \\
\hline sec-Butylbenzene & 0 of 5 & ND (1.0) & ND (1.0) & NAN & 164 & 0 of 5 \\
\hline Toluene & 0 of 5 & ND (1.0) & ND (1.0) & NAN & 9.8 & 0 of 5 \\
\hline \multicolumn{7}{|c|}{ Polycyclic Aromatic Hydrocarbons } \\
\hline Acenaphthene & 0 of 5 & $\mathrm{ND}(0.1)$ & $\mathrm{ND}(0.1)$ & NAN & 74 & 0 of 5 \\
\hline Acenaphthylene & 0 of 5 & $\mathrm{ND}(0.1)$ & ND $(0.1)$ & NAN & 307 & 0 of 5 \\
\hline Anthracene & 0 of 5 & $\mathrm{ND}(0.1)$ & $\mathrm{ND}(0.1)$ & NAN & 0.73 & 0 of 5 \\
\hline Benzo(a)anthracene & 0 of 5 & $\mathrm{ND}(0.1)$ & $\mathrm{ND}(0.1)$ & NAN & 0.027 & 0 of 5 \\
\hline Benzo(a)pyrene & 0 of 5 & $\mathrm{ND}(0.1)$ & $\mathrm{ND}(0.1)$ & NAN & 0.014 & 0 of 5 \\
\hline Benzo(b)fluoranthene & 0 of 5 & $\mathrm{ND}(0.1)$ & ND $(0.1)$ & NAN & 0.68 & 0 of 5 \\
\hline Benzo(g,h,I)perylene & 0 of 5 & ND $(0.1)$ & ND $(0.1)$ & NAN & 0.44 & 0 of 5 \\
\hline Benzo(k)fluoranthene & 0 of 5 & ND $(0.1)$ & $\mathrm{ND}(0.1)$ & NAN & 0.64 & 0 of 5 \\
\hline
\end{tabular}




\section{Table 5-3}

\section{Summary of Analytical Results and Ecological Risk Assessment Screening for Surface Water in Falls Creek, Drill Site D}

(Page 2 of 3 )

\begin{tabular}{|c|c|c|c|c|c|c|}
\hline Analyte & $\begin{array}{l}\text { Detection } \\
\text { Frequency }\end{array}$ & $\begin{array}{c}\text { Minimum } \\
\text { Detected } \\
\text { Concentration } \\
(\mu \mathrm{g} / \mathrm{L})\end{array}$ & $\begin{array}{c}\text { Maximum } \\
\text { Detected } \\
\text { Concentration } \\
(\mu \mathrm{g} / \mathrm{L})\end{array}$ & $\begin{array}{c}\text { Upland Stream } \\
\text { Background } \\
\text { Concentration }^{\mathrm{a}} \\
(\mu \mathrm{g} / \mathrm{L})\end{array}$ & $\begin{array}{l}\text { Ecological } \\
\text { Screening } \\
\text { Value } \\
(\mu \mathrm{g} / \mathrm{L})\end{array}$ & $\begin{array}{l}\text { Detection } \\
\text { Frequency } \\
\text { Above ESV }\end{array}$ \\
\hline Chrysene & 0 of 5 & $\mathrm{ND}(0.1)$ & $\mathrm{ND}(0.1)$ & NAN & 2.0 & 0 of 5 \\
\hline Dibenzo(a,h)anthracene & 0 of 5 & ND $(0.1)$ & $\mathrm{ND}(0.1)$ & NAN & 0.28 & 0 of 5 \\
\hline Fluoranthene & 0 of 5 & $\mathrm{ND}(0.1)$ & $\mathrm{ND}(0.1)$ & NAN & 15 & 0 of 5 \\
\hline Fluorene & 0 of 5 & ND $(0.1)$ & ND $(0.1)$ & NAN & 3.9 & 0 of 5 \\
\hline Indeno(1,2,3-cd)pyrene & 0 of 5 & $\mathrm{ND}(0.1)$ & ND $(0.1)$ & NAN & 0.28 & 0 of 5 \\
\hline Naphthalene & 0 of 5 & ND $(0.1)$ & $\mathrm{ND}(0.1)$ & NAN & 12 & 0 of 5 \\
\hline Phenanthrene & 0 of 5 & $\mathrm{ND}(0.1)$ & ND $(0.1)$ & NAN & 200 & 0 of 5 \\
\hline Pyrene & 0 of 5 & $\mathrm{ND}(0.1)$ & $\mathrm{ND}(0.1)$ & NAN & 10 & 0 of 5 \\
\hline \multicolumn{7}{|c|}{ Polychlorinated Biphenyls } \\
\hline Aroclor 1016 & NAV & NAV & NAV & NAN & NAV & - \\
\hline Aroclor 1221 & NAV & NAV & NAV & NAN & NAV & - \\
\hline Aroclor 1232 & NAV & NAV & NAV & NAN & NAV & - \\
\hline Aroclor 1242 & NAV & NAV & NAV & NAN & NAV & - \\
\hline Aroclor 1248 & NAV & NAV & NAV & NAN & NAV & - \\
\hline Aroclor 1254 & NAV & NAV & NAV & NAN & NAV & - \\
\hline Aroclor 1260 & NAV & NAV & NAV & NAN & NAV & - \\
\hline Aroclor 1262 & NAV & NAV & NAV & NAN & NAV & - \\
\hline Aroclor 1268 & NAV & NAV & NAV & NAN & NAV & - \\
\hline \multicolumn{7}{|c|}{ Metals } \\
\hline Aluminum & 0 of 5 & ND (50) & ND (50) & $87.1^{b}$ & 87 & 0 of 5 \\
\hline Arsenic & 0 of 5 & $\mathrm{ND}(0.1)$ & $\mathrm{ND}(0.1)$ & ND (1) & 150 & 0 of 5 \\
\hline Barium & 0 of 5 & ND (10) & ND (10) & ND (10) & 4.0 & 0 of 5 \\
\hline Beryllium & 0 of 5 & ND (1.0) & ND (1.0) & ND (1) & 0.66 & 0 of 5 \\
\hline Boron & 0 of 5 & ND (50) & ND (50) & ND (50) & 1.6 & 0 of 5 \\
\hline Cadmium & 0 of 5 & ND (1.0) & ND (1.0) & ND (1) & 2.2 & 0 of 5 \\
\hline Calcium & 5 of 5 & 3,740 & 4,990 & $8,810^{b}$ & 116,000 & 0 of 5 \\
\hline Cerium & 0 of 5 & ND (1.0) & ND (1.0) & ND (1) & 0.5 & 0 of 5 \\
\hline Cesium & 0 of 5 & ND (1.0) & ND (1.0) & ND (1) & 30 & 0 of 5 \\
\hline Chromium & 1 of 5 & ND (1.0) & 12 & $3.49^{b}$ & 74 & 0 of 5 \\
\hline
\end{tabular}




\section{Table 5-3}

\section{Summary of Analytical Results and Ecological Risk Assessment Screening for Surface Water in Falls Creek, Drill Site D}

(Page 3 of 3 )

\begin{tabular}{|c|c|c|c|c|c|c|}
\hline Analyte & $\begin{array}{l}\text { Detection } \\
\text { Frequency }\end{array}$ & $\begin{array}{c}\text { Minimum } \\
\text { Detected } \\
\text { Concentration } \\
(\mu \mathrm{g} / \mathrm{L})\end{array}$ & $\begin{array}{c}\text { Maximum } \\
\text { Detected } \\
\text { Concentration } \\
(\mu \mathrm{g} / \mathrm{L})\end{array}$ & $\begin{array}{l}\text { Upland Stream } \\
\text { Background } \\
\text { Concentration } \\
(\mu \mathrm{g} / \mathrm{L})\end{array}$ & $\begin{array}{c}\text { Ecological } \\
\text { Screening } \\
\text { Value } \\
(\mu \mathrm{g} / \mathrm{L})\end{array}$ & $\begin{array}{c}\text { Detection } \\
\text { Frequency } \\
\text { Above ESV }\end{array}$ \\
\hline Cobalt & 5 of 5 & 2.83 & 4.59 & 9.52 & 23 & 0 of 5 \\
\hline Copper & 4 of 5 & ND (1.0) & 3.3 & $13.4^{\mathrm{b}}$ & 9.0 & 0 of 5 \\
\hline Iron & 3 of 5 & ND (150) & 199 & $2,830^{b}$ & 1,000 & 0 of 5 \\
\hline Lithium & 1 of 5 & ND (1.0) & 1.04 & ND (1) & 14 & 0 of 5 \\
\hline Magnesium & 5 of 5 & 3,650 & 4,350 & 5,250 & 82,000 & 0 of 5 \\
\hline Manganese & 5 of 5 & 10.4 & 17.1 & 748 & 120 & 0 of 5 \\
\hline Molybdenum & 1 of 5 & ND (1.0) & 1.01 & ND (1) & 370 & 0 of 5 \\
\hline Nickel & 2 of 5 & ND (1.0) & 1.59 & $1.69^{b}$ & 52 & 0 of 5 \\
\hline Potassium & 5 of 5 & 1,910 & 2,270 & $1,190^{b}$ & 53,000 & 0 of 5 \\
\hline Selenium & 0 of 5 & ND (1.0) & ND (1.0) & ND (1) & 5.0 & 0 of 5 \\
\hline Strontium & 5 of 5 & 31.9 & 36.9 & $44.1^{\mathrm{b}}$ & 1,500 & 0 of 5 \\
\hline Thallium & 0 of 5 & ND (1.0) & ND (1.0) & $2.13^{b}$ & 12 & 0 of 5 \\
\hline Thorium & 0 of 5 & ND (1.0) & ND (1.0) & $1.14^{b}$ & NAV & 0 of 5 \\
\hline Titanium & 5 of 5 & 1.25 & 2.37 & $2.37^{b}$ & 29 & 0 of 5 \\
\hline Uranium & 0 of 5 & ND (1.0) & ND (1.0) & ND (1) & 2.6 & 0 of 5 \\
\hline Vanadium & 5 of 5 & 1.62 & 2.37 & $1.44^{b}$ & 20 & 0 of 5 \\
\hline Zinc & 5 of 5 & 11 & 23.3 & $12.8^{\mathrm{b}}$ & 120 & 0 of 5 \\
\hline
\end{tabular}

${ }^{a}$ Background concentrations, which are presented only for metals, are upper tolerance limits (UTL) unless otherwise noted.

${ }^{b}$ This metal was not detected in a sufficient number of reference stream samples to allow calculation of a valid UTL. The concentration shown is the maximum detected concentration in the appropriate background samples.

ND - Analyte not detected at a concentration above the reporting limit, which is shown in parentheses.

NAV - Not available

NAN - Not analyzed

$\mu \mathrm{g} / \mathrm{L}$ - Micrograms per liter 
Table 5-4

\section{Summary of Analytical Results and Ecological Risk Assessment Screening for Sediment in Falls Creek, Drill Site D}

(Page 1 of 3 )

\begin{tabular}{|c|c|c|c|c|c|c|}
\hline Analyte & $\begin{array}{l}\text { Detection } \\
\text { Frequency }\end{array}$ & $\begin{array}{c}\text { Minimum } \\
\text { Detected } \\
\text { Concentration } \\
(\mathbf{m g} / \mathbf{k g})\end{array}$ & $\begin{array}{c}\text { Maximum } \\
\text { Detected } \\
\text { Concentration } \\
(\mathbf{m g} / \mathbf{k g})\end{array}$ & $\begin{array}{c}\text { Upland Stream } \\
\text { Background } \\
\text { Concentration } \\
\text { (mg/kg) }\end{array}$ & $\begin{array}{l}\text { Ecological } \\
\text { Screening } \\
\text { Value }(\mathbf{m g} / \mathbf{k g})\end{array}$ & $\begin{array}{c}\text { Detection } \\
\text { Frequency } \\
\text { Above ESV }\end{array}$ \\
\hline $\begin{array}{l}\text { Diesel-Range } \\
\text { Hydrocarbons }\end{array}$ & 1 of 5 & ND (4.0) & 176 & NAN & $640^{\mathrm{C}}$ & 0 of 5 \\
\hline \multicolumn{7}{|c|}{ Volatile Organic Compounds } \\
\hline 1,2,4-Trimethylbenzene & NAV & NAV & NAV & NAN & NAV & - \\
\hline 1,3,5-Trimethylbenzene & NAV & NAV & NAV & NAN & NAV & - \\
\hline 2-Butanone & NAV & NAV & NAV & NAN & NAV & - \\
\hline Acetone & NAV & NAV & NAV & NAN & NAV & - \\
\hline Benzene & NAV & NAV & NAV & NAN & NAV & - \\
\hline Carbon disulfide & NAV & NAV & NAV & NAN & NAV & - \\
\hline Ethylbenzene & NAV & NAV & NAV & NAN & NAV & - \\
\hline Isopropylbenzene & NAV & NAV & NAV & NAN & NAV & - \\
\hline m,p-Xylene & NAV & NAV & NAV & NAN & NAV & - \\
\hline Methylene chloride & NAV & NAV & NAV & NAN & NAV & - \\
\hline n-Butylbenzene & NAV & NAV & NAV & NAN & NAV & - \\
\hline n-Propylbenzene & NAV & NAV & NAV & NAN & NAV & - \\
\hline Naphthalene & NAV & NAV & NAV & NAN & NAV & - \\
\hline o-Xylene & NAV & NAV & NAV & NAN & NAV & - \\
\hline p-Isopropyltoluene & NAV & NAV & NAV & NAN & NAV & - \\
\hline sec-Butylbenzene & NAV & NAV & NAV & NAN & NAV & - \\
\hline Toluene & NAV & NAV & NAV & NAN & NAV & - \\
\hline \multicolumn{7}{|c|}{ Polycyclic Aromatic Hydrocarbons } \\
\hline Acenaphthene & 0 of 5 & ND (0.0033) & ND (0.0143) & NAN & $4.91^{\mathrm{C}}$ & 0 of 5 \\
\hline Acenaphthylene & 0 of 5 & ND (0.0033) & ND (0.0143) & NAN & $4.52^{\mathrm{C}}$ & 0 of 5 \\
\hline Anthracene & 1 of 5 & ND (0.0033) & 0.0161 & NAN & $5.94^{\mathrm{C}}$ & 0 of 5 \\
\hline Benzo(a)anthracene & 1 of 5 & ND (0.0033) & 0.034 & NAN & $8.41^{\mathrm{C}}$ & 0 of 5 \\
\hline Benzo(a)pyrene & 1 of 5 & ND (0.0033) & 0.0501 & NAN & $9.65^{\mathrm{c}}$ & 0 of 5 \\
\hline Benzo(b)fluoranthene & 1 of 5 & ND (0.0033) & 0.0876 & NAN & $9.79^{C}$ & 0 of 5 \\
\hline Benzo(g,h,l)perylene & 1 of 5 & ND (0.0033) & 0.0143 & NAN & $10.95^{\mathrm{C}}$ & 0 of 5 \\
\hline Benzo(k)fluoranthene & 1 of 5 & ND (0.0033) & 0.0322 & NAN & $9.81^{\mathrm{C}}$ & 0 of 5 \\
\hline
\end{tabular}


Table 5-4

\section{Summary of Analytical Results and Ecological Risk Assessment Screening for Sediment in Falls Creek, Drill Site D}

(Page 2 of 3 )

\begin{tabular}{|c|c|c|c|c|c|c|}
\hline Analyte & $\begin{array}{l}\text { Detection } \\
\text { Frequency }\end{array}$ & $\begin{array}{c}\text { Minimum } \\
\text { Detected } \\
\text { Concentration } \\
(\mathbf{m g} / \mathbf{k g})\end{array}$ & $\begin{array}{c}\text { Maximum } \\
\text { Detected } \\
\text { Concentration } \\
(\mathbf{m g} / \mathrm{kg})\end{array}$ & $\begin{array}{c}\text { Upland Stream } \\
\text { Background } \\
\text { Concentration } \\
(\mathrm{mg} / \mathrm{kg})\end{array}$ & $\begin{array}{l}\text { Ecological } \\
\text { Screening } \\
\text { Value }(\mathbf{m g} / \mathbf{k g})\end{array}$ & $\begin{array}{c}\text { Detection } \\
\text { Frequency } \\
\text { Above ESV }\end{array}$ \\
\hline Chrysene & 1 of 5 & ND $(0.0033)$ & 0.0215 & NAN & $8.44^{\mathrm{C}}$ & 0 of 5 \\
\hline Dibenzo(a,h)anthracene & 0 of 5 & ND (0.0033) & ND (0.0143) & NAN & $11.23^{\mathrm{C}}$ & 0 of 5 \\
\hline Fluoranthene & 1 of 5 & ND (0.0033) & 0.0232 & NAN & $7.07^{\mathrm{C}}$ & 0 of 5 \\
\hline Fluorene & 1 of 5 & ND (0.0033) & 0.0125 & NAN & $5.38^{\mathrm{C}}$ & 0 of 5 \\
\hline Indeno(1,2,3-cd)pyrene & 1 of 5 & ND $(0.0033)$ & 0.0197 & NAN & $11.15^{\mathrm{c}}$ & 0 of 5 \\
\hline Naphthalene & 0 of 5 & ND $(0.0033)$ & ND (0.0143) & NAN & $3.85^{\mathrm{C}}$ & 0 of 5 \\
\hline Phenanthrene & 1 of 5 & ND (0.0033) & 0.125 & NAN & $5.96^{\mathrm{C}}$ & 0 of 5 \\
\hline Pyrene & 1 of 5 & ND (0.0033) & 0.0554 & NAN & $6.97^{\mathrm{C}}$ & 0 of 5 \\
\hline \multicolumn{7}{|c|}{ Polychlorinated Biphenyls } \\
\hline Aroclor 1016 & NAV & NAV & NAV & NAN & NAV & - \\
\hline Aroclor 1221 & NAV & NAV & NAV & NAN & NAV & - \\
\hline Aroclor 1232 & NAV & NAV & NAV & NAN & NAV & - \\
\hline Aroclor 1242 & NAV & NAV & NAV & NAN & NAV & - \\
\hline Aroclor 1248 & NAV & NAV & NAV & NAN & NAV & - \\
\hline Aroclor 1254 & NAV & NAV & NAV & NAN & NAV & - \\
\hline Aroclor 1260 & 2 of 3 & ND (0.033) & 0.82 & NAN & $0.07^{c}$ & 2 of 3 \\
\hline Aroclor 1262 & NAV & NAV & NAV & NAN & NAV & - \\
\hline Aroclor 1268 & NAV & NAV & NAV & NAN & NAV & - \\
\hline \multicolumn{7}{|c|}{ Metals } \\
\hline Aluminum & 5 of 5 & 9,490 & 38,200 & 47,500 & 58,000 & 0 of 5 \\
\hline Arsenic & 5 of 5 & 1.62 & 10 & 30.5 & 6.0 & 2 of 5 \\
\hline Barium & 5 of 5 & 53 & 181 & 468 & NAV & \\
\hline Beryllium & 0 of 5 & ND (0.5) & ND (2.17) & ND & NAV & \\
\hline Boron & 5 of 5 & 17.2 & 163 & $6.42^{b}$ & NAV & \\
\hline Cadmium & 0 of 5 & ND (0.5) & ND (2.17) & ND & 0.6 & 0 of 5 \\
\hline Calcium & 5 of 5 & 1,700 & 7,410 & 7,720 & NAV & \\
\hline Cerium & 5 of 5 & 3.71 & 14.6 & 13 & NAV & \\
\hline Cesium & 0 of 5 & ND (0.5) & ND (2.17) & ND & NAV & \\
\hline Chromium & 5 of 5 & 20.6 & 226 & 18.9 & 26 & 3 of 5 \\
\hline
\end{tabular}




\section{Table 5-4}

\section{Summary of Analytical Results and Ecological Risk Assessment Screening for Sediment in Falls Creek, Drill Site D}

(Page 3 of 3 )

\begin{tabular}{|c|c|c|c|c|c|c|}
\hline Analyte & $\begin{array}{l}\text { Detection } \\
\text { Frequency }\end{array}$ & $\begin{array}{c}\text { Minimum } \\
\text { Detected } \\
\text { Concentration } \\
(\mathbf{m g} / \mathbf{k g})\end{array}$ & $\begin{array}{c}\text { Maximum } \\
\text { Detected } \\
\text { Concentration } \\
(\mathbf{m g} / \mathbf{k g})\end{array}$ & $\begin{array}{c}\text { Upland Stream } \\
\text { Background } \\
\text { Concentration }{ }^{\mathrm{a}} \\
(\mathrm{mg} / \mathrm{kg})\end{array}$ & $\begin{array}{c}\text { Ecological } \\
\text { Screening } \\
\text { Value }(\mathbf{m g} / \mathbf{k g})\end{array}$ & $\begin{array}{l}\text { Detection } \\
\text { Frequency } \\
\text { Above ESV }\end{array}$ \\
\hline Cobalt & 5 of 5 & 4.62 & 23.5 & 37.3 & NAV & \\
\hline Copper & 5 of 5 & 21.5 & 93.1 & 82.4 & 16 & 5 of 5 \\
\hline Iron & 5 of 5 & 8,960 & 85,500 & 123,000 & 20,000 & 3 of 5 \\
\hline Lithium & 5 of 5 & 2.51 & 16.6 & 22 & NAV & \\
\hline Magnesium & 5 of 5 & 1,660 & 17,300 & 17,000 & NAV & \\
\hline Manganese & 5 of 5 & 256 & 2,310 & 26,500 & 460 & 4 of 5 \\
\hline Molybdenum & 1 of 5 & ND (1.34) & 0.555 & ND & NAV & \\
\hline Nickel & 5 of 5 & 11.4 & 21.9 & 23.8 & 16 & 2 of 5 \\
\hline Potassium & 5 of 5 & 175 & 1,060 & 1,290 & NAV & \\
\hline Selenium & 0 of 5 & ND (0.5) & ND (2.17) & $3.15^{b}$ & NAV & \\
\hline Strontium & 5 of 5 & 19.8 & 93.7 & 207 & NAV & \\
\hline Thallium & 0 of 5 & ND $(0.5)$ & ND (2.17) & ND & NAV & \\
\hline Thorium & 0 of 5 & ND (0.5) & ND (2.17) & ND & NAV & \\
\hline Titanium & 5 of 5 & 376 & 1,590 & 1,480 & NAV & \\
\hline Uranium & 1 of 5 & $\mathrm{ND}(0.5)$ & 2.49 & ND & NAV & \\
\hline Vanadium & 5 of 5 & 49.2 & 247 & 336 & NAV & \\
\hline Zinc & 5 of 5 & 82 & 256 & 185 & 120 & 2 of 5 \\
\hline Total Organic Carbon & 5 of 5 & 11,600 & 82,700 & NAN & & \\
\hline
\end{tabular}

${ }^{\mathrm{a}}$ Background concentrations, which are presented only for metals, are upper tolerance limits (UTL) unless otherwise noted.

${ }^{b}$ This metal was not detected in a sufficient number of reference stream samples to allow calculation of a valid UTL. The concentration shown is the maximum detected concentration in the appropriate background samples.

${ }^{\mathrm{c}} \mathrm{S}$ creening concentration is based on $1 \%$ organic carbon.

ND - Analyte not detected at a concentration above the reporting limit, which is shown in parentheses.

NAV - Not available

NAN - Not analyzed

$\mathrm{mg} / \mathrm{kg}$ - Milligrams per kilogram 


\section{Table 5-5}

\section{Summary of Analytical Results and Ecological Risk Assessment Screening for Surface Water in Drill Site D Lake}

(Page 1 of 3 )

\begin{tabular}{|c|c|c|c|c|c|c|}
\hline Analyte & $\begin{array}{l}\text { Detection } \\
\text { Frequency }\end{array}$ & $\begin{array}{c}\text { Minimum } \\
\text { Detected } \\
\text { Concentration } \\
(\mu \mathrm{g} / \mathrm{L})\end{array}$ & $\begin{array}{c}\text { Maximum } \\
\text { Detected } \\
\text { Concentration } \\
(\mu \mathrm{g} / \mathrm{L})\end{array}$ & $\begin{array}{c}\text { Upland Stream } \\
\text { Background } \\
\text { Concentration }{ }^{\mathrm{a}} \\
(\mu \mathrm{g} / \mathrm{L})\end{array}$ & $\begin{array}{l}\text { Ecological } \\
\text { Screening } \\
\text { Value } \\
(\mu \mathrm{g} / \mathrm{L})\end{array}$ & $\begin{array}{l}\text { Detection } \\
\text { Frequency } \\
\text { Above ESV }\end{array}$ \\
\hline $\begin{array}{l}\text { Diesel-Range } \\
\text { Hydrocarbons }\end{array}$ & 3 of 3 & 56.2 & 119 & NAN & 15 & 3 of 3 \\
\hline \multicolumn{7}{|c|}{ Volatile Organic Compounds } \\
\hline 1,2,4-Trimethylbenzene & 0 of 3 & ND (1.0) & ND (1.0) & NAN & 33 & 0 of 3 \\
\hline 1,3,5-Trimethylbenzene & 0 of 3 & ND (1.0) & ND (1.0) & NAN & 33 & 0 of 3 \\
\hline 2-Butanone & 0 of 3 & ND (20) & ND (20) & NAN & 14,000 & 0 of 3 \\
\hline Acetone & 0 of 3 & ND (20) & ND (20) & NAN & 1,500 & 0 of 3 \\
\hline Benzene & 0 of 3 & ND (1.0) & ND (1.0) & NAN & 130 & 0 of 3 \\
\hline Carbon disulfide & 0 of 3 & ND (1.0) & ND (1.0) & NAN & 0.92 & 0 of 3 \\
\hline Ethylbenzene & 0 of 3 & ND (1.0) & ND (1.0) & NAN & 7.3 & 0 of 3 \\
\hline Isopropylbenzene & 0 of 3 & ND (1.0) & ND (1.0) & NAN & 2.6 & 0 of 3 \\
\hline $\mathrm{m}, \mathrm{p}$-Xylene & 0 of 3 & ND (2.0) & ND (2.0) & NAN & 13 & 0 of 3 \\
\hline Methylene chloride & 0 of 3 & ND (3.17) & ND (4.42) & NAN & 2,200 & 0 of 3 \\
\hline n-Butylbenzene & 0 of 3 & ND (1.0) & ND (1.0) & NAN & 164 & 0 of 3 \\
\hline n-Propylbenzene & 0 of 3 & ND (1.0) & ND (1.0) & NAN & 6.0 & 0 of 3 \\
\hline Naphthalene & 0 of 3 & ND (1.0) & ND (1.0) & NAN & 12 & 0 of 3 \\
\hline o-Xylene & 0 of 3 & ND (1.0) & ND (1.0) & NAN & 13 & 0 of 3 \\
\hline p-Isopropyltoluene & 0 of 3 & ND (1.0) & ND (1.0) & NAN & 26 & 0 of 3 \\
\hline sec-Butylbenzene & 0 of 3 & ND (1.0) & ND (1.0) & NAN & 164 & 0 of 3 \\
\hline Toluene & 0 of 3 & ND (1.0) & ND (1.0) & NAN & 9.8 & 0 of 3 \\
\hline \multicolumn{7}{|c|}{ Polycyclic Aromatic Hydrocarbons } \\
\hline Acenaphthene & 0 of 3 & $\mathrm{ND}(0.1)$ & $\mathrm{ND}(0.1)$ & NAN & 74 & 0 of 3 \\
\hline Acenaphthylene & 0 of 3 & $\mathrm{ND}(0.1)$ & ND $(0.1)$ & NAN & 307 & 0 of 3 \\
\hline Anthracene & 0 of 3 & $\mathrm{ND}(0.1)$ & $\mathrm{ND}(0.1)$ & NAN & 0.73 & 0 of 3 \\
\hline Benzo(a)anthracene & 0 of 3 & $\mathrm{ND}(0.1)$ & $\mathrm{ND}(0.1)$ & NAN & 0.027 & 0 of 3 \\
\hline Benzo(a)pyrene & 0 of 3 & $\mathrm{ND}(0.1)$ & $\mathrm{ND}(0.1)$ & NAN & 0.014 & 0 of 3 \\
\hline Benzo(b)fluoranthene & 0 of 3 & $\mathrm{ND}(0.1)$ & ND $(0.1)$ & NAN & 0.68 & 0 of 3 \\
\hline Benzo(g,h,I)perylene & 0 of 3 & ND $(0.1)$ & ND $(0.1)$ & NAN & 0.44 & 0 of 3 \\
\hline Benzo(k)fluoranthene & 0 of 3 & ND $(0.1)$ & $\mathrm{ND}(0.1)$ & NAN & 0.64 & 0 of 3 \\
\hline
\end{tabular}




\section{Table 5-5}

\section{Summary of Analytical Results and Ecological Risk Assessment Screening for Surface Water in Drill Site D Lake}

(Page 2 of 3 )

\begin{tabular}{|c|c|c|c|c|c|c|}
\hline Analyte & $\begin{array}{l}\text { Detection } \\
\text { Frequency }\end{array}$ & $\begin{array}{c}\text { Minimum } \\
\text { Detected } \\
\text { Concentration } \\
(\mu \mathrm{g} / \mathrm{L})\end{array}$ & $\begin{array}{c}\text { Maximum } \\
\text { Detected } \\
\text { Concentration } \\
(\mu \mathrm{g} / \mathrm{L})\end{array}$ & $\begin{array}{c}\text { Upland Stream } \\
\text { Background } \\
\text { Concentration }{ }^{\mathrm{a}} \\
(\mu \mathrm{g} / \mathrm{L})\end{array}$ & $\begin{array}{l}\text { Ecological } \\
\text { Screening } \\
\text { Value } \\
(\mu \mathrm{g} / \mathrm{L})\end{array}$ & $\begin{array}{l}\text { Detection } \\
\text { Frequency } \\
\text { Above ESV }\end{array}$ \\
\hline Chrysene & 0 of 3 & $\mathrm{ND}(0.1)$ & $\mathrm{ND}(0.1)$ & NAN & 2.0 & 0 of 3 \\
\hline Dibenzo(a,h)anthracene & 0 of 3 & ND $(0.1)$ & $\mathrm{ND}(0.1)$ & NAN & 0.28 & 0 of 3 \\
\hline Fluoranthene & 0 of 3 & $\mathrm{ND}(0.1)$ & $\mathrm{ND}(0.1)$ & NAN & 15 & 0 of 3 \\
\hline Fluorene & 0 of 3 & ND $(0.1)$ & ND $(0.1)$ & NAN & 3.9 & 0 of 3 \\
\hline Indeno(1,2,3-cd)pyrene & 0 of 3 & $\mathrm{ND}(0.1)$ & ND $(0.1)$ & NAN & 0.28 & 0 of 3 \\
\hline Naphthalene & 0 of 3 & ND $(0.1)$ & $\mathrm{ND}(0.1)$ & NAN & 12 & 0 of 3 \\
\hline Phenanthrene & 0 of 3 & ND $(0.1)$ & ND $(0.1)$ & NAN & 200 & 0 of 3 \\
\hline Pyrene & 0 of 3 & $\mathrm{ND}(0.1)$ & $\mathrm{ND}(0.1)$ & NAN & 10 & 0 of 3 \\
\hline \multicolumn{7}{|c|}{ Polychlorinated Biphenyls } \\
\hline Aroclor 1016 & NAV & NAV & NAV & NAN & NAV & - \\
\hline Aroclor 1221 & NAV & NAV & NAV & NAN & NAV & - \\
\hline Aroclor 1232 & NAV & NAV & NAV & NAN & NAV & - \\
\hline Aroclor 1242 & NAV & NAV & NAV & NAN & NAV & - \\
\hline Aroclor 1248 & NAV & NAV & NAV & NAN & NAV & - \\
\hline Aroclor 1254 & NAV & NAV & NAV & NAN & NAV & - \\
\hline Aroclor 1260 & NAV & NAV & NAV & NAN & NAV & - \\
\hline Aroclor 1262 & NAV & NAV & NAV & NAN & NAV & - \\
\hline Aroclor 1268 & NAV & NAV & NAV & NAN & NAV & - \\
\hline \multicolumn{7}{|c|}{ Metals } \\
\hline Aluminum & 2 of 3 & ND (50) & 186 & $87.1^{b}$ & 87 & 2 of 3 \\
\hline Arsenic & 0 of 3 & ND (1.0) & ND (1.0) & ND (1) & 150 & 0 of 3 \\
\hline Barium & 0 of 3 & ND (10) & ND (10) & ND (10) & 4.0 & 0 of 3 \\
\hline Beryllium & 0 of 3 & ND (1.0) & ND (1.0) & ND (1) & 0.66 & 0 of 3 \\
\hline Boron & 0 of 3 & ND (50) & ND (50) & ND (50) & 1.6 & 0 of 3 \\
\hline Cadmium & 0 of 3 & ND (1.0) & ND (1.0) & ND (1) & 2.2 & 0 of 3 \\
\hline Calcium & 3 of 3 & 4,700 & 5,700 & $8,810^{b}$ & 116,000 & 0 of 3 \\
\hline Cerium & 0 of 3 & ND (1.0) & ND (1.0) & ND (1) & 0.5 & 0 of 3 \\
\hline Cesium & 0 of 3 & ND (1.0) & ND (1.0) & ND (1) & 30 & 0 of 3 \\
\hline Chromium & 3 of 3 & 10.5 & 11.5 & $3.49^{b}$ & 74 & 0 of 3 \\
\hline
\end{tabular}




\section{Table 5-5}

\section{Summary of Analytical Results and Ecological Risk Assessment Screening for Surface Water in Drill Site D Lake}

(Page 3 of 3 )

\begin{tabular}{|c|c|c|c|c|c|c|}
\hline Analyte & $\begin{array}{l}\text { Detection } \\
\text { Frequency }\end{array}$ & $\begin{array}{c}\text { Minimum } \\
\text { Detected } \\
\text { Concentration } \\
(\mu \mathrm{g} / \mathrm{L})\end{array}$ & $\begin{array}{c}\text { Maximum } \\
\text { Detected } \\
\text { Concentration } \\
(\mu \mathrm{g} / \mathrm{L})\end{array}$ & $\begin{array}{l}\text { Upland Stream } \\
\text { Background } \\
\text { Concentration }^{\text {a }} \\
(\mu \mathrm{g} / \mathrm{L})\end{array}$ & $\begin{array}{c}\text { Ecological } \\
\text { Screening } \\
\text { Value } \\
(\mu \mathrm{g} / \mathrm{L})\end{array}$ & $\begin{array}{l}\text { Detection } \\
\text { Frequency } \\
\text { Above ESV }\end{array}$ \\
\hline Cobalt & 3 of 3 & 4.66 & 5.18 & 9.52 & 23 & 0 of 3 \\
\hline Copper & 3 of 3 & 3.33 & 3.63 & $13.4^{\mathrm{b}}$ & 9.0 & 0 of 3 \\
\hline Iron & 0 of 3 & ND (150) & ND (150) & $2,830^{b}$ & 1,000 & 0 of 3 \\
\hline Lithium & 3 of 3 & 1.12 & 1.18 & ND (1) & 14 & 0 of 3 \\
\hline Magnesium & 3 of 3 & 4,220 & 4,340 & 5,250 & 82,000 & 0 of 3 \\
\hline Manganese & 3 of 3 & 17.2 & 28.9 & 748 & 120 & 0 of 3 \\
\hline Molybdenum & 0 of 3 & ND (1.0) & ND (1.0) & ND (1) & 370 & 0 of 3 \\
\hline Nickel & 3 of 3 & 1.6 & 1.92 & $1.69^{b}$ & 52 & 0 of 3 \\
\hline Potassium & 3 of 3 & 1,310 & 2,080 & $1,190^{b}$ & 53,000 & 0 of 3 \\
\hline Selenium & 0 of 3 & ND (1.0) & ND (1.0) & ND (1) & 5.0 & 0 of 3 \\
\hline Strontium & 3 of 3 & 38.4 & 39.9 & $44.1^{b}$ & 1,500 & 0 of 3 \\
\hline Thallium & 0 of 3 & ND (1.0) & ND (1.0) & $2.13^{b}$ & 12 & 0 of 3 \\
\hline Thorium & 0 of 3 & ND (1.0) & ND (1.0) & $1.14^{\mathrm{b}}$ & NAV & 0 of 3 \\
\hline Titanium & 3 of 3 & 2.46 & 8.83 & $2.37^{b}$ & 29 & 0 of 3 \\
\hline Uranium & 0 of 3 & ND (1.0) & ND (1.0) & ND (1) & 2.6 & 0 of 3 \\
\hline Vanadium & 3 of 3 & 1.44 & 1.65 & $1.44^{\mathrm{b}}$ & 20 & 0 of 3 \\
\hline Zinc & 0 of 3 & ND (10) & ND (10) & $12.8^{b}$ & 120 & 0 of 3 \\
\hline
\end{tabular}

${ }^{a}$ Background concentrations, which are presented only for metals, are upper tolerance limits (UTL) unless otherwise noted.

${ }^{b}$ This metal was not detected in a sufficient number of reference stream samples to allow calculation of a valid UTL. The concentration shown is the maximum detected concentration in the appropriate background samples.

ND - Analyte not detected at a concentration above the reporting limit, which is shown in parentheses.

NAV - Not available

NAN - Not analyzed

$\mu \mathrm{g} / \mathrm{L}$ - Micrograms per liter 
Table 5-6

Summary of Analytical Results and Ecological Risk Assessment
Screening for Sediment in Drill Site D Lake

(Page 1 of 3 )

\begin{tabular}{|c|c|c|c|c|c|c|}
\hline Analyte & $\begin{array}{l}\text { Detection } \\
\text { Frequency }\end{array}$ & $\begin{array}{c}\text { Minimum } \\
\text { Detected } \\
\text { Concentration } \\
(\mathbf{m g} / \mathbf{k g})\end{array}$ & $\begin{array}{l}\text { Maximum } \\
\text { Detected } \\
\text { Concentration } \\
(\mathrm{mg} / \mathrm{kg})\end{array}$ & $\begin{array}{l}\text { Upland Stream } \\
\text { Background } \\
\text { Concentration }{ }^{\mathrm{a}} \\
(\mathrm{mg} / \mathrm{kg})\end{array}$ & $\begin{array}{c}\text { Ecological } \\
\text { Screening } \\
\text { Value } \\
(\mathrm{mg} / \mathrm{kg})\end{array}$ & $\begin{array}{l}\text { Detection } \\
\text { Frequency } \\
\text { Above ESV }\end{array}$ \\
\hline $\begin{array}{l}\text { Diesel-Range } \\
\text { Hydrocarbons }\end{array}$ & 5 of 5 & 66.2 & 146 & NAN & $640^{\mathrm{C}}$ & 0 of 5 \\
\hline \multicolumn{7}{|c|}{ Volatile Organic Compounds } \\
\hline 1,2,4-Trimethylbenzene & 0 of 5 & ND (0.005) & ND (0.0248) & NAN & $1.2^{\mathrm{C}}$ & 0 of 5 \\
\hline 1,3,5-Trimethylbenzene & 0 of 5 & ND (0.005) & ND (0.0248) & NAN & $0.76^{\mathrm{C}}$ & 0 of 5 \\
\hline 2-Butanone & 1 of 5 & ND $(0.01)$ & 0.0496 & NAN & 14 & 0 of 5 \\
\hline Acetone & 0 of 5 & ND $(0.4)$ & ND (13.9) & NAN & 1.5 & 0 of 5 \\
\hline Benzene & 0 of 5 & ND (0.005) & ND (0.0248) & NAN & $0.34^{\mathrm{C}}$ & 0 of 5 \\
\hline Carbon disulfide & 0 of 5 & ND (0.018) & ND (0.197) & NAN & 0.001 & 0 of 5 \\
\hline Ethylbenzene & 0 of 5 & ND (0.005) & ND (0.0248) & NAN & $1.4^{\mathrm{c}}$ & 0 of 5 \\
\hline Isopropylbenzene & 0 of 5 & ND (0.005) & ND (0.0248) & NAN & $0.1^{c}$ & 0 of 5 \\
\hline m,p-Xylene & 0 of 5 & ND (0.005) & ND (0.0248) & NAN & $0.12^{\mathrm{C}}$ & 0 of 5 \\
\hline Methylene chloride & 0 of 5 & ND (0.005) & ND (0.0248) & NAN & $0.5^{c}$ & 0 of 5 \\
\hline n-Butylbenzene & 0 of 5 & ND (0.005) & ND (0.0248) & NAN & $22^{c}$ & 0 of 5 \\
\hline n-Propylbenzene & 0 of 5 & ND (0.005) & ND (0.0248) & NAN & $0.25^{\mathrm{c}}$ & 0 of 5 \\
\hline Naphthalene & 0 of 5 & ND (0.005) & ND (0.0248) & NAN & $3.85^{\mathrm{c}}$ & 0 of 5 \\
\hline o-Xylene & 0 of 5 & ND (0.005) & ND (0.0248) & NAN & $0.12^{\mathrm{C}}$ & 0 of 5 \\
\hline p-Isopropyltoluene & 0 of 5 & ND (0.005) & ND (0.0248) & NAN & $2.8^{\mathrm{C}}$ & 0 of 5 \\
\hline sec-Butylbenzene & 0 of 5 & ND (0.005) & ND $(0.0248)$ & NAN & $24^{c}$ & 0 of 5 \\
\hline Toluene & 0 of 5 & ND (0.005) & ND (0.0248) & NAN & $2.5^{c}$ & 0 of 5 \\
\hline \multicolumn{7}{|c|}{ Polycyclic Aromatic Hydrocarbons } \\
\hline Acenaphthene & 0 of 5 & ND (0.0033) & ND (0.0163) & NAN & $4.91^{\mathrm{c}}$ & 0 of 5 \\
\hline Acenaphthylene & 0 of 5 & ND (0.0033) & ND (0.0163) & NAN & $4.52^{c}$ & 0 of 5 \\
\hline Anthracene & 1 of 5 & ND (0.0033) & 0.0216 & NAN & $5.94^{\mathrm{C}}$ & 0 of 5 \\
\hline Benzo(a)anthracene & 0 of 5 & ND (0.0033) & ND (0.0163) & NAN & $8.41^{\mathrm{c}}$ & 0 of 5 \\
\hline Benzo(a)pyrene & 0 of 5 & ND (0.0033) & ND (0.0163) & NAN & $9.65^{\mathrm{C}}$ & 0 of 5 \\
\hline Benzo(b)fluoranthene & 0 of 5 & ND (0.0033) & ND (0.0163) & NAN & $9.79^{c}$ & 0 of 5 \\
\hline Benzo(ghi)perylene & 0 of 5 & ND (0.0033) & ND (0.0163) & NAN & $10.95^{c}$ & 0 of 5 \\
\hline Benzo(k)fluoranthene & 0 of 5 & ND (0.0033) & ND (0.0163) & NAN & $9.81^{\mathrm{c}}$ & 0 of 5 \\
\hline
\end{tabular}


Table 5-6

Summary of Analytical Results and Ecological Risk Assessment
Screening for Sediment in Drill Site D Lake

(Page 2 of 3 )

\begin{tabular}{|c|c|c|c|c|c|c|}
\hline Analyte & $\begin{array}{l}\text { Detection } \\
\text { Frequency }\end{array}$ & $\begin{array}{c}\text { Minimum } \\
\text { Detected } \\
\text { Concentration } \\
(\mathbf{m g} / \mathbf{k g})\end{array}$ & $\begin{array}{l}\text { Maximum } \\
\text { Detected } \\
\text { Concentration } \\
(\mathrm{mg} / \mathrm{kg})\end{array}$ & $\begin{array}{l}\text { Upland Stream } \\
\text { Background } \\
\text { Concentration }{ }^{\mathrm{a}} \\
(\mathrm{mg} / \mathrm{kg})\end{array}$ & $\begin{array}{c}\text { Ecological } \\
\text { Screening } \\
\text { Value } \\
(\mathrm{mg} / \mathrm{kg})\end{array}$ & $\begin{array}{l}\text { Detection } \\
\text { Frequency } \\
\text { Above ESV }\end{array}$ \\
\hline Chrysene & 0 of 5 & ND (0.0033) & ND (0.0163) & NAN & $8.44^{\mathrm{C}}$ & 0 of 5 \\
\hline Dibenzo(a,h)anthracene & 0 of 5 & ND $(0.0033)$ & ND (0.0163) & NAN & $11.23^{\mathrm{C}}$ & 0 of 5 \\
\hline Fluoranthene & 0 of 5 & ND (0.0033) & ND (0.0163) & NAN & $7.07^{c}$ & 0 of 5 \\
\hline Fluorene & 2 of 5 & ND (0.0033) & 0.0432 & NAN & $5.38^{\mathrm{C}}$ & 0 of 5 \\
\hline Indeno(1,2,3-cd)pyrene & 0 of 5 & ND (0.0033) & ND (0.0163) & NAN & $11.15^{\mathrm{c}}$ & 0 of 5 \\
\hline Naphthalene & NAV & NAV & NAV & NAN & NAV & - \\
\hline Phenanthrene & 4 of 5 & 0.00588 & 0.207 & NAN & $5.96^{\mathrm{C}}$ & 0 of 5 \\
\hline Pyrene & 2 of 5 & ND (0.0033) & 0.036 & NAN & $6.97^{\mathrm{C}}$ & 0 of 5 \\
\hline \multicolumn{7}{|c|}{ Polychlorinated Biphenyls } \\
\hline Aroclor 1016 & NAV & NAV & NAV & NAN & NAV & - \\
\hline Aroclor 1221 & NAV & NAV & NAV & NAN & NAV & - \\
\hline Aroclor 1232 & NAV & NAV & NAV & NAN & NAV & - \\
\hline Aroclor 1242 & NAV & NAV & NAV & NAN & NAV & - \\
\hline Aroclor 1248 & NAV & NAV & NAV & NAN & NAV & - \\
\hline Aroclor 1254 & NAV & NAV & NAV & NAN & NAV & - \\
\hline Aroclor 1260 & NAV & NAV & NAV & NAN & NAV & - \\
\hline Aroclor 1262 & NAV & NAV & NAV & NAN & NAV & - \\
\hline Aroclor 1268 & NAV & NAV & NAV & NAN & NAV & - \\
\hline \multicolumn{7}{|c|}{ Metals } \\
\hline Aluminum & 5 of 5 & 14,400 & 22,000 & 47,500 & 58,000 & 0 of 5 \\
\hline Arsenic & 4 of 5 & ND $(0.5)$ & 5.08 & 30.5 & 6 & 0 of 5 \\
\hline Barium & 5 of 5 & 38.1 & 515 & 468 & NAV & \\
\hline Beryllium & 0 of 5 & ND $(0.5)$ & ND (2.48) & ND & NAV & \\
\hline Boron & 0 of 5 & ND (2.5) & ND (12.4) & $6.42^{\mathrm{b}}$ & NAV & \\
\hline Cadmium & 0 of 5 & ND (0.5) & ND (2.48) & ND & 0.6 & 0 of 5 \\
\hline Calcium & 5 of 5 & 6,710 & 10,500 & 7,720 & NAV & \\
\hline Cerium & 5 of 5 & 9.45 & 61.4 & 13 & NAV & \\
\hline Cesium & 0 of 5 & ND $(0.5)$ & ND (2.48) & ND & NAV & \\
\hline Chromium & 5 of 5 & 16.1 & 695 & 18.9 & 26 & 4 of 5 \\
\hline
\end{tabular}


Table 5-6

Summary of Analytical Results and Ecological Risk Assessment
Screening for Sediment in Drill Site D Lake

(Page 3 of 3 )

\begin{tabular}{|c|c|c|c|c|c|c|}
\hline Analyte & $\begin{array}{l}\text { Detection } \\
\text { Frequency }\end{array}$ & $\begin{array}{c}\text { Minimum } \\
\text { Detected } \\
\text { Concentration } \\
(\mathbf{m g} / \mathbf{k g})\end{array}$ & $\begin{array}{c}\text { Maximum } \\
\text { Detected } \\
\text { Concentration } \\
(\mathrm{mg} / \mathrm{kg})\end{array}$ & $\begin{array}{l}\text { Upland Stream } \\
\text { Background } \\
\text { Concentration }{ }^{a} \\
(\mathrm{mg} / \mathbf{k g})\end{array}$ & $\begin{array}{c}\text { Ecological } \\
\text { Screening } \\
\text { Value } \\
(\mathbf{m g} / \mathbf{k g})\end{array}$ & $\begin{array}{l}\text { Detection } \\
\text { Frequency } \\
\text { Above ESV }\end{array}$ \\
\hline Cobalt & 5 of 5 & 2.63 & 9.83 & 37.3 & NAV & \\
\hline Copper & 5 of 5 & 25.7 & 98.3 & 82.4 & 16 & 5 of 5 \\
\hline Iron & 5 of 5 & 21,600 & 32,700 & 123,000 & 20,000 & 5 of 5 \\
\hline Lithium & 5 of 5 & 1.83 & 6.31 & 21.7 & NAV & \\
\hline Magnesium & 5 of 5 & 881 & 8,370 & 17,000 & NAV & \\
\hline Manganese & 5 of 5 & 98.7 & 783 & 26,500 & 460 & 4 of 5 \\
\hline Molybdenum & 0 of 5 & ND (0.5) & ND (3.6) & ND & NAV & \\
\hline Nickel & 5 of 5 & 5.78 & 15.9 & 23.8 & 16 & 0 of 5 \\
\hline Potassium & 5 of 5 & 216 & 2,020 & 1,290 & NAV & \\
\hline Selenium & 0 of 5 & ND (0.5) & ND (2.48) & $3.15^{b}$ & NAV & \\
\hline Strontium & 5 of 5 & 59.4 & 200 & 207 & NAV & \\
\hline Thallium & 0 of 5 & ND (0.5) & ND (2.48) & ND & NAV & \\
\hline Thorium & 5 of 5 & 0.91 & 16.8 & ND & NAV & \\
\hline Titanium & 5 of 5 & 416 & 1,950 & 1,480 & NAV & \\
\hline Uranium & 4 of 5 & ND (0.5) & 6.22 & ND & NAV & \\
\hline Vanadium & 5 of 5 & 32 & 99.7 & 336 & NAV & \\
\hline Zinc & 5 of 5 & 13.6 & 73.4 & 185 & 120 & 0 of 5 \\
\hline
\end{tabular}

${ }^{a}$ Background concentrations, which are presented only for metals, are upper tolerance limits (UTL) unless otherwise noted.

${ }^{b}$ This metal was not detected in a sufficient number of reference stream samples to allow calculation of a valid UTL. The concentration shown is the maximum detected concentration in the appropriate background samples.

${ }^{\mathrm{c}}$ Screening concentration is based on $1 \%$ organic carbon.

ND - Analyte not detected at a concentration above the reporting limit, which is shown in parentheses.

NAV - Not available

NAN - Not analyzed

$\mathrm{mg} / \mathrm{kg}$ - Milligrams per kilogram 
Table 5-7

\section{Summary of Analytical Results and Ecological Risk Assessment Screening for Surface Water in Drill Site E Stream}

(Page 1 of 3 )

\begin{tabular}{|c|c|c|c|c|c|c|}
\hline Analyte & $\begin{array}{l}\text { Detection } \\
\text { Frequency }\end{array}$ & $\begin{array}{c}\text { Minimum } \\
\text { Detected } \\
\text { Concentration } \\
(\mu \mathrm{g} / \mathrm{L})\end{array}$ & $\begin{array}{c}\text { Maximum } \\
\text { Detected } \\
\text { Concentration } \\
(\mu \mathrm{g} / \mathrm{L})\end{array}$ & $\begin{array}{c}\text { Upland Stream } \\
\text { Background } \\
\text { Concentration }{ }^{\mathrm{a}} \\
(\mu \mathrm{g} / \mathrm{L})\end{array}$ & $\begin{array}{c}\text { Ecological } \\
\text { Screening } \\
\text { Value } \\
(\mu \mathrm{g} / L)\end{array}$ & $\begin{array}{l}\text { Detection } \\
\text { Frequency } \\
\text { Above ESV }\end{array}$ \\
\hline $\begin{array}{l}\text { Diesel-Range } \\
\text { Hydrocarbons }\end{array}$ & 5 of 6 & ND (50) & 104 & NAN & 15 & 5 of 6 \\
\hline \multicolumn{7}{|c|}{ Volatile Organic Compounds } \\
\hline 1,2,4-Trimethylbenzene & 0 of 6 & ND (1.0) & ND (1.0) & NAN & 33 & 0 of 6 \\
\hline 1,3,5-Trimethylbenzene & 0 of 6 & ND (1.0) & ND (1.0) & NAN & 33 & 0 of 6 \\
\hline 2-Butanone & 0 of 6 & ND (20) & ND (20) & NAN & 14,000 & 0 of 6 \\
\hline Acetone & 0 of 6 & ND (20) & ND (20) & NAN & 1,500 & 0 of 6 \\
\hline Benzene & 0 of 6 & ND (1) & ND (1) & NAN & 130 & 0 of 6 \\
\hline Carbon disulfide & 0 of 6 & ND (1) & ND (1) & NAN & 0.92 & 0 of 6 \\
\hline Ethylbenzene & 0 of 6 & ND (1) & ND (1) & NAN & 7.3 & 0 of 6 \\
\hline Isopropylbenzene & 0 of 6 & ND (1) & ND (1) & NAN & 2.6 & 0 of 6 \\
\hline $\mathrm{m}, \mathrm{p}$-Xylene & 0 of 6 & ND (2) & ND (2) & NAN & 13 & 0 of 6 \\
\hline Methylene chloride & 0 of 6 & ND (2.0) & ND (7.94) & NAN & 2,200 & 0 of 6 \\
\hline n-Butylbenzene & 0 of 6 & ND (1) & ND (1) & NAN & 164 & 0 of 6 \\
\hline n-Propylbenzene & 0 of 6 & ND (1) & ND (1) & NAN & 6 & 0 of 6 \\
\hline Naphthalene & 0 of 6 & ND (1.0) & ND (1.0) & NAN & 12 & 0 of 6 \\
\hline o-Xylene & 0 of 6 & ND (1) & ND (1) & NAN & 13 & 0 of 6 \\
\hline p-Isopropyltoluene & 0 of 6 & ND (1.0) & ND (1.0) & NAN & 26 & 0 of 6 \\
\hline sec-Butylbenzene & 0 of 6 & ND (1) & ND (1) & NAN & 164 & 0 of 6 \\
\hline Toluene & 0 of 6 & ND (1) & ND (1) & NAN & 9.8 & 0 of 6 \\
\hline \multicolumn{7}{|c|}{ Polycyclic Aromatic Hydrocarbons } \\
\hline Acenaphthene & 0 of 6 & $\mathrm{ND}(0.1)$ & $\mathrm{ND}(0.1)$ & NAN & 74 & 0 of 6 \\
\hline Acenaphthylene & 0 of 6 & ND $(0.1)$ & $\mathrm{ND}(0.1)$ & NAN & 307 & 0 of 6 \\
\hline Anthracene & 0 of 6 & $\mathrm{ND}(0.1)$ & ND $(0.1)$ & NAN & 0.73 & 0 of 6 \\
\hline Benzo(a)anthracene & 0 of 6 & $\mathrm{ND}(0.1)$ & $\mathrm{ND}(0.1)$ & NAN & 0.027 & 0 of 6 \\
\hline Benzo(a)pyrene & 0 of 6 & $\mathrm{ND}(0.1)$ & $\mathrm{ND}(0.1)$ & NAN & 0.014 & 0 of 6 \\
\hline Benzo(b)fluoranthene & 0 of 6 & ND $(0.1)$ & ND $(0.1)$ & NAN & 0.68 & 0 of 6 \\
\hline Benzo(g,h,I)perylene & 0 of 6 & ND $(0.1)$ & ND $(0.1)$ & NAN & 0.44 & 0 of 6 \\
\hline Benzo(k)fluoranthene & 0 of 6 & $\mathrm{ND}(0.1)$ & $\mathrm{ND}(0.1)$ & NAN & 0.64 & 0 of 6 \\
\hline
\end{tabular}


Table 5-7

\section{Summary of Analytical Results and Ecological Risk Assessment Screening for Surface Water in Drill Site E Stream}

(Page 2 of 3)

\begin{tabular}{|c|c|c|c|c|c|c|}
\hline Analyte & $\begin{array}{l}\text { Detection } \\
\text { Frequency }\end{array}$ & $\begin{array}{c}\text { Minimum } \\
\text { Detected } \\
\text { Concentration } \\
(\mu \mathrm{g} / \mathrm{L})\end{array}$ & $\begin{array}{c}\text { Maximum } \\
\text { Detected } \\
\text { Concentration } \\
(\mu \mathrm{g} / \mathrm{L})\end{array}$ & $\begin{array}{c}\text { Upland Stream } \\
\text { Background } \\
\text { Concentration }{ }^{\mathrm{a}} \\
(\mu \mathrm{g} / \mathrm{L})\end{array}$ & $\begin{array}{c}\text { Ecological } \\
\text { Screening } \\
\text { Value } \\
(\mu \mathrm{g} / L)\end{array}$ & $\begin{array}{l}\text { Detection } \\
\text { Frequency } \\
\text { Above ESV }\end{array}$ \\
\hline Chrysene & 0 of 6 & ND $(0.1)$ & ND $(0.1)$ & NAN & 2 & 0 of 6 \\
\hline Dibenzo(a,h)anthracene & 0 of 6 & ND $(0.1)$ & ND $(0.1)$ & NAN & 0.28 & 0 of 6 \\
\hline Fluoranthene & 0 of 6 & ND $(0.1)$ & ND $(0.1)$ & NAN & 15 & 0 of 6 \\
\hline Fluorene & 0 of 6 & $\mathrm{ND}(0.1)$ & ND $(0.1)$ & NAN & 3.9 & 0 of 6 \\
\hline Indeno(1,2,3-c,d)pyrene & 0 of 6 & $\mathrm{ND}(0.1)$ & ND $(0.1)$ & NAN & 0.28 & 0 of 6 \\
\hline Naphthalene & 0 of 6 & ND $(0.1)$ & ND $(0.1)$ & NAN & 12 & 0 of 6 \\
\hline Phenanthrene & 0 of 6 & ND $(0.1)$ & ND $(0.1)$ & NAN & 200 & 0 of 6 \\
\hline Pyrene & 0 of 6 & $\mathrm{ND}(0.1)$ & ND $(0.1)$ & NAN & 10 & 0 of 6 \\
\hline \multicolumn{7}{|c|}{ Polychlorinated Biphenyls } \\
\hline Aroclor 1016 & NAV & NAV & NAV & NAN & NAV & - \\
\hline Aroclor 1221 & NAV & NAV & NAV & NAN & NAV & - \\
\hline Aroclor 1232 & NAV & NAV & NAV & NAN & NAV & - \\
\hline Aroclor 1242 & NAV & NAV & NAV & NAN & NAV & - \\
\hline Aroclor 1248 & NAV & NAV & NAV & NAN & NAV & - \\
\hline Aroclor 1254 & NAV & NAV & NAV & NAN & NAV & - \\
\hline Aroclor 1260 & NAV & NAV & NAV & NAN & NAV & - \\
\hline Aroclor 1262 & NAV & NAV & NAV & NAN & NAV & - \\
\hline Aroclor 1268 & NAV & NAV & NAV & NAN & NAV & - \\
\hline \multicolumn{7}{|c|}{ Metals } \\
\hline Aluminum & 5 of 6 & ND (50) & 105 & $87.1^{\mathrm{b}}$ & 87 & 2 of 6 \\
\hline Arsenic & 0 of 6 & ND (1.0) & ND (1.0) & ND (1) & 150 & 0 of 6 \\
\hline Barium & 0 of 6 & ND (10) & ND (10) & ND (10) & 4.0 & 0 of 6 \\
\hline Beryllium & 0 of 6 & ND (1.0) & ND (1.0) & ND (1) & 0.66 & 0 of 6 \\
\hline Boron & 0 of 6 & ND (50) & ND (50) & ND (50) & 1.6 & 0 of 6 \\
\hline Cadmium & 0 of 6 & ND (1.0) & ND (1.0) & ND (1) & 2.2 & 0 of 6 \\
\hline Calcium & 6 of 6 & 2,140 & 3,780 & $8,810^{b}$ & 116,000 & 0 of 6 \\
\hline Cerium & 0 of 6 & ND (1.0) & ND (1.0) & ND (1) & 0.5 & 0 of 6 \\
\hline Cesium & 0 of 6 & ND (1.0) & ND (1.0) & ND (1) & 30 & 0 of 6 \\
\hline Chromium & 0 of 6 & ND (1.0) & ND (1.0) & $3.49^{b}$ & 74 & 0 of 6 \\
\hline
\end{tabular}


Table 5-7

\section{Summary of Analytical Results and Ecological Risk Assessment Screening for Surface Water in Drill Site E Stream}

(Page 3 of 3 )

\begin{tabular}{|c|c|c|c|c|c|c|}
\hline Analyte & $\begin{array}{l}\text { Detection } \\
\text { Frequency }\end{array}$ & $\begin{array}{c}\text { Minimum } \\
\text { Detected } \\
\text { Concentration } \\
(\mu \mathrm{g} / \mathrm{L})\end{array}$ & $\begin{array}{c}\text { Maximum } \\
\text { Detected } \\
\text { Concentration } \\
(\mu \mathrm{g} / \mathrm{L})\end{array}$ & $\begin{array}{c}\text { Upland Stream } \\
\text { Background } \\
\text { Concentration }^{\text {a }} \\
(\mu \mathrm{g} / \mathrm{L})\end{array}$ & $\begin{array}{c}\text { Ecological } \\
\text { Screening } \\
\text { Value } \\
(\mu \mathrm{g} / \mathrm{L})\end{array}$ & $\begin{array}{c}\text { Detection } \\
\text { Frequency } \\
\text { Above ESV }\end{array}$ \\
\hline Cobalt & 5 of 6 & ND (1.0) & 4.74 & 9.52 & 23 & 0 of 6 \\
\hline Copper & 5 of 6 & ND (1.0) & 2.85 & $13.4^{\mathrm{b}}$ & 9.0 & 0 of 6 \\
\hline Iron & 1 of 6 & ND (150) & 150 & $2,830^{b}$ & 1,000 & 0 of 6 \\
\hline Lithium & 0 of 6 & ND (1.0) & ND (1.0) & ND (1) & 14 & 0 of 6 \\
\hline Magnesium & 6 of 6 & 1,690 & 2,850 & 5,250 & 82,000 & 0 of 6 \\
\hline Manganese & 6 of 6 & 8.09 & 19.9 & 748 & 120 & 0 of 6 \\
\hline Molybdenum & 0 of 6 & ND (1.0) & ND (1.0) & ND (1) & 370 & 0 of 6 \\
\hline Nickel & 5 of 6 & ND (1.0) & 1.65 & $1.69^{b}$ & 52 & 0 of 6 \\
\hline Potassium & 6 of 6 & 947 & 1,610 & $1,190^{b}$ & 53,000 & 0 of 6 \\
\hline Selenium & 0 of 6 & ND (1.0) & ND (1.0) & ND (1) & 5.0 & 0 of 6 \\
\hline Strontium & 6 of 6 & 18.7 & 29.5 & $44.1^{b}$ & 1,500 & 0 of 6 \\
\hline Thallium & 0 of 6 & ND (1.0) & ND (1.0) & $2.13^{b}$ & 12 & 0 of 6 \\
\hline Thorium & 0 of 6 & ND (1.0) & ND (1.0) & $1.14^{b}$ & NAV & 0 of 6 \\
\hline Titanium & 5 of 6 & ND (1.0) & 7.7 & $2.37^{b}$ & 29 & 0 of 6 \\
\hline Uranium & 0 of 6 & ND (1.0) & ND (1.0) & ND (1) & 2.6 & 0 of 6 \\
\hline Vanadium & 3 of 6 & ND (1.0) & 2.25 & $1.44^{b}$ & 20 & 0 of 6 \\
\hline Zinc & 3 of 6 & ND (1.0) & 13.7 & $12.8^{\mathrm{b}}$ & 120 & 0 of 6 \\
\hline
\end{tabular}

${ }^{\mathrm{a} B a c k g r o u n d}$ concentrations, which are presented only for metals, are upper tolerance limits (UTL) unless otherwise noted.

${ }^{b}$ This metal was not detected in a sufficient number of reference stream samples to allow calculation of a valid UTL. The concentration shown is the maximum detected concentration in the appropriate background samples.

ND - Analyte not detected at a concentration above the reporting limit, which is shown in parentheses.

NAV - Not available

NAN - Not analyzed

$\mu \mathrm{g} / \mathrm{L}$ - Micrograms per liter 
Table 5-8

Summary of Analytical Results and Ecological Risk Assessment Screening for Sediment in Drill Site E Stream

(Page 1 of 3 )

\begin{tabular}{|c|c|c|c|c|c|c|}
\hline Analyte & $\begin{array}{l}\text { Detection } \\
\text { Frequency }\end{array}$ & $\begin{array}{c}\text { Minimum } \\
\text { Detected } \\
\text { Concentration } \\
(\mathbf{m g} / \mathbf{k g})\end{array}$ & $\begin{array}{c}\text { Maximum } \\
\text { Detected } \\
\text { Concentration } \\
(\mathbf{m g} / \mathbf{k g})\end{array}$ & $\begin{array}{c}\text { Upland Stream } \\
\text { Background } \\
\text { Concentration } \\
\text { (mg/kg) }\end{array}$ & $\begin{array}{c}\text { Ecological } \\
\text { Screening } \\
\text { Value }(\mathbf{m g} / \mathbf{k g})\end{array}$ & $\begin{array}{l}\text { Detection } \\
\text { Frequency } \\
\text { Above ESV }\end{array}$ \\
\hline $\begin{array}{l}\text { Diesel-Range } \\
\text { Hydrocarbons }\end{array}$ & 1 of 5 & ND (4.0) & 22.1 & NAN & $640^{\mathrm{C}}$ & 0 of 5 \\
\hline \multicolumn{7}{|c|}{ Volatile Organic Compounds } \\
\hline 1,2,4-Trimethylbenzene & 0 of 1 & ND $(0.0641)$ & ND $(0.0641)$ & NAN & $1.2^{\mathrm{c}}$ & 0 of 1 \\
\hline 1,3,5-Trimethylbenzene & 0 of 1 & ND (0.0641) & ND $(0.0641)$ & NAN & $0.76^{\mathrm{C}}$ & 0 of 1 \\
\hline 2-Butanone & 0 of 1 & ND (0.128) & $\mathrm{ND}(0.128)$ & NAN & 14 & 0 of 1 \\
\hline Acetone & 0 of 1 & ND (1.07) & ND (1.07) & NAN & 1.5 & 0 of 1 \\
\hline Benzene & 0 of 1 & ND $(0.0641)$ & ND $(0.0641)$ & NAN & $0.34^{\mathrm{C}}$ & 0 of 1 \\
\hline Carbon disulfide & 0 of 1 & ND $(0.0641)$ & ND $(0.0641)$ & NAN & 0.001 & 0 of 1 \\
\hline Ethylbenzene & 0 of 1 & ND $(0.0641)$ & ND $(0.0641)$ & NAN & $1.4^{\mathrm{c}}$ & 0 of 1 \\
\hline Isopropylbenzene & 0 of 1 & ND $(0.0641)$ & ND $(0.0641)$ & NAN & $0.1^{c}$ & 0 of 1 \\
\hline m,p-Xylene & 0 of 1 & ND (0.0641) & ND (0.0641) & NAN & 0.12 & 0 of 1 \\
\hline Methylene chloride & 0 of 1 & ND (0.0641) & ND $(0.0641)$ & NAN & $0.5^{\mathrm{c}}$ & 0 of 1 \\
\hline n-Butylbenzene & 0 of 1 & ND (0.0641) & ND (0.0641) & NAN & $22^{c}$ & 0 of 1 \\
\hline n-Propylbenzene & 0 of 1 & ND (0.0641) & ND (0.0641) & NAN & $0.25^{\mathrm{c}}$ & 0 of 1 \\
\hline Naphthalene & 0 of 1 & ND (0.0641) & ND $(0.0641)$ & NAN & $3.85^{\mathrm{C}}$ & 0 of 1 \\
\hline o-Xylene & 0 of 1 & ND $(0.0641)$ & ND $(0.0641)$ & NAN & $0.12^{\mathrm{C}}$ & 0 of 1 \\
\hline p-Isopropyltoluene & 0 of 1 & ND $(0.0641)$ & ND $(0.0641)$ & NAN & $2.8^{\mathrm{c}}$ & 0 of 1 \\
\hline sec-Butylbenzene & 0 of 1 & ND $(0.0641)$ & ND $(0.0641)$ & NAN & $24^{c}$ & 0 of 1 \\
\hline Toluene & 0 of 1 & ND $(0.0641)$ & ND $(0.0641)$ & NAN & $2.5^{c}$ & 0 of 1 \\
\hline \multicolumn{7}{|c|}{ Polycyclic Aromatic Hydrocarbons } \\
\hline Acenaphthene & 1 of 5 & ND (0.0033) & 0.0103 & NAN & $4.91^{\mathrm{c}}$ & 0 of 5 \\
\hline Acenaphthylene & 0 of 5 & ND (0.0033) & ND (0.0176) & NAN & $4.52^{c}$ & 0 of 5 \\
\hline Anthracene & 0 of 5 & ND (0.0033) & ND (0.0176) & NAN & $5.94^{\mathrm{C}}$ & 0 of 5 \\
\hline Benzo(a)anthracene & 0 of 5 & ND (0.0033) & ND (0.0176) & NAN & $8.41^{\mathrm{c}}$ & 0 of 5 \\
\hline Benzo(a)pyrene & 0 of 5 & ND (0.0033) & ND (0.0176) & NAN & $9.65^{\mathrm{c}}$ & 0 of 5 \\
\hline Benzo(b)fluoranthene & 0 of 5 & ND (0.0033) & ND (0.0176) & NAN & $9.79^{C}$ & 0 of 5 \\
\hline Benzo(g,h,i)perylene & 0 of 5 & ND (0.0033) & ND $(0.0176)$ & NAN & $10.95^{c}$ & 0 of 5 \\
\hline Benzo(k)fluoranthene & 0 of 5 & ND (0.0033) & ND $(0.0176)$ & NAN & $9.81^{c}$ & 0 of 5 \\
\hline
\end{tabular}


Table 5-8

Summary of Analytical Results and Ecological Risk Assessment Screening for Sediment in Drill Site E Stream

(Page 2 of 3 )

\begin{tabular}{|c|c|c|c|c|c|c|}
\hline Analyte & $\begin{array}{l}\text { Detection } \\
\text { Frequency }\end{array}$ & $\begin{array}{c}\text { Minimum } \\
\text { Detected } \\
\text { Concentration } \\
(\mathbf{m g} / \mathbf{k g})\end{array}$ & $\begin{array}{l}\text { Maximum } \\
\text { Detected } \\
\text { Concentration } \\
(\mathrm{mg} / \mathrm{kg})\end{array}$ & $\begin{array}{l}\text { Upland Stream } \\
\text { Background } \\
\text { Concentration }{ }^{\mathrm{a}} \\
(\mathrm{mg} / \mathrm{kg})\end{array}$ & $\begin{array}{l}\text { Ecological } \\
\text { Screening } \\
\text { Value }(\mathbf{m g} / \mathbf{k g})\end{array}$ & $\begin{array}{c}\text { Detection } \\
\text { Frequency } \\
\text { Above ESV }\end{array}$ \\
\hline Chrysene & 0 of 5 & ND (0.0033) & ND (0.0176) & NAN & $8.44^{\mathrm{C}}$ & 0 of 5 \\
\hline Dibenzo(a,h)anthracene & 0 of 5 & ND (0.0033) & ND $(0.0176)$ & NAN & $11.23^{\mathrm{C}}$ & 0 of 5 \\
\hline Fluoranthene & 0 of 5 & ND (0.0033) & ND $(0.0176)$ & NAN & $7.07^{\mathrm{C}}$ & 0 of 5 \\
\hline Fluorene & 1 of 5 & ND (0.0033) & 0.0274 & NAN & $5.38^{c}$ & 0 of 5 \\
\hline Indeno(1,2,3-c,d)pyrene & 0 of 5 & ND (0.0033) & ND (0.0176) & NAN & $11.15^{\mathrm{c}}$ & 0 of 5 \\
\hline Naphthalene & 0 of 2 & ND (0.00694) & ND (0.00701) & NAN & $3.85^{\mathrm{C}}$ & 0 of 5 \\
\hline Phenanthrene & 1 of 5 & ND (0.0033) & 0.0702 & NAN & $5.96^{\mathrm{C}}$ & 0 of 5 \\
\hline Pyrene & 0 of 5 & ND (0.0033) & ND $(0.0176)$ & NAN & $6.97^{\mathrm{C}}$ & 0 of 5 \\
\hline \multicolumn{7}{|c|}{ Polychlorinated Biphenyls } \\
\hline Aroclor 1016 & NAV & NAV & NAV & NAN & NAV & - \\
\hline Aroclor 1221 & NAV & NAV & NAV & NAN & NAV & - \\
\hline Aroclor 1232 & NAV & NAV & NAV & NAN & NAV & - \\
\hline Aroclor 1242 & NAV & NAV & NAV & NAN & NAV & - \\
\hline Aroclor 1248 & NAV & NAV & NAV & NAN & NAV & - \\
\hline Aroclor 1254 & NAV & NAV & NAV & NAN & NAV & - \\
\hline Aroclor 1260 & NAV & NAV & NAV & NAN & NAV & - \\
\hline Aroclor 1262 & NAV & NAV & NAV & NAN & NAV & - \\
\hline Aroclor 1268 & NAV & NAV & NAV & NAN & NAV & - \\
\hline \multicolumn{7}{|c|}{ Metals } \\
\hline Aluminum & 5 of 5 & 11,700 & 25,100 & 47,500 & 58,000 & 0 of 5 \\
\hline Arsenic & 5 of 5 & 1.25 & 3.64 & 30.5 & 6 & 0 of 5 \\
\hline Barium & 5 of 5 & 36.5 & 76.7 & 468 & NAV & \\
\hline Beryllium & 0 of 5 & ND $(0.5)$ & ND (2.67) & ND & NAV & \\
\hline Boron & 0 of 5 & ND (2.5) & ND (13.4) & $6.42^{b}$ & NAV & \\
\hline Cadmium & 0 of 5 & ND (0.5) & ND (2.67) & ND & 0.6 & 0 of 5 \\
\hline Calcium & 5 of 5 & 1,950 & 5,370 & 7,720 & NAV & \\
\hline Cerium & 5 of 5 & 8.03 & 19.7 & 13 & NAV & \\
\hline Cesium & 0 of 5 & ND $(0.5)$ & ND (2.67) & ND & NAV & \\
\hline Chromium & 5 of 5 & 9.49 & 41.1 & 18.9 & 26 & 1 of 5 \\
\hline
\end{tabular}


Table 5-8 Summary of Analytical Results and Ecological Risk Assessment
Screening for Sediment in Drill Site E Stream

(Page 3 of 3 )

\begin{tabular}{|c|c|c|c|c|c|c|}
\hline Analyte & $\begin{array}{l}\text { Detection } \\
\text { Frequency }\end{array}$ & $\begin{array}{c}\text { Minimum } \\
\text { Detected } \\
\text { Concentration } \\
(\mathrm{mg} / \mathrm{kg})\end{array}$ & $\begin{array}{c}\text { Maximum } \\
\text { Detected } \\
\text { Concentration } \\
(\mathbf{m g} / \mathrm{kg})\end{array}$ & $\begin{array}{l}\text { Upland Stream } \\
\text { Background } \\
\text { Concentration }{ }^{\mathrm{a}} \\
(\mathrm{mg} / \mathrm{kg})\end{array}$ & $\begin{array}{l}\text { Ecological } \\
\text { Screening } \\
\text { Value }(\mathbf{m g} / \mathbf{k g})\end{array}$ & $\begin{array}{l}\text { Detection } \\
\text { Frequency } \\
\text { Above ESV }\end{array}$ \\
\hline Cobalt & 5 of 5 & 4.03 & 18.5 & 37.3 & NAV & \\
\hline Copper & 5 of 5 & 20.6 & 68 & 82.4 & 16 & 5 of 5 \\
\hline Iron & 5 of 5 & 13,700 & 31,000 & 123,000 & 20,000 & 4 of 5 \\
\hline Lithium & 5 of 5 & 3.03 & 8.74 & 21.7 & NAV & \\
\hline Magnesium & 5 of 5 & 1,310 & 6,950 & 17,000 & NAV & \\
\hline Manganese & 5 of 5 & 82.5 & 1,260 & 26,500 & 460 & 3 of 5 \\
\hline Molybdenum & 1 of 5 & ND (1.05) & 1.02 & ND & NAV & \\
\hline Nickel & 5 of 5 & 6.28 & 32.5 & 23.8 & 16 & 2 of 5 \\
\hline Potassium & 5 of 5 & 123 & 556 & 1,290 & NAV & \\
\hline Selenium & 3 of 5 & ND (1.05) & 3.31 & $3.15^{b}$ & NAV & \\
\hline Strontium & 5 of 5 & 24.6 & 51.4 & 207 & NAV & \\
\hline Thallium & 0 of 5 & ND (0.5) & ND (2.67) & ND & NAV & \\
\hline Thorium & 0 of 5 & ND (0.5) & ND (2.67) & ND & NAV & \\
\hline Titanium & 5 of 5 & 471 & 1,010 & 1,480 & NAV & \\
\hline Uranium & 0 of 5 & ND (0.5) & ND (2.67) & ND & NAV & \\
\hline Vanadium & 5 of 5 & 63.3 & 90.6 & 336 & NAV & \\
\hline Zinc & 4 of 5 & 36.1 & 59.3 & 185 & 120 & 0 of 5 \\
\hline Total Organic Carbon & 5 of 5 & 10,300 & 103,000 & NAN & & \\
\hline
\end{tabular}

${ }^{a}$ Background concentrations, which are presented only for metals, are upper tolerance limits (UTL) unless otherwise noted.

${ }^{\mathrm{b}}$ This metal was not detected in a sufficient number of reference stream samples to allow calculation of a valid UTL. The concentration shown is the maximum detected concentration in the appropriate background samples.

${ }^{\mathrm{c}} \mathrm{S}$ creening concentration is based on $1 \%$ organic carbon.

ND - Analyte not detected at a concentration above the reporting limit, which is shown in parentheses.

NAV - Not available

NAN - Not analyzed

$\mathrm{mg} / \mathrm{kg}$ - Milligrams per kilogram 
Table 5-9

\section{Summary of Analytical Results and Ecological Risk Assessment Screening for Surface Water in Limpet Creek, Drill Site F}

(Page 1 of 3 )

\begin{tabular}{|c|c|c|c|c|c|c|}
\hline Analyte & $\begin{array}{l}\text { Detection } \\
\text { Frequency }\end{array}$ & $\begin{array}{c}\text { Minimum } \\
\text { Detected } \\
\text { Concentration } \\
(\mu \mathrm{g} / \mathrm{L})\end{array}$ & $\begin{array}{c}\text { Maximum } \\
\text { Detected } \\
\text { Concentration } \\
(\mu \mathrm{g} / \mathrm{L})\end{array}$ & $\begin{array}{c}\text { Upland Stream } \\
\text { Background } \\
\text { Concentration }^{\mathrm{a}} \\
(\mu \mathrm{g} / \mathrm{L})\end{array}$ & $\begin{array}{c}\text { Ecological } \\
\text { Screening } \\
\text { Value } \\
(\mu \mathrm{g} / \mathrm{L})\end{array}$ & $\begin{array}{l}\text { Detection } \\
\text { Frequency } \\
\text { Above ESV }\end{array}$ \\
\hline $\begin{array}{l}\text { Diesel-Range } \\
\text { Hydrocarbons }\end{array}$ & 0 of 5 & ND (50) & ND (50) & NAN & 15 & 0 of 5 \\
\hline \multicolumn{7}{|c|}{ Volatile Organic Compounds } \\
\hline 1,2,4-Trimethylbenzene & 0 of 5 & ND (1.0) & ND (1.0) & NAN & 33 & 0 of 5 \\
\hline 1,3,5-Trimethylbenzene & 0 of 5 & ND (1.0) & ND (1.0) & NAN & 33 & 0 of 5 \\
\hline 2-Butanone & 0 of 5 & ND (20) & ND (20) & NAN & 14,000 & 0 of 5 \\
\hline Acetone & 0 of 5 & ND (20) & ND (20) & NAN & 1,500 & 0 of 5 \\
\hline Benzene & 0 of 5 & ND (1.0) & ND (1.0) & NAN & 130 & 0 of 5 \\
\hline Carbon disulfide & 0 of 5 & ND (1.0) & ND (1.0) & NAN & 0.92 & 0 of 5 \\
\hline Ethylbenzene & 0 of 5 & ND (1.0) & ND (1.0) & NAN & 7.3 & 0 of 5 \\
\hline Isopropylbenzene & 0 of 5 & ND (1.0) & ND (1.0) & NAN & 2.6 & 0 of 5 \\
\hline $\mathrm{m}, \mathrm{p}$-Xylene & 0 of 5 & ND (2.0) & ND (2.0) & NAN & 13 & 0 of 5 \\
\hline Methylene chloride & 0 of 5 & ND (2.0) & ND (4.83) & NAN & 2,200 & 0 of 5 \\
\hline n-Butylbenzene & 0 of 5 & ND (1.0) & ND (1.0) & NAN & 164 & 0 of 5 \\
\hline n-Propylbenzene & 0 of 5 & ND (1.0) & ND (1.0) & NAN & 6.0 & 0 of 5 \\
\hline Naphthalene & 0 of 5 & ND (1.0) & ND (1.0) & NAN & 12 & 0 of 5 \\
\hline o-Xylene & 0 of 5 & ND (1.0) & ND (1.0) & NAN & 13 & 0 of 5 \\
\hline p-Isopropyltoluene & 0 of 5 & ND (1.0) & ND (1.0) & NAN & 26 & 0 of 5 \\
\hline sec-Butylbenzene & 0 of 5 & ND (1.0) & ND (1.0) & NAN & 164 & 0 of 5 \\
\hline Toluene & 0 of 5 & ND (1.0) & ND (1.0) & NAN & 9.8 & 0 of 5 \\
\hline \multicolumn{7}{|c|}{ Polycyclic Aromatic Hydrocarbons } \\
\hline Acenaphthene & 0 of 5 & ND $(0.1)$ & $\mathrm{ND}(0.1)$ & NAN & 74 & 0 of 5 \\
\hline Acenaphthylene & 0 of 5 & $\mathrm{ND}(0.1)$ & $\mathrm{ND}(0.1)$ & NAN & 307 & 0 of 5 \\
\hline Anthracene & 0 of 5 & ND $(0.1)$ & $\mathrm{ND}(0.1)$ & NAN & 0.73 & 0 of 5 \\
\hline Benzo(a)anthracene & 0 of 5 & ND $(0.1)$ & $\mathrm{ND}(0.1)$ & NAN & 0.027 & 0 of 5 \\
\hline Benzo(a)pyrene & 0 of 5 & $\mathrm{ND}(0.1)$ & $\mathrm{ND}(0.1)$ & NAN & 0.014 & 0 of 5 \\
\hline Benzo(b)fluoranthene & 0 of 5 & $\mathrm{ND}(0.1)$ & $\mathrm{ND}(0.1)$ & NAN & 0.68 & 0 of 5 \\
\hline Benzo(g,h,l)perylene & 0 of 5 & ND $(0.1)$ & $\mathrm{ND}(0.1)$ & NAN & 0.44 & 0 of 5 \\
\hline Benzo(k)fluoranthene & 0 of 5 & $\mathrm{ND}(0.1)$ & $\mathrm{ND}(0.1)$ & NAN & 0.64 & 0 of 5 \\
\hline
\end{tabular}


Table 5-9

\section{Summary of Analytical Results and Ecological Risk Assessment Screening for Surface Water in Limpet Creek, Drill Site F}

(Page 2 of 3 )

\begin{tabular}{|c|c|c|c|c|c|c|}
\hline Analyte & $\begin{array}{l}\text { Detection } \\
\text { Frequency }\end{array}$ & $\begin{array}{c}\text { Minimum } \\
\text { Detected } \\
\text { Concentration } \\
(\mu \mathrm{g} / \mathrm{L})\end{array}$ & $\begin{array}{c}\text { Maximum } \\
\text { Detected } \\
\text { Concentration } \\
(\mu \mathrm{g} / \mathrm{L})\end{array}$ & $\begin{array}{c}\text { Upland Stream } \\
\text { Background } \\
\text { Concentration }^{\mathrm{a}} \\
(\mu \mathrm{g} / \mathrm{L})\end{array}$ & $\begin{array}{c}\text { Ecological } \\
\text { Screening } \\
\text { Value } \\
(\mu \mathrm{g} / \mathrm{L})\end{array}$ & $\begin{array}{l}\text { Detection } \\
\text { Frequency } \\
\text { Above ESV }\end{array}$ \\
\hline Chrysene & 0 of 5 & ND $(0.1)$ & ND $(0.1)$ & NAN & 2.0 & 0 of 5 \\
\hline Dibenzo(a,h)anthracene & 0 of 5 & ND $(0.1)$ & ND $(0.1)$ & NAN & 0.28 & 0 of 5 \\
\hline Fluoranthene & 0 of 5 & $\mathrm{ND}(0.1)$ & $\mathrm{ND}(0.1)$ & NAN & 15 & 0 of 5 \\
\hline Fluorene & 0 of 5 & ND $(0.1)$ & $\mathrm{ND}(0.1)$ & NAN & 3.9 & 0 of 5 \\
\hline Indeno(1,2,3-c,d)pyrene & 0 of 5 & $\mathrm{ND}(0.1)$ & $\mathrm{ND}(0.1)$ & NAN & 0.28 & 0 of 5 \\
\hline Naphthalene & 0 of 5 & ND $(0.1)$ & ND $(0.1)$ & NAN & 12 & 0 of 5 \\
\hline Phenanthrene & 0 of 5 & ND $(0.1)$ & ND $(0.1)$ & NAN & 200 & 0 of 5 \\
\hline Pyrene & 0 of 5 & ND $(0.1)$ & $\mathrm{ND}(0.1)$ & NAN & 10 & 0 of 5 \\
\hline \multicolumn{7}{|c|}{ Polychlorinated Biphenyls } \\
\hline Aroclor 1016 & NAV & NAV & NAV & NAN & NAV & - \\
\hline Aroclor 1221 & NAV & NAV & NAV & NAN & NAV & - \\
\hline Aroclor 1232 & NAV & NAV & NAV & NAN & NAV & - \\
\hline Aroclor 1242 & NAV & NAV & NAV & NAN & NAV & - \\
\hline Aroclor 1248 & NAV & NAV & NAV & NAN & NAV & - \\
\hline Aroclor 1254 & NAV & NAV & NAV & NAN & NAV & - \\
\hline Aroclor 1260 & NAV & NAV & NAV & NAN & NAV & - \\
\hline Aroclor 1262 & NAV & NAV & NAV & NAN & NAV & - \\
\hline Aroclor 1268 & NAV & NAV & NAV & NAN & NAV & - \\
\hline \multicolumn{7}{|c|}{ Metals } \\
\hline Aluminum & 0 of 5 & ND (50) & ND (50) & $87.1^{b}$ & 87 & 0 of 5 \\
\hline Arsenic & 0 of 5 & ND (1.0) & ND (1.0) & ND (1) & 150 & 0 of 5 \\
\hline Barium & 0 of 5 & ND (10) & ND (10) & ND (10) & 4.0 & 0 of 5 \\
\hline Beryllium & 0 of 5 & ND (1.0) & ND (1.0) & ND (1) & 0.66 & 0 of 5 \\
\hline Boron & 0 of 5 & ND (50) & ND (50) & ND (50) & 1.6 & 0 of 5 \\
\hline Cadmium & 0 of 5 & ND (1.0) & ND (1.0) & ND (1) & 2.2 & 0 of 5 \\
\hline Calcium & 5 of 5 & 3,160 & 3,540 & $8,810^{b}$ & 116,000 & 0 of 5 \\
\hline Cerium & 0 of 5 & ND (1.0) & ND (1.0) & ND (1) & 0.5 & 0 of 5 \\
\hline Cesium & 0 of 5 & ND (1.0) & ND (1.0) & ND (1) & 30 & 0 of 5 \\
\hline Chromium & 0 of 5 & ND (1.0) & ND (1.0) & $3.49^{b}$ & 74 & 0 of 5 \\
\hline
\end{tabular}


Table 5-9

\section{Summary of Analytical Results and Ecological Risk Assessment Screening for Surface Water in Limpet Creek, Drill Site F}

(Page 3 of 3 )

\begin{tabular}{|c|c|c|c|c|c|c|}
\hline Analyte & $\begin{array}{l}\text { Detection } \\
\text { Frequency }\end{array}$ & $\begin{array}{c}\text { Minimum } \\
\text { Detected } \\
\text { Concentration } \\
(\mu \mathrm{g} / \mathrm{L})\end{array}$ & $\begin{array}{c}\text { Maximum } \\
\text { Detected } \\
\text { Concentration } \\
(\mu \mathrm{g} / \mathrm{L})\end{array}$ & $\begin{array}{c}\text { Upland Stream } \\
\text { Background } \\
\text { Concentration } \\
(\mu \mathrm{g} / \mathrm{L})\end{array}$ & $\begin{array}{c}\text { Ecological } \\
\text { Screening } \\
\text { Value } \\
(\mu \mathrm{g} / \mathrm{L})\end{array}$ & $\begin{array}{c}\text { Detection } \\
\text { Frequency } \\
\text { Above ESV }\end{array}$ \\
\hline Cobalt & 4 of 5 & ND (1.0) & 4.91 & 9.52 & 23 & 0 of 5 \\
\hline Copper & 3 of 5 & ND (1.0) & 1.85 & $13.4^{b}$ & 9.0 & 0 of 5 \\
\hline Iron & 2 of 5 & ND (150) & 165 & $2,830^{b}$ & 1,000 & 0 of 5 \\
\hline Lithium & 0 of 5 & ND (1.0) & ND (1.0) & ND (1) & 14 & 0 of 5 \\
\hline Magnesium & 5 of 5 & 2,700 & 3,330 & 5,250 & 82,000 & 0 of 5 \\
\hline Manganese & 5 of 5 & 2.92 & 21.3 & 748 & 120 & 0 of 5 \\
\hline Molybdenum & 0 of 5 & ND (1.0) & ND (1.0) & ND (1) & 370 & 0 of 5 \\
\hline Nickel & 4 of 5 & ND (1.0) & 1.64 & $1.69^{\mathrm{b}}$ & 52 & 0 of 5 \\
\hline Potassium & 5 of 5 & 1,160 & 1,640 & $1,190^{b}$ & 53,000 & 0 of 5 \\
\hline Selenium & 0 of 5 & ND (1.0) & ND (1.0) & ND (1) & 5.0 & 0 of 5 \\
\hline Strontium & 5 of 5 & 32.8 & 35.8 & $44.1^{b}$ & 1,500 & 0 of 5 \\
\hline Thallium & 0 of 5 & ND (1.0) & ND (1.0) & $2.13^{b}$ & 12 & 0 of 5 \\
\hline Thorium & 0 of 5 & ND (1.0) & ND (1.0) & $1.14^{b}$ & NAV & 0 of 5 \\
\hline Titanium & 5 of 5 & 1.16 & 2.29 & $2.37^{b}$ & 29 & 0 of 5 \\
\hline Uranium & 0 of 5 & ND (1.0) & ND (1.0) & ND (1) & 2.6 & 0 of 5 \\
\hline Vanadium & 4 of 5 & ND (1.0) & 2.02 & $1.44^{b}$ & 20 & 0 of 5 \\
\hline Zinc & 4 of 5 & ND (1.0) & 60.8 & $12.8^{\mathrm{b}}$ & 120 & 0 of 5 \\
\hline
\end{tabular}

${ }^{a}$ Background concentrations, which are presented only for metals, are upper tolerance limits (UTL) unless otherwise noted.

${ }^{b}$ This metal was not detected in a sufficient number of reference stream samples to allow calculation of a valid UTL. The concentration shown is the maximum detected concentration in the appropriate background samples.

ND - Analyte not detected at a concentration above the reporting limit, which is shown in parentheses.

NAV - Not available

NAN - Not analyzed

$\mu \mathrm{g} / \mathrm{L}$ - Micrograms per liter 
Table 5-10

\section{Summary of Analytical Results and Ecological Risk Assessment Screening for Sediment in Limpet Creek, Drill Site F}

(Page 1 of 3 )

\begin{tabular}{|c|c|c|c|c|c|c|}
\hline Analyte & $\begin{array}{l}\text { Detection } \\
\text { Frequency }\end{array}$ & $\begin{array}{c}\text { Minimum } \\
\text { Detected } \\
\text { Concentration } \\
(\mathbf{m g} / \mathbf{k g})\end{array}$ & $\begin{array}{c}\text { Maximum } \\
\text { Detected } \\
\text { Concentration } \\
(\mathbf{m g} / \mathbf{k g})\end{array}$ & $\begin{array}{l}\text { Upland Stream } \\
\text { Background } \\
\text { Concentration }{ }^{\mathrm{a}} \\
(\mathrm{mg} / \mathrm{kg})\end{array}$ & $\begin{array}{c}\text { Ecological } \\
\text { Screening } \\
\text { Value } \\
(\mathrm{mg} / \mathrm{kg})\end{array}$ & $\begin{array}{l}\text { Detection } \\
\text { Frequency } \\
\text { Above ESV }\end{array}$ \\
\hline $\begin{array}{l}\text { Diesel-Range } \\
\text { Hydrocarbons }\end{array}$ & 0 of 5 & ND (10.3) & ND (19.8) & NAN & $640^{\mathrm{C}}$ & 0 of 5 \\
\hline \multicolumn{7}{|c|}{ Volatile Organic Compounds } \\
\hline 1,2,4-Trimethylbenzene & 0 of 1 & ND (0.0244) & ND (0.0244) & NAN & $1.2^{\mathrm{C}}$ & 0 of 1 \\
\hline 1,3,5-Trimethylbenzene & 0 of 1 & ND (0.0244) & ND (0.0244) & NAN & $0.76^{\mathrm{C}}$ & 0 of 1 \\
\hline 2-Butanone & 0 of 1 & ND (0.0489) & ND (0.0489) & NAN & 14 & 0 of 1 \\
\hline Acetone & 0 of 1 & ND $(0.564)$ & ND $(0.564)$ & NAN & 1.5 & 0 of 1 \\
\hline Benzene & 0 of 1 & ND (0.0244) & ND (0.0244) & NAN & $0.34^{c}$ & 0 of 1 \\
\hline Carbon disulfide & 0 of 1 & ND (0.0426) & ND (0.0426) & NAN & 0.001 & 0 of 1 \\
\hline Ethylbenzene & 0 of 1 & ND (0.0244) & ND (0.0244) & NAN & $1.4^{\mathrm{C}}$ & 0 of 1 \\
\hline Isopropylbenzene & 0 of 1 & ND (0.0244) & ND $(0.0244)$ & NAN & $0.1^{\mathrm{c}}$ & 0 of 1 \\
\hline $\mathrm{m}, \mathrm{p}$-Xylene & 0 of 1 & ND (0.0244) & ND $(0.0244)$ & NAN & $0.12^{\mathrm{C}}$ & 0 of 1 \\
\hline Methylene chloride & 0 of 1 & ND (0.0244) & ND (0.0244) & NAN & $0.5^{\mathrm{c}}$ & 0 of 1 \\
\hline n-Butylbenzene & 0 of 1 & ND (0.0244) & ND (0.0244) & NAN & $22^{c}$ & 0 of 1 \\
\hline n-Propylbenzene & 0 of 1 & ND (0.0244) & ND (0.0244) & NAN & $0.25^{\mathrm{c}}$ & 0 of 1 \\
\hline Naphthalene & 0 of 1 & ND (0.0244) & ND (0.0244) & NAN & $3.85^{\mathrm{C}}$ & 0 of 1 \\
\hline o-Xylene & 0 of 1 & ND (0.0244) & ND (0.0244) & NAN & $0.12^{\mathrm{C}}$ & 0 of 1 \\
\hline p-Isopropyltoluene & 0 of 1 & ND (0.0244) & ND (0.0244) & NAN & $2.8^{\mathrm{C}}$ & 0 of 1 \\
\hline sec-Butylbenzene & 0 of 1 & ND $(0.0244)$ & ND $(0.0244)$ & NAN & $24^{c}$ & 0 of 1 \\
\hline Toluene & 0 of 1 & ND (0.0244) & ND (0.0244) & NAN & $2.5^{\mathrm{c}}$ & 0 of 1 \\
\hline \multicolumn{7}{|c|}{ Polycyclic Aromatic Hydrocarbons } \\
\hline Acenaphthene & 0 of 5 & ND (0.00849) & ND (0.0163) & NAN & $4.91^{\mathrm{c}}$ & 0 of 5 \\
\hline Acenaphthylene & 0 of 5 & ND (0.00849) & ND (0.0163) & NAN & $4.52^{c}$ & 0 of 5 \\
\hline Anthracene & 0 of 5 & ND (0.00849) & ND (0.0163) & NAN & $5.94^{\mathrm{C}}$ & 0 of 5 \\
\hline Benzo(a)anthracene & 0 of 5 & ND (0.00849) & ND (0.0163) & NAN & $8.41^{\mathrm{c}}$ & 0 of 5 \\
\hline Benzo(a)pyrene & 0 of 5 & ND (0.00849) & ND (0.0163) & NAN & $9.65^{\mathrm{c}}$ & 0 of 5 \\
\hline Benzo(b)fluoranthene & 0 of 5 & ND (0.00849) & ND (0.0163) & NAN & $9.79^{C}$ & 0 of 5 \\
\hline Benzo(g,h,l)perylene & 0 of 5 & ND (0.00849) & ND (0.0163) & NAN & $10.95^{\mathrm{c}}$ & 0 of 5 \\
\hline Benzo(k)fluoranthene & 0 of 5 & ND (0.00849) & ND (0.0163) & NAN & $9.81^{\mathrm{C}}$ & 0 of 5 \\
\hline
\end{tabular}


Table 5-10

\section{Summary of Analytical Results and Ecological Risk Assessment Screening for Sediment in Limpet Creek, Drill Site F}

(Page 2 of 3 )

\begin{tabular}{|c|c|c|c|c|c|c|}
\hline Analyte & $\begin{array}{l}\text { Detection } \\
\text { Frequency }\end{array}$ & $\begin{array}{c}\text { Minimum } \\
\text { Detected } \\
\text { Concentration } \\
(\mathbf{m g} / \mathbf{k g})\end{array}$ & $\begin{array}{c}\text { Maximum } \\
\text { Detected } \\
\text { Concentration } \\
(\mathbf{m g} / \mathbf{k g})\end{array}$ & $\begin{array}{l}\text { Upland Stream } \\
\text { Background } \\
\text { Concentration }{ }^{\mathrm{a}} \\
(\mathrm{mg} / \mathrm{kg})\end{array}$ & $\begin{array}{c}\text { Ecological } \\
\text { Screening } \\
\text { Value } \\
(\mathrm{mg} / \mathrm{kg})\end{array}$ & $\begin{array}{l}\text { Detection } \\
\text { Frequency } \\
\text { Above ESV }\end{array}$ \\
\hline Chrysene & 0 of 5 & ND (0.00849) & ND (0.0163) & NAN & $8.44^{\mathrm{C}}$ & 0 of 5 \\
\hline Dibenzo(a,h)anthracene & 0 of 5 & ND (0.00849) & ND (0.0163) & NAN & $11.23^{\mathrm{C}}$ & 0 of 5 \\
\hline Fluoranthene & 0 of 5 & ND (0.00849) & ND (0.0163) & NAN & $7.07^{\mathrm{C}}$ & 0 of 5 \\
\hline Fluorene & 0 of 5 & ND (0.00849) & ND (0.0163) & NAN & $5.38^{\mathrm{C}}$ & 0 of 5 \\
\hline Indeno(1,2,3-c,d)pyrene & 0 of 5 & ND (0.00849) & ND $(0.0163)$ & NAN & $11.15^{\mathrm{c}}$ & 0 of 5 \\
\hline Naphthalene & 0 of 5 & ND (0.00849) & ND $(0.0163)$ & NAN & $3.85^{\mathrm{C}}$ & 0 of 5 \\
\hline Phenanthrene & 0 of 5 & ND (0.00849) & ND (0.0163) & NAN & $5.96^{\mathrm{C}}$ & 0 of 5 \\
\hline Pyrene & 0 of 5 & ND (0.00849) & ND (0.0163) & NAN & $6.97^{\mathrm{c}}$ & 0 of 5 \\
\hline \multicolumn{7}{|c|}{ Polychlorinated Biphenyls } \\
\hline Aroclor 1016 & NAV & NAV & NAV & NAN & NAV & - \\
\hline Aroclor 1221 & NAV & NAV & NAV & NAN & NAV & - \\
\hline Aroclor 1232 & NAV & NAV & NAV & NAN & NAV & - \\
\hline Aroclor 1242 & NAV & NAV & NAV & NAN & NAV & - \\
\hline Aroclor 1248 & NAV & NAV & NAV & NAN & NAV & - \\
\hline Aroclor 1254 & NAV & NAV & NAV & NAN & NAV & - \\
\hline Aroclor 1260 & NAV & NAV & NAV & NAN & NAV & - \\
\hline Aroclor 1262 & NAV & NAV & NAV & NAN & NAV & - \\
\hline Aroclor 1268 & NAV & NAV & NAV & NAN & NAV & - \\
\hline \multicolumn{7}{|c|}{ Metals } \\
\hline Aluminum & 5 of 5 & 15,500 & 25,100 & 47,500 & 58,000 & 0 of 5 \\
\hline Arsenic & 4 of 5 & ND (2.47) & 4.16 & 30.5 & 6.0 & 0 of 5 \\
\hline Barium & 5 of 5 & 36.8 & 93.1 & 468 & NAV & \\
\hline Beryllium & 0 of 5 & ND (1.29) & ND (2.47) & ND & NAV & \\
\hline Boron & 3 of 5 & ND (6.57) & 89.3 & $6.42^{b}$ & NAV & \\
\hline Cadmium & 0 of 5 & ND (1.29) & ND (2.47) & ND & 0.6 & 0 of 5 \\
\hline Calcium & 5 of 5 & 3,360 & 7,300 & 7,720 & NAV & \\
\hline Cerium & 5 of 5 & 5.55 & 12.9 & 13 & NAV & \\
\hline Cesium & 0 of 5 & ND (1.29) & ND (2.47) & ND & NAV & \\
\hline Chromium & 5 of 5 & 7.66 & 9.81 & 18.9 & 26 & 0 of 5 \\
\hline
\end{tabular}


Table 5-10

\section{Summary of Analytical Results and Ecological Risk Assessment Screening for Sediment in Limpet Creek, Drill Site F}

(Page 3 of 3 )

\begin{tabular}{|c|c|c|c|c|c|c|}
\hline Analyte & $\begin{array}{l}\text { Detection } \\
\text { Frequency }\end{array}$ & $\begin{array}{c}\text { Minimum } \\
\text { Detected } \\
\text { Concentration } \\
(\mathbf{m g} / \mathbf{k g})\end{array}$ & $\begin{array}{c}\text { Maximum } \\
\text { Detected } \\
\text { Concentration } \\
(\mathrm{mg} / \mathrm{kg})\end{array}$ & $\begin{array}{l}\text { Upland Stream } \\
\text { Background } \\
\text { Concentration } \\
(\mathrm{mg} / \mathrm{kg})\end{array}$ & $\begin{array}{c}\text { Ecological } \\
\text { Screening } \\
\text { Value } \\
(\mathrm{mg} / \mathrm{kg})\end{array}$ & $\begin{array}{l}\text { Detection } \\
\text { Frequency } \\
\text { Above ESV }\end{array}$ \\
\hline Cobalt & 5 of 5 & 6.67 & 16.6 & 37.3 & NAV & \\
\hline Copper & 5 of 5 & 16.8 & 57.6 & 82.4 & 16 & 5 of 5 \\
\hline Iron & 5 of 5 & 10,400 & 64,800 & 123,000 & 20,000 & 3 of 5 \\
\hline Lithium & 5 of 5 & 4.08 & 5.59 & 21.7 & NAV & \\
\hline Magnesium & 5 of 5 & 750 & 3,340 & 17,000 & NAV & \\
\hline Manganese & 5 of 5 & 91.2 & 1,250 & 26,500 & 460 & 1 of 5 \\
\hline Molybdenum & 1 of 5 & ND (1.31) & 1.63 & ND & NAV & \\
\hline Nickel & 5 of 5 & 4.74 & 7.71 & 23.8 & 16 & 0 of 5 \\
\hline Potassium & 5 of 5 & 201 & 260 & 1,290 & NAV & \\
\hline Selenium & 3 of 5 & ND (1.31) & 2.52 & $3.15^{b}$ & NAV & \\
\hline Strontium & 5 of 5 & 23.6 & 37.4 & 207 & NAV & \\
\hline Thallium & 0 of 5 & ND (1.29) & ND (2.47) & ND & NAV & \\
\hline Thorium & 0 of 5 & ND (1.29) & ND (2.47) & ND & NAV & \\
\hline Titanium & 5 of 5 & 865 & 1,370 & 1,480 & NAV & \\
\hline Uranium & 0 of 5 & ND (1.29) & ND (2.47) & ND & NAV & \\
\hline Vanadium & 5 of 5 & 92.5 & 165 & 336 & NAV & \\
\hline Zinc & 5 of 5 & 39.2 & 257 & 185 & 120 & 1 of 5 \\
\hline Total Organic Carbon & 5 of 5 & 19,200 & 72,500 & & & \\
\hline
\end{tabular}

${ }^{a}$ Background concentrations, which are presented only for metals, are upper tolerance limits (UTL) unless otherwise noted.

${ }^{\mathrm{b}}$ This metal was not detected in a sufficient number of reference stream samples to allow calculation of a valid UTL. The concentration shown is the maximum detected concentration in the appropriate background samples.

${ }^{\mathrm{c}}$ Screening concentration is based on $1 \%$ organic carbon.

ND - Analyte not detected at a concentration above the reporting limit, which is shown in parentheses.

NAV - Not available

NAN - Not analyzed

$\mathrm{mg} / \mathrm{kg}$ - Milligrams per kilogram 
Table 5-11

Summary of Analytical Results and Ecological Risk Assessment
Screening for Surface Water in Bridge Creek, Long Shot Site

(Page 1 of 3 )

\begin{tabular}{|c|c|c|c|c|c|c|}
\hline Analyte & $\begin{array}{l}\text { Detection } \\
\text { Frequency }\end{array}$ & $\begin{array}{c}\text { Minimum } \\
\text { Detected } \\
\text { Concentration } \\
(\mu \mathrm{g} / \mathrm{L})\end{array}$ & $\begin{array}{c}\text { Maximum } \\
\text { Detected } \\
\text { Concentration } \\
(\mu \mathrm{g} / \mathrm{L})\end{array}$ & $\begin{array}{l}\text { Upland Stream } \\
\text { Background } \\
\text { Concentration }{ }^{\mathrm{a}} \\
(\mu \mathrm{g} / \mathrm{L})\end{array}$ & $\begin{array}{c}\text { Ecological } \\
\text { Screening } \\
\text { Value } \\
(\mu \mathrm{g} / \mathrm{L})\end{array}$ & $\begin{array}{l}\text { Detection } \\
\text { Frequency } \\
\text { Above ESV }\end{array}$ \\
\hline $\begin{array}{l}\text { Diesel-Range } \\
\text { Hydrocarbons }\end{array}$ & 3 of 5 & ND (50) & 77.2 & NAN & 15 & 3 of 5 \\
\hline \multicolumn{7}{|c|}{ Volatile Organic Compounds } \\
\hline 1,2,4-Trimethylbenzene & 0 of 5 & ND (1.0) & ND (1.0) & NAN & 33 & 0 of 5 \\
\hline 1,3,5-Trimethylbenzene & 0 of 5 & ND (1.0) & ND (1.0) & NAN & 33 & 0 of 5 \\
\hline 2-Butanone & 0 of 5 & ND (20) & ND (20) & NAN & 14,000 & 0 of 5 \\
\hline Acetone & 0 of 5 & ND (20) & ND (20) & NAN & 1,500 & 0 of 5 \\
\hline Benzene & 0 of 5 & ND (1.0) & ND (1.0) & NAN & 130 & 0 of 5 \\
\hline Carbon disulfide & 0 of 5 & ND (1.0) & ND (1.0) & NAN & 0.92 & 0 of 5 \\
\hline Ethylbenzene & 0 of 5 & ND (1.0) & ND (1.0) & NAN & 7.3 & 0 of 5 \\
\hline Isopropylbenzene & 0 of 5 & ND (1.0) & ND (1.0) & NAN & 2.6 & 0 of 5 \\
\hline m,p-Xylene & 0 of 5 & ND (2.0) & ND (2.0) & NAN & 13 & 0 of 5 \\
\hline Methylene chloride & 0 of 5 & ND (2.0) & ND (5.95) & NAN & 2,200 & 0 of 5 \\
\hline n-Butylbenzene & 0 of 5 & ND (1.0) & ND (1.0) & NAN & 164 & 0 of 5 \\
\hline n-Propylbenzene & 0 of 5 & ND (1.0) & ND (1.0) & NAN & 6.0 & 0 of 5 \\
\hline Naphthalene & 0 of 5 & ND (1.0) & ND (1.0) & NAN & 12 & 0 of 5 \\
\hline o-Xylene & 0 of 5 & ND (1.0) & ND (1.0) & NAN & 13 & 0 of 5 \\
\hline p-Isopropyltoluene & 0 of 5 & ND (1.0) & ND (1.0) & NAN & 26 & 0 of 5 \\
\hline sec-Butylbenzene & 0 of 5 & ND (1.0) & ND (1.0) & NAN & 164 & 0 of 5 \\
\hline Toluene & 0 of 5 & ND (1.0) & ND (1.0) & NAN & 9.8 & 0 of 5 \\
\hline \multicolumn{7}{|c|}{ Polycyclic Aromatic Hydrocarbons } \\
\hline Acenaphthene & 0 of 5 & ND $(0.1)$ & $\mathrm{ND}(0.1)$ & NAN & 74 & 0 of 5 \\
\hline Acenaphthylene & 0 of 5 & $\mathrm{ND}(0.1)$ & $\mathrm{ND}(0.1)$ & NAN & 307 & 0 of 5 \\
\hline Anthracene & 0 of 5 & ND $(0.1)$ & $\mathrm{ND}(0.1)$ & NAN & 0.73 & 0 of 5 \\
\hline Benzo(a)anthracene & 0 of 5 & $\mathrm{ND}(0.1)$ & ND $(0.1)$ & NAN & 0.027 & 0 of 5 \\
\hline Benzo(a)pyrene & 0 of 5 & $\mathrm{ND}(0.1)$ & $\mathrm{ND}(0.1)$ & NAN & 0.014 & 0 of 5 \\
\hline Benzo(b)fluoranthene & 0 of 5 & $\mathrm{ND}(0.1)$ & $\mathrm{ND}(0.1)$ & NAN & 0.68 & 0 of 5 \\
\hline Benzo(g,h,i)perylene & 0 of 5 & $\mathrm{ND}(0.1)$ & $\mathrm{ND}(0.1)$ & NAN & 0.44 & 0 of 5 \\
\hline Benzo(k)fluoranthene & 0 of 5 & $\mathrm{ND}(0.1)$ & $\mathrm{ND}(0.1)$ & NAN & 0.64 & 0 of 5 \\
\hline
\end{tabular}


Table 5-11

\section{Summary of Analytical Results and Ecological Risk Assessment Screening for Surface Water in Bridge Creek, Long Shot Site} (Page 2 of 3 )

\begin{tabular}{|c|c|c|c|c|c|c|}
\hline Analyte & $\begin{array}{l}\text { Detection } \\
\text { Frequency }\end{array}$ & $\begin{array}{c}\text { Minimum } \\
\text { Detected } \\
\text { Concentration } \\
(\mu \mathrm{g} / \mathrm{L})\end{array}$ & $\begin{array}{c}\text { Maximum } \\
\text { Detected } \\
\text { Concentration } \\
(\mu \mathrm{g} / \mathrm{L})\end{array}$ & $\begin{array}{c}\text { Upland Stream } \\
\text { Background } \\
\text { Concentration }^{\mathrm{a}} \\
(\mu \mathrm{g} / \mathrm{L})\end{array}$ & $\begin{array}{c}\text { Ecological } \\
\text { Screening } \\
\text { Value } \\
(\mu \mathrm{g} / \mathrm{L})\end{array}$ & $\begin{array}{l}\text { Detection } \\
\text { Frequency } \\
\text { Above ESV }\end{array}$ \\
\hline Chrysene & 0 of 5 & ND $(0.1)$ & ND $(0.1)$ & NAN & 2.0 & 0 of 5 \\
\hline Dibenzo(a,h)anthracene & 0 of 5 & $\mathrm{ND}(0.1)$ & $\mathrm{ND}(0.1)$ & NAN & 0.28 & 0 of 5 \\
\hline Fluoranthene & 0 of 5 & $\mathrm{ND}(0.1)$ & $\mathrm{ND}(0.1)$ & NAN & 15 & 0 of 5 \\
\hline Fluorene & 0 of 5 & ND $(0.1)$ & $\mathrm{ND}(0.1)$ & NAN & 3.9 & 0 of 5 \\
\hline Indeno(1,2,3-c,d)pyrene & 0 of 5 & $\mathrm{ND}(0.1)$ & $\mathrm{ND}(0.1)$ & NAN & 0.28 & 0 of 5 \\
\hline Naphthalene & 0 of 5 & ND $(0.1)$ & $\mathrm{ND}(0.1)$ & NAN & 12 & 0 of 5 \\
\hline Phenanthrene & 0 of 5 & $\mathrm{ND}(0.1)$ & ND $(0.1)$ & NAN & 200 & 0 of 5 \\
\hline Pyrene & 0 of 5 & $\mathrm{ND}(0.1)$ & $\mathrm{ND}(0.1)$ & NAN & 10 & 0 of 5 \\
\hline \multicolumn{7}{|c|}{ Polychlorinated Biphenyls } \\
\hline Aroclor 1016 & NAV & NAV & NAV & NAN & NAV & - \\
\hline Aroclor 1221 & NAV & NAV & NAV & NAN & NAV & - \\
\hline Aroclor 1232 & NAV & NAV & NAV & NAN & NAV & - \\
\hline Aroclor 1242 & NAV & NAV & NAV & NAN & NAV & - \\
\hline Aroclor 1248 & NAV & NAV & NAV & NAN & NAV & - \\
\hline Aroclor 1254 & NAV & NAV & NAV & NAN & NAV & - \\
\hline Aroclor 1260 & NAV & NAV & NAV & NAN & NAV & - \\
\hline Aroclor 1262 & NAV & NAV & NAV & NAN & NAV & - \\
\hline Aroclor 1268 & NAV & NAV & NAV & NAN & NAV & - \\
\hline \multicolumn{7}{|c|}{ Metals } \\
\hline Aluminum & 4 of 5 & ND (50) & 85.1 & 269 & 87 & 0 of 5 \\
\hline Arsenic & 0 of 5 & ND (1.0) & ND (1.0) & ND (1) & 150 & 0 of 5 \\
\hline Barium & 0 of 5 & ND (10) & ND (10) & ND (10) & 4.0 & 0 of 5 \\
\hline Beryllium & 0 of 5 & ND (1.0) & ND (1.0) & ND (1) & 0.66 & 0 of 5 \\
\hline Boron & 0 of 5 & ND (50) & ND (50) & ND (50) & 1.6 & 0 of 5 \\
\hline Cadmium & 0 of 5 & ND (1.0) & ND (1.0) & ND (1) & 2.2 & 0 of 5 \\
\hline Calcium & 5 of 5 & 3,700 & 7,250 & $4,320^{b}$ & 116,000 & 0 of 5 \\
\hline Cerium & 0 of 5 & ND (1.0) & ND (1.0) & ND (1) & 0.5 & 0 of 5 \\
\hline Cesium & 0 of 5 & ND (1.0) & ND (1.0) & ND (1) & 30 & 0 of 5 \\
\hline Chromium & 0 of 5 & ND (1.0) & ND (1.0) & $2.25^{\mathrm{b}}$ & 74 & 0 of 5 \\
\hline
\end{tabular}




\section{Table 5-11}

\section{Summary of Analytical Results and Ecological Risk Assessment Screening for Surface Water in Bridge Creek, Long Shot Site} (Page 3 of 3 )

\begin{tabular}{|c|c|c|c|c|c|c|}
\hline Analyte & $\begin{array}{l}\text { Detection } \\
\text { Frequency }\end{array}$ & $\begin{array}{c}\text { Minimum } \\
\text { Detected } \\
\text { Concentration } \\
(\mu \mathrm{g} / \mathrm{L})\end{array}$ & $\begin{array}{c}\text { Maximum } \\
\text { Detected } \\
\text { Concentration } \\
(\mu \mathrm{g} / \mathrm{L})\end{array}$ & $\begin{array}{c}\text { Upland Stream } \\
\text { Background } \\
\text { Concentration } \\
(\mu \mathrm{g} / L)\end{array}$ & $\begin{array}{c}\text { Ecological } \\
\text { Screening } \\
\text { Value } \\
(\mu \mathrm{g} / \mathrm{L})\end{array}$ & $\begin{array}{c}\text { Detection } \\
\text { Frequency } \\
\text { Above ESV }\end{array}$ \\
\hline Cobalt & 3 of 5 & ND (1.0) & 1.28 & 3.35 & 23 & 0 of 5 \\
\hline Copper & 2 of 5 & ND (1.0) & 1.78 & 10.8 & 9.0 & 0 of 5 \\
\hline Iron & 5 of 5 & 199 & 535 & 993 & 1,000 & 0 of 5 \\
\hline Lithium & 0 of 5 & ND (1.0) & ND (1.0) & $1.17^{\mathrm{b}}$ & 14 & 0 of 5 \\
\hline Magnesium & 5 of 5 & 3,170 & 4,750 & $4,690^{b}$ & 82,000 & 0 of 5 \\
\hline Manganese & 5 of 5 & 7.24 & 52.1 & 267 & 120 & 0 of 5 \\
\hline Molybdenum & 0 of 5 & ND (1.0) & ND (1.0) & ND (1) & 370 & 0 of 5 \\
\hline Nickel & 4 of 5 & ND (1.0) & 1.32 & ND (1) & 52 & 0 of 5 \\
\hline Potassium & 4 of 5 & ND (200) & 1,100 & 2,620 & 53,000 & 0 of 5 \\
\hline Selenium & 0 of 5 & ND (1.0) & ND (1.0) & ND (1) & 5.0 & 0 of 5 \\
\hline Strontium & 5 of 5 & 22.1 & 33.7 & 36.1 & 1,500 & 0 of 5 \\
\hline Thallium & 0 of 5 & ND (1.0) & ND (1.0) & $7.07^{\mathrm{b}}$ & 12 & 0 of 5 \\
\hline Thorium & 0 of 5 & ND (1.0) & ND (1.0) & ND (1) & NAV & 0 of 5 \\
\hline Titanium & 5 of 5 & 1.38 & 3.85 & 6.13 & 29 & 0 of 5 \\
\hline Uranium & 0 of 5 & ND (1.0) & ND (1.0) & ND (1) & 2.6 & 0 of 5 \\
\hline Vanadium & 1 of 5 & ND (1.0) & 1.03 & $4.43^{b}$ & 20 & 0 of 5 \\
\hline Zinc & 1 of 5 & ND (10) & 12.2 & $50.3^{b}$ & 120 & 0 of 5 \\
\hline
\end{tabular}

${ }^{a}$ Background concentrations, which are presented only for metals, are upper tolerance limits (UTL) unless otherwise noted.

${ }^{b}$ This metal was not detected in a sufficient number of reference stream samples to allow calculation of a valid UTL. The concentration shown is the maximum detected concentration in the appropriate background samples.

ND - Analyte not detected at a concentration above the reporting limit, which is shown in parentheses.

NAV - Not available

NAN - Not analyzed

$\mu \mathrm{g} / \mathrm{L}$ - Micrograms per liter 
Table 5-12

\section{Summary of Analytical Results and Ecological Risk Assessment Screening for Sediment in Bridge Creek, Long Shot Site}

(Page 1 of 3 )

\begin{tabular}{|c|c|c|c|c|c|c|}
\hline Analyte & $\begin{array}{l}\text { Detection } \\
\text { Frequency }\end{array}$ & $\begin{array}{c}\text { Minimum } \\
\text { Detected } \\
\text { Concentration } \\
(\mathbf{m g} / \mathbf{k g})\end{array}$ & $\begin{array}{c}\text { Maximum } \\
\text { Detected } \\
\text { Concentration } \\
(\mathbf{m g} / \mathbf{k g})\end{array}$ & $\begin{array}{l}\text { Lowland Stream } \\
\text { Background } \\
\text { Concentration }{ }^{\mathrm{a}} \\
(\mathrm{mg} / \mathrm{kg})\end{array}$ & $\begin{array}{c}\text { Ecological } \\
\text { Screening } \\
\text { Value } \\
(\mathrm{mg} / \mathrm{kg})\end{array}$ & $\begin{array}{l}\text { Detection } \\
\text { Frequency } \\
\text { Above ESV }\end{array}$ \\
\hline $\begin{array}{l}\text { Diesel-Range } \\
\text { Hydrocarbons }\end{array}$ & 0 of 5 & ND (15.2) & ND (64.5) & NAN & $640^{c}$ & 0 of 5 \\
\hline \multicolumn{7}{|c|}{ Volatile Organic Compounds } \\
\hline 1,2,4-Trimethylbenzene & 0 of 1 & ND $(0.071)$ & ND $(0.071)$ & NAN & $1.2^{\mathrm{c}}$ & 0 of 1 \\
\hline 1,3,5-Trimethylbenzene & 0 of 1 & ND $(0.071)$ & ND $(0.071)$ & NAN & $0.76^{c}$ & 0 of 1 \\
\hline 2-Butanone & 0 of 1 & ND (0.142) & ND (0.142) & NAN & 14 & 0 of 1 \\
\hline Benzene & 0 of 1 & ND (0.071) & ND $(0.071)$ & NAN & $0.34^{\mathrm{C}}$ & 0 of 1 \\
\hline Acetone & 0 of 1 & ND (0.101) & ND (0.101) & NAN & 1.5 & 0 of 1 \\
\hline Carbon disulfide & 0 of 1 & ND (0.071) & $\mathrm{ND}(0.071)$ & NAN & 0.001 & 0 of 1 \\
\hline Ethylbenzene & 0 of 1 & ND $(0.071)$ & ND $(0.071)$ & NAN & $1.4^{\mathrm{C}}$ & 0 of 1 \\
\hline Isopropylbenzene & 0 of 1 & ND $(0.071)$ & ND $(0.071)$ & NAN & $0.1^{c}$ & 0 of 1 \\
\hline m,p-Xylene & 0 of 1 & ND $(0.071)$ & ND $(0.071)$ & NAN & $0.12^{\mathrm{c}}$ & 0 of 1 \\
\hline Methylene chloride & 0 of 1 & ND $(0.071)$ & ND (0.071) & NAN & $0.5^{\mathrm{c}}$ & 0 of 1 \\
\hline n-Butylbenzene & 0 of 1 & ND $(0.071)$ & ND $(0.071)$ & NAN & $22^{\mathrm{C}}$ & 0 of 1 \\
\hline n-Propylbenzene & 0 of 1 & ND $(0.071)$ & ND $(0.071)$ & NAN & $0.25^{\mathrm{c}}$ & 0 of 1 \\
\hline Naphthalene & 0 of 1 & ND $(0.071)$ & ND $(0.071)$ & NAN & $3.85^{\mathrm{c}}$ & 0 of 1 \\
\hline o-Xylene & 0 of 1 & ND $(0.071)$ & ND $(0.071)$ & NAN & $0.12^{\mathrm{C}}$ & 0 of 1 \\
\hline p-Isopropyltoluene & 0 of 1 & ND $(0.071)$ & ND $(0.071)$ & NAN & $2.8^{c}$ & 0 of 1 \\
\hline sec-Butylbenzene & 0 of 1 & ND $(0.071)$ & ND $(0.071)$ & NAN & $24^{c}$ & 0 of 1 \\
\hline Toluene & 0 of 1 & ND $(0.071)$ & ND $(0.071)$ & NAN & $2.5^{\mathrm{c}}$ & 0 of 1 \\
\hline \multicolumn{7}{|c|}{ Polycyclic Aromatic Hydrocarbons } \\
\hline Acenaphthene & 0 of 5 & ND $(0.0126)$ & ND $(0.0532)$ & NAN & $4.91^{\mathrm{c}}$ & 0 of 5 \\
\hline Acenaphthylene & 0 of 5 & ND $(0.0126)$ & ND (0.0532) & NAN & $4.52^{\mathrm{c}}$ & 0 of 5 \\
\hline Anthracene & 0 of 5 & ND (0.0126) & ND (0.0532) & NAN & $5.94^{c}$ & 0 of 5 \\
\hline Benzo(a)anthracene & 0 of 5 & ND $(0.0126)$ & ND (0.0532) & NAN & $8.41^{c}$ & 0 of 5 \\
\hline Benzo(a)pyrene & 0 of 5 & ND $(0.0126)$ & ND (0.0532) & NAN & $9.65^{c}$ & 0 of 5 \\
\hline Benzo(b)fluoranthene & 0 of 5 & ND $(0.0126)$ & ND (0.0532) & NAN & $9.79^{c}$ & 0 of 5 \\
\hline Benzo(g,h,i)perylene & 0 of 5 & ND $(0.0126)$ & ND (0.0532) & NAN & $10.95^{c}$ & 0 of 5 \\
\hline Benzo(k)fluoranthene & 0 of 5 & ND $(0.0126)$ & ND (0.0532) & NAN & $9.81^{\mathrm{c}}$ & 0 of 5 \\
\hline
\end{tabular}


Table 5-12

\section{Summary of Analytical Results and Ecological Risk Assessment Screening for Sediment in Bridge Creek, Long Shot Site}

(Page 2 of 3 )

\begin{tabular}{|c|c|c|c|c|c|c|}
\hline Analyte & $\begin{array}{l}\text { Detection } \\
\text { Frequency }\end{array}$ & $\begin{array}{c}\text { Minimum } \\
\text { Detected } \\
\text { Concentration } \\
(\mathbf{m g} / \mathbf{k g})\end{array}$ & $\begin{array}{c}\text { Maximum } \\
\text { Detected } \\
\text { Concentration } \\
(\mathbf{m g} / \mathbf{k g})\end{array}$ & $\begin{array}{l}\text { Lowland Stream } \\
\text { Background } \\
\text { Concentration }{ }^{a} \\
(\mathrm{mg} / \mathrm{kg})\end{array}$ & $\begin{array}{c}\text { Ecological } \\
\text { Screening } \\
\text { Value } \\
(\mathrm{mg} / \mathrm{kg})\end{array}$ & $\begin{array}{l}\text { Detection } \\
\text { Frequency } \\
\text { Above ESV }\end{array}$ \\
\hline Chrysene & 0 of 5 & ND $(0.0126)$ & ND (0.0532) & NAN & $8.44^{\mathrm{C}}$ & 0 of 5 \\
\hline Dibenzo(a,h)anthracene & 0 of 5 & ND $(0.0126)$ & ND (0.0532) & NAN & $11.23^{\mathrm{C}}$ & 0 of 5 \\
\hline Fluoranthene & 0 of 5 & ND $(0.0126)$ & ND (0.0532) & NAN & $7.07^{\mathrm{C}}$ & 0 of 5 \\
\hline Fluorene & 0 of 5 & ND $(0.0126)$ & ND (0.0532) & NAN & $5.38^{\mathrm{C}}$ & 0 of 5 \\
\hline Indeno(1,2,3-c,d)pyrene & 0 of 5 & ND $(0.0126)$ & ND (0.0532) & NAN & $11.15^{\mathrm{c}}$ & 0 of 5 \\
\hline Naphthalene & 0 of 5 & ND $(0.0126)$ & ND (0.0532) & NAN & $3.85^{\mathrm{c}}$ & 0 of 5 \\
\hline Phenanthrene & 0 of 5 & ND (0.0126) & ND (0.0532) & NAN & $5.96^{c}$ & 0 of 5 \\
\hline Pyrene & 0 of 5 & ND (0.0126) & ND $(0.0532)$ & NAN & $6.97^{c}$ & 0 of 5 \\
\hline \multicolumn{7}{|c|}{ Polychlorinated Biphenyls } \\
\hline Aroclor 1016 & NAV & NAV & NAV & NAN & NAV & - \\
\hline Aroclor 1221 & NAV & NAV & NAV & NAN & NAV & - \\
\hline Aroclor 1232 & NAV & NAV & NAV & NAN & NAV & - \\
\hline Aroclor 1242 & NAV & NAV & NAV & NAN & NAV & - \\
\hline Aroclor 1248 & NAV & NAV & NAV & NAN & NAV & - \\
\hline Aroclor 1254 & NAV & NAV & NAV & NAN & NAV & - \\
\hline Aroclor 1260 & NAV & NAV & NAV & NAN & NAV & - \\
\hline Aroclor 1262 & NAV & NAV & NAV & NAN & NAV & - \\
\hline Aroclor 1268 & NAV & NAV & NAV & NAN & NAV & - \\
\hline \multicolumn{7}{|c|}{ Metals } \\
\hline Aluminum & 5 of 5 & 11,200 & 63,900 & 45,400 & 58,000 & 1 of 5 \\
\hline Arsenic & 5 of 5 & 5.42 & 16.7 & 100 & 6.0 & 4 of 5 \\
\hline Barium & 4 of 5 & ND (80.6) & 271 & 469 & NAV & 4 of 5 \\
\hline Beryllium & 0 of 5 & ND (1.9) & ND (8.06) & ND & NAV & \\
\hline Boron & 0 of 5 & ND (9.51) & ND (40.3) & $7.18^{b}$ & NAV & \\
\hline Cadmium & 0 of 5 & ND (1.9) & ND (8.06) & $0.608^{b}$ & 0.6 & \\
\hline Calcium & 5 of 5 & 3,930 & 8,770 & 7,300 & NAV & \\
\hline Cerium & 5 of 5 & 7.11 & 11.9 & 14.2 & NAV & \\
\hline Cesium & 0 of 5 & ND (1.9) & ND (8.06) & ND & NAV & \\
\hline Chromium & 4 of 5 & 5.82 & 10 & 11.8 & 26 & \\
\hline
\end{tabular}


Table 5-12

\section{Summary of Analytical Results and Ecological Risk Assessment Screening for Sediment in Bridge Creek, Long Shot Site}

(Page 3 of 3 )

\begin{tabular}{|c|c|c|c|c|c|c|}
\hline Analyte & $\begin{array}{l}\text { Detection } \\
\text { Frequency }\end{array}$ & $\begin{array}{c}\text { Minimum } \\
\text { Detected } \\
\text { Concentration } \\
(\mathbf{m g} / \mathbf{k g})\end{array}$ & $\begin{array}{c}\text { Maximum } \\
\text { Detected } \\
\text { Concentration } \\
(\mathbf{m g} / \mathbf{k g})\end{array}$ & $\begin{array}{c}\text { Lowland Stream } \\
\text { Background } \\
\text { Concentration }{ }^{\mathrm{a}} \\
(\mathrm{mg} / \mathrm{kg})\end{array}$ & $\begin{array}{l}\text { Ecological } \\
\text { Screening } \\
\text { Value } \\
(\mathrm{mg} / \mathrm{kg})\end{array}$ & $\begin{array}{l}\text { Detection } \\
\text { Frequency } \\
\text { Above ESV }\end{array}$ \\
\hline Cobalt & 5 of 5 & 8.35 & 20.3 & 43.7 & NAV & \\
\hline Copper & 5 of 5 & 32.2 & 68.4 & 71.1 & 16 & 5 of 5 \\
\hline Iron & 5 of 5 & 39,400 & 98,600 & 155,000 & 20,000 & 5 of 5 \\
\hline Lithium & 4 of 5 & ND (8.06) & 18 & 48.8 & NAV & \\
\hline Magnesium & 5 of 5 & 1,930 & 12,300 & 9,320 & NAV & \\
\hline Manganese & 5 of 5 & 373 & 6,320 & 20,700 & 460 & 4 of 5 \\
\hline Molybdenum & 1 of 5 & ND (1.9) & 8.47 & $5.5^{\mathrm{b}}$ & NAV & \\
\hline Nickel & 4 of 5 & 6.83 & 9.8 & 13.6 & 16 & \\
\hline Potassium & 5 of 5 & 432 & 4,050 & 1,390 & NAV & \\
\hline Selenium & 2 of 5 & ND (1.9) & 8.86 & $5.44^{b}$ & NAV & \\
\hline Strontium & 5 of 5 & 66.4 & 178 & 164 & NAV & \\
\hline Thallium & 0 of 5 & ND (1.9) & ND (8.06) & $1^{b}$ & NAV & \\
\hline Thorium & 0 of 5 & ND (1.9) & ND (8.06) & $0.5^{\mathrm{b}}$ & NAV & \\
\hline Titanium & 5 of 5 & 440 & 1,460 & 3,010 & NAV & \\
\hline Uranium & 0 of 5 & ND (1.9) & ND (8.06) & $4.13^{b}$ & NAV & \\
\hline Vanadium & 5 of 5 & 109 & 294 & 734 & NAV & \\
\hline Zinc & 5 of 5 & 98.6 & 138 & 434 & 120 & 2 of 5 \\
\hline Total Organic Carbon & 5 of 5 & 43,300 & 194,000 & NAN & & \\
\hline
\end{tabular}

${ }^{\mathrm{a}}$ Background concentrations, which are presented only for metals, are upper tolerance limits (UTL) unless otherwise noted.

${ }^{b}$ This metal was not detected in a sufficient number of reference stream samples to allow calculation of a valid UTL. The concentration shown is the maximum detected concentration in the appropriate background samples.

${ }^{c}$ Screening concentration is based on $1 \%$ organic carbon.

ND - Analyte not detected at a concentration above the reporting limit, which is shown in parentheses.

NAV - Not available

NAN - Not analyzed

$\mathrm{mg} / \mathrm{kg}$ - Milligrams per kilogram 
Table 5-13

\section{Summary of Analytical Results and Ecological Risk Assessment Screening for Surface Water in Reed Pond, Long Shot Site}

(Page 1 of 3 )

\begin{tabular}{|c|c|c|c|c|c|c|}
\hline Analyte & $\begin{array}{l}\text { Detection } \\
\text { Frequency }\end{array}$ & $\begin{array}{c}\text { Minimum } \\
\text { Detected } \\
\text { Concentration } \\
(\mu \mathrm{g} / \mathrm{L})\end{array}$ & $\begin{array}{c}\text { Maximum } \\
\text { Detected } \\
\text { Concentration } \\
(\mu \mathrm{g} / \mathrm{L})\end{array}$ & $\begin{array}{c}\text { Lowland } \\
\text { Stream } \\
\text { Background } \\
\text { Concentration }^{\mathrm{a}} \\
(\mu \mathrm{g} / \mathrm{L})\end{array}$ & $\begin{array}{c}\text { Ecological } \\
\text { Screening } \\
\text { Value (ESV) } \\
(\mu \mathrm{g} / \mathrm{L})\end{array}$ & $\begin{array}{c}\text { Detection } \\
\text { Frequency } \\
\text { Above ESV }\end{array}$ \\
\hline $\begin{array}{l}\text { Diesel-Range } \\
\text { Hydrocarbons }\end{array}$ & 2 of 2 & 113 & 137 & NAN & 15 & 2 of 2 \\
\hline \multicolumn{7}{|c|}{ Volatile Organic Compounds } \\
\hline 1,2,4-Trimethylbenzene & 0 of 2 & ND (1.0) & ND (1.0) & NAN & 33 & 0 of 2 \\
\hline 1,3,5-Trimethylbenzene & 0 of 2 & ND (1.0) & ND (1.0) & NAN & 33 & 0 of 2 \\
\hline 2-Butanone & 0 of 2 & ND (20) & ND (20) & NAN & 14,000 & 0 of 2 \\
\hline Acetone & 0 of 2 & ND (20) & ND (20) & NAN & 1,500 & 0 of 2 \\
\hline Benzene & 0 of 2 & ND (1) & ND (1) & NAN & 130 & 0 of 2 \\
\hline Carbon disulfide & 0 of 2 & ND (1.0) & ND (1.0) & NAN & 0.92 & 0 of 2 \\
\hline Ethylbenzene & 0 of 2 & ND (1.0) & ND (1.0) & NAN & 7.3 & 0 of 2 \\
\hline Isopropylbenzene & 0 of 2 & ND (1.0) & ND (1.0) & NAN & 2.6 & 0 of 2 \\
\hline m,p-Xylene & 0 of 2 & ND (2.0) & ND (2.0) & NAN & 13 & 0 of 2 \\
\hline Methylene chloride & 0 of 2 & ND (2.47) & ND (3.04) & NAN & 2,200 & 0 of 2 \\
\hline n-Butylbenzene & 0 of 2 & ND (1.0) & ND (1.0) & NAN & 164 & 0 of 2 \\
\hline n-Propylbenzene & 0 of 2 & ND (1.0) & ND (1.0) & NAN & 6.0 & 0 of 2 \\
\hline Naphthalene & 0 of 2 & ND (1.0) & ND (1.0) & NAN & 12 & 0 of 2 \\
\hline o-Xylene & 0 of 2 & ND (1.0) & ND (1.0) & NAN & 13 & 0 of 2 \\
\hline p-Isopropyltoluene & 0 of 2 & ND (1.0) & ND (1.0) & NAN & 26 & 0 of 2 \\
\hline sec-Butylbenzene & 0 of 2 & ND (1.0) & ND (1.0) & NAN & 164 & 0 of 2 \\
\hline Toluene & 0 of 2 & ND (1.0) & ND (1.0) & NAN & 9.8 & 0 of 2 \\
\hline \multicolumn{7}{|c|}{ Polycyclic Aromatic Hydrocarbons } \\
\hline Acenaphthene & 0 of 2 & ND $(0.1)$ & ND $(0.1)$ & NAN & 74 & 0 of 2 \\
\hline Acenaphthylene & 0 of 2 & $\mathrm{ND}(0.1)$ & ND $(0.1)$ & NAN & 307 & 0 of 2 \\
\hline Anthracene & 0 of 2 & $\mathrm{ND}(0.1)$ & $\mathrm{ND}(0.1)$ & NAN & 0.73 & 0 of 2 \\
\hline Benzo(a)anthracene & 0 of 2 & ND $(0.1)$ & ND $(0.1)$ & NAN & 0.027 & 0 of 2 \\
\hline Benzo(a)pyrene & 0 of 2 & ND $(0.1)$ & ND $(0.1)$ & NAN & 0.014 & 0 of 2 \\
\hline Benzo(b)fluoranthene & 0 of 2 & ND $(0.1)$ & $\mathrm{ND}(0.1)$ & NAN & 0.68 & 0 of 2 \\
\hline Benzo(g,h,i)perylene & 0 of 2 & ND $(0.1)$ & ND $(0.1)$ & NAN & 0.44 & 0 of 2 \\
\hline
\end{tabular}


Table 5-13

\section{Summary of Analytical Results and Ecological Risk Assessment Screening for Surface Water in Reed Pond, Long Shot Site}

(Page 2 of 3 )

\begin{tabular}{|c|c|c|c|c|c|c|}
\hline Analyte & $\begin{array}{l}\text { Detection } \\
\text { Frequency }\end{array}$ & $\begin{array}{c}\text { Minimum } \\
\text { Detected } \\
\text { Concentration } \\
(\mu \mathrm{g} / L)\end{array}$ & $\begin{array}{c}\text { Maximum } \\
\text { Detected } \\
\text { Concentration } \\
(\mu \mathrm{g} / \mathrm{L})\end{array}$ & $\begin{array}{c}\text { Lowland } \\
\text { Stream } \\
\text { Background } \\
\text { Concentration }{ }^{\mathrm{a}} \\
(\mu \mathrm{g} / \mathrm{L})\end{array}$ & $\begin{array}{c}\text { Ecological } \\
\text { Screening } \\
\text { Value (ESV) } \\
(\mu \mathrm{g} / \mathrm{L})\end{array}$ & $\begin{array}{c}\text { Detection } \\
\text { Frequency } \\
\text { Above ESV }\end{array}$ \\
\hline Benzo(k)fluoranthene & 0 of 2 & $\mathrm{ND}(0.1)$ & $\mathrm{ND}(0.1)$ & NAN & 0.64 & 0 of 2 \\
\hline Chrysene & 0 of 2 & $\mathrm{ND}(0.1)$ & $\mathrm{ND}(0.1)$ & NAN & 2.0 & 0 of 2 \\
\hline Dibenzo(a,h)anthracene & 0 of 2 & ND $(0.1)$ & $\mathrm{ND}(0.1)$ & NAN & 0.28 & 0 of 2 \\
\hline Fluoranthene & 0 of 2 & ND $(0.1)$ & $\mathrm{ND}(0.1)$ & NAN & 15 & 0 of 2 \\
\hline Fluorene & 0 of 2 & ND $(0.1)$ & $\mathrm{ND}(0.1)$ & NAN & 3.9 & 0 of 2 \\
\hline Indeno(1,2,3-c,d)pyrene & 0 of 2 & ND $(0.1)$ & $\mathrm{ND}(0.1)$ & NAN & 0.28 & 0 of 2 \\
\hline Naphthalene & 0 of 2 & $\mathrm{ND}(0.1)$ & $\mathrm{ND}(0.1)$ & NAN & 12 & 0 of 2 \\
\hline Phenanthrene & 0 of 2 & $\mathrm{ND}(0.1)$ & $\mathrm{ND}(0.1)$ & NAN & 200 & 0 of 2 \\
\hline Pyrene & 0 of 2 & $\mathrm{ND}(0.1)$ & $\mathrm{ND}(0.1)$ & NAN & 10 & 0 of 2 \\
\hline \multicolumn{7}{|c|}{ Polychlorinated Biphenyls } \\
\hline Aroclor 1016 & NAV & NAV & NAV & NAN & NAV & - \\
\hline Aroclor 1221 & NAV & NAV & NAV & NAN & NAV & - \\
\hline Aroclor 1232 & NAV & NAV & NAV & NAN & NAV & - \\
\hline Aroclor 1242 & NAV & NAV & NAV & NAN & NAV & - \\
\hline Aroclor 1248 & NAV & NAV & NAV & NAN & NAV & - \\
\hline Aroclor 1254 & NAV & NAV & NAV & NAN & NAV & - \\
\hline Aroclor 1260 & NAV & NAV & NAV & NAN & NAV & - \\
\hline Aroclor 1262 & NAV & NAV & NAV & NAN & NAV & - \\
\hline Aroclor 1268 & NAV & NAV & NAV & NAN & NAV & - \\
\hline \multicolumn{7}{|c|}{ Metals } \\
\hline Aluminum & 1 of 2 & ND (50) & 82.2 & 269 & 87 & 0 of 2 \\
\hline Arsenic & 0 of 2 & ND (1.0) & ND (1.0) & ND (1) & 150 & 0 of 2 \\
\hline Barium & 2 of 2 & 49.4 & 53 & ND (10) & 4.0 & 2 of 2 \\
\hline Beryllium & 0 of 2 & ND (1.0) & ND (1.0) & ND (1) & 0.66 & 0 of 2 \\
\hline Boron & 0 of 2 & ND (50) & ND (50) & ND (50) & 1.6 & 0 of 2 \\
\hline Cadmium & 0 of 2 & ND (1.0) & ND (1.0) & ND (1) & 2.2 & 0 of 2 \\
\hline Calcium & 2 of 2 & 3,420 & 7,330 & $4,320^{b}$ & 116,000 & 0 of 2 \\
\hline Cerium & 0 of 2 & ND (1.0) & ND (1.0) & ND (1) & 0.5 & 0 of 2 \\
\hline
\end{tabular}


Table 5-13

\section{Summary of Analytical Results and Ecological Risk Assessment Screening for Surface Water in Reed Pond, Long Shot Site}

(Page 3 of 3 )

\begin{tabular}{|c|c|c|c|c|c|c|}
\hline Analyte & $\begin{array}{l}\text { Detection } \\
\text { Frequency }\end{array}$ & $\begin{array}{c}\text { Minimum } \\
\text { Detected } \\
\text { Concentration } \\
(\mu \mathrm{g} / \mathrm{L})\end{array}$ & $\begin{array}{c}\text { Maximum } \\
\text { Detected } \\
\text { Concentration } \\
(\mu \mathrm{g} / \mathrm{L})\end{array}$ & $\begin{array}{c}\text { Lowland } \\
\text { Stream } \\
\text { Background } \\
\text { Concentration }{ }^{\mathrm{a}} \\
(\mu \mathrm{g} / \mathrm{L})\end{array}$ & $\begin{array}{c}\text { Ecological } \\
\text { Screening } \\
\text { Value (ESV) } \\
(\mu \mathrm{g} / \mathrm{L})\end{array}$ & $\begin{array}{l}\text { Detection } \\
\text { Frequency } \\
\text { Above ESV }\end{array}$ \\
\hline Cesium & 0 of 2 & ND (1.0) & ND (1.0) & ND (1) & 30 & 0 of 2 \\
\hline Chromium & 2 of 2 & 1.15 & 1.4 & $2.25^{\mathrm{b}}$ & 74 & 0 of 2 \\
\hline Cobalt & 0 of 2 & ND (1.0) & ND (1.0) & 3.35 & 23 & 0 of 2 \\
\hline Copper & 1 of 2 & ND (1.0) & 1.04 & 10.8 & 9.0 & 0 of 2 \\
\hline Iron & 2 of 2 & 370 & 571 & 993 & 1,000 & 0 of 2 \\
\hline Lithium & 0 of 2 & ND (1.0) & ND (1.0) & $1.17^{b}$ & 14 & 0 of 2 \\
\hline Magnesium & 2 of 2 & 2,310 & 3,870 & $4,690^{b}$ & 82,000 & 0 of 2 \\
\hline Manganese & 2 of 2 & 6.85 & 42.4 & 267 & 120 & 0 of 2 \\
\hline Molybdenum & 0 of 2 & ND (1.0) & ND (1.0) & ND (1) & 370 & 0 of 2 \\
\hline Nickel & 0 of 2 & ND (1.0) & ND (1.0) & ND (1) & 52 & 0 of 2 \\
\hline Potassium & 2 of 2 & 1,300 & 1,360 & 2,620 & 53,000 & 0 of 2 \\
\hline Selenium & 0 of 2 & ND (1.0) & ND (1.0) & ND (1) & 5.0 & 0 of 2 \\
\hline Strontium & 2 of 2 & 19.5 & 25.9 & 36.1 & 1,500 & 0 of 2 \\
\hline Thallium & 0 of 2 & ND (1.0) & ND (1.0) & $7.07^{b}$ & 12 & 0 of 2 \\
\hline Thorium & 0 of 2 & ND (1.0) & ND (1.0) & ND (1) & NAV & \\
\hline Titanium & 2 of 2 & 1.47 & 2.1 & 6.13 & 29 & 0 of 2 \\
\hline Uranium & 0 of 2 & ND (1.0) & ND (1.0) & ND (1) & 2.6 & 0 of 2 \\
\hline Vanadium & 0 of 2 & ND (1.0) & ND (1.0) & $4.43^{b}$ & 20 & 0 of 2 \\
\hline Zinc & 0 of 2 & ND (10) & ND (10) & $50.3^{b}$ & 120 & 0 of 2 \\
\hline
\end{tabular}

${ }^{\mathrm{a} B}$ Background concentrations, which are presented only for metals, are upper tolerance limits (UTL) unless otherwise noted.

${ }^{b}$ This metal was not detected in a sufficient number of reference stream samples to allow calculation of a valid UTL. The concentration shown is the maximum detected concentration in the appropriate background samples.

ND - Analyte not detected at a concentration above the reporting limit, which is shown in parentheses.

NAV - Not available

NAN - Not analyzed

$\mu \mathrm{g} / \mathrm{L}$ - Micrograms per liter 


\section{Table 5-14}

\section{Summary of Analytical Results and Ecological Risk Assessment Screening for Sediment in Reed Pond, Long Shot Site}

(Page 1 of 3 )

\begin{tabular}{|c|c|c|c|c|c|c|}
\hline Analyte & $\begin{array}{l}\text { Detection } \\
\text { Frequency }\end{array}$ & $\begin{array}{c}\text { Minimum } \\
\text { Detected } \\
\text { Concentration } \\
(\mathrm{mg} / \mathrm{kg})\end{array}$ & $\begin{array}{c}\text { Maximum } \\
\text { Detected } \\
\text { Concentration } \\
(\mathbf{m g} / \mathrm{kg})\end{array}$ & $\begin{array}{c}\text { Lowland } \\
\text { Stream } \\
\text { Background } \\
\text { Concentration }{ }^{\mathrm{a}} \\
(\mathrm{mg} / \mathbf{k g})\end{array}$ & $\begin{array}{c}\text { Ecological } \\
\text { Screening } \\
\text { Value } \\
(\mathrm{mg} / \mathrm{kg})\end{array}$ & $\begin{array}{l}\text { Detection } \\
\text { Frequency } \\
\text { Above ESV }\end{array}$ \\
\hline $\begin{array}{l}\text { Diesel-Range } \\
\text { Hydrocarbons }\end{array}$ & 1 of 2 & ND (27.4) & 42.6 & NAN & $640^{c}$ & 0 of 2 \\
\hline \multicolumn{7}{|c|}{ Volatile Organic Compounds } \\
\hline 1,2,4-Trimethylbenzene & 0 of 2 & ND (0.0188) & ND $(0.0533)$ & NAN & $1.2^{\mathrm{C}}$ & 0 of 2 \\
\hline 1,3,5-Trimethylbenzene & 0 of 2 & ND $(0.0188)$ & ND $(0.0533)$ & NAN & $0.76^{\mathrm{c}}$ & 0 of 2 \\
\hline 2-Butanone & 2 of 2 & 0.0575 & ND $(0.0533)$ & NAN & 14 & 0 of 2 \\
\hline Acetone & 2 of 2 & 0.38 & 1.02 & NAN & 1.5 & 0 of 2 \\
\hline Benzene & 2 of 2 & ND $(0.0188)$ & ND $(0.0533)$ & NAN & $0.34^{\mathrm{c}}$ & 0 of 2 \\
\hline Carbon disulfide & 0 of 2 & ND $(0.0188)$ & ND $(0.0533)$ & NAN & 0.001 & 0 of 2 \\
\hline Ethylbenzene & 0 of 2 & ND $(0.0188)$ & ND $(0.0533)$ & NAN & $1.4^{\mathrm{C}}$ & 0 of 2 \\
\hline Isopropylbenzene & 0 of 2 & ND (0.0188) & ND (0.0533) & NAN & $0.1^{c}$ & 0 of 2 \\
\hline m,p-Xylene & 0 of 2 & ND $(0.0188)$ & ND (0.0533) & NAN & $0.12^{\mathrm{C}}$ & 0 of 2 \\
\hline Methylene chloride & 0 of 2 & ND (0.0188) & ND (0.0533) & NAN & $0.5^{\mathrm{c}}$ & 0 of 2 \\
\hline n-Butylbenzene & 0 of 2 & ND (0.0188) & ND (0.0533) & NAN & $22^{\mathrm{C}}$ & 0 of 2 \\
\hline n-Propylbenzene & 0 of 2 & ND (0.0188) & ND (0.0533) & NAN & $0.25^{\mathrm{C}}$ & 0 of 2 \\
\hline Naphthalene & 0 of 2 & ND $(0.0188)$ & ND (0.0533) & NAN & $3.85^{\mathrm{c}}$ & 0 of 2 \\
\hline o-Xylene & 0 of 2 & ND $(0.0188)$ & ND (0.0533) & NAN & $0.12^{c}$ & 0 of 2 \\
\hline p-Isopropyltoluene & 0 of 2 & ND (0.0188) & ND (0.0533) & NAN & $2.8^{\mathrm{C}}$ & 0 of 2 \\
\hline sec-Butylbenzene & 0 of 2 & ND (0.0188) & ND (0.0533) & NAN & $24^{c}$ & 0 of 2 \\
\hline Toluene & 1 of 2 & ND $(0.0434)$ & ND (0.0533) & NAN & $2.5^{\mathrm{C}}$ & 0 of 2 \\
\hline \multicolumn{7}{|c|}{ Polycyclic Aromatic Hydrocarbons } \\
\hline Acenaphthene & 0 of 2 & ND (0.0226) & ND $(0.0333)$ & NAN & $4.91^{\mathrm{c}}$ & 0 of 2 \\
\hline Acenaphthylene & 0 of 2 & ND (0.0226) & ND (0.0333) & NAN & $4.52^{\mathrm{C}}$ & 0 of 2 \\
\hline Anthracene & 0 of 2 & ND (0.0333) & ND (0.0226) & NAN & $5.94^{\mathrm{C}}$ & 0 of 2 \\
\hline Benzo(a)anthracene & 0 of 2 & ND $(0.0333)$ & ND $(0.0226)$ & NAN & $8.41^{c}$ & 0 of 2 \\
\hline Benzo(a)pyrene & 0 of 2 & ND (0.0333) & ND $(0.0226)$ & NAN & $9.65^{\mathrm{c}}$ & 0 of 2 \\
\hline Benzo(b)fluoranthene & 0 of 2 & ND (0.0333) & ND (0.0226) & NAN & $9.79^{C}$ & 0 of 2 \\
\hline Benzo(g,h,i)perylene & 0 of 2 & ND $(0.0333)$ & ND $(0.0226)$ & NAN & $10.95^{\mathrm{C}}$ & 0 of 2 \\
\hline
\end{tabular}




\section{Table 5-14}

\section{Summary of Analytical Results and Ecological Risk Assessment Screening for Sediment in Reed Pond, Long Shot Site}

(Page 2 of 3 )

\begin{tabular}{|c|c|c|c|c|c|c|}
\hline Analyte & $\begin{array}{l}\text { Detection } \\
\text { Frequency }\end{array}$ & $\begin{array}{c}\text { Minimum } \\
\text { Detected } \\
\text { Concentration } \\
(\mathbf{m g} / \mathbf{k g})\end{array}$ & $\begin{array}{c}\text { Maximum } \\
\text { Detected } \\
\text { Concentration } \\
(\mathbf{m g} / \mathbf{k g})\end{array}$ & $\begin{array}{c}\text { Lowland } \\
\text { Stream } \\
\text { Background } \\
\text { Concentration }{ }^{\mathrm{a}} \\
(\mathrm{mg} / \mathbf{k g})\end{array}$ & $\begin{array}{c}\text { Ecological } \\
\text { Screening } \\
\text { Value } \\
(\mathrm{mg} / \mathrm{kg})\end{array}$ & $\begin{array}{l}\text { Detection } \\
\text { Frequency } \\
\text { Above ESV }\end{array}$ \\
\hline Benzo(k)fluoranthene & 0 of 2 & ND (0.0333) & ND $(0.0226)$ & NAN & $9.81^{\mathrm{C}}$ & 0 of 2 \\
\hline Chrysene & 0 of 2 & ND (0.0333) & ND (0.0226) & NAN & $8.44^{\mathrm{C}}$ & 0 of 2 \\
\hline Dibenzo(a,h)anthracene & 0 of 2 & ND $(0.0333)$ & ND $(0.0226)$ & NAN & $11.23^{\mathrm{C}}$ & 0 of 2 \\
\hline Fluoranthene & 0 of 2 & ND (0.0333) & ND $(0.0226)$ & NAN & $7.07^{\mathrm{c}}$ & 0 of 2 \\
\hline Fluorene & 0 of 2 & ND $(0.0226)$ & ND (0.0333) & NAN & $5.38^{\mathrm{C}}$ & 0 of 2 \\
\hline Indeno(1,2,3-c,d)pyrene & 0 of 2 & ND (0.0333) & ND $(0.0226)$ & NAN & $11.15^{\mathrm{C}}$ & 0 of 2 \\
\hline Naphthalene & 0 of 2 & ND (0.0226) & ND (0.0333) & NAN & $3.85^{\mathrm{C}}$ & 0 of 2 \\
\hline Phenanthrene & 0 of 2 & ND $(0.0226)$ & ND $(0.0333)$ & NAN & $5.96^{\mathrm{C}}$ & 0 of 2 \\
\hline Pyrene & 0 of 2 & ND (0.0333) & ND (0.0226) & NAN & $6.97^{\mathrm{C}}$ & 0 of 2 \\
\hline \multicolumn{7}{|c|}{ Polychlorinated Biphenyls } \\
\hline Aroclor 1016 & NAV & NAV & NAV & NAN & NAV & - \\
\hline Aroclor 1221 & NAV & NAV & NAV & NAN & NAV & - \\
\hline Aroclor 1232 & NAV & NAV & NAV & NAN & NAV & - \\
\hline Aroclor 1242 & NAV & NAV & NAV & NAN & NAV & - \\
\hline Aroclor 1248 & NAV & NAV & NAV & NAN & NAV & - \\
\hline Aroclor 1254 & NAV & NAV & NAV & NAN & NAV & - \\
\hline Aroclor 1260 & NAV & NAV & NAV & NAN & NAV & - \\
\hline Aroclor 1262 & NAV & NAV & NAV & NAN & NAV & - \\
\hline Aroclor 1268 & NAV & NAV & NAV & NAN & NAV & - \\
\hline \multicolumn{7}{|c|}{ Metals } \\
\hline Aluminum & 2 of 2 & 10,000 & 13,100 & 45,400 & 58,000 & 0 of 2 \\
\hline Arsenic & 1 of 2 & ND (3.42) & 7.92 & 100 & 6.0 & 1 of 2 \\
\hline Barium & 1 of 2 & ND (50.5) & 43.6 & 469 & NAV & \\
\hline Beryllium & 0 of 2 & ND (3.42) & ND (5.05) & ND & NAV & \\
\hline Boron & 0 of 2 & $\mathrm{ND}(17.1)$ & ND (25.3) & $7.18^{b}$ & NAV & \\
\hline Cadmium & 0 of 2 & ND (3.42) & ND (5.05) & $0.608^{b}$ & 0.6 & 0 of 2 \\
\hline Calcium & 2 of 2 & 3,570 & 9,230 & 7,300 & NAV & \\
\hline Cerium & 2 of 2 & 3.87 & 5.72 & 14.2 & NAV & \\
\hline
\end{tabular}


Table 5-14

\section{Summary of Analytical Results and Ecological Risk Assessment Screening for Sediment in Reed Pond, Long Shot Site}

(Page 3 of 3 )

\begin{tabular}{|c|c|c|c|c|c|c|}
\hline Analyte & $\begin{array}{l}\text { Detection } \\
\text { Frequency }\end{array}$ & $\begin{array}{c}\text { Minimum } \\
\text { Detected } \\
\text { Concentration } \\
(\mathbf{m g} / \mathbf{k g})\end{array}$ & $\begin{array}{c}\text { Maximum } \\
\text { Detected } \\
\text { Concentration } \\
(\mathbf{m g} / \mathbf{k g})\end{array}$ & $\begin{array}{c}\text { Lowland } \\
\text { Stream } \\
\text { Background } \\
\text { Concentration } \\
\text { (mg/kg) }\end{array}$ & $\begin{array}{c}\text { Ecological } \\
\text { Screening } \\
\text { Value } \\
(\mathrm{mg} / \mathrm{kg})\end{array}$ & $\begin{array}{c}\text { Detection } \\
\text { Frequency } \\
\text { Above ESV }\end{array}$ \\
\hline Cesium & 1 of 2 & ND (3.42) & 5.72 & ND & NAV & \\
\hline Chromium & 1 of 2 & ND (3.42) & 9.71 & 11.8 & 26 & 0 of 2 \\
\hline Cobalt & 2 of 2 & 3.99 & 7.57 & 43.7 & NAV & \\
\hline Copper & 2 of 2 & 27.4 & 47.5 & 71.1 & 16 & 2 of 2 \\
\hline Iron & 2 of 2 & 4,910 & 5,890 & 155,000 & 20,000 & 0 of 2 \\
\hline Lithium & 1 of 2 & ND (5.05) & 3.53 & 48.8 & NAV & \\
\hline Magnesium & 2 of 2 & 1,480 & 1,790 & 9,320 & NAV & \\
\hline Manganese & 2 of 2 & 97.7 & 221 & 20,700 & 460 & 0 of 2 \\
\hline Molybdenum & 0 of 2 & ND (3.42) & ND (5.05) & $5.5^{\mathrm{b}}$ & NAV & \\
\hline Nickel & 1 of 2 & ND (3.42) & 8.04 & 13.6 & 16 & 0 of 2 \\
\hline Potassium & 2 of 2 & 108 & 145 & 1,390 & NAV & \\
\hline Selenium & 0 of 2 & ND (3.42) & ND (5.05) & $5.44^{b}$ & NAV & \\
\hline Strontium & 2 of 2 & 41 & 46.9 & 164 & NAV & \\
\hline Thallium & 0 of 2 & ND (3.42) & ND (5.05) & $1^{b}$ & NAV & \\
\hline Thorium & 0 of 2 & ND (3.42) & ND (5.05) & $0.5^{\mathrm{b}}$ & NAV & \\
\hline Titanium & 2 of 2 & 752 & 1,280 & 3,010 & NAV & \\
\hline Uranium & 0 of 2 & ND (3.42) & ND (5.05) & $4.13^{b}$ & NAV & \\
\hline Vanadium & 2 of 2 & 46.6 & 340 & 734 & NAV & \\
\hline Zinc & 0 of 2 & ND (34.2) & ND (50.5) & 434 & 120 & 0 of 2 \\
\hline
\end{tabular}

${ }^{\mathrm{a} B}$ Background concentrations, which are presented only for metals, are upper tolerance limits (UTL) unless otherwise noted.

${ }^{b}$ This metal was not detected in a sufficient number of reference stream samples to allow calculation of a valid UTL. The concentration shown is the maximum detected concentration in the appropriate background samples.

${ }^{\mathrm{c}}$ Screening concentration is based on $1 \%$ organic carbon.

ND - Analyte not detected at a concentration above the reporting limit, which is shown in parentheses.

NAV - Not available

NAN - Not analyzed

$\mathrm{mg} / \mathrm{kg}$ - Milligrams per kilogram 
Table 5-15

\section{Summary of Analytical Results and Ecological Risk Assessment Screening for Surface Water in Cloudberry Creek, Long Shot Site} (Page 1 of 3 )

\begin{tabular}{|c|c|c|c|c|c|c|}
\hline Analyte & $\begin{array}{l}\text { Detection } \\
\text { Frequency }\end{array}$ & $\begin{array}{c}\text { Minimum } \\
\text { Detected } \\
\text { Concentration } \\
(\mu \mathrm{g} / \mathrm{L})\end{array}$ & $\begin{array}{c}\text { Maximum } \\
\text { Detected } \\
\text { Concentration } \\
(\mu \mathrm{g} / \mathrm{L})\end{array}$ & $\begin{array}{c}\text { Lowland } \\
\text { Stream } \\
\text { Background } \\
\text { Concentration } \\
(\mu \mathrm{g} / L)\end{array}$ & $\begin{array}{l}\text { Ecological } \\
\text { Screening } \\
\text { Value }(\mu \mathrm{g} / L)\end{array}$ & $\begin{array}{c}\text { Detection } \\
\text { Frequency } \\
\text { Above ESV }\end{array}$ \\
\hline $\begin{array}{l}\text { Diesel-Range } \\
\text { Hydrocarbons }\end{array}$ & 2 of 5 & ND (50) & 54.6 & NAN & 15 & 2 of 5 \\
\hline \multicolumn{7}{|c|}{ Volatile Organic Compounds } \\
\hline 1,2,4-Trimethylbenzene & 0 of 5 & ND (1.0) & ND (1.0) & NAN & 33 & 0 of 5 \\
\hline 1,3,5-Trimethylbenzene & 0 of 5 & ND (1.0) & ND (1.0) & NAN & 33 & 0 of 5 \\
\hline 2-Butanone & 0 of 5 & ND (20) & ND (20) & NAN & 14,000 & 0 of 5 \\
\hline Acetone & 0 of 5 & ND (20) & ND (20) & NAN & 1,500 & 0 of 5 \\
\hline Benzene & 0 of 5 & ND (1) & ND (1) & NAN & 130 & 0 of 5 \\
\hline Carbon disulfide & 0 of 5 & ND (1.0) & ND (1.0) & NAN & 0.92 & 0 of 5 \\
\hline Ethylbenzene & 0 of 5 & ND (1.0) & ND (1.0) & NAN & 7.3 & 0 of 5 \\
\hline Isopropylbenzene & 0 of 5 & ND (1.0) & ND (1.0) & NAN & 2.6 & 0 of 5 \\
\hline $\mathrm{m}, \mathrm{p}$-Xylene & 0 of 5 & ND (2.0) & ND (2.0) & NAN & 13 & 0 of 5 \\
\hline Methylene chloride & 0 of 5 & ND (2.0) & ND (5.14) & NAN & 2,200 & 0 of 5 \\
\hline n-Butylbenzene & 0 of 5 & ND (1.0) & ND (1.0) & NAN & 164 & 0 of 5 \\
\hline n-Propylbenzene & 0 of 5 & ND (1.0) & ND (1.0) & NAN & 6 & 0 of 5 \\
\hline Naphthalene & 0 of 5 & ND (1.0) & ND (1.0) & NAN & 12 & 0 of 5 \\
\hline o-Xylene & 0 of 5 & ND (1.0) & ND (1.0) & NAN & 13 & 0 of 5 \\
\hline p-Isopropyltoluene & 0 of 5 & ND (1.0) & ND (1.0) & NAN & 26 & 0 of 5 \\
\hline sec-Butylbenzene & 0 of 5 & ND (1.0) & ND (1.0) & NAN & 164 & 0 of 5 \\
\hline Toluene & 0 of 5 & ND (1.0) & ND (1.0) & NAN & 9.8 & 0 of 5 \\
\hline \multicolumn{7}{|c|}{ Polycyclic Aromatic Hydrocarbons } \\
\hline Acenaphthene & 0 of 5 & ND $(0.1)$ & ND $(0.1)$ & NAN & 74 & 0 of 5 \\
\hline Acenaphthylene & 0 of 5 & ND $(0.1)$ & ND $(0.1)$ & NAN & 307 & 0 of 5 \\
\hline Anthracene & 0 of 5 & ND $(0.1)$ & ND $(0.1)$ & NAN & 0.73 & 0 of 5 \\
\hline Bezo(a)anthracene & 0 of 5 & ND $(0.1)$ & ND $(0.1)$ & NAN & 0.66 & 0 of 5 \\
\hline Benzo(a)pyrene & 0 of 5 & ND $(0.1)$ & ND $(0.1)$ & NAN & 0.014 & 0 of 5 \\
\hline Benzo(b)fluoranthene & 0 of 5 & ND $(0.1)$ & ND $(0.1)$ & NAN & 0.68 & 0 of 5 \\
\hline Benzo(g,h,l)perylene & 0 of 5 & ND $(0.1)$ & ND $(0.1)$ & NAN & 0.44 & 0 of 5 \\
\hline
\end{tabular}


Table 5-15

Summary of Analytical Results and Ecological Risk Assessment Screening for Surface Water in Cloudberry Creek, Long Shot Site

(Page 2 of 3 )

\begin{tabular}{|c|c|c|c|c|c|c|}
\hline Analyte & $\begin{array}{l}\text { Detection } \\
\text { Frequency }\end{array}$ & $\begin{array}{c}\text { Minimum } \\
\text { Detected } \\
\text { Concentration } \\
(\mu \mathrm{g} / \mathrm{L})\end{array}$ & $\begin{array}{c}\text { Maximum } \\
\text { Detected } \\
\text { Concentration } \\
(\mu \mathrm{g} / \mathrm{L})\end{array}$ & $\begin{array}{c}\text { Lowland } \\
\text { Stream } \\
\text { Background } \\
\text { Concentration }{ }^{\mathrm{a}} \\
(\mu \mathrm{g} / \mathrm{L})\end{array}$ & $\begin{array}{l}\text { Ecological } \\
\text { Screening } \\
\text { Value }(\mu \mathrm{g} / \mathrm{L})\end{array}$ & $\begin{array}{c}\text { Detection } \\
\text { Frequency } \\
\text { Above ESV }\end{array}$ \\
\hline Benzo(k)fluoranthene & 0 of 5 & $\mathrm{ND}(0.1)$ & $\mathrm{ND}(0.1)$ & NAN & 0.64 & 0 of 5 \\
\hline Chrysene & 0 of 5 & $\mathrm{ND}(0.1)$ & ND $(0.1)$ & NAN & 2.0 & 0 of 5 \\
\hline Dibenzo(a,h)anthracene & 0 of 5 & ND $(0.1)$ & ND $(0.1)$ & NAN & 0.28 & 0 of 5 \\
\hline Fluoranthene & 0 of 5 & ND $(0.1)$ & ND $(0.1)$ & NAN & 15 & 0 of 5 \\
\hline Fluorene & 0 of 5 & $\mathrm{ND}(0.1)$ & $\mathrm{ND}(0.1)$ & NAN & 3.9 & 0 of 5 \\
\hline Ideno(1,2,3-c,d)pyrene & 0 of 5 & ND $(0.1)$ & ND $(0.1)$ & NAN & 3.9 & 0 of 5 \\
\hline Naphthalene & 0 of 5 & $\mathrm{ND}(0.1)$ & $\mathrm{ND}(0.1)$ & NAN & 12 & 0 of 5 \\
\hline Phenanthrene & 0 of 5 & $\mathrm{ND}(0.1)$ & $\mathrm{ND}(0.1)$ & NAN & 200 & 0 of 5 \\
\hline Pyrene & 0 of 5 & ND $(0.1)$ & $\mathrm{ND}(0.1)$ & NAN & 10 & 0 of 5 \\
\hline \multicolumn{7}{|c|}{ Polychlorinated Biphenyls } \\
\hline Aroclor 1016 & NAV & NAV & NAV & NAN & NAV & - \\
\hline Aroclor 1221 & NAV & NAV & NAV & NAN & NAV & - \\
\hline Aroclor 1232 & NAV & NAV & NAV & NAN & NAV & - \\
\hline Aroclor 1242 & NAV & NAV & NAV & NAN & NAV & - \\
\hline Aroclor 1248 & NAV & NAV & NAV & NAN & NAV & - \\
\hline Aroclor 1254 & NAV & NAV & NAV & NAN & NAV & - \\
\hline Aroclor 1260 & NAV & NAV & NAV & NAN & NAV & - \\
\hline Aroclor 1262 & NAV & NAV & NAV & NAN & NAV & - \\
\hline Aroclor 1268 & NAV & NAV & NAV & NAN & NAV & - \\
\hline \multicolumn{7}{|c|}{ Metals } \\
\hline Aluminum & 3 of 5 & ND (50) & 77.1 & 269 & 87 & 0 of 5 \\
\hline Arsenic & 0 of 5 & ND (1.0) & ND (1.0) & ND (1) & 150 & 0 of 5 \\
\hline Barium & 0 of 5 & ND (10) & ND (10) & ND (10) & 4.0 & 0 of 5 \\
\hline Beryllium & 0 of 5 & ND (1.0) & ND (1.0) & ND (1) & 0.66 & 0 of 5 \\
\hline Boron & 0 of 5 & ND (50) & ND (50) & ND (50) & 1.6 & 0 of 5 \\
\hline Cadmium & 0 of 5 & ND (1.0) & ND (1.0) & ND (1) & 2.2 & 0 of 5 \\
\hline Calcium & 5 of 5 & 3,490 & 4,300 & $4,320^{b}$ & 116,000 & 0 of 5 \\
\hline Cerium & 0 of 5 & ND (1.0) & ND (1.0) & ND (1) & 0.5 & 0 of 5 \\
\hline
\end{tabular}


Table 5-15

Summary of Analytical Results and Ecological Risk Assessment
Screening for Surface Water in Cloudberry Creek, Long Shot Site (Page 3 of 3 )

\begin{tabular}{|c|c|c|c|c|c|c|}
\hline Analyte & $\begin{array}{l}\text { Detection } \\
\text { Frequency }\end{array}$ & $\begin{array}{c}\text { Minimum } \\
\text { Detected } \\
\text { Concentration } \\
(\mu \mathrm{g} / \mathrm{L})\end{array}$ & $\begin{array}{c}\text { Maximum } \\
\text { Detected } \\
\text { Concentration } \\
(\mu \mathrm{g} / \mathrm{L})\end{array}$ & $\begin{array}{c}\text { Lowland } \\
\text { Stream } \\
\text { Background } \\
\text { Concentration }^{\mathrm{a}} \\
(\mu \mathrm{g} / \mathrm{L})\end{array}$ & $\begin{array}{c}\text { Ecological } \\
\text { Screening } \\
\text { Value }(\mu \mathrm{g} / \mathrm{L})\end{array}$ & $\begin{array}{c}\text { Detection } \\
\text { Frequency } \\
\text { Above ESV }\end{array}$ \\
\hline Cesium & 0 of 5 & ND (1.0) & ND (1.0) & ND (1) & 30 & 0 of 5 \\
\hline Chromium & 0 of 5 & ND (1.0) & ND (1.0) & $2.25^{\mathrm{b}}$ & 74 & 0 of 5 \\
\hline Cobalt & 3 of 5 & ND (1.0) & 2 & 3.35 & 23 & 0 of 5 \\
\hline Copper & 5 of 5 & 1.79 & 3.19 & 10.8 & 9.0 & 0 of 5 \\
\hline Iron & 5 of 5 & 196 & 349 & 993 & 1,000 & 0 of 5 \\
\hline Lithium & 1 of 5 & ND (1.0) & 1.21 & $1.17^{\mathrm{b}}$ & 14 & 0 of 5 \\
\hline Magnesium & 5 of 5 & 3,610 & 3,910 & $4,690^{b}$ & 82,000 & 0 of 5 \\
\hline Manganese & 5 of 5 & 5.84 & 35.4 & 267 & 120 & 0 of 5 \\
\hline Molybdenum & 0 of 5 & ND (1.0) & ND (1.0) & ND (1) & 370 & 0 of 5 \\
\hline Nickel & 3 of 5 & ND (1.0) & 1.27 & ND (1) & 52 & 0 of 5 \\
\hline Potassium & 5 of 5 & 915 & 1,480 & 2,620 & 53,000 & 0 of 5 \\
\hline Selenium & 0 of 5 & ND (1.0) & ND (1.0) & ND (1) & 5.0 & 0 of 5 \\
\hline Strontium & 5 of 5 & 1.85 & 20.8 & 36.1 & 1,500 & 0 of 5 \\
\hline Thallium & 0 of 5 & ND (1.0) & ND (1.0) & $7.07^{b}$ & 12 & 0 of 5 \\
\hline Thorium & 0 of 5 & ND (1.0) & ND (1.0) & ND (1) & NAV & \\
\hline Titanium & 5 of 5 & 1.72 & 3.13 & 6.13 & 29 & 0 of 5 \\
\hline Uranium & 0 of 5 & ND (1.0) & ND (1.0) & ND (1) & 2.6 & 0 of 5 \\
\hline Vanadium & 4 of 5 & ND (1.0) & 2.87 & $4.43^{b}$ & 20 & 0 of 5 \\
\hline Zinc & 1 of 5 & ND (10) & 17 & $50.3^{b}$ & 120 & 0 of 5 \\
\hline
\end{tabular}

${ }^{\mathrm{a} B}$ Background concentrations, which are presented only for metals, are upper tolerance limits (UTL) unless otherwise noted.

${ }^{\mathrm{b}}$ This metal was not detected in a sufficient number of reference stream samples to allow calculation of a valid UTL. The concentration shown is the maximum detected concentration in the appropriate background samples.

ND - Analyte not detected at a concentration above the reporting limit, which is shown in parentheses.

NAV - Not available

NAN - Not analyzed

$\mu \mathrm{g} / \mathrm{L}$ - Micrograms per liter 


\section{Table 5-16}

\section{Summary of Analytical Results and Ecological Risk Assessment Screening for Sediment in Cloudberry Creek, Long Shot Site}

(Page 1 of 3 )

\begin{tabular}{|c|c|c|c|c|c|c|}
\hline Analyte & $\begin{array}{l}\text { Detection } \\
\text { Frequency }\end{array}$ & $\begin{array}{c}\text { Minimum } \\
\text { Detected } \\
\text { Concentration } \\
(\mathrm{mg} / \mathrm{kg})\end{array}$ & $\begin{array}{c}\text { Maximum } \\
\text { Detected } \\
\text { Concentration } \\
(\mathbf{m g} / \mathbf{k g})\end{array}$ & $\begin{array}{c}\text { Lowland } \\
\text { Stream } \\
\text { Background } \\
\text { Concentration }{ }^{\mathrm{a}} \\
(\mathrm{mg} / \mathrm{kg})\end{array}$ & $\begin{array}{c}\text { Ecological } \\
\text { Screening } \\
\text { Value } \\
(\mathrm{mg} / \mathrm{kg})\end{array}$ & $\begin{array}{c}\text { Detection } \\
\text { Frequency } \\
\text { Above } \\
\text { ESV }\end{array}$ \\
\hline $\begin{array}{l}\text { Diesel-Range } \\
\text { Hydrocarbons }\end{array}$ & 0 of 6 & ND (12.1) & ND (50.5) & NAN & $640^{\mathrm{C}}$ & 0 of 6 \\
\hline \multicolumn{7}{|c|}{ Volatile Organic Compounds } \\
\hline 1,2,4-Trimethylbenzene & 0 of 1 & ND (0.0389) & ND (0.0389) & NAN & $1.2^{\mathrm{C}}$ & 0 of 1 \\
\hline 1,3,5-Trimethylbenzene & 0 of 1 & ND (0.0389) & ND (0.0389) & NAN & $0.76^{\mathrm{c}}$ & 0 of 1 \\
\hline 2-Butanone & 0 of 1 & ND (0.0778) & ND $(0.0778)$ & NAN & 14 & 0 of 1 \\
\hline Acetone & 0 of 1 & ND $(0.907)$ & ND (0.907) & NAN & 1.5 & 0 of 1 \\
\hline Benzene & 0 of 1 & ND (0.0389) & ND (0.0389) & NAN & 0.34 & 0 of 1 \\
\hline Carbon disulfide & 0 of 1 & ND (0.0494) & ND $(0.0494)$ & NAN & 0.001 & 0 of 1 \\
\hline Ethylbenzene & 0 of 1 & ND (0.0389) & ND (0.0389) & NAN & $1.4^{\mathrm{C}}$ & 0 of 1 \\
\hline Isopropylbenzene & 0 of 1 & ND (0.0389) & ND (0.0389) & NAN & $0.10^{C}$ & 0 of 1 \\
\hline m,p-Xylene & 0 of 1 & ND (0.0389) & ND (0.0389) & NAN & $0.12^{\mathrm{C}}$ & 0 of 1 \\
\hline Methylene chloride & 0 of 1 & ND (0.0389) & ND (0.0389) & NAN & $0.5^{\mathrm{c}}$ & 0 of 1 \\
\hline n-Butylbenzene & 0 of 1 & ND (0.0389) & ND (0.0389) & NAN & $22^{c}$ & 0 of 1 \\
\hline n-Propylbenzene & 0 of 1 & ND (0.0389) & ND (0.0389) & NAN & $0.25^{\mathrm{C}}$ & 0 of 1 \\
\hline Naphthalene & 0 of 1 & ND (0.0389) & ND (0.0389) & NAN & $3.85^{c}$ & 0 of 1 \\
\hline o-Xylene & 0 of 1 & ND (0.0389) & ND (0.0389) & NAN & $0.12^{c}$ & 0 of 1 \\
\hline p-Isopropyltoluene & 0 of 1 & ND (0.0389) & ND (0.0389) & NAN & $2.8^{\mathrm{C}}$ & 0 of 1 \\
\hline sec-Butylbenzene & 0 of 1 & ND (0.0389) & ND (0.0389) & NAN & $24^{c}$ & 0 of 1 \\
\hline Toluene & 0 of 1 & ND (0.0389) & ND (0.0389) & NAN & $2.5^{\mathrm{c}}$ & 0 of 1 \\
\hline \multicolumn{7}{|c|}{ Polycyclic Aromatic Hydrocarbons } \\
\hline Acenaphthene & 0 of 6 & ND (0.00996) & ND $(0.0417)$ & NAN & $4.91^{\mathrm{C}}$ & 0 of 6 \\
\hline Acenaphthylene & 0 of 6 & ND (0.00996) & ND $(0.0417)$ & NAN & $4.52^{c}$ & 0 of 6 \\
\hline Anthracene & 0 of 6 & ND $(0.00996)$ & ND $(0.0417)$ & NAN & $5.94^{\mathrm{C}}$ & 0 of 6 \\
\hline Benzo(a)anthracene & 0 of 6 & ND (0.00996) & ND (0.0417) & NAN & $8.41^{c}$ & 0 of 6 \\
\hline Benzo(a)pyrene & 0 of 6 & ND $(0.00996)$ & ND $(0.0417)$ & NAN & $9.65^{\mathrm{C}}$ & 0 of 6 \\
\hline Benzo(b)fluoranthene & 0 of 6 & ND (0.00996) & ND (0.0417) & NAN & $9.79^{C}$ & 0 of 6 \\
\hline Benzo(g,h,l)perylene & 0 of 6 & ND (0.00996) & ND $(0.0417)$ & NAN & $10.95^{\mathrm{C}}$ & 0 of 6 \\
\hline
\end{tabular}


Table 5-16

\section{Summary of Analytical Results and Ecological Risk Assessment Screening for Sediment in Cloudberry Creek, Long Shot Site}

(Page 2 of 3 )

\begin{tabular}{|c|c|c|c|c|c|c|}
\hline Analyte & $\begin{array}{l}\text { Detection } \\
\text { Frequency }\end{array}$ & $\begin{array}{c}\text { Minimum } \\
\text { Detected } \\
\text { Concentration } \\
(\mathrm{mg} / \mathrm{kg})\end{array}$ & $\begin{array}{c}\text { Maximum } \\
\text { Detected } \\
\text { Concentration } \\
(\mathbf{m g} / \mathbf{k g})\end{array}$ & $\begin{array}{c}\text { Lowland } \\
\text { Stream } \\
\text { Background } \\
\text { Concentration }{ }^{\mathrm{a}} \\
(\mathrm{mg} / \mathrm{kg})\end{array}$ & $\begin{array}{c}\text { Ecological } \\
\text { Screening } \\
\text { Value } \\
(\mathrm{mg} / \mathrm{kg})\end{array}$ & $\begin{array}{l}\text { Detection } \\
\text { Frequency } \\
\text { Above } \\
\text { ESV }\end{array}$ \\
\hline Benzo(k)fluoranthene & 0 of 6 & ND (0.00996) & ND $(0.0417)$ & NAN & $9.81^{\mathrm{C}}$ & 0 of 6 \\
\hline Chrysene & 0 of 6 & ND (0.00996) & ND (0.0417) & NAN & $8.44^{\mathrm{C}}$ & 0 of 6 \\
\hline Dibenzo(a,h)anthracene & 0 of 6 & ND (0.00996) & ND (0.0417) & NAN & $11.23^{\mathrm{C}}$ & 0 of 6 \\
\hline Fluoranthene & 0 of 6 & ND (0.00996) & ND $(0.0417)$ & NAN & $7.07^{\mathrm{C}}$ & 0 of 6 \\
\hline Fluorene & 0 of 6 & ND (0.00996) & ND (0.0417) & NAN & $5.38^{c}$ & 0 of 6 \\
\hline Ideno(1,2,3-c,d)pyrene & 0 of 6 & ND (0.00996) & ND (0.0417) & NAN & $1.2^{\mathrm{c}}$ & 0 of 6 \\
\hline Naphthalene & 0 of 6 & ND (0.00996) & ND (0.0417) & NAN & $3.85^{\mathrm{c}}$ & 0 of 6 \\
\hline Phenanthrene & 0 of 6 & ND (0.00996) & ND $(0.0417)$ & NAN & $5.96^{\mathrm{C}}$ & 0 of 6 \\
\hline Pyrene & 0 of 6 & ND (0.00996) & ND $(0.0417)$ & NAN & $6.97^{\mathrm{C}}$ & 0 of 6 \\
\hline \multicolumn{7}{|c|}{ Polychlorinated Biphenyls } \\
\hline Aroclor 1016 & NAV & NAV & NAV & NAN & NAV & - \\
\hline Aroclor 1221 & NAV & NAV & NAV & NAN & NAV & - \\
\hline Aroclor 1232 & NAV & NAV & NAV & NAN & NAV & - \\
\hline Aroclor 1242 & NAV & NAV & NAV & NAN & NAV & - \\
\hline Aroclor 1248 & NAV & NAV & NAV & NAN & NAV & - \\
\hline Aroclor 1254 & NAV & NAV & NAV & NAN & NAV & - \\
\hline Aroclor 1260 & NAV & NAV & NAV & NAN & NAV & - \\
\hline Aroclor 1262 & NAV & NAV & NAV & NAN & NAV & - \\
\hline Aroclor 1268 & NAV & NAV & NAV & NAN & NAV & - \\
\hline \multicolumn{7}{|c|}{ Metals } \\
\hline Aluminum & 6 of 6 & 17,100 & 41,800 & 45,400 & 58,000 & 0 of 6 \\
\hline Arsenic & 5 of 6 & 3.58 & 9.89 & 100 & 6.0 & 4 of 6 \\
\hline Barium & 6 of 6 & 68.5 & 151 & 469 & NAV & \\
\hline Beryllium & 1 of 6 & ND (1.51) & 2.9 & ND & NAV & \\
\hline Boron & 1 of 6 & ND (10) & 159 & $7.18^{\mathrm{b}}$ & NAV & \\
\hline Cadmium & 1 of 6 & ND (1.51) & 3.17 & $0.608^{b}$ & 0.6 & 1 of 6 \\
\hline Calcium & 6 of 6 & 5,460 & 8,010 & 7,300 & NAV & \\
\hline Cerium & 6 of 6 & 6.83 & 12 & 14.2 & NAV & \\
\hline
\end{tabular}


Table 5-16

\section{Summary of Analytical Results and Ecological Risk Assessment Screening for Sediment in Cloudberry Creek, Long Shot Site}

(Page 3 of 3 )

\begin{tabular}{|c|c|c|c|c|c|c|}
\hline Analyte & $\begin{array}{l}\text { Detection } \\
\text { Frequency }\end{array}$ & $\begin{array}{c}\text { Minimum } \\
\text { Detected } \\
\text { Concentration } \\
(\mathbf{m g} / \mathbf{k g})\end{array}$ & $\begin{array}{c}\text { Maximum } \\
\text { Detected } \\
\text { Concentration } \\
(\mathbf{m g} / \mathbf{k g})\end{array}$ & $\begin{array}{c}\text { Lowland } \\
\text { Stream } \\
\text { Background } \\
\text { Concentration } \\
\text { (mg/kg) }\end{array}$ & $\begin{array}{c}\text { Ecological } \\
\text { Screening } \\
\text { Value } \\
(\mathrm{mg} / \mathrm{kg})\end{array}$ & $\begin{array}{c}\text { Detection } \\
\text { Frequency } \\
\text { Above } \\
\text { ESV }\end{array}$ \\
\hline Cesium & 1 of 6 & ND (1.51) & 3.51 & ND & NAV & \\
\hline Chromium & 5 of 6 & 5.55 & 11.3 & 11.8 & 26 & 0 of 6 \\
\hline Cobalt & 6 of 6 & 8.33 & 24.6 & 43.7 & NAV & \\
\hline Copper & 6 of 6 & 52.6 & 71.7 & 71.1 & 16 & 6 of 6 \\
\hline Iron & 6 of 6 & 18,700 & 79,900 & 155,000 & 20,000 & 5 of 6 \\
\hline Lithium & 6 of 6 & 10.2 & 17 & 48.8 & NAV & \\
\hline Magnesium & 6 of 6 & 7,490 & 16,800 & 9,320 & NAV & \\
\hline Manganese & 6 of 6 & 140 & 5,160 & 20,700 & 460 & 5 of 6 \\
\hline Molybdenum & 1 of 6 & $\mathrm{ND}(1.51)$ & 3.79 & $5.5^{b}$ & NAV & \\
\hline Nickel & 6 of 6 & 6.86 & 9.67 & 13.6 & 16 & 0 of 6 \\
\hline Potassium & 6 of 6 & 537 & 2,630 & 1,390 & NAV & \\
\hline Selenium & 1 of 6 & ND (1.51) & 4.55 & $5.44^{b}$ & NAV & \\
\hline Strontium & 6 of 6 & 50.8 & 221 & 164 & NAV & \\
\hline Thallium & 1 of 6 & ND (1.51) & 4.08 & $1^{b}$ & NAV & \\
\hline Thorium & 0 of 6 & $\mathrm{ND}(1.51)$ & $\mathrm{ND}(6.31)$ & $0.5^{b}$ & NAV & \\
\hline Titanium & 6 of 6 & 639 & 1,640 & 3,010 & NAV & \\
\hline Uranium & 0 of 6 & $\mathrm{ND}(1.51)$ & ND (6.31) & $4.13^{b}$ & NAV & \\
\hline Vanadium & 6 of 6 & 121 & 230 & 734 & NAV & \\
\hline Zinc & 6 of 6 & 57.8 & 106 & 434 & 120 & 0 of 6 \\
\hline Total Organic Carbon & 6 of 6 & 29,900 & 220,000 & NAN & & \\
\hline
\end{tabular}

${ }^{a}$ Background concentrations, which are presented only for metals, are upper tolerance limits (UTL) unless otherwise noted.

${ }^{b}$ This metal was not detected in a sufficient number of reference stream samples to allow calculation of a valid UTL. The concentration shown is the maximum detected concentration in the appropriate background samples.

${ }^{\mathrm{c}}$ Screening concentration is based on $1 \%$ organic carbon.

ND - Analyte not detected at a concentration above the reporting limit, which is shown in parentheses.

NAV - Not available

NAN - Not analyzed

$\mathrm{mg} / \mathrm{kg}$ - Milligrams per kilogram 
Table 5-17

\section{Summary of Analytical Results and Ecological Risk Assessment Screening for Surface Water in Rainbow Creek, Long Shot Site}

(Page 1 of 3 )

\begin{tabular}{|c|c|c|c|c|c|c|}
\hline Analyte & $\begin{array}{l}\text { Detection } \\
\text { Frequency }\end{array}$ & $\begin{array}{c}\text { Minimum } \\
\text { Detected } \\
\text { Concentration } \\
(\mu \mathrm{g} / \mathrm{L})\end{array}$ & $\begin{array}{c}\text { Maximum } \\
\text { Detected } \\
\text { Concentration } \\
(\mu \mathrm{g} / L)\end{array}$ & $\begin{array}{c}\text { Lowland } \\
\text { Stream } \\
\text { Background } \\
\text { Concentration }{ }^{\mathrm{a}} \\
(\mu \mathrm{g} / \mathrm{L})\end{array}$ & $\begin{array}{l}\text { Ecological } \\
\text { Screening } \\
\text { Value }(\mu g / L)\end{array}$ & $\begin{array}{c}\text { Detection } \\
\text { Frequency } \\
\text { Above ESV }\end{array}$ \\
\hline $\begin{array}{l}\text { Diesel-Range } \\
\text { Hydrocarbons }\end{array}$ & 4 of 5 & ND (50) & 121 & NAN & 15 & 4 of 5 \\
\hline \multicolumn{7}{|c|}{ Volatile Organic Compounds } \\
\hline 1,2,4-Trimethylbenzene & 0 of 5 & ND (1.0) & ND (1.0) & NAN & 33 & 0 of 5 \\
\hline 1,3,5-Trimethylbenzene & 0 of 5 & ND (1.0) & ND (1.0) & NAN & 33 & 0 of 5 \\
\hline 2-Butanone & 0 of 5 & ND (20) & ND (20) & NAN & 14,000 & 0 of 5 \\
\hline Acetone & 0 of 5 & ND (20) & ND (20) & NAN & 1,500 & 0 of 5 \\
\hline Benzene & 0 of 5 & ND (1.0) & ND (1.0) & NAN & 130 & 0 of 5 \\
\hline Carbon disulfide & 0 of 5 & ND (1.0) & ND (1.0) & NAN & 0.92 & 0 of 5 \\
\hline Ethylbenzene & 0 of 5 & ND (1.0) & ND (1.0) & NAN & 7.3 & 0 of 5 \\
\hline Isopropylbenzene & 0 of 5 & ND (1.0) & ND (1.0) & NAN & 2.6 & 0 of 5 \\
\hline $\mathrm{m}, \mathrm{p}$-Xylene & 0 of 5 & ND (2.0) & ND (2.0) & NAN & 13 & 0 of 5 \\
\hline Methylene chloride & 0 of 5 & ND (4.64) & ND (10.3) & NAN & 2,200 & 0 of 5 \\
\hline n-Butylbenzene & 0 of 5 & ND (1.0) & ND (1.0) & NAN & 164 & 0 of 5 \\
\hline n-Propylbenzene & 0 of 5 & ND (1.0) & ND (1.0) & NAN & 6.0 & 0 of 5 \\
\hline Naphthalene & 0 of 5 & ND (1.0) & ND (1.0) & NAN & 12 & 0 of 5 \\
\hline o-Xylene & 0 of 5 & ND (1.0) & ND (1.0) & NAN & 13 & 0 of 5 \\
\hline p-Isopropyltoluene & 0 of 5 & ND (1.0) & ND (1.0) & NAN & 26 & 0 of 5 \\
\hline sec-Butylbenzene & 0 of 5 & ND (1.0) & ND (1.0) & NAN & 164 & 0 of 5 \\
\hline Toluene & 0 of 5 & ND (1.0) & ND (1.0) & NAN & 9.8 & 0 of 5 \\
\hline \multicolumn{7}{|c|}{ Polycyclic Aromatic Hydrocarbons } \\
\hline Acenaphthene & 0 of 5 & ND $(0.1)$ & ND $(0.1)$ & NAN & 74 & 0 of 5 \\
\hline Acenaphthylene & 0 of 5 & ND $(0.1)$ & ND $(0.1)$ & NAN & 307 & 0 of 5 \\
\hline Anthracene & 0 of 5 & ND $(0.1)$ & ND $(0.1)$ & NAN & 0.73 & 0 of 5 \\
\hline Benzo(a)anthracene & 0 of 5 & ND $(0.1)$ & ND $(0.1)$ & NAN & 0.027 & 0 of 5 \\
\hline Benzo(a)pyrene & 0 of 5 & ND $(0.1)$ & ND $(0.1)$ & NAN & 0.014 & 0 of 5 \\
\hline Benzo(b)fluoranthene & 0 of 5 & ND $(0.1)$ & ND $(0.1)$ & NAN & 0.68 & 0 of 5 \\
\hline Benzo(g,h,l)perylene & 0 of 5 & ND $(0.1)$ & ND $(0.1)$ & NAN & 0.44 & 0 of 5 \\
\hline
\end{tabular}


Table 5-17

\section{Summary of Analytical Results and Ecological Risk Assessment Screening for Surface Water in Rainbow Creek, Long Shot Site}

(Page 2 of 3 )

\begin{tabular}{|c|c|c|c|c|c|c|}
\hline Analyte & $\begin{array}{l}\text { Detection } \\
\text { Frequency }\end{array}$ & $\begin{array}{c}\text { Minimum } \\
\text { Detected } \\
\text { Concentration } \\
(\mu \mathrm{g} / \mathrm{L})\end{array}$ & $\begin{array}{c}\text { Maximum } \\
\text { Detected } \\
\text { Concentration } \\
(\mu \mathrm{g} / \mathrm{L})\end{array}$ & $\begin{array}{c}\text { Lowland } \\
\text { Stream } \\
\text { Background } \\
\text { Concentration }{ }^{\mathrm{a}} \\
(\mu \mathrm{g} / \mathrm{L})\end{array}$ & $\begin{array}{l}\text { Ecological } \\
\text { Screening } \\
\text { Value }(\mu \mathrm{g} / \mathrm{L})\end{array}$ & $\begin{array}{c}\text { Detection } \\
\text { Frequency } \\
\text { Above ESV }\end{array}$ \\
\hline Benzo(k)fluoranthene & 0 of 5 & ND $(0.1)$ & ND $(0.1)$ & NAN & 0.64 & 0 of 5 \\
\hline Chrysene & 0 of 5 & ND $(0.1)$ & ND $(0.1)$ & NAN & 2.0 & 0 of 5 \\
\hline Dibenzo(a,h)anthracene & 0 of 5 & ND $(0.1)$ & $\mathrm{ND}(0.1)$ & NAN & 0.28 & 0 of 5 \\
\hline Fluoranthene & 0 of 5 & ND $(0.1)$ & ND $(0.1)$ & NAN & 15 & 0 of 5 \\
\hline Fluorene & 0 of 5 & ND $(0.1)$ & ND $(0.1)$ & NAN & 3.9 & 0 of 5 \\
\hline Indeno(1,2,3-c,d)pyrene & 0 of 5 & ND $(0.1)$ & ND $(0.1)$ & NAN & 0.28 & 0 of 5 \\
\hline Naphthalene & 0 of 5 & ND $(0.1)$ & $\mathrm{ND}(0.1)$ & NAN & 12 & 0 of 5 \\
\hline Phenanthrene & 0 of 5 & ND $(0.1)$ & ND $(0.1)$ & NAN & 200 & 0 of 5 \\
\hline Pyrene & 0 of 5 & ND $(0.1)$ & $\mathrm{ND}(0.1)$ & NAN & 10 & 0 of 5 \\
\hline \multicolumn{7}{|c|}{ Polychlorinated Biphenyls } \\
\hline Aroclor 1016 & NAV & NAV & NAV & NAN & NAV & - \\
\hline Aroclor 1221 & NAV & NAV & NAV & NAN & NAV & - \\
\hline Aroclor 1232 & NAV & NAV & NAV & NAN & NAV & - \\
\hline Aroclor 1242 & NAV & NAV & NAV & NAN & NAV & - \\
\hline Aroclor 1248 & NAV & NAV & NAV & NAN & NAV & - \\
\hline Aroclor 1254 & NAV & NAV & NAV & NAN & NAV & - \\
\hline Aroclor 1260 & NAV & NAV & NAV & NAN & NAV & - \\
\hline Aroclor 1262 & NAV & NAV & NAV & NAN & NAV & - \\
\hline Aroclor 1268 & NAV & NAV & NAV & NAN & NAV & - \\
\hline \multicolumn{7}{|c|}{ Metals } \\
\hline Aluminum & 0 of 5 & ND (50) & ND (50) & 269 & 87 & 0 of 5 \\
\hline Arsenic & 0 of 5 & ND (1.0) & ND (1.0) & ND (1) & 150 & 0 of 5 \\
\hline Barium & 0 of 5 & ND (10) & ND (10) & ND (10) & 4.0 & 0 of 5 \\
\hline Beryllium & 0 of 5 & ND (1.0) & ND (1.0) & ND (1) & 0.66 & 0 of 5 \\
\hline Boron & 0 of 5 & ND (50) & ND (50) & ND (50) & 1.6 & 0 of 5 \\
\hline Cadmium & 0 of 5 & ND (1.0) & ND (1.0) & ND (1) & 2.2 & 0 of 5 \\
\hline Calcium & 5 of 5 & 5,530 & 8,220 & $4,320^{b}$ & 116,000 & 0 of 5 \\
\hline Cerium & 0 of 5 & ND (1.0) & ND (1.0) & ND (1) & 0.5 & 0 of 5 \\
\hline
\end{tabular}


Table 5-17

\section{Summary of Analytical Results and Ecological Risk Assessment Screening for Surface Water in Rainbow Creek, Long Shot Site}

(Page 3 of 3 )

\begin{tabular}{|c|c|c|c|c|c|c|}
\hline Analyte & $\begin{array}{l}\text { Detection } \\
\text { Frequency }\end{array}$ & $\begin{array}{c}\text { Minimum } \\
\text { Detected } \\
\text { Concentration } \\
(\mu \mathrm{g} / \mathrm{L})\end{array}$ & $\begin{array}{c}\text { Maximum } \\
\text { Detected } \\
\text { Concentration } \\
(\mu \mathrm{g} / \mathrm{L})\end{array}$ & $\begin{array}{c}\text { Lowland } \\
\text { Stream } \\
\text { Background } \\
\text { Concentration }{ }^{\text {a }} \\
(\mu \mathrm{g} / \mathrm{L})\end{array}$ & $\begin{array}{l}\text { Ecological } \\
\text { Screening } \\
\text { Value }(\mu g / L)\end{array}$ & $\begin{array}{l}\text { Detection } \\
\text { Frequency } \\
\text { Above ESV }\end{array}$ \\
\hline Cesium & 0 of 5 & ND (1.0) & ND (1.0) & ND (1) & 30 & 0 of 5 \\
\hline Chromium & 0 of 5 & ND (1.0) & ND (1.0) & $2.25^{\mathrm{b}}$ & 74 & 0 of 5 \\
\hline Cobalt & 2 of 5 & ND (1.0) & 2.48 & 3.35 & 23 & 0 of 5 \\
\hline Copper & 3 of 5 & ND (1.0) & 17.7 & 10.8 & 9.0 & 1 of 5 \\
\hline Iron & 4 of 5 & ND (150) & 321 & 993 & 1,000 & 0 of 5 \\
\hline Lithium & 0 of 5 & ND (1.0) & ND (1.0) & $1.17^{\mathrm{b}}$ & 14 & 0 of 5 \\
\hline Magnesium & 5 of 5 & 4,160 & 5,350 & $4,690^{b}$ & 82,000 & 0 of 5 \\
\hline Manganese & 5 of 5 & 7.74 & 44.4 & 267 & 120 & 0 of 5 \\
\hline Molybdenum & 0 of 5 & ND (1.0) & ND (1.0) & ND (1) & 370 & 0 of 5 \\
\hline Nickel & 0 of 5 & ND (1.0) & ND (1.0) & ND (1) & 52 & 0 of 5 \\
\hline Potassium & 5 of 5 & 898 & 1,270 & 2,620 & 53,000 & 0 of 5 \\
\hline Selenium & 0 of 5 & ND (1.0) & ND (1.0) & ND (1) & 5.0 & 0 of 5 \\
\hline Strontium & 5 of 5 & 35 & 58.3 & 36.1 & 1,500 & 0 of 5 \\
\hline Thallium & 0 of 5 & ND (1.0) & ND (1.0) & $7.07^{\mathrm{b}}$ & 12 & 0 of 5 \\
\hline Thorium & 0 of 5 & ND (1.0) & ND (1.0) & ND (1) & NAV & 0 of 5 \\
\hline Titanium & 5 of 5 & 1.09 & 1.94 & 6.13 & 29 & 0 of 5 \\
\hline Uranium & 0 of 5 & ND (1.0) & ND (1.0) & ND (1) & 2.6 & 0 of 5 \\
\hline Vanadium & 1 of 5 & ND (1.0) & 2.12 & $4.43^{b}$ & 20 & 0 of 5 \\
\hline Zinc & 1 of 5 & ND (10) & 23.3 & $50.3^{b}$ & 120 & 0 of 5 \\
\hline
\end{tabular}

${ }^{\mathrm{a} B a c k g r o u n d}$ concentrations, which are presented only for metals, are upper tolerance limits (UTL) unless otherwise noted.

${ }^{b}$ This metal was not detected in a sufficient number of reference stream samples to allow calculation of a valid UTL. The concentration shown is the maximum detected concentration in the appropriate background samples.

ND - Analyte not detected at a concentration above the reporting limit, which is shown in parentheses.

NAV - Not available

NAN - Not analyzed

$\mu \mathrm{g} / \mathrm{L}$ - Micrograms per liter 


\section{Table 5-18}

\section{Summary of Analytical Results and Ecological Risk Assessment Screening for Sediment in Rainbow Creek, Long Shot Site}

(Page 1 of 3)

\begin{tabular}{|c|c|c|c|c|c|c|}
\hline Analyte & $\begin{array}{l}\text { Detection } \\
\text { Frequency }\end{array}$ & $\begin{array}{c}\text { Minimum } \\
\text { Detected } \\
\text { Concentration } \\
(\mathrm{mg} / \mathrm{kg})\end{array}$ & $\begin{array}{c}\text { Maximum } \\
\text { Detected } \\
\text { Concentration } \\
(\mathbf{m g} / \mathbf{k g})\end{array}$ & $\begin{array}{c}\text { Lowland } \\
\text { Stream } \\
\text { Background } \\
\text { Concentration }{ }^{\mathrm{a}} \\
(\mathrm{mg} / \mathrm{kg})\end{array}$ & $\begin{array}{c}\text { Ecological } \\
\text { Screening } \\
\text { Value } \\
(\mathrm{mg} / \mathrm{kg})\end{array}$ & $\begin{array}{c}\text { Detection } \\
\text { Frequency } \\
\text { Above ESV }\end{array}$ \\
\hline $\begin{array}{l}\text { Diesel-Range } \\
\text { Hydrocarbons }\end{array}$ & 4 of 5 & ND (19.9) & 2,110 & NAN & $640^{c}$ & 0 of 5 \\
\hline \multicolumn{7}{|c|}{ Volatile Organic Compounds } \\
\hline 1,2,4-Trimethylbenzene & NAV & NAV & NAV & NAN & NAV & - \\
\hline 1,3,5-Trimethylbenzene & NAV & NAV & NAV & NAN & NAV & - \\
\hline 2-Butanone & NAV & NAV & NAV & NAN & NAV & - \\
\hline Acetone & NAV & NAV & NAV & NAN & NAV & - \\
\hline Benzene & NAV & NAV & NAV & NAN & NAV & - \\
\hline Carbon disulfide & NAV & NAV & NAV & NAN & NAV & - \\
\hline Ethylbenzene & NAV & NAV & NAV & NAN & NAV & - \\
\hline Isopropylbenzene & NAV & NAV & NAV & NAN & NAV & - \\
\hline $\mathrm{m}, \mathrm{p}$-Xylene & NAV & NAV & NAV & NAN & NAV & - \\
\hline Methylene chloride & NAV & NAV & NAV & NAN & NAV & - \\
\hline n-Butylbenzene & NAV & NAV & NAV & NAN & NAV & - \\
\hline n-Propylbenzene & NAV & NAV & NAV & NAN & NAV & - \\
\hline Naphthalene & NAV & NAV & NAV & NAN & NAV & - \\
\hline o-Xylene & NAV & NAV & NAV & NAN & NAV & - \\
\hline p-Isopropyltoluene & NAV & NAV & NAV & NAN & NAV & - \\
\hline sec-Butylbenzene & NAV & NAV & NAV & NAN & NAV & - \\
\hline Toluene & NAV & NAV & NAV & NAN & NAV & - \\
\hline \multicolumn{7}{|c|}{ Polycyclic Aromatic Hydrocarbons } \\
\hline Acenaphthene & 0 of 5 & ND $(0.0164)$ & ND $(0.0454)$ & NAN & $4.91^{c}$ & 0 of 5 \\
\hline Acenaphthylene & 0 of 5 & ND $(0.0164)$ & ND $(0.0454)$ & NAN & $4.52^{\mathrm{C}}$ & 0 of 5 \\
\hline Anthracene & 0 of 5 & ND $(0.0164)$ & ND $(0.0454)$ & NAN & $5.94^{\mathrm{c}}$ & 0 of 5 \\
\hline Benzo(a)anthracene & 0 of 5 & ND (0.0164) & ND $(0.0454)$ & NAN & $8.41^{\mathrm{c}}$ & 0 of 5 \\
\hline Benzo(a)pyrene & 0 of 5 & ND (0.0164) & ND $(0.0454)$ & NAN & $9.65^{\mathrm{c}}$ & 0 of 5 \\
\hline Benzo(b)fluoranthene & 0 of 5 & ND $(0.0164)$ & ND $(0.0454)$ & NAN & $9.79^{C}$ & 0 of 5 \\
\hline Benzo(g,h,l)perylene & 0 of 5 & ND (0.0164) & ND $(0.0454)$ & NAN & $10.95^{\mathrm{C}}$ & 0 of 5 \\
\hline
\end{tabular}




\section{Table 5-18}

\section{Summary of Analytical Results and Ecological Risk Assessment Screening for Sediment in Rainbow Creek, Long Shot Site}

(Page 2 of 3 )

\begin{tabular}{|c|c|c|c|c|c|c|}
\hline Analyte & $\begin{array}{l}\text { Detection } \\
\text { Frequency }\end{array}$ & $\begin{array}{c}\text { Minimum } \\
\text { Detected } \\
\text { Concentration } \\
(\mathbf{m g} / \mathbf{k g})\end{array}$ & $\begin{array}{c}\text { Maximum } \\
\text { Detected } \\
\text { Concentration } \\
(\mathrm{mg} / \mathrm{kg})\end{array}$ & $\begin{array}{c}\text { Lowland } \\
\text { Stream } \\
\text { Background } \\
\text { Concentration }{ }^{a} \\
(\mathbf{m g} / \mathbf{k g})\end{array}$ & $\begin{array}{l}\text { Ecological } \\
\text { Screening } \\
\text { Value } \\
(\mathrm{mg} / \mathrm{kg})\end{array}$ & $\begin{array}{l}\text { Detection } \\
\text { Frequency } \\
\text { Above ESV }\end{array}$ \\
\hline Benzo(k)fluoranthene & 0 of 5 & ND (0.0164) & ND (0.0454) & NAN & $9.81^{c}$ & 0 of 5 \\
\hline Chrysene & 0 of 5 & ND (0.0164) & ND (0.0454) & NAN & $8.44^{\mathrm{C}}$ & 0 of 5 \\
\hline Dibenzo(a,h)anthracene & 0 of 5 & ND (0.0164) & ND (0.0454) & NAN & $11.23^{\mathrm{c}}$ & 0 of 5 \\
\hline Fluoranthene & 0 of 5 & ND $(0.0164)$ & ND $(0.0454)$ & NAN & $7.07^{\mathrm{C}}$ & 0 of 5 \\
\hline Fluorene & 0 of 5 & ND (0.0164) & ND (0.0454) & NAN & $5.38^{c}$ & 0 of 5 \\
\hline Indeno(1,2,3-c,d)pyrene & 0 of 5 & ND (0.0164) & ND (0.0454) & NAN & $11.15^{\mathrm{c}}$ & 0 of 5 \\
\hline Naphthalene & 0 of 5 & ND (0.0164) & ND (0.0454) & NAN & $3.85^{c}$ & 0 of 5 \\
\hline Phenanthrene & 0 of 5 & ND (0.0164) & ND (0.0454) & NAN & $5.96^{c}$ & 0 of 5 \\
\hline Pyrene & 0 of 5 & ND (0.0164) & ND (0.0454) & NAN & $6.97^{\mathrm{C}}$ & 0 of 5 \\
\hline \multicolumn{7}{|c|}{ Polychlorinated Biphenyls } \\
\hline Aroclor 1016 & NAV & NAV & NAV & NAN & NAV & - \\
\hline Aroclor 1221 & NAV & NAV & NAV & NAN & NAV & - \\
\hline Aroclor 1232 & NAV & NAV & NAV & NAN & NAV & - \\
\hline Aroclor 1242 & NAV & NAV & NAV & NAN & NAV & - \\
\hline Aroclor 1248 & NAV & NAV & NAV & NAN & NAV & - \\
\hline Aroclor 1254 & NAV & NAV & NAV & NAN & NAV & \\
\hline Aroclor 1260 & 1 of 2 & ND (0.056) & 0.46 & NAN & $0.07^{c}$ & 1 of 2 \\
\hline Aroclor 1262 & NAV & NAV & NAV & NAN & NAV & - \\
\hline Aroclor 1268 & NAV & NAV & NAV & NAN & NAV & - \\
\hline \multicolumn{7}{|c|}{ Metals } \\
\hline Aluminum & 5 of 5 & 3,990 & 35,400 & 45,400 & 58,000 & 0 of 5 \\
\hline Arsenic & 3 of 5 & ND (3.67) & 32.1 & 100 & 6 & 3 of 5 \\
\hline Barium & 4 of 5 & ND (68.3) & 266 & 469 & NAV & \\
\hline Beryllium & 0 of 5 & ND (2.48) & ND (6.87) & ND & NAV & \\
\hline Boron & 1 of 5 & ND (18.3) & 18.2 & $7.18^{b}$ & NAV & \\
\hline Cadmium & 0 of 5 & ND (2.48) & ND (6.87) & $0.608^{b}$ & 0.6 & 0 of 5 \\
\hline Calcium & 5 of 5 & 2,880 & 7,300 & 7,300 & NAV & \\
\hline Cerium & 4 of 5 & ND (6.83) & 13.1 & 14.2 & NAV & \\
\hline
\end{tabular}


Table 5-18

\section{Summary of Analytical Results and Ecological Risk Assessment Screening for Sediment in Rainbow Creek, Long Shot Site} (Page 3 of 3 )

\begin{tabular}{|c|c|c|c|c|c|c|}
\hline Analyte & $\begin{array}{l}\text { Detection } \\
\text { Frequency }\end{array}$ & $\begin{array}{c}\text { Minimum } \\
\text { Detected } \\
\text { Concentration } \\
(\mathrm{mg} / \mathrm{kg})\end{array}$ & $\begin{array}{c}\text { Maximum } \\
\text { Detected } \\
\text { Concentration } \\
(\mathbf{m g} / \mathbf{k g})\end{array}$ & $\begin{array}{c}\text { Lowland } \\
\text { Stream } \\
\text { Background } \\
\text { Concentration } \\
\text { (mg/kg) }\end{array}$ & $\begin{array}{c}\text { Ecological } \\
\text { Screening } \\
\text { Value } \\
(\mathrm{mg} / \mathrm{kg})\end{array}$ & $\begin{array}{c}\text { Detection } \\
\text { Frequency } \\
\text { Above ESV }\end{array}$ \\
\hline Cesium & 0 of 5 & ND (2.48) & ND (6.87) & ND & NAV & \\
\hline Chromium & 4 of 5 & ND (6.83) & 38.8 & 11.8 & 26 & 2 of 5 \\
\hline Cobalt & 3 of 5 & ND (6.83) & 28 & 43.7 & NAV & \\
\hline Copper & 5 of 5 & 13 & 61.4 & 71.1 & 16 & 4 of 5 \\
\hline Iron & 5 of 5 & 8,070 & 192,000 & 155,000 & 20,000 & 3 of 5 \\
\hline Lithium & 2 of 5 & ND (5.13) & 9.52 & 48.8 & NAV & \\
\hline Magnesium & 5 of 5 & 1,890 & 7,650 & 9,320 & NAV & \\
\hline Manganese & 5 of 5 & 196 & 14,700 & 20,700 & 460 & 4 of 5 \\
\hline Molybdenum & 0 of 5 & ND (2.48) & ND (6.87) & $5.5^{\mathrm{b}}$ & NAV & \\
\hline Nickel & 3 of 5 & 5.67 & 9.66 & 13.6 & 16 & 0 of 5 \\
\hline Potassium & 5 of 5 & 324 & 748 & 1,390 & NAV & \\
\hline Selenium & 0 of 5 & ND (2.48) & ND (6.87) & $5.44^{b}$ & NAV & \\
\hline Strontium & 5 of 5 & 59.5 & 94.1 & 164 & NAV & \\
\hline Thallium & 0 of 5 & ND (2.48) & ND (6.87) & $1^{\mathrm{b}}$ & NAV & \\
\hline Thorium & 0 of 5 & ND (2.48) & ND (6.87) & $0.5^{\mathrm{b}}$ & NAV & \\
\hline Titanium & 5 of 5 & 285 & 1,550 & 3,010 & NAV & \\
\hline Uranium & 0 of 5 & ND (2.48) & ND (6.87) & $4.13^{b}$ & NAV & \\
\hline Vanadium & 5 of 5 & 54.7 & 296 & 734 & NAV & \\
\hline Zinc & 2 of 5 & ND (36.7) & 142 & 434 & 120 & 1 of 5 \\
\hline Total Organic Carbon & 5 of 5 & 63,300 & 284,000 & NAN & & \\
\hline
\end{tabular}

${ }^{a}$ Background concentrations, which are presented only for metals, are upper tolerance limits (UTL) unless otherwise noted.

${ }^{b}$ This metal was not detected in a sufficient number of reference stream samples to allow calculation of a valid UTL. The concentration shown is the maximum detected concentration in the appropriate background samples.

${ }^{\mathrm{c}}$ Screening concentration is based on $1 \%$ organic carbon.

ND - Analyte not detected at a concentration above the reporting limit, which is shown in parentheses.

NAV - Not available

NAN - Not analyzed

$\mathrm{mg} / \mathrm{kg}$ - Milligrams per kilogram 
Table 5-19

Summary of Analytical Results and Ecological Risk Assessment Screening for Surface Water in Clevenger Creek, Milrow Site

(Page 1 of 3 )

\begin{tabular}{|c|c|c|c|c|c|c|}
\hline Analyte & $\begin{array}{l}\text { Detection } \\
\text { Frequency }\end{array}$ & $\begin{array}{c}\text { Minimum } \\
\text { Detected } \\
\text { Concentration } \\
(\mu \mathrm{g} / \mathrm{L})\end{array}$ & $\begin{array}{c}\text { Maximum } \\
\text { Detected } \\
\text { Concentration } \\
(\mu \mathrm{g} / \mathrm{L})\end{array}$ & $\begin{array}{c}\text { Lowland Stream } \\
\text { Background } \\
\text { Concentration }{ }^{\mathrm{a}} \\
(\mu \mathrm{g} / \mathrm{L})\end{array}$ & $\begin{array}{l}\text { Ecological } \\
\text { Screening } \\
\text { Value }(\mu \mathrm{g} / \mathrm{L})\end{array}$ & $\begin{array}{l}\text { Detection } \\
\text { Frequency } \\
\text { Above ESV }\end{array}$ \\
\hline $\begin{array}{l}\text { Diesel-Range } \\
\text { Hydrocarbons }\end{array}$ & 0 of 5 & ND (98.9) & ND (225) & NAN & 15 & 0 of 5 \\
\hline \multicolumn{7}{|c|}{ Volatile Organic Compounds } \\
\hline 1,2,4-Trimethylbenzene & 0 of 5 & ND (1) & ND (1) & NAN & 33 & 0 of 5 \\
\hline 1,3,5-Trimethylbenzene & 0 of 5 & ND (1) & ND (1) & NAN & 33 & 0 of 5 \\
\hline 2-Butanone & 0 of 5 & ND (20) & ND (20) & NAN & 14,000 & 0 of 5 \\
\hline Acetone & 0 of 5 & ND (20) & ND (20) & NAN & 1,500 & 0 of 5 \\
\hline Benzene & 0 of 5 & ND (1) & ND (1) & NAN & 130 & 0 of 5 \\
\hline Carbon disulfide & 0 of 5 & ND (1.0) & ND (1.0) & NAN & 0.92 & 0 of 5 \\
\hline Ethylbenzene & 0 of 5 & ND (1) & ND (1) & NAN & 7.3 & 0 of 5 \\
\hline Isopropylbenzene & 0 of 5 & ND (1) & ND (1) & NAN & 2.6 & 0 of 5 \\
\hline $\mathrm{m}, \mathrm{p}$-Xylene & 0 of 5 & ND (2) & ND (2) & NAN & 13 & 0 of 5 \\
\hline Methylene chloride & 0 of 5 & ND (2.0) & ND (4.51) & NAN & 2,200 & 0 of 5 \\
\hline n-Butylbenzene & 0 of 5 & ND (1) & ND (1) & NAN & 164 & 0 of 5 \\
\hline n-Propylbenzene & 0 of 5 & ND (1) & ND (1) & NAN & 6.0 & 0 of 5 \\
\hline Naphthalene & 0 of 5 & ND (1) & ND (1) & NAN & 12 & 0 of 5 \\
\hline o-Xylene & 0 of 5 & ND (1) & ND (1) & NAN & 13 & 0 of 5 \\
\hline p-Isopropyltoluene & 0 of 5 & ND (1) & ND (1) & NAN & 26 & 0 of 5 \\
\hline sec-Butylbenzene & 0 of 5 & ND (1) & ND (1) & NAN & 164 & 0 of 5 \\
\hline Toluene & 0 of 5 & ND (1) & ND (1) & NAN & 9.8 & 0 of 5 \\
\hline \multicolumn{7}{|c|}{ Polycyclic Aromatic Hydrocarbons } \\
\hline Acenaphthene & 0 of 5 & $\mathrm{ND}(0.1)$ & ND $(0.1)$ & NAN & 74 & 0 of 5 \\
\hline Acenaphthylene & 0 of 5 & ND $(0.1)$ & ND $(0.1)$ & NAN & 307 & 0 of 5 \\
\hline Anthracene & 0 of 5 & ND $(0.1)$ & ND $(0.1)$ & NAN & 0.73 & 0 of 5 \\
\hline Benzo(a)anthracene & 0 of 5 & $\mathrm{ND}(0.1)$ & ND $(0.1)$ & NAN & 0.027 & 0 of 5 \\
\hline Benzo(a)pyrene & 0 of 5 & ND $(0.1)$ & ND $(0.1)$ & NAN & 0.014 & 0 of 5 \\
\hline Benzo(b)fluoranthene & 0 of 5 & ND $(0.1)$ & ND $(0.1)$ & NAN & 0.68 & 0 of 5 \\
\hline Benzo(k)fluoranthene & 0 of 5 & $\mathrm{ND}(0.1)$ & ND $(0.1)$ & NAN & 0.64 & 0 of 5 \\
\hline Benzo(g,h,i)perylene & 0 of 5 & ND $(0.1)$ & ND $(0.1)$ & NAN & 0.44 & 0 of 5 \\
\hline
\end{tabular}


Table 5-19

Summary of Analytical Results and Ecological Risk Assessment Screening for Surface Water in Clevenger Creek, Milrow Site

(Page 2 of 3 )

\begin{tabular}{|c|c|c|c|c|c|c|}
\hline Analyte & $\begin{array}{l}\text { Detection } \\
\text { Frequency }\end{array}$ & $\begin{array}{c}\text { Minimum } \\
\text { Detected } \\
\text { Concentration } \\
(\mu \mathrm{g} / \mathrm{L})\end{array}$ & $\begin{array}{c}\text { Maximum } \\
\text { Detected } \\
\text { Concentration } \\
(\mu \mathrm{g} / \mathrm{L})\end{array}$ & $\begin{array}{c}\text { Lowland Stream } \\
\text { Background } \\
\text { Concentration }{ }^{\mathrm{a}} \\
(\mu \mathrm{g} / \mathrm{L})\end{array}$ & $\begin{array}{l}\text { Ecological } \\
\text { Screening } \\
\text { Value }(\mu g / L)\end{array}$ & $\begin{array}{l}\text { Detection } \\
\text { Frequency } \\
\text { Above ESV }\end{array}$ \\
\hline Chrysene & 0 of 5 & $\mathrm{ND}(0.1)$ & $\mathrm{ND}(0.1)$ & NAN & 2.0 & 0 of 5 \\
\hline Dibenzo(a,h)anthracene & 0 of 5 & $\mathrm{ND}(0.1)$ & ND $(0.1)$ & NAN & 0.28 & 0 of 5 \\
\hline Fluoranthene & 0 of 5 & $\mathrm{ND}(0.1)$ & $\mathrm{ND}(0.1)$ & NAN & 15 & 0 of 5 \\
\hline Fluorene & 0 of 5 & ND $(0.1)$ & ND $(0.1)$ & NAN & 3.9 & 0 of 5 \\
\hline Indeno(1,2,3-c,d)pyrene & 0 of 5 & $\mathrm{ND}(0.1)$ & $\mathrm{ND}(0.1)$ & NAN & 3.9 & 0 of 5 \\
\hline Naphthalene & 0 of 5 & $\mathrm{ND}(0.1)$ & $\mathrm{ND}(0.1)$ & NAN & 12 & 0 of 5 \\
\hline Phenanthrene & 0 of 5 & $\mathrm{ND}(0.1)$ & $\mathrm{ND}(0.1)$ & NAN & 200 & 0 of 5 \\
\hline Pyrene & 0 of 5 & ND $(0.1)$ & $\mathrm{ND}(0.1)$ & NAN & 10 & 0 of 5 \\
\hline \multicolumn{7}{|c|}{ Polychlorinated Biphenyls } \\
\hline Aroclor 1016 & NAV & NAV & NAV & NAN & NAV & - \\
\hline Aroclor 1221 & NAV & NAV & NAV & NAN & NAV & - \\
\hline Aroclor 1232 & NAV & NAV & NAV & NAN & NAV & - \\
\hline Aroclor 1242 & NAV & NAV & NAV & NAN & NAV & - \\
\hline Aroclor 1248 & NAV & NAV & NAV & NAN & NAV & - \\
\hline Aroclor 1254 & NAV & NAV & NAV & NAN & NAV & - \\
\hline Aroclor 1260 & NAV & NAV & NAV & NAN & NAV & - \\
\hline Aroclor 1262 & NAV & NAV & NAV & NAN & NAV & - \\
\hline Aroclor 1268 & NAV & NAV & NAV & NAN & NAV & - \\
\hline \multicolumn{7}{|c|}{ Metals } \\
\hline Aluminum & 5 of 5 & 75.8 & 625 & 269 & 87 & 3 of 5 \\
\hline Arsenic & 3 of 5 & ND (10) & 1.85 & ND (1) & 150 & 0 of 5 \\
\hline Barium & 0 of 5 & ND (10) & ND (10) & ND (10) & 4.0 & 0 of 5 \\
\hline Beryllium & 0 of 5 & ND (1.0) & ND (1.0) & ND (1) & 0.66 & 0 of 5 \\
\hline Boron & 2 of 5 & ND (50) & 88.7 & ND (50) & 1.6 & 2 of 5 \\
\hline Cadmium & 0 of 5 & ND (1.0) & ND (1.0) & ND (1) & 2.2 & 0 of 5 \\
\hline Calcium & 5 of 5 & 3,340 & 4,470 & $4,320^{b}$ & 116,000 & 0 of 5 \\
\hline Cerium & 0 of 5 & ND (1.0) & ND (1.0) & ND (1) & 0.5 & 0 of 5 \\
\hline Cesium & 0 of 5 & ND (1.0) & ND (1.0) & ND (1) & 30 & 0 of 5 \\
\hline Chromium & 1 of 5 & ND (1.0) & 1.25 & $2.25^{b}$ & 74 & 0 of 5 \\
\hline
\end{tabular}


Table 5-19

\section{Summary of Analytical Results and Ecological Risk Assessment Screening for Surface Water in Clevenger Creek, Milrow Site} (Page 3 of 3 )

\begin{tabular}{|c|c|c|c|c|c|c|}
\hline Analyte & $\begin{array}{l}\text { Detection } \\
\text { Frequency }\end{array}$ & $\begin{array}{c}\text { Minimum } \\
\text { Detected } \\
\text { Concentration } \\
(\mu \mathrm{g} / \mathrm{L})\end{array}$ & $\begin{array}{c}\text { Maximum } \\
\text { Detected } \\
\text { Concentration } \\
(\mu \mathrm{g} / \mathrm{L})\end{array}$ & $\begin{array}{c}\text { Lowland Stream } \\
\text { Background } \\
\text { Concentration }^{\mathrm{a}} \\
(\mu \mathrm{g} / \mathrm{L})\end{array}$ & $\begin{array}{l}\text { Ecological } \\
\text { Screening } \\
\text { Value }(\mu \mathrm{g} / \mathrm{L})\end{array}$ & $\begin{array}{l}\text { Detection } \\
\text { Frequency } \\
\text { Above ESV }\end{array}$ \\
\hline Cobalt & 3 of 5 & ND (1.0) & 4.32 & 3.35 & 23 & 0 of 5 \\
\hline Copper & 5 of 5 & 3.7 & 11.8 & 10.8 & 9.0 & 2 of 5 \\
\hline Iron & 5 of 5 & 336 & 1,070 & 993 & 1,000 & 1 of 5 \\
\hline Lithium & 1 of 5 & ND (1.0) & 1.14 & $1.17^{b}$ & 14 & 0 of 5 \\
\hline Magnesium & 5 of 5 & 2,110 & 3,250 & $4,690^{\mathrm{b}}$ & 82,000 & 0 of 5 \\
\hline Manganese & 5 of 5 & 12.6 & 27.2 & 267 & 120 & 0 of 5 \\
\hline Molybdenum & 0 of 5 & ND (1.0) & ND (1.0) & ND (1) & 370 & 0 of 5 \\
\hline Nickel & 2 of 5 & ND (1.0) & 1.97 & ND (1) & 52 & 0 of 5 \\
\hline Potassium & 5 of 5 & 1,570 & 2,730 & 2,620 & 53,000 & 0 of 5 \\
\hline Selenium & 0 of 5 & ND (1.0) & ND (1.0) & ND (1) & 5.0 & 0 of 5 \\
\hline Strontium & 5 of 5 & 10.5 & 18.9 & 36.1 & 1,500 & 0 of 5 \\
\hline Thallium & 0 of 5 & ND (1.0) & ND (1.0) & $7.07^{b}$ & 12 & 0 of 5 \\
\hline Thorium & 0 of 5 & ND (1.0) & ND (1.0) & ND (1) & NAV & \\
\hline Titanium & 5 of 5 & 2.79 & 123 & 6.13 & 29 & 3 of 5 \\
\hline Uranium & 0 of 5 & ND (1.0) & ND (1.0) & ND (1) & 2.6 & 0 of 5 \\
\hline Vanadium & 4 of 5 & ND (1.0) & 4.44 & $4.43^{b}$ & 20 & 0 of 5 \\
\hline Zinc & 4 of 5 & ND (10) & 44.9 & $50.3^{b}$ & 120 & 0 of 5 \\
\hline
\end{tabular}

${ }^{a}$ Background concentrations, which are presented only for metals, are upper tolerance limits (UTL) unless otherwise noted.

${ }^{b}$ This metal was not detected in a sufficient number of reference stream samples to allow calculation of a valid UTL. The concentration shown is the maximum detected concentration in the appropriate background samples.

ND - Analyte not detected at a concentration above the reporting limit, which is shown in parentheses.

NAV - Not available

NAN - Not analyzed

$\mu \mathrm{g} / \mathrm{L}$ - Micrograms per liter 
Table 5-20

\section{Summary of Analytical Results and Ecological Risk Assessment Screening for Sediment in Clevenger Creek, Milrow Site}

(Page 1 of 3 )

\begin{tabular}{|c|c|c|c|c|c|c|}
\hline Analyte & $\begin{array}{l}\text { Detection } \\
\text { Frequency }\end{array}$ & $\begin{array}{c}\text { Minimum } \\
\text { Detected } \\
\text { Concentration } \\
(\mathrm{mg} / \mathrm{kg})\end{array}$ & $\begin{array}{c}\text { Maximum } \\
\text { Detected } \\
\text { Concentration } \\
(\mathrm{mg} / \mathrm{kg})\end{array}$ & $\begin{array}{c}\text { Lowland } \\
\text { Stream } \\
\text { Background } \\
\text { Concentration }{ }^{\mathrm{a}} \\
(\mathrm{mg} / \mathbf{k g})\end{array}$ & $\begin{array}{c}\text { Ecological } \\
\text { Screening } \\
\text { Value } \\
(\mathrm{mg} / \mathrm{kg})\end{array}$ & $\begin{array}{c}\text { Detection } \\
\text { Frequency } \\
\text { Above ESV }\end{array}$ \\
\hline $\begin{array}{l}\text { Diesel-Range } \\
\text { Hydrocarbons }\end{array}$ & 4 of 6 & 29.6 & 542 & NAN & $640^{c}$ & 0 of 6 \\
\hline \multicolumn{7}{|c|}{ Volatile Organic Compounds } \\
\hline 1,2,4-Trimethylbenzene & 0 of 2 & ND $(0.0085)$ & ND (0.045) & NAN & $1.2^{\mathrm{C}}$ & 0 of 2 \\
\hline 1,3,5-Trimethylbenzene & 0 of 2 & ND (0.0085) & ND $(0.045)$ & NAN & $0.76^{c}$ & 0 of 2 \\
\hline 2-Butanone & 0 of 2 & ND $(0.017)$ & ND (0.09) & NAN & 14 & 0 of 2 \\
\hline Acetone & 0 of 2 & ND (1.64) & ND $(0.4)$ & NAN & 1.5 & 0 of 2 \\
\hline Benzene & 0 of 2 & ND $(0.0085)$ & ND (0.045) & NAN & $0.34^{c}$ & 0 of 2 \\
\hline Carbon disulfide & 0 of 2 & ND $(0.0901)$ & ND $(0.318)$ & NAN & 0.001 & 0 of 2 \\
\hline Ethylbenzene & 0 of 2 & ND $(0.0085)$ & ND $(0.045)$ & NAN & $1.4^{\mathrm{C}}$ & 0 of 2 \\
\hline Isopropylbenzene & 0 of 2 & ND (0.0085) & ND (0.045) & NAN & $0.1^{\mathrm{C}}$ & 0 of 2 \\
\hline m,p-Xylene & 0 of 2 & ND (0.0085) & ND (0.045) & NAN & $0.12^{\mathrm{C}}$ & 0 of 2 \\
\hline Methylene chloride & 0 of 2 & ND (0.0085) & ND (0.045) & NAN & $0.5^{\mathrm{c}}$ & 0 of 2 \\
\hline n-Butylbenzene & 0 of 2 & ND (0.0085) & ND (0.045) & NAN & $22^{c}$ & 0 of 2 \\
\hline n-Propylbenzene & 0 of 2 & ND (0.0085) & ND (0.045) & NAN & $0.25^{\mathrm{c}}$ & 0 of 2 \\
\hline Naphthalene & 0 of 2 & ND (0.0085) & ND (0.045) & NAN & $3.85^{c}$ & 0 of 2 \\
\hline o-Xylene & 0 of 2 & ND (0.0085) & ND (0.045) & NAN & $0.12^{c}$ & 0 of 2 \\
\hline p-Isopropyltoluene & 0 of 2 & ND (0.0085) & ND (0.045) & NAN & $2.8^{c}$ & 0 of 2 \\
\hline sec-Butylbenzene & 0 of 2 & ND (0.0085) & ND (0.045) & NAN & $24^{\mathrm{C}}$ & 0 of 2 \\
\hline Toluene & 0 of 2 & ND (0.0085) & ND (0.045) & NAN & $2.5^{\mathrm{c}}$ & 0 of 2 \\
\hline \multicolumn{7}{|c|}{ Polycyclic Aromatic Hydrocarbons } \\
\hline Acenaphthene & 0 of 6 & ND $(0.00702)$ & ND (0.0232) & NAN & $4.91^{\mathrm{C}}$ & 0 of 6 \\
\hline Acenaphthylene & 0 of 6 & ND (0.00702) & ND (0.0232) & NAN & $4.52^{c}$ & 0 of 6 \\
\hline Anthracene & 0 of 6 & ND $(0.00702)$ & ND (0.0232) & NAN & $5.94^{\mathrm{C}}$ & 0 of 6 \\
\hline Benzo(a)anthracene & 0 of 6 & ND (0.00702) & ND (0.0232) & NAN & $8.41^{c}$ & 0 of 6 \\
\hline Benzo(a)pyrene & 0 of 6 & ND $(0.00702)$ & ND (0.0232) & NAN & $9.65^{\mathrm{C}}$ & 0 of 6 \\
\hline Benzo(b)fluoranthene & 0 of 6 & ND (0.00702) & ND (0.0232) & NAN & $9.79^{\mathrm{C}}$ & 0 of 6 \\
\hline Benzo(k)fluoranthene & 0 of 6 & ND (0.00702) & ND (0.0232) & NAN & $9.81^{\mathrm{c}}$ & 0 of 6 \\
\hline
\end{tabular}


Table 5-20

\section{Summary of Analytical Results and Ecological Risk Assessment Screening for Sediment in Clevenger Creek, Milrow Site}

(Page 2 of 3 )

\begin{tabular}{|c|c|c|c|c|c|c|}
\hline Analyte & $\begin{array}{l}\text { Detection } \\
\text { Frequency }\end{array}$ & $\begin{array}{c}\text { Minimum } \\
\text { Detected } \\
\text { Concentration } \\
(\mathbf{m g} / \mathbf{k g})\end{array}$ & $\begin{array}{c}\text { Maximum } \\
\text { Detected } \\
\text { Concentration } \\
(\mathbf{m g} / \mathbf{k g})\end{array}$ & $\begin{array}{c}\text { Lowland } \\
\text { Stream } \\
\text { Background } \\
\text { Concentration }{ }^{\mathrm{a}} \\
(\mathbf{m g} / \mathbf{k g})\end{array}$ & $\begin{array}{c}\text { Ecological } \\
\text { Screening } \\
\text { Value } \\
(\mathrm{mg} / \mathrm{kg})\end{array}$ & $\begin{array}{c}\text { Detection } \\
\text { Frequency } \\
\text { Above ESV }\end{array}$ \\
\hline Benzo(g,h,i)perylene & 0 of 6 & ND (0.00702) & ND $(0.0232)$ & NAN & $10.95^{\mathrm{C}}$ & 0 of 6 \\
\hline Chrysene & 0 of 6 & ND (0.00702) & ND (0.0232) & NAN & $8.44^{c}$ & 0 of 6 \\
\hline Dibenzo(a,h)anthracene & 0 of 6 & ND (0.00702) & ND (0.0232) & NAN & $11.23^{\mathrm{C}}$ & 0 of 6 \\
\hline Fluoranthene & 0 of 6 & ND (0.00702) & ND (0.0232) & NAN & $7.07^{\mathrm{C}}$ & 0 of 6 \\
\hline Fluorene & 0 of 6 & ND (0.00702) & ND (0.0232) & NAN & $5.38^{\mathrm{C}}$ & 0 of 6 \\
\hline Indeno(1,2,3-c,d)pyrene & 0 of 6 & ND $(0.00702)$ & ND (0.0232) & NAN & $11.15^{\mathrm{C}}$ & 0 of 6 \\
\hline Naphthalene & 0 of 6 & ND (0.00702) & ND (0.0232) & NAN & $3.85^{c}$ & 0 of 6 \\
\hline Phenanthrene & 0 of 6 & ND $(0.00702)$ & ND $(0.0232)$ & NAN & $5.96^{c}$ & 0 of 6 \\
\hline Pyrene & 0 of 6 & ND (0.00702) & ND (0.0232) & NAN & $6.97^{\mathrm{C}}$ & 0 of 6 \\
\hline \multicolumn{7}{|c|}{ Polychlorinated Biphenyls } \\
\hline Aroclor 1016 & NAV & NAV & NAV & NAN & NAV & - \\
\hline Aroclor 1221 & NAV & NAV & NAV & NAN & NAV & - \\
\hline Aroclor 1232 & NAV & NAV & NAV & NAN & NAV & - \\
\hline Aroclor 1242 & NAV & NAV & NAV & NAN & NAV & - \\
\hline Aroclor 1248 & NAV & NAV & NAV & NAN & NAV & - \\
\hline Aroclor 1254 & NAV & NAV & NAV & NAN & NAV & - \\
\hline Aroclor 1260 & NAV & NAV & NAV & NAN & NAV & - \\
\hline Aroclor 1262 & NAV & NAV & NAV & NAN & NAV & - \\
\hline Aroclor 1268 & NAV & NAV & NAV & NAN & NAV & - \\
\hline \multicolumn{7}{|c|}{ Metals } \\
\hline Aluminum & 6 of 6 & 16,000 & 90,600 & 45,400 & 58,000 & 1 of 6 \\
\hline Arsenic & 6 of 6 & 6.05 & 48.9 & 100 & 6.0 & 6 of 6 \\
\hline Barium & 6 of 6 & 104 & 355 & 469 & NAV & \\
\hline Beryllium & 0 of 6 & ND (1.06) & ND (4.02) & ND & NAV & \\
\hline Boron & 4 of 4 & 76.8 & 198 & $7.18^{b}$ & NAV & \\
\hline Cadmium & 0 of 6 & ND (1.06) & ND (4.02) & $0.608^{b}$ & 0.6 & 0 of 6 \\
\hline Calcium & 6 of 6 & 2,450 & 20,200 & 7,300 & NAV & \\
\hline Cerium & 6 of 6 & 7.58 & 30.9 & 14.2 & NAV & \\
\hline
\end{tabular}


Table 5-20

\section{Summary of Analytical Results and Ecological Risk Assessment Screening for Sediment in Clevenger Creek, Milrow Site}

(Page 3 of 3 )

\begin{tabular}{|c|c|c|c|c|c|c|}
\hline Analyte & $\begin{array}{l}\text { Detection } \\
\text { Frequency }\end{array}$ & $\begin{array}{c}\text { Minimum } \\
\text { Detected } \\
\text { Concentration } \\
(\mathbf{m g} / \mathbf{k g})\end{array}$ & $\begin{array}{c}\text { Maximum } \\
\text { Detected } \\
\text { Concentration } \\
(\mathrm{mg} / \mathrm{kg})\end{array}$ & $\begin{array}{c}\text { Lowland } \\
\text { Stream } \\
\text { Background } \\
\text { Concentration } \\
\text { (mg/kg) }\end{array}$ & $\begin{array}{c}\text { Ecological } \\
\text { Screening } \\
\text { Value } \\
(\mathrm{mg} / \mathrm{kg})\end{array}$ & $\begin{array}{c}\text { Detection } \\
\text { Frequency } \\
\text { Above ESV }\end{array}$ \\
\hline Cesium & 2 of 6 & 1.8 & 4.26 & ND & NAV & \\
\hline Chromium & 6 of 6 & 9.8 & 24.7 & 11.8 & 26 & 0 of 6 \\
\hline Cobalt & 6 of 6 & 13.5 & 38.3 & 43.7 & NAV & \\
\hline Copper & 6 of 6 & 40.6 & 247 & 71.1 & 16 & 6 of 6 \\
\hline Iron & 6 of 6 & 36,000 & 108,000 & 155,000 & 20,000 & 6 of 6 \\
\hline Lithium & 6 of 6 & 5.84 & 41.7 & 48.8 & NAV & \\
\hline Magnesium & 6 of 6 & 5,280 & 37,500 & 9,320 & NAV & \\
\hline Manganese & 6 of 6 & 297 & 9,020 & 20,700 & 460 & 5 of 6 \\
\hline Molybdenum & 0 of 6 & $\mathrm{ND}(1.06)$ & ND (4.02) & $5.5^{b}$ & NAV & \\
\hline Nickel & 6 of 6 & 5.47 & 18.9 & 13.6 & 16 & 1 of 6 \\
\hline Potassium & 6 of 6 & 359 & 3,560 & 1,390 & NAV & \\
\hline Selenium & 0 of 6 & ND (1.06) & ND (4.02) & $5.44^{b}$ & NAV & \\
\hline Strontium & 6 of 6 & 44.4 & 609 & 164 & NAV & \\
\hline Thallium & 0 of 6 & ND (1.06) & ND (4.02) & $1.0^{\mathrm{b}}$ & NAV & \\
\hline Thorium & 0 of 6 & $\mathrm{ND}(1.06)$ & ND (4.02) & $0.5^{\mathrm{b}}$ & NAV & \\
\hline Titanium & 6 of 6 & 552 & 5,760 & 3,010 & NAV & \\
\hline Uranium & 0 of 6 & ND (1.06) & ND (4.02) & $4.13^{b}$ & NAV & \\
\hline Vanadium & 6 of 6 & 151 & 420 & 734 & NAV & \\
\hline Zinc & 6 of 6 & 90.5 & 208 & 434 & 120 & 3 of 6 \\
\hline Total Organic Carbon & 6 of 6 & 44,900 & 178,000 & NAN & & \\
\hline
\end{tabular}

${ }^{a}$ Background concentrations, which are presented only for metals, are upper tolerance limits (UTL) unless otherwise noted.

${ }^{b}$ This metal was not detected in a sufficient number of reference stream samples to allow calculation of a valid UTL. The concentration shown is the maximum detected concentration in the appropriate background samples.

${ }^{\mathrm{c}}$ Screening concentration is based on $1 \%$ organic carbon.

ND - Analyte not detected at a concentration above the reporting limit, which is shown in parentheses.

NAV - Not available

NAN - Not analyzed

$\mathrm{mg} / \mathrm{kg}$ - Milligrams per kilogram 
Table 5-21

\section{Summary of Analytical Results and Ecological Risk Assessment Screening for Surface Water in Heart Lake, Milrow Site}

(Page 1 of 3 )

\begin{tabular}{|c|c|c|c|c|c|c|}
\hline Analyte & $\begin{array}{l}\text { Detection } \\
\text { Frequency }\end{array}$ & $\begin{array}{c}\text { Minimum } \\
\text { Detected } \\
\text { Concentration } \\
(\mu \mathrm{g} / \mathrm{L})\end{array}$ & $\begin{array}{c}\text { Maximum } \\
\text { Detected } \\
\text { Concentration } \\
(\mu \mathrm{g} / \mathrm{L})\end{array}$ & $\begin{array}{c}\text { Lowland } \\
\text { Stream } \\
\text { Background } \\
\text { Concentration } \\
(\mu \mathrm{g} / \mathrm{L})\end{array}$ & $\begin{array}{l}\text { Ecological } \\
\text { Screening } \\
\text { Value }(\mu g / L)\end{array}$ & $\begin{array}{l}\text { Detection } \\
\text { Frequency } \\
\text { Above ESV }\end{array}$ \\
\hline $\begin{array}{l}\text { Diesel-Range } \\
\text { Hydrocarbons }\end{array}$ & 1 of 3 & ND (220) & 56.8 & NAN & 15 & 1 of 3 \\
\hline \multicolumn{7}{|c|}{ Volatile Organic Compounds } \\
\hline 1,2,4-Trimethylbenzene & 0 of 3 & ND (1) & ND (1) & NAN & 33 & 0 of 3 \\
\hline 1,3,5-Trimethylbenzene & 0 of 3 & ND (1) & ND (1) & NAN & 33 & 0 of 3 \\
\hline 2-Butanone & 0 of 3 & ND (20) & ND (20) & NAN & 14,000 & 0 of 3 \\
\hline Acetone & 0 of 3 & ND (20) & ND (20) & NAN & 1,500 & 0 of 3 \\
\hline Benzene & 0 of 3 & ND (1) & ND (1) & NAN & 130 & 0 of 3 \\
\hline Carbon disulfide & 0 of 3 & ND (1.0) & ND (1.0) & NAN & 0.92 & 0 of 3 \\
\hline Ethylbenzene & 0 of 3 & ND (1) & ND (1) & NAN & 7.3 & 0 of 3 \\
\hline Isopropylbenzene & 0 of 3 & ND (1) & ND (1) & NAN & 2.6 & 0 of 3 \\
\hline m,p-Xylene & 0 of 3 & ND (2) & ND (2) & NAN & 13 & 0 of 3 \\
\hline Methylene chloride & 0 of 3 & ND (2.0) & ND (5.48) & NAN & 2,200 & 0 of 3 \\
\hline n-Butylbenzene & 0 of 3 & ND (2) & ND (2) & NAN & 164 & 0 of 3 \\
\hline n-Propylbenzene & 0 of 3 & ND (1) & ND (1) & NAN & 6.0 & 0 of 3 \\
\hline Naphthalene & 0 of 3 & ND (1) & ND (1) & NAN & 12 & 0 of 3 \\
\hline o-Xylene & 0 of 3 & ND (1) & ND (1) & NAN & 13 & 0 of 3 \\
\hline p-isopropyltoluene & 0 of 3 & ND (1) & ND (1) & NAN & 26 & 0 of 3 \\
\hline sec-Butylbenzene & 0 of 3 & ND (1) & ND (1) & NAN & 164 & 0 of 3 \\
\hline Toluene & 0 of 3 & ND (1) & ND (1) & NAN & 938 & 0 of 3 \\
\hline \multicolumn{7}{|c|}{ Polycyclic Aromatic Hydrocarbons } \\
\hline Acenaphthene & 0 of 3 & ND $(0.1)$ & ND $(0.1)$ & NAN & 74 & 0 of 3 \\
\hline Acenaphthylene & 0 of 3 & ND $(0.1)$ & ND $(0.1)$ & NAN & 307 & 0 of 3 \\
\hline Anthracene & 0 of 3 & ND $(0.1)$ & ND $(0.1)$ & NAN & 0.73 & 0 of 3 \\
\hline Benzo(a)anthracene & 0 of 3 & ND $(0.1)$ & ND $(0.1)$ & NAN & 0.027 & 0 of 3 \\
\hline Benzo(a)pyrene & 0 of 3 & ND $(0.1)$ & $\mathrm{ND}(0.1)$ & NAN & 0.014 & 0 of 3 \\
\hline Benzo(b)fluoranthene & 0 of 3 & ND $(0.1)$ & $\mathrm{ND}(0.1)$ & NAN & 0.68 & 0 of 3 \\
\hline Benzo(g,h,i)perylene & 0 of 3 & ND $(0.1)$ & ND $(0.1)$ & NAN & 0.44 & 0 of 3 \\
\hline
\end{tabular}


Table 5-21

\section{Summary of Analytical Results and Ecological Risk Assessment Screening for Surface Water in Heart Lake, Milrow Site}

(Page 2 of 3 )

\begin{tabular}{|c|c|c|c|c|c|c|}
\hline Analyte & $\begin{array}{l}\text { Detection } \\
\text { Frequency }\end{array}$ & $\begin{array}{c}\text { Minimum } \\
\text { Detected } \\
\text { Concentration } \\
(\mu \mathrm{g} / \mathrm{L})\end{array}$ & $\begin{array}{c}\text { Maximum } \\
\text { Detected } \\
\text { Concentration } \\
(\mu \mathrm{g} / \mathrm{L})\end{array}$ & $\begin{array}{c}\text { Lowland } \\
\text { Stream } \\
\text { Background } \\
\text { Concentration } \\
(\mu \mathrm{g} / \mathrm{L})\end{array}$ & $\begin{array}{l}\text { Ecological } \\
\text { Screening } \\
\text { Value }(\mu g / L)\end{array}$ & $\begin{array}{l}\text { Detection } \\
\text { Frequency } \\
\text { Above ESV }\end{array}$ \\
\hline Benzo(k)fluoranthene & 0 of 3 & ND $(0.1)$ & ND $(0.1)$ & NAN & 0.64 & 0 of 3 \\
\hline Chrysene & 0 of 3 & ND $(0.1)$ & ND $(0.1)$ & NAN & 2.0 & 0 of 3 \\
\hline Dibenzo(a,h)anthracene & 0 of 3 & ND $(0.1)$ & ND $(0.1)$ & NAN & 0.28 & 0 of 3 \\
\hline Fluoranthene & 0 of 3 & ND $(0.1)$ & ND $(0.1)$ & NAN & 15 & 0 of 3 \\
\hline Fluorene & 0 of 3 & $\mathrm{ND}(0.1)$ & ND $(0.1)$ & NAN & 3.9 & 0 of 3 \\
\hline Indeno(1,2,3-c,d)pyrene & 0 of 3 & $\mathrm{ND}(0.1)$ & ND $(0.1)$ & NAN & 0.28 & 0 of 3 \\
\hline Naphthalene & 0 of 3 & $\mathrm{ND}(0.1)$ & $\mathrm{ND}(0.1)$ & NAN & 12 & 0 of 3 \\
\hline Phenanthrene & 0 of 3 & ND $(0.1)$ & ND $(0.1)$ & NAN & 200 & 0 of 3 \\
\hline Pyrene & 0 of 3 & ND $(0.1)$ & ND $(0.1)$ & NAN & 10 & 0 of 3 \\
\hline \multicolumn{7}{|c|}{ Polychlorinated Biphenyls } \\
\hline Aroclor 1016 & NAV & NAV & NAV & NAN & NAV & - \\
\hline Aroclor 1221 & NAV & NAV & NAV & NAN & NAV & - \\
\hline Aroclor 1232 & NAV & NAV & NAV & NAN & NAV & - \\
\hline Aroclor 1242 & NAV & NAV & NAV & NAN & NAV & - \\
\hline Aroclor 1248 & NAV & NAV & NAV & NAN & NAV & - \\
\hline Aroclor 1254 & NAV & NAV & NAV & NAN & NAV & - \\
\hline Aroclor 1260 & NAV & NAV & NAV & NAN & NAV & - \\
\hline Aroclor 1262 & NAV & NAV & NAV & NAN & NAV & - \\
\hline Aroclor 1268 & NAV & NAV & NAV & NAN & NAV & - \\
\hline \multicolumn{7}{|c|}{ Metals } \\
\hline Aluminum & 3 of 3 & 231 & 261 & 269 & 87 & 3 of 3 \\
\hline Arsenic & 0 of 3 & ND (1.0) & ND (1.0) & ND (1) & 150 & 0 of 3 \\
\hline Barium & 0 of 3 & ND (10) & ND (10) & ND (10) & 4.0 & 0 of 3 \\
\hline Beryllium & 0 of 3 & ND (1) & ND (1) & ND (1) & 0.66 & 0 of 3 \\
\hline Boron & 0 of 3 & ND (50) & ND (50) & ND (50) & 1.6 & 0 of 3 \\
\hline Cadmium & 0 of 3 & ND (1) & ND (1) & ND (1) & 2.2 & 0 of 3 \\
\hline Calcium & 3 of 3 & 1,390 & 1,490 & $4,320^{b}$ & 116,000 & 0 of 3 \\
\hline Cerium & 0 of 3 & ND (1.0) & ND (1.0) & ND (1) & 0.5 & 0 of 3 \\
\hline
\end{tabular}


Table 5-21

\section{Summary of Analytical Results and Ecological Risk Assessment Screening for Surface Water in Heart Lake, Milrow Site}

(Page 3 of 3 )

\begin{tabular}{|c|c|c|c|c|c|c|}
\hline Analyte & $\begin{array}{l}\text { Detection } \\
\text { Frequency }\end{array}$ & $\begin{array}{c}\text { Minimum } \\
\text { Detected } \\
\text { Concentration } \\
(\mu \mathrm{g} / \mathrm{L})\end{array}$ & $\begin{array}{c}\text { Maximum } \\
\text { Detected } \\
\text { Concentration } \\
(\mu \mathrm{g} / \mathrm{L})\end{array}$ & $\begin{array}{c}\text { Lowland } \\
\text { Stream } \\
\text { Background } \\
\text { Concentration } \\
(\mu \mathrm{g} / \mathrm{L})\end{array}$ & $\begin{array}{l}\text { Ecological } \\
\text { Screening } \\
\text { Value }(\mu \mathrm{g} / \mathrm{L})\end{array}$ & $\begin{array}{c}\text { Detection } \\
\text { Frequency } \\
\text { Above ESV }\end{array}$ \\
\hline Cesium & 0 of 3 & ND (1.0) & ND (1.0) & ND (1) & 30 & 0 of 3 \\
\hline Chromium & 0 of 3 & ND (1.0) & ND (1.0) & $2.25^{b}$ & 74 & 0 of 3 \\
\hline Cobalt & 2 of 3 & ND (1.0) & 1.82 & 3.35 & 23 & 0 of 3 \\
\hline Copper & 3 of 3 & 3.23 & 4.47 & 10.8 & 9.0 & 0 of 3 \\
\hline Iron & 3 of 3 & 232 & 243 & 993 & 1,000 & 0 of 3 \\
\hline Lithium & 0 of 3 & ND (1.0) & ND (1.0) & $1.17^{\mathrm{b}}$ & 14 & 0 of 3 \\
\hline Magnesium & 3 of 3 & 1,460 & 1,480 & $4,690^{b}$ & 82,000 & 0 of 3 \\
\hline Manganese & 3 of 3 & 6.54 & 6.98 & 267 & 120 & 0 of 3 \\
\hline Molybdenum & 3 of 3 & ND (1) & ND (1) & ND (1) & 370 & 0 of 3 \\
\hline Nickel & 3 of 3 & 1.41 & 1.81 & ND (1) & 52 & 0 of 3 \\
\hline Potassium & 3 of 3 & 1,110 & 1,210 & 2,620 & 53,000 & 0 of 3 \\
\hline Selenium & 0 of 3 & ND (1.0) & ND (1.0) & ND (1) & 5.0 & 0 of 3 \\
\hline Strontium & 3 of 3 & 11.6 & 12.4 & 36.1 & 1,500 & 0 of 3 \\
\hline Thallium & 0 of 3 & ND (1) & ND (1) & ND (1) & 12 & 0 of 3 \\
\hline Thorium & 0 of 3 & ND (1.0) & ND (1.0) & ND (1) & NAV & \\
\hline Titanium & 3 of 3 & 7.94 & 8.21 & 6.13 & 29 & 0 of 3 \\
\hline Uranium & 0 of 3 & ND (1) & ND (1) & ND (1) & 2.6 & 0 of 3 \\
\hline Vanadium & 0 of 3 & ND (1.0) & ND (1.0) & $4.43^{b}$ & 20 & 0 of 3 \\
\hline Zinc & 0 of 3 & ND (10) & ND (10) & $50.3^{b}$ & 120 & 0 of 3 \\
\hline
\end{tabular}

${ }^{\mathrm{a} B a c k g r o u n d}$ concentrations, which are presented only for metals, are upper tolerance limits (UTL) unless otherwise noted.

${ }^{b}$ This metal was not detected in a sufficient number of reference stream samples to allow calculation of a valid UTL. The concentration shown is the maximum detected concentration in the appropriate background samples.

ND - Analyte not detected at a concentration above the reporting limit, which is shown in parentheses.

NAV - Not available

NAN - Not analyzed

$\mu \mathrm{g} / \mathrm{L}$ - Micrograms per liter 
Table 5-22

\section{Summary of Analytical Results and Ecological Risk Assessment Screening for Sediment in Heart Lake, Milrow Site}

(Page 1 of 3 )

\begin{tabular}{|c|c|c|c|c|c|c|}
\hline Analyte & $\begin{array}{l}\text { Detection } \\
\text { Frequency }\end{array}$ & $\begin{array}{c}\text { Minimum } \\
\text { Detected } \\
\text { Concentration } \\
(\mathrm{mg} / \mathrm{kg})\end{array}$ & $\begin{array}{c}\text { Maximum } \\
\text { Detected } \\
\text { Concentration } \\
(\mathrm{mg} / \mathrm{kg})\end{array}$ & $\begin{array}{c}\text { Lowland } \\
\text { Stream } \\
\text { Background } \\
\text { Concentration } \\
\text { (mg/kg) }\end{array}$ & $\begin{array}{c}\text { Ecological } \\
\text { Screening } \\
\text { Value } \\
(\mathbf{m g} / \mathbf{k g})\end{array}$ & $\begin{array}{c}\text { Detection } \\
\text { Frequency } \\
\text { Above ESV }\end{array}$ \\
\hline $\begin{array}{l}\text { Diesel-Range } \\
\text { Hydrocarbons }\end{array}$ & 0 of 5 & ND (4.0) & ND (32.7) & NAN & $640^{\mathrm{C}}$ & 0 of 5 \\
\hline \multicolumn{7}{|c|}{ Volatile Organic Compounds } \\
\hline 1,2,4-Trimethylbenzene & 0 of 5 & ND (0.005) & ND (0.0409) & NAN & $1.2^{\mathrm{C}}$ & 0 of 5 \\
\hline 1,3,5-Trimethylbenzene & 0 of 5 & ND $(0.005)$ & ND $(0.0409)$ & NAN & $0.76^{\mathrm{C}}$ & 0 of 5 \\
\hline 2-Butanone & 3 of 5 & ND (0.01) & 0.127 & NAN & 14 & 0 of 5 \\
\hline Acetone & 0 of 5 & ND (0.07) & ND (2.24) & NAN & 1.5 & 0 of 5 \\
\hline Benzene & 0 of 5 & ND (0.005) & ND (0.0409) & NAN & $0.34^{c}$ & 0 of 5 \\
\hline Carbon disulfide & 0 of 5 & ND (0.005) & ND (0.0409) & NAN & 0.001 & 0 of 5 \\
\hline Ethylbenzene & 0 of 5 & ND $(0.005)$ & ND $(0.0409)$ & NAN & $1.4^{\mathrm{C}}$ & 0 of 5 \\
\hline Isopropylbenzene & 0 of 5 & ND $(0.005)$ & ND (0.0409) & NAN & $0.1^{\mathrm{C}}$ & 0 of 5 \\
\hline m,p-Xylene & 0 of 5 & ND (0.005) & ND (0.0409) & NAN & $0.12^{\mathrm{C}}$ & 0 of 5 \\
\hline Methylene chloride & 0 of 5 & ND (0.005) & ND (0.0409) & NAN & $0.5^{\mathrm{C}}$ & 0 of 5 \\
\hline n-Butylbenzene & 0 of 5 & ND $(0.005)$ & ND (0.0409) & NAN & $22^{c}$ & 0 of 5 \\
\hline n-Propylbenzene & 0 of 5 & ND (0.005) & ND (0.0409) & NAN & $0.25^{c}$ & 0 of 5 \\
\hline Naphthalene & 0 of 5 & ND (0.005) & ND (0.0409) & NAN & $3.85^{\mathrm{c}}$ & 0 of 5 \\
\hline o-Xylene & 0 of 5 & ND (0.005) & ND (0.0409) & NAN & $0.12^{c}$ & 0 of 5 \\
\hline p-isopropyltoluene & 0 of 5 & ND $(0.005)$ & ND (0.0409) & NAN & $2.8^{\mathrm{C}}$ & 0 of 5 \\
\hline sec-Butylbenzene & 0 of 5 & ND (0.005) & ND (0.0409) & NAN & $24^{\mathrm{C}}$ & 0 of 5 \\
\hline Toluene & 0 of 5 & ND (0.005) & ND (0.0409) & NAN & $2.5^{\mathrm{c}}$ & 0 of 5 \\
\hline \multicolumn{7}{|c|}{ Polycyclic Aromatic Hydrocarbons } \\
\hline Acenaphthene & 0 of 5 & ND (0.0033) & ND $(0.027)$ & NAN & $4.91^{\mathrm{C}}$ & 0 of 5 \\
\hline Acenaphthylene & 0 of 5 & ND (0.0033) & ND $(0.027)$ & NAN & $4.52^{\mathrm{C}}$ & 0 of 5 \\
\hline Anthracene & 0 of 5 & ND (0.0033) & ND (0.027) & NAN & $5.94^{\mathrm{C}}$ & 0 of 5 \\
\hline Benzo(a)anthracene & 0 of 5 & ND (0.0033) & ND $(0.027)$ & NAN & $8.41^{c}$ & 0 of 5 \\
\hline Benzo(a)pyrene & 0 of 5 & ND (0.0033) & ND $(0.027)$ & NAN & $9.65^{\mathrm{C}}$ & 0 of 5 \\
\hline Benzo(b)fluoranthene & 0 of 5 & ND (0.0033) & ND (0.027) & NAN & $9.79^{\mathrm{C}}$ & 0 of 5 \\
\hline Benzo(g,h,i)perylene & 0 of 5 & ND (0.0033) & ND (0.027) & NAN & $10.95^{\mathrm{c}}$ & 0 of 5 \\
\hline
\end{tabular}


Table 5-22

\section{Summary of Analytical Results and Ecological Risk Assessment Screening for Sediment in Heart Lake, Milrow Site}

(Page 2 of 3 )

\begin{tabular}{|c|c|c|c|c|c|c|}
\hline Analyte & $\begin{array}{l}\text { Detection } \\
\text { Frequency }\end{array}$ & $\begin{array}{c}\text { Minimum } \\
\text { Detected } \\
\text { Concentration } \\
(\mathrm{mg} / \mathrm{kg})\end{array}$ & $\begin{array}{c}\text { Maximum } \\
\text { Detected } \\
\text { Concentration } \\
(\mathrm{mg} / \mathrm{kg})\end{array}$ & $\begin{array}{c}\text { Lowland } \\
\text { Stream } \\
\text { Background } \\
\text { Concentration }{ }^{a} \\
(\mathbf{m g} / \mathbf{k g})\end{array}$ & $\begin{array}{c}\text { Ecological } \\
\text { Screening } \\
\text { Value } \\
(\mathrm{mg} / \mathrm{kg})\end{array}$ & $\begin{array}{c}\text { Detection } \\
\text { Frequency } \\
\text { Above ESV }\end{array}$ \\
\hline Benzo(k)fluoranthene & 0 of 5 & ND (0.0033) & ND (0.027) & NAN & $9.81^{\mathrm{C}}$ & 0 of 5 \\
\hline Chrysene & 0 of 5 & ND (0.0033) & ND $(0.027)$ & NAN & $8.44^{\mathrm{C}}$ & 0 of 5 \\
\hline Dibenzo(a,h)anthracene & 0 of 5 & ND (0.0033) & ND $(0.027)$ & NAN & $11.23^{\mathrm{C}}$ & 0 of 5 \\
\hline Fluoranthene & 0 of 5 & ND (0.0033) & ND (0.027) & NAN & $7.07^{\mathrm{C}}$ & 0 of 5 \\
\hline Fluorene & 0 of 5 & ND (0.0033) & ND (0.027) & NAN & $5.38^{C}$ & 0 of 5 \\
\hline Indeno $(1,2,3-c, d)$ pyrene & 0 of 5 & ND $(0.0033)$ & ND (0.027) & NAN & $11.15^{\mathrm{C}}$ & 0 of 5 \\
\hline Naphthalene & 0 of 5 & ND (0.0033) & ND (0.027) & NAN & $3.85^{\mathrm{C}}$ & 0 of 5 \\
\hline Phenanthrene & 0 of 5 & ND $(0.0033)$ & ND (0.027) & NAN & $5.96^{\mathrm{C}}$ & 0 of 5 \\
\hline Pyrene & 0 of 5 & ND (0.0033) & ND (0.027) & NAN & $6.97^{c}$ & 0 of 5 \\
\hline \multicolumn{7}{|c|}{ Polychlorinated Biphenyls } \\
\hline Aroclor 1016 & NAV & NAV & NAV & NAN & NAV & - \\
\hline Aroclor 1221 & NAV & NAV & NAV & NAN & NAV & - \\
\hline Aroclor 1232 & NAV & NAV & NAV & NAN & NAV & - \\
\hline Aroclor 1242 & NAV & NAV & NAV & NAN & NAV & - \\
\hline Aroclor 1248 & NAV & NAV & NAV & NAN & NAV & - \\
\hline Aroclor 1254 & NAV & NAV & NAV & NAN & NAV & - \\
\hline Aroclor 1260 & NAV & NAV & NAV & NAN & NAV & - \\
\hline Aroclor 1262 & NAV & NAV & NAV & NAN & NAV & - \\
\hline Aroclor 1268 & NAV & NAV & NAV & NAN & NAV & - \\
\hline \multicolumn{7}{|c|}{ Metals } \\
\hline Aluminum & 5 of 5 & 29,500 & 77,500 & 45,400 & 58,000 & 1 of 5 \\
\hline Arsenic & 0 of 5 & ND (0.5) & ND (4.09) & 100 & 6 & 0 of 5 \\
\hline Barium & 5 of 5 & 60.3 & 188 & 469 & NAV & \\
\hline Beryllium & 0 of 5 & ND (0.5) & ND (4.09) & ND & NAV & \\
\hline Boron & 0 of 5 & ND (2.5) & ND (20.4) & $7.18^{b}$ & NAV & \\
\hline Cadmium & 0 of 5 & ND (0.5) & ND (4.09) & $0.608^{b}$ & 0.6 & 0 of 5 \\
\hline Calcium & 5 of 5 & 1,390 & 8,130 & 7,300 & NAV & \\
\hline Cerium & 5 of 5 & 8.29 & 16.3 & 14.2 & NAV & \\
\hline
\end{tabular}


Table 5-22

\section{Summary of Analytical Results and Ecological Risk Assessment Screening for Sediment in Heart Lake, Milrow Site}

(Page 3 of 3 )

\begin{tabular}{|c|c|c|c|c|c|c|}
\hline Analyte & $\begin{array}{l}\text { Detection } \\
\text { Frequency }\end{array}$ & $\begin{array}{c}\text { Minimum } \\
\text { Detected } \\
\text { Concentration } \\
(\mathrm{mg} / \mathrm{kg})\end{array}$ & $\begin{array}{c}\text { Maximum } \\
\text { Detected } \\
\text { Concentration } \\
(\mathrm{mg} / \mathrm{kg})\end{array}$ & $\begin{array}{c}\text { Lowland } \\
\text { Stream } \\
\text { Background } \\
\text { Concentration } \\
\text { (mg/kg) }\end{array}$ & $\begin{array}{c}\text { Ecological } \\
\text { Screening } \\
\text { Value } \\
(\mathrm{mg} / \mathrm{kg})\end{array}$ & $\begin{array}{c}\text { Detection } \\
\text { Frequency } \\
\text { Above ESV }\end{array}$ \\
\hline Cesium & 0 of 5 & ND (0.5) & ND (4.09) & ND & NAV & \\
\hline Chromium & 5 of 5 & 7.76 & 21.7 & 11.8 & 26 & 0 of 5 \\
\hline Cobalt & 5 of 5 & 5.74 & 27 & 43.7 & NAV & \\
\hline Copper & 5 of 5 & 66.7 & 192 & 71.1 & 16 & 5 of 5 \\
\hline Iron & 5 of 5 & 7,660 & 66,400 & 155,000 & 20,000 & 3 of 5 \\
\hline Lithium & 5 of 5 & 10.9 & 19.1 & 48.8 & NAV & \\
\hline Magnesium & 5 of 5 & 2,510 & 29,400 & 9,320 & NAV & \\
\hline Manganese & 5 of 5 & 28.4 & 309 & 20,700 & 460 & 0 of 5 \\
\hline Molybdenum & 0 of 5 & ND (0.5) & ND (4.09) & $5.5^{\mathrm{b}}$ & NAV & \\
\hline Nickel & 5 of 5 & 6.51 & 13.6 & 13.6 & 16 & 0 of 5 \\
\hline Potassium & 5 of 5 & 258 & 1,580 & 1,390 & NAV & \\
\hline Selenium & 1 of 5 & ND (0.5) & 4.67 & $5.44^{\mathrm{b}}$ & NAV & \\
\hline Strontium & 5 of 5 & 30.7 & 196 & 164 & NAV & \\
\hline Thallium & 0 of 5 & ND (0.5) & ND (4.09) & $1.0^{\mathrm{b}}$ & NAV & \\
\hline Thorium & 1 of 5 & ND (3.06) & 0.855 & $0.5^{\mathrm{b}}$ & NAV & \\
\hline Titanium & 5 of 5 & 1,460 & 1,830 & 3,010 & NAV & \\
\hline Uranium & 0 of 5 & ND (0.5) & ND (4.09) & $4.13^{b}$ & NAV & \\
\hline Vanadium & 5 of 5 & 118 & 212 & 734 & NAV & \\
\hline Zinc & 4 of 5 & 33.6 & 71 & 434 & 120 & 0 of 5 \\
\hline
\end{tabular}

${ }^{\mathrm{a} B}$ Background concentrations, which are presented only for metals, are upper tolerance limits (UTL) unless otherwise noted.

${ }^{b}$ This metal was not detected in a sufficient number of reference stream samples to allow calculation of a valid UTL. The concentration shown is the maximum detected concentration in the appropriate background samples.

${ }^{\mathrm{c}}$ Screening concentration is based on $1 \%$ organic carbon.

ND - Analyte not detected at a concentration above the reporting limit, which is shown in parentheses.

NAV - Not available

NAN - Not analyzed

$\mathrm{mg} / \mathrm{kg}$ - Milligrams per kilogram 
Table 5-23

\section{Summary of Analytical Results and Ecological Risk Assessment Screening for Surface Water in Cannikin Lake}

(Page 1 of 3 )

\begin{tabular}{|c|c|c|c|c|c|c|}
\hline Analyte & $\begin{array}{l}\text { Detection } \\
\text { Frequency }\end{array}$ & $\begin{array}{c}\text { Minimum } \\
\text { Detected } \\
\text { Concentration } \\
(\mu \mathrm{g} / \mathrm{L})\end{array}$ & $\begin{array}{c}\text { Maximum } \\
\text { Detected } \\
\text { Concentration } \\
(\mu \mathrm{g} / \mathrm{L})\end{array}$ & $\begin{array}{c}\text { Lowland } \\
\text { Stream } \\
\text { Background } \\
\text { Concentration } \\
(\mu \mathrm{g} / \mathrm{L})\end{array}$ & $\begin{array}{l}\text { Ecological } \\
\text { Screening } \\
\text { Value }(\mu g / L)\end{array}$ & $\begin{array}{c}\text { Detection } \\
\text { Frequency } \\
\text { Above ESV }\end{array}$ \\
\hline $\begin{array}{l}\text { Diesel-Range } \\
\text { Hydrocarbons }\end{array}$ & 3 of 3 & 57.8 & 72.2 & NAN & 15 & 3 of 3 \\
\hline \multicolumn{7}{|c|}{ Volatile Organic Compounds } \\
\hline 1,2,4-Trimethylbenzene & 0 of 3 & ND (1.0) & ND (1.0) & NAN & 33 & 0 of 3 \\
\hline 1,3,5-Trimethylbenzene & 0 of 3 & ND (1.0) & ND (1.0) & NAN & 33 & 0 of 3 \\
\hline 2-Butanone & 0 of 3 & ND (20) & ND (20) & NAN & 14,000 & 0 of 3 \\
\hline Acetone & 0 of 3 & ND (20) & ND (20) & NAN & 1,500 & 0 of 3 \\
\hline Benzene & 0 of 3 & ND (1) & ND (1) & NAN & 130 & 0 of 3 \\
\hline Carbon disulfide & 0 of 3 & ND (1.0) & ND (1.0) & NAN & 0.92 & 0 of 3 \\
\hline Ethylbenzene & 0 of 3 & ND (1.0) & ND (1.0) & NAN & 7.3 & 0 of 3 \\
\hline Isopropylbenzene & 0 of 3 & ND (1.0) & ND (1.0) & NAN & 2.6 & 0 of 3 \\
\hline m,p-Xylene & 0 of 3 & ND (2.0) & ND (2.0) & NAN & 13 & 0 of 3 \\
\hline Methylene chloride & 0 of 3 & ND (2.03) & ND (5.34) & NAN & 2,200 & 0 of 3 \\
\hline n-Butylbenzene & 0 of 3 & ND (1.0) & ND (1.0) & NAN & 164 & 0 of 3 \\
\hline n-Propylbenzene & 0 of 3 & ND (1.0) & ND (1.0) & NAN & 6 & 0 of 3 \\
\hline Naphthalene & 0 of 3 & ND (1.0) & ND (1.0) & NAN & 12 & 0 of 3 \\
\hline o-Xylene & 0 of 3 & ND (1.0) & ND (1.0) & NAN & 13 & 0 of 3 \\
\hline p-Isopropyltoluene & 0 of 3 & ND (1.0) & ND (1.0) & NAN & 26 & 0 of 3 \\
\hline sec-Butylbenzene & 0 of 3 & ND (1.0) & ND (1.0) & NAN & 164 & 0 of 3 \\
\hline Toluene & 0 of 3 & ND (1.0) & ND (1.0) & NAN & 9.8 & 0 of 3 \\
\hline \multicolumn{7}{|c|}{ Polycyclic Aromatic Hydrocarbons } \\
\hline Acenaphthene & 0 of 3 & ND $(0.1)$ & ND $(0.1)$ & NAN & 74 & 0 of 3 \\
\hline Acenaphthylene & 0 of 3 & ND $(0.1)$ & ND $(0.1)$ & NAN & 307 & 0 of 3 \\
\hline Anthracene & 0 of 3 & ND $(0.1)$ & ND $(0.1)$ & NAN & 0.73 & 0 of 3 \\
\hline Benzo(a)anthracene & 0 of 3 & ND $(0.1)$ & ND $(0.1)$ & NAN & 0.027 & 0 of 3 \\
\hline Benzo(a)pyrene & 0 of 3 & ND $(0.1)$ & ND $(0.1)$ & NAN & 0.014 & 0 of 3 \\
\hline Benzo(b)fluoranthene & 0 of 3 & ND $(0.1)$ & ND $(0.1)$ & NAN & 0.68 & 0 of 3 \\
\hline Benzo(g,h,i)perylene & 0 of 3 & ND $(0.1)$ & ND $(0.1)$ & NAN & 0.44 & 0 of 3 \\
\hline
\end{tabular}


Table 5-23

\section{Summary of Analytical Results and Ecological Risk Assessment Screening for Surface Water in Cannikin Lake}

(Page 2 of 3 )

\begin{tabular}{|c|c|c|c|c|c|c|}
\hline Analyte & $\begin{array}{l}\text { Detection } \\
\text { Frequency }\end{array}$ & $\begin{array}{c}\text { Minimum } \\
\text { Detected } \\
\text { Concentration } \\
(\mu \mathrm{g} / \mathrm{L})\end{array}$ & $\begin{array}{c}\text { Maximum } \\
\text { Detected } \\
\text { Concentration } \\
(\mu \mathrm{g} / \mathrm{L})\end{array}$ & $\begin{array}{c}\text { Lowland } \\
\text { Stream } \\
\text { Background } \\
\text { Concentration } \\
(\mu \mathrm{g} / \mathrm{L})\end{array}$ & $\begin{array}{l}\text { Ecological } \\
\text { Screening } \\
\text { Value }(\mu g / L)\end{array}$ & $\begin{array}{c}\text { Detection } \\
\text { Frequency } \\
\text { Above ESV }\end{array}$ \\
\hline Benzo(k)fluoranthene & 0 of 3 & ND $(0.1)$ & ND $(0.1)$ & NAN & 0.64 & 0 of 3 \\
\hline Chrysene & 0 of 3 & ND $(0.1)$ & ND $(0.1)$ & NAN & 2.0 & 0 of 3 \\
\hline Dibenzo(a,h)anthracene & 0 of 3 & $\mathrm{ND}(0.1)$ & $\mathrm{ND}(0.1)$ & NAN & 0.3 & 0 of 3 \\
\hline Fluoranthene & 0 of 3 & ND $(0.1)$ & ND $(0.1)$ & NAN & 15 & 0 of 3 \\
\hline Fluorene & 0 of 3 & ND $(0.1)$ & ND $(0.1)$ & NAN & 3.9 & 0 of 3 \\
\hline Indeno(1,2,3-c,d)pyrene & 0 of 3 & ND $(0.1)$ & ND $(0.1)$ & NAN & 3.9 & 0 of 3 \\
\hline Naphthalene & 0 of 3 & ND $(0.1)$ & ND $(0.1)$ & NAN & 12 & 0 of 3 \\
\hline Phenanthrene & 0 of 3 & ND $(0.1)$ & ND $(0.1)$ & NAN & 200 & 0 of 3 \\
\hline Pyrene & 0 of 3 & ND $(0.1)$ & ND $(0.1)$ & NAN & 10 & 0 of 3 \\
\hline \multicolumn{7}{|c|}{ Polychlorinated Biphenyls } \\
\hline Aroclor 1016 & 0 of 3 & ND $(0.5)$ & ND $(0.5)$ & NAN & 0.014 & 0 of 3 \\
\hline Aroclor 1221 & 0 of 3 & ND (0.5) & ND (0.5) & NAN & 0.014 & 0 of 3 \\
\hline Aroclor 1232 & 0 of 3 & ND $(0.5)$ & ND $(0.5)$ & NAN & 0.014 & 0 of 3 \\
\hline Aroclor 1242 & 0 of 3 & ND $(0.5)$ & ND (0.5) & NAN & 0.014 & 0 of 3 \\
\hline Aroclor 1248 & 0 of 3 & ND (0.5) & ND $(0.5)$ & NAN & 0.014 & 0 of 3 \\
\hline Aroclor 1260 & 0 of 3 & ND (0.5) & ND (0.5) & NAN & 0.014 & 0 of 3 \\
\hline Aroclor 1262 & 0 of 3 & ND (0.5) & ND $(0.5)$ & NAN & 0.014 & 0 of 3 \\
\hline Aroclor 1268 & 0 of 3 & ND $(0.5)$ & ND (0.5) & NAN & 0.014 & 0 of 3 \\
\hline \multicolumn{7}{|c|}{ Metals } \\
\hline Aluminum & 3 of 3 & 77.8 & 102 & 269 & 87 & 2 of 3 \\
\hline Arsenic & 3 of 3 & 6.81 & 7.53 & ND (1) & 150 & 0 of 3 \\
\hline Barium & 0 of 3 & ND (10) & ND (10) & ND (10) & 4.0 & 0 of 3 \\
\hline Beryllium & 0 of 3 & ND (1) & ND (1) & ND (1) & 0.7 & 0 of 3 \\
\hline Boron & 3 of 3 & 429 & 468 & ND (50) & 1.6 & 3 of 3 \\
\hline Cadmium & 0 of 3 & ND (1) & ND (1) & ND (1) & 2.2 & 0 of 3 \\
\hline Calcium & 3 of 3 & 5,250 & 5,500 & $4,320^{b}$ & 116,000 & 0 of 3 \\
\hline Cerium & 0 of 3 & ND (1) & ND (1) & ND (1) & 0.50 & 0 of 3 \\
\hline Cesium & 0 of 3 & ND (1) & ND (1) & ND (1) & 30 & 0 of 3 \\
\hline
\end{tabular}


Table 5-23

\section{Summary of Analytical Results and Ecological Risk Assessment Screening for Surface Water in Cannikin Lake}

(Page 3 of 3 )

\begin{tabular}{|c|c|c|c|c|c|c|}
\hline Analyte & $\begin{array}{l}\text { Detection } \\
\text { Frequency }\end{array}$ & $\begin{array}{c}\text { Minimum } \\
\text { Detected } \\
\text { Concentration } \\
(\mu \mathrm{g} / \mathrm{L})\end{array}$ & $\begin{array}{c}\text { Maximum } \\
\text { Detected } \\
\text { Concentration } \\
(\mu \mathrm{g} / \mathrm{L})\end{array}$ & $\begin{array}{c}\text { Lowland } \\
\text { Stream } \\
\text { Background } \\
\text { Concentration }{ }^{\mathrm{a}} \\
(\mu \mathrm{g} / \mathrm{L})\end{array}$ & $\begin{array}{l}\text { Ecological } \\
\text { Screening } \\
\text { Value }(\mu g / L)\end{array}$ & $\begin{array}{c}\text { Detection } \\
\text { Frequency } \\
\text { Above ESV }\end{array}$ \\
\hline Chromium & 3 of 3 & 1.91 & 2.05 & $2.25^{b}$ & 74 & 0 of 3 \\
\hline Cobalt & 2 of 3 & ND (1.0) & 4.81 & 3.35 & 23 & 0 of 3 \\
\hline Copper & 3 of 3 & 1.66 & 2.5 & 10.8 & 9.0 & 0 of 3 \\
\hline Iron & 3 of 3 & 222 & 242 & 993 & 1,000 & 0 of 3 \\
\hline Lithium & 0 of 3 & ND (1.0) & ND (1.0) & $1.17^{\mathrm{b}}$ & 14 & 0 of 3 \\
\hline Magnesium & 3 of 3 & 1,840 & 1,890 & $4,690^{b}$ & 82,000 & 0 of 3 \\
\hline Manganese & 3 of 3 & 17.4 & 28.2 & 267 & 120 & 0 of 3 \\
\hline Molybdenum & 3 of 3 & ND (1) & ND (1) & ND (1) & 370 & 0 of 3 \\
\hline Nickel & 3 of 3 & ND (1) & 1.2 & ND (1) & 52 & 0 of 3 \\
\hline Potassium & 3 of 3 & 689 & 773 & 2,620 & 53,000 & 0 of 3 \\
\hline Selenium & 0 of 3 & ND (1) & ND (1) & ND (1) & 5.0 & 0 of 3 \\
\hline Strontium & 3 of 3 & 28.8 & 29.8 & 36.1 & 1,500 & 0 of 3 \\
\hline Thallium & 1 of 3 & ND (1.0) & 1.27 & $7.07^{b}$ & 12 & 0 of 3 \\
\hline Thorium & 0 of 3 & ND (1) & ND (1) & ND (1) & NAV & 0 of 3 \\
\hline Titanium & 3 of 3 & 2.81 & 4.1 & 6.13 & 29 & 0 of 3 \\
\hline Uranium & 0 of 3 & ND (1) & ND (1) & ND (1) & 2.6 & 0 of 3 \\
\hline Vanadium & 3 of 3 & 5.04 & 5.31 & $4.43^{b}$ & 20 & 0 of 3 \\
\hline Zinc & 0 of 3 & ND (10) & ND (10) & $50.3^{b}$ & 120 & 0 of 3 \\
\hline
\end{tabular}

${ }^{\mathrm{a}}$ Background concentrations, which are presented only for metals, are upper tolerance limits (UTL) unless otherwise noted.

${ }^{b}$ This metal was not detected in a sufficient number of reference stream samples to allow calculation of a valid UTL. The concentration shown is the maximum detected concentration in the appropriate background samples.

ND - Analyte not detected at a concentration above the reporting limit, which is shown in parentheses.

NAV - Not available

NAN - Not analyzed

$\mu \mathrm{g} / \mathrm{L}$ - Micrograms per liter 
Table 5-24

Summary of Analytical Results and Ecological Risk Assessment Screening for Sediment in Cannikin Lake

(Page 1 of 3 )

\begin{tabular}{|c|c|c|c|c|c|c|}
\hline Analyte & $\begin{array}{l}\text { Detection } \\
\text { Frequency }\end{array}$ & $\begin{array}{c}\text { Minimum } \\
\text { Detected } \\
\text { Concentration } \\
(\mathbf{m g} / \mathbf{k g})\end{array}$ & $\begin{array}{c}\text { Maximum } \\
\text { Detected } \\
\text { Concentration } \\
(\mathrm{mg} / \mathrm{kg})\end{array}$ & $\begin{array}{l}\text { Lowland } \\
\text { Stream } \\
\text { Background } \\
\text { Concentration }{ }^{\mathrm{a}} \\
(\mathrm{mg} / \mathrm{kg})\end{array}$ & $\begin{array}{c}\text { Ecological } \\
\text { Screening } \\
\text { Value } \\
(\mathbf{m g} / \mathbf{k g})\end{array}$ & $\begin{array}{c}\text { Detection } \\
\text { Frequency } \\
\text { Above ESV }\end{array}$ \\
\hline \multicolumn{7}{|c|}{ Petroleum Hydrocarbons } \\
\hline $\begin{array}{l}\text { Gasoline-Range } \\
\text { Hydrocarbons }\end{array}$ & 1 of 3 & ND (5.0) & 25.9 & NAN & $1.2^{c}$ & 1 of 3 \\
\hline $\begin{array}{l}\text { Diesel-Range } \\
\text { Hydrocarbons }\end{array}$ & 3 of 3 & 110 & 4,710 & NAN & $640^{\mathrm{C}}$ & 0 of 3 \\
\hline \multicolumn{7}{|c|}{ Volatile Organic Compounds } \\
\hline 1,2,4-Trimethylbenzene & 2 of 3 & ND (0.493) & 5.16 & NAN & $1.2^{\mathrm{c}}$ & 1 of 3 \\
\hline 1,3,5-Trimethylbenzene & 1 of 3 & $\mathrm{ND}(0.15)$ & 1.21 & NAN & $0.76^{\mathrm{C}}$ & 1 of 3 \\
\hline 2-Butanone & 0 of 3 & ND (3.5) & ND (12.3) & NAN & 14 & 0 of 3 \\
\hline Acetone & 0 of 3 & ND (3.5) & ND (12.3) & NAN & 1.5 & 0 of 3 \\
\hline Benzene & 0 of 3 & ND $(0.14)$ & ND $(0.493)$ & NAN & $0.34^{\mathrm{C}}$ & 0 of 3 \\
\hline Carbon disulfide & 0 of 3 & ND $(0.7)$ & ND (2.47) & NAN & 0.001 & 0 of 3 \\
\hline Ethylbenzene & 1 of 3 & $\mathrm{ND}(0.15)$ & 0.186 & NAN & $1.4^{\mathrm{c}}$ & 0 of 3 \\
\hline Isopropylbenzene & 0 of 3 & ND $(0.14)$ & ND (0.493) & NAN & $0.10^{\mathrm{c}}$ & 0 of 3 \\
\hline m,p-Xylene & 1 of 3 & ND (0.3) & 0.836 & NAN & $0.12^{c}$ & 1 of 3 \\
\hline Methylene chloride & 0 of 3 & ND $(0.7)$ & ND (2.47) & NAN & $0.5^{c}$ & 0 of 3 \\
\hline n-Butylbenzene & 2 of 3 & 0.311 & 0.496 & NAN & $22^{c}$ & 2 of 3 \\
\hline n-Propylbenzene & 2 of 3 & 0.15 & 0.369 & NAN & $0.25^{\mathrm{C}}$ & 2 of 3 \\
\hline Naphthalene & 1 of 3 & ND $(0.15)$ & 5.53 & NAN & $3.85^{\mathrm{c}}$ & 1 of 3 \\
\hline o-Xylene & 1 of 3 & $\mathrm{ND}(0.15)$ & 0.498 & NAN & $0.12^{c}$ & 1 of 3 \\
\hline p-Isopropyltoluene & 2 of 3 & 0.282 & 0.304 & NAN & $2.8^{\mathrm{C}}$ & 0 of 3 \\
\hline sec-Butylbenzene & 2 of 3 & 0.202 & 0.233 & NAN & $24^{c}$ & 0 of 3 \\
\hline Toluene & 0 of 3 & ND (0.14) & ND (0.493) & NAN & $2.5^{\mathrm{c}}$ & 0 of 3 \\
\hline \multicolumn{7}{|c|}{ Polycyclic Aromatic Hydrocarbons } \\
\hline Acenaphthene & 2 of 3 & ND (0.00798) & 1.89 & NAN & $4.91^{\mathrm{C}}$ & 0 of 3 \\
\hline Acenaphthylene & 0 of 3 & ND (0.00798) & ND (1.0) & NAN & $4.52^{\mathrm{C}}$ & 0 of 3 \\
\hline Anthracene & 2 of 3 & ND (0.00798) & 0.403 & NAN & $5.94^{c}$ & 0 of 3 \\
\hline Benzo(a)anthracene & 0 of 3 & ND (0.00798) & ND (0.033) & NAN & $8.41^{\mathrm{C}}$ & 0 of 3 \\
\hline Benzo(a)pyrene & 0 of 3 & ND (0.00798) & ND (0.033) & NAN & $9.65^{c}$ & 0 of 3 \\
\hline
\end{tabular}


Table 5-24

Summary of Analytical Results and Ecological Risk Assessment Screening for Sediment in Cannikin Lake

(Page 2 of 3 )

\begin{tabular}{|c|c|c|c|c|c|c|}
\hline Analyte & $\begin{array}{l}\text { Detection } \\
\text { Frequency }\end{array}$ & $\begin{array}{c}\text { Minimum } \\
\text { Detected } \\
\text { Concentration } \\
(\mathbf{m g} / \mathbf{k g})\end{array}$ & $\begin{array}{c}\text { Maximum } \\
\text { Detected } \\
\text { Concentration } \\
(\mathbf{m g} / \mathbf{k g})\end{array}$ & $\begin{array}{c}\text { Lowland } \\
\text { Stream } \\
\text { Background } \\
\text { Concentration }{ }^{\mathrm{a}} \\
(\mathrm{mg} / \mathrm{kg})\end{array}$ & $\begin{array}{c}\text { Ecological } \\
\text { Screening } \\
\text { Value } \\
(\mathrm{mg} / \mathrm{kg})\end{array}$ & $\begin{array}{c}\text { Detection } \\
\text { Frequency } \\
\text { Above ESV }\end{array}$ \\
\hline Benzo(b)fluoranthene & 0 of 3 & ND (0.00798) & ND (0.033) & NAN & $9.79^{C}$ & 0 of 3 \\
\hline Benzo(g,h,i)perylene & 0 of 3 & ND (0.00798) & ND $(0.033)$ & NAN & $10.95^{\mathrm{C}}$ & 0 of 3 \\
\hline Benzo(k)fluoranthene & 0 of 3 & ND (0.00798) & ND (0.033) & NAN & $9.81^{\mathrm{C}}$ & 0 of 3 \\
\hline Chrysene & 0 of 3 & ND (0.00798) & ND (0.033) & NAN & $8.44^{\mathrm{C}}$ & 0 of 3 \\
\hline Dibenzo(a,h)anthracene & 0 of 3 & ND (0.00798) & ND (0.033) & NAN & $11.23^{\mathrm{c}}$ & 0 of 3 \\
\hline Fluoranthene & 2 of 3 & ND (0.00798) & 0.227 & NAN & $7.07^{\mathrm{C}}$ & 0 of 3 \\
\hline Fluorene & 2 of 3 & ND (0.00798) & 4.87 & NAN & $5.38^{\mathrm{C}}$ & 0 of 3 \\
\hline Indeno(1,2,3-c,d)pyrene & 0 of 3 & ND (0.00798) & ND $(0.033)$ & NAN & $11.15^{\mathrm{c}}$ & 0 of 3 \\
\hline Naphthalene & 2 of 3 & ND (0.00798) & 1.4 & NAN & $3.85^{\mathrm{C}}$ & 0 of 3 \\
\hline Phenanthrene & 2 of 3 & ND (0.00798) & 12.1 & NAN & $5.96^{\mathrm{C}}$ & 1 of 3 \\
\hline Pyrene & 2 of 3 & ND (0.00798) & 0.34 & NAN & $6.97^{\mathrm{C}}$ & 0 of 3 \\
\hline \multicolumn{7}{|c|}{ Polychlorinated Biphenyls } \\
\hline Aroclor 1016 & 0 of 3 & ND (0.05) & ND (0.05) & NAN & $0.07^{c}$ & 0 of 3 \\
\hline Aroclor 1221 & 0 of 3 & ND (0.05) & ND (0.05) & NAN & $0.07^{\mathrm{C}}$ & 0 of 3 \\
\hline Aroclor 1232 & 0 of 3 & ND (0.05) & ND (0.05) & NAN & $0.07^{\mathrm{C}}$ & 0 of 3 \\
\hline Aroclor 1242 & 0 of 3 & ND (0.05) & ND (0.05) & NAN & $0.07^{C}$ & 0 of 3 \\
\hline Aroclor 1248 & 1 of 3 & ND (0.05) & 0.0605 & NAN & $0.07^{C}$ & 1 of 3 \\
\hline Aroclor 1260 & 2 of 3 & 0.104 & 0.107 & NAN & $0.07^{c}$ & 2 of 3 \\
\hline Aroclor 1262 & 0 of 3 & ND (0.05) & ND (0.05) & NAN & $0.07^{c}$ & 0 of 3 \\
\hline Aroclor 1268 & 0 of 3 & ND (0.05) & ND (0.05) & NAN & $0.07^{c}$ & 0 of 3 \\
\hline \multicolumn{7}{|c|}{ Metals } \\
\hline Aluminum & 3 of 3 & 29,300 & 55,900 & 45,400 & 58,000 & 0 of 3 \\
\hline Arsenic & 3 of 3 & 9.15 & 20.4 & 100 & 6.0 & 3 of 3 \\
\hline Barium & 3 of 3 & 60 & 198 & 469 & NAV & \\
\hline Beryllium & 0 of 3 & ND (1.21) & ND (2.5) & ND & NAV & \\
\hline Boron & 3 of 3 & 7.01 & 15.6 & $7.18^{b}$ & NAV & \\
\hline Cadmium & 1 of 3 & ND (0.5) & 0.876 & $0.608^{b}$ & 0.6 & 1 of 3 \\
\hline Calcium & 3 of 3 & 3,300 & 25,900 & 7,300 & NAV & \\
\hline
\end{tabular}


Table 5-24

Summary of Analytical Results and Ecological Risk Assessment Screening for Sediment in Cannikin Lake

(Page 3 of 3)

\begin{tabular}{|c|c|c|c|c|c|c|}
\hline Analyte & $\begin{array}{l}\text { Detection } \\
\text { Frequency }\end{array}$ & $\begin{array}{c}\text { Minimum } \\
\text { Detected } \\
\text { Concentration } \\
(\mathbf{m g} / \mathbf{k g})\end{array}$ & $\begin{array}{c}\text { Maximum } \\
\text { Detected } \\
\text { Concentration } \\
(\mathbf{m g} / \mathbf{k g})\end{array}$ & $\begin{array}{c}\text { Lowland } \\
\text { Stream } \\
\text { Background } \\
\text { Concentration }{ }^{\mathrm{a}} \\
(\mathrm{mg} / \mathrm{kg})\end{array}$ & $\begin{array}{c}\text { Ecological } \\
\text { Screening } \\
\text { Value } \\
(\mathrm{mg} / \mathrm{kg})\end{array}$ & $\begin{array}{c}\text { Detection } \\
\text { Frequency } \\
\text { Above ESV }\end{array}$ \\
\hline Cerium & 3 of 3 & 4.73 & 21.1 & 14.2 & NAV & \\
\hline Cesium & 1 of 3 & ND (0.5) & 1.11 & ND & NAV & \\
\hline Chromium & 3 of 3 & 13.8 & 257 & 11.8 & 26 & 2 of 3 \\
\hline Cobalt & 3 of 3 & 8.62 & 21.6 & 43.7 & NAV & \\
\hline Copper & 3 of 3 & 65.6 & 203 & 71.1 & 16 & 3 of 3 \\
\hline Iron & 3 of 3 & 36,900 & 55,900 & 155,000 & 20,000 & 3 of 3 \\
\hline Lithium & 3 of 3 & 14.7 & 15.8 & 48.8 & NAV & \\
\hline Magnesium & 3 of 3 & 11,400 & 23,300 & 9,320 & NAV & \\
\hline Molybdenum & 0 of 3 & ND (1.21) & ND (2.5) & $5.5^{\mathrm{b}}$ & NAV & \\
\hline Manganese & 3 of 3 & 357 & 956 & 20,700 & 460 & 2 of 3 \\
\hline Nickel & 3 of 3 & 17.5 & 66.9 & 13.6 & 16 & 3 of 3 \\
\hline Potassium & 3 of 3 & 379 & 1,290 & 1,390 & NAV & \\
\hline Selenium & 3 of 3 & 0.707 & 1.64 & $5.44^{\mathrm{b}}$ & NAV & \\
\hline Strontium & 3 of 3 & 32.3 & 251 & 164 & NAV & \\
\hline Thallium & 0 of 3 & ND (0.5) & ND (1.21) & $1^{b}$ & NAV & \\
\hline Thorium & 2 of 3 & ND (1.21) & 2.81 & $0.5^{b}$ & NAV & \\
\hline Titanium & 3 of 3 & 798 & 1,360 & 3,010 & NAV & \\
\hline Uranium & 2 of 3 & ND (1.21) & 1.32 & $4.13^{b}$ & NAV & \\
\hline Vanadium & 3 of 3 & 118 & 164 & 734 & NAV & \\
\hline Zinc & 3 of 3 & 53.9 & 78.9 & 434 & 120 & 0 of 3 \\
\hline
\end{tabular}

${ }^{\mathrm{a} B a c k g r o u n d}$ concentrations, which are presented only for metals, are upper tolerance limits (UTL) unless otherwise noted.

${ }^{\mathrm{b}}$ This metal was not detected in a sufficient number of reference stream samples to allow calculation of a valid UTL. The concentration shown is the maximum detected concentration in the appropriate background samples.

${ }^{\mathrm{c}} \mathrm{Screening}$ concentration is based on $1 \%$ organic carbon.

ND - Analyte not detected at a concentration above the reporting limit, which is shown in parentheses.

NAV - Not available

NAN - Not analyzed

$\mathrm{mg} / \mathrm{kg}$ - Milligrams per kilogram 
Table 5-25

Summary of Analytical Results and Ecological Risk Assessment Screening for Surface Water in White Alice Creek, Cannikin Site

(Page 1 of 3 )

\begin{tabular}{|c|c|c|c|c|c|c|}
\hline Analyte & $\begin{array}{l}\text { Detection } \\
\text { Frequency }\end{array}$ & $\begin{array}{c}\text { Minimum } \\
\text { Detected } \\
\text { Concentration } \\
(\mu \mathrm{g} / \mathrm{L})\end{array}$ & $\begin{array}{c}\text { Maximum } \\
\text { Detected } \\
\text { Concentration } \\
(\mu \mathrm{g} / \mathrm{L})\end{array}$ & $\begin{array}{c}\text { Lowland } \\
\text { Stream } \\
\text { Background } \\
\text { Concentration }^{\mathrm{a}} \\
(\mu \mathrm{g} / \mathrm{L})\end{array}$ & $\begin{array}{l}\text { Ecological } \\
\text { Screening } \\
\text { Value }(\mu g / L)\end{array}$ & $\begin{array}{c}\text { Detection } \\
\text { Frequency } \\
\text { Above ESV }\end{array}$ \\
\hline $\begin{array}{l}\text { Diesel-Range } \\
\text { Hydrocarbons }\end{array}$ & 5 of 5 & 65.6 & 95.3 & NAN & 15 & 5 of 5 \\
\hline \multicolumn{7}{|c|}{ Volatile Organic Compounds } \\
\hline 1,2,4-Trimethylbenzene & 0 of 5 & ND (1.0) & ND (1.0) & NAN & 33 & 0 of 5 \\
\hline 1,3,5-Trimethylbenzene & 0 of 5 & ND (1.0) & ND (1.0) & NAN & 33 & 0 of 5 \\
\hline 2-Butanone & 0 of 5 & ND (20) & ND (20) & NAN & 14,000 & 0 of 5 \\
\hline Acetone & 0 of 5 & ND (20) & ND (20) & NAN & 1,500 & 0 of 5 \\
\hline Benzene & 0 of 5 & ND (1.0) & ND (1.0) & NAN & 130 & 0 of 5 \\
\hline Carbon disulfide & 0 of 5 & ND (1.0) & ND (1.0) & NAN & 0.92 & 0 of 5 \\
\hline Ethylbenzene & 0 of 5 & ND (1.0) & ND (1.0) & NAN & 7.3 & 0 of 5 \\
\hline Isopropylbenzene & 0 of 5 & ND (1.0) & ND (1.0) & NAN & 2.6 & 0 of 5 \\
\hline m,p-Xylene & 0 of 5 & ND (2.0) & ND (2.0) & NAN & 13 & 0 of 5 \\
\hline Methylene chloride & 0 of 5 & ND (2.0) & ND (5.59) & NAN & 2,200 & 0 of 5 \\
\hline n-Butylbenzene & 0 of 5 & ND (1.0) & ND (1.0) & NAN & 164 & 0 of 5 \\
\hline n-Propylbenzene & 0 of 5 & ND (1.0) & ND (1.0) & NAN & 6.0 & 0 of 5 \\
\hline Naphthalene & 0 of 5 & ND (1.0) & ND (1.0) & NAN & 164 & 0 of 5 \\
\hline o-Xylene & 0 of 5 & ND (1.0) & ND (1.0) & NAN & 13 & 0 of 5 \\
\hline p-Isopropyltoluene & 0 of 5 & ND (1.0) & ND (1.0) & NAN & 26 & 0 of 5 \\
\hline sec-Butylbenzene & 0 of 5 & ND (1.0) & ND (1.0) & NAN & 164 & 0 of 5 \\
\hline Toluene & 0 of 5 & ND (1.0) & ND (1.0) & NAN & 9.8 & 0 of 5 \\
\hline \multicolumn{7}{|c|}{ Polycyclic Aromatic Hydrocarbons } \\
\hline Acenaphthene & 0 of 5 & ND $(0.1)$ & $\mathrm{ND}(0.1)$ & NAN & 74 & 0 of 5 \\
\hline Acenaphthylene & 0 of 5 & ND $(0.1)$ & ND $(0.1)$ & NAN & 307 & 0 of 5 \\
\hline Anthracene & 0 of 5 & ND $(0.1)$ & $\mathrm{ND}(0.1)$ & NAN & 0.73 & 0 of 5 \\
\hline Benzo(a)anthracene & 0 of 5 & ND $(0.1)$ & ND $(0.1)$ & NAN & 0.027 & 0 of 5 \\
\hline Benzo(a)pyrene & 0 of 5 & $\mathrm{ND}(0.1)$ & $\mathrm{ND}(0.1)$ & NAN & 0.014 & 0 of 5 \\
\hline Benzo(b)fluoranthene & 0 of 5 & ND $(0.1)$ & $\mathrm{ND}(0.1)$ & NAN & 0.68 & 0 of 5 \\
\hline Benzo(g,h,i)perylene & 0 of 5 & ND $(0.1)$ & ND $(0.1)$ & NAN & 0.44 & 0 of 5 \\
\hline
\end{tabular}


Table 5-25

Summary of Analytical Results and Ecological Risk Assessment Screening for Surface Water in White Alice Creek, Cannikin Site

(Page 2 of 3 )

\begin{tabular}{|c|c|c|c|c|c|c|}
\hline Analyte & $\begin{array}{l}\text { Detection } \\
\text { Frequency }\end{array}$ & $\begin{array}{c}\text { Minimum } \\
\text { Detected } \\
\text { Concentration } \\
(\mu \mathrm{g} / \mathrm{L})\end{array}$ & $\begin{array}{c}\text { Maximum } \\
\text { Detected } \\
\text { Concentration } \\
(\mu \mathrm{g} / \mathrm{L})\end{array}$ & $\begin{array}{c}\text { Lowland } \\
\text { Stream } \\
\text { Background } \\
\text { Concentration }{ }^{\mathrm{a}} \\
(\mu \mathrm{g} / \mathrm{L})\end{array}$ & $\begin{array}{l}\text { Ecological } \\
\text { Screening } \\
\text { Value }(\mu g / L)\end{array}$ & $\begin{array}{c}\text { Detection } \\
\text { Frequency } \\
\text { Above ESV }\end{array}$ \\
\hline Benzo(k)fluoranthene & 0 of 5 & $\mathrm{ND}(0.1)$ & $\mathrm{ND}(0.1)$ & NAN & 0.64 & 0 of 5 \\
\hline Chrysene & 0 of 5 & ND $(0.1)$ & $\mathrm{ND}(0.1)$ & NAN & 2.0 & 0 of 5 \\
\hline Dibenzo(a,h)anthracene & 0 of 5 & $\mathrm{ND}(0.1)$ & $\mathrm{ND}(0.1)$ & NAN & 0.3 & 0 of 5 \\
\hline Fluoranthene & 0 of 5 & $\mathrm{ND}(0.1)$ & $\mathrm{ND}(0.1)$ & NAN & 15 & 0 of 5 \\
\hline Fluorene & 0 of 5 & ND $(0.1)$ & $\mathrm{ND}(0.1)$ & NAN & 3.9 & 0 of 5 \\
\hline Indeno(1,2,3-c,d)pyrene & 0 of 5 & $\mathrm{ND}(0.1)$ & ND $(0.1)$ & NAN & 0.28 & 0 of 5 \\
\hline Naphthalene & 0 of 5 & ND $(0.1)$ & $\mathrm{ND}(0.1)$ & NAN & 12 & 0 of 5 \\
\hline Phenanthrene & 0 of 5 & ND $(0.1)$ & $\mathrm{ND}(0.1)$ & NAN & 200 & 0 of 5 \\
\hline Pyrene & 0 of 5 & ND $(0.1)$ & $\mathrm{ND}(0.1)$ & NAN & 10 & 0 of 5 \\
\hline \multicolumn{7}{|c|}{ Polychlorinated Biphenyls } \\
\hline Aroclor 1016 & NAV & NAV & NAV & NAN & NAV & - \\
\hline Aroclor 1221 & NAV & NAV & NAV & NAN & NAV & - \\
\hline Aroclor 1232 & NAV & NAV & NAV & NAN & NAV & - \\
\hline Aroclor 1242 & NAV & NAV & NAV & NAN & NAV & - \\
\hline Aroclor 1248 & NAV & NAV & NAV & NAN & NAV & - \\
\hline Aroclor 1254 & NAV & NAV & NAV & NAN & NAV & - \\
\hline Aroclor 1260 & NAV & NAV & NAV & NAN & NAV & - \\
\hline Aroclor 1262 & NAV & NAV & NAV & NAN & NAV & - \\
\hline Aroclor 1268 & NAV & NAV & NAV & NAN & NAV & - \\
\hline \multicolumn{7}{|c|}{ Metals } \\
\hline Aluminum & 4 of 5 & ND (50) & 97.4 & 269 & 87 & 1 of 5 \\
\hline Arsenic & 2 of 5 & ND (1.0) & 6.42 & ND (1) & 150 & 0 of 5 \\
\hline Barium & 0 of 5 & ND (10) & ND (10) & ND (10) & 4.0 & 0 of 5 \\
\hline Beryllium & 0 of 5 & ND (1) & ND (1) & ND (1) & 0.7 & 0 of 5 \\
\hline Boron & 2 of 5 & ND (50) & 424 & ND (50) & 1.6 & 2 of 5 \\
\hline Cadmium & 0 of 5 & ND (1.0) & ND (1.0) & ND (1) & 2.2 & 0 of 5 \\
\hline Calcium & 5 of 5 & 3,280 & 5,550 & $4,320^{b}$ & 116,000 & 0 of 5 \\
\hline Cerium & 0 of 5 & ND (1.0) & ND (1.0) & ND (1) & 0.5 & 0 of 5 \\
\hline
\end{tabular}


Table 5-25

Summary of Analytical Results and Ecological Risk Assessment Screening for Surface Water in White Alice Creek, Cannikin Site

(Page 3 of 3 )

\begin{tabular}{|c|c|c|c|c|c|c|}
\hline Analyte & $\begin{array}{l}\text { Detection } \\
\text { Frequency }\end{array}$ & $\begin{array}{c}\text { Minimum } \\
\text { Detected } \\
\text { Concentration } \\
(\mu \mathrm{g} / \mathrm{L})\end{array}$ & $\begin{array}{c}\text { Maximum } \\
\text { Detected } \\
\text { Concentration } \\
(\mu \mathrm{g} / \mathrm{L})\end{array}$ & $\begin{array}{c}\text { Lowland } \\
\text { Stream } \\
\text { Background } \\
\text { Concentration }{ }^{\mathrm{a}} \\
(\mu \mathrm{g} / \mathrm{L})\end{array}$ & $\begin{array}{l}\text { Ecological } \\
\text { Screening } \\
\text { Value }(\mu \mathrm{g} / \mathrm{L})\end{array}$ & $\begin{array}{l}\text { Detection } \\
\text { Frequency } \\
\text { Above ESV }\end{array}$ \\
\hline Cesium & 0 of 5 & ND (1.0) & ND (1.0) & ND (1) & 30 & 0 of 5 \\
\hline Chromium & 0 of 5 & ND (1.0) & ND (1.0) & $2.25^{\mathrm{b}}$ & 74 & 0 of 5 \\
\hline Cobalt & 5 of 5 & 1.09 & 1.54 & 3.35 & 23 & 0 of 5 \\
\hline Copper & 5 of 5 & 1.2 & 2.01 & 10.8 & 9.0 & 0 of 5 \\
\hline Iron & 4 of 5 & ND (150) & 266 & 993 & 1,000 & 0 of 5 \\
\hline Lithium & 0 of 5 & ND (1.0) & ND (1.0) & $1.17^{b}$ & 14 & 0 of 5 \\
\hline Magnesium & 5 of 5 & 1,880 & 3,090 & $4,690^{b}$ & 82,000 & 0 of 5 \\
\hline Manganese & 5 of 5 & 7.3 & 21.5 & 267 & 120 & 0 of 5 \\
\hline Molybdenum & 0 of 5 & ND (1) & ND (1) & ND (1) & 370 & 0 of 5 \\
\hline Nickel & 0 of 5 & ND (1.0) & ND (1.0) & ND (1) & 52 & 0 of 5 \\
\hline Potassium & 5 of 5 & 217 & 564 & 2,620 & 53,000 & 0 of 5 \\
\hline Selenium & 1 of 5 & ND (1.0) & 1.1 & ND (1) & 5.0 & 0 of 5 \\
\hline Strontium & 5 of 5 & 22.4 & 33 & 36.1 & 1,500 & 0 of 5 \\
\hline Thallium & 0 of 5 & ND (1.0) & ND (1.0) & $7.07^{b}$ & 12 & 0 of 5 \\
\hline Thorium & 0 of 5 & ND (1.0) & ND (1.0) & ND (1) & NAV & 0 of 5 \\
\hline Titanium & 5 of 5 & 1.41 & 2.96 & 6.13 & 29 & 0 of 5 \\
\hline Uranium & 0 of 5 & ND (1.0) & ND (1.0) & ND (1) & 2.6 & 0 of 5 \\
\hline Vanadium & 4 of 5 & ND (1.0) & 4.94 & $4.43^{b}$ & 20 & 0 of 5 \\
\hline Zinc & 0 of 5 & ND (10) & ND (10) & $50.3^{b}$ & 120 & 0 of 5 \\
\hline
\end{tabular}

${ }^{\mathrm{a} B}$ Background concentrations, which are presented only for metals, are upper tolerance limits (UTL) unless otherwise noted.

${ }^{b}$ This metal was not detected in a sufficient number of reference stream samples to allow calculation of a valid UTL. The concentration shown is the maximum detected concentration in the appropriate background samples.

ND - Analyte not detected at a concentration above the reporting limit, which is shown in parentheses.

NAV - Not available

NAN - Not analyzed

$\mu \mathrm{g} / \mathrm{L}$ - Micrograms per liter 
Table 5-26

\section{Summary of Analytical Results and Ecological Risk Assessment Screening for Sediment in White Alice Creek, Cannikin Site}

(Page 1 of 3 )

\begin{tabular}{|c|c|c|c|c|c|c|}
\hline Analyte & $\begin{array}{l}\text { Detection } \\
\text { Frequency }\end{array}$ & $\begin{array}{c}\text { Minimum } \\
\text { Detected } \\
\text { Concentration } \\
(\mathbf{m g} / \mathbf{k g})\end{array}$ & $\begin{array}{c}\text { Maximum } \\
\text { Detected } \\
\text { Concentration } \\
(\mathbf{m g} / \mathbf{k g})\end{array}$ & $\begin{array}{l}\text { Lowland Stream } \\
\text { Background } \\
\text { Concentration }{ }^{\mathrm{a}} \\
(\mathrm{mg} / \mathrm{kg})\end{array}$ & $\begin{array}{c}\text { Ecological } \\
\text { Screening } \\
\text { Value } \\
(\mathrm{mg} / \mathrm{kg})\end{array}$ & $\begin{array}{l}\text { Detection } \\
\text { Frequency } \\
\text { Above ESV }\end{array}$ \\
\hline \multicolumn{7}{|c|}{ Petroleum Hydrocarbons } \\
\hline $\begin{array}{l}\text { Diesel-Range } \\
\text { Hydrocarbons }\end{array}$ & 5 of 5 & 32.6 & 116 & NAN & $640^{\mathrm{C}}$ & 0 of 5 \\
\hline \multicolumn{7}{|c|}{ Volatile Organic Compounds } \\
\hline 1,2,4-Trimethylbenzene & 0 of 1 & ND $(0.078)$ & ND $(0.078)$ & NAN & $1.2^{\mathrm{C}}$ & 0 of 1 \\
\hline 1,3,5-Trimethylbenzene & 0 of 1 & ND (0.078) & ND $(0.078)$ & NAN & $0.7^{\mathrm{C}}$ & 0 of 1 \\
\hline 2-Butanone & 0 of 1 & ND $(0.156)$ & ND $(0.156)$ & NAN & 14 & 0 of 1 \\
\hline Acetone & 0 of 1 & ND (8.91) & ND (8.91) & NAN & 1.5 & 0 of 1 \\
\hline Benzene & 0 of 1 & ND $(0.078)$ & ND $(0.078)$ & NAN & $0.34^{c}$ & 0 of 1 \\
\hline Carbon disulfide & 0 of 1 & ND $(0.078)$ & ND $(0.078)$ & NAN & 0.001 & 0 of 1 \\
\hline Ethylbenzene & 0 of 1 & ND (0.078) & ND (0.078) & NAN & $1.4^{\mathrm{C}}$ & 0 of 1 \\
\hline Isopropylbenzene & 0 of 1 & ND (0.078) & ND (0.078) & NAN & $0.1^{\mathrm{c}}$ & 0 of 1 \\
\hline m,p-Xylene & 0 of 1 & ND $(0.078)$ & ND $(0.078)$ & NAN & $0.12^{\mathrm{C}}$ & 0 of 1 \\
\hline Methylene chloride & 0 of 1 & ND $(0.078)$ & ND (0.078) & NAN & $0.5^{\mathrm{c}}$ & 0 of 1 \\
\hline n-Butylbenzene & 0 of 1 & ND (0.078) & ND (0.078) & NAN & $22^{c}$ & 0 of 1 \\
\hline n-Propylbenzene & 0 of 1 & ND $(0.078)$ & ND $(0.078)$ & NAN & $0.25^{\mathrm{C}}$ & 0 of 1 \\
\hline Naphthalene & 0 of 1 & ND (0.078) & ND (0.078) & NAN & $3.85^{c}$ & 0 of 1 \\
\hline o-Xylene & 0 of 1 & ND (0.078) & ND (0.078) & NAN & $0.12^{\mathrm{C}}$ & 0 of 1 \\
\hline p-Isopropyltoluene & 0 of 1 & ND (0.078) & ND $(0.078)$ & NAN & $2.8^{\mathrm{C}}$ & 0 of 1 \\
\hline sec-Butylbenzene & 0 of 1 & ND (0.078) & ND (0.078) & NAN & $24^{c}$ & 0 of 1 \\
\hline Toluene & 0 of 1 & ND $(0.078)$ & ND $(0.078)$ & NAN & $2.5^{\mathrm{c}}$ & 0 of 1 \\
\hline \multicolumn{7}{|c|}{ Polycyclic Aromatic Hydrocarbons } \\
\hline Acenaphthene & 0 of 5 & ND $(0.0125)$ & ND $(0.0387)$ & NAN & $4.91^{c}$ & 0 of 5 \\
\hline Acenaphthylene & 0 of 5 & ND (0.0125) & ND $(0.0387)$ & NAN & $4.52^{\mathrm{C}}$ & 0 of 5 \\
\hline Anthracene & 0 of 5 & ND (0.0125) & ND (0.0387) & NAN & $5.94^{c}$ & 0 of 5 \\
\hline Benzo(a)anthracene & 0 of 5 & ND (0.0125) & ND $(0.0387)$ & NAN & $8.41^{c}$ & 0 of 5 \\
\hline Benzo(a)pyrene & 0 of 5 & ND (0.0125) & ND $(0.0387)$ & NAN & $9.65^{c}$ & 0 of 5 \\
\hline Benzo(b)fluoranthene & 0 of 5 & $\mathrm{ND}(0.0125)$ & ND $(0.0387)$ & NAN & $9.79^{c}$ & 0 of 5 \\
\hline Benzo(g,h,i)perylene & 0 of 5 & ND (0.0125) & ND $(0.0387)$ & NAN & $10.95^{\mathrm{C}}$ & 0 of 5 \\
\hline
\end{tabular}


Table 5-26

\section{Summary of Analytical Results and Ecological Risk Assessment Screening for Sediment in White Alice Creek, Cannikin Site}

(Page 2 of 3 )

\begin{tabular}{|c|c|c|c|c|c|c|}
\hline Analyte & $\begin{array}{l}\text { Detection } \\
\text { Frequency }\end{array}$ & $\begin{array}{c}\text { Minimum } \\
\text { Detected } \\
\text { Concentration } \\
(\mathbf{m g} / \mathbf{k g})\end{array}$ & $\begin{array}{c}\text { Maximum } \\
\text { Detected } \\
\text { Concentration } \\
(\mathbf{m g} / \mathbf{k g})\end{array}$ & $\begin{array}{l}\text { Lowland Stream } \\
\text { Background } \\
\text { Concentration }{ }^{\mathrm{a}} \\
(\mathrm{mg} / \mathrm{kg})\end{array}$ & $\begin{array}{c}\text { Ecological } \\
\text { Screening } \\
\text { Value } \\
(\mathrm{mg} / \mathrm{kg})\end{array}$ & $\begin{array}{l}\text { Detection } \\
\text { Frequency } \\
\text { Above ESV }\end{array}$ \\
\hline Beno(k)fluoranthene & 0 of 5 & ND (0.0125) & ND (0.0387) & NAN & $9.81^{\mathrm{C}}$ & 0 of 5 \\
\hline Chrysene & 0 of 5 & ND (0.0125) & ND (0.0387) & NAN & $8.44^{c}$ & 0 of 5 \\
\hline Dibenzo(a,h)anthracene & 0 of 5 & ND (0.0125) & ND (0.0387) & NAN & $11.23^{\mathrm{C}}$ & 0 of 5 \\
\hline Fluoranthene & 0 of 5 & ND (0.0125) & ND $(0.0387)$ & NAN & $7.07^{c}$ & 0 of 5 \\
\hline Fluorene & 0 of 5 & ND (0.0125) & ND (0.0387) & NAN & $5.38^{\mathrm{C}}$ & 0 of 5 \\
\hline Indeno(1,2,3-c,d)pyrene & 0 of 5 & ND (0.0125) & ND (0.0387) & NAN & $11.15^{\mathrm{c}}$ & 0 of 5 \\
\hline Naphthalene & NAV & NAV & NAV & NAN & NAV & - \\
\hline Phenanthrene & 0 of 5 & ND (0.0125) & $\mathrm{ND}(0.0387)$ & NAN & $5.96^{\mathrm{C}}$ & 0 of 5 \\
\hline Pyrene & 0 of 5 & ND $(0.0125)$ & ND $(0.0387)$ & NAN & $6.97^{\mathrm{C}}$ & 0 of 5 \\
\hline \multicolumn{7}{|c|}{ Polychlorinated Biphenyls } \\
\hline Aroclor 1016 & 0 of 5 & ND (0.19) & ND $(0.586)$ & NAN & $0.07^{C}$ & 0 of 5 \\
\hline Aroclor 1221 & 0 of 5 & ND (0.19) & ND (0.586) & NAN & $0.07^{c}$ & 0 of 5 \\
\hline Aroclor 1232 & 0 of 5 & ND (0.19) & ND (0.586) & NAN & $0.07^{c}$ & 0 of 5 \\
\hline Aroclor 1242 & 0 of 5 & ND (0.19) & ND (0.586) & NAN & $0.07^{c}$ & 0 of 5 \\
\hline Aroclor 1248 & 0 of 5 & ND (0.19) & ND (0.586) & NAN & $0.07^{c}$ & 0 of 5 \\
\hline Aroclor 1254 & 0 of 5 & ND (0.19) & ND (0.586) & NAN & $0.07^{c}$ & 0 of 5 \\
\hline Aroclor 1260 & 0 of 5 & ND (0.19) & ND (0.586) & NAN & $0.07^{\mathrm{C}}$ & 0 of 5 \\
\hline Aroclor 1262 & 0 of 5 & ND (0.19) & ND (0.586) & NAN & $0.07^{\mathrm{C}}$ & 0 of 5 \\
\hline Aroclor 1268 & 0 of 5 & ND (0.19) & ND (0.586) & NAN & $0.07^{c}$ & 0 of 5 \\
\hline \multicolumn{7}{|c|}{ Metals } \\
\hline Aluminum & 5 of 5 & 6,480 & 68,300 & 45,400 & 58,000 & 2 of 5 \\
\hline Arsenic & 4 of 5 & ND (5.86) & 109 & 100 & 6.0 & 4 of 5 \\
\hline Barium & 3 of 5 & ND (58.6) & 377 & 469 & NAV & \\
\hline Beryllium & 0 of 5 & ND (5.86) & ND (94.8) & ND & NAV & \\
\hline Boron & 0 of 5 & ND (9.48) & ND (29.3) & $7.18^{b}$ & NAV & \\
\hline Cadmium & 0 of 5 & ND (1.9) & ND (5.86) & $0.608^{b}$ & 0.6 & 0 of 5 \\
\hline Calcium & 5 of 5 & 5,540 & 10,800 & 7,300 & NAV & \\
\hline Cerium & 4 of 5 & ND (5.86) & 20.9 & 14.2 & NAV & \\
\hline Cesium & 0 of 5 & ND (1.9) & ND (5.86) & ND & NAV & \\
\hline
\end{tabular}


Table 5-26

\section{Summary of Analytical Results and Ecological Risk Assessment Screening for Sediment in White Alice Creek, Cannikin Site} (Page 3 of 3 )

\begin{tabular}{|c|c|c|c|c|c|c|}
\hline Analyte & $\begin{array}{l}\text { Detection } \\
\text { Frequency }\end{array}$ & $\begin{array}{c}\text { Minimum } \\
\text { Detected } \\
\text { Concentration } \\
(\mathbf{m g} / \mathbf{k g})\end{array}$ & $\begin{array}{c}\text { Maximum } \\
\text { Detected } \\
\text { Concentration } \\
(\mathbf{m g} / \mathbf{k g})\end{array}$ & $\begin{array}{c}\text { Lowland Stream } \\
\text { Background } \\
\text { Concentration }{ }^{a} \\
(\mathrm{mg} / \mathrm{kg})\end{array}$ & $\begin{array}{c}\text { Ecological } \\
\text { Screening } \\
\text { Value } \\
(\mathrm{mg} / \mathrm{kg})\end{array}$ & $\begin{array}{l}\text { Detection } \\
\text { Frequency } \\
\text { Above ESV }\end{array}$ \\
\hline Chromium & 5 of 5 & 13.1 & 27.5 & 11.8 & 26 & 1 of 5 \\
\hline Cobalt & 4 of 5 & ND (5.86) & 38.2 & 43.7 & NAV & \\
\hline Copper & 5 of 5 & 28.7 & 168 & 71.1 & 16 & 5 of 5 \\
\hline Iron & 5 of 5 & 6,900 & 96,800 & 155,000 & 20,000 & 4 of 5 \\
\hline Lithium & 4 of 5 & ND (5.86) & 22.7 & 48.8 & NAV & \\
\hline Magnesium & 5 of 5 & 1,530 & 36,600 & 9,320 & NAV & \\
\hline Manganese & 5 of 5 & 442 & 10,300 & 20,700 & 460 & 4 of 5 \\
\hline Molybdenum & 0 of 5 & ND (1.9) & ND (5.86) & $5.5^{b}$ & NAV & \\
\hline Nickel & 5 of 5 & 6.22 & 24.9 & 13.6 & 16 & 3 of 5 \\
\hline Potassium & 5 of 5 & 249 & 3,880 & 1,390 & NAV & \\
\hline Selenium & 2 of 5 & 2.21 & 4.52 & $5.44^{b}$ & NAV & \\
\hline Strontium & 5 of 5 & 49.5 & 547 & 164 & NAV & \\
\hline Thallium & 0 of 5 & ND (1.9) & ND (5.86) & $1^{b}$ & NAV & \\
\hline Thorium & 0 of 5 & ND (1.9) & ND (5.86) & $0.5^{b}$ & NAV & \\
\hline Titanium & 5 of 5 & 228 & 2,010 & 3,010 & NAV & \\
\hline Uranium & 0 of 5 & ND (1.9) & ND (5.86) & $4.13^{b}$ & NAV & \\
\hline Vanadium & 5 of 5 & 40.6 & 645 & 734 & NAV & \\
\hline Zinc & 4 of 5 & 71.4 & 248 & 434 & 120 & 2 of 5 \\
\hline Total Organic Carbon & 5 of 5 & 8,250 & 158,000 & NAN & & \\
\hline
\end{tabular}

${ }^{\mathrm{a} B}$ Background concentrations, which are presented only for metals, are upper tolerance limits (UTL) unless otherwise noted.

${ }^{b}$ This metal was not detected in a sufficient number of reference stream samples to allow calculation of a valid UTL. The concentration shown is the maximum detected concentration in the appropriate background samples.

${ }^{\mathrm{c}}$ Screening concentration is based on $1 \%$ organic carbon.

ND - Analyte not detected at a concentration above the reporting limit, which is shown in parentheses.

NAV - Not available

NAN - Not analyzed

$\mathrm{mg} / \mathrm{kg}$ - Milligrams per kilogram 
Table 5-27

\section{Summary of Analytical Results and Ecological Risk Assessment Screening for Surface Water in Reference Stream No. 1}

(Page 1 of 3 )

\begin{tabular}{|c|c|c|c|c|c|}
\hline Analyte & $\begin{array}{l}\text { Detection } \\
\text { Frequency }\end{array}$ & $\begin{array}{c}\text { Minimum } \\
\text { Detected } \\
\text { Concentration } \\
(\mu \mathrm{g} / \mathrm{L})\end{array}$ & $\begin{array}{c}\text { Maximum } \\
\text { Detected } \\
\text { Concentration } \\
(\mu \mathrm{g} / \mathrm{L})\end{array}$ & $\begin{array}{c}\text { Ecological } \\
\text { Screening Value } \\
(\mu \mathrm{g} / \mathrm{L})\end{array}$ & $\begin{array}{c}\text { Detection } \\
\text { Frequency Above } \\
\text { ESV }\end{array}$ \\
\hline $\begin{array}{l}\text { Diesel-Range } \\
\text { Hydrocarbons }\end{array}$ & 5 of 5 & 50.8 & 146 & 15 & 5 of 5 \\
\hline \multicolumn{6}{|c|}{ Volatile Organic Compounds } \\
\hline 1,2,4-Trimethylbenzene & NAV & NAV & NAV & NAV & - \\
\hline 1,3,5-Trimethylbenzene & NAV & NAV & NAV & NAV & - \\
\hline 2-Butanone & NAV & NAV & NAV & NAV & - \\
\hline Benzene & NAV & NAV & NAV & NAV & - \\
\hline Acetone & NAV & NAV & NAV & NAV & - \\
\hline Carbon disulfide & NAV & NAV & NAV & NAV & - \\
\hline Ethylbenzene & NAV & NAV & NAV & NAV & - \\
\hline Isopropylbenzene & NAV & NAV & NAV & NAV & - \\
\hline m,p-Xylene & NAV & NAV & NAV & NAV & - \\
\hline Methylene chloride & NAV & NAV & NAV & NAV & - \\
\hline n-Butylbenzene & NAV & NAV & NAV & NAV & - \\
\hline n-Propylbenzene & NAV & NAV & NAV & NAV & - \\
\hline Naphthalene & NAV & NAV & NAV & NAV & - \\
\hline o-Xylene & NAV & NAV & NAV & NAV & - \\
\hline p-Isopropyltoluene & NAV & NAV & NAV & NAV & - \\
\hline sec-Butylbenzene & NAV & NAV & NAV & NAV & - \\
\hline Toluene & NAV & NAV & NAV & NAV & - \\
\hline \multicolumn{6}{|c|}{ Polycyclic Aromatic Hydrocarbons } \\
\hline Acenaphthene & NAV & NAV & NAV & NAV & - \\
\hline Acenaphthylene & NAV & NAV & NAV & NAV & - \\
\hline Anthracene & NAV & NAV & NAV & NAV & - \\
\hline Benzo(a)anthracene & NAV & NAV & NAV & NAV & - \\
\hline Benzo(a)pyrene & NAV & NAV & NAV & NAV & - \\
\hline Benzo(b)fluoranthene & NAV & NAV & NAV & NAV & - \\
\hline Benzo(g,h,i)perylene & NAV & NAV & NAV & NAV & - \\
\hline Benzo(k)fluoranthene & NAV & NAV & NAV & NAV & - \\
\hline
\end{tabular}


Table 5-27

\section{Summary of Analytical Results and Ecological Risk Assessment Screening for Surface Water in Reference Stream No. 1}

(Page 2 of 3 )

\begin{tabular}{|c|c|c|c|c|c|}
\hline Analyte & $\begin{array}{l}\text { Detection } \\
\text { Frequency }\end{array}$ & $\begin{array}{c}\text { Minimum } \\
\text { Detected } \\
\text { Concentration } \\
(\mu \mathrm{g} / \mathrm{L})\end{array}$ & $\begin{array}{c}\text { Maximum } \\
\text { Detected } \\
\text { Concentration } \\
(\mu \mathrm{g} / \mathrm{L})\end{array}$ & $\begin{array}{c}\text { Ecological } \\
\text { Screening Value } \\
(\mu \mathrm{g} / \mathrm{L})\end{array}$ & $\begin{array}{c}\text { Detection } \\
\text { Frequency Above } \\
\text { ESV }\end{array}$ \\
\hline Chrysene & NAV & NAV & NAV & NAV & - \\
\hline Dibenzo(a,h)anthracene & NAV & NAV & NAV & NAV & - \\
\hline Fluoranthene & NAV & NAV & NAV & NAV & - \\
\hline Fluorene & NAV & NAV & NAV & NAV & - \\
\hline Indeno(1,2,3-c,d)pyrene & NAV & NAV & NAV & NAV & - \\
\hline Naphthalene & NAV & NAV & NAV & NAV & - \\
\hline Phenanthrene & NAV & NAV & NAV & NAV & - \\
\hline Pyrene & NAV & NAV & NAV & NAV & - \\
\hline \multicolumn{6}{|c|}{ Polychlorinated Biphenyls } \\
\hline Aroclor 1016 & NAV & NAV & NAV & NAV & - \\
\hline Aroclor 1221 & NAV & NAV & NAV & NAV & - \\
\hline Aroclor 1232 & NAV & NAV & NAV & NAV & - \\
\hline Aroclor 1242 & NAV & NAV & NAV & NAV & - \\
\hline Aroclor 1248 & NAV & NAV & NAV & NAV & - \\
\hline Aroclor 1254 & NAV & NAV & NAV & NAV & - \\
\hline Aroclor 1260 & NAV & NAV & NAV & NAV & - \\
\hline Aroclor 1262 & NAV & NAV & NAV & NAV & - \\
\hline Aroclor 1268 & NAV & NAV & NAV & NAV & - \\
\hline \multicolumn{6}{|c|}{ Metals } \\
\hline Aluminum & 5 of 5 & 119 & 175 & 87 & 5 of 5 \\
\hline Arsenic & 0 of 5 & ND (1.0) & ND (1.0) & 150 & 0 of 5 \\
\hline Barium & 0 of 5 & ND (10) & ND (10) & 4.0 & 0 of 5 \\
\hline Beryllium & 0 of 5 & ND (1.0) & ND (1.0) & 0.66 & 0 of 5 \\
\hline Boron & 0 of 5 & ND (50) & ND (50) & 1.6 & 0 of 5 \\
\hline Cadmium & 0 of 5 & ND (1.0) & ND (1.0) & 2.2 & 0 of 5 \\
\hline Calcium & 5 of 5 & 1,110 & 1,440 & 116,000 & 0 of 5 \\
\hline Cerium & 0 of 5 & ND (1.0) & ND (1.0) & 0.5 & 0 of 5 \\
\hline Cesium & 0 of 5 & ND (1.0) & ND (1.0) & 30 & 0 of 5 \\
\hline Chromium & 0 of 5 & ND (1.0) & ND (1.0) & 74 & 0 of 5 \\
\hline
\end{tabular}


Table 5-27

\section{Summary of Analytical Results and Ecological Risk Assessment} Screening for Surface Water in Reference Stream No. 1

(Page 3 of 3 )

\begin{tabular}{|c|c|c|c|c|c|}
\hline Analyte & $\begin{array}{l}\text { Detection } \\
\text { Frequency }\end{array}$ & $\begin{array}{c}\text { Minimum } \\
\text { Detected } \\
\text { Concentration } \\
(\mu \mathrm{g} / \mathrm{L})\end{array}$ & $\begin{array}{c}\text { Maximum } \\
\text { Detected } \\
\text { Concentration } \\
(\mu \mathrm{g} / \mathrm{L})\end{array}$ & $\begin{array}{c}\text { Ecological } \\
\text { Screening Value } \\
(\mu \mathrm{g} / \mathrm{L})\end{array}$ & $\begin{array}{c}\text { Detection } \\
\text { Frequency Above } \\
\text { ESV }\end{array}$ \\
\hline Cobalt & 5 of 5 & 1.19 & 2.44 & 23 & 0 of 5 \\
\hline Copper & 5 of 5 & 1.16 & 5.29 & 9.0 & 0 of 5 \\
\hline Iron & 5 of 5 & 552 & 877 & 1,000 & 0 of 5 \\
\hline Lithium & 0 of 5 & ND (1.0) & ND (1.0) & 14 & 0 of 5 \\
\hline Magnesium & 5 of 5 & 1,510 & 1,630 & 82,000 & 0 of 5 \\
\hline Manganese & 5 of 5 & 8.3 & 28.4 & 120 & 0 of 5 \\
\hline Molybdenum & 0 of 5 & ND (1.0) & ND (1.0) & 370 & 0 of 5 \\
\hline Nickel & 0 of 5 & ND (1.0) & ND (1.0) & 52 & 0 of 5 \\
\hline Potassium & 5 of 5 & 264 & 800 & 53,000 & 0 of 5 \\
\hline Selenium & 0 of 5 & ND (1.0) & ND (1.0) & 5.0 & 0 of 5 \\
\hline Strontium & 5 of 5 & 11.2 & 12.5 & 1,500 & 0 of 5 \\
\hline Thallium & 0 of 5 & ND (1.0) & ND (1.0) & 12 & 0 of 5 \\
\hline Thorium & 0 of 5 & ND (1.0) & ND (1.0) & NAV & 0 of 5 \\
\hline Titanium & 5 of 5 & 3.11 & 4.34 & 29 & 0 of 5 \\
\hline Uranium & 0 of 5 & ND (1.0) & ND (1.0) & 2.6 & 0 of 5 \\
\hline Vanadium & 3 of 5 & 1.09 & 1.68 & 20 & 0 of 5 \\
\hline Zinc & 1 of 5 & ND (10) & 50.3 & 120 & 0 of 5 \\
\hline
\end{tabular}

ND - Analyte not detected at a concentration above the reporting limit, which is shown in parentheses.

NAV - Not available

$\mu \mathrm{g} / \mathrm{L}$ - Micrograms per liter 
Table 5-28

Summary of Analytical Results and Ecological Risk Assessment Screening for Sediment in Reference Stream No. 1 (Page 1 of 3 )

\begin{tabular}{|c|c|c|c|c|c|}
\hline Analyte & $\begin{array}{l}\text { Detection } \\
\text { Frequency }\end{array}$ & $\begin{array}{c}\text { Minimum } \\
\text { Detected } \\
\text { Concentration } \\
(\mathbf{m g} / \mathbf{k g})\end{array}$ & $\begin{array}{c}\text { Maximum } \\
\text { Detected } \\
\text { Concentration } \\
(\mathbf{m g} / \mathbf{k g})\end{array}$ & $\begin{array}{c}\text { Ecological } \\
\text { Screening } \\
\text { Value }(\mathbf{m g} / \mathbf{k g})\end{array}$ & $\begin{array}{l}\text { Detection } \\
\text { Frequency } \\
\text { Above ESV }\end{array}$ \\
\hline Diesel-Range Hydrocarbons & 0 of 5 & ND (18.9) & ND (27.8) & $640^{\mathrm{a}}$ & 0 of 5 \\
\hline \multicolumn{6}{|c|}{ Volatile Organic Compounds } \\
\hline 1,2,4-Trimethylbenzene & NAV & NAV & NAV & NAV & - \\
\hline 1,3,5-Trimethylbenzene & NAV & NAV & NAV & NAV & - \\
\hline 2-Butanone & NAV & NAV & NAV & NAV & - \\
\hline Benzene & NAV & NAV & NAV & NAV & - \\
\hline Acetone & NAV & NAV & NAV & NAV & - \\
\hline Carbon disulfide & NAV & NAV & NAV & NAV & - \\
\hline Ethylbenzene & NAV & NAV & NAV & NAV & - \\
\hline Isopropylbenzene & NAV & NAV & NAV & NAV & - \\
\hline m,p-Xylene & NAV & NAV & NAV & NAV & - \\
\hline Methylene chloride & NAV & NAV & NAV & NAV & - \\
\hline n-Butylbenzene & NAV & NAV & NAV & NAV & - \\
\hline n-Propylbenzene & NAV & NAV & NAV & NAV & - \\
\hline Naphthalene & NAV & NAV & NAV & NAV & - \\
\hline o-Xylene & NAV & NAV & NAV & NAV & - \\
\hline p-Isopropyltoluene & NAV & NAV & NAV & NAV & - \\
\hline sec-Butylbenzene & NAV & NAV & NAV & NAV & - \\
\hline Toluene & NAV & NAV & NAV & NAV & - \\
\hline \multicolumn{6}{|c|}{ Polycyclic Aromatic Hydrocarbons } \\
\hline Acenaphthene & NAV & NAV & NAV & NAV & - \\
\hline Acenaphthylene & NAV & NAV & NAV & NAV & - \\
\hline Anthracene & NAV & NAV & NAV & NAV & - \\
\hline Benzo(a)anthracene & NAV & NAV & NAV & NAV & - \\
\hline Benzo(a)pyrene & NAV & NAV & NAV & NAV & - \\
\hline Benzo(b)fluoranthene & NAV & NAV & NAV & NAV & - \\
\hline Benzo(g,h,i)perylene & NAV & NAV & NAV & NAV & - \\
\hline Benzo(k)fluoranthene & NAV & NAV & NAV & NAV & - \\
\hline Chrysene & NAV & NAV & NAV & NAV & - \\
\hline
\end{tabular}


Table 5-28

Summary of Analytical Results and Ecological Risk Assessment Screening for Sediment in Reference Stream No. 1 (Page 2 of 3 )

\begin{tabular}{|c|c|c|c|c|c|}
\hline Analyte & $\begin{array}{l}\text { Detection } \\
\text { Frequency }\end{array}$ & $\begin{array}{c}\text { Minimum } \\
\text { Detected } \\
\text { Concentration } \\
(\mathbf{m g} / \mathbf{k g})\end{array}$ & $\begin{array}{c}\text { Maximum } \\
\text { Detected } \\
\text { Concentration } \\
(\mathbf{m g} / \mathbf{k g})\end{array}$ & $\begin{array}{c}\text { Ecological } \\
\text { Screening } \\
\text { Value }(\mathbf{m g} / \mathbf{k g})\end{array}$ & $\begin{array}{c}\text { Detection } \\
\text { Frequency } \\
\text { Above ESV }\end{array}$ \\
\hline Dibenzo(a,h)anthracene & NAV & NAV & NAV & NAV & - \\
\hline Fluoranthene & NAV & NAV & NAV & NAV & - \\
\hline Fluorene & NAV & NAV & NAV & NAV & - \\
\hline Indeno $(1,2,3-c, d)$ pyrene & NAV & NAV & NAV & NAV & - \\
\hline Naphthalene & NAV & NAV & NAV & NAV & - \\
\hline Phenanthrene & NAV & NAV & NAV & NAV & - \\
\hline Pyrene & NAV & NAV & NAV & NAV & - \\
\hline \multicolumn{6}{|c|}{ Polychlorinated Biphenyls } \\
\hline Aroclor 1016 & 0 of 1 & ND $(0.237)$ & ND $(0.237)$ & $0.07^{\mathrm{a}}$ & 0 of 1 \\
\hline Aroclor 1221 & 0 of 1 & ND $(0.237)$ & ND $(0.237)$ & $0.07^{\mathrm{a}}$ & 0 of 1 \\
\hline Aroclor 1232 & 0 of 1 & ND $(0.237)$ & ND $(0.237)$ & $0.07^{\mathrm{a}}$ & 0 of 1 \\
\hline Aroclor 1242 & 0 of 1 & ND (0.237) & ND $(0.237)$ & $0.07^{a}$ & 0 of 1 \\
\hline Aroclor 1248 & 0 of 1 & ND (0.237) & ND (0.237) & $0.07^{a}$ & 0 of 1 \\
\hline Aroclor 1254 & 0 of 1 & ND (0.237) & ND (0.237) & $0.07^{a}$ & 0 of 1 \\
\hline Aroclor 1260 & 0 of 1 & ND (0.237) & ND (0.237) & $0.07^{\mathrm{a}}$ & 0 of 1 \\
\hline Aroclor 1262 & 0 of 1 & ND (0.237) & ND (0.237) & $0.07^{\mathrm{a}}$ & 0 of 1 \\
\hline Aroclor 1268 & 0 of 1 & ND (0.237) & ND (0.237) & $0.07^{a}$ & 0 of 1 \\
\hline \multicolumn{6}{|c|}{ Metals } \\
\hline Aluminum & 5 of 5 & 18,400 & 33,100 & 58,000 & 0 of 5 \\
\hline Arsenic & 5 of 5 & 6.63 & 21.1 & 6.0 & 5 of 5 \\
\hline Barium & 5 of 5 & 74.1 & 175 & NAV & \\
\hline Beryllium & 0 of 5 & ND (2.37) & ND (3.47) & NAV & \\
\hline Boron & 0 of 5 & ND (11.8) & ND (17.4) & NAV & \\
\hline Cadmium & 0 of 5 & ND (2.37) & ND (3.47) & 0.6 & 0 of 5 \\
\hline Calcium & 5 of 5 & 1,480 & 4,980 & NAV & \\
\hline Cerium & 5 of 5 & 8.12 & 12.6 & NAV & \\
\hline Cesium & 0 of 5 & ND (2.37) & ND (3.47) & NAV & \\
\hline Chromium & 5 of 5 & 5.66 & 8.51 & 26 & 0 of 5 \\
\hline Cobalt & 5 of 5 & 11 & 35.4 & NAV & \\
\hline
\end{tabular}


Table 5-28

Summary of Analytical Results and Ecological Risk Assessment Screening for Sediment in Reference Stream No. 1 (Page 3 of 3 )

\begin{tabular}{|c|c|c|c|c|c|}
\hline Analyte & $\begin{array}{l}\text { Detection } \\
\text { Frequency }\end{array}$ & $\begin{array}{c}\text { Minimum } \\
\text { Detected } \\
\text { Concentration } \\
(\mathbf{m g} / \mathbf{k g})\end{array}$ & $\begin{array}{c}\text { Maximum } \\
\text { Detected } \\
\text { Concentration } \\
(\mathbf{m g} / \mathbf{k g})\end{array}$ & $\begin{array}{c}\text { Ecological } \\
\text { Screening } \\
\text { Value }(\mathbf{m g} / \mathbf{k g})\end{array}$ & $\begin{array}{c}\text { Detection } \\
\text { Frequency } \\
\text { Above ESV }\end{array}$ \\
\hline Copper & 5 of 5 & 24.5 & 51.5 & 16 & 5 of 5 \\
\hline Iron & 5 of 5 & 10,400 & 81,400 & 20,000 & 4 of 5 \\
\hline Lithium & 5 of 5 & 7.02 & 15.5 & NAV & \\
\hline Magnesium & 5 of 5 & 2,690 & 8,220 & NAV & \\
\hline Manganese & 5 of 5 & 125 & 14,400 & 460 & 4 of 5 \\
\hline Molybdenum & 1 of 5 & ND (2.37) & 5.5 & NAV & \\
\hline Nickel & 5 of 5 & 4.98 & 8.04 & 16 & 0 of 5 \\
\hline Potassium & 5 of 5 & 183 & 826 & NAV & \\
\hline Selenium & 3 of 5 & ND (2.37) & 5.44 & NAV & \\
\hline Strontium & 5 of 5 & 36.5 & 101 & NAV & \\
\hline Thallium & 0 of 5 & ND (2.37) & ND (3.47) & NAV & \\
\hline Thorium & 0 of 5 & ND (2.37) & ND (3.47) & NAV & \\
\hline Titanium & 5 of 5 & 210 & 968 & NAV & \\
\hline Uranium & 1 of 5 & ND (2.37) & 4.13 & NAV & \\
\hline Vanadium & 5 of 5 & 194 & 245 & NAV & \\
\hline Zinc & 5 of 5 & 45.5 & 167 & 120 & 1 of 5 \\
\hline Total Organic Carbon & 5 of 5 & 67,100 & 98,400 & & \\
\hline
\end{tabular}

${ }^{a}$ Screening concentration is based on $1 \%$ organic carbon.

ND - Analyte not detected at a concentration above the reporting limit, which is shown in parentheses.

NAV - Not available

$\mathrm{mg} / \mathrm{kg}$ - Milligrams per kilogram 
Table 5-29

\section{Summary of Analytical Results and Ecological Risk Assessment Screening for Surface Water in Reference Stream No. 2}

(Page 1 of 3 )

\begin{tabular}{|c|c|c|c|c|c|}
\hline Analyte & $\begin{array}{l}\text { Detection } \\
\text { Frequency }\end{array}$ & $\begin{array}{c}\text { Minimum } \\
\text { Detected } \\
\text { Concentration } \\
(\mu \mathrm{g} / \mathrm{L})\end{array}$ & $\begin{array}{c}\text { Maximum } \\
\text { Detected } \\
\text { Concentration } \\
(\mu \mathrm{g} / \mathrm{L})\end{array}$ & $\begin{array}{c}\text { Ecological } \\
\text { Screening Value } \\
(\mu \mathrm{g} / \mathrm{L})\end{array}$ & $\begin{array}{c}\text { Detection } \\
\text { Frequency Above } \\
\text { ESV }\end{array}$ \\
\hline $\begin{array}{l}\text { Diesel-Range } \\
\text { Hydrocarbons }\end{array}$ & 0 of 5 & ND (71.3) & ND (147) & 15 & 0 of 5 \\
\hline \multicolumn{6}{|c|}{ Volatile Organic Compounds } \\
\hline 1,2,4-Trimethylbenzene & NAV & NAV & NAV & NAV & - \\
\hline 1,3,5-Trimethylbenzene & NAV & NAV & NAV & NAV & - \\
\hline 2-Butanone & NAV & NAV & NAV & NAV & - \\
\hline Acetone & NAV & NAV & NAV & NAV & - \\
\hline Benzene & NAV & NAV & NAV & NAV & - \\
\hline Carbon disulfide & NAV & NAV & NAV & NAV & - \\
\hline Ethylbenzene & NAV & NAV & NAV & NAV & - \\
\hline Isopropylbenzene & NAV & NAV & NAV & NAV & - \\
\hline m,p-Xylene & NAV & NAV & NAV & NAV & - \\
\hline Methylene chloride & NAV & NAV & NAV & NAV & - \\
\hline n-Butylbenzene & NAV & NAV & NAV & NAV & - \\
\hline n-Propylbenzene & NAV & NAV & NAV & NAV & - \\
\hline Naphthalene & NAV & NAV & NAV & NAV & - \\
\hline o-Xylene & NAV & NAV & NAV & NAV & - \\
\hline p-Isopropyltoluene & NAV & NAV & NAV & NAV & - \\
\hline sec-Butylbenzene & NAV & NAV & NAV & NAV & - \\
\hline Toluene & NAV & NAV & NAV & NAV & - \\
\hline \multicolumn{6}{|c|}{ Polycyclic Aromatic Hydrocarbons } \\
\hline Acenaphthene & NAV & NAV & NAV & NAV & - \\
\hline Acenaphthylene & NAV & NAV & NAV & NAV & - \\
\hline Anthracene & NAV & NAV & NAV & NAV & - \\
\hline Benzo(a)anthracene & NAV & NAV & NAV & NAV & - \\
\hline Benzo(a)pyrene & NAV & NAV & NAV & NAV & - \\
\hline Benzo(b)fluoranthene & NAV & NAV & NAV & NAV & - \\
\hline Benzo(k)fluoranthene & NAV & NAV & NAV & NAV & - \\
\hline Benzo(g,h,i)perylene & NAV & NAV & NAV & NAV & - \\
\hline
\end{tabular}


Table 5-29

\section{Summary of Analytical Results and Ecological Risk Assessment Screening for Surface Water in Reference Stream No. 2}

(Page 2 of 3 )

\begin{tabular}{|c|c|c|c|c|c|}
\hline Analyte & $\begin{array}{l}\text { Detection } \\
\text { Frequency }\end{array}$ & $\begin{array}{c}\text { Minimum } \\
\text { Detected } \\
\text { Concentration } \\
(\mu \mathrm{g} / \mathrm{L})\end{array}$ & $\begin{array}{c}\text { Maximum } \\
\text { Detected } \\
\text { Concentration } \\
(\mu \mathrm{g} / \mathrm{L})\end{array}$ & $\begin{array}{c}\text { Ecological } \\
\text { Screening Value } \\
(\mu \mathrm{g} / \mathrm{L})\end{array}$ & $\begin{array}{c}\text { Detection } \\
\text { Frequency Above } \\
\text { ESV }\end{array}$ \\
\hline Chrysene & NAV & NAV & NAV & NAV & - \\
\hline Dibenzo(a,h)anthracene & NAV & NAV & NAV & NAV & - \\
\hline Fluoranthene & NAV & NAV & NAV & NAV & - \\
\hline Fluorene & NAV & NAV & NAV & NAV & - \\
\hline Ideno(1,2,3-c,d)pyrene & NAV & NAV & NAV & NAV & - \\
\hline Naphthalene & NAV & NAV & NAV & NAV & - \\
\hline Phenanthrene & NAV & NAV & NAV & NAV & - \\
\hline Pyrene & NAV & NAV & NAV & NAV & - \\
\hline \multicolumn{6}{|c|}{ Polychlorinated Biphenyls } \\
\hline Aroclor 1016 & NAV & NAV & NAV & NAV & - \\
\hline Aroclor 1221 & NAV & NAV & NAV & NAV & - \\
\hline Aroclor 1232 & NAV & NAV & NAV & NAV & - \\
\hline Aroclor 1242 & NAV & NAV & NAV & NAV & - \\
\hline Aroclor 1248 & NAV & NAV & NAV & NAV & - \\
\hline Aroclor 1254 & NAV & NAV & NAV & NAV & - \\
\hline Aroclor 1260 & NAV & NAV & NAV & NAV & - \\
\hline Aroclor 1262 & NAV & NAV & NAV & NAV & - \\
\hline Aroclor 1268 & NAV & NAV & NAV & NAV & - \\
\hline \multicolumn{6}{|c|}{ Metals } \\
\hline Aluminum & 3 of 5 & ND (50) & 87.5 & 87 & 1 of 5 \\
\hline Arsenic & 0 of 5 & ND (1.0) & ND (1.0) & 150 & 0 of 5 \\
\hline Barium & 0 of 5 & ND (10) & ND (10) & 4.0 & 0 of 5 \\
\hline Beryllium & 0 of 5 & ND (1.0) & ND (1.0) & 0.66 & 0 of 5 \\
\hline Boron & 0 of 5 & ND (50) & ND (50) & 1.6 & 0 of 5 \\
\hline Cadmium & 0 of 5 & ND (1.0) & ND (1.0) & 2.2 & 0 of 5 \\
\hline Calcium & 5 of 5 & 3,320 & 4,320 & 116,000 & 0 of 5 \\
\hline Cerium & 0 of 5 & ND (1.0) & ND (1.0) & 0.5 & 0 of 5 \\
\hline Cesium & 0 of 5 & ND (1.0) & ND (1.0) & 30 & 0 of 5 \\
\hline Chromium & 2 of 5 & ND (1.0) & 2.25 & 74 & 0 of 5 \\
\hline
\end{tabular}


Table 5-29 Summary of Analytical Results and Ecological Risk Assessment
Screening for Surface Water in Reference Stream No. 2

(Page 3 of 3 )

\begin{tabular}{|c|c|c|c|c|c|}
\hline Analyte & $\begin{array}{l}\text { Detection } \\
\text { Frequency }\end{array}$ & $\begin{array}{c}\text { Minimum } \\
\text { Detected } \\
\text { Concentration } \\
(\mu \mathrm{g} / \mathrm{L})\end{array}$ & $\begin{array}{c}\text { Maximum } \\
\text { Detected } \\
\text { Concentration } \\
(\mu \mathrm{g} / \mathrm{L})\end{array}$ & $\begin{array}{c}\text { Ecological } \\
\text { Screening Value } \\
(\mu \mathrm{g} / \mathrm{L})\end{array}$ & $\begin{array}{c}\text { Detection } \\
\text { Frequency Above } \\
\text { ESV }\end{array}$ \\
\hline Cobalt & 3 of 5 & ND (1.0) & 1.35 & 23 & 0 of 5 \\
\hline Copper & 3 of 5 & ND (1.0) & 1.69 & 9.0 & 0 of 5 \\
\hline Iron & 5 of 5 & 315 & 618 & 1,000 & 0 of 5 \\
\hline Lithium & 1 of 5 & ND (1.0) & 1.17 & 14 & 0 of 5 \\
\hline Magnesium & 5 of 5 & 3,430 & 4,690 & 82,000 & 0 of 5 \\
\hline Manganese & 5 of 5 & 5.34 & 86 & 120 & 0 of 5 \\
\hline Molybdenum & 0 of 5 & ND (1.0) & ND (1.0) & 370 & 0 of 5 \\
\hline Nickel & 0 of 5 & ND (1.0) & ND (1.0) & 52 & 0 of 5 \\
\hline Potassium & 5 of 5 & 855 & 2,250 & 53,000 & 0 of 5 \\
\hline Selenium & 0 of 5 & ND (1.0) & ND (1.0) & 5.0 & 0 of 5 \\
\hline Strontium & 5 of 5 & 18.7 & 30.8 & 1,500 & 0 of 5 \\
\hline Thallium & 2 of 5 & ND (1.0) & 7.07 & 12 & 0 of 5 \\
\hline Thorium & 0 of 5 & ND (1.0) & ND (1.0) & NAV & 0 of 5 \\
\hline Titanium & 5 of 5 & 1.31 & 2.91 & 29 & 0 of 5 \\
\hline Uranium & 0 of 5 & ND (1.0) & ND (1.0) & 2.6 & 0 of 5 \\
\hline Vanadium & 2 of 5 & ND (1.0) & 4.43 & 20 & 0 of 5 \\
\hline Zinc & 0 of 5 & ND (10) & ND (10) & 120 & 0 of 5 \\
\hline
\end{tabular}

ND - Analyte not detected at a concentration above the reporting limit, which is shown in parentheses.

NAV - Not available

$\mu \mathrm{g} / \mathrm{L}$ - Micrograms per liter 
Table 5-30

\section{Summary of Analytical Results and Ecological Risk Assessment Screening for Sediment in Reference Stream No. 2}

(Page 1 of 3 )

\begin{tabular}{|c|c|c|c|c|c|}
\hline Analyte & $\begin{array}{l}\text { Detection } \\
\text { Frequency }\end{array}$ & $\begin{array}{c}\text { Minimum } \\
\text { Detected } \\
\text { Concentration } \\
(\mathbf{m g} / \mathbf{k g})\end{array}$ & $\begin{array}{c}\text { Maximum } \\
\text { Detected } \\
\text { Concentration } \\
(\mathbf{m g} / \mathbf{k g})\end{array}$ & $\begin{array}{l}\text { Ecological } \\
\text { Screening } \\
\text { Value }(\mathbf{m g} / \mathbf{k g})\end{array}$ & $\begin{array}{l}\text { Detection } \\
\text { Frequency } \\
\text { Above ESV }\end{array}$ \\
\hline Diesel-Range Hydrocarbons & 4 of 6 & 9.47 & 15.3 & $640^{\mathrm{a}}$ & 0 of 6 \\
\hline \multicolumn{6}{|c|}{ Volatile Organic Compounds } \\
\hline 1,2,4-Trimethylbenzene & NAV & NAV & NAV & NAV & - \\
\hline 1,3,5-Trimethylbenzene & NAV & NAV & NAV & NAV & - \\
\hline 2-Butanone & NAV & NAV & NAV & NAV & - \\
\hline Acetone & NAV & NAV & NAV & NAV & - \\
\hline Benzene & NAV & NAV & NAV & NAV & - \\
\hline Carbon disulfide & NAV & NAV & NAV & NAV & - \\
\hline Ethylbenzene & NAV & NAV & NAV & NAV & - \\
\hline Isopropylbenzene & NAV & NAV & NAV & NAV & - \\
\hline m,p-Xylene & NAV & NAV & NAV & NAV & - \\
\hline Methylene chloride & NAV & NAV & NAV & NAV & - \\
\hline n-Butylbenzene & NAV & NAV & NAV & NAV & - \\
\hline n-Propylbenzene & NAV & NAV & NAV & NAV & - \\
\hline Naphthalene & NAV & NAV & NAV & NAV & - \\
\hline o-Xylene & NAV & NAV & NAV & NAV & - \\
\hline p-Isopropyltoluene & NAV & NAV & NAV & NAV & - \\
\hline sec-Butylbenzene & NAV & NAV & NAV & NAV & - \\
\hline Toluene & NAV & NAV & NAV & NAV & - \\
\hline \multicolumn{6}{|c|}{ Polycyclic Aromatic Hydrocarbons } \\
\hline Acenaphthene & NAV & NAV & NAV & NAV & - \\
\hline Acenaphthylene & NAV & NAV & NAV & NAV & - \\
\hline Anthracene & NAV & NAV & NAV & NAV & - \\
\hline Benzo(a)anthracene & NAV & NAV & NAV & NAV & - \\
\hline Benzo(a)pyrene & NAV & NAV & NAV & NAV & - \\
\hline Benzo(b)fluoranthene & NAV & NAV & NAV & NAV & - \\
\hline Benzo(k)fluoranthene & NAV & NAV & NAV & NAV & - \\
\hline Benzo(g,h,i)perylene & NAV & NAV & NAV & NAV & - \\
\hline Chrysene & NAV & NAV & NAV & NAV & - \\
\hline
\end{tabular}


Table 5-30

\section{Summary of Analytical Results and Ecological Risk Assessment Screening for Sediment in Reference Stream No. 2}

(Page 2 of 3 )

\begin{tabular}{|c|c|c|c|c|c|}
\hline Analyte & $\begin{array}{l}\text { Detection } \\
\text { Frequency }\end{array}$ & $\begin{array}{c}\text { Minimum } \\
\text { Detected } \\
\text { Concentration } \\
(\mathrm{mg} / \mathrm{kg})\end{array}$ & $\begin{array}{c}\text { Maximum } \\
\text { Detected } \\
\text { Concentration } \\
(\mathbf{m g} / \mathbf{k g})\end{array}$ & $\begin{array}{c}\text { Ecological } \\
\text { Screening } \\
\text { Value }(\mathbf{m g} / \mathbf{k g})\end{array}$ & $\begin{array}{c}\text { Detection } \\
\text { Frequency } \\
\text { Above ESV }\end{array}$ \\
\hline Dibenzo(a,h)anthracene & NAV & NAV & NAV & NAV & - \\
\hline Fluoranthene & NAV & NAV & NAV & NAV & - \\
\hline Fluorene & NAV & NAV & NAV & NAV & - \\
\hline Ideno(1,2,3-c,d)pyrene & NAV & NAV & NAV & NAV & - \\
\hline Naphthalene & NAV & NAV & NAV & NAV & - \\
\hline Phenanthrene & NAV & NAV & NAV & NAV & - \\
\hline Pyrene & NAV & NAV & NAV & NAV & - \\
\hline \multicolumn{6}{|c|}{ Polychlorinated Biphenyls } \\
\hline Aroclor 1016 & NAV & NAV & NAV & NAV & - \\
\hline Aroclor 1221 & NAV & NAV & NAV & NAV & - \\
\hline Aroclor 1232 & NAV & NAV & NAV & NAV & - \\
\hline Aroclor 1242 & NAV & NAV & NAV & NAV & - \\
\hline Aroclor 1248 & NAV & NAV & NAV & NAV & - \\
\hline Aroclor 1254 & NAV & NAV & NAV & NAV & - \\
\hline Aroclor 1260 & NAV & NAV & NAV & NAV & - \\
\hline Aroclor 1262 & NAV & NAV & NAV & NAV & - \\
\hline Aroclor 1268 & NAV & NAV & NAV & NAV & - \\
\hline \multicolumn{6}{|c|}{ Metals } \\
\hline Aluminum & 6 of 6 & 14,200 & 35,000 & 58,000 & 0 of 6 \\
\hline Arsenic & 6 of 6 & 2.48 & 40.3 & 6.0 & 3 of 6 \\
\hline Barium & 5 of 6 & 149 & 339 & NAV & \\
\hline Beryllium & 0 of 6 & ND (0.5) & ND (6.83) & NAV & \\
\hline Boron & 2 of 6 & 5.42 & 7.18 & NAV & \\
\hline Cadmium & 1 of 6 & ND (1.27) & 0.608 & 0.6 & 1 of 6 \\
\hline Calcium & 6 of 6 & 1,950 & 5,860 & NAV & \\
\hline Cerium & 6 of 6 & 5.91 & 8.72 & NAV & \\
\hline Cesium & 0 of 6 & ND (0.5) & ND (1.39) & NAV & \\
\hline Chromium & 6 of 6 & 3.4 & 7.57 & 26 & 0 of 6 \\
\hline Cobalt & 6 of 6 & 8.75 & 31.7 & NAV & \\
\hline
\end{tabular}




\section{Table 5-30}

\section{Summary of Analytical Results and Ecological Risk Assessment Screening for Sediment in Reference Stream No. 2} (Page 3 of 3 )

\begin{tabular}{|c|c|c|c|c|c|}
\hline Analyte & $\begin{array}{l}\text { Detection } \\
\text { Frequency }\end{array}$ & $\begin{array}{c}\text { Minimum } \\
\text { Detected } \\
\text { Concentration } \\
(\mathrm{mg} / \mathrm{kg})\end{array}$ & $\begin{array}{c}\text { Maximum } \\
\text { Detected } \\
\text { Concentration } \\
(\mathrm{mg} / \mathrm{kg})\end{array}$ & $\begin{array}{c}\text { Ecological } \\
\text { Screening } \\
\text { Value }(\mathbf{m g} / \mathbf{k g})\end{array}$ & $\begin{array}{l}\text { Detection } \\
\text { Frequency } \\
\text { Above ESV }\end{array}$ \\
\hline Copper & 6 of 6 & 21.5 & 59.3 & 16 & 6 of 6 \\
\hline Iron & 6 of 6 & 37,700 & 137,000 & 20,000 & 6 of 6 \\
\hline Lithium & 6 of 6 & 6.52 & 37.8 & NAV & \\
\hline Magnesium & 6 of 6 & 4,010 & 6,150 & NAV & \\
\hline Manganese & 6 of 6 & 3,300 & 46,300 & 460 & 6 of 6 \\
\hline Molybdenum & 2 of 6 & ND (1.27) & 5 & NAV & \\
\hline Nickel & 6 of 6 & 3.15 & 11.6 & 16 & 0 of 6 \\
\hline Potassium & 6 of 6 & 441 & 1,180 & NAV & \\
\hline Selenium & 1 of 6 & ND (1.27) & 0.951 & NAV & \\
\hline Strontium & 6 of 6 & 45.4 & 148 & NAV & \\
\hline Thallium & 1 of 6 & ND (1.27) & 1 & NAV & \\
\hline Thorium & 1 of 6 & ND (1.27) & 0.5 & NAV & \\
\hline Titanium & 6 of 6 & 976 & 2,130 & NAV & \\
\hline Uranium & 2 of 6 & ND (1.27) & 2.46 & NAV & \\
\hline Vanadium & 6 of 6 & 77.6 & 479 & NAV & \\
\hline Zinc & 6 of 6 & 55.3 & 292 & 120 & 1 of 6 \\
\hline Total Organic Carbon & 6 of 6 & 12,400 & 23,300 & & \\
\hline
\end{tabular}

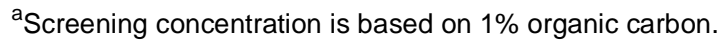

ND - Analyte not detected at a concentration above the reporting limit, which is shown in parentheses.

NAV - Not available

$\mathrm{mg} / \mathrm{kg}$ - Milligrams per kilogram 
Table 5-31

\section{Summary of Analytical Results and Ecological Risk Assessment} Screening for Surface Water in Reference Stream No. 3

(Page 1 of 3 )

\begin{tabular}{|c|c|c|c|c|c|}
\hline Analyte & $\begin{array}{l}\text { Detection } \\
\text { Frequency }\end{array}$ & $\begin{array}{c}\text { Minimum } \\
\text { Detected } \\
\text { Concentration } \\
(\mu \mathrm{g} / \mathrm{L})\end{array}$ & $\begin{array}{c}\text { Maximum } \\
\text { Detected } \\
\text { Concentration } \\
(\mu \mathrm{g} / \mathrm{L})\end{array}$ & $\begin{array}{c}\text { Ecological } \\
\text { Screening Value } \\
(\mu \mathrm{g} / \mathrm{L})\end{array}$ & $\begin{array}{c}\text { Detection } \\
\text { Frequency Above } \\
\text { ESV }\end{array}$ \\
\hline $\begin{array}{l}\text { Diesel-Range } \\
\text { Hydrocarbons }\end{array}$ & 2 of 5 & ND (50) & 66.5 & 15 & 2 of 5 \\
\hline \multicolumn{6}{|c|}{ Volatile Organic Compounds } \\
\hline 1,2,4-Trimethylbenzene & NAV & NAV & NAV & NAV & - \\
\hline 1,3,5-Trimethylbenzene & NAV & NAV & NAV & NAV & - \\
\hline 2-Butanone & NAV & NAV & NAV & NAV & - \\
\hline Acetone & NAV & NAV & NAV & NAV & - \\
\hline Benzene & NAV & NAV & NAV & NAV & - \\
\hline Carbon disulfide & NAV & NAV & NAV & NAV & - \\
\hline Ethylbenzene & NAV & NAV & NAV & NAV & - \\
\hline Isopropylbenzene & NAV & NAV & NAV & NAV & - \\
\hline m,p-Xylene & NAV & NAV & NAV & NAV & - \\
\hline Methylene chloride & NAV & NAV & NAV & NAV & - \\
\hline n-Butylbenzene & NAV & NAV & NAV & NAV & - \\
\hline n-Propylbenzene & NAV & NAV & NAV & NAV & - \\
\hline Naphthalene & NAV & NAV & NAV & NAV & - \\
\hline o-Xylene & NAV & NAV & NAV & NAV & - \\
\hline p-Isopropyltoluene & NAV & NAV & NAV & NAV & - \\
\hline sec-Butylbenzene & NAV & NAV & NAV & NAV & - \\
\hline Toluene & NAV & NAV & NAV & NAV & - \\
\hline \multicolumn{6}{|c|}{ Polycyclic Aromatic Hydrocarbons } \\
\hline Acenaphthene & NAV & NAV & NAV & NAV & - \\
\hline Acenaphthylene & NAV & NAV & NAV & NAV & - \\
\hline Anthracene & NAV & NAV & NAV & NAV & - \\
\hline Benzo(a)anthracene & NAV & NAV & NAV & NAV & - \\
\hline Benzo(a)pyrene & NAV & NAV & NAV & NAV & - \\
\hline Benzo(b)fluoranthene & NAV & NAV & NAV & NAV & - \\
\hline Benzo(k)fluoranthene & NAV & NAV & NAV & NAV & - \\
\hline Benzo(g,h,i)perylene & NAV & NAV & NAV & NAV & - \\
\hline
\end{tabular}


Table 5-31

Summary of Analytical Results and Ecological Risk Assessment Screening for Surface Water in Reference Stream No. 3

(Page 2 of 3 )

\begin{tabular}{|c|c|c|c|c|c|}
\hline Analyte & $\begin{array}{l}\text { Detection } \\
\text { Frequency }\end{array}$ & $\begin{array}{c}\text { Minimum } \\
\text { Detected } \\
\text { Concentration } \\
(\mu \mathrm{g} / \mathrm{L})\end{array}$ & $\begin{array}{c}\text { Maximum } \\
\text { Detected } \\
\text { Concentration } \\
(\mu \mathrm{g} / \mathrm{L})\end{array}$ & $\begin{array}{c}\text { Ecological } \\
\text { Screening Value } \\
(\mu \mathrm{g} / \mathrm{L})\end{array}$ & $\begin{array}{c}\text { Detection } \\
\text { Frequency Above } \\
\text { ESV }\end{array}$ \\
\hline Chrysene & NAV & NAV & NAV & NAV & - \\
\hline Dibenzo(a,h)anthracene & NAV & NAV & NAV & NAV & - \\
\hline Fluoranthene & NAV & NAV & NAV & NAV & - \\
\hline Fluorene & NAV & NAV & NAV & NAV & - \\
\hline Indeno(1,2,3-c,d)pyrene & NAV & NAV & NAV & NAV & - \\
\hline Naphthalene & NAV & NAV & NAV & NAV & - \\
\hline Phenanthrene & NAV & NAV & NAV & NAV & - \\
\hline Pyrene & NAV & NAV & NAV & NAV & - \\
\hline \multicolumn{6}{|c|}{ Polychlorinated Biphenyls } \\
\hline Aroclor 1016 & NAV & NAV & NAV & NAV & - \\
\hline Aroclor 1221 & NAV & NAV & NAV & NAV & - \\
\hline Aroclor 1232 & NAV & NAV & NAV & NAV & - \\
\hline Aroclor 1242 & NAV & NAV & NAV & NAV & - \\
\hline Aroclor 1248 & NAV & NAV & NAV & NAV & - \\
\hline Aroclor 1254 & NAV & NAV & NAV & NAV & - \\
\hline Aroclor 1260 & NAV & NAV & NAV & NAV & - \\
\hline Aroclor 1262 & NAV & NAV & NAV & NAV & - \\
\hline Aroclor 1268 & NAV & NAV & NAV & NAV & - \\
\hline \multicolumn{6}{|c|}{ Metals } \\
\hline Aluminum & 3 of 5 & ND (50) & 87.1 & 87 & 1 of 5 \\
\hline Arsenic & 0 of 5 & ND (1.0) & ND (1.0) & 150 & 0 of 5 \\
\hline Barium & 0 of 5 & ND (10) & ND (10) & 4.0 & 0 of 5 \\
\hline Beryllium & 0 of 5 & ND (1.0) & ND (1.0) & 0.66 & 0 of 5 \\
\hline Boron & 0 of 5 & ND (50) & ND (50) & 1.6 & 0 of 5 \\
\hline Cadmium & 0 of 5 & ND (1.0) & ND (1.0) & 2.2 & 0 of 5 \\
\hline Calcium & 5 of 5 & 4,460 & 5,900 & 116,000 & 0 of 5 \\
\hline Cerium & 0 of 5 & ND (1.0) & ND (1.0) & 0.5 & 0 of 5 \\
\hline Cesium & 0 of 5 & ND (1.0) & ND (1.0) & 30 & 0 of 5 \\
\hline Chromium & 5 of 5 & 1.54 & 3.49 & 74 & 0 of 5 \\
\hline
\end{tabular}




\section{Table 5-31}

\section{Summary of Analytical Results and Ecological Risk Assessment Screening for Surface Water in Reference Stream No. 3}

(Page 3 of 3 )

\begin{tabular}{|c|c|c|c|c|c|}
\hline Analyte & $\begin{array}{l}\text { Detection } \\
\text { Frequency }\end{array}$ & $\begin{array}{c}\text { Minimum } \\
\text { Detected } \\
\text { Concentration } \\
(\mu \mathrm{g} / \mathrm{L})\end{array}$ & $\begin{array}{c}\text { Maximum } \\
\text { Detected } \\
\text { Concentration } \\
(\mu \mathrm{g} / \mathrm{L})\end{array}$ & $\begin{array}{c}\text { Ecological } \\
\text { Screening Value } \\
(\mu \mathrm{g} / \mathrm{L})\end{array}$ & $\begin{array}{c}\text { Detection } \\
\text { Frequency Above } \\
\text { ESV }\end{array}$ \\
\hline Cobalt & 4 of 5 & ND (1.0) & 7.67 & 23 & 0 of 5 \\
\hline Copper & 5 of 5 & 1.09 & 13.4 & 9.0 & 1 of 5 \\
\hline Iron & 5 of 5 & 208 & 2,830 & 1,000 & 1 of 5 \\
\hline Lithium & 0 of 5 & ND (1.0) & ND (1.0) & 14 & 0 of 5 \\
\hline Magnesium & 5 of 5 & 3,510 & 4,670 & 82,000 & 0 of 5 \\
\hline Manganese & 5 of 5 & 6.82 & 353 & 120 & 1 of 5 \\
\hline Molybdenum & 0 of 5 & ND (1.0) & ND (1.0) & 370 & 0 of 5 \\
\hline Nickel & 4 of 5 & ND (1.0) & 1.69 & 52 & 0 of 5 \\
\hline Potassium & 5 of 5 & 742 & 1,190 & 53,000 & 0 of 5 \\
\hline Selenium & 0 of 5 & ND (1.0) & ND (1.0) & 5.0 & 0 of 5 \\
\hline Strontium & 5 of 5 & 39.5 & 43.8 & 1,500 & 0 of 5 \\
\hline Thallium & 1 of 5 & ND (1.0) & 2.13 & 12 & 0 of 5 \\
\hline Thorium & 0 of 5 & ND (1.0) & ND (1.0) & NAV & 0 of 5 \\
\hline Titanium & 5 of 5 & 1.74 & 2.37 & 29 & 0 of 5 \\
\hline Uranium & 0 of 5 & ND (1.0) & ND (1.0) & 2.6 & 0 of 5 \\
\hline Vanadium & 2 of 5 & ND (1.0) & 1.44 & 20 & 0 of 5 \\
\hline Zinc & 2 of 5 & ND (1.0) & 12.8 & 120 & 0 of 5 \\
\hline
\end{tabular}

ND - Analyte not detected at a concentration above the reporting limit, which is shown in parentheses.

NAV - Not available

$\mu \mathrm{g} / \mathrm{L}$ - Micrograms per liter 
Table 5-32

\section{Summary of Analytical Results and Ecological Risk Assessment Screening for Sediment in Reference Stream No. 3}

(Page 1 of 3 )

\begin{tabular}{|c|c|c|c|c|c|}
\hline Analyte & $\begin{array}{l}\text { Detection } \\
\text { Frequency }\end{array}$ & $\begin{array}{c}\text { Minimum } \\
\text { Detected } \\
\text { Concentration } \\
(\mathbf{m g} / \mathbf{k g})\end{array}$ & $\begin{array}{c}\text { Maximum } \\
\text { Detected } \\
\text { Concentration } \\
(\mathbf{m g} / \mathbf{k g})\end{array}$ & $\begin{array}{l}\text { Ecological } \\
\text { Screening } \\
\text { Value }(\mathbf{m g} / \mathbf{k g})\end{array}$ & $\begin{array}{c}\text { Detection } \\
\text { Frequency } \\
\text { Above ESV }\end{array}$ \\
\hline Diesel-Range Hydrocarbons & 1 of 6 & ND (10.2) & 206 & $640^{a}$ & 0 of 6 \\
\hline \multicolumn{6}{|c|}{ Volatile Organic Compounds } \\
\hline 1,2,4-Trimethylbenzene & NAV & NAV & NAV & NAV & - \\
\hline 1,3,5-Trimethylbenzene & NAV & NAV & NAV & NAV & - \\
\hline 2-Butanone & NAV & NAV & NAV & NAV & - \\
\hline Acetone & NAV & NAV & NAV & NAV & - \\
\hline Benzene & NAV & NAV & NAV & NAV & - \\
\hline Carbon disulfide & NAV & NAV & NAV & NAV & - \\
\hline Ethylbenzene & NAV & NAV & NAV & NAV & - \\
\hline Isopropylbenzene & NAV & NAV & NAV & NAV & - \\
\hline m,p-Xylene & NAV & NAV & NAV & NAV & - \\
\hline Methylene chloride & NAV & NAV & NAV & NAV & - \\
\hline n-Butylbenzene & NAV & NAV & NAV & NAV & - \\
\hline n-Propylbenzene & NAV & NAV & NAV & NAV & - \\
\hline Naphthalene & NAV & NAV & NAV & NAV & - \\
\hline o-Xylene & NAV & NAV & NAV & NAV & - \\
\hline p-Isopropyltoluene & NAV & NAV & NAV & NAV & - \\
\hline sec-Butylbenzene & NAV & NAV & NAV & NAV & - \\
\hline Toluene & NAV & NAV & NAV & NAV & - \\
\hline \multicolumn{6}{|c|}{ Polycyclic Aromatic Hydrocarbons } \\
\hline Acenaphthene & NAV & NAV & NAV & NAV & - \\
\hline Acenaphthylene & NAV & NAV & NAV & NAV & - \\
\hline Anthracene & NAV & NAV & NAV & NAV & - \\
\hline Benzo(a)anthracene & NAV & NAV & NAV & NAV & - \\
\hline Benzo(a)pyrene & NAV & NAV & NAV & NAV & - \\
\hline Benzo(b)fluoranthene & NAV & NAV & NAV & NAV & - \\
\hline Benzo(k)fluoranthene & NAV & NAV & NAV & NAV & - \\
\hline Benzo(g,h,i)perylene & NAV & NAV & NAV & NAV & - \\
\hline Chrysene & NAV & NAV & NAV & NAV & - \\
\hline
\end{tabular}


Table 5-32

\section{Summary of Analytical Results and Ecological Risk Assessment Screening for Sediment in Reference Stream No. 3}

(Page 2 of 3 )

\begin{tabular}{|c|c|c|c|c|c|}
\hline Analyte & $\begin{array}{l}\text { Detection } \\
\text { Frequency }\end{array}$ & $\begin{array}{c}\text { Minimum } \\
\text { Detected } \\
\text { Concentration } \\
(\mathrm{mg} / \mathrm{kg})\end{array}$ & $\begin{array}{c}\text { Maximum } \\
\text { Detected } \\
\text { Concentration } \\
(\mathbf{m g} / \mathbf{k g})\end{array}$ & $\begin{array}{c}\text { Ecological } \\
\text { Screening } \\
\text { Value }(\mathbf{m g} / \mathbf{k g})\end{array}$ & $\begin{array}{c}\text { Detection } \\
\text { Frequency } \\
\text { Above ESV }\end{array}$ \\
\hline Dibenzo(a,h)anthracene & NAV & NAV & NAV & NAV & - \\
\hline Fluoranthene & NAV & NAV & NAV & NAV & - \\
\hline Fluorene & NAV & NAV & NAV & NAV & - \\
\hline Indeno(1,2,3-c,d)pyrene & NAV & NAV & NAV & NAV & - \\
\hline Naphthalene & NAV & NAV & NAV & NAV & - \\
\hline Phenanthrene & NAV & NAV & NAV & NAV & - \\
\hline Pyrene & NAV & NAV & NAV & NAV & - \\
\hline \multicolumn{6}{|c|}{ Polychlorinated Biphenyls } \\
\hline Aroclor 1016 & 0 of 2 & ND $(0.127)$ & ND $(0.145)$ & $0.07^{\mathrm{a}}$ & 0 of 2 \\
\hline Aroclor 1221 & 0 of 2 & ND $(0.127)$ & ND (0.145) & $0.07^{\mathrm{a}}$ & 0 of 2 \\
\hline Aroclor 1232 & 0 of 2 & ND $(0.127)$ & ND (0.145) & $0.07^{\mathrm{a}}$ & 0 of 2 \\
\hline Aroclor 1242 & 0 of 2 & ND $(0.127)$ & ND (0.145) & $0.07^{\mathrm{a}}$ & 0 of 2 \\
\hline Aroclor 1248 & 0 of 2 & ND (0.127) & ND (0.145) & $0.07^{\mathrm{a}}$ & 0 of 2 \\
\hline Aroclor 1254 & 0 of 2 & ND $(0.127)$ & ND (0.145) & $0.07^{\mathrm{a}}$ & 0 of 2 \\
\hline Aroclor 1260 & 0 of 2 & ND (0.127) & ND (0.145) & $0.07^{\mathrm{a}}$ & 0 of 2 \\
\hline Aroclor 1262 & 0 of 2 & ND (0.127) & ND (0.145) & $0.07^{a}$ & 0 of 2 \\
\hline Aroclor 1268 & 0 of 2 & ND (0.127) & ND (0.145) & $0.07^{a}$ & 0 of 2 \\
\hline \multicolumn{6}{|c|}{ Metals } \\
\hline Aluminum & 6 of 6 & 7,400 & 33,100 & 58,000 & 0 of 6 \\
\hline Arsenic & 6 of 6 & 11.2 & 23.6 & 6.0 & 6 of 6 \\
\hline Barium & 6 of 6 & 91.1 & 232 & NAV & \\
\hline Beryllium & 0 of 6 & ND (1.27) & ND (3.57) & NAV & \\
\hline Boron & 1 of 6 & ND (7.25) & 6.42 & NAV & \\
\hline Cadmium & 0 of 6 & ND (1.27) & ND (3.57) & 0.6 & 0 of 6 \\
\hline Calcium & 6 of 6 & 4,200 & 6,510 & NAV & \\
\hline Cerium & 6 of 6 & 3.79 & 10.9 & NAV & \\
\hline Cesium & 0 of 6 & ND (1.27) & ND (3.57) & NAV & \\
\hline Chromium & 6 of 6 & 4.62 & 9.94 & 26 & 0 of 6 \\
\hline Cobalt & 6 of 6 & 8.83 & 27.6 & NAV & \\
\hline
\end{tabular}


Table 5-32

\section{Summary of Analytical Results and Ecological Risk Assessment Screening for Sediment in Reference Stream No. 3}

(Page 3 of 3 )

\begin{tabular}{|c|c|c|c|c|c|}
\hline Analyte & $\begin{array}{l}\text { Detection } \\
\text { Frequency }\end{array}$ & $\begin{array}{c}\text { Minimum } \\
\text { Detected } \\
\text { Concentration } \\
(\mathbf{m g} / \mathbf{k g})\end{array}$ & $\begin{array}{c}\text { Maximum } \\
\text { Detected } \\
\text { Concentration } \\
(\mathbf{m g} / \mathbf{k g})\end{array}$ & $\begin{array}{c}\text { Ecological } \\
\text { Screening } \\
\text { Value }(\mathbf{m g} / \mathbf{k g})\end{array}$ & $\begin{array}{c}\text { Detection } \\
\text { Frequency } \\
\text { Above ESV }\end{array}$ \\
\hline Copper & 6 of 6 & 16 & 57.9 & 16 & 5 of 6 \\
\hline Iron & 6 of 6 & 33,400 & 104,000 & 20,000 & 6 of 6 \\
\hline Lithium & 5 of 6 & ND (3.57) & 15.2 & NAV & \\
\hline Magnesium & 6 of 6 & 1,280 & 12,200 & NAV & \\
\hline Manganese & 6 of 6 & 724 & 13,300 & 460 & 6 of 6 \\
\hline Molybdenum & 0 of 6 & ND (1.27) & ND (3.57) & NAV & \\
\hline Nickel & 6 of 6 & 5.16 & 17.9 & 16 & 1 of 6 \\
\hline Potassium & 6 of 6 & 179 & 678 & NAV & \\
\hline Selenium & 2 of 6 & ND (1.27) & 3.15 & NAV & \\
\hline Strontium & 6 of 6 & 45.9 & 181 & NAV & \\
\hline Thallium & 0 of 6 & ND (1.27) & ND (3.57) & NAV & \\
\hline Thorium & 0 of 6 & ND (1.27) & ND (3.57) & NAV & \\
\hline Titanium & 6 of 6 & 285 & 1,070 & NAV & \\
\hline Uranium & 0 of 6 & ND (1.27) & ND (3.57) & NAV & \\
\hline Vanadium & 6 of 6 & 126 & 263 & NAV & \\
\hline Zinc & 6 of 6 & 42.3 & 162 & 120 & 1 of 6 \\
\hline Total Organic Carbon & 6 of 6 & 40,500 & 159,000 & & \\
\hline
\end{tabular}

${ }^{a}$ Screening concentration is based on $1 \%$ organic carbon.

ND - Analyte not detected at a concentration above the reporting limit, which is shown in parentheses.

NAV - Not available

$\mathrm{mg} / \mathrm{kg}$ - Milligrams per kilogram 
Table 5-33

\section{Summary of Analytical Results and Ecological Risk Assessment Screening for Surface Water in Reference Stream No. 4}

(Page 1 of 3 )

\begin{tabular}{|c|c|c|c|c|c|}
\hline Analyte & $\begin{array}{l}\text { Detection } \\
\text { Frequency }\end{array}$ & $\begin{array}{c}\text { Minimum } \\
\text { Detected } \\
\text { Concentration } \\
(\mu \mathrm{g} / \mathrm{L})\end{array}$ & $\begin{array}{c}\text { Maximum } \\
\text { Detected } \\
\text { Concentration } \\
(\mu \mathrm{g} / \mathrm{L})\end{array}$ & $\begin{array}{c}\text { Ecological } \\
\text { Screening Value } \\
(\mu \mathrm{g} / \mathrm{L})\end{array}$ & $\begin{array}{c}\text { Detection Frequency } \\
\text { Above ESV }\end{array}$ \\
\hline $\begin{array}{l}\text { Diesel-Range } \\
\text { Hydrocarbons }\end{array}$ & 2 of 6 & ND (50) & 59.5 & 15 & 2 of 6 \\
\hline \multicolumn{6}{|c|}{ Volatile Organic Compounds } \\
\hline 1,2,4-Trimethylbenzene & NAV & NAV & NAV & NAV & - \\
\hline 1,3,5-Trimethylbenzene & NAV & NAV & NAV & NAV & - \\
\hline 2-Butanone & NAV & NAV & NAV & NAV & - \\
\hline Acetone & NAV & NAV & NAV & NAV & - \\
\hline Benzene & NAV & NAV & NAV & NAV & - \\
\hline Carbon disulfide & NAV & NAV & NAV & NAV & - \\
\hline Ethylbenzene & NAV & NAV & NAV & NAV & - \\
\hline Isopropylbenzene & NAV & NAV & NAV & NAV & - \\
\hline m,p-Xylene & NAV & NAV & NAV & NAV & - \\
\hline Methylene chloride & NAV & NAV & NAV & NAV & - \\
\hline n-Butylbenzene & NAV & NAV & NAV & NAV & - \\
\hline n-Propylbenzene & NAV & NAV & NAV & NAV & - \\
\hline Naphthalene & NAV & NAV & NAV & NAV & - \\
\hline o-Xylene & NAV & NAV & NAV & NAV & - \\
\hline p-Isopropyltoluene & NAV & NAV & NAV & NAV & - \\
\hline sec-Butylbenzene & NAV & NAV & NAV & NAV & - \\
\hline Toluene & NAV & NAV & NAV & NAV & - \\
\hline \multicolumn{6}{|c|}{ Polycyclic Aromatic Hydrocarbons } \\
\hline Acenaphthene & NAV & NAV & NAV & NAV & - \\
\hline Acenaphthylene & NAV & NAV & NAV & NAV & - \\
\hline Anthracene & NAV & NAV & NAV & NAV & - \\
\hline Benzo(a)anthracene & NAV & NAV & NAV & NAV & - \\
\hline Benzo(a)pyrene & NAV & NAV & NAV & NAV & - \\
\hline Benzo(b)fluoranthene & NAV & NAV & NAV & NAV & - \\
\hline Benzo(k)fluoranthene & NAV & NAV & NAV & NAV & - \\
\hline Benzo(g,h,i)perylene & NAV & NAV & NAV & NAV & - \\
\hline
\end{tabular}


Table 5-33

\section{Summary of Analytical Results and Ecological Risk Assessment Screening for Surface Water in Reference Stream No. 4}

(Page 2 of 3 )

\begin{tabular}{|c|c|c|c|c|c|}
\hline Analyte & $\begin{array}{l}\text { Detection } \\
\text { Frequency }\end{array}$ & $\begin{array}{c}\text { Minimum } \\
\text { Detected } \\
\text { Concentration } \\
(\mu \mathrm{g} / \mathrm{L})\end{array}$ & $\begin{array}{c}\text { Maximum } \\
\text { Detected } \\
\text { Concentration } \\
(\mu \mathrm{g} / \mathrm{L})\end{array}$ & $\begin{array}{c}\text { Ecological } \\
\text { Screening Value } \\
(\mu \mathrm{g} / \mathrm{L})\end{array}$ & $\begin{array}{c}\text { Detection Frequency } \\
\text { Above ESV }\end{array}$ \\
\hline Chrysene & NAV & NAV & NAV & NAV & - \\
\hline Dibenzo(a,h)anthracene & NAV & NAV & NAV & NAV & - \\
\hline Fluoranthene & NAV & NAV & NAV & NAV & - \\
\hline Fluorene & NAV & NAV & NAV & NAV & - \\
\hline Indeno(1,2,3-c,d)pyrene & NAV & NAV & NAV & NAV & - \\
\hline Naphthalene & NAV & NAV & NAV & NAV & - \\
\hline Phenanthrene & NAV & NAV & NAV & NAV & - \\
\hline Pyrene & NAV & NAV & NAV & NAV & - \\
\hline \multicolumn{6}{|c|}{ Polychlorinated Biphenyls } \\
\hline Aroclor 1016 & NAV & NAV & NAV & NAV & - \\
\hline Aroclor 1221 & NAV & NAV & NAV & NAV & - \\
\hline Aroclor 1232 & NAV & NAV & NAV & NAV & - \\
\hline Aroclor 1242 & NAV & NAV & NAV & NAV & - \\
\hline Aroclor 1248 & NAV & NAV & NAV & NAV & - \\
\hline Aroclor 1254 & NAV & NAV & NAV & NAV & - \\
\hline Aroclor 1260 & NAV & NAV & NAV & NAV & - \\
\hline Aroclor 1262 & NAV & NAV & NAV & NAV & - \\
\hline Aroclor 1268 & NAV & NAV & NAV & NAV & - \\
\hline \multicolumn{6}{|c|}{ Metals } \\
\hline Aluminum & 0 of 6 & ND (50) & ND (50) & 87 & 0 of 6 \\
\hline Arsenic & 0 of 6 & ND (1.0) & ND (1.0) & 150 & 0 of 6 \\
\hline Barium & 0 of 6 & ND (10) & ND (10) & 4.0 & 0 of 6 \\
\hline Beryllium & 0 of 6 & ND (1.0) & ND (1.0) & 0.66 & 0 of 6 \\
\hline Boron & 0 of 6 & ND (50) & ND (50) & 1.6 & 0 of 6 \\
\hline Cadmium & 0 of 6 & ND (1.0) & ND (1.0) & 2.2 & 0 of 6 \\
\hline Calcium & 6 of 6 & 7,150 & 8,810 & 116,000 & 0 of 6 \\
\hline Cerium & 0 of 6 & ND (1.0) & ND (1.0) & 0.5 & 0 of 6 \\
\hline Cesium & 0 of 6 & ND (1.0) & ND (1.0) & 30 & 0 of 6 \\
\hline Chromium & 0 of 6 & ND (1.0) & ND (1.0) & 74 & 0 of 6 \\
\hline
\end{tabular}


Table 5-33

\section{Summary of Analytical Results and Ecological Risk Assessment Screening for Surface Water in Reference Stream No. 4}

(Page 3 of 3 )

\begin{tabular}{|c|c|c|c|c|c|}
\hline Analyte & $\begin{array}{l}\text { Detection } \\
\text { Frequency }\end{array}$ & $\begin{array}{c}\text { Minimum } \\
\text { Detected } \\
\text { Concentration } \\
(\mu \mathrm{g} / \mathrm{L})\end{array}$ & $\begin{array}{c}\text { Maximum } \\
\text { Detected } \\
\text { Concentration } \\
(\mu \mathrm{g} / \mathrm{L})\end{array}$ & $\begin{array}{c}\text { Ecological } \\
\text { Screening Value } \\
(\mu \mathrm{g} / \mathrm{L})\end{array}$ & $\begin{array}{c}\text { Detection Frequency } \\
\text { Above ESV }\end{array}$ \\
\hline Cobalt & 5 of 6 & ND (1.0) & 4.24 & 23 & 0 of 6 \\
\hline Copper & 0 of 6 & ND (1.0) & ND (1.0) & 9.0 & 0 of 6 \\
\hline Iron & 0 of 6 & ND (150) & ND (150) & 1,000 & 0 of 6 \\
\hline Lithium & 0 of 6 & ND (1.0) & ND (1.0) & 14 & 0 of 6 \\
\hline Magnesium & 6 of 6 & 2,740 & 3,320 & 82,000 & 0 of 6 \\
\hline Manganese & 6 of 6 & 1.3 & 14.8 & 120 & 0 of 6 \\
\hline Molybdenum & 0 of 6 & ND (1.0) & ND (1.0) & 370 & 0 of 6 \\
\hline Nickel & 1 of 6 & ND (1.0) & 1.07 & 52 & 0 of 6 \\
\hline Potassium & 6 of 6 & 778 & 906 & 53,000 & 0 of 6 \\
\hline Selenium & 0 of 6 & ND (1.0) & ND (1.0) & 5.0 & 0 of 6 \\
\hline Strontium & 6 of 6 & 32.9 & 44.1 & 1,500 & 0 of 6 \\
\hline Thallium & 0 of 6 & ND (1.0) & ND (1.0) & 12 & 0 of 6 \\
\hline Thorium & 1 of 6 & ND (1.0) & 1.14 & NAV & 0 of 6 \\
\hline Titanium & 1 of 6 & ND (1.0) & 1.04 & 29 & 0 of 6 \\
\hline Uranium & 0 of 6 & ND (1.0) & ND (1.0) & 2.6 & 0 of 6 \\
\hline Vanadium & 0 of 6 & ND (1.0) & ND (1.0) & 20 & 0 of 6 \\
\hline Zinc & 2 of 6 & ND (10) & 12.5 & 120 & 0 of 6 \\
\hline
\end{tabular}

ND - Analyte not detected at a concentration above the reporting limit, which is shown in parentheses.

NAV - Not available

$\mu \mathrm{g} / \mathrm{L}$ - Micrograms per liter 
Table 5-34

\section{Summary of Analytical Results and Ecological Risk Assessment Screening for Sediment in Reference Stream No. 4}

(Page 1 of 3 )

\begin{tabular}{|c|c|c|c|c|c|}
\hline Analyte & $\begin{array}{l}\text { Detection } \\
\text { Frequency }\end{array}$ & $\begin{array}{c}\text { Minimum } \\
\text { Detected } \\
\text { Concentration } \\
(\mathbf{m g} / \mathbf{k g})\end{array}$ & $\begin{array}{c}\text { Maximum } \\
\text { Detected } \\
\text { Concentration } \\
(\mathbf{m g} / \mathbf{k g})\end{array}$ & $\begin{array}{c}\text { Ecological } \\
\text { Screening Value } \\
(\mathrm{mg} / \mathrm{kg})\end{array}$ & $\begin{array}{c}\text { Detection } \\
\text { Frequency Above } \\
\text { ESV }\end{array}$ \\
\hline $\begin{array}{l}\text { Diesel-Range } \\
\text { Hydrocarbons }\end{array}$ & 4 of 5 & 6.59 & 10.7 & $640^{\mathrm{a}}$ & 0 of 5 \\
\hline \multicolumn{6}{|c|}{ Volatile Organic Compounds } \\
\hline 1,2,4-Trimethylbenzene & NAV & NAV & NAV & NAV & - \\
\hline 1,3,5-Trimethylbenzene & NAV & NAV & NAV & NAV & - \\
\hline 2-Butanone & NAV & NAV & NAV & NAV & - \\
\hline Acetone & NAV & NAV & NAV & NAV & - \\
\hline Benzene & NAV & NAV & NAV & NAV & - \\
\hline Carbon disulfide & NAV & NAV & NAV & NAV & - \\
\hline Ethylbenzene & NAV & NAV & NAV & NAV & - \\
\hline Isopropylbenzene & NAV & NAV & NAV & NAV & - \\
\hline m,p-Xylene & NAV & NAV & NAV & NAV & - \\
\hline Methylene chloride & NAV & NAV & NAV & NAV & - \\
\hline n-Butylbenzene & NAV & NAV & NAV & NAV & - \\
\hline n-Propylbenzene & NAV & NAV & NAV & NAV & - \\
\hline Naphthalene & NAV & NAV & NAV & NAV & - \\
\hline o-Xylene & NAV & NAV & NAV & NAV & - \\
\hline p-Isopropyltoluene & NAV & NAV & NAV & NAV & - \\
\hline sec-Butylbenzene & NAV & NAV & NAV & NAV & - \\
\hline Toluene & NAV & NAV & NAV & NAV & - \\
\hline \multicolumn{6}{|c|}{ Polycyclic Aromatic Hydrocarbons } \\
\hline Acenaphthene & NAV & NAV & NAV & NAV & - \\
\hline Acenaphthylene & NAV & NAV & NAV & NAV & - \\
\hline Anthracene & NAV & NAV & NAV & NAV & - \\
\hline Benzo(a)anthracene & NAV & NAV & NAV & NAV & - \\
\hline Benzo(a)pyrene & NAV & NAV & NAV & NAV & - \\
\hline Benzo(b)fluoranthene & NAV & NAV & NAV & NAV & - \\
\hline Benzo(k)fluoranthene & NAV & NAV & NAV & NAV & - \\
\hline Benzo(g,h,i)perylene & NAV & NAV & NAV & NAV & - \\
\hline
\end{tabular}


Table 5-34

\section{Summary of Analytical Results and Ecological Risk Assessment} Screening for Sediment in Reference Stream No. 4

(Page 2 of 3 )

\begin{tabular}{|c|c|c|c|c|c|}
\hline Analyte & $\begin{array}{l}\text { Detection } \\
\text { Frequency }\end{array}$ & $\begin{array}{c}\text { Minimum } \\
\text { Detected } \\
\text { Concentration } \\
(\mathbf{m g} / \mathbf{k g})\end{array}$ & $\begin{array}{c}\text { Maximum } \\
\text { Detected } \\
\text { Concentration } \\
(\mathrm{mg} / \mathrm{kg})\end{array}$ & $\begin{array}{c}\text { Ecological } \\
\text { Screening Value } \\
(\mathrm{mg} / \mathrm{kg})\end{array}$ & $\begin{array}{c}\text { Detection } \\
\text { Frequency Above } \\
\text { ESV }\end{array}$ \\
\hline Chrysene & NAV & NAV & NAV & NAV & - \\
\hline Dibenzo(a,h)anthracene & NAV & NAV & NAV & NAV & - \\
\hline Fluoranthene & NAV & NAV & NAV & NAV & - \\
\hline Fluorene & NAV & NAV & NAV & NAV & - \\
\hline Indeno(1,2,3-c,d)pyrene & NAV & NAV & NAV & NAV & - \\
\hline Naphthalene & NAV & NAV & NAV & NAV & - \\
\hline Phenanthrene & NAV & NAV & NAV & NAV & - \\
\hline Pyrene & NAV & NAV & NAV & NAV & - \\
\hline \multicolumn{6}{|c|}{ Polychlorinated Biphenyls } \\
\hline Aroclor 1016 & 0 of 3 & ND (0.05) & ND (0.05) & $0.07^{\mathrm{a}}$ & 0 of 3 \\
\hline Aroclor 1221 & 0 of 3 & ND (0.05) & ND (0.05) & $0.07^{a}$ & 0 of 3 \\
\hline Aroclor 1232 & 0 of 3 & ND $(0.05)$ & ND (0.05) & $0.07^{\mathrm{a}}$ & 0 of 3 \\
\hline Aroclor 1242 & 0 of 3 & ND (0.05) & ND (0.05) & $0.07^{\mathrm{a}}$ & 0 of 3 \\
\hline Aroclor 1248 & 0 of 3 & ND (0.05) & ND (0.05) & $0.07^{a}$ & 0 of 3 \\
\hline Aroclor 1254 & 0 of 3 & ND (0.05) & ND (0.05) & $0.07^{a}$ & 0 of 3 \\
\hline Aroclor 1260 & 0 of 3 & ND (0.05) & ND (0.05) & $0.07^{\mathrm{a}}$ & 0 of 3 \\
\hline Aroclor 1262 & 0 of 3 & ND (0.05) & ND (0.05) & $0.07^{\mathrm{a}}$ & 0 of 3 \\
\hline Aroclor 1268 & 0 of 3 & ND (0.05) & ND (0.05) & $0.07^{\mathrm{a}}$ & 0 of 3 \\
\hline \multicolumn{6}{|c|}{ Metals } \\
\hline Aluminum & 5 of 5 & 15,600 & 28,400 & 58,000 & 0 of 5 \\
\hline Arsenic & 5 of 5 & 0.982 & 3.01 & 6.0 & 0 of 5 \\
\hline Barium & 5 of 5 & 38.5 & 44.3 & NAV & \\
\hline Beryllium & 0 of 5 & ND (0.5) & ND (56.2) & NAV & \\
\hline Boron & 0 of 5 & ND (2.5) & ND (7.73) & NAV & \\
\hline Cadmium & 0 of 5 & ND (0.5) & ND (1.55) & 0.6 & 0 of 5 \\
\hline Calcium & 5 of 5 & 2,380 & 5,320 & NAV & \\
\hline Cerium & 5 of 5 & 6.29 & 9.41 & NAV & \\
\hline Cesium & 0 of 5 & ND (0.5) & ND (1.55) & NAV & \\
\hline Chromium & 5 of 5 & 9.82 & 17.6 & 26 & 0 of 5 \\
\hline
\end{tabular}


Table 5-34

\section{Summary of Analytical Results and Ecological Risk Assessment Screening for Sediment in Reference Stream No. 4}

(Page 3 of 3 )

\begin{tabular}{|c|c|c|c|c|c|}
\hline Analyte & $\begin{array}{l}\text { Detection } \\
\text { Frequency }\end{array}$ & $\begin{array}{c}\text { Minimum } \\
\text { Detected } \\
\text { Concentration } \\
(\mathbf{m g} / \mathbf{k g})\end{array}$ & $\begin{array}{c}\text { Maximum } \\
\text { Detected } \\
\text { Concentration } \\
(\mathbf{m g} / \mathrm{kg})\end{array}$ & $\begin{array}{c}\text { Ecological } \\
\text { Screening Value } \\
(\mathrm{mg} / \mathrm{kg})\end{array}$ & $\begin{array}{c}\text { Detection } \\
\text { Frequency Above } \\
\text { ESV }\end{array}$ \\
\hline Cobalt & 5 of 5 & 8.77 & 17.7 & NAV & \\
\hline Copper & 5 of 5 & 21.8 & 41 & 16 & 5 of 5 \\
\hline Iron & 5 of 5 & 26,000 & 54,200 & 20,000 & 5 of 5 \\
\hline Lithium & 5 of 5 & 6.2 & 15 & NAV & \\
\hline Magnesium & 5 of 5 & 5,710 & 11,200 & NAV & \\
\hline Manganese & 5 of 5 & 755 & 4,450 & 460 & 5 of 5 \\
\hline Molybdenum & 0 of 5 & ND (0.5) & ND (1.55) & NAV & \\
\hline Nickel & 5 of 5 & 8.05 & 14.8 & 16 & 0 of 5 \\
\hline Potassium & 5 of 5 & 181 & 371 & NAV & \\
\hline Selenium & 5 of 5 & 0.536 & 2.4 & NAV & \\
\hline Strontium & 5 of 5 & 17.6 & 46.8 & NAV & \\
\hline Thallium & 0 of 5 & ND $(0.5)$ & ND (1.55) & NAV & \\
\hline Thorium & 0 of 5 & ND (0.5) & ND (1.55) & NAV & \\
\hline Titanium & 5 of 5 & 313 & 537 & NAV & \\
\hline Uranium & 0 of 5 & ND (0.5) & ND (1.55) & NAV & \\
\hline Vanadium & 5 of 5 & 47.7 & 90.4 & NAV & \\
\hline Zinc & 5 of 5 & 53.1 & 109 & 120 & 0 of 5 \\
\hline Total Organic Carbon & 5 of 5 & 4,150 & 38,000 & & \\
\hline
\end{tabular}

${ }^{\mathrm{a}}$ Screening concentration is based on $1 \%$ organic carbon.

ND - Analyte not detected at a concentration above the reporting limit, which is shown in parentheses.

NAV - Not available

$\mathrm{mg} / \mathrm{kg}$ - Milligrams per kilogram 
The PAHs were not detected in any of the surface water samples on site. Detection limits for PAHs were lower than the ESVs. Therefore, PAHs were not considered COPECs for surface water.

Analytical results of PCBs in surface water are only available for Cannikin Lake. Though PCBs were not detected in any of the three surface water samples, the detection limits $(0.5 \mu \mathrm{g} / \mathrm{L})$ were greater than the ESV of $0.014 \mu \mathrm{g} / \mathrm{L}$. Since these are considered bioaccumulative compounds, PCBs were retained as COPECs for surface water.

\subsection{Inorganics}

Several metals were detected in some or all of the surface water samples: aluminum, arsenic, barium, boron, calcium, chromium, cobalt, copper, iron, lithium, magnesium, manganese, molybdenum, nickel, potassium, strontium, thallium, titanium, vanadium, and zinc.

Aluminum was detected rarely above the ESV of $87 \mu \mathrm{g} / \mathrm{L}$. The background concentration for aluminum is $87.1 \mu \mathrm{g} / \mathrm{L}$ for upland streams and $269 \mu \mathrm{g} / \mathrm{L}$ for lowland streams. In the lowland streams, aluminum was only detected above the background concentration and ESV in two of the five samples from Clevenger Creek (maximum concentration $625 \mu \mathrm{g} / \mathrm{L}$ ). In the upland streams, in two of six samples from Drill Site E Stream (maximum concentration $105 \mu \mathrm{g} / \mathrm{L}$ ) and in two of three samples from Drill Site D Lake (maximum concentration $186 \mu \mathrm{g} / \mathrm{L}$ ).

Barium was only detected in surface water from Reed Pond ( $49.4 \mu \mathrm{g} / \mathrm{L}$ and $53 \mu \mathrm{g} / \mathrm{L})$. These were both above the ESV of $4 \mu \mathrm{g} / \mathrm{L}$. Though not detected elsewhere, the detection limit (10 $\mu \mathrm{g} / \mathrm{L})$ was two and a half times greater than the ESV. Also, barium was not detected in background samples.

Boron was only detected in surface water from Cannikin Lake (maximum concentration $468 \mu \mathrm{g} / \mathrm{L}$ ), Clevenger Creek (maximum concentration $88.7 \mu \mathrm{g} / \mathrm{L}$ ), and White Alice Creek (maximum concentration $424 \mu \mathrm{g} / \mathrm{L})$. These were greater than the ESV of $1.6 \mu \mathrm{g} / \mathrm{L}$. Though not detected in most samples, the detection limit (50 $\mu \mathrm{g} / \mathrm{L})$ was more than 20 times the ESV.

Copper was detected in most samples, but rarely above the ESV of $9 \mu \mathrm{g} / \mathrm{L}$ and the background concentration (10.8 $\mu \mathrm{g} / \mathrm{L}$ for lowland streams and $13.4 \mu \mathrm{g} / \mathrm{L}$ for upland streams). These occurred in one sample from Clevenger Creek $(11.8 \mu \mathrm{g} / \mathrm{L})$ and in one sample from Rainbow Creek $(17.7 \mu \mathrm{g} / \mathrm{L})$. 
Iron was detected in most samples, but only once above the ESV of $1000 \mu \mathrm{g} / \mathrm{L}$. It was detected in one sample from Clevenger Creek $(1,070 \mu \mathrm{g} / \mathrm{L})$ above the ESV; the background concentration for lowland streams is $993 \mu \mathrm{g} / \mathrm{L}$.

Titanium was detected in most samples. However, it was only detected in Clevenger Creek at concentrations greater than the ESV of $29 \mu \mathrm{g} / \mathrm{L}$. Three of five surface water samples had concentrations greater than the ESV with a maximum concentration of $123 \mu \mathrm{g} / \mathrm{L}$. The background concentration of titanium for lowland streams is $6.13 \mu \mathrm{g} / \mathrm{L}$.

Though beryllium and cerium were not detected in any surface water samples, their detection limits $(1 \mu \mathrm{g} / \mathrm{L})$ were greater than the ESVs $0.64 \mu \mathrm{g} / \mathrm{L}$ and $0.5 \mu \mathrm{g} / \mathrm{L}$, respectively.

Though a few metals were detected above the ESVs, these were not considered to be related to site operations and drilling muds. Additionally, none of the detected metals are considered to be bioaccumulative. Therefore, no metals were considered to be COPECs for surface water.

\subsubsection{Sediment}

Sediment samples were collected from Falls Creek, Drill Site D Lake, Drill Site E Stream, Limpet Creek, Bridge Creek, Reed Pond, Cloudberry Creek, Rainbow Creek, Clevenger Creek, Heart Lake, Cannikin Lake, White Alice Creek, and four reference streams. Analyses conducted on some or all of these samples were inorganics, PAHs, VOCs, PCBs, DRO, and total organic carbon (TOC). The identification of COPECs for sediment is presented below.

\subsection{Organics}

The DROs were detected in sediment samples from Cannikin Lake, Clevenger Creek, Drill Site D Lake, Drill Site E Stream, Falls Creek, Rainbow Creek, Reed Pond, and White Alice Creek. However, none of these were above the ESV of 640 milligrams per percent (mg/\%) OC. Thus, DROs were not considered to be COPECs for sediment.

The PAHs were detected in sediment samples from Cannikin Lake, Drill Site D Lake, Drill Site E Stream, and Falls Creek. None of these were above the ESVs for individual PAHs. However, since 
PAHs may bioaccumulate in the food chain, PAHs were retained as COPECs for sediment in the ecological risk assessment.

Analysis for PCBs in sediment was only conducted for samples from Cannikin Lake, White Alice Creek, Rainbow Creek, and Falls Creek. Aroclor 1248 and aroclor 1260 were detected in Cannikin Lake (maximum concentrations of $0.185 \mathrm{mg} / \mathrm{kg}$ and $0.107 \mathrm{mg} / \mathrm{kg}$ ) but not in White Alice Creek. The ESV for PCBs is $0.07 \mathrm{mg} / \%$ OC. No organic carbon analysis was conducted for Cannikin Lake which drains the lake, so the detected concentrations cannot be compared to the ESV. Based on the TOC analyses for White Alice Creek, the detection limits were sufficiently low in all but one of the samples to assume that PCBs are not present in White Alice Creek above the ESVs. Aroclor 1260 was detected in two of three samples from Falls Creek $(0.82 \mathrm{mg} / \mathrm{kg}$ and $0.04 \mathrm{mg} / \mathrm{kg})$ and in one of the two samples from Rainbow Creek at a concentration of $0.46 \mathrm{mg} / \mathrm{kg}$. No analysis of TOC in collocated samples was conducted for these samples. Since PCBs were detected above ESVs conservatively based on 1 percent TOC and because PCBs are bioaccumulative, PCBs were retained as COPECs for sediment.

The VOCs were detected most frequently in Cannikin Lake samples, but were also detected in Heart Lake, Reed Pond, and Drill Site D Lake. Most of the ESVs for VOCs are TOC-based. However, no TOC analysis was conducted on pond and lake samples. Therefore, actual comparisons to ESVs could not be conducted, and detected concentrations were conservatively compared to 1 percent TOC-based ESVs. In Cannikin Lake, 1,2,4-trimethylbenzene was detected in one sample $(5.16 \mathrm{mg} / \mathrm{kg})$ above the ESV $(1.2 \mathrm{mg} / \mathrm{kg}), 1,3,5$-trimethylbenzene was detected in one sample $(1.21 \mathrm{mg} / \mathrm{kg})$ above the $\mathrm{ESV}(0.76 \mathrm{mg} / \mathrm{kg}), m, p$-xylene and $o$-xylene were detected in one sample $(0.836 \mathrm{mg} / \mathrm{kg}$ and $0.498 \mathrm{mg} / \mathrm{kg}$, respectively) above the ESV $0.12 \mathrm{mg} / \mathrm{kg}$, and n-propylbenzene was detected in one sample $(0.369 \mathrm{mg} / \mathrm{kg})$ above the ESV $(0.25 \mathrm{mg} / \mathrm{kg})$. Acetone, ethylbenzene, n-butylbenzene, $\mathrm{p}$-isopropyltoluene, and sec-butylbenzene were also detected, but not above the ESVs. In Heart Lake, 2-butanone was detected in three of five samples, but not above the ESVs. In Reed Pond, 2-butanone, acetone, and toluene were detected in sediment, but none were above the ESVs. In Drill Site D Lake, 2-butanone was detected in one of five samples but not above the ESV, and carbon disulfide was detected in four of five samples at concentrations $(0.0253 \mathrm{mg} / \mathrm{kg}-$ $0.197 \mathrm{mg} / \mathrm{kg}$ ) above the ESV (0.001 $\mathrm{mg} / \%$ OC). Carbon disulfide is a common laboratory contaminant and is also not considered to be related to site activities; thus, it was not retained as a 
COPEC. However, the BTEX compounds (benzene, toluene, ethylbenzene, and xylene) could be considered to be site related. Therefore, trimethylbenzene, xylene, and propylbenzene were retained as COPECs.

\subsection{Inorganics}

Many of the inorganics were detected in all the sediment samples, and are likely associated with natural background levels. The screening for inorganic COPECs in sediment is discussed below.

Aluminum was detected above the ESV $(58,000 \mathrm{mg} / \mathrm{kg})$ and above background in one sample from Bridge Creek (63,900 mg/kg), one sample from Clevenger Creek $(90,600 \mathrm{mg} / \mathrm{kg})$, one sample from Heart Lake (77,500 mg/kg), and two samples from White Alice Creek (60,100 mg/kg and $68,300 \mathrm{mg} / \mathrm{kg})$.

Though arsenic was detected in many samples above the ESV of $6 \mathrm{mg} / \mathrm{kg}$, it was detected above background (100 mg/kg for lowland streams and $30.5 \mathrm{mg} / \mathrm{kg}$ for upland streams) in only one sample from White Alice Creek (109 mg/kg).

Likewise, barium was detected in only one sample at a concentration above both the ESV $(6 \mathrm{mg} / \mathrm{kg})$ and the background concentration $(468 \mathrm{mg} / \mathrm{kg})$. Barium was detected in one of five samples from Drill Site D Lake at a concentration of $515 \mathrm{mg} / \mathrm{kg}$.

Cadmium was detected in only one sample from Cannikin Lake $(0.876 \mathrm{mg} / \mathrm{kg})$ and one sample from Cloudberry Creek $(3.17 \mathrm{mg} / \mathrm{kg})$ above the ESV $(0.6 \mathrm{mg} / \mathrm{kg})$ and the background $(0.608 \mathrm{mg} / \mathrm{kg}$ for upland streams). However, other samples may have contained cadmium above the ESV, but the detection limits for many of the samples was as much as an order of magnitude greater than the ESV.

Chromium was detected in many of the samples, but less frequently above the ESV of $26 \mathrm{mg} / \mathrm{kg}$. Chromium was detected in two of three samples in Cannikin Lake (253 mg/kg and $253 \mathrm{mg} / \mathrm{kg}$ ), two of five samples from Rainbow Creek $(33.2 \mathrm{mg} / \mathrm{kg}$ and $38.8 \mathrm{mg} / \mathrm{kg})$, one of five samples from White Alice Creek (27.5 mg/kg), four of five samples from Drill Site D Lake (564 mg/kg, $695 \mathrm{mg} / \mathrm{kg}$, $421 \mathrm{mg} / \mathrm{kg}$, and $406 \mathrm{mg} / \mathrm{kg})$, one of five samples from Drill Site E Stream $(41.1 \mathrm{mg} / \mathrm{kg})$, and three of five samples from Falls Creek (226 mg/kg, $36.2 \mathrm{mg} / \mathrm{kg}$, and $31.2 \mathrm{mg} / \mathrm{kg}$ ). 
Copper was detected in most samples. Many detections were above the ESV of $16 \mathrm{mg} / \mathrm{kg}$, and several were above the background concentrations $(71.1 \mathrm{mg} / \mathrm{kg}$ for lowland streams and $82.4 \mathrm{mg} / \mathrm{kg}$ for upland streams). Copper was detected in two samples from Cannikin Lake (157 mg/kg and $203 \mathrm{mg} / \mathrm{kg}$ ), two samples from Clevenger Creek (247 mg/kg and $94.2 \mathrm{mg} / \mathrm{kg}$ ), one sample from Cloudberry Creek (71.7 mg/kg), four samples from Heart lake $(74.3 \mathrm{mg} / \mathrm{kg}, 73.4 \mathrm{mg} / \mathrm{kg}, 192 \mathrm{mg} / \mathrm{kg}$, and $75.2 \mathrm{mg} / \mathrm{kg}$ ), two samples from White Alice Creek (127 mg/kg and $168 \mathrm{mg} / \mathrm{kg}$ ), two samples from Drill Site D Lake (96.6 mg/kg and $98.3 \mathrm{mg} / \mathrm{kg}$ ), and one sample from Falls Creek (93.1 mg/kg) above the background concentration and the ESV.

Iron was detected in most samples. Many detections were above the ESV of $20,000 \mathrm{mg} / \mathrm{kg}$, but only once above the background concentrations $(155,000 \mathrm{mg} / \mathrm{kg}$ for lowland streams and 123,000 $\mathrm{mg} / \mathrm{kg}$ for upland streams). Iron was detected in one sample from Rainbow Creek $(192,000 \mathrm{mg} / \mathrm{kg})$ above the background concentrations.

Nickel was also detected in most of the samples. The ESV for nickel is $16 \mathrm{mg} / \mathrm{kg}$. The background concentrations for nickel are $13.6 \mathrm{mg} / \mathrm{kg}$ for lowland streams and $23.8 \mathrm{mg} / \mathrm{kg}$ for upland streams. Nickel was detected in all three samples from Cannikin Lake $(66.9 \mathrm{mg} / \mathrm{kg}, 18.6 \mathrm{mg} / \mathrm{kg}$, and $17.5 \mathrm{mg} / \mathrm{kg})$, one of six samples from Clevenger Creek $(18.9 \mathrm{mg} / \mathrm{kg})$, three of six samples from White Alice Creek (22.1 mg/kg, $17.2 \mathrm{mg} / \mathrm{kg}$, and $24.9 \mathrm{mg} / \mathrm{kg})$, and one of five samples from Drill Site E Stream $(32.5 \mathrm{mg} / \mathrm{kg})$ above the ESV and background concentration.

Zinc was detected in most sediment samples. The ESV for zinc is $120 \mathrm{mg} / \mathrm{kg}$ and the background concentrations are $434 \mathrm{mg} / \mathrm{kg}$ for lowland streams and $185 \mathrm{mg} / \mathrm{kg}$ for upland streams. Only two samples from Falls Creek (256 mg/kg and $231 \mathrm{mg} / \mathrm{kg}$ ) and one sample from Limpet Creek $(257 \mathrm{mg} / \mathrm{kg}$ ) exceeded both the ESV and background concentration.

Manganese was detected in samples above the ESV, but all were below the background concentrations. Beryllium was detected in one sample, but it was below the ESV. Cobalt, lithium, and vanadium were detected in samples below background concentrations; there were no ESVs found for these metals. Boron, calcium, cerium, magnesium, molybdenum, potassium, selenium, strontium, thallium, thorium, titanium, and uranium were detected in some or all of the samples. These were found in some samples at concentrations above background. However, no ESVs were found for these metals. 
Though several metals were found above ESV values and above background (i.e., aluminum, arsenic, barium, cadmium, chromium, copper, iron, nickel, and zinc), only chromium is associated with drilling muds. Therefore, chromium is retained as a COPEC in sediments for the ecological risk assessment.

\subsection{Problem Formulation and Preliminary Ecological Effects Evaluation}

Deep drilling operations on Amchitka Island required the use of large volumes of drilling muds. These muds were contained in mud pits, but historically had releases to the streams, lakes, and ponds on site. Contaminants associated with drilling muds (e.g., chromium, PAHs, BTEX compounds, and DROs) as well as PCBs may have impacted the surface water and sediments of these surface water bodies. Ecological receptors may be exposed to this contamination through direct contact, ingestion of surface water, incidental ingestion of sediments, and ingestion of prey and plants which have accumulated contaminants at high enough levels that they present a risk to the environment. Through the conceptual site model, sensitive environmental receptors were identified as well as potentially complete exposure pathways. Assessment end points were identified as the following:

- The potential for significant adverse effects on benthic invertebrate community abundance and diversity

- The potential for significant adverse effects on plant species abundance, diversity, and primary production

- The potential for significant adverse effects on freshwater fish abundance

- The potential for significant adverse effects on waterfowl abundance

- The potential for significant adverse effects on Bald Eagle abundance

Both sediment and surface water ecological screening values were identified in Section 5.1. Concentrations of contaminants in these media were compared to screening values. COPECs were identified through this screening that may have been related to site activities, are greater than background concentrations, and exceed ESVs, or are bioaccumulative and may have impacts through food chain transfer. These COPECs were DROs, PAHs, PCBs, several VOCs, and chromium. Since COPECs were identified, an ecological risk assessment is warranted. The remainder of this section of the Work Plan presents the methods to be used to conduct the ecological risk assessment. 


\subsection{Measurement End Points and Assessment Methods}

A measurement end point is a measurable ecological characteristic incorporating a measure of biological effects that can be associated with the identified assessment end points. In this section, the measurement end points for each of the identified ecological receptors/assessment end points are presented.

\subsubsection{Benthic Invertebrate Communities}

The assessment end point for this ecological risk assessment is the potential for significant adverse effects on benthic invertebrate community abundance and diversity. Evaluation of risks to the benthic invertebrate community will be based on a weight of evidence approach typically referred to as the sediment quality triad (Long and Chapman, 1985). This incorporates measurements of chemistry, toxicity, and biology. The measurement end points to be used are the following:

- Contaminant exposure concentrations in sediment exceeding toxicity values indicative of impacts

- Significant toxicity to benthic invertebrates in laboratory toxicity tests with whole sediment samples

- Significant adverse differences in benthic community indices (i.e., Family Biotic Index, Ephemeroptera-Plecoptera-Trichoptera (EPT) relative abundance, and Species Diversity)

The results of the laboratory toxicity tests and the benthic community analysis, as well as the chemical analyses for those samples, are presented as an appendix in the draft site characterization report (Shannon \& Wilson, 1999) and will be incorporated into the ecological risk assessment.

\subsubsection{Aquatic Plants}

The assessment end point for this ecological risk assessment is the potential for significant adverse effects on plant species abundance, diversity, and primary production. The dominant plant species providing habitat and a food source to wildlife and fish are aquatic mosses and water milfoils. The measurement end point for this assessment end point is contaminant exposure concentrations in excess of relevant surface water toxicity values. 
Relevant surface water toxicity values will be derived from literature sources including EPA's ambient water quality criteria documents and the AQUIRE database. When possible, toxicity values representing these families of aquatic plants will be used. Potential risk will be evaluated through the use of a HQ defined as the following:

$$
\mathrm{HQ}=\frac{\mathrm{EEC}}{\text { Benchmark }}
$$

where:

HQ $\quad=$ Hazard Quotient

EEC $\quad=$ Estimated environmental concentration

Benchmark $=$ Relevant toxicity value.

\subsubsection{Fish Population}

The assessment end point for this ecological risk assessment is the potential for significant adverse effects on freshwater fish abundance. The Landlocked Dolly Varden was selected as the indicator receptor since it is likely to have the greatest degree of exposure to site contaminants. However, effects on other salmonid species will be investigated regarding possible species-specific differences in sensitivity. The measurement end point is significant adverse effects on survival, growth, or reproduction as measured by the following methods: (1) a comparison of exposure concentrations to appropriate toxicity values, and (2) a comparison of tissue residue values with residue effect thresholds.

Relevant surface water toxicity values will be derived from literature sources including EPA's ambient water quality criteria documents and the AQUIRE database. Relevant residue effect thresholds will be obtained, where possible, from the open literature. When possible, toxicity values as closely representing this family of fish will be used. Potential risk will be evaluated through the use of a HQ defined as the following:

$$
\mathrm{HQ}=\frac{\text { EEC }}{\text { Benchmark }}
$$


and

$$
\mathrm{HQ}=\frac{\text { Tissue Concentration }}{\mathrm{RET}}
$$

where:

$\begin{array}{ll}\text { HQ } & =\text { Hazard Quotient } \\ \text { EEC } & =\text { Estimated environmental concentration } \\ \text { Benchmark } & =\text { Relevant toxicity value } \\ \text { RET } & =\text { Residue effect threshold. }\end{array}$

\subsubsection{Waterfowl}

The assessment end point for this ecological risk assessment is the potential for significant adverse effects on waterfowl abundance. The measurement end point is significant adverse effects on survival, reproduction, or growth of Green-Winged Teal or Aleutian Canada Goose. To assess this end point, the dose to these species for each of the COPECs will be modeled and compared to appropriate toxicity reference values.

Both the Green-Winged Teal and the Aleutian Canada Goose are assumed to be potentially exposed to contaminants at the ponds and lakes but not at the streams due to their small size and flow characteristics. The dose for each of these receptors will be represented by the cumulative dose from all appropriate exposure routes. For the Green-Winged Teal, these are ingestion of aquatic plants, ingestion of water column invertebrates, ingestion of surface water, incidental ingestion of sediment, ingestion of rooted aquatic plants, and ingestion of benthic invertebrates. For the Aleutian Canada Goose, the exposure routes are the same as that for the Green-Winged Teal except that the Aleutian Canada Goose is exclusively herbivorous and would not be exposed through ingestion of water column or benthic invertebrates.

Appropriate concentrations of contaminants in surface water and sediment have been measured; concentrations of contaminants in aquatic plants and invertebrates will be estimated based on surface water and sediment concentrations and appropriate media-to-biota transfer factors as found in the literature. 
Potential risk will be evaluated through the use of a HQ defined as the following:

$$
\mathrm{HQ}=\frac{\text { Dose }}{\text { Benchmark }}
$$

The benchmark values for wildlife (i.e., Green-Winged Teal, Aleutian Canada Goose, and Bald Eagle) are referred to as toxicity reference values (TRV) and these are expressed in terms of a contaminant dose to the organism. The TRVs focus on growth, survival, and reproduction of species. Generally, TRVs are not available for specific contaminant-receptor combinations for the ecological receptors identified in the ecological risk assessment. Thus, data on surrogate species is often used. The NOAEL is assumed to be appropriate for deriving wildlife TRVs for the initial stages of an ecological risk assessment since it provides the greatest degree of protection to the receptor species (ADEC, 2000a).

Toxicity information will be gathered from available sources, such as the Toxicological Benchmarks for Wildlife: 1996 Revision (Sample et al., 1996) and the open literature. Since the toxicity information usually available is provided for test species, which are not the same as the wildlife receptor species identified in the ecological risk assessment, the NOAELs for the test species need to be used to derive the wildlife TRVs for the receptor species. Studies have shown that sensitivity to toxic chemicals usually varies among different species of mammals as a function of body size. This is due to the fact that many physiological functions, such as metabolic rates, are related to body size such that smaller species have higher metabolic rates and are more resistant to toxic chemicals because of more rapid detoxification. However, research by Mineau et al. (1996) suggests that scaling factors, as developed for mammals to account for body size differences, are not appropriate for extrapolation between different species of birds. They found that for 29 of the 37 chemicals they investigated, the ratio of the NOAELs between species was not significantly different than one. Thus, the scaling factor for birds is assumed to be one (Sample et al., 1996). This means that the NOAEL for the wildlife bird receptor species is equal to the NOAEL for the test bird species (i.e., the body-weight normalized NOAEL dose are the same for birds regardless of body size).

Chronic NOAELs for the test species used to derive TRVs for the wildlife receptor species are not always available. Instead, test end points may be chronic or acute, or reported as LOAELs or even 
$\mathrm{LD}_{50} \mathrm{~s}$. In such cases, test species chronic NOAELs are conservatively derived from the reported test end points through the application of safety factors or uncertainty factors (UF). In deriving wildlife TRVs, the UFs will be the following (ADEC, 2000a):

- Chronic NOEL - UF = 1

- Chronic NOAEL - UF = 1-2

- Chronic LOEL - UF = 5

- Chronic LOAEL - UF = 5-10

- Subchronic NOEL - UF = 5

- Subchronic NOAEL - UF = 5-10

- Subchronic LOEL - UF $=25$

- Subchronic LOAEL - UF = 25-50

- Acute NOEL - UF = 20

- Acute NOAEL - UF = 20-40

- Acute LOEL - UF = 100

- Acute LOAEL - UF = 100-200

- Acute $\mathrm{LD}_{50}-\mathrm{UF}=250$

\subsubsection{Bald Eagle}

The assessment end point for this ecological risk assessment is the potential for significant adverse effects on Bald Eagle abundance. The measurement end point is significant adverse effects on survival, reproduction, or growth of the Bald Eagle. To assess this end point, the dose to the Bald Eagle for each of the COPECs will be modeled and compared to appropriate toxicity reference values.

The Bald Eagle consumes fish, birds, and mammals in its diet. Due to the small size of fish throughout most of the ponds, lakes, and streams on Amchitka Island, the Bald Eagle would only be expected to feed on fish from Cannikin Lake. However, they may feed on Green-Winged Teal or other small waterfowl from any of the ponds and lakes. Ingestion of surface water or incidental ingestion of sediment may also occur in any of the ponds and lakes, but is not likely in the streams since Bald Eagles are not expected to use the streams as feeding grounds. Therefore, the exposure concentrations from ponds and lakes will be used in dose modeling. The dose will be represented by the cumulative dose from all appropriate exposure routes.

Appropriate concentrations of contaminants in surface water and sediment have been measured; concentrations of contaminants in fish at Cannikin Lake have also been measured. Concentrations in 
waterfowl will be estimated based on surface water and sediment concentrations and appropriate media-to-biota transfer factors as found in the literature.

Potential risk will be evaluated through the use of a HQ defined as the following:

$$
\mathrm{HQ}=\frac{\text { Dose }}{\text { Benchmark }}
$$

\subsection{Uncertainty Analysis}

The uncertainty analysis will consist of a qualitative discussion of uncertainties involved in each step of the risk assessment process. Where possible, a qualitative description of the magnitude (e.g., high, medium, low) and direction (i.e., over-estimation, under-estimation) of the bias associated with the uncertainty will be specified. 


\subsection{Data Usability and Potential Data Gaps}

In this section, an evaluation of the available data is presented. In order to be used for risk assessment, the data must represent accurate, chemical-specific concentrations which will allow for an evaluation of risk. Though all the data have been validated and deemed usable, three issues require further discussion within this section:

- The absence of data for certain COPECs in certain surface water bodies

- Analytical detection limits that are greater than screening values

- Data flagged with a 'J' qualifier, particularly for PCB analysis exceeding holding times

\subsection{Data Gaps}

Table 6-1 presents a summary of the existing surface water, sediment, and fish tissue chemistry data, toxicity data, and benthic community analysis data for each of the ponds, lakes, and streams being assessed in the ecological risk assessment. The discussion below assesses what data is available and what is not, how the existing data will be used for the ecological risk assessment, and whether data gaps exist which must be filled in order to complete the ecological risk assessment. Table 6-2 presents a summary of the complete exposure routes for each ecological receptor for each of the ponds, lakes, and streams.

Benthic invertebrates will be assessed through the sediment quality triad approach which requires chemistry, toxicity, and biology data. Data for all three components are available for all streams. However, only chemistry data is available for Heart Lake, Reed Pond, Cannikin Lake, and Drill Site D Lake. The potential risk in the lakes and ponds will be evaluated through extrapolation of creek results to ponds and lakes based on chemical data. Relevant toxicity benchmark values for organic compounds are based on organic carbon normalization. Unfortunately, TOC analysis was not conducted for many of the ponds and lakes. For the ponds and lakes, a generic site TOC will be assumed as the average TOC of all existing pond, lake, and lowland stream (i.e., not fast flowing) TOC data. If individual samples in a pond, lake, or stream are missing TOC data, then an average TOC for that pond, lake, stream, or stream reach will be used.

The ingestion of benthic invertebrates is also an exposure route for Green-Winged Teal. The exposure concentration in benthic invertebrates will be estimated based on sediment concentrations 
Table 6-1

Summary of Available Data

(Page 1 of 3 )

\begin{tabular}{|c|c|c|c|c|c|c|c|c|c|}
\hline Site Description & $\begin{array}{l}\text { Sample } \\
\text { Type }\end{array}$ & $\begin{array}{c}\text { No. of } \\
\text { Samples }\end{array}$ & DRO & voc & PAHs & Metals & PCBs & TOC & $\%$ Lipids \\
\hline \multicolumn{10}{|c|}{ Milrow Sites } \\
\hline \multirow{2}{*}{$\begin{array}{l}\text { Milrow Pond } \\
\text { (Heart Lake) }\end{array}$} & Water & 3 & $\checkmark$ & $\checkmark$ & $\checkmark$ & $\checkmark$ & & & \\
\hline & Sediment & 5 & $\checkmark$ & $\checkmark$ & $\checkmark$ & $\checkmark$ & & & \\
\hline \multirow{7}{*}{ Clevenger Creek } & Water & 5 & $\checkmark$ & $\checkmark$ & $\checkmark$ & $\checkmark$ & & & \\
\hline & Sediment & $4+$ Dup & $\checkmark$ & & $\checkmark$ & $\checkmark$ & & $\checkmark$ & \\
\hline & Sediment & 1 & $\checkmark$ & $\checkmark$ & $\checkmark$ & $v$ & & $\checkmark$ & \\
\hline & Sediment & Dup & & $\checkmark$ & & & & & \\
\hline & Benthos & 3 & NA & NA & NA & NA & NA & & \\
\hline & Toxicity & 3 & NA & NA & NA & NA & NA & & \\
\hline & Fish & 3 & & & $\checkmark$ & $\boldsymbol{V}$ & $\checkmark$ & & (1) \\
\hline \multicolumn{10}{|c|}{ Long Shot Sites } \\
\hline \multirow{6}{*}{ Bridge Creek } & Water & 5 & $\checkmark$ & $\checkmark$ & $\checkmark$ & $v$ & & & \\
\hline & Sediment & 4 & $\checkmark$ & & $\checkmark$ & $\checkmark$ & & $\checkmark$ & \\
\hline & Sediment & 1 & $\checkmark$ & $\checkmark$ & $\checkmark$ & $\checkmark$ & & $\checkmark$ & \\
\hline & Benthos & 3 & NA & NA & NA & NA & NA & & \\
\hline & Toxicity & 3 & NA & NA & NA & NA & NA & & \\
\hline & Fish & 2 & & & $\checkmark$ & $\checkmark$ & $\checkmark$ & & \\
\hline \multirow{7}{*}{ Rainbow Creek } & Water & 5 & $v$ & 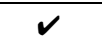 & $\checkmark$ & $\checkmark$ & & & \\
\hline & Sediment & 5 & $\boldsymbol{V}$ & $\mathrm{MeOH}$ & $\checkmark$ & $\checkmark$ & & $\checkmark$ & \\
\hline & Sediment & 2 & & & & & $\checkmark$ & & \\
\hline & Benthos & 3 & NA & NA & NA & NA & NA & & \\
\hline & Toxicity & 3 & NA & NA & NA & NA & NA & & \\
\hline & Fish & 1 & & & $\checkmark$ & $\checkmark$ & $\checkmark$ & & \\
\hline & Fish & 1 & & & & 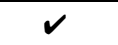 & & & \\
\hline \multirow{6}{*}{ Cloudberry Creek } & Water & 5 & $v$ & $\checkmark$ & $\checkmark$ & $\checkmark$ & & & \\
\hline & Sediment & 4 + Dup & $\checkmark$ & & $\checkmark$ & $\checkmark$ & & $\checkmark$ & \\
\hline & Sediment & 1 & $\checkmark$ & $v$ & $\checkmark$ & $\checkmark$ & & $\checkmark$ & \\
\hline & Benthos & 3 & NA & NA & NA & NA & NA & & \\
\hline & Toxicity & 3 & NA & NA & NA & NA & NA & & \\
\hline & Fish & 3 & & & $\checkmark$ & $\checkmark$ & $\checkmark$ & & \\
\hline \multirow{2}{*}{ Reed Pond } & Water & 2 & $\checkmark$ & $\checkmark$ & $\checkmark$ & $\checkmark$ & & & \\
\hline & Sediment & 2 & $\checkmark$ & $\checkmark$ & $\checkmark$ & $\checkmark$ & & & \\
\hline Long Shot Pond & Visual $^{a}$ & & & & & & & & \\
\hline
\end{tabular}


Table 6-1

Summary of Available Data

(Page 2 of 3 )

\begin{tabular}{|c|c|c|c|c|c|c|c|c|c|}
\hline Site Description & $\begin{array}{l}\text { Sample } \\
\text { Type }\end{array}$ & $\begin{array}{l}\text { No. of } \\
\text { Samples }\end{array}$ & DRO & voc & PAHs & Metals & PCBs & TOC & $\%$ Lipids \\
\hline \multicolumn{10}{|c|}{ Cannikin Site } \\
\hline \multirow{6}{*}{ White Alice Creek } & Water & 5 & $\checkmark$ & $\checkmark$ & $\checkmark$ & $\checkmark$ & & & \\
\hline & Sediment & 4 & $\checkmark$ & & $\checkmark$ & $\checkmark$ & $\checkmark$ & $\checkmark$ & \\
\hline & Sediment & 1 & $\checkmark$ & $\checkmark$ & $\checkmark$ & $\checkmark$ & $\checkmark$ & $\checkmark$ & \\
\hline & Benthos & 5 & NA & NA & NA & NA & NA & & \\
\hline & Toxicity & 5 & NA & NA & NA & NA & NA & & \\
\hline & Fish & $4+$ Dup & & & $\checkmark$ & $\checkmark$ & $\checkmark$ & & (1) \\
\hline \multirow{3}{*}{ Cannikin Lake } & Water & 3 & 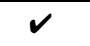 & $\checkmark$ & $\checkmark$ & 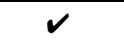 & $\checkmark$ & & \\
\hline & Sediment & 3 & $\checkmark$ & $\checkmark$ & $\checkmark$ & $\checkmark$ & $\checkmark$ & & \\
\hline & Fish & $1+$ Dup & & & $\checkmark$ & $\checkmark$ & 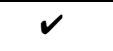 & & $\checkmark$ \\
\hline \multicolumn{10}{|c|}{ Drill Site D } \\
\hline \multirow{2}{*}{ Site D Lake } & Water & 3 & $v$ & $\checkmark$ & $\checkmark$ & $v$ & & & \\
\hline & Sediment & 5 & $\checkmark$ & $v$ & $\checkmark$ & $\checkmark$ & & & \\
\hline \multirow{7}{*}{ Falls Creek } & Water & 5 & $\checkmark$ & $\checkmark$ & $\checkmark$ & $\checkmark$ & & & \\
\hline & Sediment & 4 & $\boldsymbol{V}$ & $\mathrm{MeOH}$ & $\checkmark$ & $\checkmark$ & & $\checkmark$ & \\
\hline & Sediment & 1 & $\checkmark$ & & $\checkmark$ & $\checkmark$ & & $\checkmark$ & \\
\hline & Sediment & 3 & & & & & $\checkmark$ & & \\
\hline & Benthos & 3 & NA & NA & NA & NA & NA & & \\
\hline & Toxicity & 3 & NA & NA & NA & NA & NA & & \\
\hline & Fish & 2 & & & $\checkmark$ & $v$ & $\checkmark$ & & \\
\hline \multicolumn{10}{|c|}{ Drill Site F } \\
\hline \multirow{6}{*}{ Limpet Creek } & Water & 5 & $v$ & $\checkmark$ & $\checkmark$ & $v$ & & & \\
\hline & Sediment & 1 & $v$ & $\checkmark$ & $\checkmark$ & $\checkmark$ & & $\checkmark$ & \\
\hline & Sediment & 4 & $v$ & & $\checkmark$ & 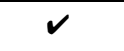 & & $\checkmark$ & \\
\hline & Benthos & 3 & NA & NA & NA & NA & NA & & \\
\hline & Toxicity & 3 & NA & NA & NA & NA & NA & & \\
\hline & Fish & 3 & & & $\checkmark$ & $\checkmark$ & $\checkmark$ & ‘ & (1) \\
\hline $\begin{array}{l}\text { Western Drainage } \\
\text { (lake and stream) }\end{array}$ & Visual $^{a}$ & & & & & & & & \\
\hline \multicolumn{10}{|c|}{ Drill Site E } \\
\hline \multirow{6}{*}{ Site E Stream } & Water & $5+$ Dup & $\checkmark$ & $v$ & $\checkmark$ & $\checkmark$ & & & \\
\hline & Sediment & 1 & $v$ & $\checkmark$ & $\checkmark$ & $\checkmark$ & & $\checkmark$ & \\
\hline & Sediment & 4 & $v$ & & $v$ & $\checkmark$ & & $v$ & \\
\hline & Benthos & 3 & NA & NA & NA & NA & NA & & \\
\hline & Toxicity & 3 & NA & NA & NA & NA & NA & & \\
\hline & Fish & 3 & & & $\checkmark$ & $\checkmark$ & $\checkmark$ & & \\
\hline Milepost 13 Lake & Visual $^{\mathrm{a}}$ & & & & & & & & \\
\hline
\end{tabular}


Table 6-1

Summary of Available Data

(Page 3 of 3 )

\begin{tabular}{|c|c|c|c|c|c|c|c|c|c|}
\hline Site Description & $\begin{array}{c}\text { Sample } \\
\text { Type }\end{array}$ & $\begin{array}{c}\text { No. of } \\
\text { Samples }\end{array}$ & DRO & VOC & PAHs & Metals & PCBs & TOC & \% Lipids \\
\hline Milepost 14 Lake & Visual $^{\mathrm{a}}$ & & & & & & & & \\
\hline \multirow{6}{*}{ Reference Stream 1} & Water & 5 & $\checkmark$ & & & $\checkmark$ & & & \\
\hline & Sediment & 1 & $\checkmark$ & & & 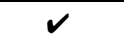 & $\checkmark$ & $\checkmark$ & \\
\hline & Sediment & 4 & $\checkmark$ & & & $\checkmark$ & & $\checkmark$ & \\
\hline & Benthos & 3 & NA & NA & NA & NA & NA & & \\
\hline & Toxicity & 3 & NA & NA & NA & NA & NA & & \\
\hline & Fish & 4 & & & $\checkmark$ & $\checkmark$ & $\checkmark$ & & \\
\hline \multirow{5}{*}{ Reference Stream 2} & Water & 5 & $v$ & & & $v$ & & & \\
\hline & Sediment & $5+$ Dup & $\checkmark$ & & & $\checkmark$ & & $\checkmark$ & \\
\hline & Benthos & 3 & NA & NA & NA & NA & NA & & \\
\hline & Toxicity & 3 & NA & NA & NA & NA & NA & & \\
\hline & Fish & 3 & & & $\checkmark$ & $\checkmark$ & $\checkmark$ & & \\
\hline \multirow{6}{*}{ Reference Stream 3} & Water & 5 & $\checkmark$ & & & $\checkmark$ & & & \\
\hline & Sediment & 2 & $\boldsymbol{v}$ & & & $\boldsymbol{v}$ & $\checkmark$ & $\checkmark$ & \\
\hline & Sediment & $3+$ Dup & $\checkmark$ & & & $\checkmark$ & & $\checkmark$ & \\
\hline & Benthos & 3 & NA & NA & NA & NA & NA & & \\
\hline & Toxicity & 3 & NA & NA & NA & NA & NA & & \\
\hline & Fish & $3+$ dup & & & $\checkmark$ & $\checkmark$ & $\checkmark$ & & \\
\hline \multirow{6}{*}{ Reference Stream 4} & Water & $5+$ Dup & $\checkmark$ & & & $\checkmark$ & & & \\
\hline & Sediment & 3 & $\checkmark$ & & & $\checkmark$ & $\checkmark$ & $\checkmark$ & \\
\hline & Sediment & 2 & $\checkmark$ & & & $\checkmark$ & & $\checkmark$ & \\
\hline & Benthos & 3 & NA & NA & NA & NA & NA & & \\
\hline & Toxicity & 3 & NA & NA & NA & NA & NA & & \\
\hline & Fish & 3 & & & $\checkmark$ & $\checkmark$ & $\checkmark$ & & (1) \\
\hline
\end{tabular}

${ }^{a}$ No drilling mud, diesel odors, or sheen were observed; no analytical samples were collected.

(1) Measurements of percent lipids reported for one sample only.

$\mathrm{MeOH}$ - Only the methanol extraction procedure was used for VOC analysis. Results not used in risk screening.

NA - Not analyzed 
Table 6-2

Complete Exposure Routes for Ecological Receptors

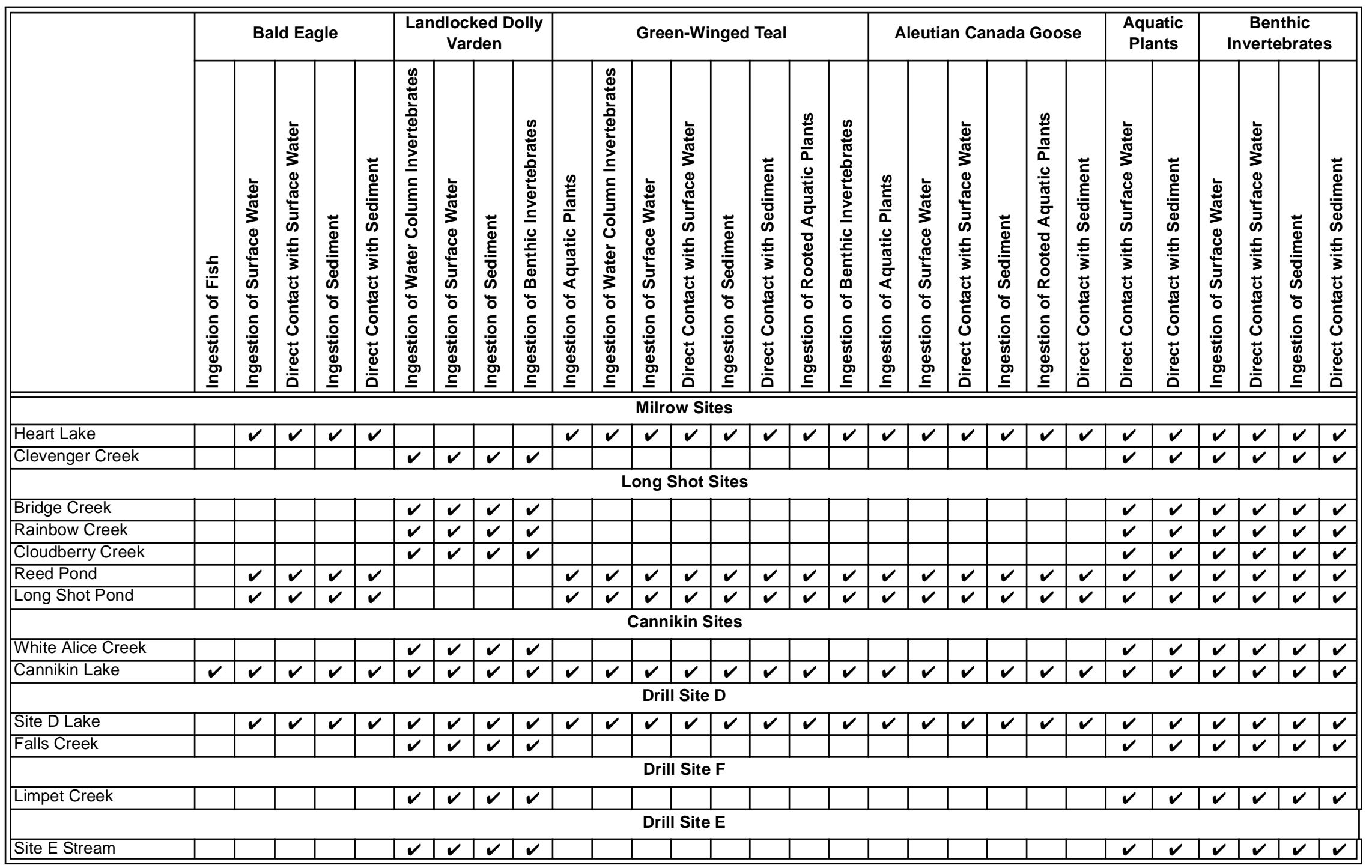


and media-to-biota transfer factors. The PCB sediment data are missing for most of the ponds and lakes where this exposure is applicable. The PCB sediment data are available only for Cannikin Lake, White Alice Creek, Rainbow Creek, and Falls Creek. For PCBs, the existing data will be assumed to conservatively represent the remaining sites. This assumption will be further addressed in the uncertainty analysis.

Risks to Landlocked Dolly Varden will be evaluated through comparisons of surface water concentrations and tissue residue concentrations, with appropriate benchmarks. This is applicable for all of the ponds, lakes, and streams. Though surface water data is available for all of the surface water bodies, tissue residue data is not available for all areas. For these areas, surface water or sediment data will be used with appropriate media-to-biota transfer factors to estimate fish tissue residue concentrations. Additionally, none of the fish tissue data includes analysis for VOCs or DRO. However, these latter COPECs are not considered to be bioaccumulative; therefore, they do not represent a significant data gap.

The ingestion of fish from Cannikin Lake is an exposure route for the Bald Eagle, and appropriate fish tissue data from Cannikin Lake exists. Additionally, surface water, sediment, and waterfowl ingestion at the other ponds and lakes are also exposure routes for the Bald Eagle. Chemistry data for surface water and sediment is available for these lakes and ponds, and media-to-biota transfer factors will be used to estimate waterfowl concentrations. The PCB data for surface water and sediment is only available for Cannikin Lake; the data for Cannikin Lake, along with that for the three aforementioned streams, will be used to represent Bald Eagle exposure in the other ponds and lakes.

Exposure routes for both Green-Winged Teal and Aleutian Canada Goose include ingestion of aquatic plants. Aquatic plant concentrations are to be estimated based on surface water or sediment concentrations and appropriate media-to-biota transfer factors. The PCB data, by which to estimate plant tissue concentrations, is missing for most of the water bodies. However, PCBs are not expected to bioaccumulate in vegetative tissues. Thus, this does not represent a significant data gap in the evaluation of PCB accumulation in water fowl.

No data exist for Milepost 13 Lake, Milepost 14 Lake, or the pond and stream which make up the western drainage of Drill Site F. These were examined visually and there was no evidence of contamination (e.g., oily sheens or drilling mud) or of a migration pathway through which to expect 
contamination. Thus, though these water bodies are not addressed in the risk assessments, they do not represent significant data gaps.

\subsection{Detection Limits Above Ecological Screening Values}

In Section 5.1, a comparison of media concentrations for surface water and sediment to appropriate ESVs was conducted. The compounds where detection limits were elevated above the ESVs were identified.

In surface water, detection limits for $\mathrm{DRO}, \mathrm{PCBs}$, boron, beryllium, and cerium were above the ESVs. Though DRO is retained as a COPEC, the ecological risk assessment will be conducted on individual chemicals (e.g., individual VOCs and PAHs) and the detection limits for these were lower than the ESVs. Thus, the detection limit for surface water DRO does not pose a problem. Though PCBs were not detected in surface water, the detection limit for PCBs is not sufficient for a direct evaluation of risk from surface water. However, both sediment and fish tissue PCB data does exist for several ponds, lakes, and streams by which an adequate evaluation of risk can be made. The lack of adequate surface water PCB data will be addressed in the uncertainty evaluation. Boron, beryllium, and cerium are not considered to be related to site-related activities and drilling muds. Therefore, the elevated detection limits do not represent a significant data gap.

In sediment, no COPECs were identified as having detection limits above the ESVs. However, since PCB ESVs and bioaccumulation potential are based on TOC data, there may be individual data points for which the individual detection limits are above the sample-specific ESVs. A review of the data has revealed that the PCB data for sediments was only reported if it was above the quantitation limit, and not if it was above the detection limit but below the quantitation limit. The PCB data for both fish and sediment are currently being evaluated, and data to be used in ecological risk assessment will be based on these lower detection limits.

\section{3 'J'-Qualified Data}

Both the laboratory and data validator may qualify data with a ' $\mathrm{J}$ ' indicating uncertainty in the reported concentration but not in its assigned identity. There are many reasons why data may be qualified as estimated (e.g., data detected below quantitation limit but above method detection limit). 
Per EPA guidance (EPA, 1989), 'J'-qualified data should be used in the quantitative risk assessment. Some of the PCB data is ' $\mathrm{J}$ '-flagged due to holding time exceedance. Due to the persistent nature of PCBs, this data was deemed usable during validation and will be included in the risk assessment. 


\subsection{References}

ADEC, see Alaska Department of Environmental Conservation.

AEC, see U.S. Atomic Energy Commission.

Alaska Department of Environmental Conservation. 1998. Technical Guidance Document on Determination of Background Concentrations. Juneau, AK: Division of Spill Prevention and Response, Contaminated Sites Remediation Program.

Alaska Department of Environmental Conservation. 1999a. 18 AAC 70, "Water Quality Standards as Amended through May 27, 1999." Anchorage, AK: Alaska Department of Environmental Conservation.

Alaska Department of Environmental Conservation. 1999b. 18 AAC 75, "Articles 3 and 9, Oil and Hazardous Substance Pollution Control Regulations," 18 AAC 75.325, Site Cleanup Rules: Purpose, Applicability, and General Provisions.

Alaska Department of Environmental Conservation. 1999c. 18 AAC 75, "Articles 3 and 9, Oil and Hazardous Substance Pollution Control Regulations," 18 AAC 75.341, Soil Cleanup Levels: Tables. Anchorage, AK: Alaska Department of Environmental Conservation.

Alaska Department of Environmental Conservation. 2000a. Risk Assessment Procedures Manual. Juneau, AK: Division of Spill Prevention and Response, Contaminated Sites Remediation Program.

Alaska Department of Environmental Conservation. 2000b. Guidance for Cleanup of Petroleum Contaminated Sites. Juneau, AK: Division of Spill Prevention and Response, Contaminated Sites Remediation Program.

AQUIRE. 2000. On-line database search for toxicity data performed on November 20, 2000 at www.epa.gov/ecotox.

DOE/NV, see U.S. Department of Energy, Nevada Operations Office.

EPA, see U.S. Environmental Protection Agency.

Fuller, R.G., and J.B. Kirkwood. 1977. "Ecological consequences of nuclear testing." In The Environment of Amchitka Island, Alaska, M.L. Merritt and R.G. Fuller (eds), ERDA Report TID-26712, pp. 627-650.

Gilbert, R.O. 1987. Statistical Methods for Environmental Pollution Monitoring. New York, NY: Van Nostrand Reinhold. 
Integrated Risk Information Services. 2000. On-line database maintained by EPA. Author, Add date accessed and URL address here.

Jones, D.S., G.W. Suter, II, and R. N. Hull. 1997. Toxicological Benchmarks for Screening Contaminants of Potential Concern for Effects on Sediment-Associated Biota: 1997 Revision. Oak Ridge, TN: Oak Ridge National Laboratory.

Long, E.R., and P.M. Chapman. 1985. "A sediment quality triad: measures of sediment contamination, toxicity and infaunal community composition in Puget Sound." In Marine Pollution Bulletin, Vol. 16, pp. 405-415.

MacDonald, D.D., S.L. Smith, M.P. Wong, and P. Mudroch. 1992. The Development of Canadian Marine Environmental Quality Guidelines. Ottawa, Ontario, Canada: Ecosystem Sciences and Evaluation Directorate, Eco-Health Branch.

Merritt, M.L. 1977. "History, 1741-1967." In The Environment of Amchitka Island, Alaska, M.L. Merritt and R.G. Fuller (eds), ERDA Report TID-26712, pp. 115-140.

Mineau, P., B.T. Collins, and A. Baril. 1996. "On the use of scaling factors to improve interspecies extrapolation of acute toxicity in birds." In Reg. Toxicology and Pharmacology.

New York State Department of Environmental Conservation. 2000. Ambient Water Quality Standards and Guidance Values Groundwater Effluent Limitations. Albany, NY: Division of Water.

Persaud, D., R. Jaagumagi, and A. Hayton. 1992. Guidelines for the Protection and Management of Aquatic Sediment Quality in Ontario. Toronto, Ontario, Canada: Ontario Ministry of the Environment, Water Resources Branch.

Sample, B.E., D.M. Opresko, and G.W. Suter, II. 1996. Toxicological Benchmarks for Wildlife: 1996 Revision. Oak Ridge, TN: Oak Ridge National Laboratory.

Shannon \& Wilson, Inc. 1999. Department of Energy 1998 Drill Site Characterization Report Amchitka Island, Alaska (in prepublication review). Prepared for the U.S. Department of Energy, Nevada Operations Office under contract to U.S. Army Corps of Engineers, Alaska District.

Suter, G.W., II, and C.L. Tsao. 1996. Toxicological Benchmarks for Screening Potential Contaminants of Concern for Effects on Aquatic Biota: 1996 Revision. Oak Ridge, TN: Oak Ridge National Laboratory.

Terres, J.K. 1991. The Audubon Society Encyclopedia of North American Birds, 1109 p. Avenel, NJ: Random House. 
U.S. Atomic Energy Commission. 1972. "Planning Directive: Demobilization, Restoration, Monitoring, Amchitka Island Test Area," Nevada Operations Office, NVO-107.

U.S. Environmental Protection Agency. 1989. Risk Assessment Guidance for Superfund, Volume I, Human Health Evaluation Manual, (Part A). Washington, DC: Office of Emergency and Remedial Response.

U.S. Environmental Protection Agency. 1991. Role of Baseline Risk Assessment in Superfund Remedy Selection Decisions, OSWER Directive 9355.0-30. Washington, DC: Office of Solid Waste and Emergency Response.

U.S. Environmental Protection Agency. 1992. Framework for Ecological Risk Assessment, EPA/630/R-92/001. Washington, DC: Risk Assessment Forum.

U.S. Environmental Protection Agency. 1996. Draft, EPA Region 10 Supplemental Risk Assessment Guidance for Superfund. Seattle, WA: Office of Environmental Assessment, Risk Evaluation Unit.

U.S. Environmental Protection Agency. 1997a. Health Effects Assessment Summary Tables, EPA/540/R-97/036. Washington, DC: Office of Solid Waste and Emergency Response.

U.S. Environmental Protection Agency. 1997b. EPA Region 10 Supplemental Ecological Risk Assessment Guidance for Superfund, EPA/910/R-97/005. Seattle, WA: Office of Environmental Assessment, Risk Evaluation Unit.

U.S. Environmental Protection Agency. 1997c. Ecological Risk Assessment Guidance for Superfund: Process for Designing and Conducting Ecological Risk Assessments, EPA/540/R-97/006. Edison, NJ: Office of Solid Waste and Emergency Response.

U.S. Environmental Protection Agency. 1998a. Risk Assessment Guidance for Superfund, Volume 1: Human Health Evaluation Manual, Supplemental Guidance, Dermal Risk Assessment, Interim Guidance. Washington, DC: Office of Emergency and Remedial Response.

U.S. Environmental Protection Agency. 1998b. EPA Proposed Guidelines for Ecological Risk Assessment, EPA/630/R-95/002F. Washington, DC: Risk Assessment Form.

U.S. Environmental Protection Agency. 1999. National Recommended Water Quality Criteria, EPA/822/Z-99/001. Washington, DC: Office of Water.

U.S. Environmental Protection Agency. 2000a. Draft, Equilibrium Partitioning Sediment Guidelines (ESGs) for the Protection of Benthic Organisms: PAH Mixtures. Washington, DC: Office of Science and Technology.

U.S. Environmental Protection Agency. 2000b. Region III Risk-Based Concentration Table. Accessed December, 2000. http://www.epa/gov/reg3hwmd/risk/rbc-1000.pdf 
U.S. Geological Survey. 1975. “Amchitka Island Quadrangle Map.” In The Environment of Amchitka Island, Alaska. Edited by M.L. Merritt and R.G. Fuller in 1977. Washington, DC: Energy Research and Development Administration.

Valdez, R.A., W.T. Helm, and J.M. Neubold. 1977. "Aquatic Ecology." In The Environment of Amchitka Island, Alaska, M.L. Merritt and R.G. Fuller (eds), ERDA Report TID-26712, pp. 287-314.

White, C.M., B. Emison, and F.S.L. Williamson. 1971. "Dynamics of Raptor Populations on Amchitka Island." In BioScience, Vol. 21, pp. 623-627.

White, C.M., F.S.L. Williamson, and W.B. Emison. 1977. "Avifaunal Investigations.” In The Environment of Amchitka Island, Alaska, M.L. Merritt and R.G. Fuller (eds), IERDA Report TID-26712, pp 227-260. 


\section{Appendix A}

\section{Site and Sample Location Maps}




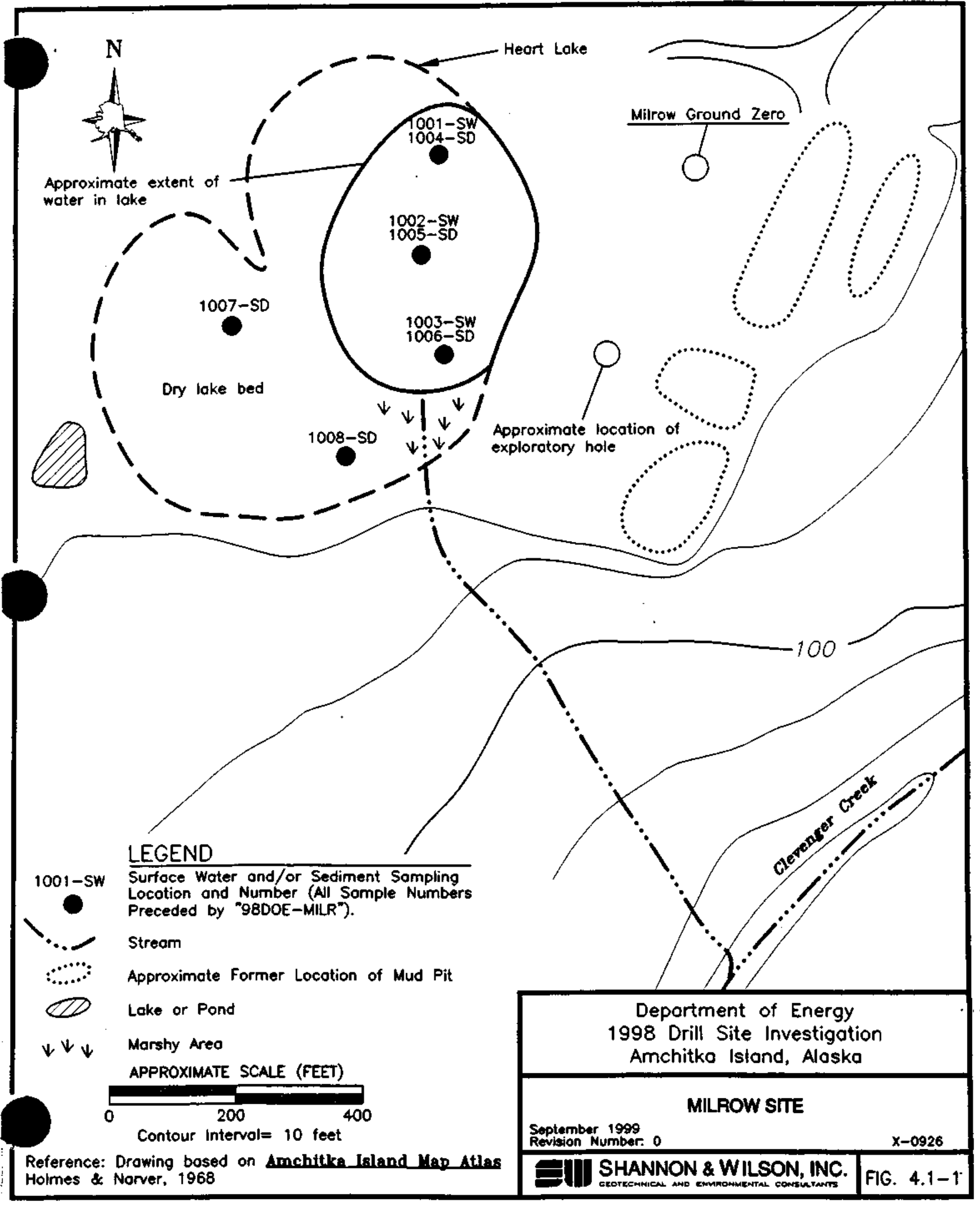




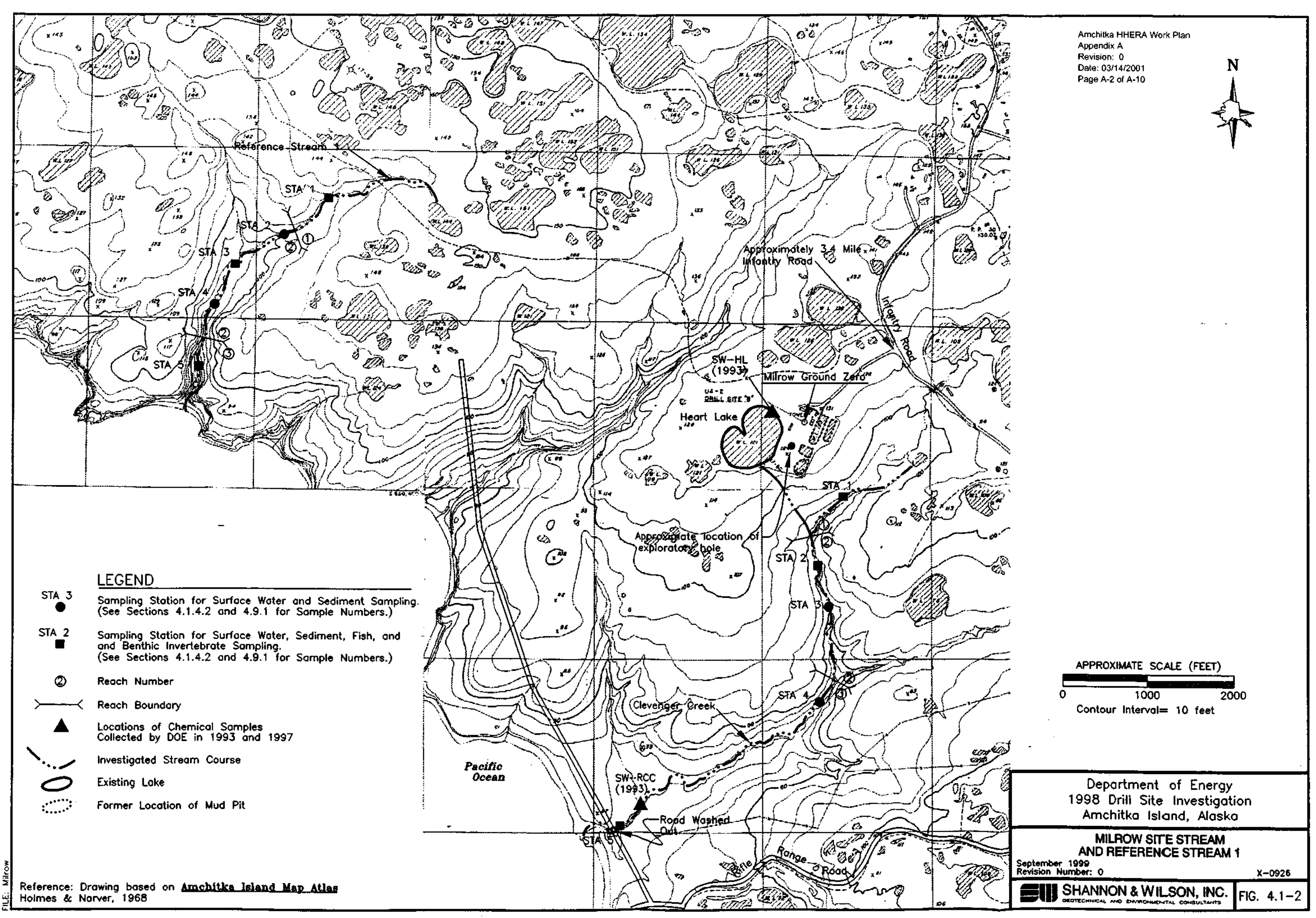


Page A-3 of A-10

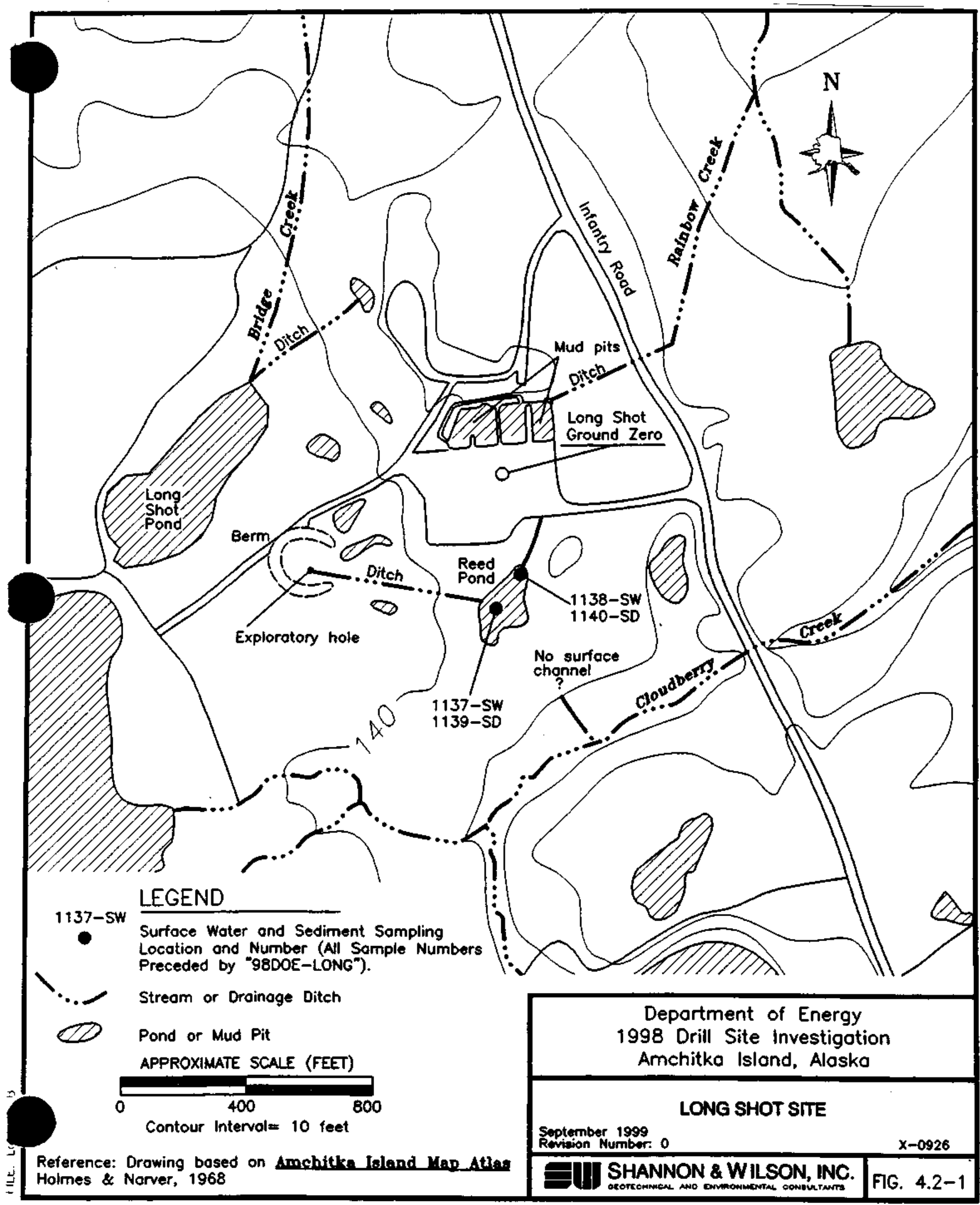




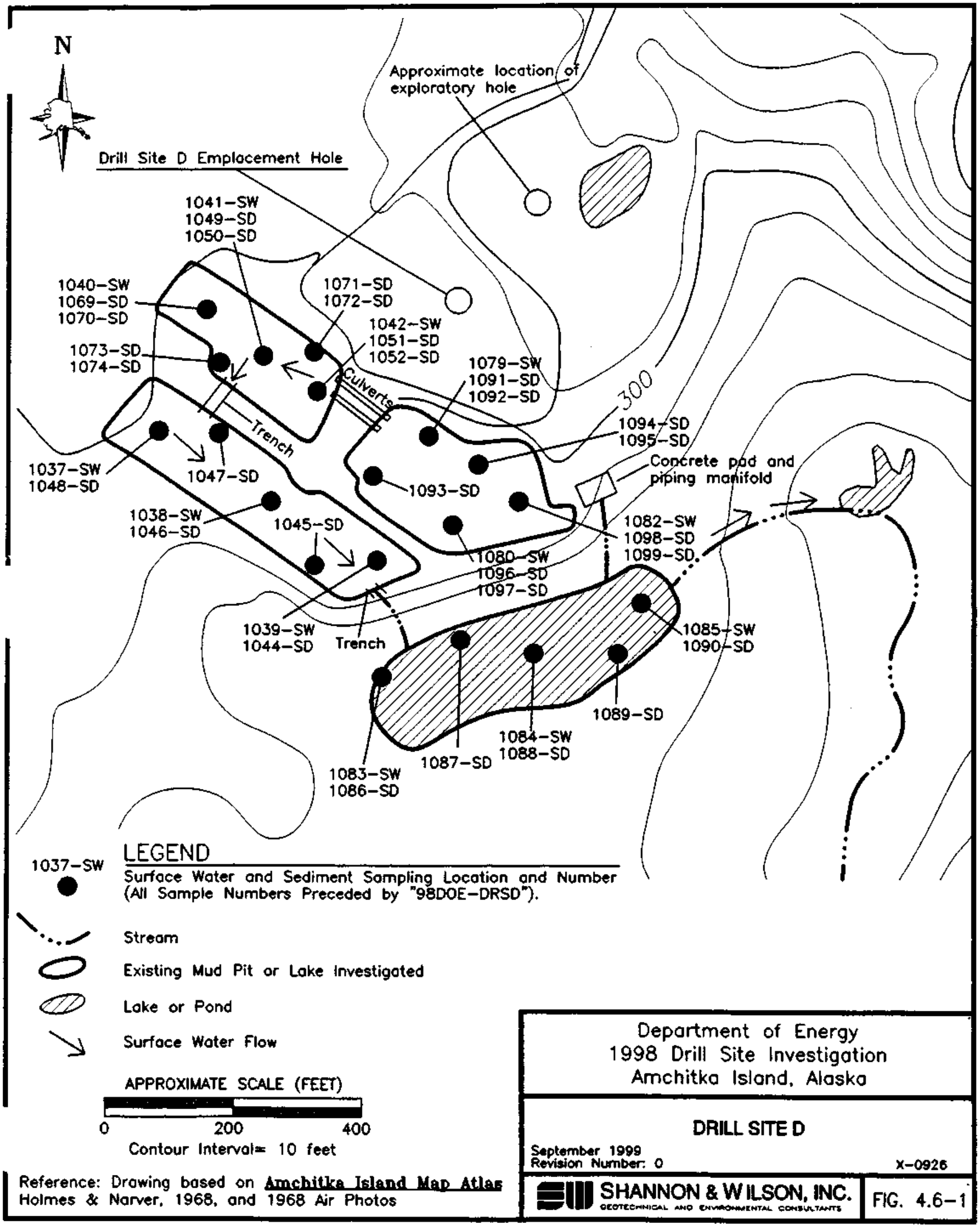




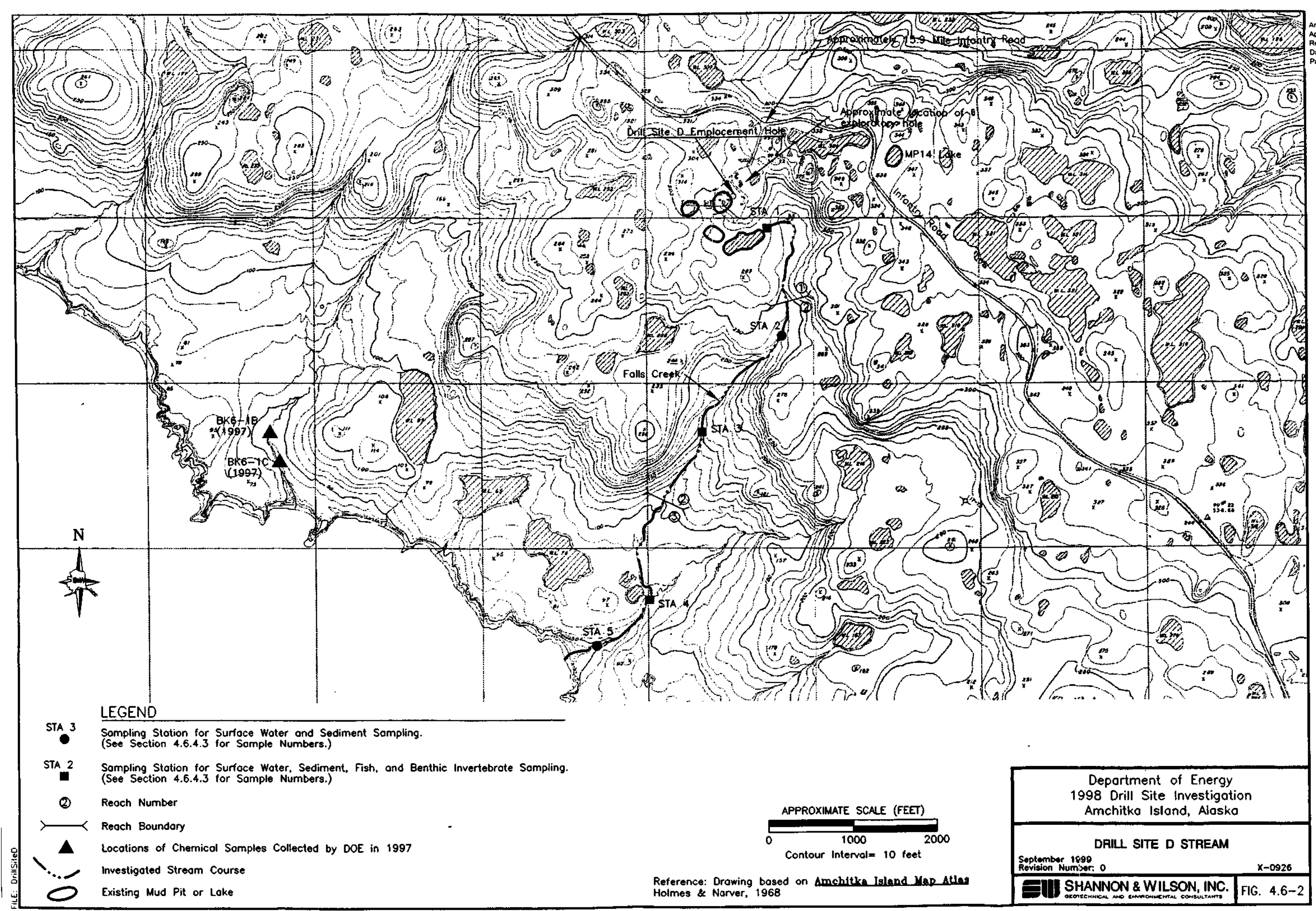




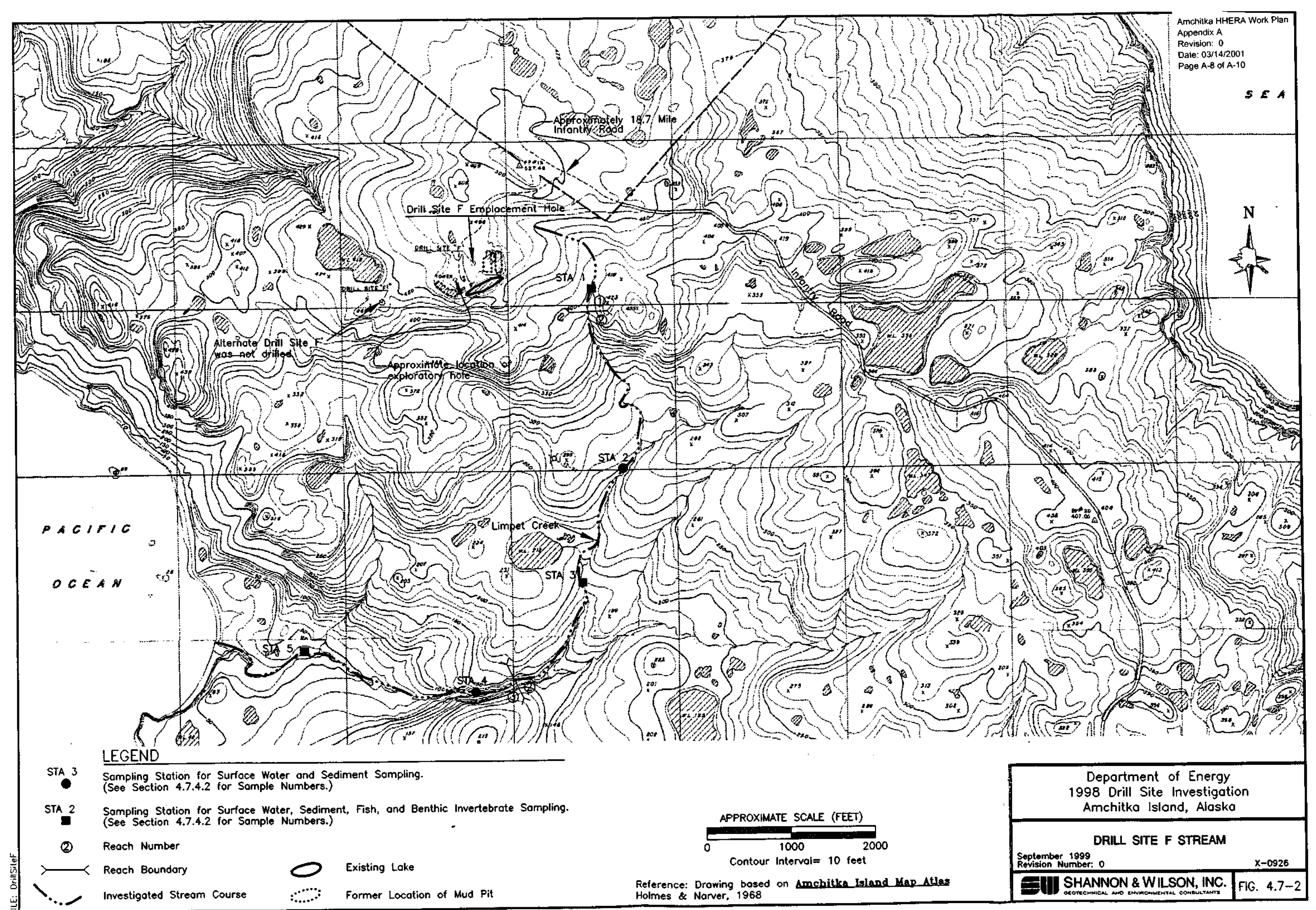




\section{Distribution}

\section{$\underline{\text { Copies }}$}

Sabrina D. Lawrence

1 (Controlled)

Environmental Restoration Division

DOE/Nevada Operations Office

P.O. Box 98518, M/S 505

Las Vegas, NV 89193-8518

Monica Sanchez

Environmental Restoration Division

1 (Controlled)

DOE/Nevada Operations Office

P.O. Box 98518, M/S 505

Las Vegas, NV 89193-8518

Michael Giblin

1 (Controlled)

Environmental Restoration Division

DOE/Nevada Operations Office

P.O. Box 98518, M/S 505

Las Vegas, NV 89193-8518

Stephanie Pingree

2 (Controlled)

Risk Assessment Division

Alaska Department of Environmental Conservation

410 Willoughby Ave, Suite 105

Juneau, AK 99801-1795

Michael Murray

1 (Controlled)

IT Corporation

2200 Cottontail Lane

Somerset, NJ 08873

David Stahl, P.E.

1 (Controlled)

IT Corporation

2 (Uncontrolled)

P.O. Box 93838

Las Vegas, NV 89193-3838

U.S. Department of Energy

1 (Uncontrolled, electronic copy)

Office of Scientific and Technical Information

P.O. Box 62

Oak Ridge, TN 37831 
DOE/Nevada Operation Office

Public Reading Facility

P.O. Box 98521

Las Vegas, NV 89193-8521

Technical Information Resource Center

DOE/Nevada Operations Office

P.O. Box 98518, M/S 505

Las Vegas, NV 89193-85181

Central Files

IT Corporation

P.O. Box 93838

Las Vegas, NV 89193-3838
1 (Uncontrolled)

1 (Uncontrolled)

3 (Uncontrolled) 